2. To: (Receiving Organization)

Remote System and Sensor Applications

5. Proj./Prog./Dept./Div.:

Sensor/Analytical Technology

Projects

8. Originator Remarks:

Documents contain 1971-1974 data record inventory logs for

BY-Tank Farm.

11. Receiver Remarks:

3. From: (Jriginating Organization)

Remote System and Sensor Applications

6. Cog. Engr.:

F. R. Reich $89800 / N 2183$
4. Related EOT NO.:

N/A

7. Purchase Order No.:

N/A

9. Equip./Component No.:

N/A

10. Systen/8ldg./Facility:

$N / A$

12. Major Assm. Dwg. No.:

N/A

13. Permit/Permit Application No.: N/A

14. Required Response Date: N/A

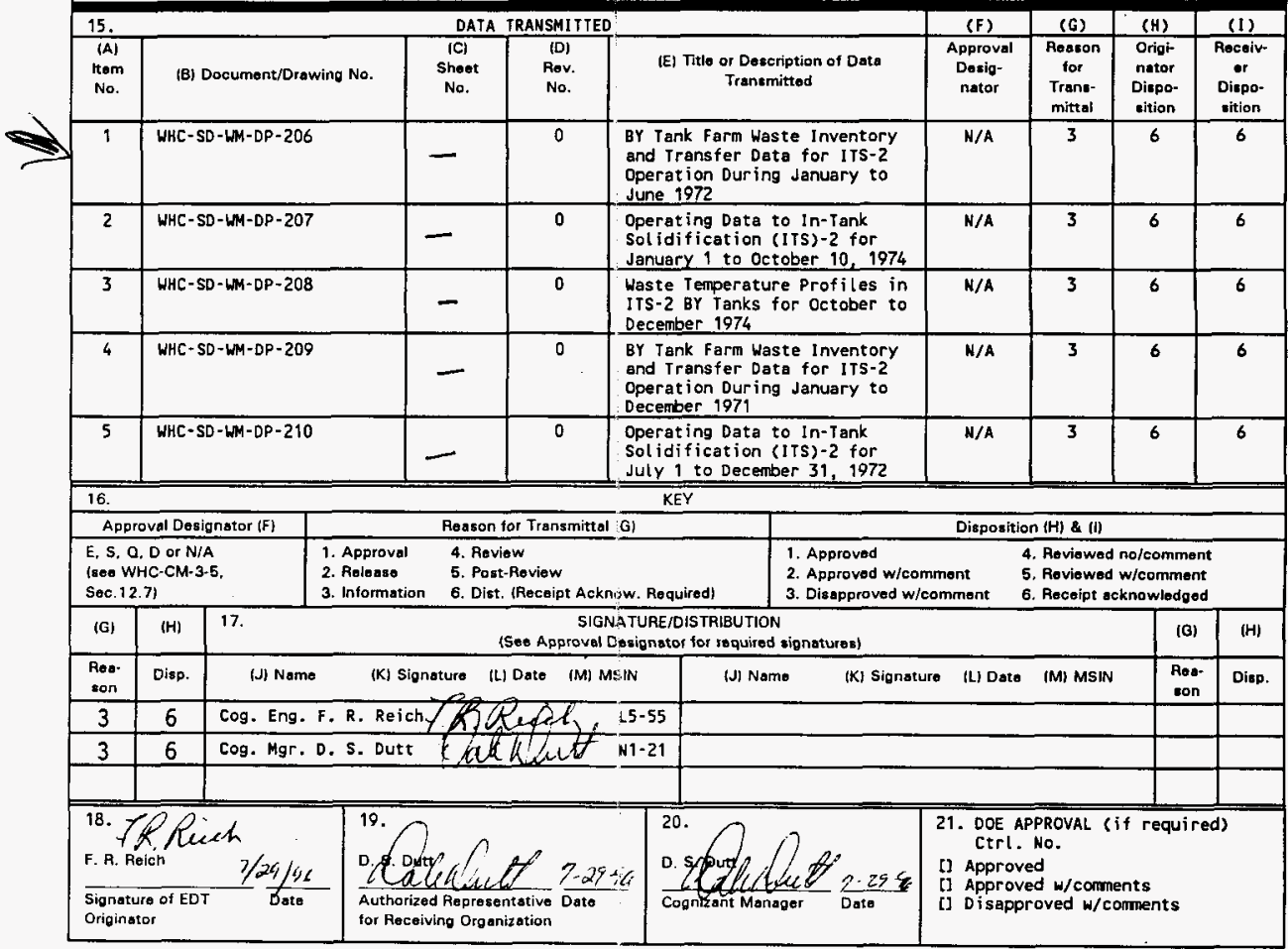

80-7400-172-2(04/94) GEF097 


\title{
BY Tank Farm Waste Inventory and Transfer Data for ITS-2 Operation During January to June 1972
}

\author{
F. R. Reich \\ Westinghouse Hanford Company, Richland, WA 99352 \\ U.S. Department of Energy Contract DE-AC06-87RL10930

$\begin{array}{llll}\text { EDT/ECN: } & 617410 & \text { Un: } 721 \\ \text { Org Code: } & 8 A 800 & \text { Charge Code: } & \text { N2183 } \\ \text { B\&R Code: } & \text { EW4010000 } & \text { Total Pages: } & 187\end{array}$

Key Words: BY Tanks, Pump Activity, Pump Inventory, Daily Record Inventory, Waste Inventory, Transfer Data, ITS-2 Operation

Abstract: Daily record inventory of pumping activities and liquid level changes including occasional operations comments for the BY Tank Farm. Waste inventory and transfar data for ITS-2 operation during January to June 1972.

\section{BEST AMALLABE COPY}

TRADEMARK DISCLAIMER. Reference herein to any specific commercial product, process, or service by trade name, trademark, manufacturer, or otherwise, does not necessarily constitute or imoly its endorsenent, recommendation, or favoring by the United states Government or any agency thereof or its contractors or subcontractors.

Printed in the United States of America. To obtain copies of this document, contact: WHC/Bcs Document Control Services, P.0. Box 1970, Mailstop H6-08, Richland WA 99352, Phone (509) 372-2420: Fax (509) 376-4989.
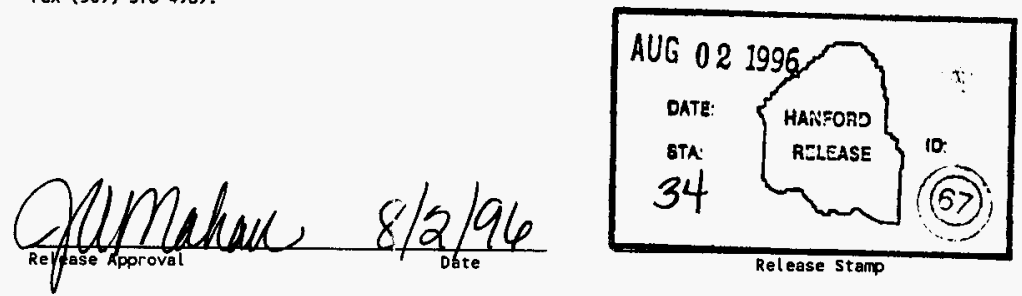

Approved for Public Release 
Document Date: None given

Document Number: None given

RHA BOX Number: 58107

File Number: None given

Title:

ITS-2 INVENTORY AND PUMPING

Description:

150 PAGE BROWN FOLDER CONTAINING COMPLETED DATA SHEETS AND SIX YELLOW FILE DIVIDERS.

\section{Comments:}

DAILY RECORO INVENTORY OF PUMPING ACTIVITIES AND LIQUID LEVEL CHANGES INCLUDES OCCASIONAL OPERATIONS COMMENT (I.E. FLUSHED, CAN NOT READ, CONTINUED, RECYCLED, ROUTED TO ..., AND PUMP ACTIVITY COMMENTS)

Addressee, Company:

Author:

Company:

Tank \#:

241-BX-103

241-BX-110

$241-B X-111$

$241-B Y-102$

241-BY 103

24I-EY-104

$241-B Y-105$

$241-B Y-106$

241-BY-107

$241-B Y-108$

$241-B Y-109$

$241-B Y-110$

$241-B Y-112$

Keywords:

PUMP ACTIVITY

PUMP INVENTORY

References: $N$

\# References: None given

Action Required: $N$

Topic: None given

Action Taken: None given

User: RM SCHWARZ

User Date: : 02-04-92 09:20:46

Reviewer: STEVENS MW

Review Date: 04-15-91

Checked By: None given

Checked Date: None given

WTS Key: 139

Document Type: DS 


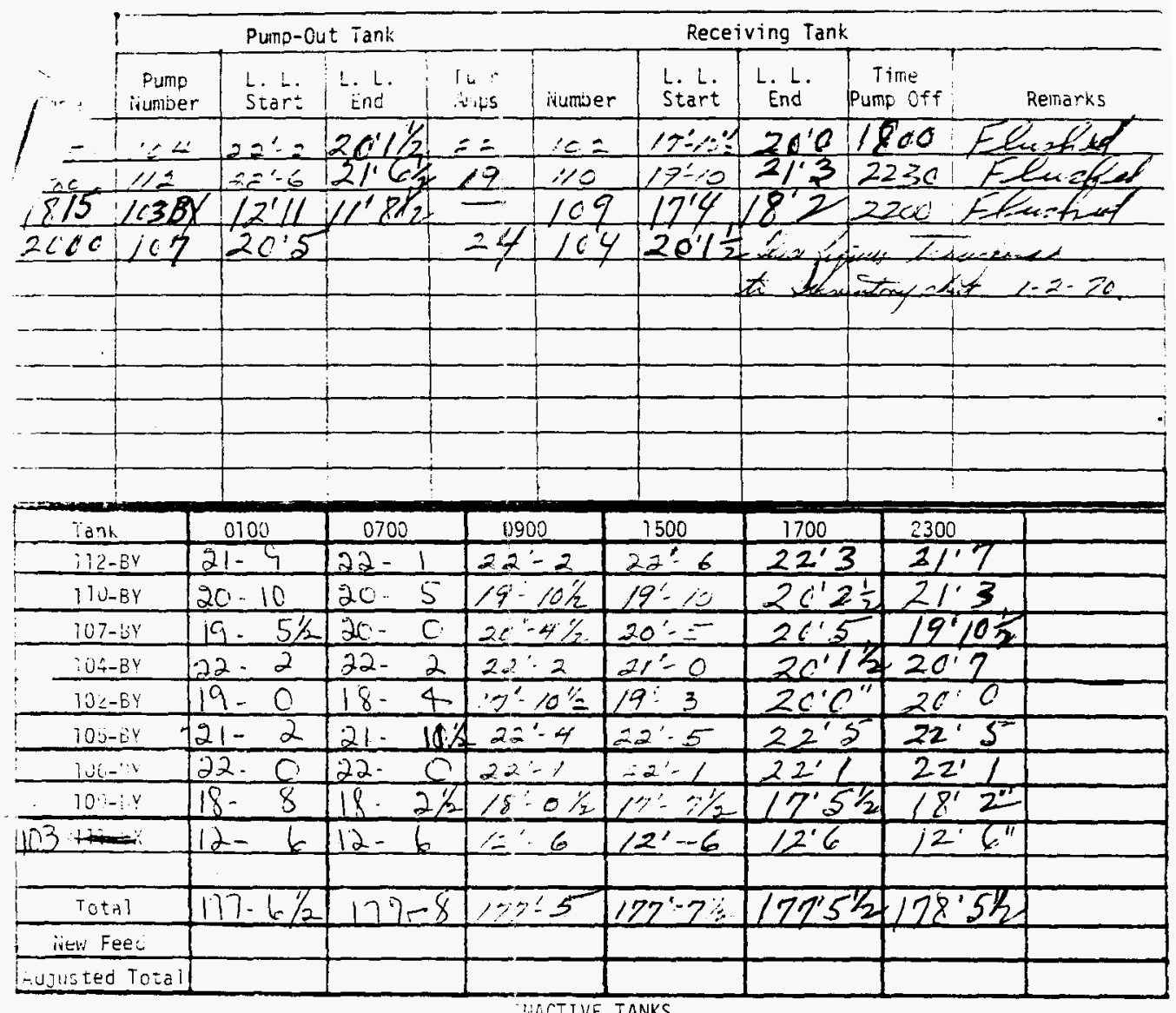
WACTIVE TANKS

\begin{tabular}{|l|l|l|l|l|l|}
\hline $108-B y$ & & & & \\
\hline
\end{tabular}




\section{$-\quad$ WHC-SD-WM-DP-206}

iTs-2 IIIVENTORY rill PUMPIHG Late $1.2-70^{\text {ReDO }}$

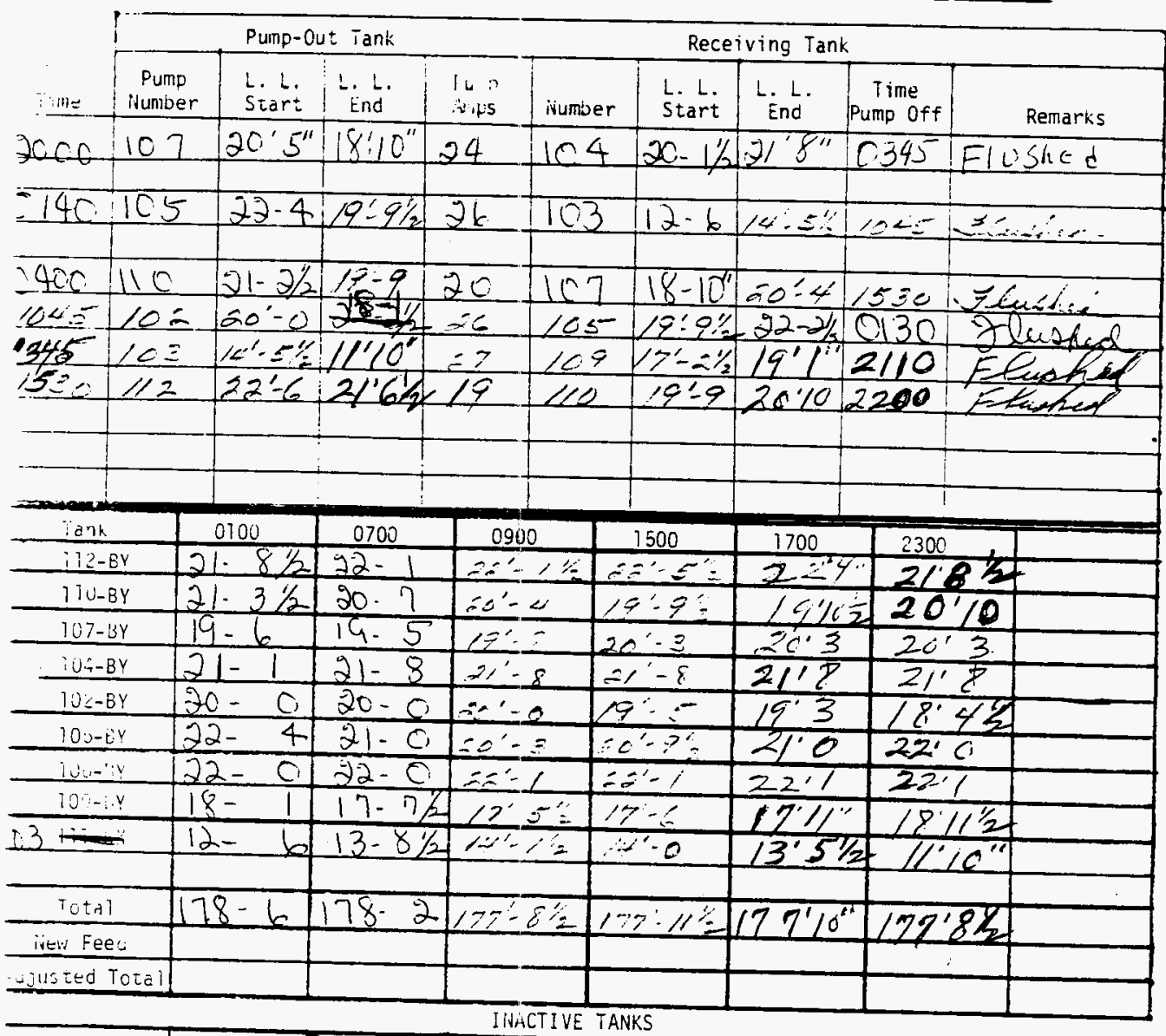

\begin{tabular}{r|}
\hline $168-13 y$ \\
\hline \\
\hline
\end{tabular}

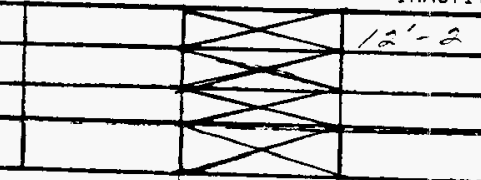

Heat iracus

0700
$22-$
20
$19-$
$21-$
$20-$
$21-$
$22-$
$17-$
$13-$
18.

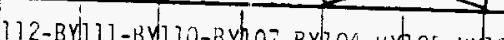

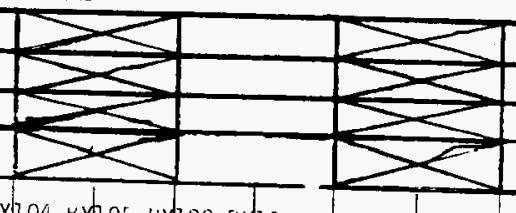

(Lidy Sitift)

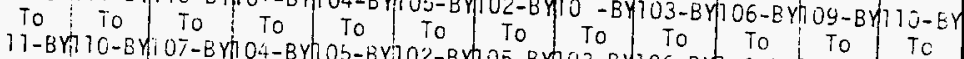

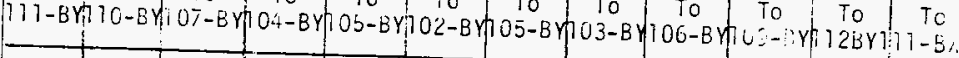




\begin{tabular}{|c|c|c|c|c|c|c|c|c|}
\hline \multirow[b]{2}{*}{$\vdots$ 픈 } & \multicolumn{3}{|c|}{ Puinp-Out Tank } & \multicolumn{4}{|c|}{ Receiving Tank } & \multirow[b]{2}{*}{ Remarks } \\
\hline & $\begin{array}{l}\text { Pump } \\
\text { Humber }\end{array}$ & $\begin{array}{l:l}\text { L. L. } & \text { L. L. } \\
\text { Start } & \text { End }\end{array}$ & $\begin{array}{l}\text { Iin } \\
\text { inits } \\
\end{array}$ & Number & $\begin{array}{l}\text { L. L. } \\
\text { Start }\end{array}$ & L. L. & $\begin{array}{l}\text { Time } \\
\text { Pump off }\end{array}$ & \\
\hline 0130 & $10: 5$ & $22-2 / 219^{\prime} 6^{\prime \prime}$ & 27 & 10.3 & $11-10$ & $1310^{\prime \prime}$ & 0045 & Elusine: \\
\hline$=3$ & 15.4 & $\rightarrow 1-8,100^{\circ}<2^{\prime \prime \prime}$ & 26 & $15: 2$ & $13-1$ & $19^{\prime} 8^{\prime \prime}$ & $0 \operatorname{ctcos} 30$ & ENOHED \\
\hline $2: 5$ & $10 \%$ & $20^{\prime} 32^{\prime}, 59$ & $2-$ & $12=$ & 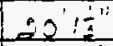 & $21^{\prime}<2$ & $2610^{-}$ & $76=25$ \\
\hline 1320 & 103 & $13^{\prime} 10^{\prime \prime}<2^{\prime} 9$ & 26 & 109 & $12^{\prime} \mu^{\prime \prime}$ & $18^{\prime} 7$ & 1620 & $\Rightarrow m+1-1=$ \\
\hline 132 & 112 & $22^{\prime} 6^{\prime \prime}+2,9$ & 18 & 110 & $20^{\prime} 9^{\prime \prime}$ & $22 \div \frac{1}{2}$ & $2.36 i$ & 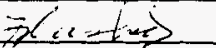 \\
\hline 163 & 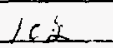 & $19-8+m=0$ & $t+$ & 164 & $19+8$ & & & \\
\hline 326 & $103.8 X$ & $1191107 \%$ & & $109 p_{i 1}$ & $18^{\prime} \cdot 5$ & $\angle 9 \geqslant 2$ & $+220 \mathrm{~T}$ & $a x=-1$ \\
\hline$\therefore 312$ & 110 & $2.2^{\prime} i^{2}=1$ & & 67 & $18 \cdot 9$ & TrAas & Jen & Data 1.4-72 \\
\hline & & & & & & & & \\
\hline & & & & & & & & \\
\hline & & & & & & & & \\
\hline
\end{tabular}

\begin{tabular}{|c|c|c|c|c|c|c|c|}
\hline ans & 0100 & 0700 & 0900 & 1500 & 1700 & 2300 & \\
\hline i12-BY & $21-10$ & $22-1 / 2$ & $2+1$ & $\operatorname{san}^{1} 4$ & $22^{1} 3$ & $21^{\prime} 9$ & \\
\hline $1 T U-B Y$ & $20-9 / 2$ & $2 x-9 / 2$ & $0,0^{\prime}$ & $10^{\prime} 11$ & $11^{\prime} 01$ & $22^{\prime}<2$ & \\
\hline $107-3 Y$ & $50-3 / 2$ & $20-3 / 2$ & $\therefore$ & $19^{\prime} \quad 10$ & $6 !$ & $15^{\circ} 9$ & \\
\hline$O A-B Y$ & $21 \cdot 8$ & $20-6 / 2$ & $0.2^{\prime}$ & $20^{\prime} \quad 6$ & 209 & $21^{\prime} 6 \frac{1}{2}=$ & \\
\hline $10 \div-B y$ & $18-1$ & $19-6$ & $19^{\prime \prime} 8^{\prime \prime}$ & $15^{\prime} 8$ & $\angle G^{\prime} \quad \bar{Y}$ & 618 & \\
\hline TOD-EY & $22-21$ & $20-6$ & $0^{\prime \prime} \sigma^{\prime \prime}$ & $19^{1} 8$ & $\angle 9^{\prime} T$ & $\angle 9^{\prime} \delta$ & \\
\hline$i v_{0}^{\prime}-: y$ & $22-1$ & $\frac{22}{25}-1$ & 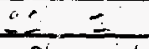 & $\therefore$ & $2 a \quad 0$ & $22^{\prime} 1$ & \\
\hline $10-y$ & $18-9$ & $18-4$ & $\varepsilon^{\prime} \div$ & 18 & 11 & $19^{\prime} 2$ & \\
\hline $103+i=$ & $11-10$ & $13-2 / 2$ & $22=$ & $1 \frac{1}{3}$ & $12^{\circ} 9$ & $12^{\prime} 9$ & \\
\hline Total & $177-6 / 2$ & $77-4 / 2$ & $176^{\prime} 82^{\prime}$ & $176^{\prime} 62^{1}$ & $126^{\circ} 3$ & $122^{\circ} 4$ & \\
\hline ivew Feec & & & & & & & \\
\hline ijusted & & & & & & & \\
\hline
\end{tabular}

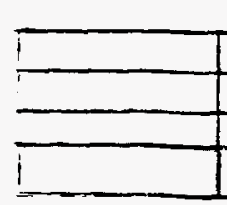

4心at Traces

(jay Siifi)

\section{IACT IVE TANKS}

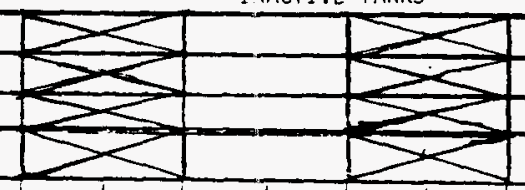

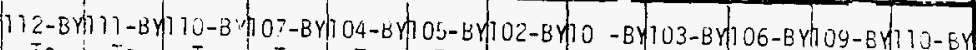
To To To To To To To to To To

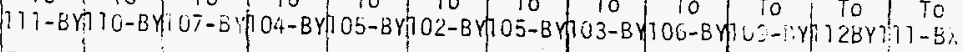




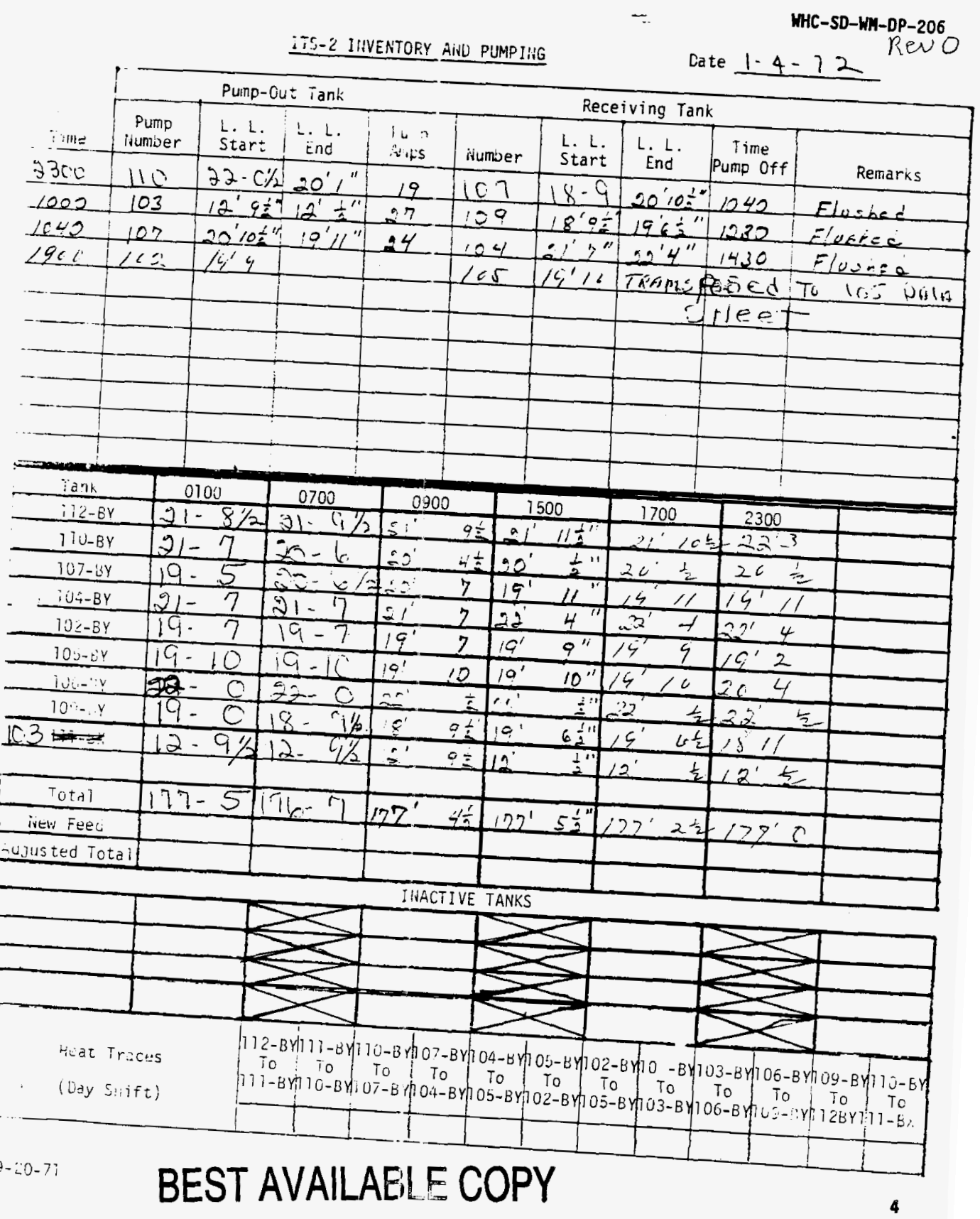




\section{WHC-SD-WH-DP-206 \\ ITS-2 IIIVENTORY AIND PUMPIIIG \\ Cate $1.5-71$ RevO}

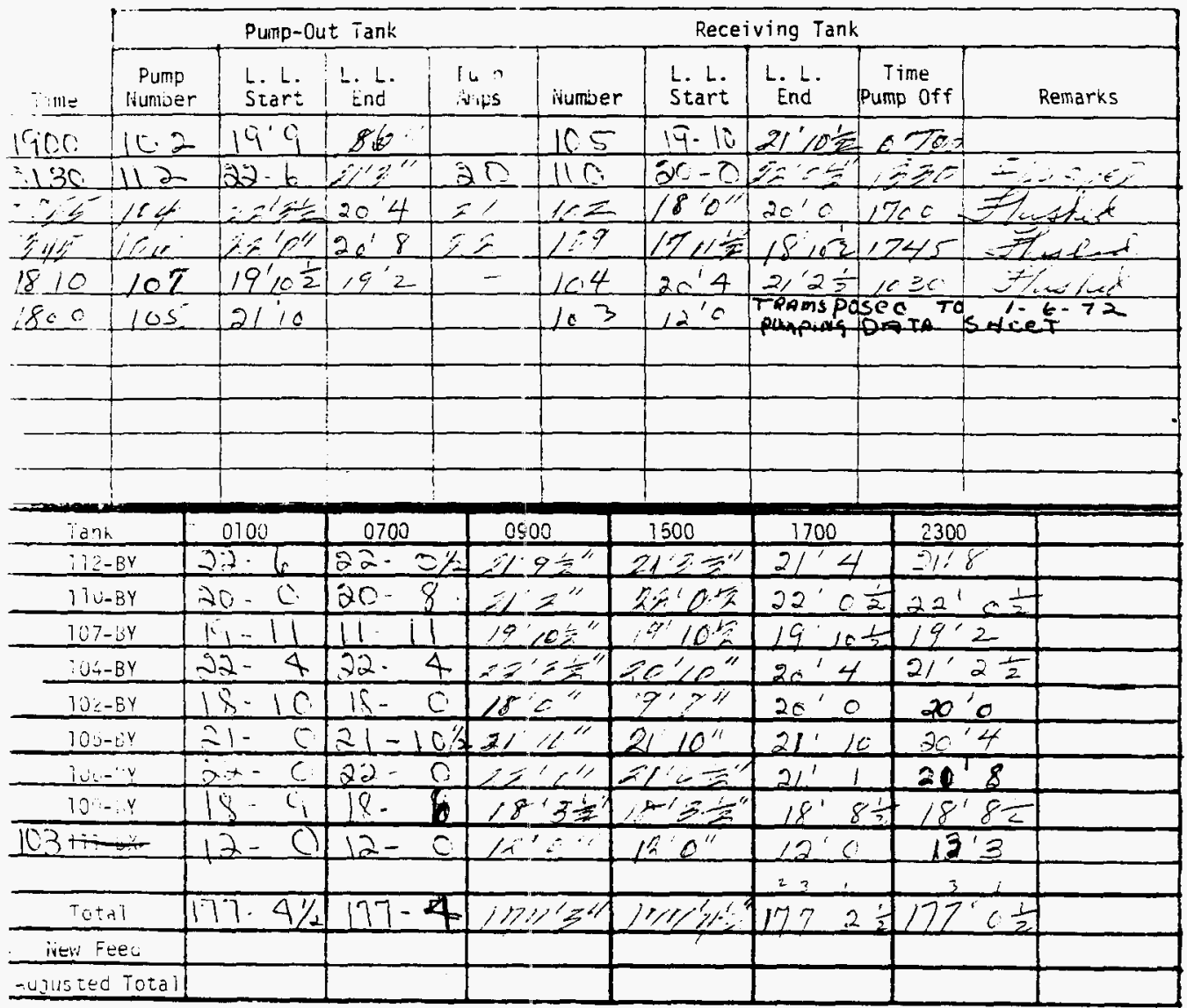

IHACTIVE TANKS

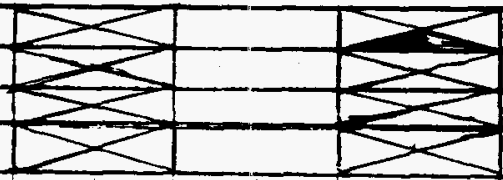

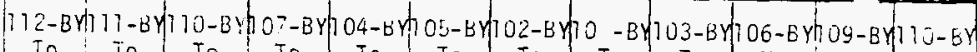

Hat Traces

To To To $_{0}$ To To $_{0}$ To

(Day $5:$ if $\tau$ )

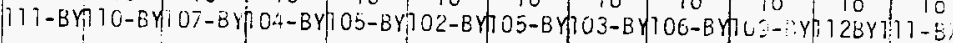


Cate $1-6.72$

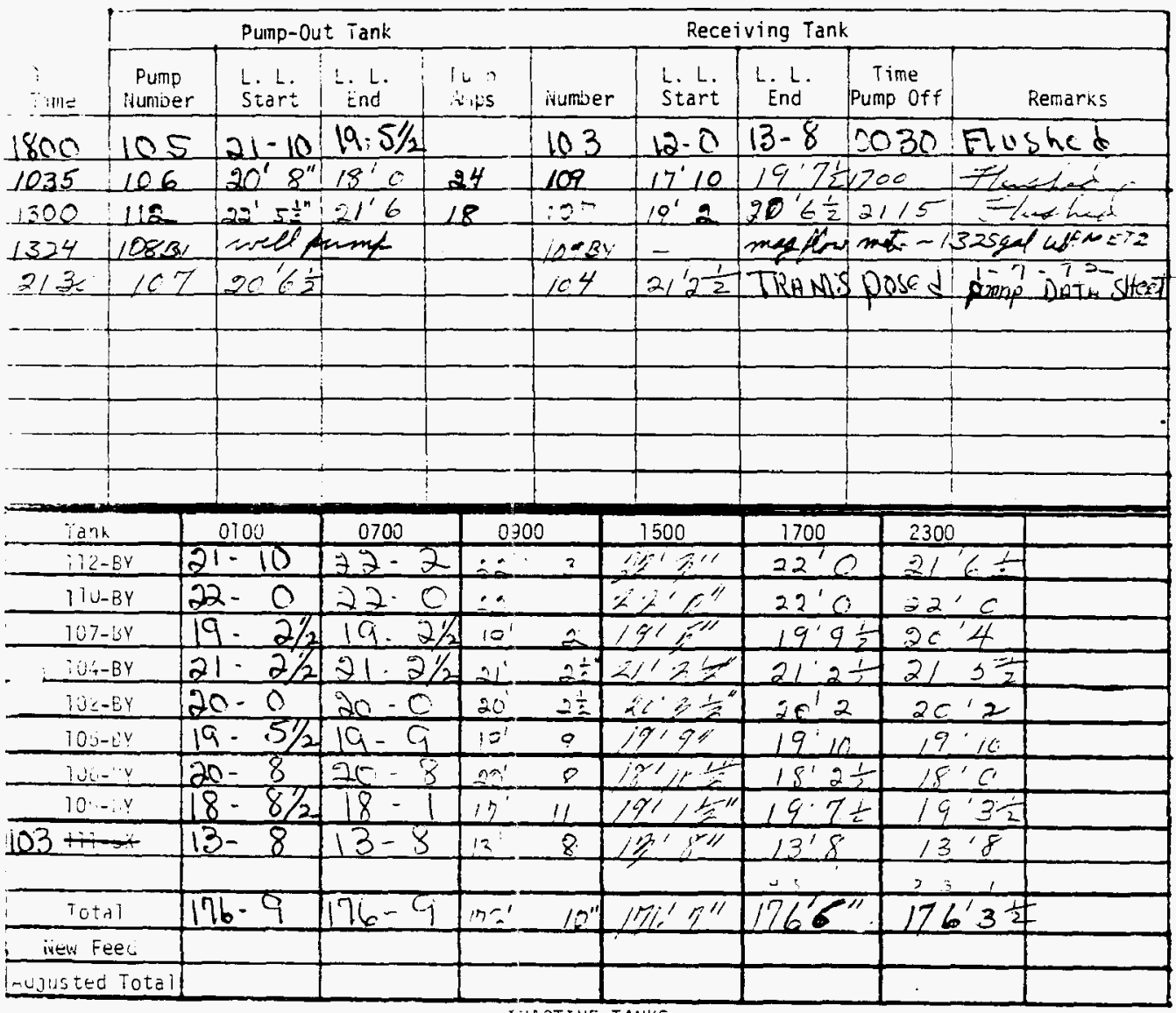

INACTIVE TANKS

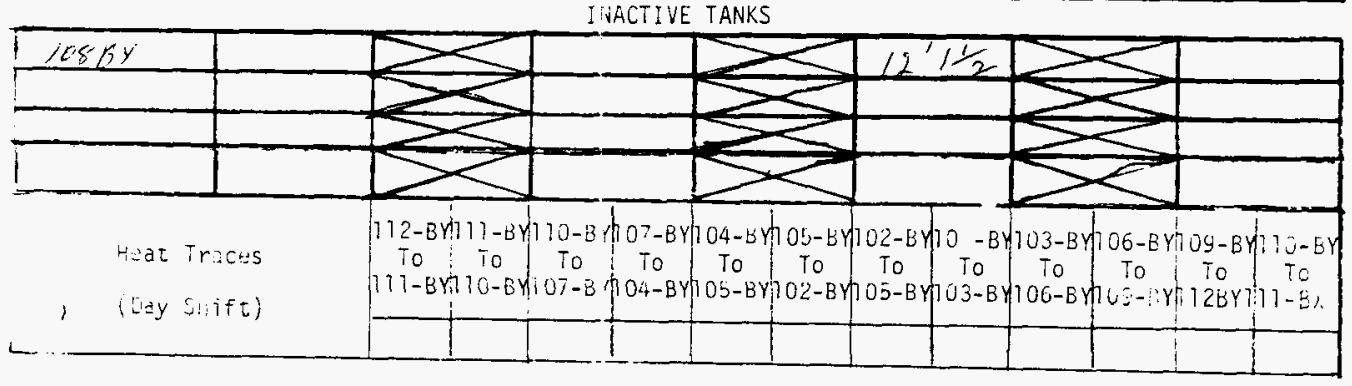




\section{$-$ \\ WHC-SD-WM-DP-206}

ITS-2 IIVENTORY AiNU PUMPING

Cate 1- i 3 RevO

\begin{tabular}{c|c|c|c}
\multicolumn{3}{c}{ Pump-Out Tank } \\
\hline $\begin{array}{c}\text { Pump } \\
\text { Humber }\end{array}$ & L.L. L. & L. L. & I I $n$ \\
Stand & ivips
\end{tabular}
Receiving Tank

\begin{tabular}{l|l|l|}
\hline L. L. & L. L. & Time \\
\hline
\end{tabular}

Start End Pump off

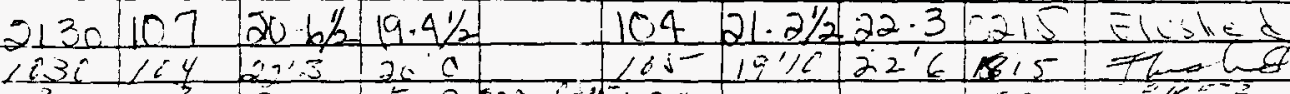

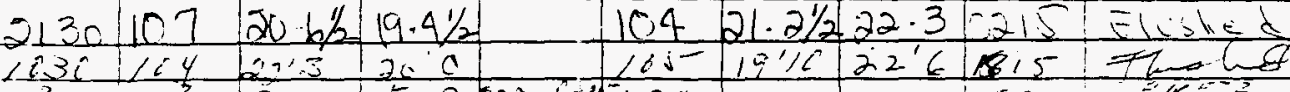

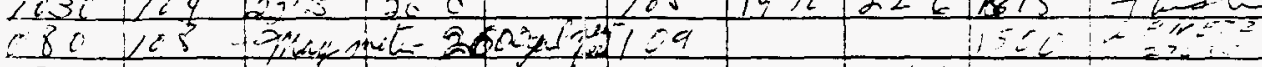

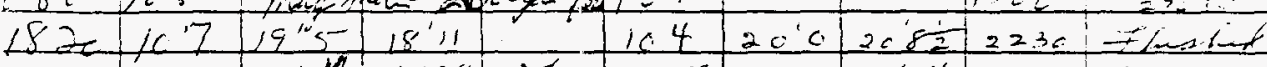

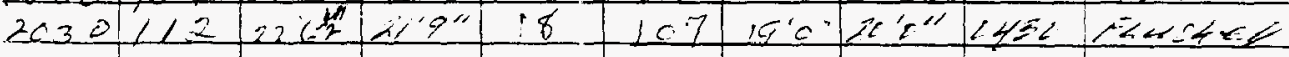

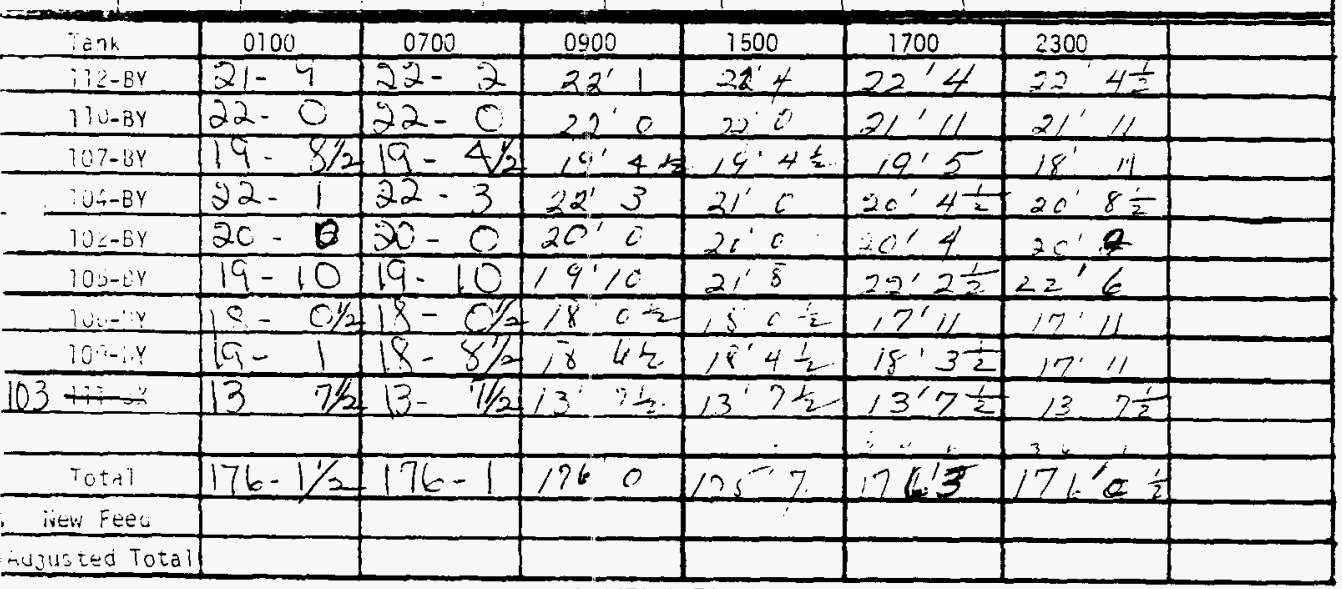

\section{IHACTIVE TANKS}

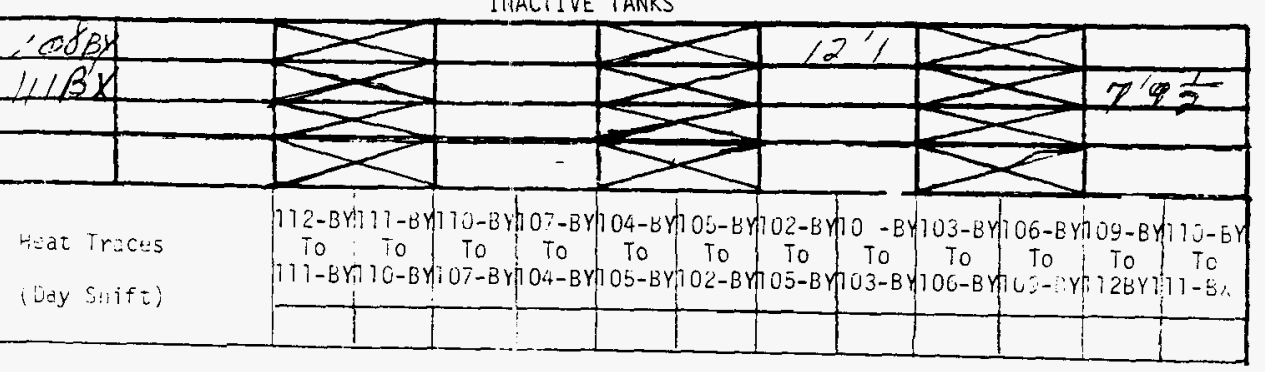




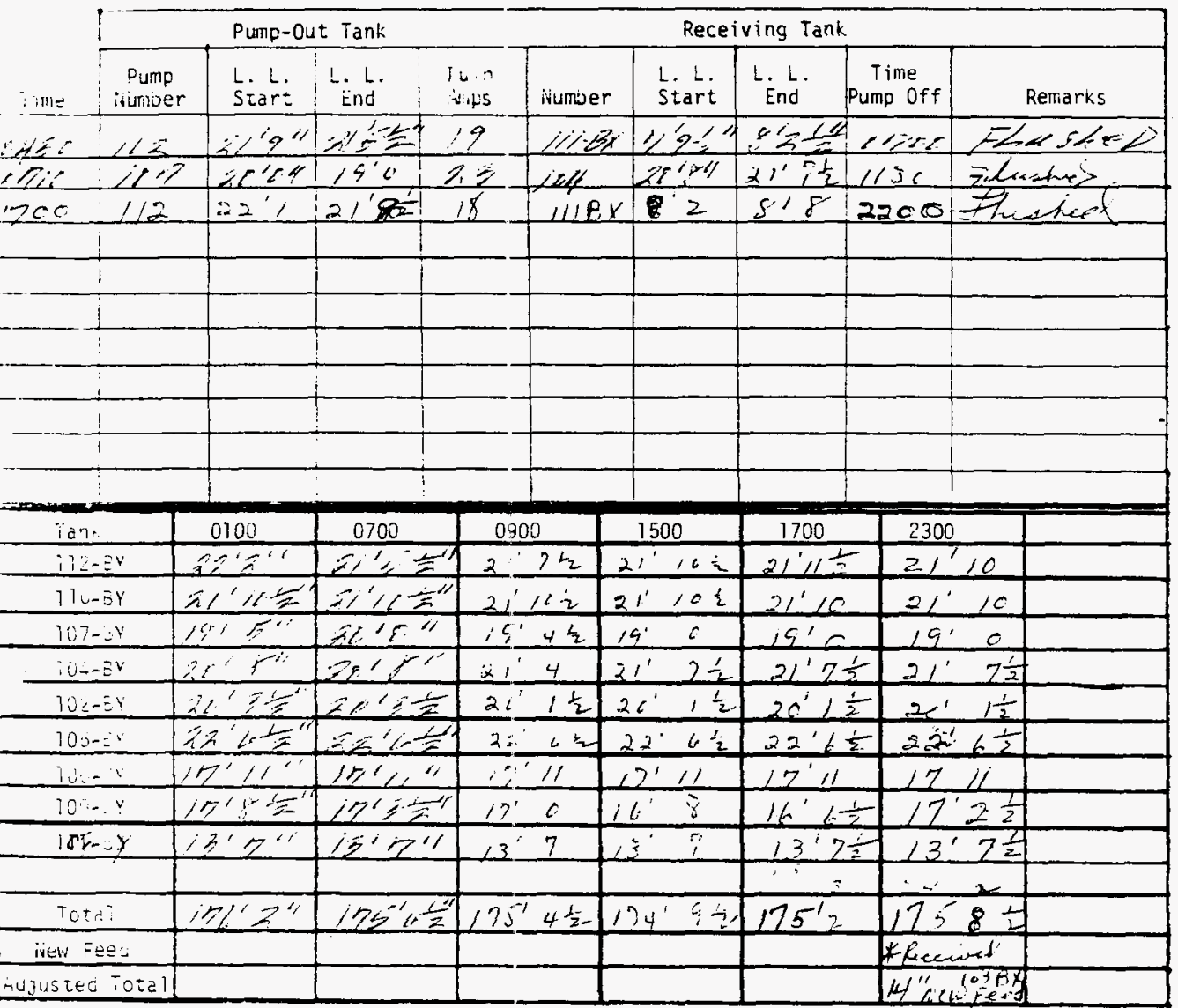

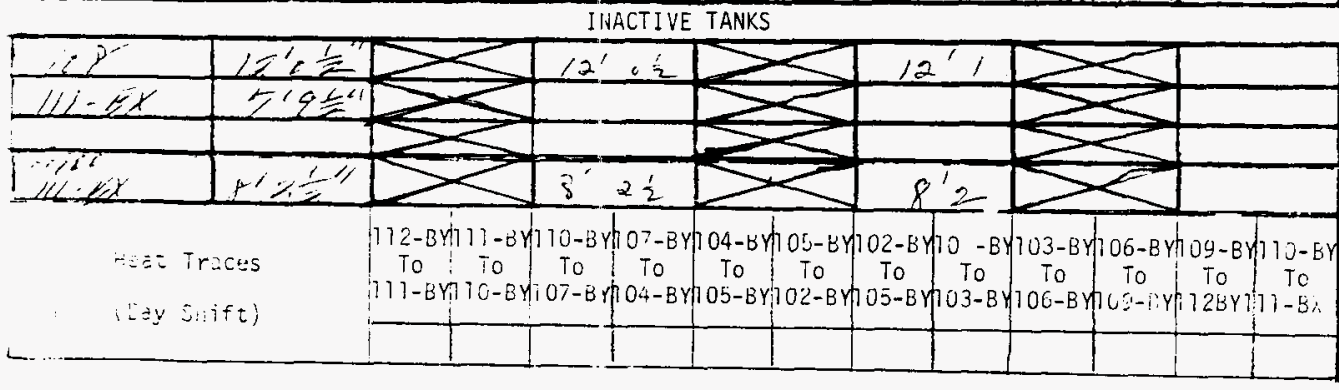


ITS-2 IIVENTORY AIVL PUMPING

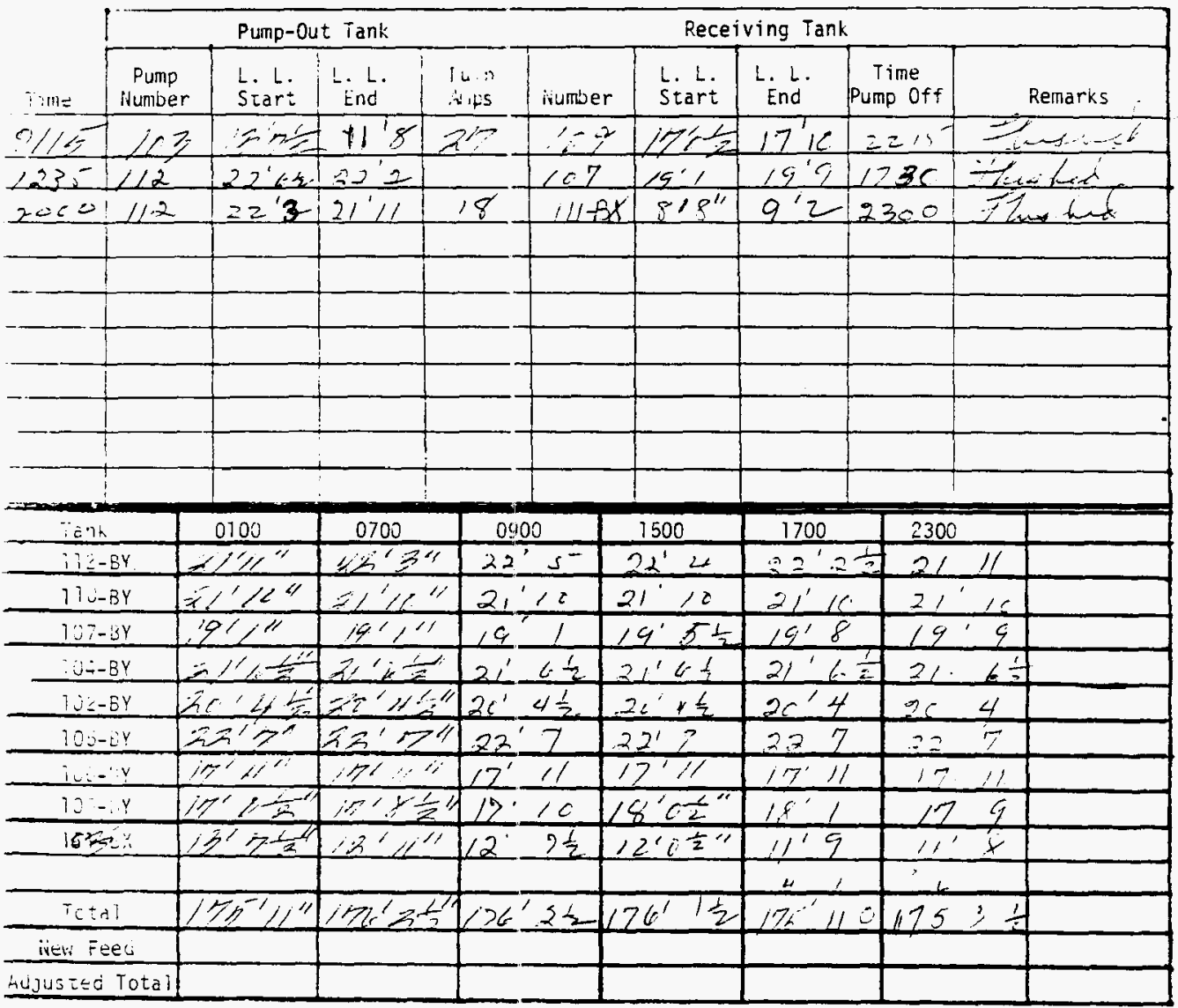

\section{IINACTIVE TANKS}

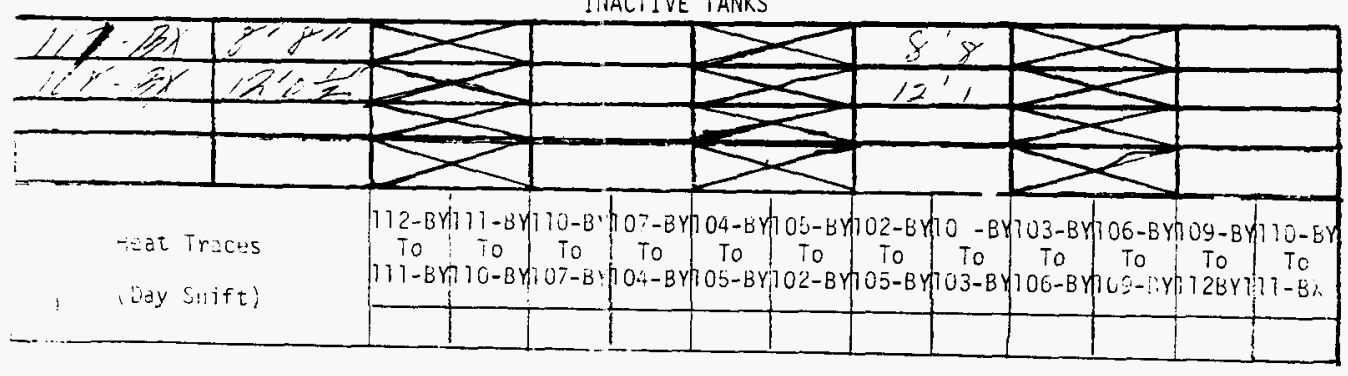




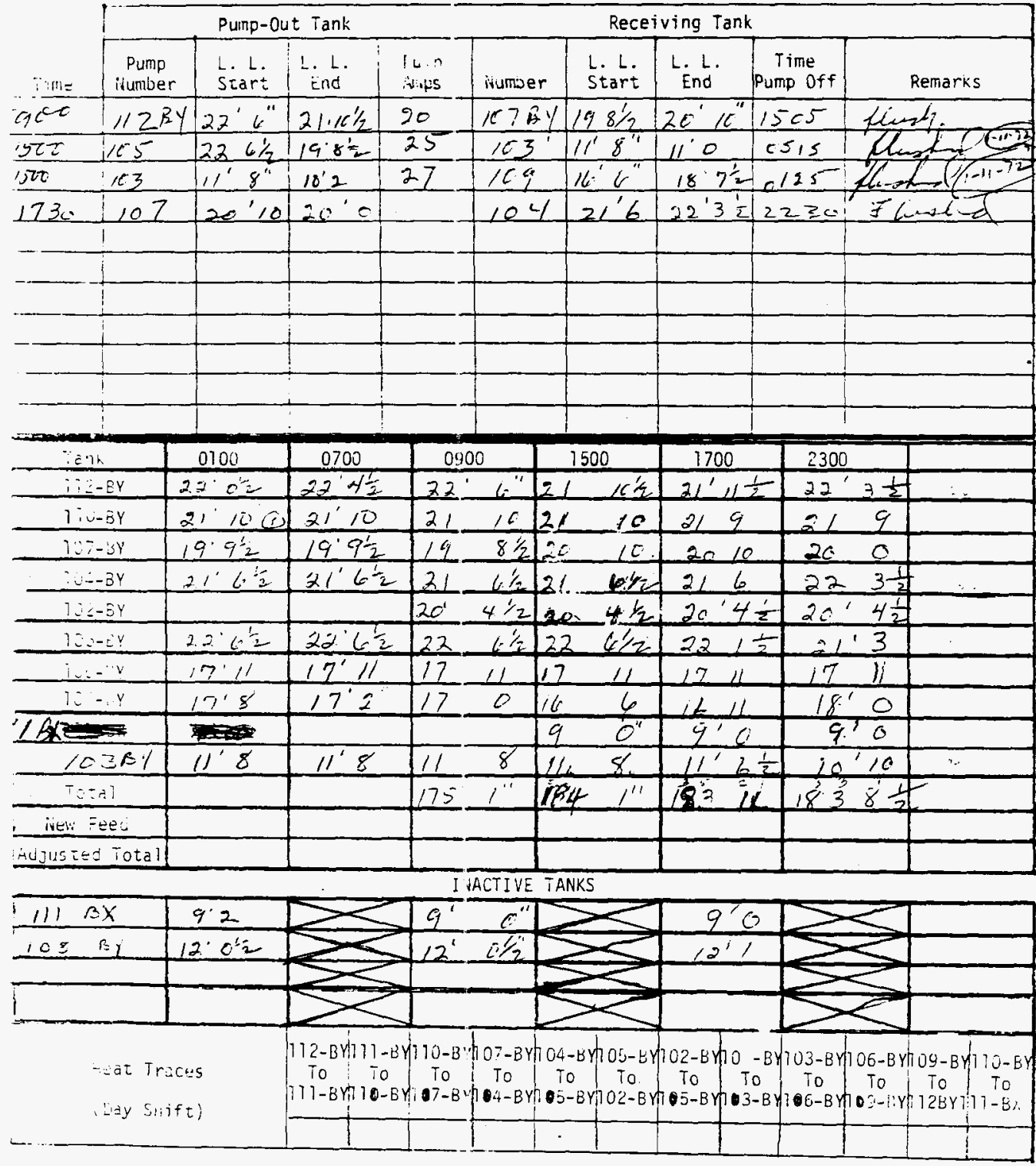




\section{$=\quad$ WHC-SD-MH-DP-206}

IT-2 IIVENTORY AIV PUMPIHG

Cate $1-11->2$ RevO

Pump-Out Tank

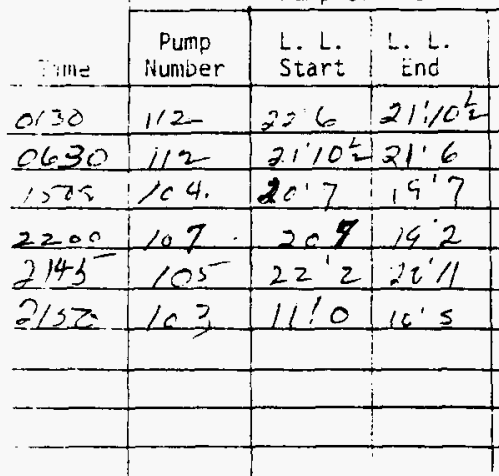

Receiving Tank

\begin{tabular}{|c|c|c|c|c|}
\hline ivumber & $\begin{array}{l}\text { L. L. } \\
\text { Start }\end{array}$ & $\begin{array}{l}\text { L. L. } \\
\text { End }\end{array}$ & $\begin{array}{c}\text { Time } \\
\text { Pump off }\end{array}$ & Remarks \\
\hline${ }_{111}^{5} x$ & $\frac{2}{2}$ & $9^{\prime} / 1 \frac{2}{2}$ & 0630 & \\
\hline 107 & $20^{\circ} 0$ & $2<1$ & $\sqrt{105}$ & \\
\hline 165 & $19^{\prime} 25$ & $2 \cdot 3$ & 652 & \\
\hline 10.4 & 2014 & $26^{\prime} / 1 / 2$ & $T_{1} \bar{C}=$ & \\
\hline 103 & $11^{\circ} 0$ & 1163 & $1 / 62$ & \\
\hline 109 & $12^{\prime} 3$ & $15: 2$ & $6^{\prime} 5^{\prime 2}=72$ & \\
\hline
\end{tabular}

\begin{tabular}{|c|c|c|c|c|c|c|c|}
\hline$a \eta k$ & 0100 & 0700 & 0900 & 1500 & 1700 & 2300 & \\
\hline$\because 72-6 Y$ & $27^{\circ}-\frac{1}{2}$ & $2.1^{\prime} 10 \div$ & $\hat{2}$ & $21^{\prime} 95$ & $F 21^{\prime} 11$ & $22 \quad 3 \frac{1}{2}$ & \\
\hline 1]U-BY & 2110 & 21,0 & $21^{\circ}-12$ & 2110 & 21 & 2119 & \\
\hline $107-3 Y$ & $20 \quad 0$ & 200 & $26^{\circ}, 3.2$ & $20^{\prime} 7$ & 207 & $20^{\prime} 5$ & \\
\hline$O U_{4} \div-B Y$ & $22: 3 \div$ & $=2^{\circ}-3 \frac{1}{2}$ & $22^{\prime} \quad 3 \frac{1}{2}$ & $22^{\prime} 3 \frac{1}{2}$ & $219 \frac{1}{2}$ & 204 & \\
\hline $30 \leq-6 Y$ & $20^{\circ} 5^{\circ}$ & $20^{\prime}=6$ & $20 \quad 6$ & $20^{\circ} 6$ & $30^{\prime} \mathrm{C}$ & $20^{\prime} 6$ & \\
\hline $103-E Y$ & $20^{3} 11$ & $19^{\circ} 7 \frac{1}{2}$ & $19^{\prime} \quad 2 \frac{1}{2}$ & $19^{\prime} 7 \frac{1}{2}$ & 206 & 22 & \\
\hline$y=-v$ & 17.11 & 1711 & 1211 & $17^{\prime} 11$ & 1711 & $17^{\prime} 11$ & \\
\hline $10-y$ & 1817 & $18^{\circ} 3$ & $\sqrt{5}, 2$ & $17^{\prime}$ & 177 & 175 & \\
\hline $111-4$. & $9 \cdot 2$ & $9^{\prime} / / 2$ & $911 \frac{1}{2}$ & 9113 & 11 & $9^{\prime}$ & \\
\hline $10: B y$ & $10 \% \frac{1}{2}$ & $11^{\prime} 0$ & $11^{\prime} \quad 0$ & 110 & 110 & 0 & \\
\hline Total & $1839 \frac{1}{2}$ & $183^{\prime} 3$ & 153 & 183.3 & $18^{2} 51$ & 183 & \\
\hline iven Feec & & & & & & & \\
\hline - -justed Total & & & & & & & \\
\hline
\end{tabular}

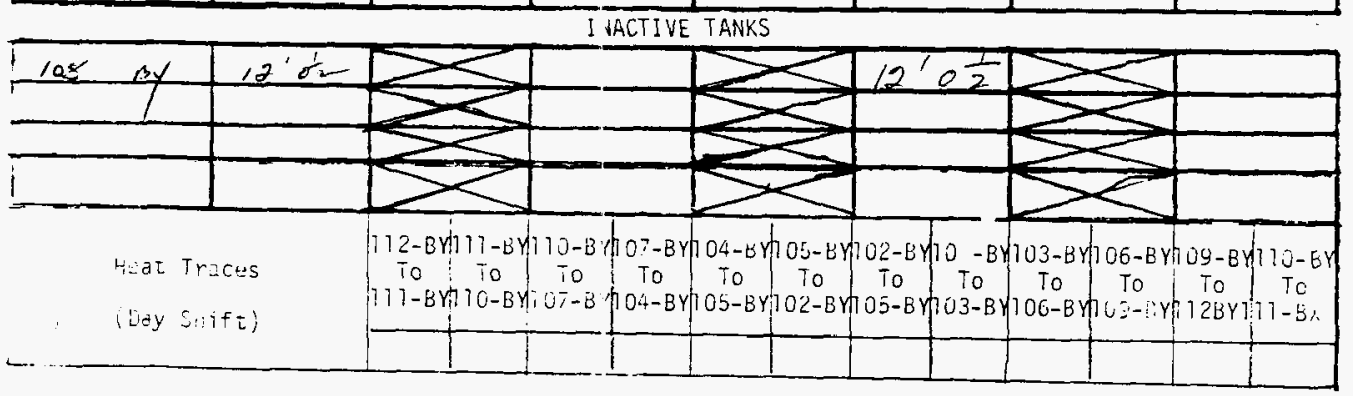




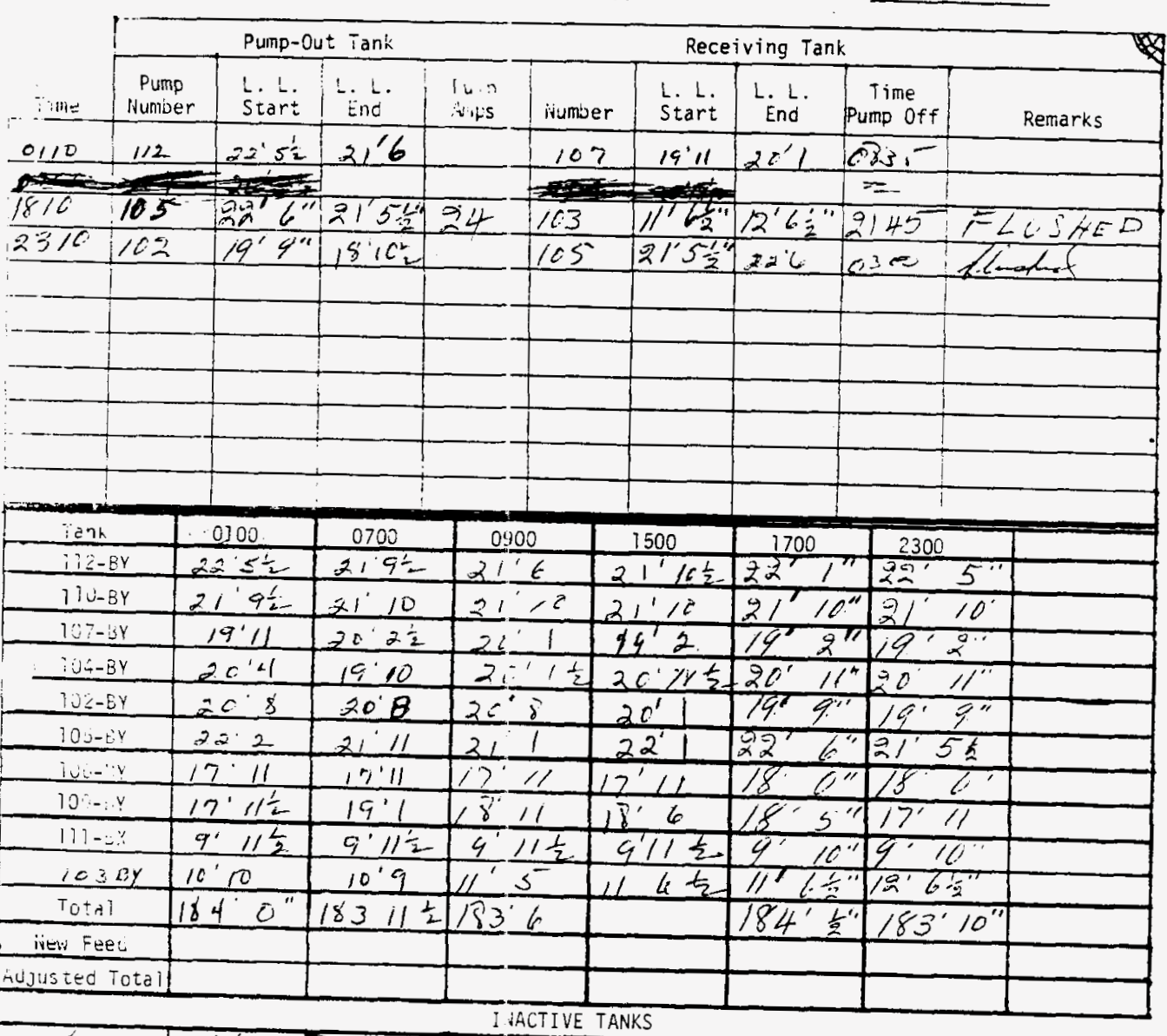

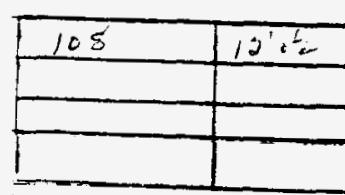

Hat Traces

$(i) y S \operatorname{sift})$

2
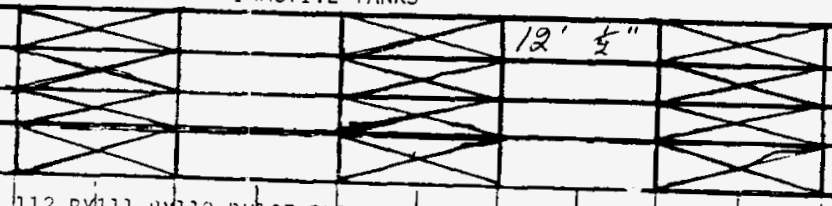

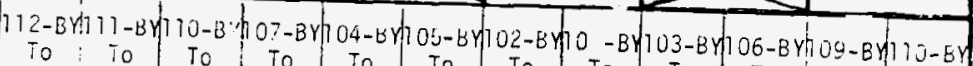

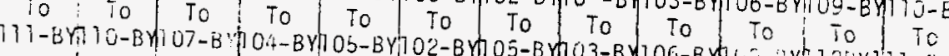

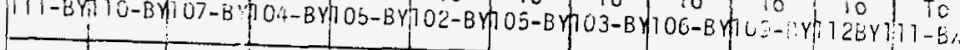

$9-20-7 ?$ 


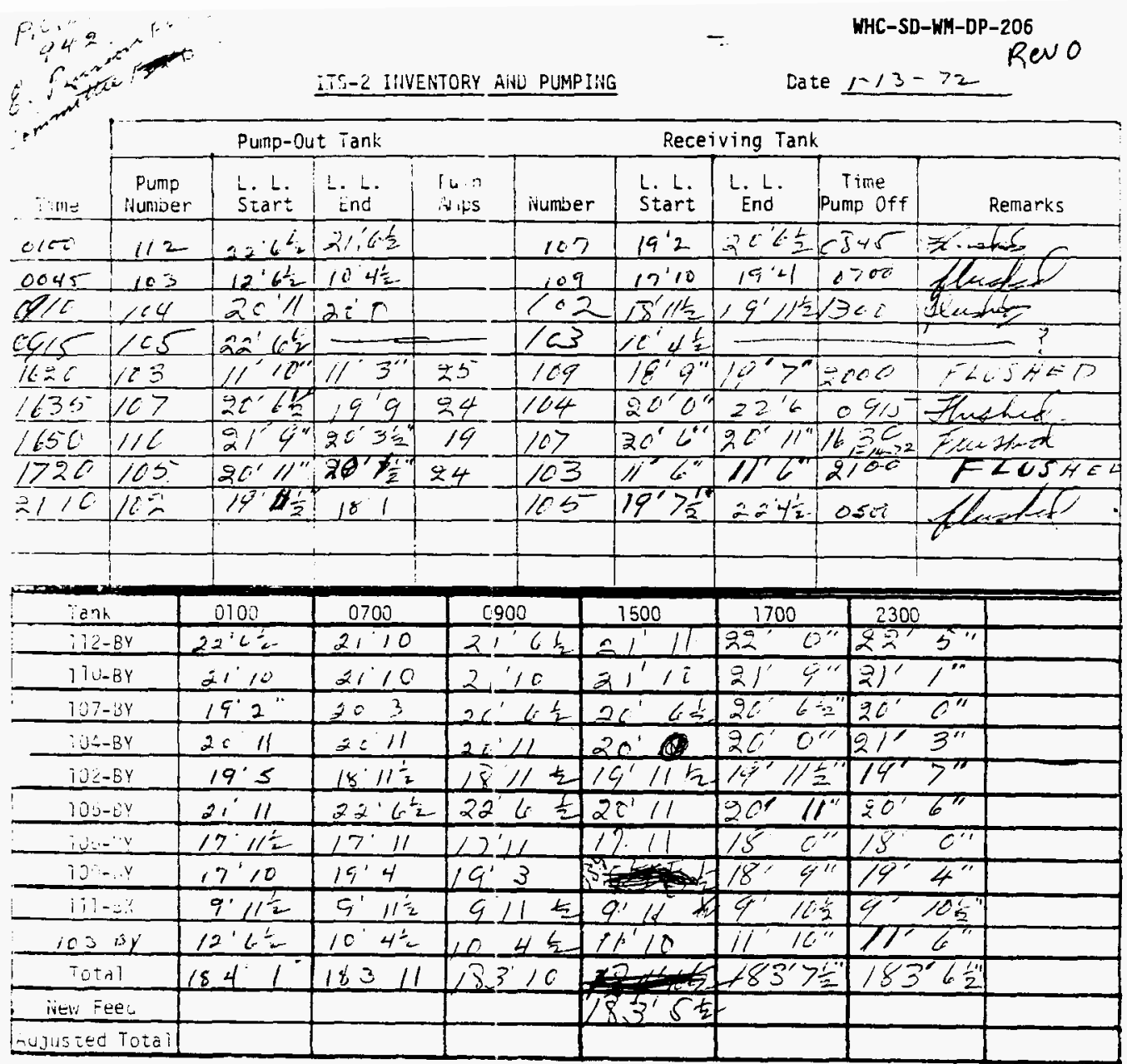

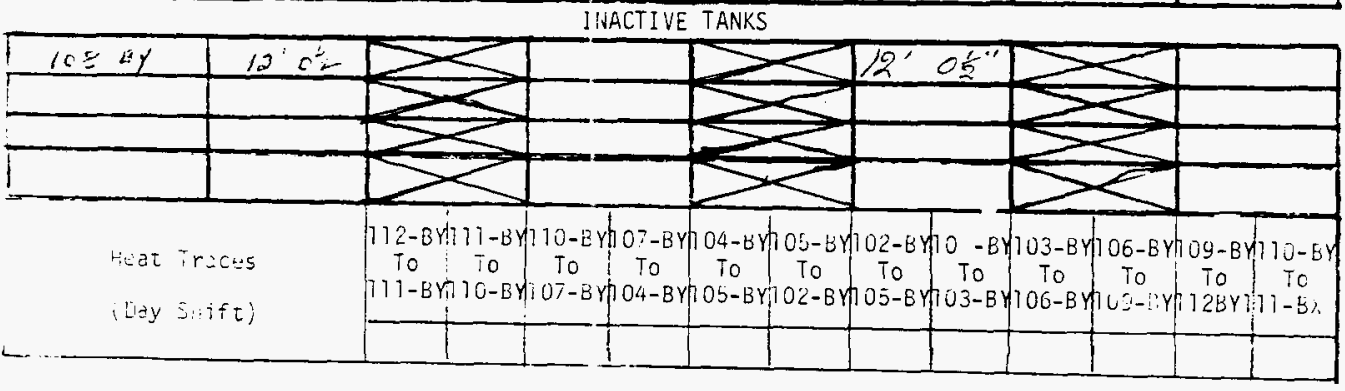

BESTA AII ABLE COPY 


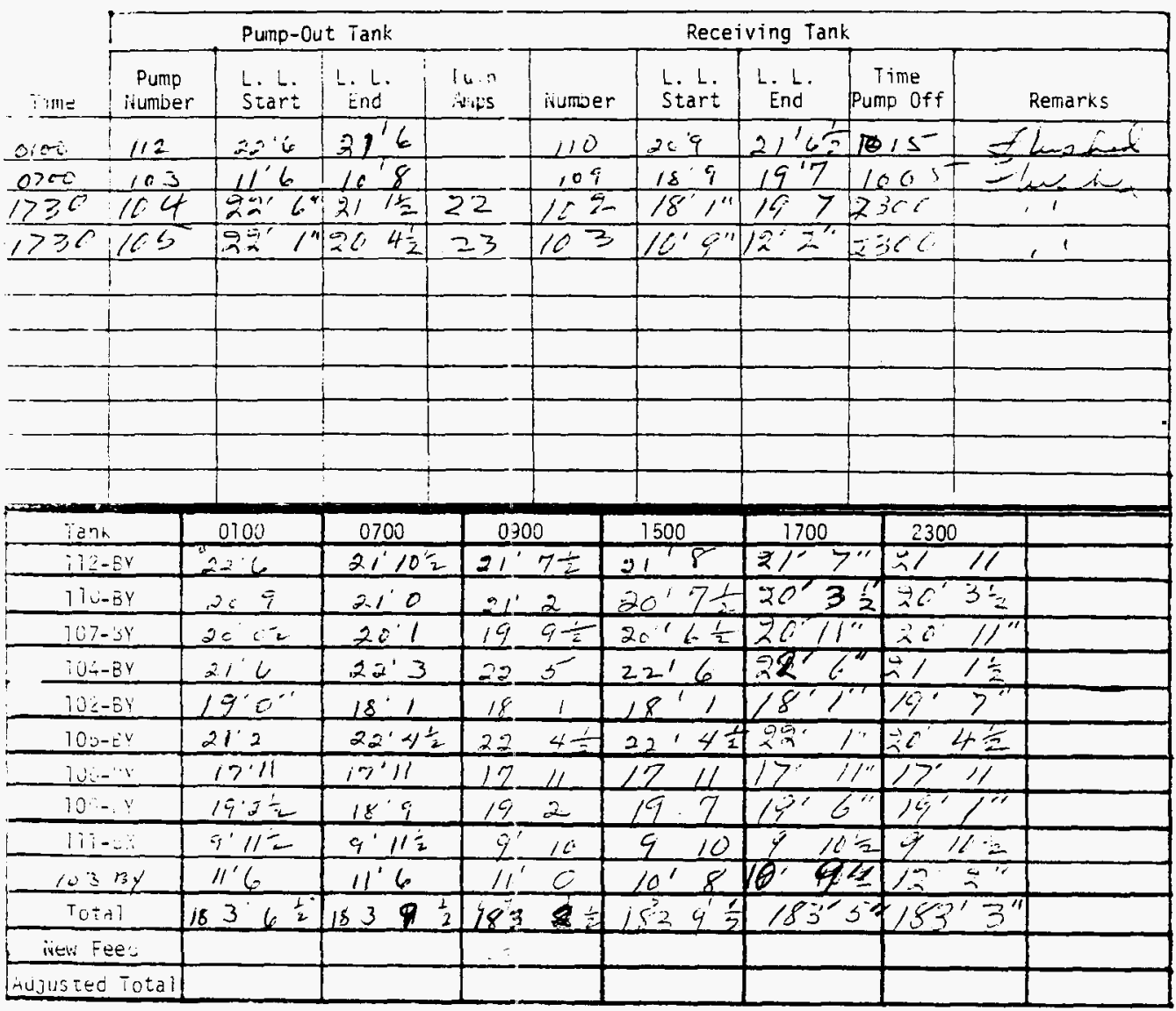

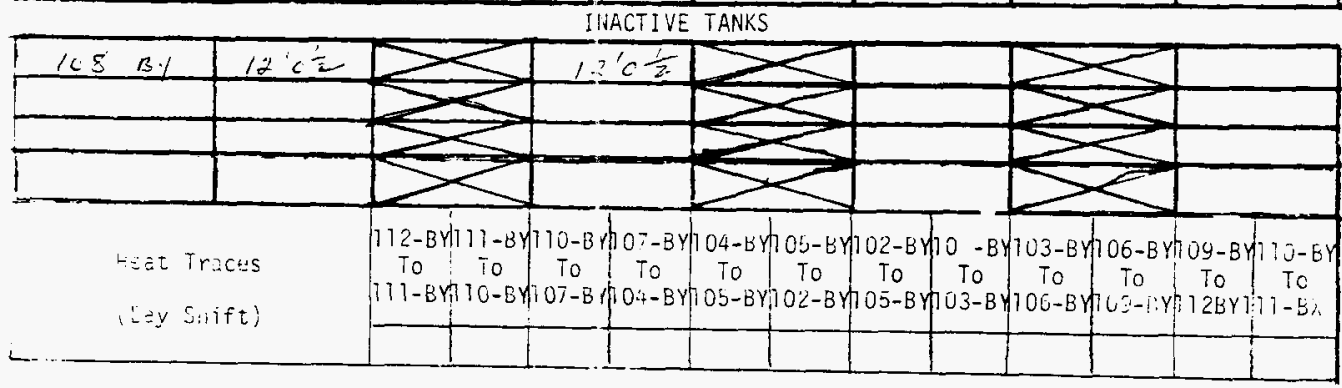


Pump-Out Tank

\begin{tabular}{l|l|l} 
Pump & L. L. & L. L.
\end{tabular}

lumber Start End

$\because \cdots 1=$

$22 \pi ? 11036 \mathrm{x}$

$0950+10384 \quad 12-2$
Receiving Tank

L. L. L. L. Time

Start End Pump off $1 / 09$ By $17^{\circ}, \frac{1}{2} 17^{\circ}, 1 / 2-030$

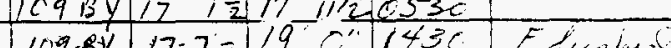

\begin{tabular}{|c|c|c|c|c|c|c|c|c|c|}
\hline ianh & \multicolumn{2}{|c|}{0100} & \multicolumn{2}{|c|}{0700} & 0900 & 1500 & 1700 & 2300 & \\
\hline$i 12-B Y$ & 21 & $7 \frac{1}{2}$ & & $c^{\prime \prime}$ & 22,3 & $\because 2^{\prime} \quad 5 "$ & $2 \alpha$ & 22 & \\
\hline $11 U-B Y$ & 22 & 1 & & $y^{\prime \prime}$ & $22,1 "$ & $22^{\prime} 1$ & $2^{\prime \prime} l^{\prime \prime}$ & Q⿱一𫝀口 & \\
\hline $107-3 Y$ & & 10 & & 10 & 26 & $20^{\prime} 10^{\prime \prime}$ & 42 & 200 & \\
\hline$O U_{4}-B Y$ & 21 & 1 & 21 & 1 & 21 & $21^{\prime} 1$ & $21^{\prime} 1^{\prime \prime}$ & $2 / 1$ & \\
\hline $10 \leq-8 Y$ & 19 & 7 & 19 & 7 & 19 & $19^{\prime} 7^{\prime}$ & $4 \%$ & $1 y^{\prime} 7^{\prime}$ & \\
\hline 10y-tiY & 20 & 4 & $x$ & 6 & 26 & $2 L^{\prime} 6^{\prime \prime}$ & 20150 & $20^{7} l_{1}$ & \\
\hline $1 U_{6-1}$ & 17 & 11 & 17 & it & $17^{\prime}$ & 1711 & $17 / 1^{\prime \prime}$ & $17^{\prime} \quad 11$ & \\
\hline $10 .-1 . \gamma$ & & 3 & 17 & 10 & 8 & $19^{\circ} \mathrm{C}$ & 140 & $10^{\prime} \quad 6$ & \\
\hline $111--1$ & & $16 \frac{1}{3}$ & & $10 \frac{1}{2}$ & $1 L^{\prime} / 2$ & $c^{\prime} 11^{\prime \prime}$ & $7^{\prime} 16 \frac{1}{2}$ & $41,16 \frac{1}{2}$ & \\
\hline $1636 y$ & 12 & 2 & 12 & 2 & 12 & $10^{\prime} 6^{\prime \prime}$ & $10^{\prime} b 2$ & 1062 & \\
\hline Totil & 183 & $11 "$ & 182 & $1 c^{\prime} / 2$ & $193^{\prime \prime} 112^{\prime \prime}$ & $183^{\circ} / 12$ & $183^{\prime} 8$ & 183.8 & \\
\hline ivew Feec & & & & $z^{\prime \prime}$ & & & & & \\
\hline ied Tota & & & & & & & & & \\
\hline
\end{tabular}

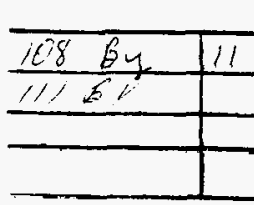

Hat Traces

(Lizy Siift)

I. ACTIVE TANKS

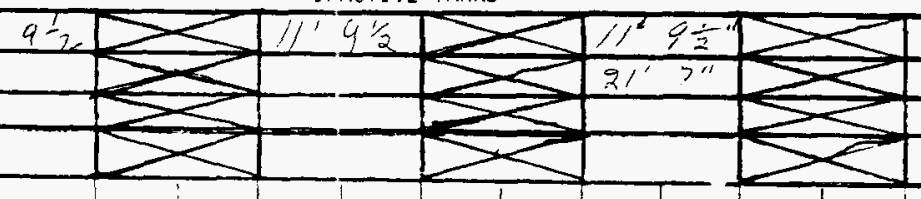

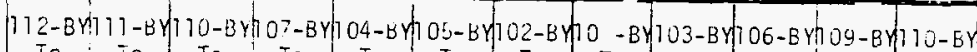

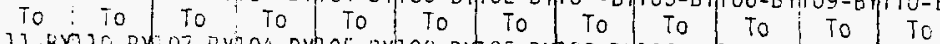

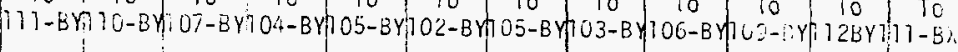




\section{WHC-SD-WIM-DP-206 \\ iT-2 IIVENTORY AND PUMPIIG

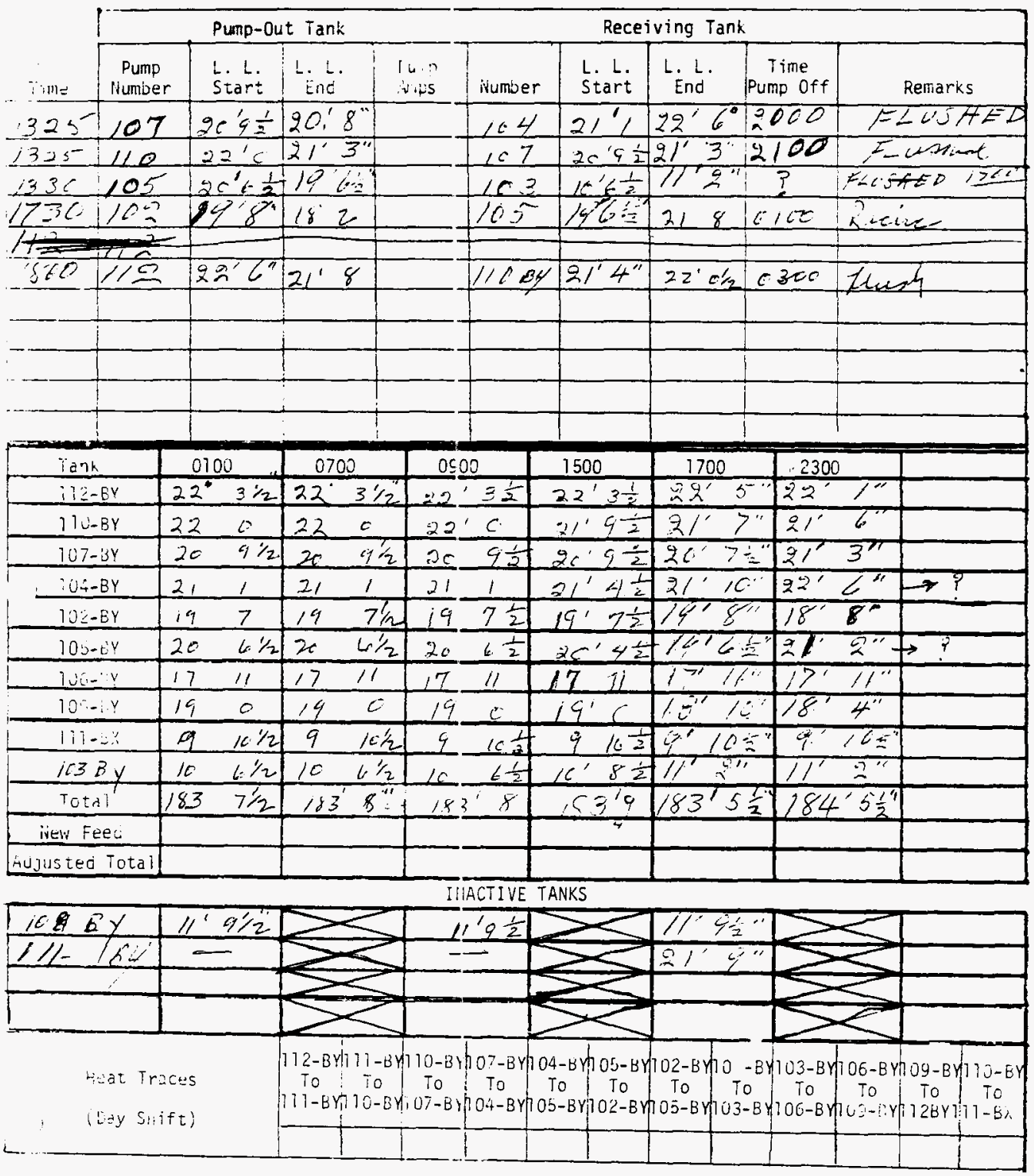




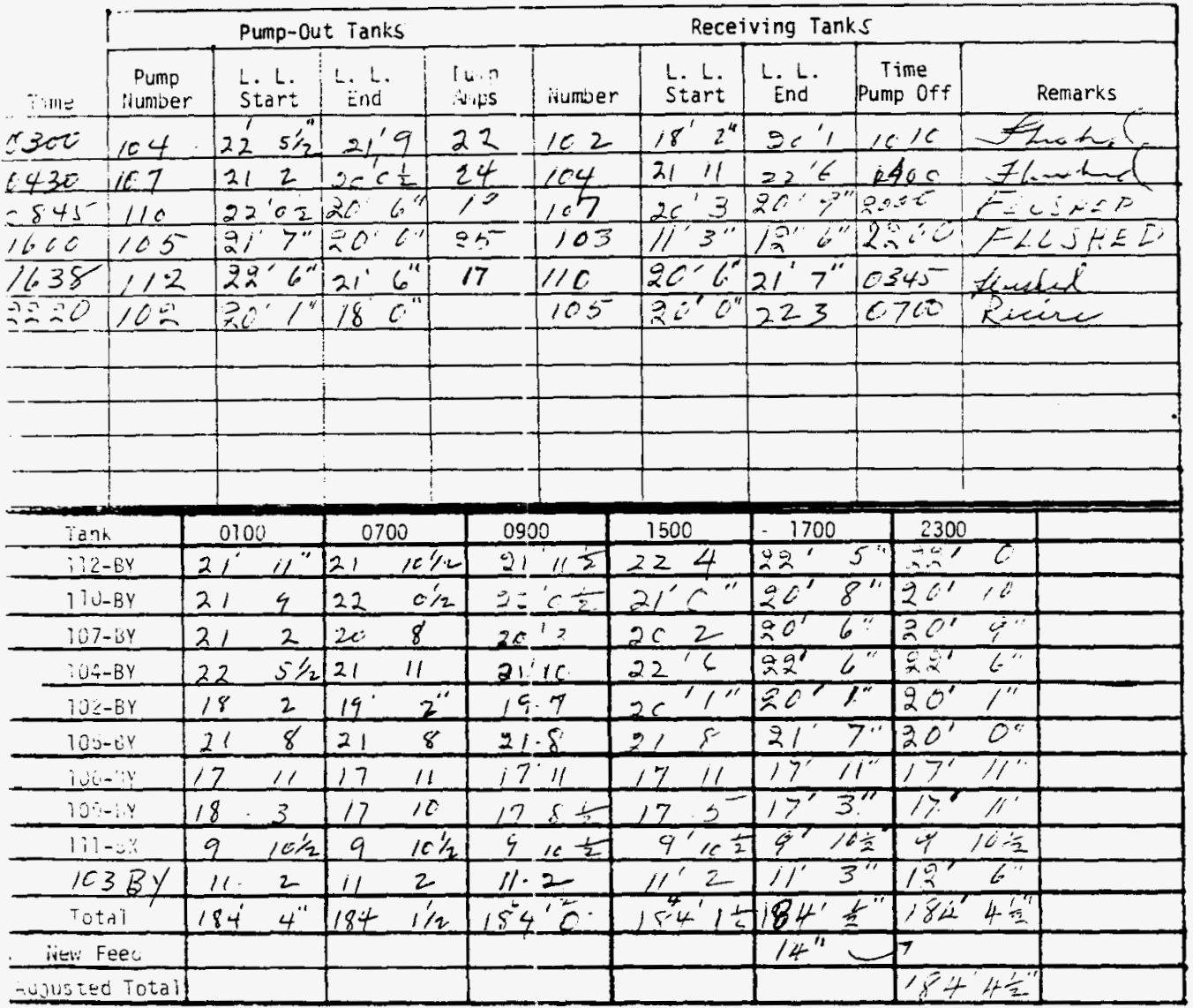

II.ACTIVE TANKS

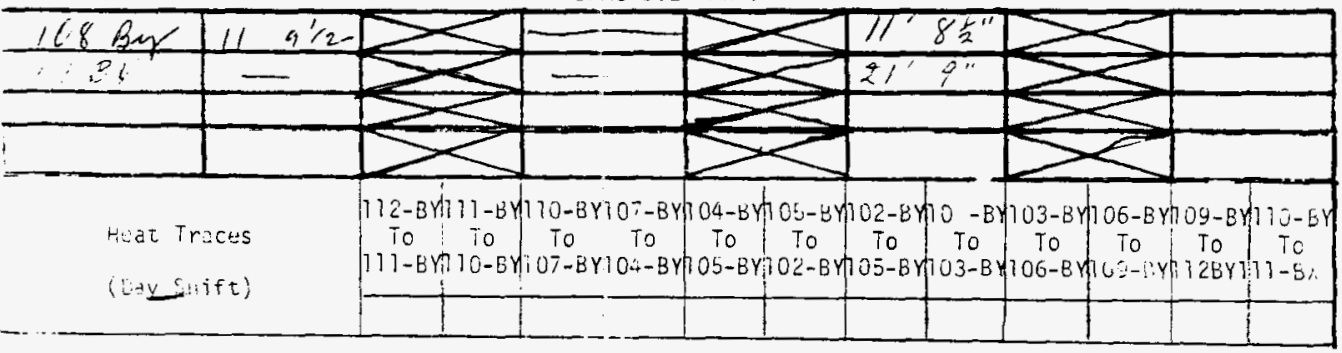




\section{ITS-2 IIIVENTORY AiNL PUMPIRG

\begin{tabular}{|c|c|c|c|c|c|c|c|c|c|}
\hline \multirow[b]{2}{*}{ nille } & \multicolumn{4}{|c|}{ Pump-Out Tank } & \multicolumn{4}{|c|}{ Receiving Tank } & \multirow[b]{2}{*}{ Remarks } \\
\hline & $\begin{array}{l}\text { Pump } \\
\text { iduniber }\end{array}$ & $\begin{array}{l}\text { L. L. } \\
\text { Start }\end{array}$ & 1. L. & $\begin{array}{l}\text { Iuin } \\
\text { irips }\end{array}$ & irumier & $\begin{array}{l}\text { L. L. } \\
\text { Start }\end{array}$ & $\begin{array}{l}\text { L. L. } \\
\text { End }\end{array}$ & $\begin{array}{c}\text { Time } \\
\text { Pump 0ff }\end{array}$ & \\
\hline 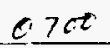 & 104 & $22^{\prime} 4^{\prime \prime}$ & $20,-7$ & 22 & 162 & $18 \quad 5$ & $S c-0$ & 1400 & $\exists\left(L_{C} 5 A \varepsilon D\right.$ \\
\hline 670 & 103 & $12^{\prime} 6^{\prime \prime}$ & $\mu^{\prime} L^{\prime \prime}$ & 24 & 109 & 172 & $10^{\circ} \mathrm{in}^{\prime \prime}$ & 1130 & Elusnos \\
\hline $110: 5$ & 105 & $22-3$ & $19^{\circ} 6^{\prime \prime}$ & 260 & 103 & $11-1$ & $11^{\prime} 7 \div$ & 2030 & $F l u s h=2$ \\
\hline $120=$ & 102 & $20^{\prime} \varepsilon^{\frac{1}{2}}$ & $19^{\prime}, 1 / 2$ & 2 & 104 & $\therefore 0^{\prime} \div \div 11$ & $22^{\circ}-x^{6} / 2$ & 0200 & timatid \\
\hline 2030 & $\because=$ & $22^{1} 2^{11}$ & $22: 3 \frac{1}{2}$ & 19 & $1118 x$ & $9^{\prime}\left(1 \frac{1}{2}\right)$ & ic $8^{\prime \prime}$ & 0100 & $E=\pi i l E<17$ \\
\hline $\operatorname{cis} 20$ & $10 \hat{~}$ & $19^{\prime} 11^{\frac{1}{2}}$ & 15.9 & - & 105 & $90^{\circ} 6^{\circ}$ & $z 25$ & 1110 & $71 \ldots$ \\
\hline 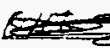 & & 372 & & 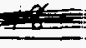 & 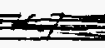 & $\Rightarrow$ & & & \\
\hline & & & & & & & & & \\
\hline & & & & & & & & & \\
\hline & & & & & & & & & \\
\hline & & & & & & & & & \\
\hline
\end{tabular}

\begin{tabular}{|c|c|c|c|c|c|c|c|c|c|}
\hline iank & \multicolumn{2}{|c|}{0100} & \multicolumn{2}{|c|}{0700} & $0 \leq 00$ & 1500 & 1700 & 2300 & \\
\hline $37-3 Y$ & 21 & $10 / 2$ & 21 & $81 / 2$ & $2 l^{\prime} 9^{\prime \prime}$ & $x^{\prime} 2^{\prime} 3$ & $\operatorname{san}^{\circ} \quad 45^{\circ}$ & $2 a^{\prime}$ & \\
\hline $17 \backsim-B Y$ & 21 & 1 & 21 & 7 & $21^{\prime} 7^{\prime}$ & $21^{\prime} 7^{\prime}$ & $5 i$ & $\mathrm{si}^{\prime}$ & \\
\hline $307-54$ & 20 & $5 / 2$ & 20 & $8 / 2$ & $20^{\prime} \quad 8 \%$ & $20^{\prime} 8 \%$ & $\div 0^{\circ} \quad 8 \div$ & $6 \div$ & \\
\hline$B O \div-B Y$ & 22 & $\varepsilon$ & 22 & 6 & $21^{\prime} 11^{\prime \prime}$ & 20.7 & $20^{\prime} 7 \frac{1}{2}$ & $0 \div$ & \\
\hline $10 \div-8 y$ & 19 & 4 & 18 & 2 & $1877^{\prime \prime}$ & $20 \cdot 0$ & $19^{\prime} \quad 11 \frac{1}{2}$ & $10 \div$ & \\
\hline $100-5$ & 26 & i & $2 z^{\prime}$ & 3 & $22^{\prime} 3$ & $21-6$ & $90^{\prime} \quad 9^{\prime \prime}$ & 10 & \\
\hline $30-1$ & 17 & ii & 17 & 11 & 17.11 & $17^{\prime} 11$ & 191 & $\therefore$ & \\
\hline $10-y$ & 17 & 4 & 17 & 2 & $17^{\prime} 8^{\prime \prime}$ & $19^{\circ} 2$ & $18^{\prime} \quad 19=$ & 10 & \\
\hline $1 i i-\therefore$ & & $121=$ & 9 & $161 / 2$ & $911 / 2$ & $q^{\prime} 161 / 2$ & $5^{\prime} \quad 11 \div$ & $10^{\circ}$ & \\
\hline 1636 & 12 & 4 & 13 & 6 & $11.9 \frac{1}{2}$ & $11-0$ & $i^{1} 5^{-1}$ & $11 !$ & \\
\hline Total & 184 & $3 / 2$ & 184 & 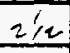 & $184^{\circ} \quad 5^{\circ}$ & $184^{\prime} 5^{\prime \prime}$ & $184^{\prime \prime} \quad 1 \frac{7}{2}$ & $153^{\prime}$ & \\
\hline iitw Fee' & & & & & & & & & \\
\hline qujustec Total & & & & & & & & & \\
\hline
\end{tabular}

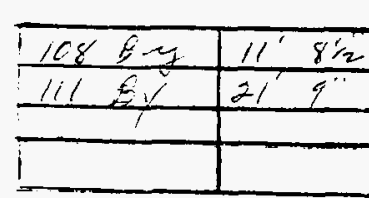

Heat Traces

i.y Sulift)

\section{IIIACTIVE TANKS}

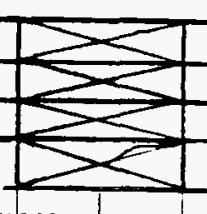

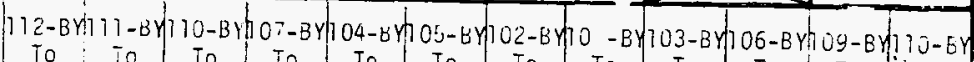

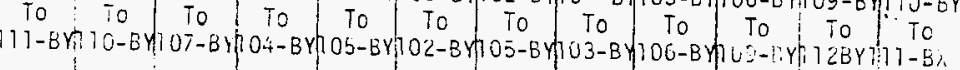

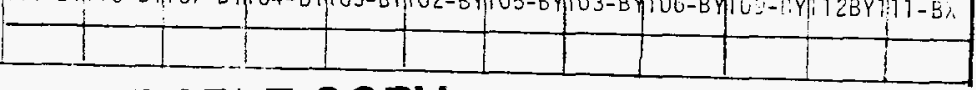




\begin{tabular}{|c|c|c|c|c|c|c|c|c|c|c|}
\hline \multirow[b]{2}{*}{ ans } & \multicolumn{3}{|c|}{ Pump-Out Tank } & \multicolumn{5}{|c|}{ Receiving Tank } & \multirow{2}{*}{\multicolumn{2}{|c|}{ Remarks }} \\
\hline & $\begin{array}{l}\text { Pump } \\
\text { ivmber }\end{array}$ & $\begin{array}{l}\text { L. L. } \\
\text { Start }\end{array}$ & 1.1 . L & irumber & $\begin{array}{l}\text { L. L. } \\
\text { Start }\end{array}$ & \multirow{2}{*}{$\begin{array}{l}\text { L. L. } \\
\text { End } \\
2 \sigma^{\prime} 4 \frac{1}{2}\end{array}$} & \multicolumn{2}{|c|}{$\begin{array}{c}\text { Time } \\
\text { Pump off }\end{array}$} & & \\
\hline 0110 & 112 & $=23 \frac{1}{2}$ & $21^{\prime} 5^{\prime} /$ & 107 & $192^{\prime \prime}$ & & \multicolumn{2}{|c|}{ 10906 } & \multicolumn{2}{|c|}{$72 \mathrm{ch}$} \\
\hline 0815 & 104 & $22^{1} 51,2$ & 504 & 102 & $18,5 \frac{1}{5}$ & $19^{\prime} 11^{\prime \prime}$ & \multicolumn{2}{|c|}{$1 / 720$} & \multicolumn{2}{|c|}{ Fiusiné } \\
\hline 1225 & 105 & $122-5$ & $20^{\circ} 9 \frac{1}{2}^{\prime}=$ & 103 & $11-1$ & $12^{\prime}=2^{\prime}$ & \multicolumn{2}{|c|}{1099} & $5:$ & Exsé \\
\hline 1700 & 107 & $20^{\prime} 33^{\circ}$ & $\angle 8, C$ & 104 & $20^{\prime} 4^{\prime \prime}$ & 219 & & 3 & $=10$ & $\leq 4 \leq 1$ \\
\hline $2+30$ & 102 & $19^{\prime} U^{\prime \prime}$ & $185^{\circ}$ & $12=$ & $=x^{\prime} \varepsilon \frac{1}{2}$ & $22^{\circ} 5^{\prime}$ & & 415 & & $=$ \\
\hline & & & & & & & & & & \\
\hline & & & & & & & & & & \\
\hline & & & & & & & & & & \\
\hline & & & & & & & & & & \\
\hline & & & & & & & & & & \\
\hline iank & & 0100 & 0700 & 0400 & 1500 & 1700 & & 2300 & & \\
\hline i12- & & $22 \quad 3 / 2$ & $8 / 2$ & $2,16 \frac{1}{2}$ & $2 / 5-\frac{7}{2}$ & : :1 & 6 & ? & in! & \\
\hline $110-$ & & $2161 / 2$ & $21 \quad 6 \% 2$ & $2: 6$ & $21^{\prime} 6$ & $21^{\prime}$ & $2 "$ & il & $e^{-1}$ & \\
\hline $107-$ & & $19^{\circ} 2^{\prime}$ & $20 \quad 0$ & $26 \quad 42$ & $20^{\prime} 4 \frac{1}{2}$ & an: & $2 \pm^{4}$ & $19^{\prime}$ & $1 "$ & \\
\hline $30 \div-$ & & $22 \quad 3 / 2$ & $22 \quad 5 \frac{1}{2}$ & $2=5 \frac{-1}{2}$ & $2018 \div$ & $290^{\circ}$ & $4 "$ & $\cdot 1^{\prime}$ & $E \div 1$ & \\
\hline $10 \div-$ & & 1911 & 182 & $14^{\prime} 5^{\frac{1}{2}}$ & $19^{\circ} 5$ & $10^{\prime}$ & $1^{\prime \prime}$ & $19^{1}$ & $9 \div$ & \\
\hline $100-$ & & $196 / 2$ & 21 & 2.9 & $21^{\prime} 10$ & $\because$ & $\angle$ & $2 i$ & 11 & \\
\hline ivi- & & 1711 & $17 \quad 1 \%$ & $17 \div / 1$ & $17^{\prime} 11$ & 17 & $\div$ & .7 & $11 \div$ & \\
\hline $10:-$ & & 194 & $18 \quad 27 / 2$ & $11^{\prime} 7 \frac{1}{2}$ & $187 \frac{1}{2}$ & 115 & $7^{\prime \prime}$ & $i 2$ & $: " 1$ & \\
\hline $111-$ & & $10^{\circ} 8^{\prime \prime}$ & 10 & $16 \div 6$ & $10^{\prime} 6$ & $10^{\circ}$ & $8^{\prime \prime}$ & $10^{1}$ & 8 & \\
\hline 103 & $6 y$ & $10 \quad 11$ & 10 & $H^{\prime} 1$ & 11 & $11^{1}$ & $1 "$ & $\therefore$ & $7 \div$ & \\
\hline Tota & & $183^{\circ} 7^{\prime \prime}$ & $18 \overline{3}$ & $184+\frac{1}{2}$ & $18^{6} 4^{\prime} 1^{2}$ & 184 & $=1$ & $134^{\prime}$ & $=\frac{1}{2}$ & \\
\hline iver $\mathrm{Fe}$ & & & & & & & & & & \\
\hline ted & Total & & & & & & & & & \\
\hline
\end{tabular}

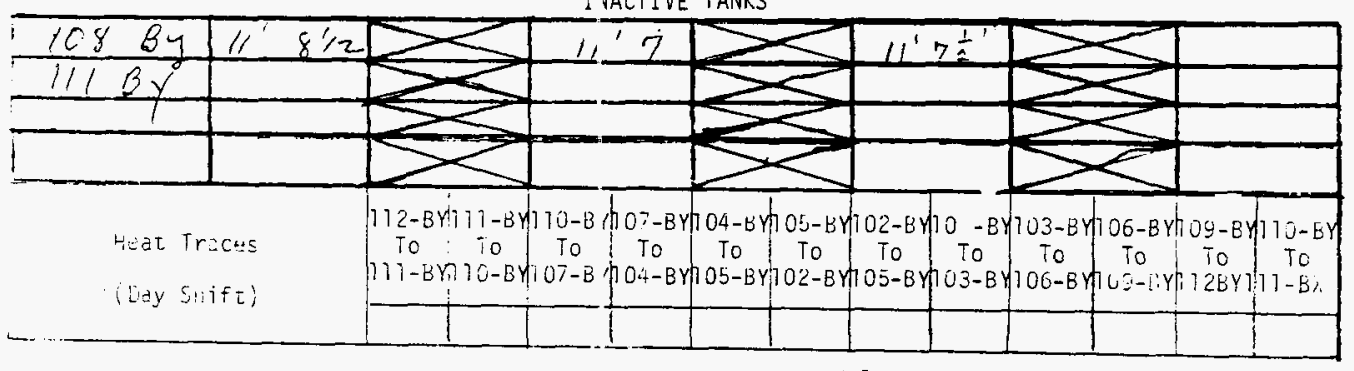




\section{$-\quad$ MHC-SD-WH-DP-206}

IIS-2 IHVENTORY AIN PUHPING Date $1-21-72$ ReNO

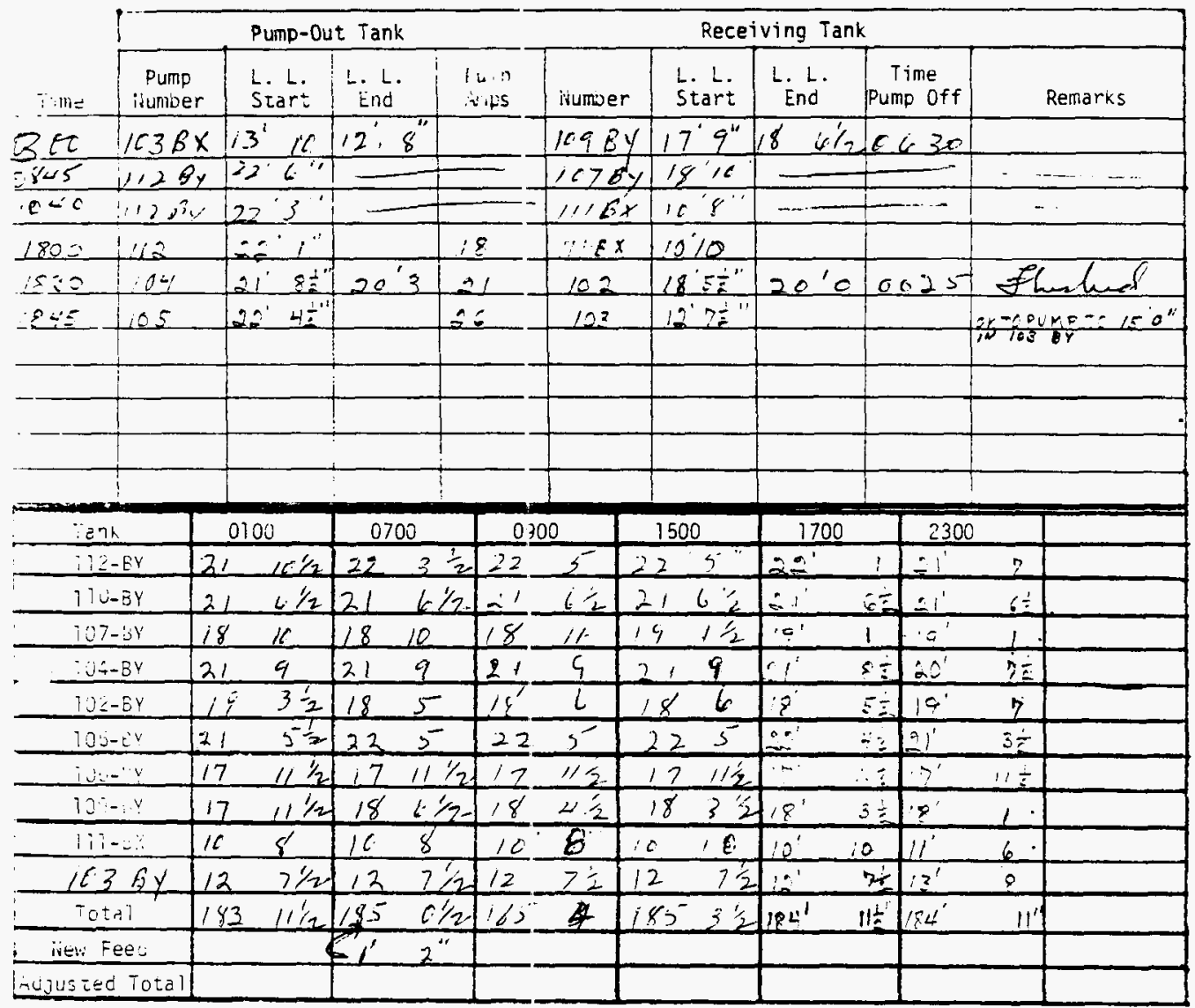

I IACTIVE TANKS

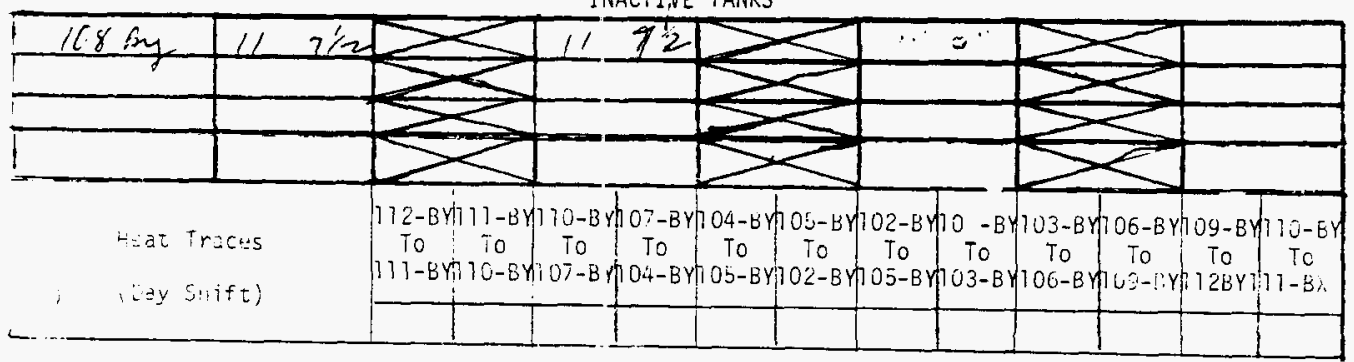

9.00-77 BEST AVAILABL 5 C.OPY 


\section{WHC-SD-WM-DP-206}

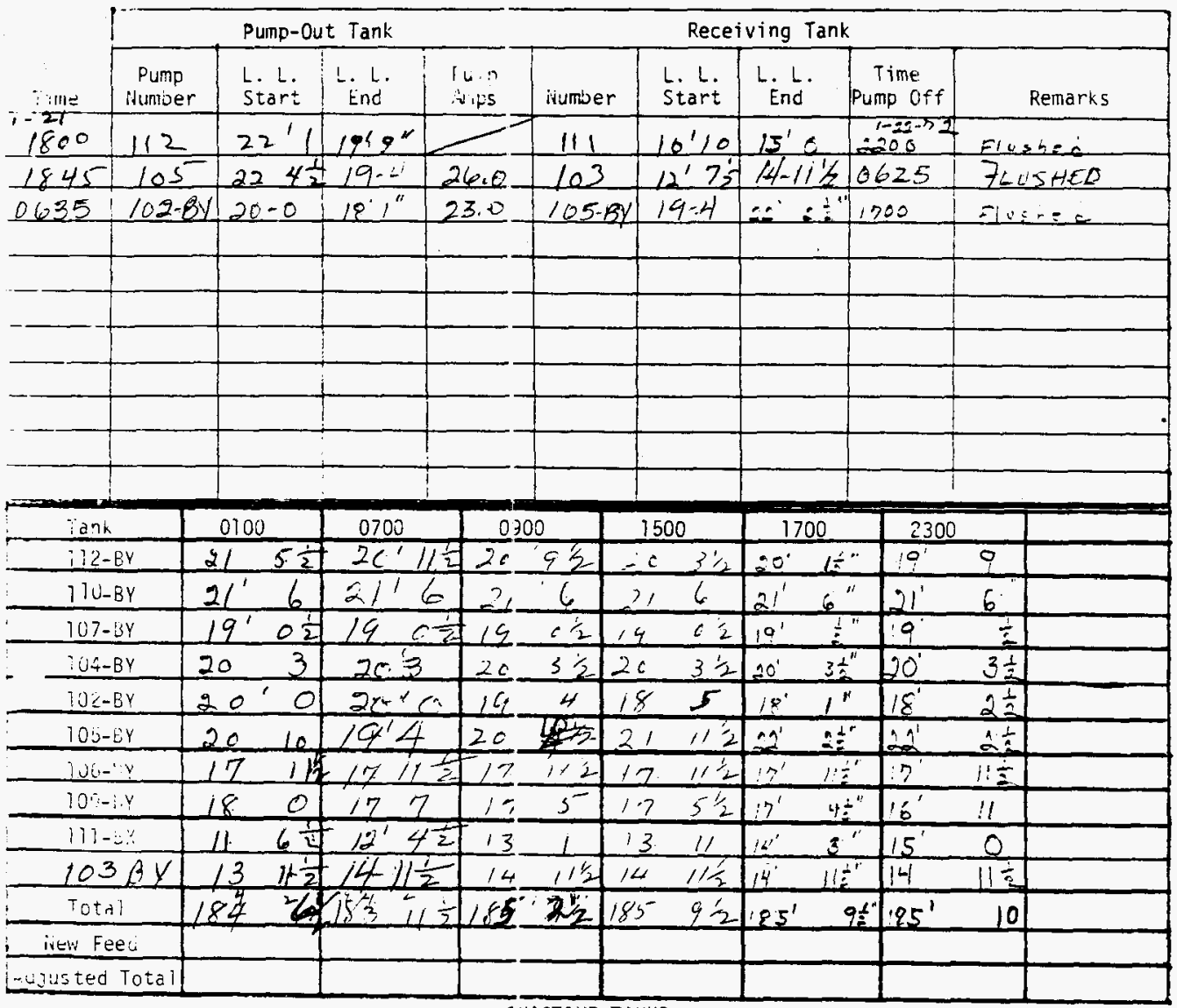

I IACTIVE TANKS

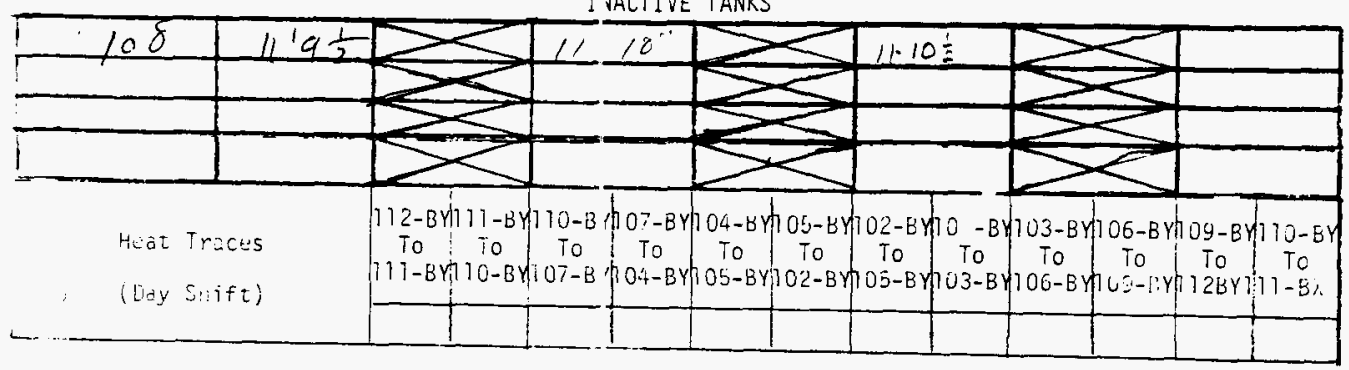




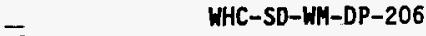

ZiS-2 IIVENTORY FIN PUMPING

Date

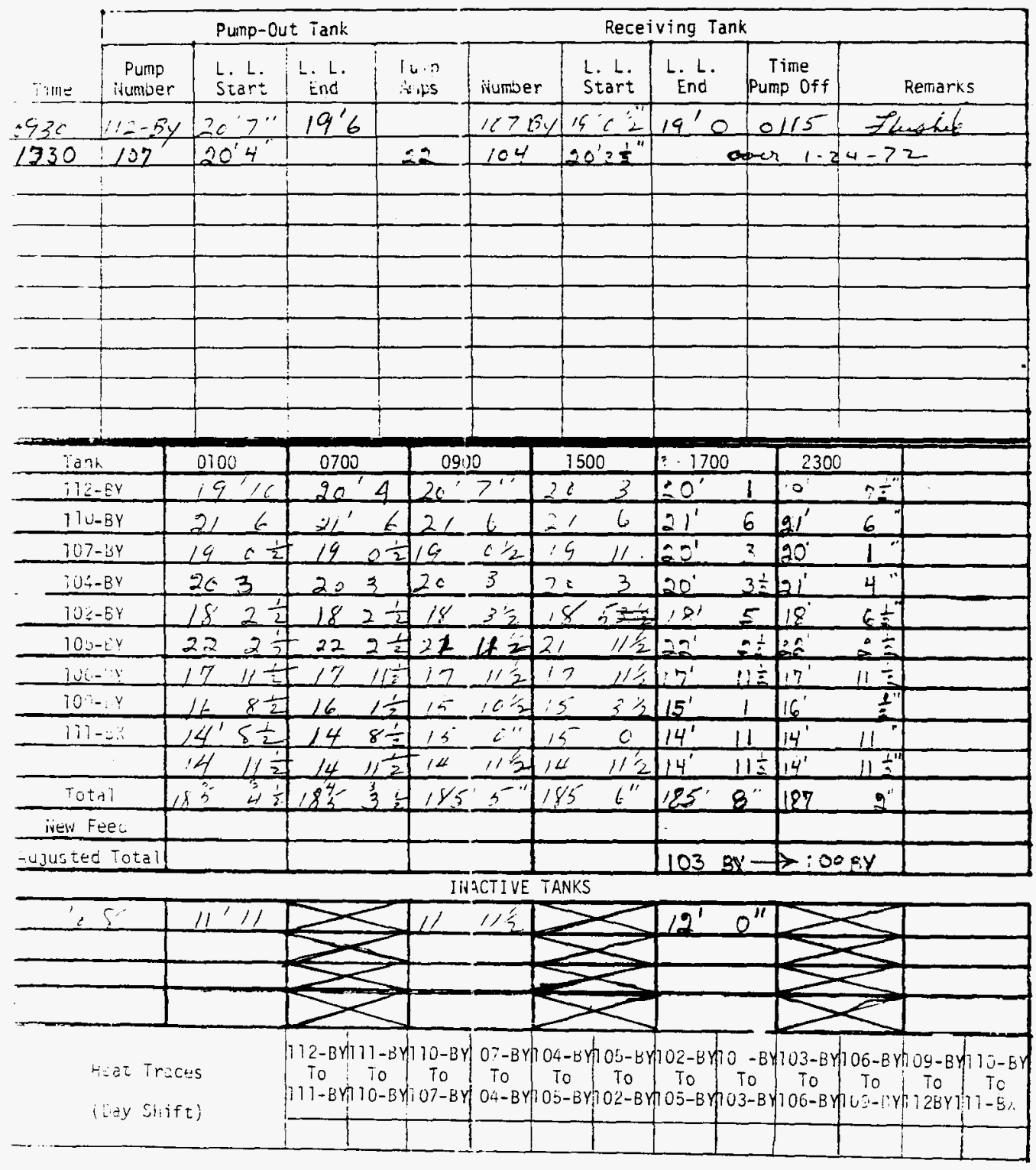

9:0:077 BEST AVAIIL $\wedge \sim$ COPY 


\begin{tabular}{|c|c|c|}
\hline \multirow[b]{2}{*}{$\because H E$} & \multicolumn{2}{|r|}{ Pump-Out Tank } \\
\hline & $\begin{array}{l}\text { Pump } \\
\text { Number }\end{array}$ & $\begin{array}{l}\text { L. L. } \\
\text { Start }\end{array}$ \\
\hline 1780 & 107 & $20^{\prime} 4: a^{\prime}, \frac{1}{2}$ \\
\hline 61000 & 104 & $22^{\prime}{ }^{\prime}+z^{\prime} x^{\circ}$ \\
\hline 13,2 & 103 & $15^{-} 6$ \\
\hline & & \\
\hline & & \\
\hline & & \\
\hline & & \\
\hline & & \\
\hline & & \\
\hline & & \\
\hline
\end{tabular}

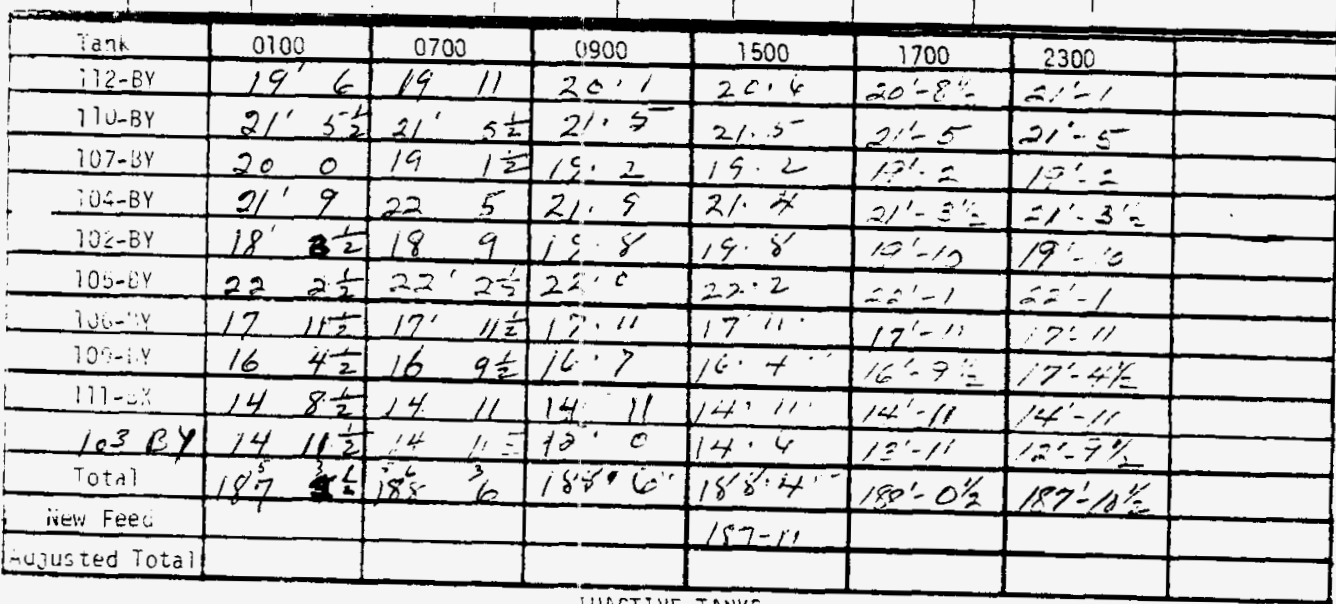

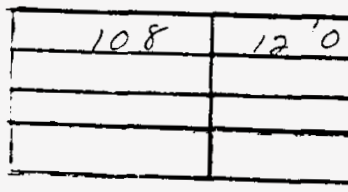

Heat Troces

(Liay Sitift)

INACTIVE TANKS

Receiving Tank

\begin{tabular}{l|l|l} 
L. L. L. L. & Time
\end{tabular}

Start End Pump off

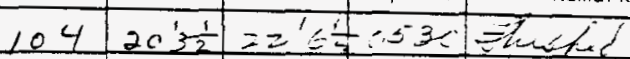

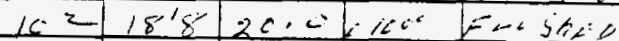
$16816 \cdot 2 \cdot$

.




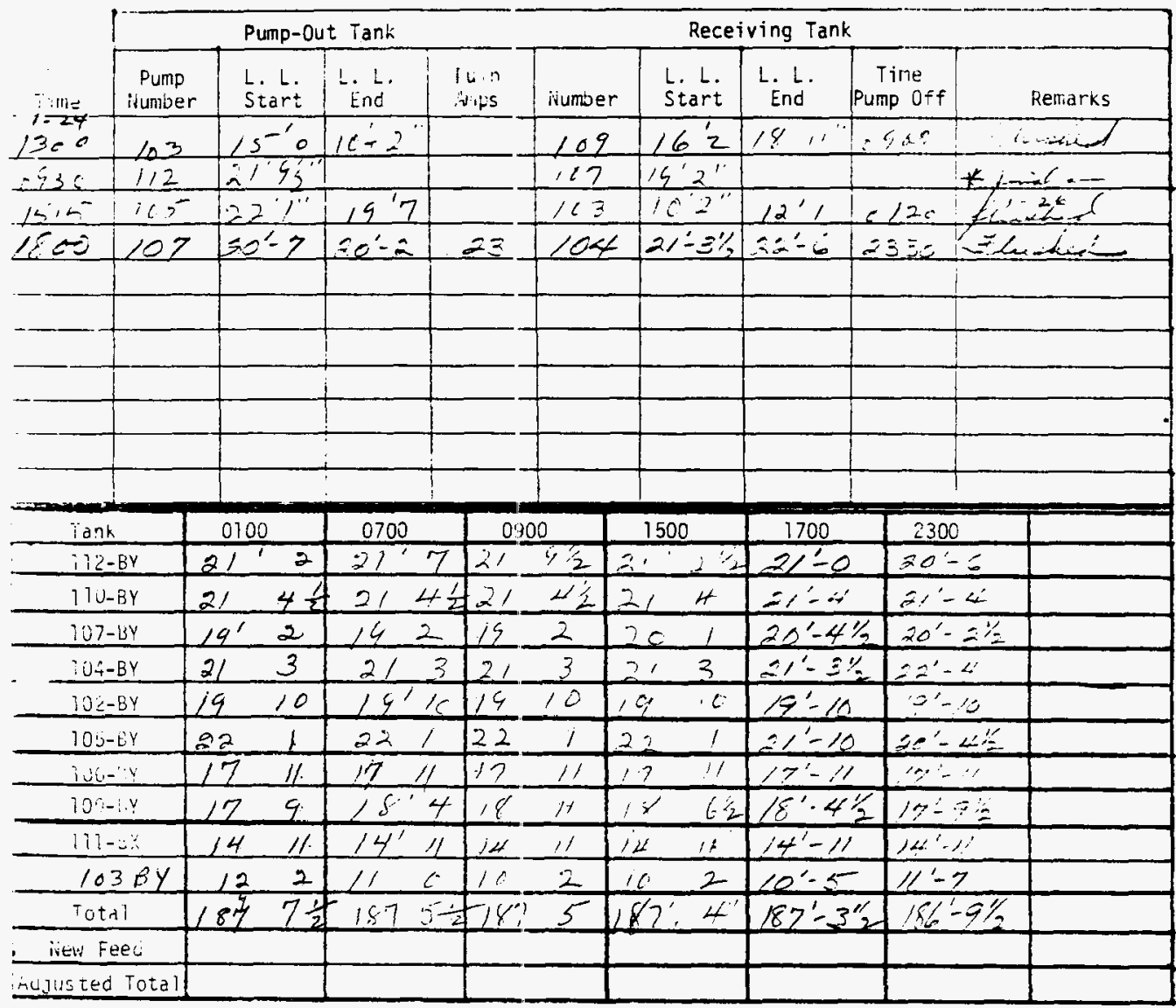

\section{IACT IVE TANKS}

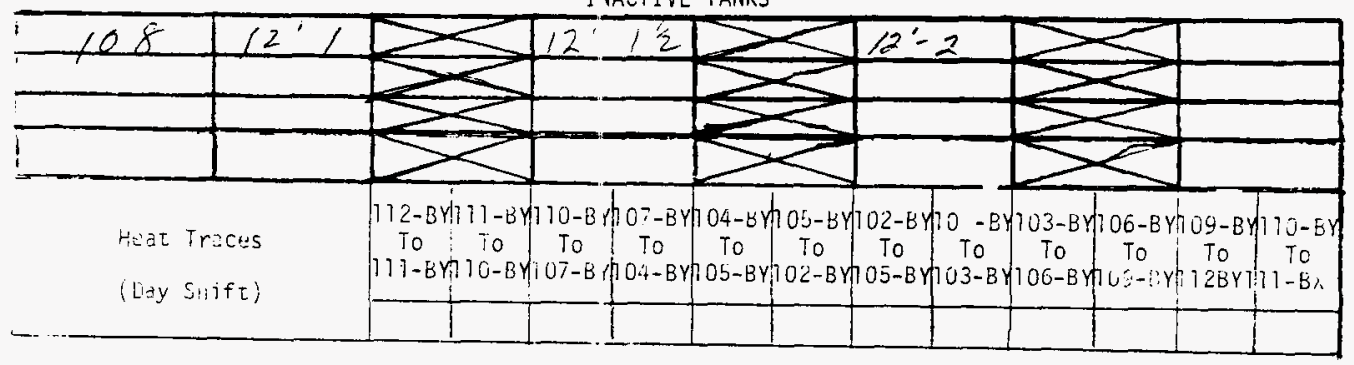




\section{Pump-out Tank}

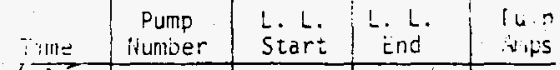

6930

- 25

$-1515$

0.125102

12

$213^{3}+22$

$22^{\prime}, 19^{\prime} ?$

0125102

$12^{\prime} \perp 1410^{\prime \prime}$

4600,124

$191018 \%$

$22^{\prime} 5129^{\prime} 11^{\prime \prime}$

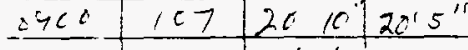

cy3o 112 20'7\% 1: ${ }^{\circ}$

1243

$226^{\prime \prime}$

\begin{tabular}{|c|c|c|c|c|}
\hline ivumer & $\begin{array}{l}\text { L. L. } \\
\text { Start }\end{array}$ & L. L. & $\begin{array}{c}\text { Time } \\
\text { Pump off }\end{array}$ & Remarks \\
\hline 107 & 192 & 9cis & 0315 & $=7 x \cos$ \\
\hline 10.3 & $10^{\prime} 2$ & 121 & $0 / 2$ & 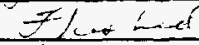 \\
\hline $10 \%$ & $17^{\prime} 7$ & $189 / 2$ & 0940 & $y_{i}=i$ - 1. \\
\hline 105 & 197 & 2253 & 1200 & 11 \\
\hline 102 & $18^{\prime} 4 \frac{1}{2}$ & $10^{i} 11^{i}$ & 1525 & $26+4=0$ \\
\hline 184 & $225^{\prime \prime}$ & $22,5 \%$ & 2360 & Eimentas \\
\hline 127 & $20^{\circ}, 0^{\prime \prime}$ & $20^{\prime} 5^{\prime \prime}$ & $23+6$ & $2 \alpha_{2}+t_{1} x_{2}$ \\
\hline 123 & $511^{\prime \prime}$ & & & $1-27-9 z$ \\
\hline
\end{tabular}

\begin{tabular}{|c|c|c|c|c|c|c|c|}
\hline anh & 0100 & 0700 & 0900 & 1500 & 1700 & 2300 & \\
\hline$i 12-8 Y$ & $20^{\prime} 42$ & 204 & $20-74$ & $2 c$ & $2 i^{11}$ & $1 a^{\prime} 7^{\prime}$ & \\
\hline $11 U-B Y$ & 21 & $21^{\prime} 4$ & 21 & 21 & $21^{\prime} 4$ " & $21^{\prime} 4 \cdots$ & \\
\hline $107-6 Y$ & $20 \quad 4=$ & 2010 & 26 & 20 & $20^{\circ} 5^{11}$ & $20^{\prime} 5^{\prime \prime}$ & \\
\hline$\therefore U \div-B Y$ & $=25$ & 223 & 225 & $2221 / 2$ & $22^{\prime} 0^{\prime \prime}$ & $225 \%$ & \\
\hline $10 \leq-5 Y$ & 19 & $18^{\prime} 9$ & +12 & 16 & $19^{\prime}-5$ & $19.10^{\prime \prime}$ & \\
\hline $100-e^{2}$ & 19 & $213 \frac{1}{2}$ & 21 & 22 & $22^{\prime} 0^{\circ}$ & $20^{\circ} 0^{\circ}$ & \\
\hline $7 v i-1$ & $17^{\prime} 11$ & 1711 & 11 & 12 & $17^{\prime} 11^{\prime \prime}$ & $17^{1} 11^{11}$ & \\
\hline $10-2 y$ & $\angle 7^{\prime} \quad 8$ & 182 & $15^{\prime}$ & 18 & $16^{\circ} 11 / 2$ & 121312 & \\
\hline $111-3.1$ & $14^{\circ} \quad 11$ & $14 \quad 11$ & 14 & 14 & $14^{\prime} 11^{\prime \prime}$ & $14^{1} 11^{\prime \prime}$ & \\
\hline $103 B Y$ & $1110 \frac{1}{2}$ & 1011 & 12 & 11 & $10^{\prime} 4^{\prime \prime 2}$ & $11^{\prime} 6$ & \\
\hline Total & $186 \quad 5 \frac{1}{2}$ & 186101 & $186-3$ & 865 & $186^{\prime} 3^{\prime \prime}$ & $1 S l^{\prime} k^{\prime \prime}$ & \\
\hline ivew Fees & & & & & & & \\
\hline Dujusted Total & & & & & & & \\
\hline
\end{tabular}

INACTIVE TANKS

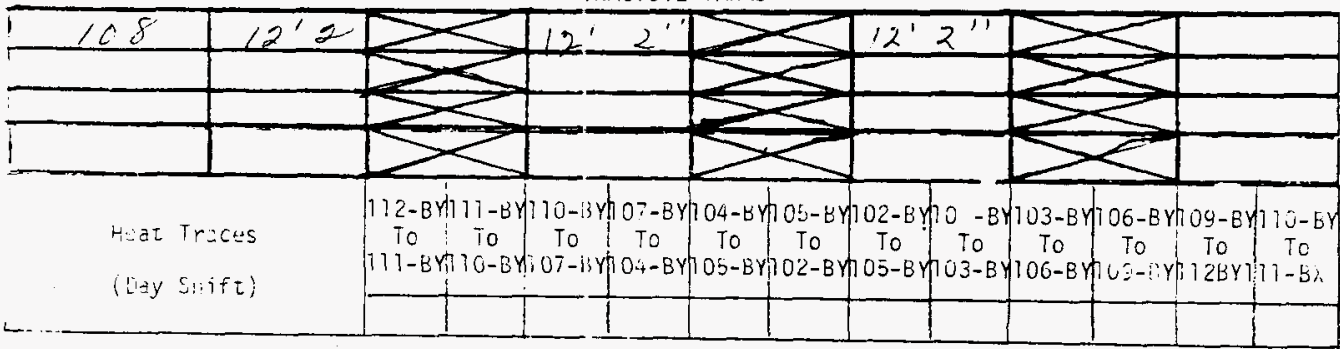




\section{- WHC-SD-WM-DP-206}

RLV 0

ITS-2 IIIVENTORY IIIU PUMPIIIG

Cate $1-22-4 z$

Pump-Out Tank

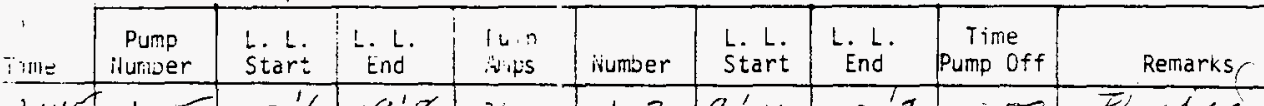

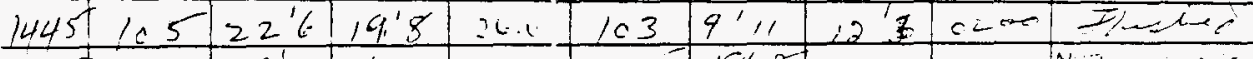

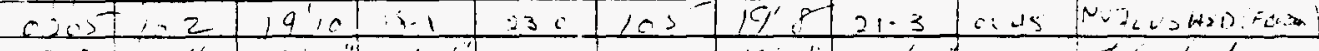

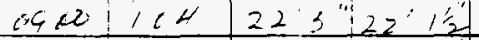

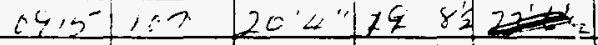

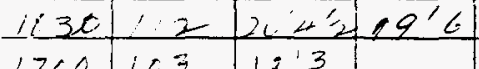
$-1760+103$ \begin{tabular}{l|l|l|}
2030 & 105 & 212 \\
\hline
\end{tabular} $=\therefore 1 / 07 \quad 20^{\circ} 20^{\prime} 5^{\prime \prime}$
25

$1<2$
$=16422^{\prime} 5^{\prime}=$ $25^{\prime \prime}=2 \sum \sum i^{\circ}$

I. 642
Thand

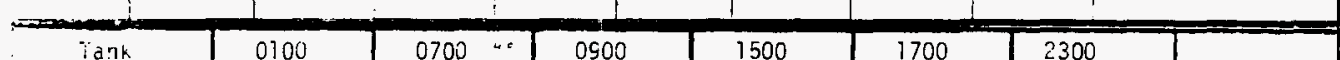

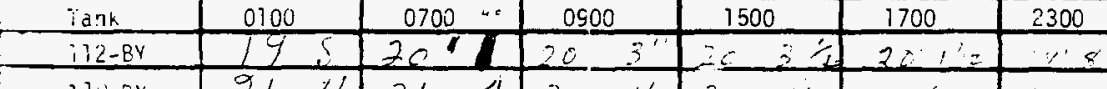
\begin{tabular}{l|ll|l|l|l|l|l|l|l}
\hline $11 U-B Y$ & 214 & 4 & 21 & 21 & 4 & 21 & 4 & 2,4 & 4
\end{tabular}

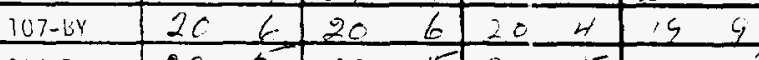
\begin{tabular}{l|ll|ll|l|l|l|l|l}
$104-B Y$ & 22 & 5 & 22 & 5 & 22 & 5 & 22 & $1 / 2$ & 22 \\
\hline
\end{tabular}

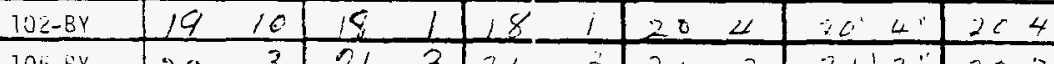

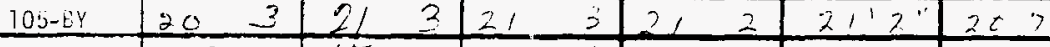

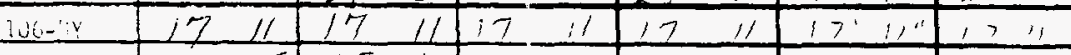

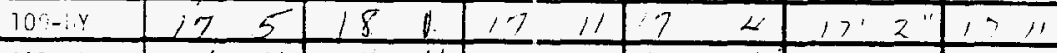

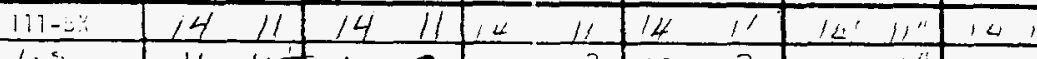

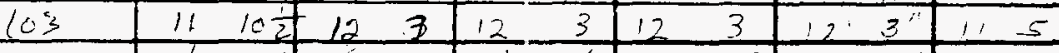

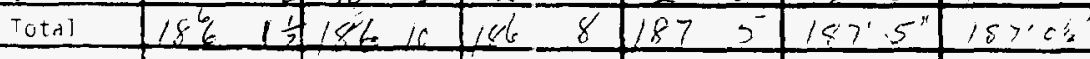
; ivew Feec aujusted Totai
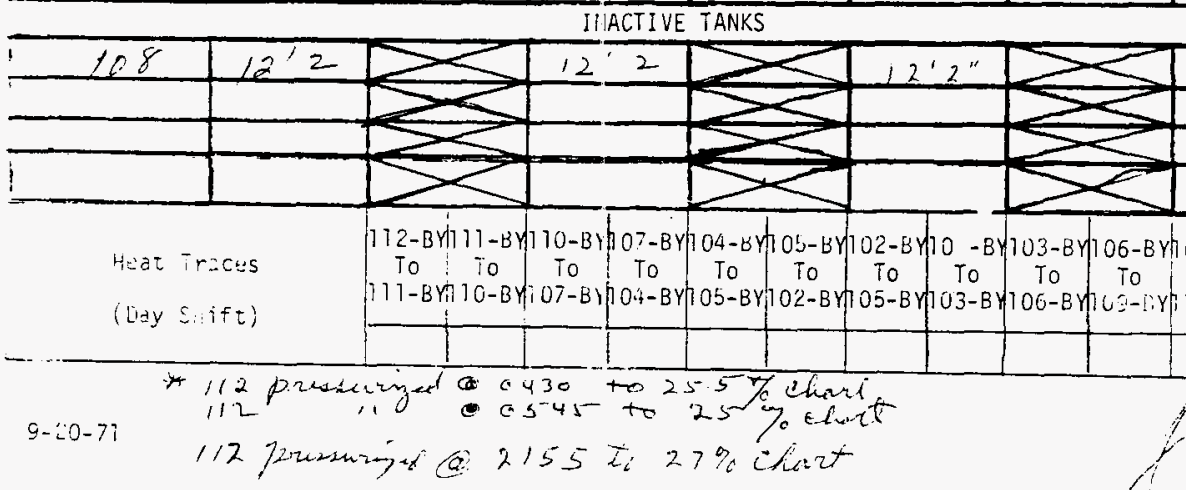
Pump-0ut Tank

\begin{tabular}{|c|c|c|c|c|c|c|c|c|c|}
\hline $7 m=$ & $\begin{array}{l}\text { Pump } \\
\text { Humioer }\end{array}$ & $\begin{array}{l}\text { L. L. } \\
\text { Start }\end{array}$ & E. L. L. & $\begin{array}{l}\text { Iun } \\
\therefore \text { ins }\end{array}$ & ivumber & $\begin{array}{l}\text { L. L. } \\
\text { Start }\end{array}$ & L. L. & $\begin{array}{c}\text { Time } \\
\text { Pump off }\end{array}$ & Remarks \\
\hline $\begin{array}{l}1-29 \\
1260\end{array}$ & 163 & $12^{\prime} 3$ & 1014 & & 169 & $17^{\prime} 2$ & $18^{\prime} 10$ & $03-4=$ & $J / L+L$ \\
\hline 2030 & tes & $2 / 2$ & $19 ; 2$ & & 103 & $11^{\prime} 4$ & $11^{\prime} 6$ & Eis & $\vec{J} L_{c}-1-d=d$ \\
\hline 0200 & LOE- & $20^{\prime} 4$ & & & $1=5$ & 19525 & & & \\
\hline $102=$ & 104 & $2 a^{\prime}-7$ & & $=1$ & $10=$ & $18: 5$ & & & \\
\hline 1115 & 105 & $22^{\prime}-1$ & 21,1, & $\approx 3.5$ & 103 & $10 \div 5$ & $12 \cdot 5$ & $223<$ & Eustes \\
\hline 1400 & 107 & $20: 10 \div$ & & $\therefore 3$ & $10 \div$ & $21-6:=$ & \multicolumn{3}{|c|}{ TPQnsposke'd To } \\
\hline 1726 & 112 & $20^{\prime} i^{\prime}$ & & & 107 & $20^{\circ} 2^{\circ}$ & 175 & \multicolumn{2}{|c|}{ INventoe: 1-24- } \\
\hline 2030 & 103 & $12^{\circ} \mathrm{C}$ & & & 109 & $17^{\prime} 7^{1 / 2}$ & & & \\
\hline
\end{tabular}

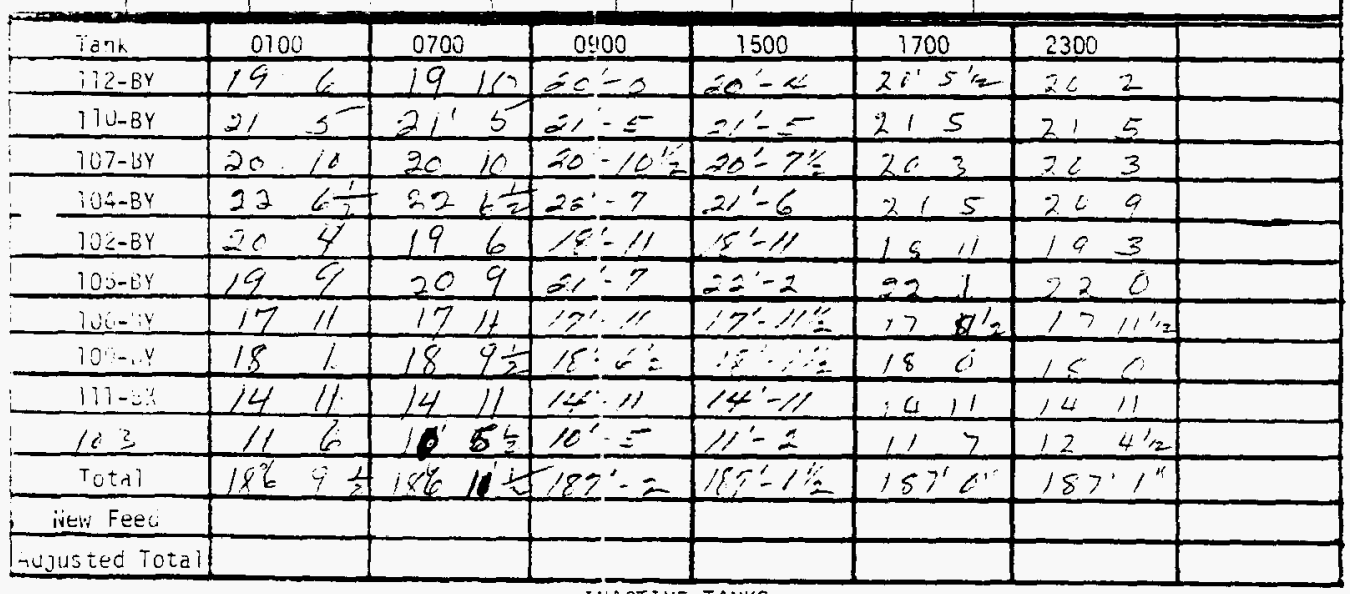

IIIACTIVE TANKS

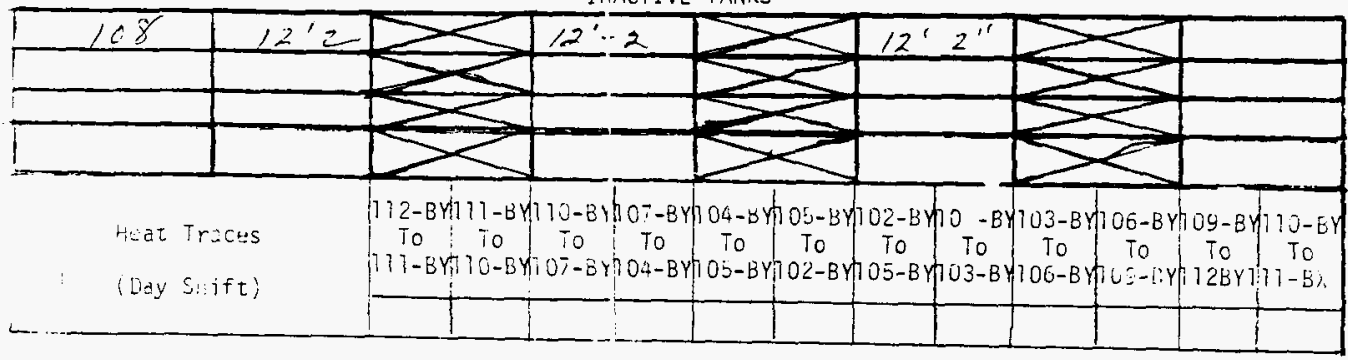


Pump L. L. I. L. L.

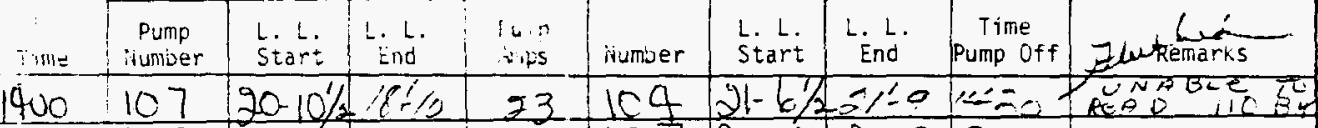

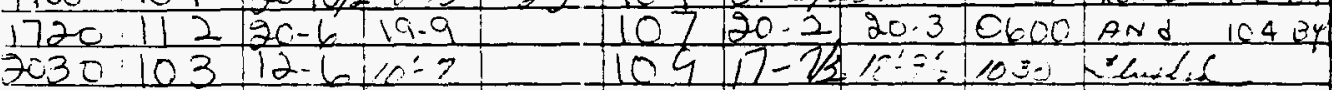

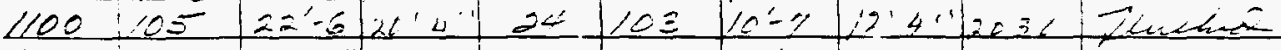
1520112 $1550+1: 3$

$\frac{261 " 6}{12 ! 5}$

Receiving Tank $16501 / 62 \quad 261 / 2$

AHNS Pused Io 26.5 inve wtop $y$ SHEeT $1-30-72$

\begin{tabular}{|c|c|c|c|c|c|c|c|}
\hline ank & 0100 & 0700 & 0400 & 1500 & 1700 & 2300 & \\
\hline i12-EV & 20 & $19-9$ & $19=$ & $60: 5$ & $26 \quad 5 \%$ & 204 & \\
\hline $11 \cup-\overline{ }$ & $81-5$ & $21-5$ & 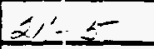 & $21-5$ & 21 & 215 & $T_{B}^{A} R^{P} \sum_{E}$ \\
\hline $107-54$ & $20-3$ & 20.3 & $20=0$ & $1: \because 2$ & 16 & $\div 5$ & \\
\hline$I U \div-B Y$ & $20-7$ & $20-4$ & 20.7 & $\div-1$ & 219 & 219 & \\
\hline $1010-5 y$ & $19-4$ & $2 c-c / 2$ & $300^{\circ}=$ & $5 e^{\prime}-c$ & 50 & 195 & \\
\hline $100-e y$ & $22-6$ & $22-6$ & $E=-6$ & $51-7$ & $211 / 2$ & 2110 & \\
\hline iving & $117-11$ & $17-11$ & $17 \%)$ & $\because \because \because$ & $17 \quad 11 / 1 / 2$ & $17111=$ & \\
\hline$\checkmark 10:-y$ & $\mid A-11$ & $18-$ & $10^{\prime}-r_{2}^{\prime}$ & $15: 4$ & $16 \quad 1 /{ }_{2}$ & $154 \%$ & \\
\hline $117-2$ & $14-11$ & $14-11$ & $14^{\prime} 10^{\prime}:$ & $14-2=$ & $1410^{1 / 2}$ & $14 \quad 1612$ & \\
\hline $103 B 6$ & $12-9$ & $11-6$ & $10^{\prime}: 8$ & $11=6 \%$ & 120 & $\therefore 10$ & \\
\hline Totai & $157=-2$ & $18 b-8$ & $186 \div 7$ & $\therefore 6 \div-7$ & $156^{\prime} 6^{\prime \prime}$ & 186512 & \\
\hline ivew Fee, & & & & & & & \\
\hline ujusted iotal & & & & & & & \\
\hline
\end{tabular}

IVACTIVE TANKS

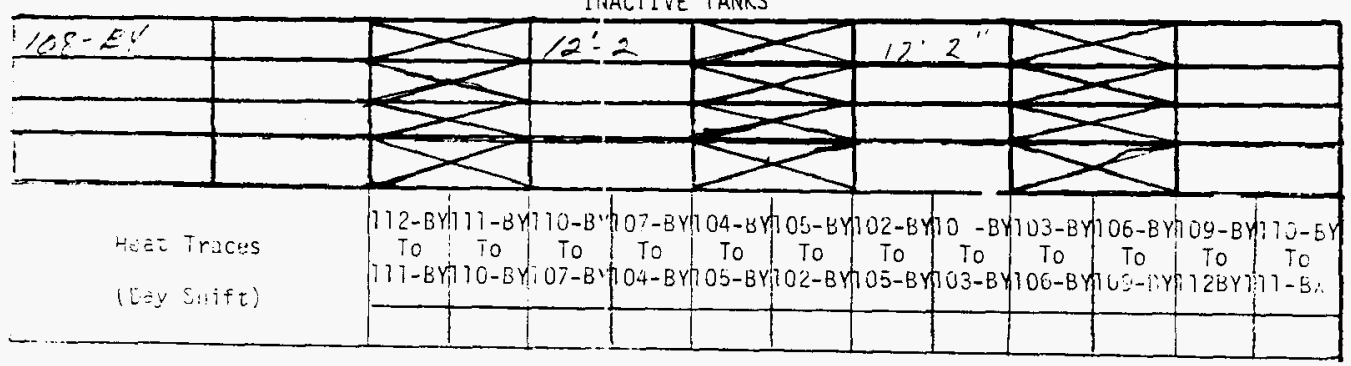




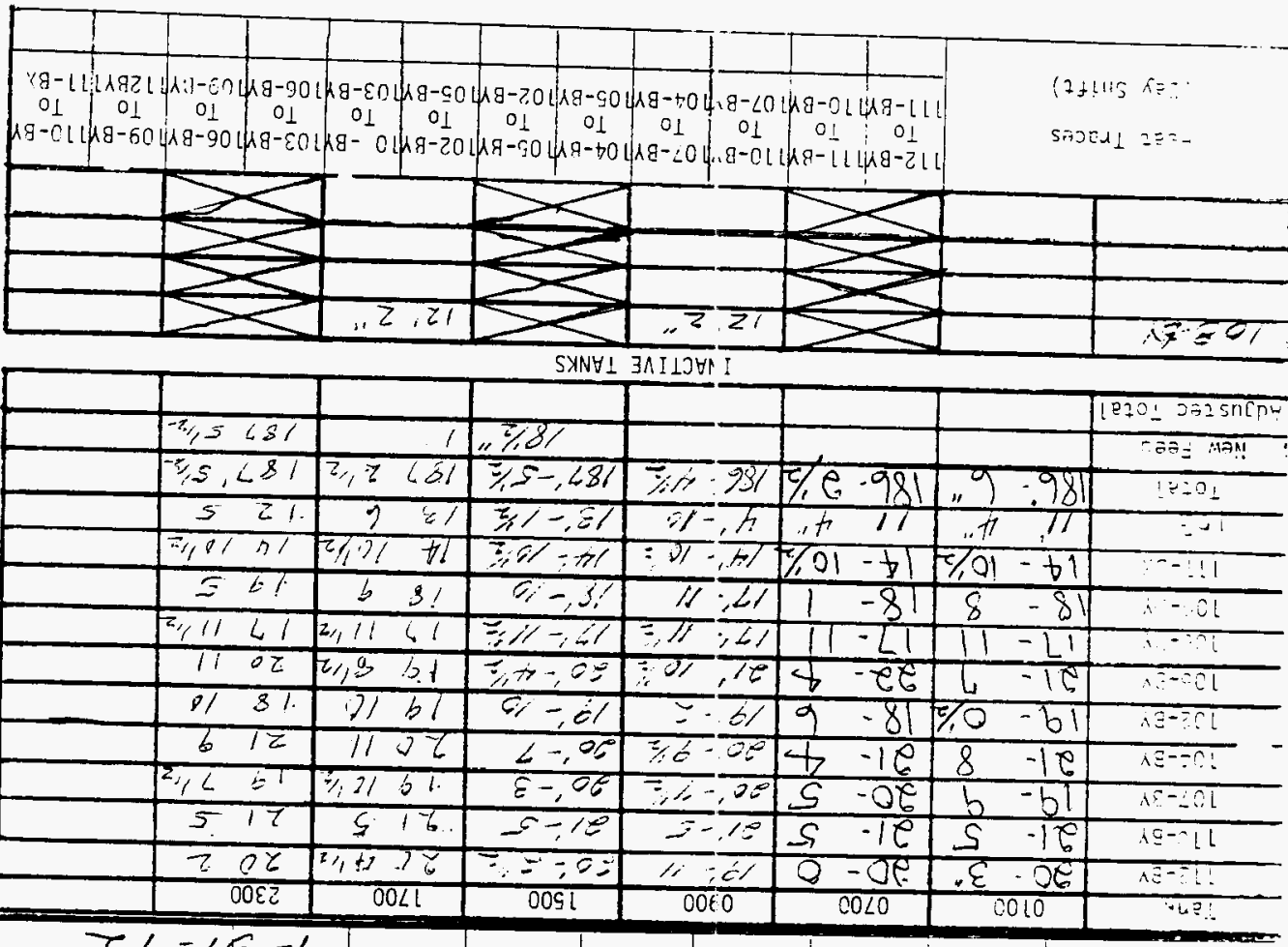

C<-1E-1

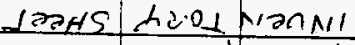

a) pasod ontyly

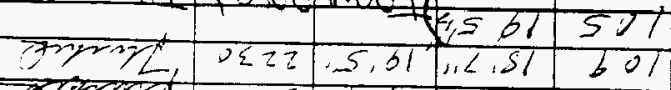

erpery?

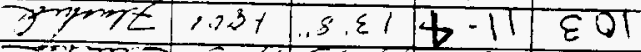

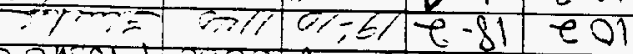

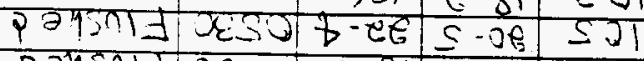

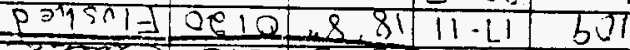

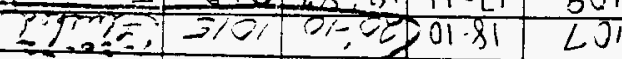

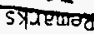

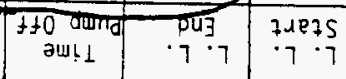

yue1 Бu!n!ลวәy

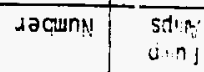

\section{1}

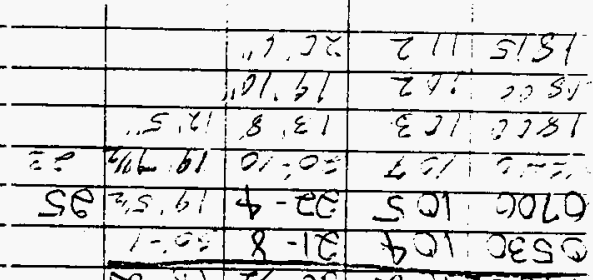

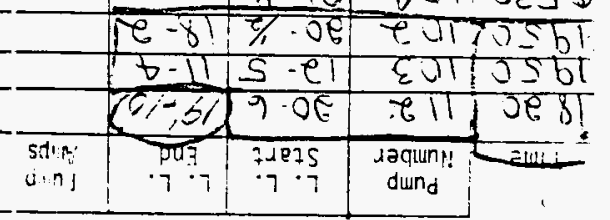
yue 1 zno-dund 
Punp-0ut Tank

Pump L. L. L.L. L I L. :

Number Start
End $1800110219^{\prime} 10018.0$

1815

0320

es3o

$\angle 209$

186116

1700

$196 \%$ 2 $22^{\prime} 45$

\section{Receiving Tank}

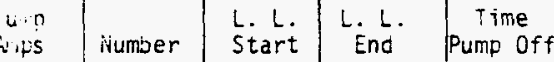

$105195 \% 22.20320$ Elosked

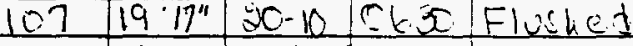

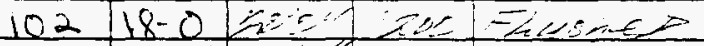

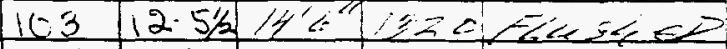
104 19' Cré 19'TIPANSPOSEd To $16917^{\prime} 9^{\prime \prime}$

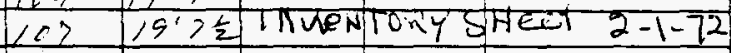

\begin{tabular}{|c|c|c|c|c|c|c|c|}
\hline Tank & 0100 & 0700 & 0900 & 1500 & 1700 & 2300 & \\
\hline$i Y Z-E Y$ & $20-0$ & 19.6 & 1981 & $201 \div 1$ & $21^{\circ} 3^{\prime \prime}$ & $2<\quad \dot{k}^{\prime \prime}$ & \\
\hline $11 \cup-\bar{B} Y$ & $21-$ & $21-5$ & 2 $z^{\prime \prime \prime}$ & $k 12^{-11}$ & 21,5 & 215 & \\
\hline $107-3 \gamma$ & $20-0$ & $2 c-1 d$ & $2818 \frac{1}{2}$ & $\operatorname{sic}^{\prime} y^{\prime}{ }^{\prime \prime}$ & 1911 & $1971 /=$ & \\
\hline $104-B Y$ & $21-9 \%$ & $21-0$ & $7^{\prime} b^{\prime \prime}$ & 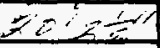 & 207 & 31.612 & \\
\hline $10<-5 y$ & $98-43$ & $18-8$ & $144^{\prime \prime \prime}$ & $20^{\prime} 2^{\prime \prime}$ & $1<5$ & 185 & \\
\hline $100-t^{2}$ & $21-7$ & $21-10$ & 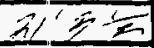 & 1775 & $=0 \quad 4$ & 2110 & \\
\hline $7 v c-1 y$ & $17-11 / 2$ & $17-11 \% / 2$ & $12^{\prime \prime}$ & 180 & $18^{\circ} c^{\circ}$ & 150 & \\
\hline $105-y$ & $19-3$ & $18-8$ & $5<2$ & $186 i^{\prime \prime}$ & 179 & 158 & \\
\hline $117-\infty$ & $1410 \%$ & $14-10 \%$ & $410=$ & in lo 10 & $11^{\circ} 7^{\prime \prime}$ & 14 & \\
\hline 103 & $12-51 / 2$ & 12 & 造要 & $\angle H^{\circ}$ is & $i$ & 1210 & \\
\hline Total & $187-83 / 4$ & $187-7$ & $18 \% 5$ & $28 r^{\prime}{ }^{2}=$ & $192^{\prime} 0^{\circ}$ & $1861+12$ & \\
\hline ivew Fee' & & & & & & & \\
\hline LuJusted Total & & & & & & & \\
\hline
\end{tabular}

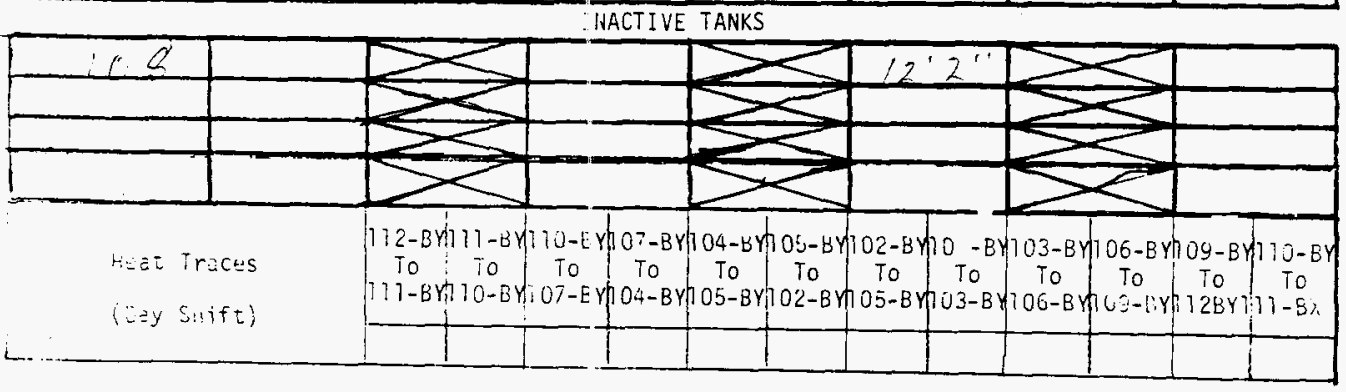


Pump-Out Tank

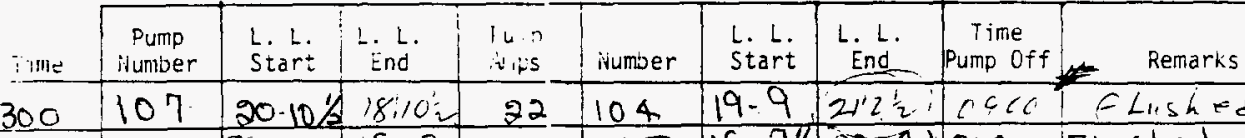
$1500: 102.20-0,18-0$, $\begin{array}{lllll}1700 & 103 & 14-6 & 11.8 \%\end{array}$ $1900 \quad 112 \cdot 20 \cdot 4 \% 19-5 \% / 2$ 0100104.22 .0121212 $0340 \quad 105.22-3 / 319^{\prime} 4$ $13301102 \quad 20^{\prime} 0: 18^{\prime 3}$ $1860103 B X$ $2100112-20^{\prime} 6^{\prime \prime}$ \begin{tabular}{l|l|l|}
2245 & 24 & $212 / 2$ \\
\hline
\end{tabular}
Receiving Tank

$105 \quad 19-73222=4$ d 0100 Flushed $10917-9$ \%9-4 10330 . Flushed $107 \quad 19-7 / 19-4) 520$ Flushed $10218-0 \mid 201018901=1 \mathrm{msl} d$ $103-11-8 / 2 / \pi^{1 / 2}-1320$ $10514^{\prime 4} 21^{\prime} 4^{\prime \prime} 2220$ Funder $10918^{\prime} 1^{\prime} 27^{\prime} 8^{\prime} 8^{\prime \prime}-150$ 107 is iź Transposed 70 $10218^{\prime} 3^{\prime \prime}$ iventain street 2-2-72

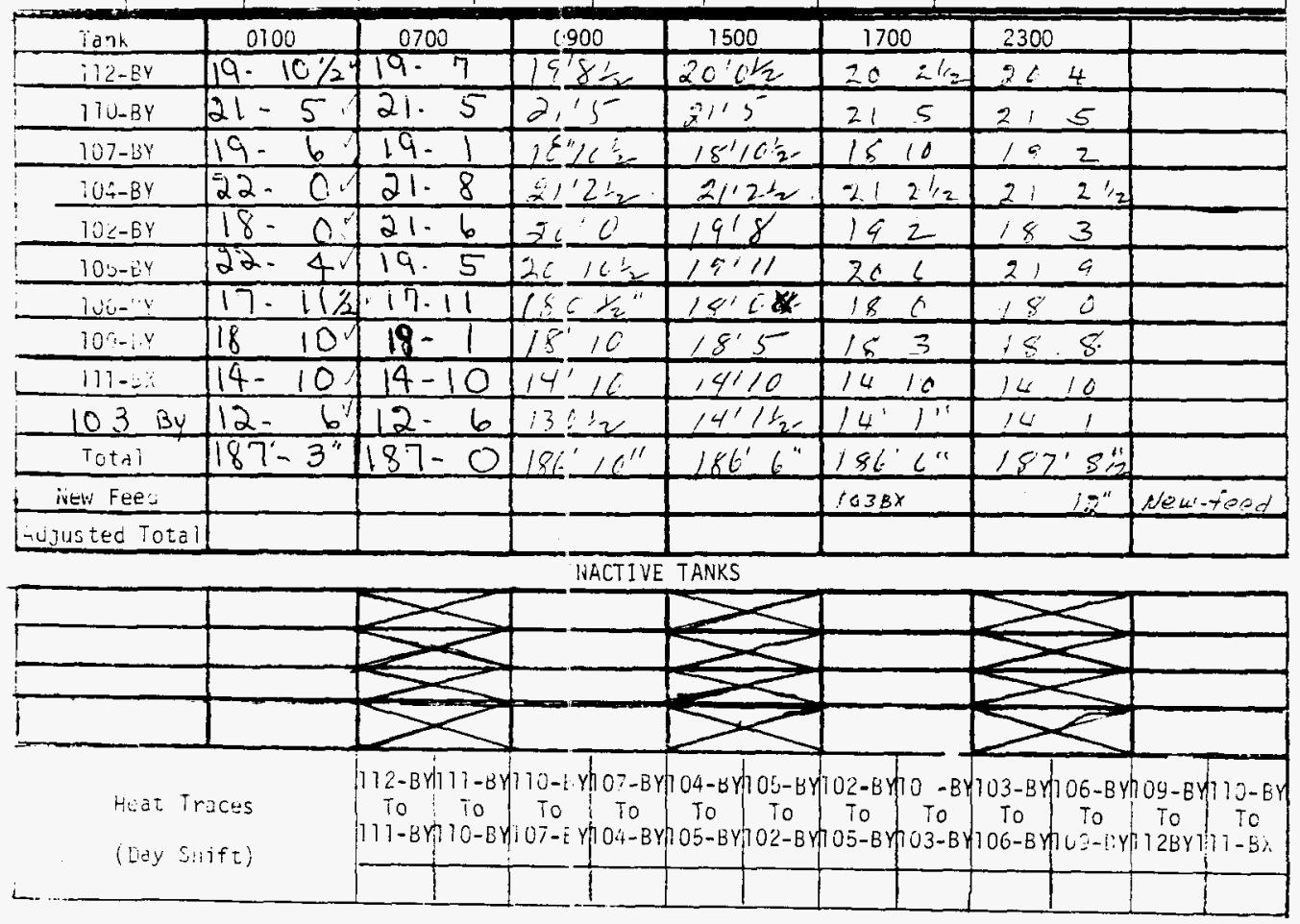


Date $2-2-7 \ddot{2}=$

Pump-0ut Tank

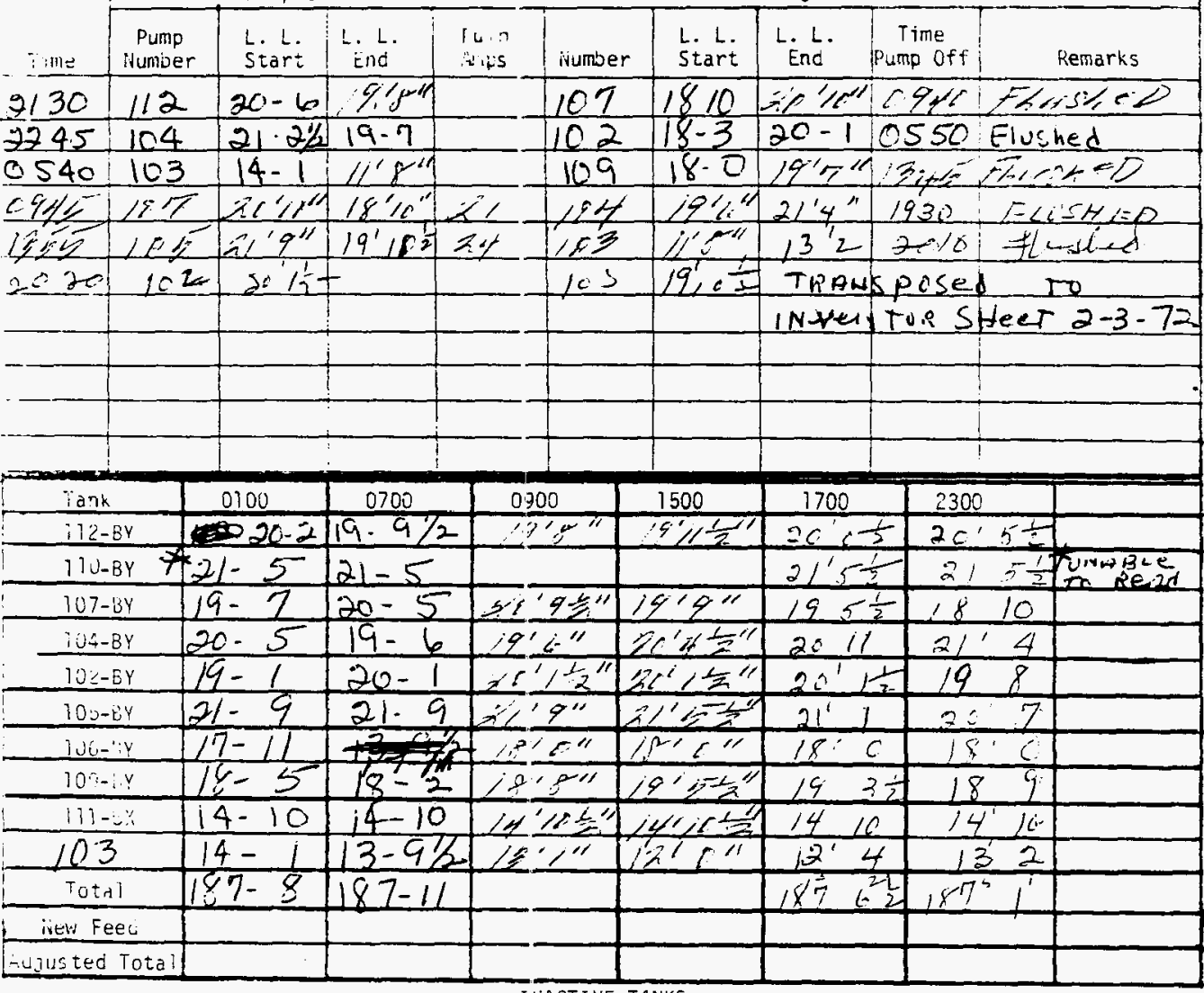

I: TACTIVE TANKS

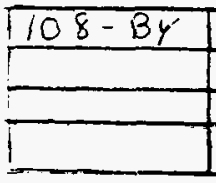

Heat iraces

( Day $\operatorname{sifift}$

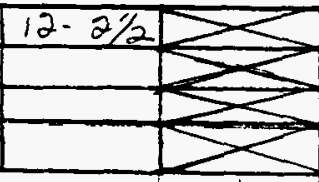

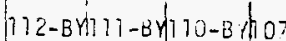

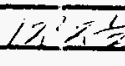
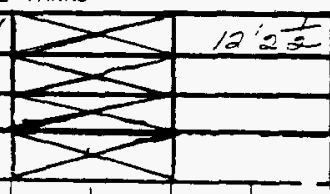

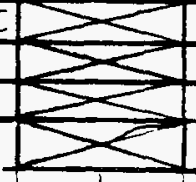

To

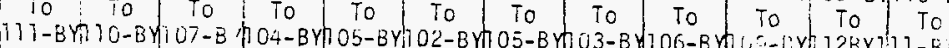

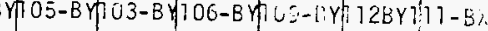

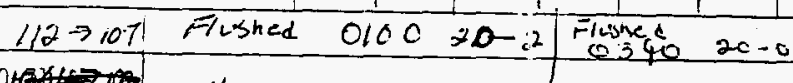

$9-\because 9047$

BES"-AVALLABLE COPY 
WHC-SD-WM-DP-206 $\mathrm{ReVO}$

cate $2-3-72$

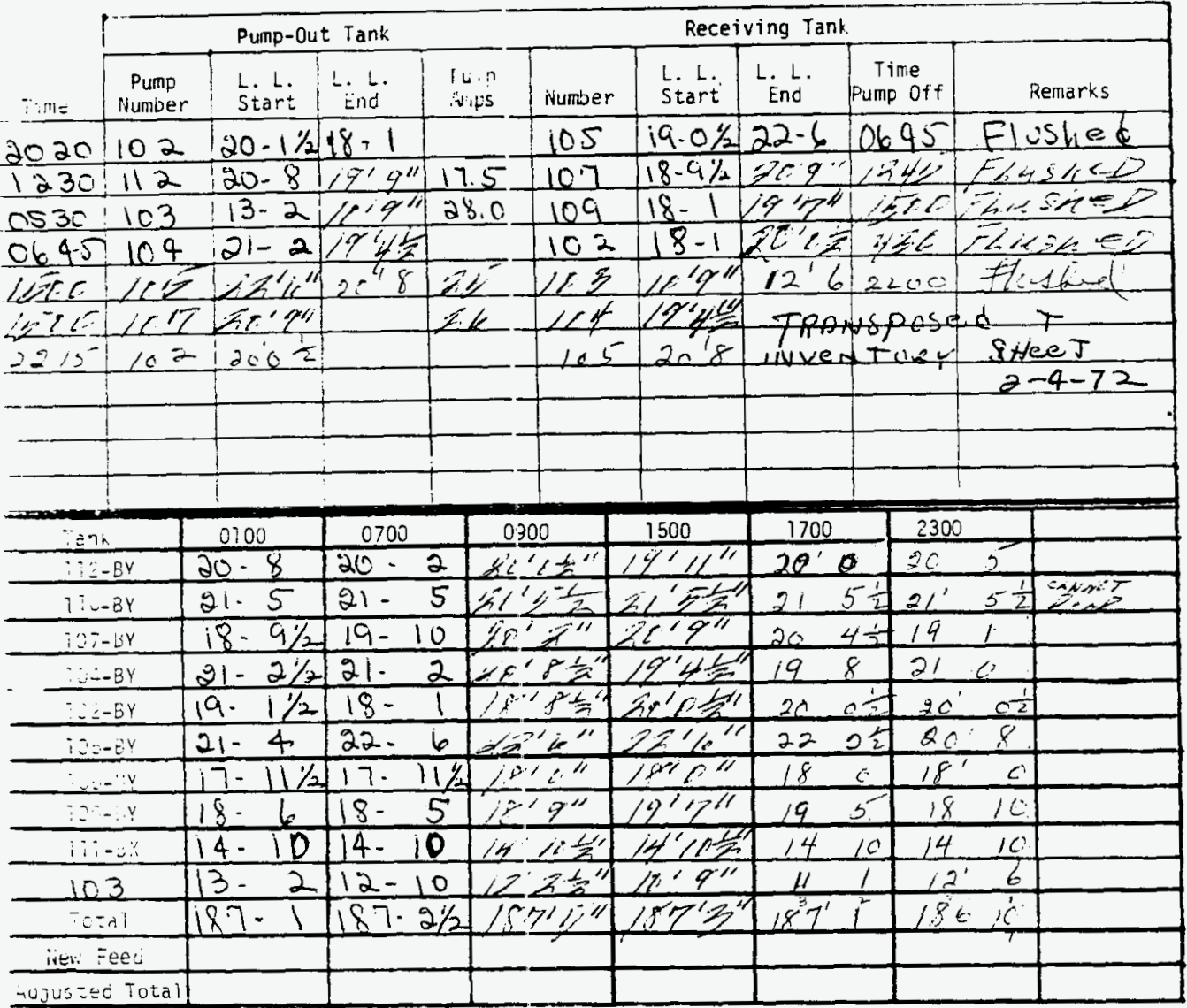

I TACTIVE TANKS

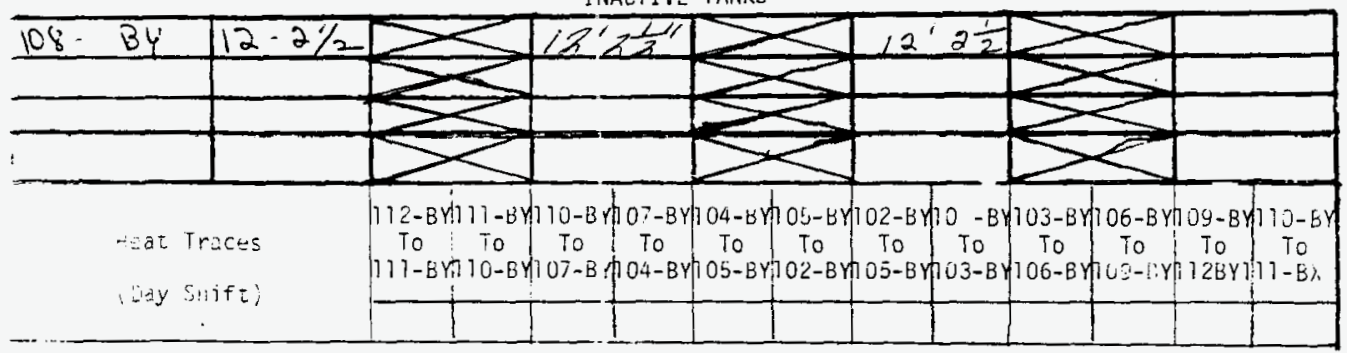




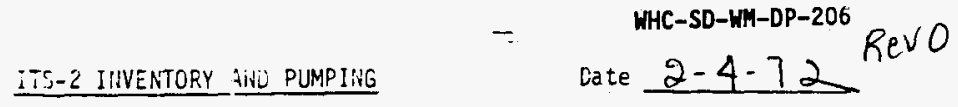

Pump-Out Tank

Receiving Tank

\begin{tabular}{|c|c|c|c|c|c|c|c|c|c|}
\hline mis & $\begin{array}{l}\text { Pump } \\
\text { Number }\end{array}$ & $\begin{array}{l}\text { L. L. } \\
\text { Start }\end{array}$ & E. L. & $\begin{array}{l}f \text { lon } \\
\text { dips }\end{array}$ & ivumber & $\begin{array}{l}\text { L. L. } \\
\text { Start }\end{array}$ & End & $\begin{array}{c}\text { Time } \\
\text { Pump off }\end{array}$ & Remarks \\
\hline 500 & 107 & 20.9 & 18.8 & 26 & 104 & $19.4 \%$ & $2 i-4$ & $01 \infty$ & Flushed \\
\hline 2215 & 102 & $20-c \%$ & 18.6 & & 105 & 20.8 & 22.5 & 9600 & Elusked \\
\hline Q 100 & 112 & $50-7$ & $20^{\prime}-0$ & $i 7$ & 107 & $18-8$ & $19^{\prime} 11$ & 1500 & $x<x<x$ \\
\hline 0615 & 10,4 & $21-4$ & $19-10$ & & 102 & $18-6$ & $20^{\circ}-0^{\prime}$ & 1238 & 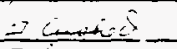 \\
\hline & 107 & $19: 11$ & $19^{\circ} 32$ & & 104 & $19^{\prime \prime 1}$ & $22^{\prime} 7$ & 1036 & 74006 \\
\hline 2100 & 112 & $200^{\prime} 6$ & $19^{\circ}:$ & & 107 & 193 & $192^{\circ}$ & $11=:$ & $-\ldots,-$ \\
\hline
\end{tabular}

\begin{tabular}{|c|c|c|c|c|c|c|c|}
\hline iank & 0100 & 0700 & $0 \leq 00$ & 1500 & 1700 & 2300 & \\
\hline$i 32-8 V$ & $20-7$ & $20-3$ & $20^{\prime} 1^{\prime \prime}$ & $20^{\prime} 1^{\prime \prime}$ & 20 & $20=$ & \\
\hline $1 T U-8 Y$ & $21-5$ & $21-5$ & $21^{\prime} 5^{\prime \prime}$ & $=12$ & $215 \frac{1}{2}$ & $21^{\prime}$ & \\
\hline $307-3 Y$ & $18-8$ & $19=$ & $14 " 16 "$ & +411 & 19 & $194 \frac{1}{2}$ & \\
\hline$Z U \div-B Y$ & $21-4$ & $21-$ & $\geq 1$ & 9.11 & 11 & $20 \quad 8$ & \\
\hline $10 \div-B Y$ & $19-4$ & $18=$ & $18^{5} 46$ & $=0^{\prime} 0^{\prime \prime}$ & 200 & $20^{\prime} 0$ & \\
\hline $100-0 y$ & $21-6$ & $92=$ & $\Sigma^{\prime} E^{\prime \prime}$ & $=25^{\prime \prime}$ & 225 & $2=5$ & \\
\hline ivén & $17-11 / 2$ & 17. & $1^{\prime}, \quad 11 \leq 0$ & 1711 & $17 \quad 11$ & $17 \quad 11$ & \\
\hline $101-i . y$ & $18-8$ & 18. & $17: 11$ & 178 & $174 \frac{1}{2}$ & $16 \quad 10$ & \\
\hline $117-2 \%$ & $14-10$ & $19-10$ & $44^{\prime}, 0^{\prime \prime}$ & $14^{\prime} 10^{\prime \prime}$ & 1410 & $14 \quad 10$ & \\
\hline 103 & $12 \cdot 6$ & $12-6$ & 12.6 & $12^{\circ} \cdot 6^{\prime \prime}$ & 126 & 126 & \\
\hline Total & $186-9$ & $186-8$ & $185^{\prime}-10^{\prime \prime}$ & $136^{\prime}-83$ & $1 \times 67$ & 1865 & \\
\hline iiew Feeci & & & & & & & \\
\hline rujusted Total & & & & & & & \\
\hline
\end{tabular}

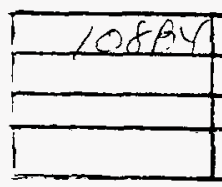

Heat Traces

( Day Sitifi)
IIIACTIVE TANKS
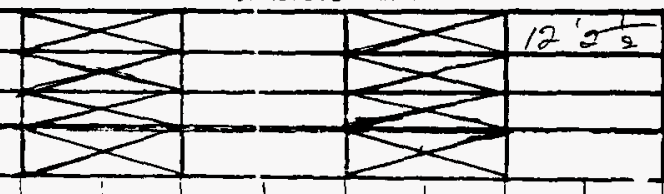

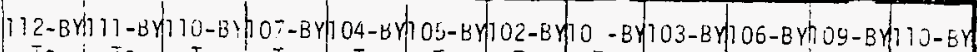
To to to To to To To To To To to to to to

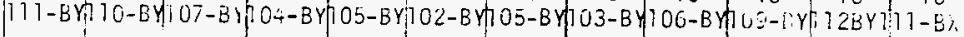




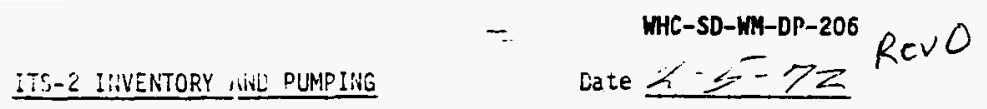

\begin{tabular}{|c|c|c|c|c|c|c|c|c|c|}
\hline & \multicolumn{4}{|c|}{ Pump-Out Tank } & \multicolumn{4}{|c|}{ Receiving Tank } & \\
\hline me & $\begin{array}{l}\text { Pump } \\
\text { Nuniber }\end{array}$ & $\begin{array}{l}\text { L. L. } \\
\text { Start }\end{array}$ & $\begin{array}{l}\text { 1. L. } \\
\text { End }\end{array}$ & $\begin{array}{l}\text { lin } \\
\text { inips }\end{array}$ & ivumier & $\begin{array}{l}\text { L. L. } \\
\text { Start }\end{array}$ & L. L. & $\begin{array}{l}\text { Time } \\
\text { Pump off }\end{array}$ & Remarks \\
\hline 216.6 & 112 & $1 x^{\prime} 2=\frac{1}{5}$ & 1612 & 27 & 107 & $127 \leq$ & $18 \%$ & icise & Eleuzhed \\
\hline 638 & 105 & 2215 & $19=$ & & 163 & $10 \%$ & $129^{\circ}$ & 二 & - $\ldots \ldots \ldots$ \\
\hline $2 i \leq r$ & is 2 & $26^{\prime} \div$ & 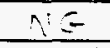 & & $i<5$ & $\angle \dot{G}^{\prime} \ddot{i}$ & $21 \quad 10 \%$ & $05 \times 5$ & $51-6]$ \\
\hline 2250 & 103 & $12^{\prime} 4$ & $10^{\prime} 9$ & & 109 & 19110 & Puppiog & in- & $\therefore$ \\
\hline & & & & & & & & & \\
\hline & & & & & & & & & \\
\hline & & & & & & & & & \\
\hline & & & & & & & & & \\
\hline & & & & & & & & & \\
\hline & & & & & & & & $\sim$ & \\
\hline & & & & & & & & & \\
\hline
\end{tabular}

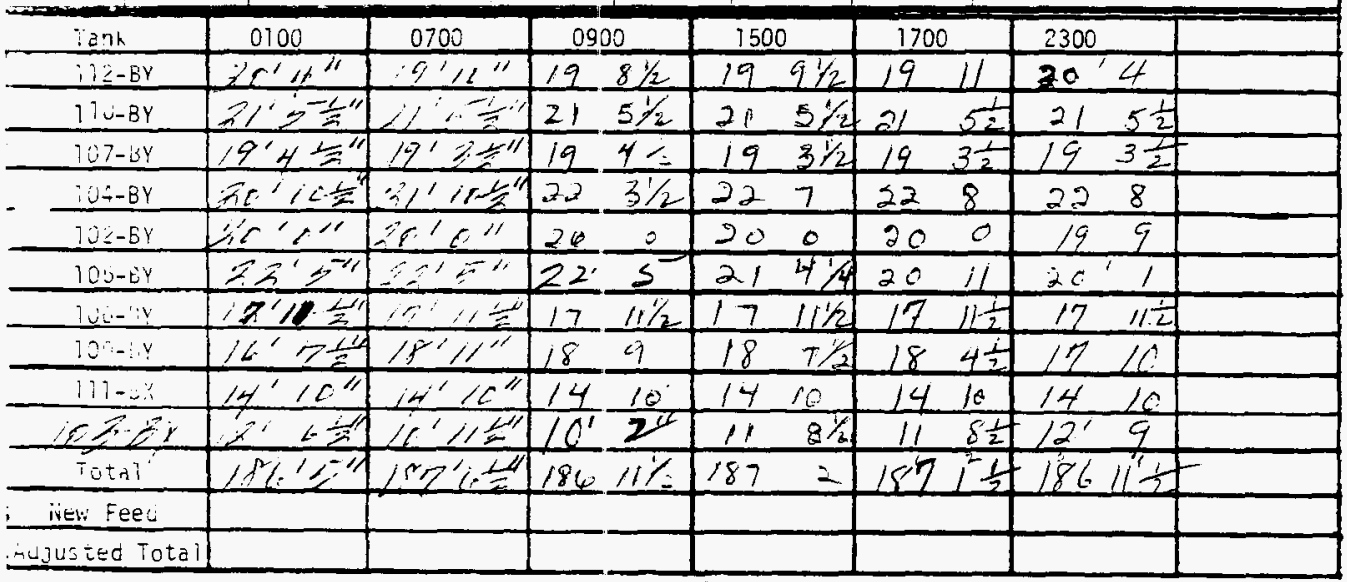

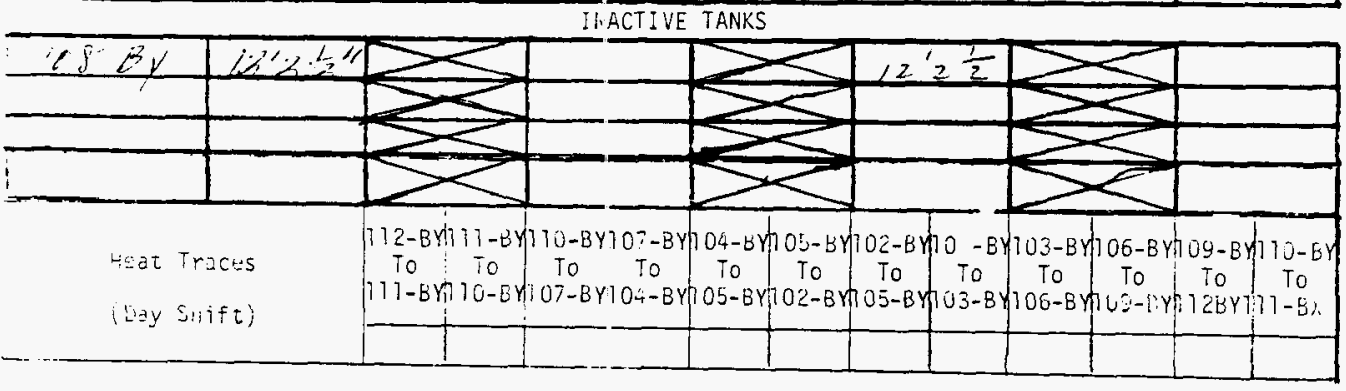




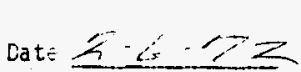

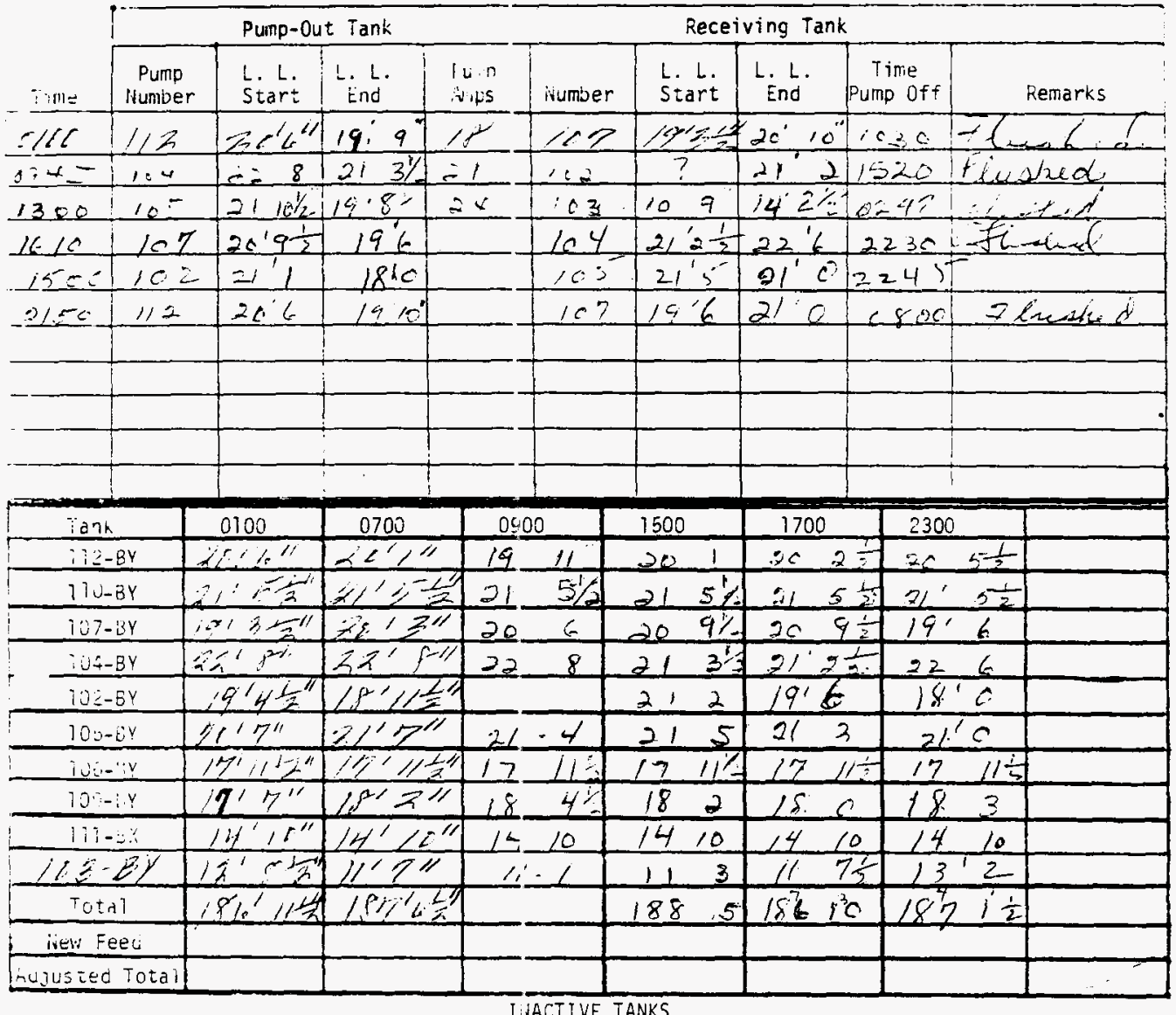

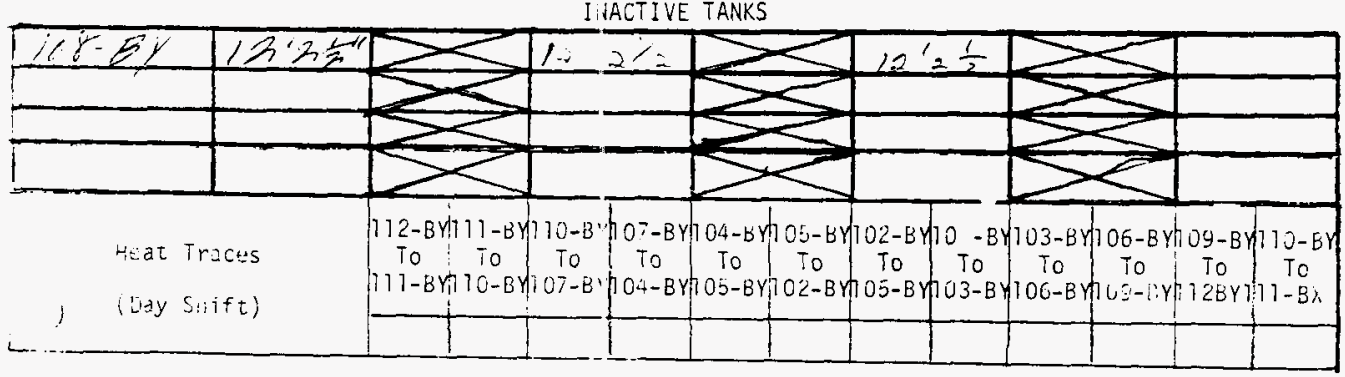




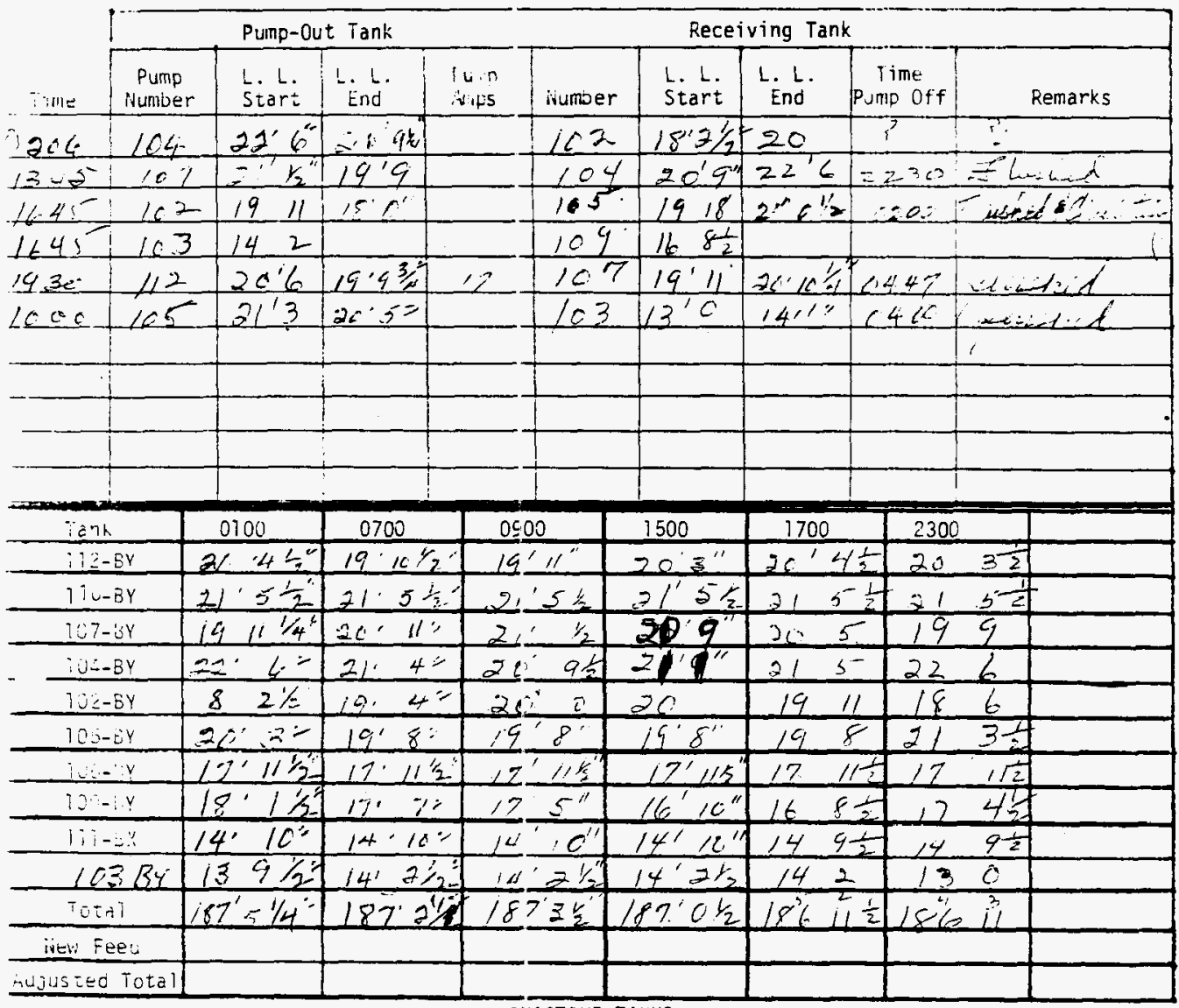

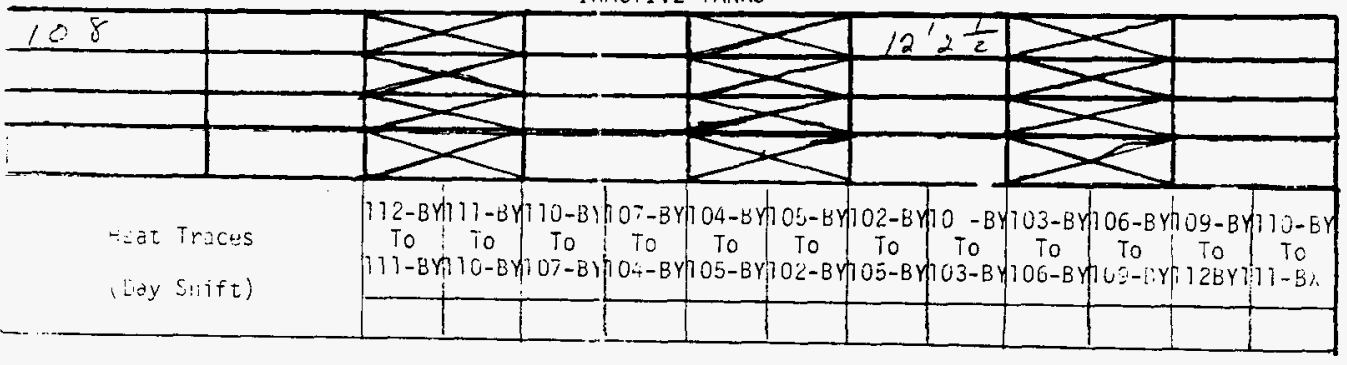




\begin{tabular}{|c|c|c|c|c|c|c|c|c|c|}
\hline \multirow[b]{2}{*}{ yinte } & \multicolumn{5}{|c|}{ Pump-0ut Tank $2 \alpha^{\prime} C^{\prime \prime}$} & \multicolumn{3}{|c|}{ Receiving Tank } & \multirow[b]{2}{*}{ Remarks } \\
\hline & $\begin{array}{c}\text { Pump } \\
\text { ivuniver }\end{array}$ & $\begin{array}{l}\text { L. L. } \\
\text { Start }\end{array}$ & $\begin{array}{c}\text { 1. } L . \\
\text { End }\end{array}$ & $\begin{array}{l}\text { I Li, n } \\
\text { inips } \\
\end{array}$ & isumber & $\begin{array}{l}\text { L. L. } \\
\text { Start }\end{array}$ & $\begin{array}{l}\text { L. L. } \\
\text { End }\end{array}$ & $\begin{array}{c}\text { Time } \\
\text { Pump off }\end{array}$ & \\
\hline$(50)$ & $\therefore<4$ & $=2^{\circ} 6^{\prime \prime}$ & $5 \div$ & 21 & 16281 & $18: 0^{\prime \prime}$ & $20^{\circ} \circ$ & 1306 & $\pm \operatorname{lng} h$ \\
\hline 1310 & 107 & $20 \quad 10 \frac{1}{2}$ & & 23 & $i c x$ & $20=1 / 2$ & $22 ; 7=1$ & $\angle<\approx<1$ & \\
\hline 1510 & $11=$ & $2 c^{\circ} \prime \prime$ & $19^{\prime} c^{\circ}$ & 22 & $\therefore=7$ & $20 \div 2$ & $20^{\prime} 7^{\prime}$ & in 4 ic & 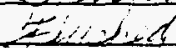 \\
\hline 1634 & 102 & $20^{\circ} 0$ & $18^{\prime} 0^{\prime \prime}{ }^{\prime}$ & & 105 & $20^{15 \frac{1}{2}}$ & in fir & 1255 & \\
\hline $163 c$ & $105^{5}$ & $2 c^{\prime}=\frac{1}{5}$ & $19^{\prime} 6^{\prime \prime}$ & & 103 & $12^{\prime} 4$ & $14^{\prime} 21$ & 1465 & \\
\hline & & & & & & & & & -5 \\
\hline
\end{tabular}

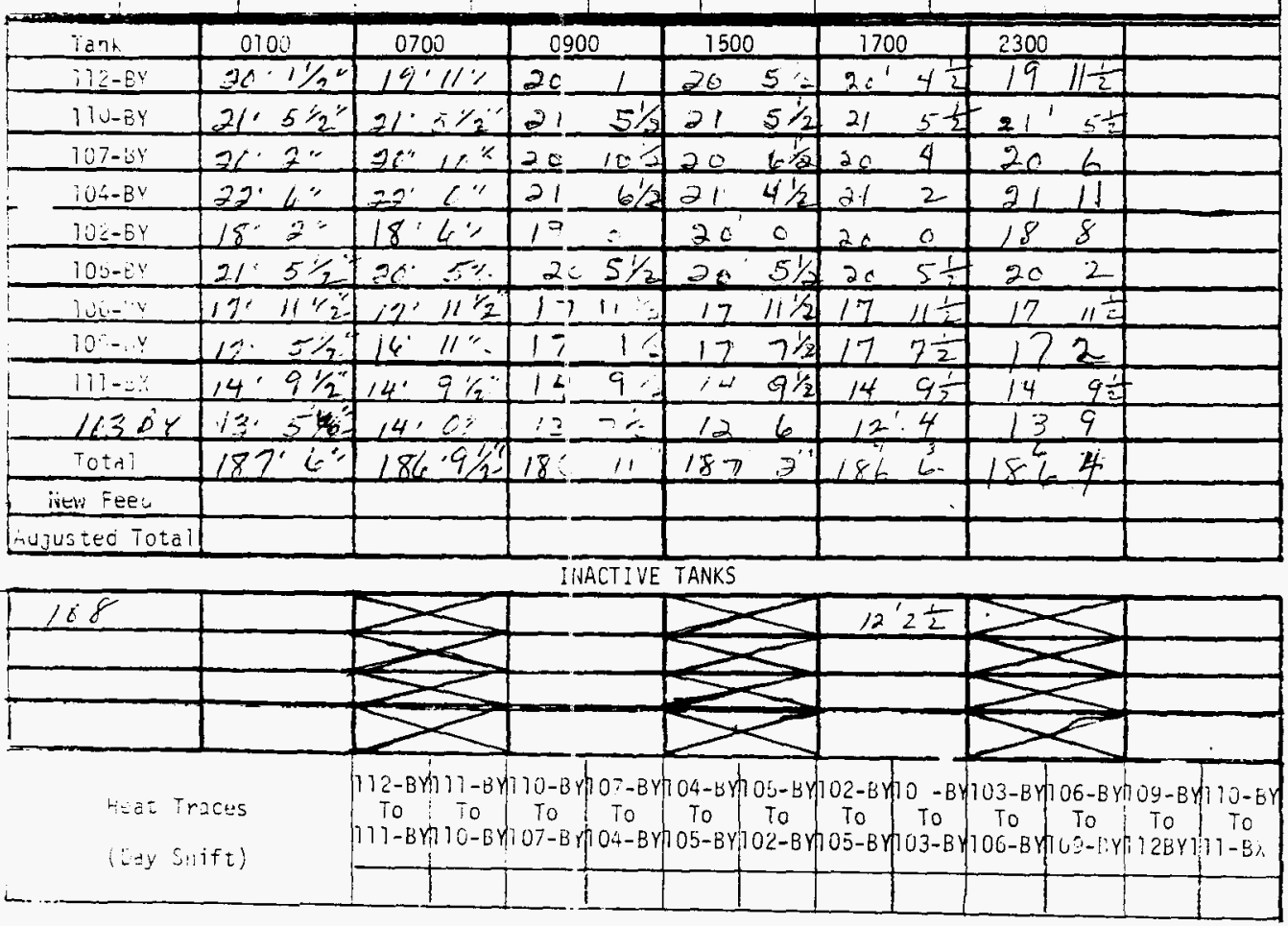




$$
-\quad \quad H C-52-W M-D P-206
$$

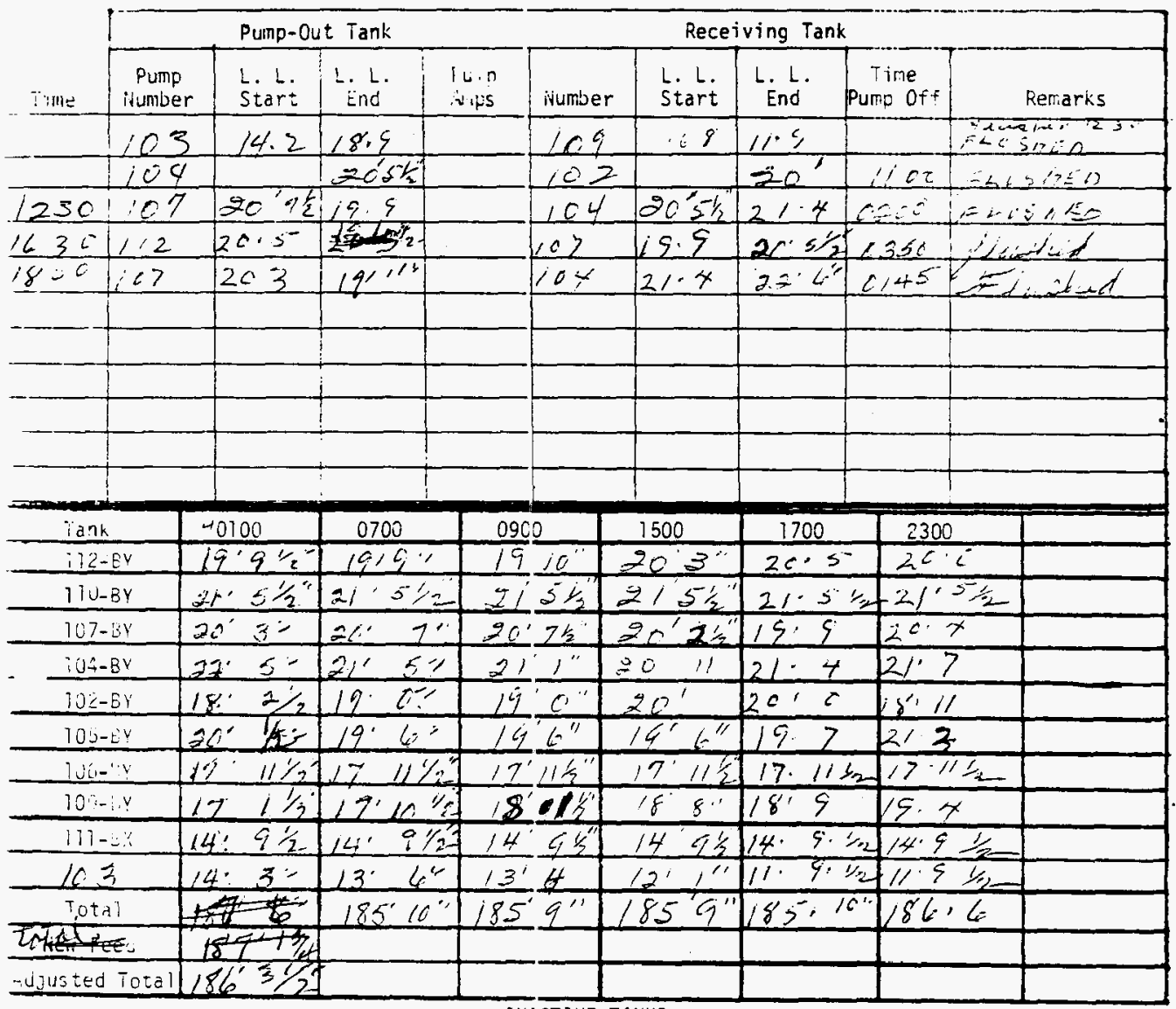

\section{IINFCTIVE TANKS}
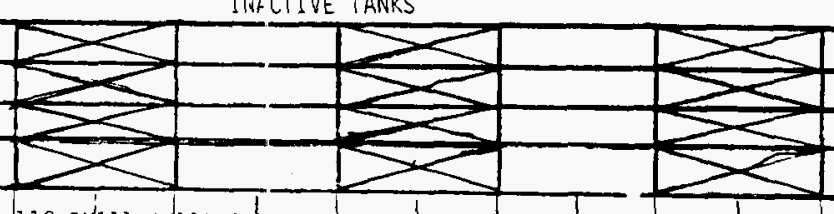

Hidez Traces

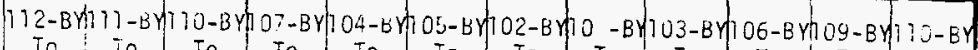

(Lizy sitift)

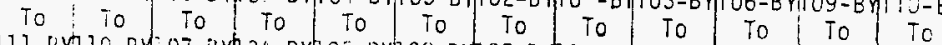

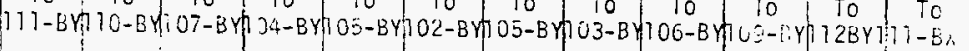

(Lify sitif 


$$
\text { -WUC-SD-WM-DP-206 ReVO }
$$

IIS-2 IIIVENTORY IHU PUMPING

Cate $z=10-\overline{2}$
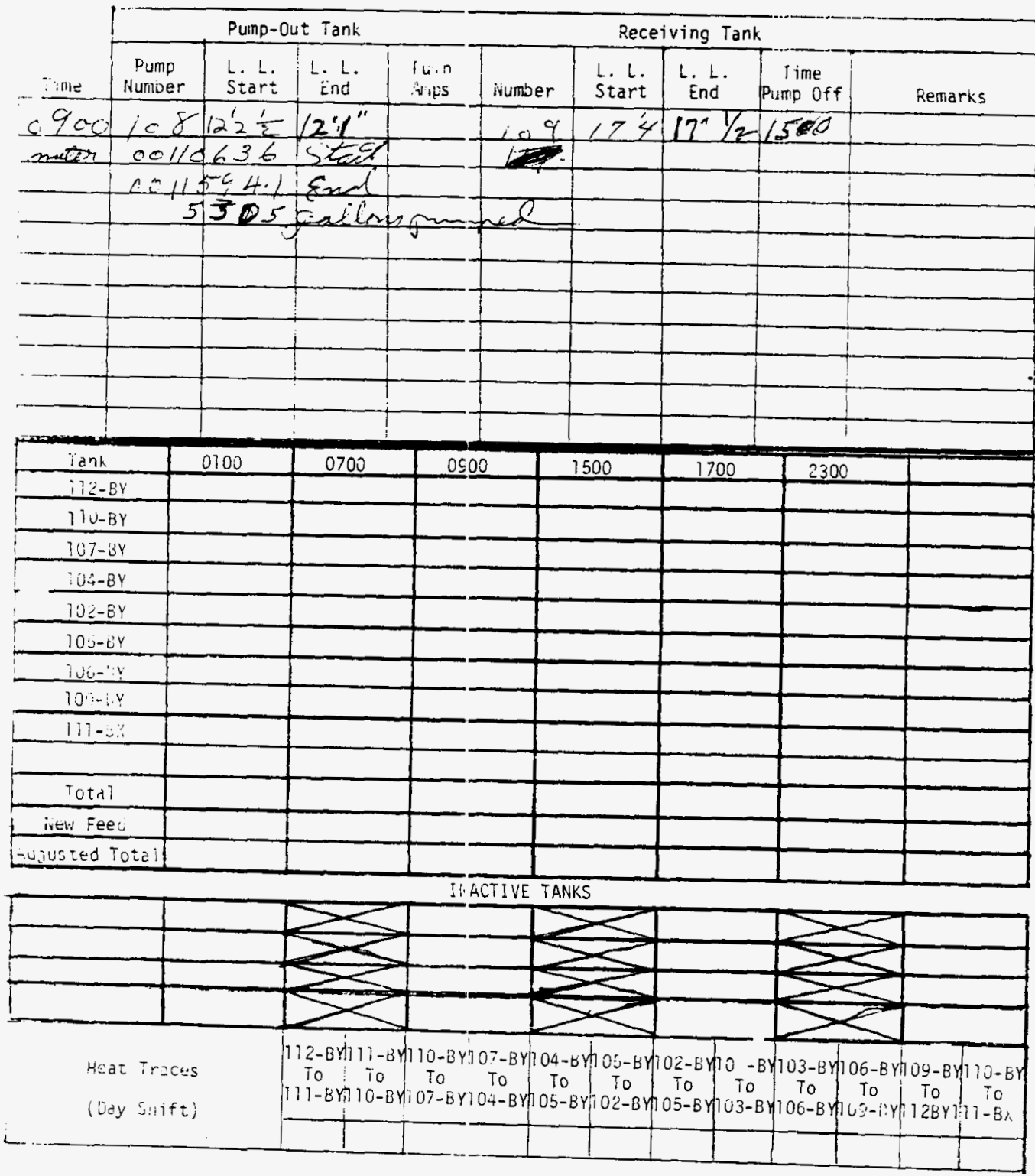

BEST AVAILARI F COPY 


\section{- WHC-SD-WM-DP-206}

iTs-2 IIVENTORY AINU PUMPING

Date ₹ー1ーグ́⿱

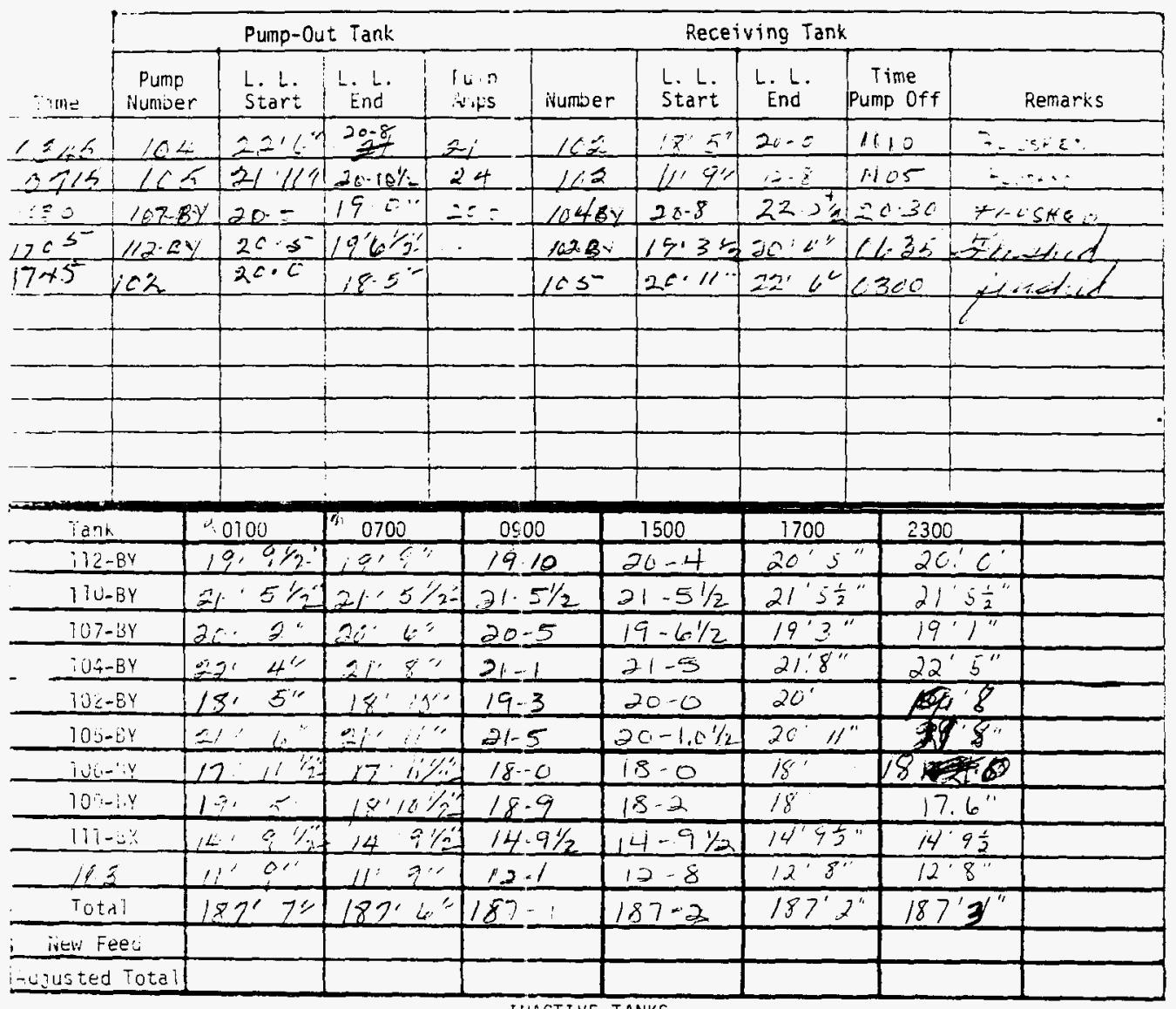

\section{IIIACTIVE TANKS}

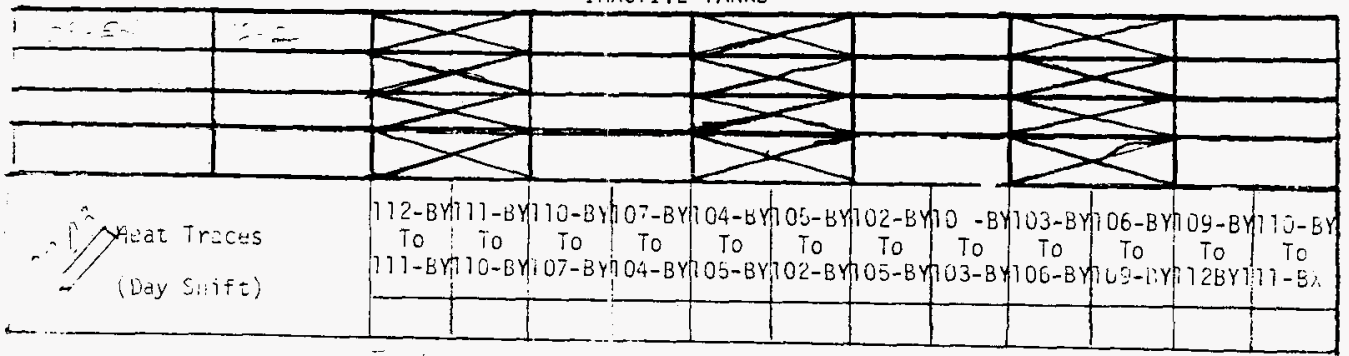




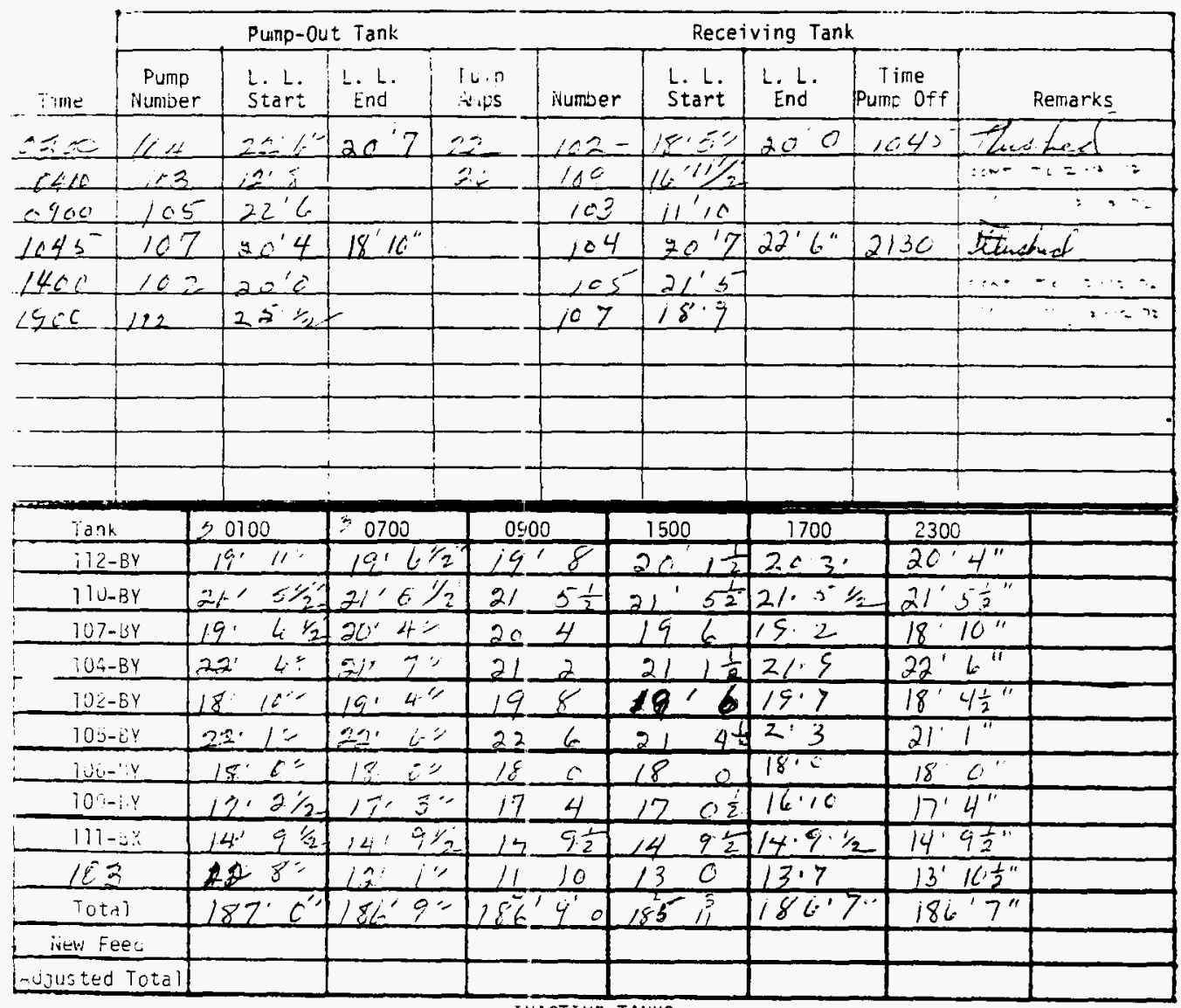

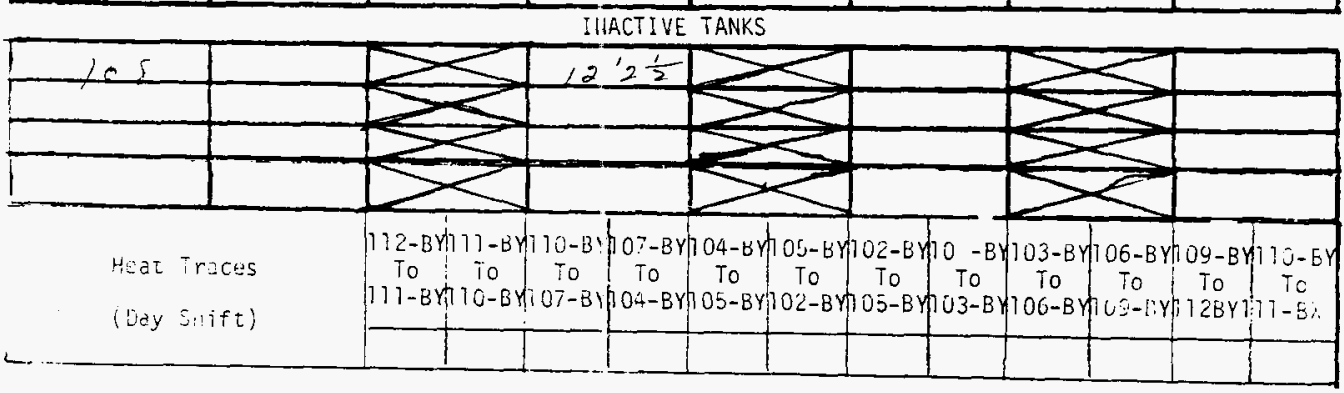




\section{$-\quad$ WHC-SD-WH-DP-206}

RevO

iT $5-2$ IINENTORY AINL PUMPIING

Date $=-2 \cdot 72$

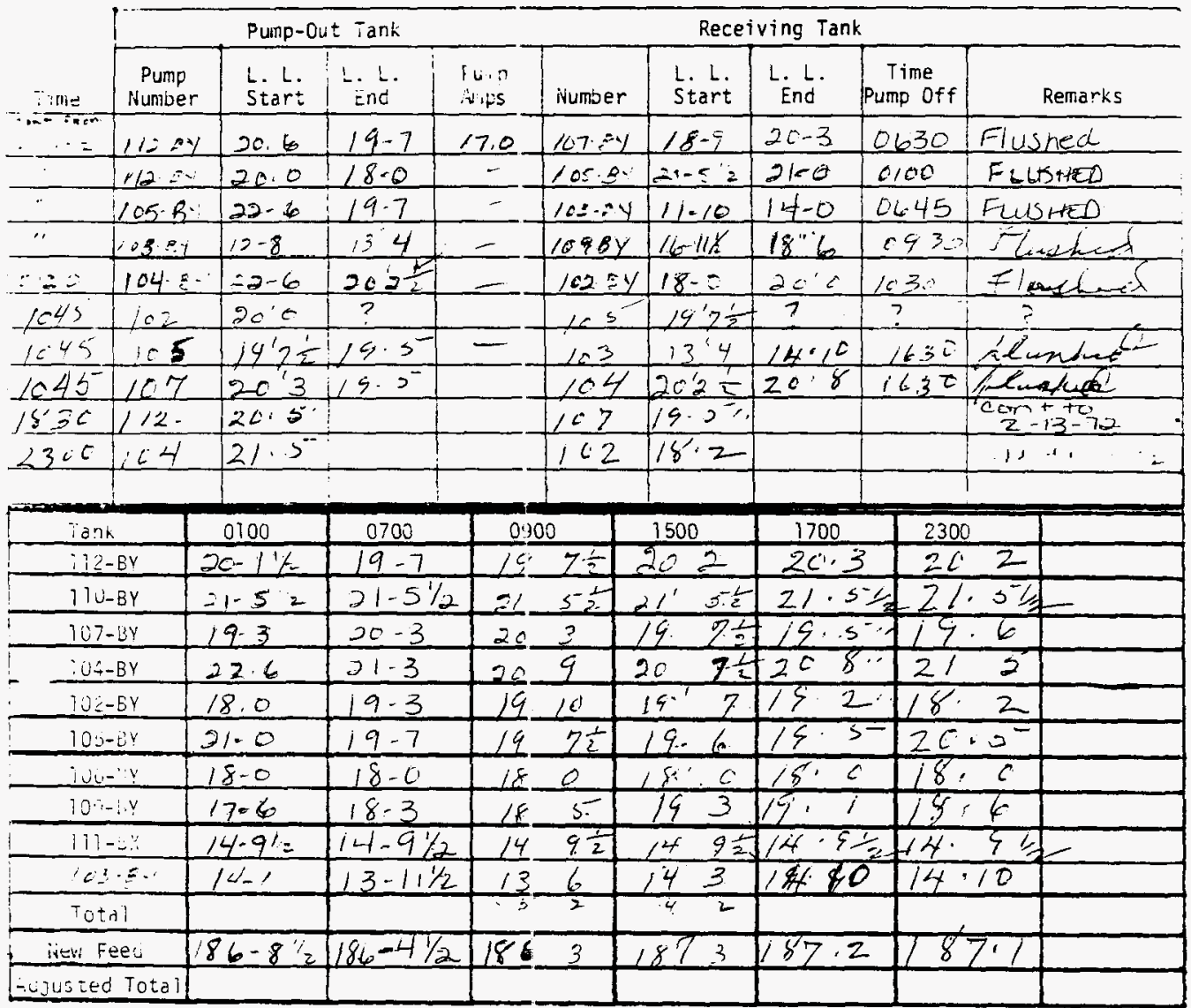

I ACTIVE TANKS

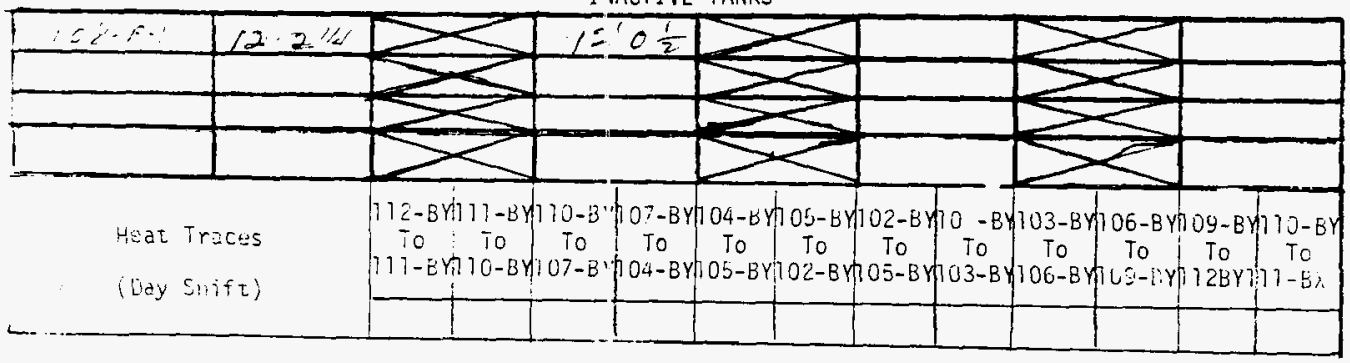

$9-00=77$

BEST AVAII ABLE COPY 
Date $2-13-72$

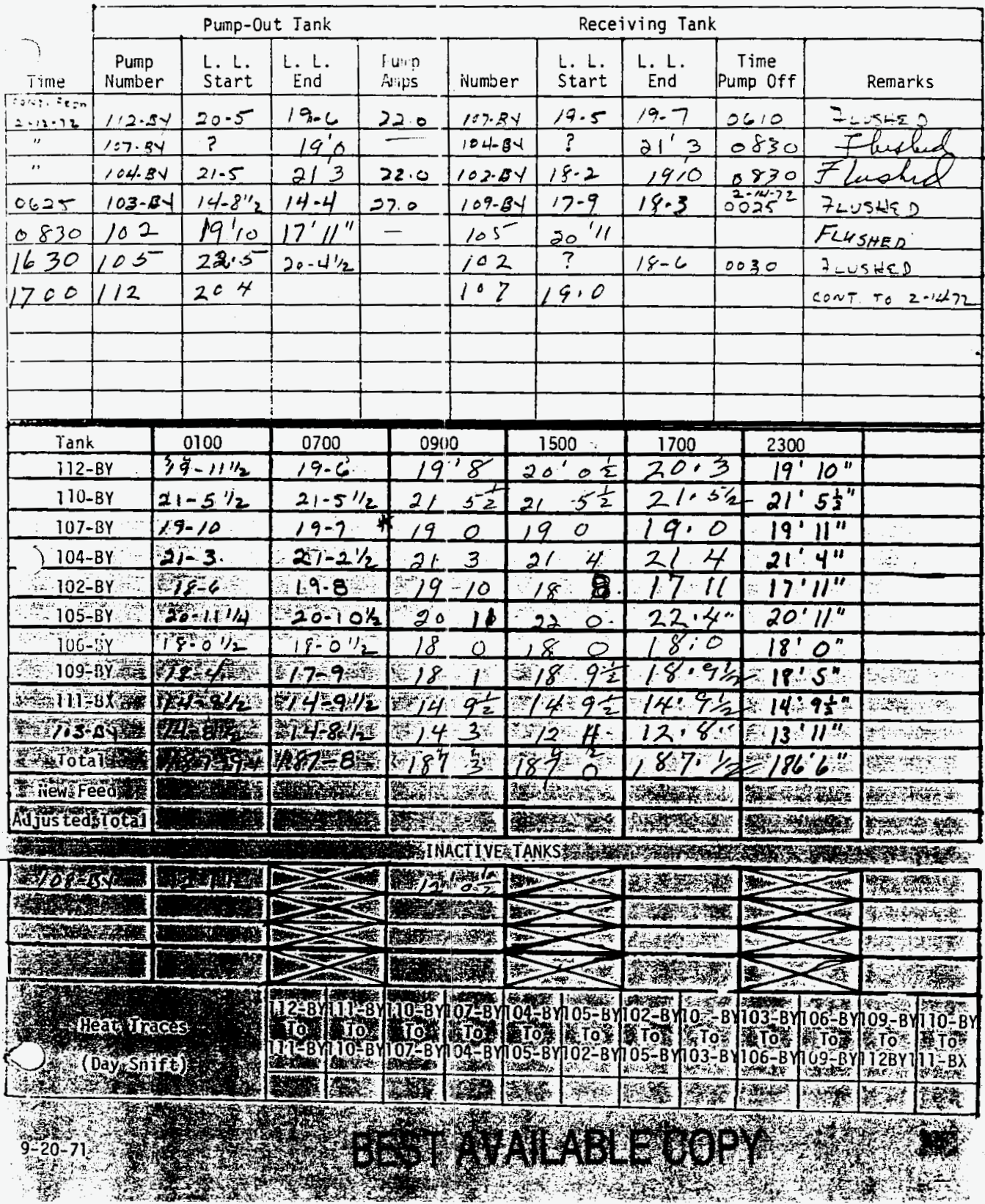




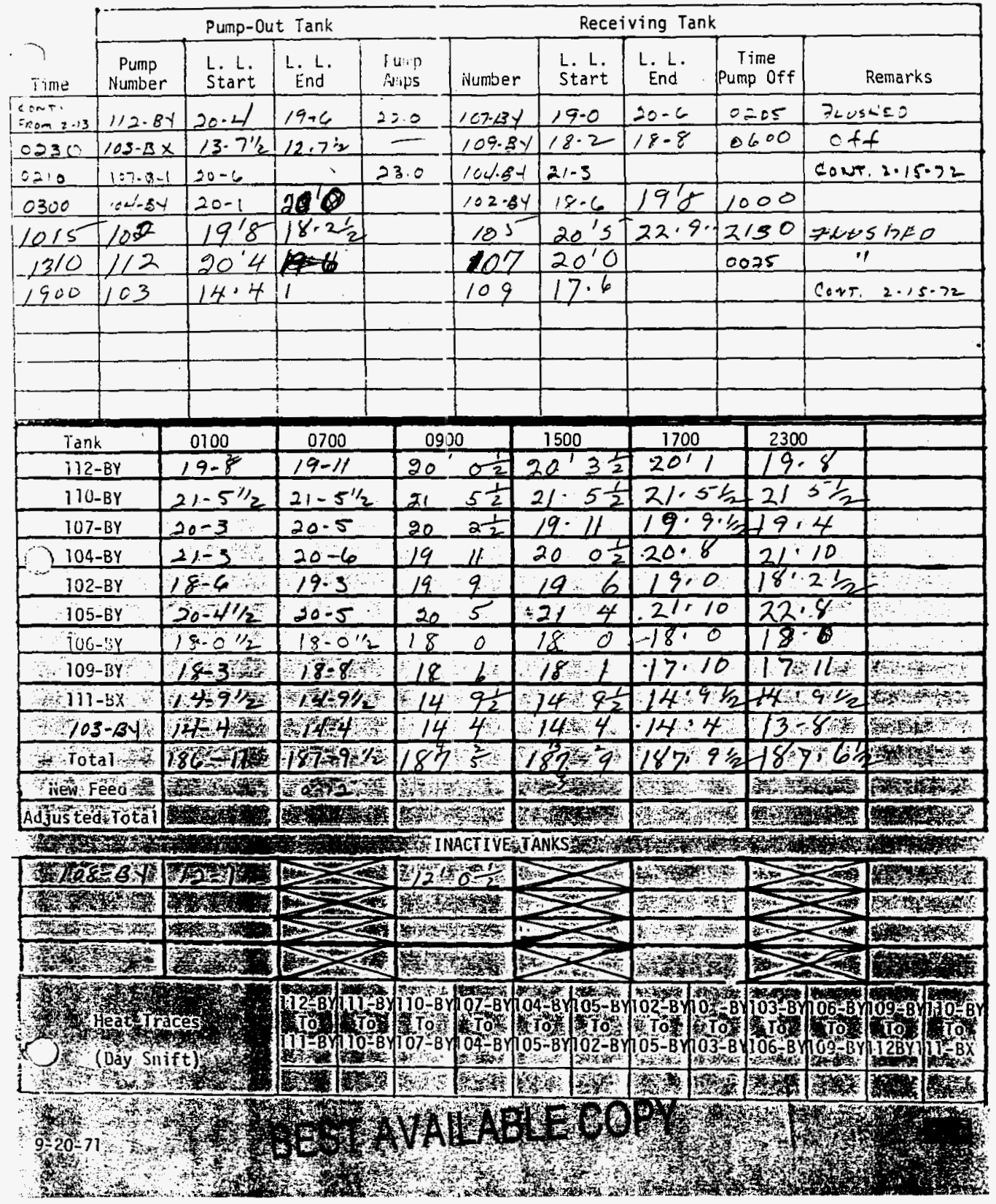


Date $2-15 \cdot 72$

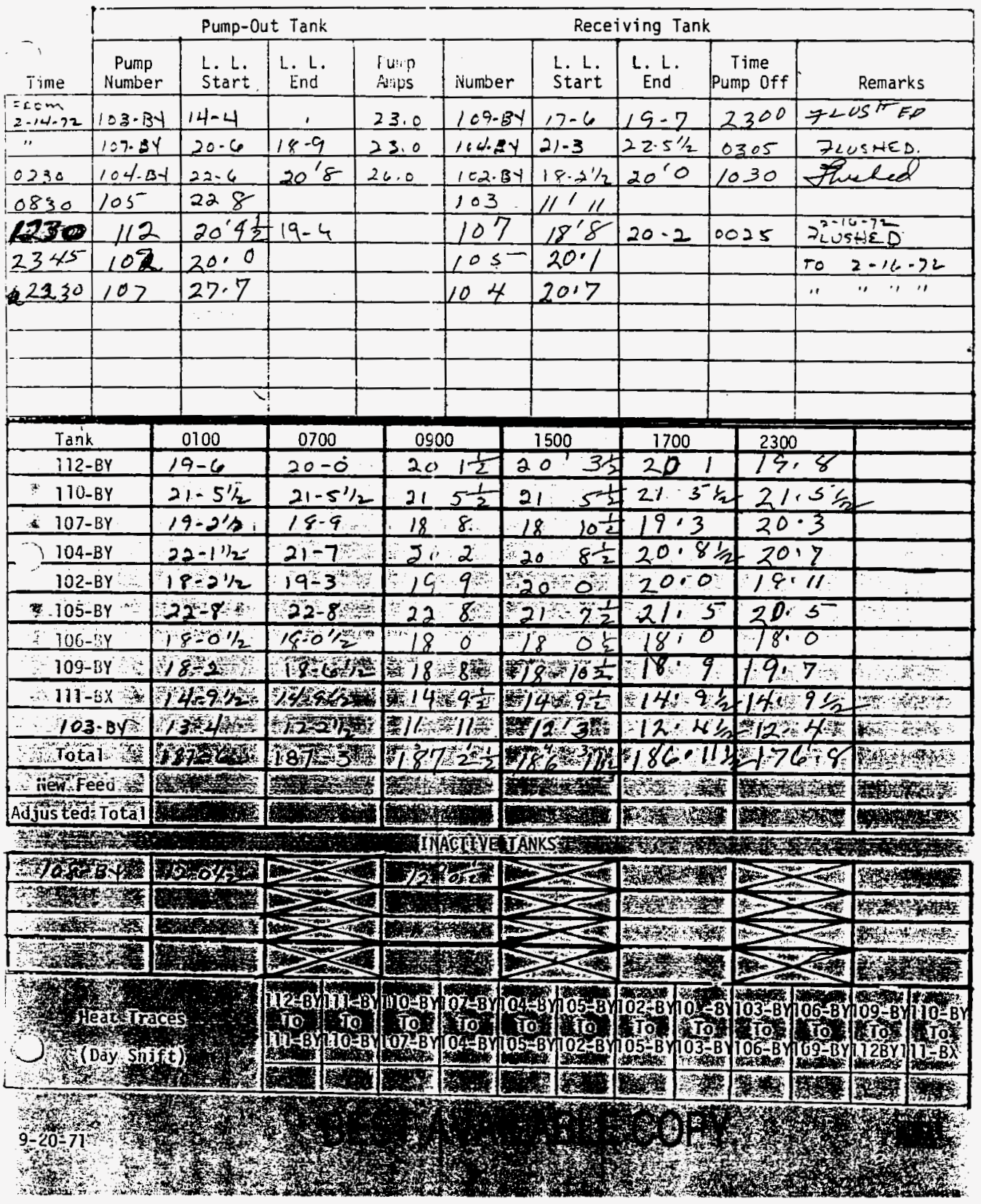


ITS-2 IIVENTORY FIND PUMPING

Date $2-16-72$

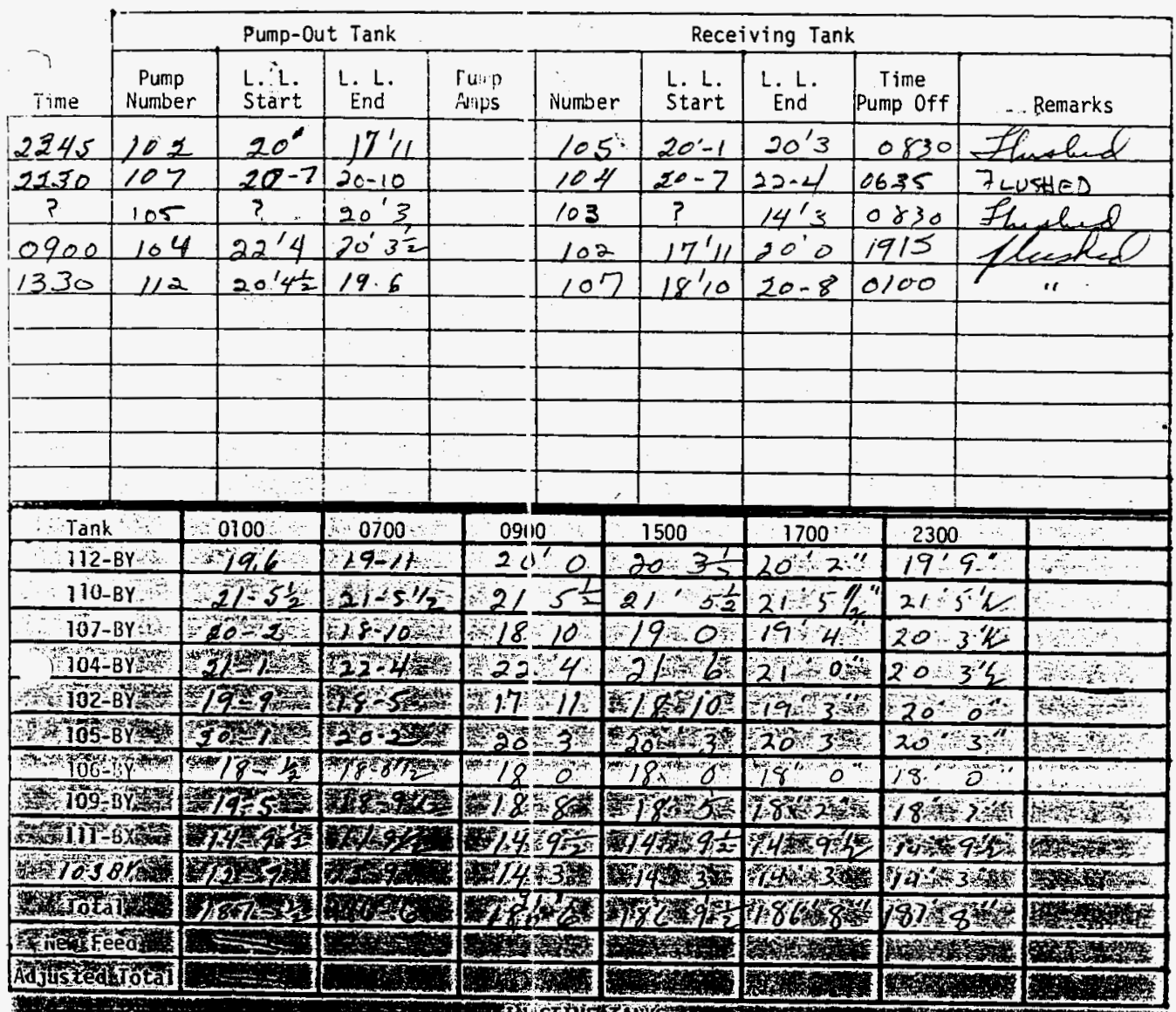

IN CT ME TANS

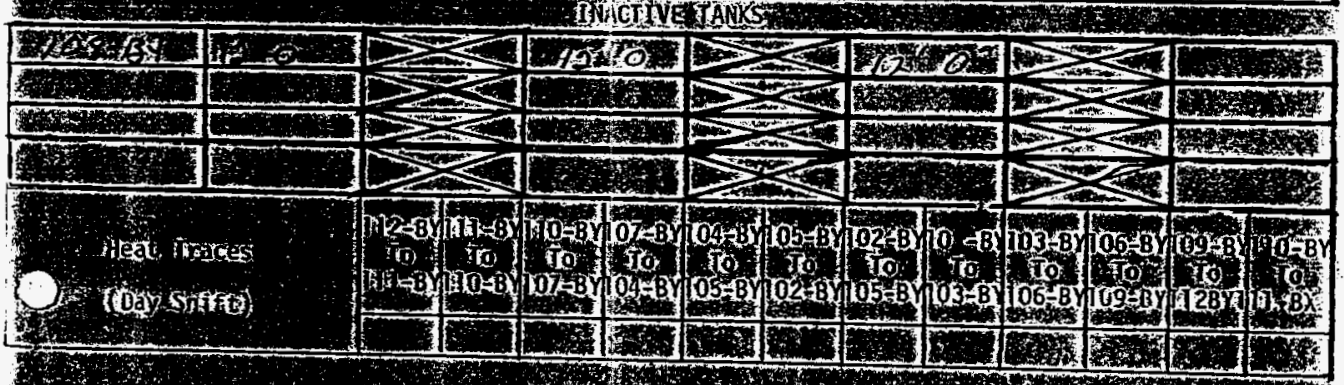




\section{-. WHC-SD-WM-DP-206}

ITS-2 IINENTORY IIIU PUMPIING

Date $2-17-72$

Pump-out Tank

Pump L.L. L.L. I I.n

Number Star

clec 102 20-0 18

$0415+20-0 / 2$

$12151 / 144$

1615103

140

1500 $21^{\prime \prime \prime}=0^{\prime \prime 2}=\frac{13}{01}$

$14^{\prime} 3^{\prime \prime}$

$22^{\prime} 5^{\prime}$

106
Receiving Tank

\begin{tabular}{|c|c|c|c|c|}
\hline iumber & $\begin{array}{l}\text { L. L. } \\
\text { Start }\end{array}$ & L. L. & $\begin{array}{l}\text { Time } \\
\text { Pump off }\end{array}$ & Remarks \\
\hline 105 & $2:-3$ & $22^{\prime} 5^{\prime \prime}$ & is is & Therack \\
\hline 104 & $3<-2$ & $\therefore 153$ & $133:$ & ZLisatud \\
\hline $1<2$ & $18^{\prime}$ & 260 & $19 / 5$ & 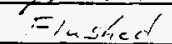 \\
\hline 169 & $\angle 7^{\prime} 7^{\prime}$ & & & \\
\hline$\angle c 3$ & $13^{\prime} 4^{\prime \prime}$ & & & \\
\hline 107 & $189=$ & & & \\
\hline & & & & \\
\hline & & & & \\
\hline & & & & \\
\hline
\end{tabular}

\begin{tabular}{|c|c|c|c|c|c|c|c|}
\hline$a n h$ & 0100 & 0700 & 0930 & 1500 & 1700 & 2300 & \\
\hline$i 12-B^{2}$ & $19-6$ & $19-16$ & $\therefore 01$ & $20-4$ & $20^{\prime} 46$ & $2 c$ & \\
\hline $17 \mathrm{U}-\mathrm{BY}$ & $2 /-5$ & $2<-5$ & 215 & 21 & $210^{\circ}$ & 21 & \\
\hline $107-3 y$ & $20-6$ & $-20-2$ & $19 \div \%$ & $18 \quad 95$ & 132 & 19.10 & \\
\hline$\because U \div-B Y$ & $20-2$ & $2 z-9$ & 211 & $21^{\prime} 1^{\prime \prime}$ & $207 \%$ & $=0 \quad 24$ & \\
\hline$\therefore \quad 10<-5 Y$ & $2 c-c$ & $t e-k \frac{1}{1}$ & 183 & 1810 & 19.5 & 200 & \\
\hline $100-E Y$ & $2<-3$ & $21-8^{2} \frac{1}{2}$ & 221 & $22^{\prime} 5^{\prime \prime}$ & 21.100 & $20 \quad r 2$ & \\
\hline ivis-'y & $1 \varepsilon-c$ & $18-6$ & $18=0$ & $18 \quad 0$ & $1 / 2$ & 1812 & \\
\hline $10 \%=i, y$ & $18-8$ & $\angle 8-1$ & $171+5$ & $1 x$ & 18 & $18 \quad 9 \%$ & \\
\hline $111-2.1$ & $14-95$ & $\angle 4-9 i_{2}$ & 1495 & $14 ?: 2$ & 1,4912 & is $\quad y^{\prime} b$ & \\
\hline 16385 & $14-3$ & $14 \cdot 3$ & 143 & $13^{\prime} 4^{\prime \prime}$ & 13 & 136 & \\
\hline Total & 187.82 & $167-6 \frac{1}{2}$ & $18 x$ & $\angle 8=$ & 187 & $187^{\prime} 8$ & \\
\hline ivew Feen & & & & & & & \\
\hline Diusted Total & & & & & & & \\
\hline
\end{tabular}

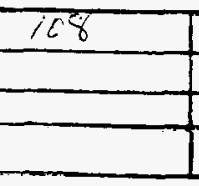

Hed Tracts

(iay Stift)
INACTIVE TANKS
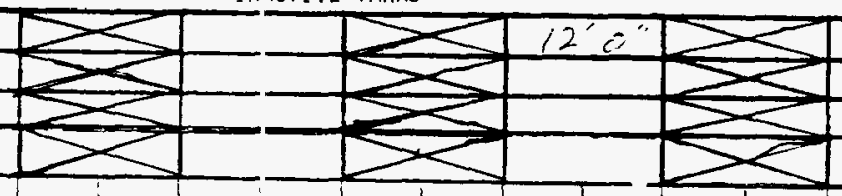

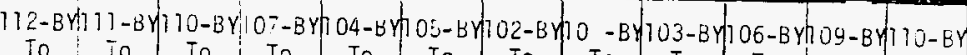

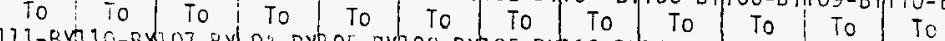

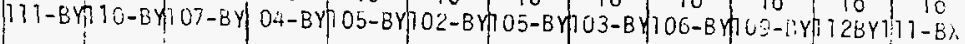

santed1/2-By@0630

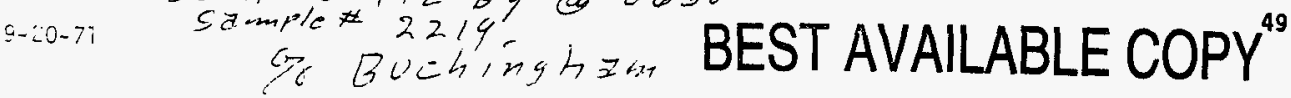




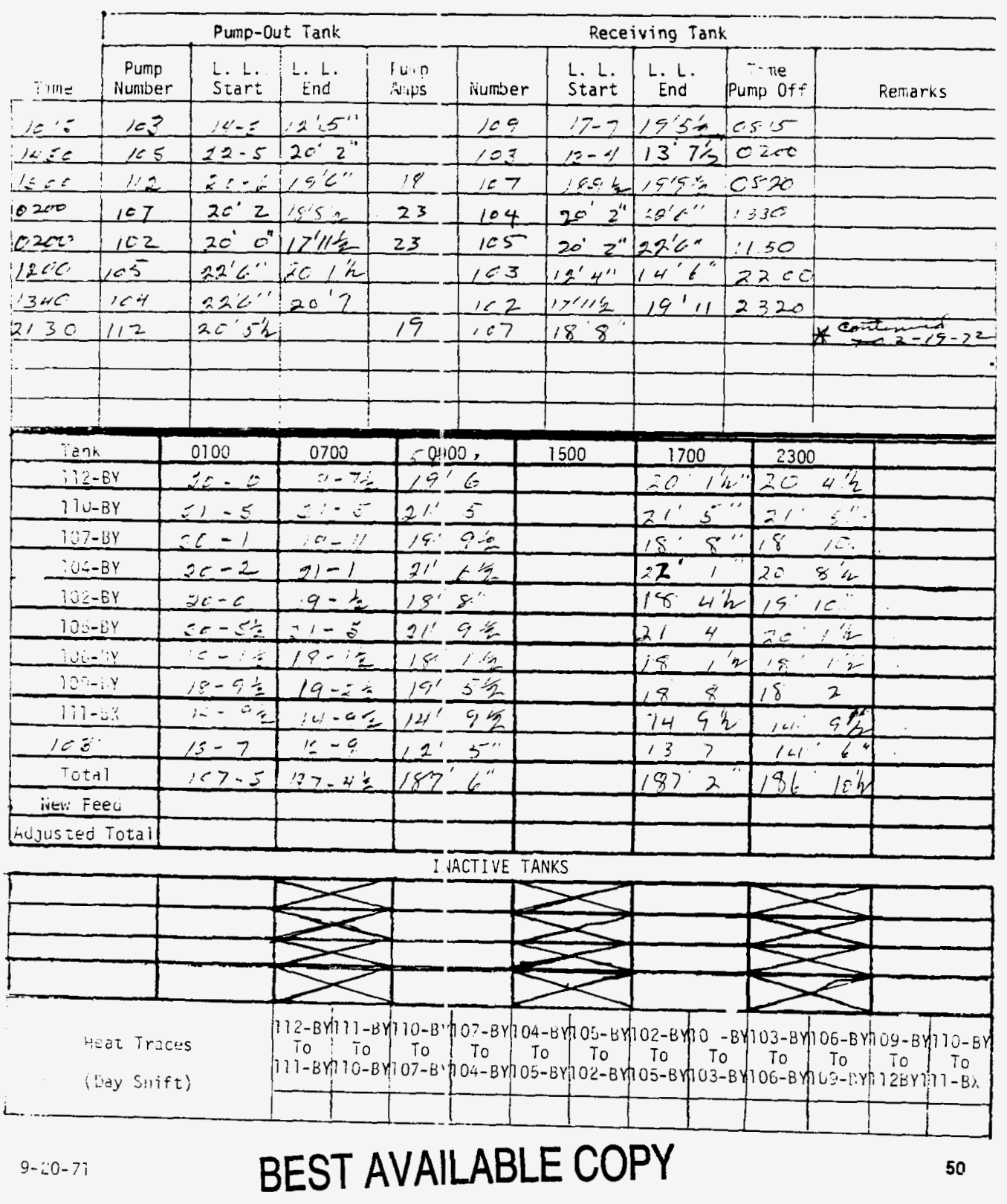




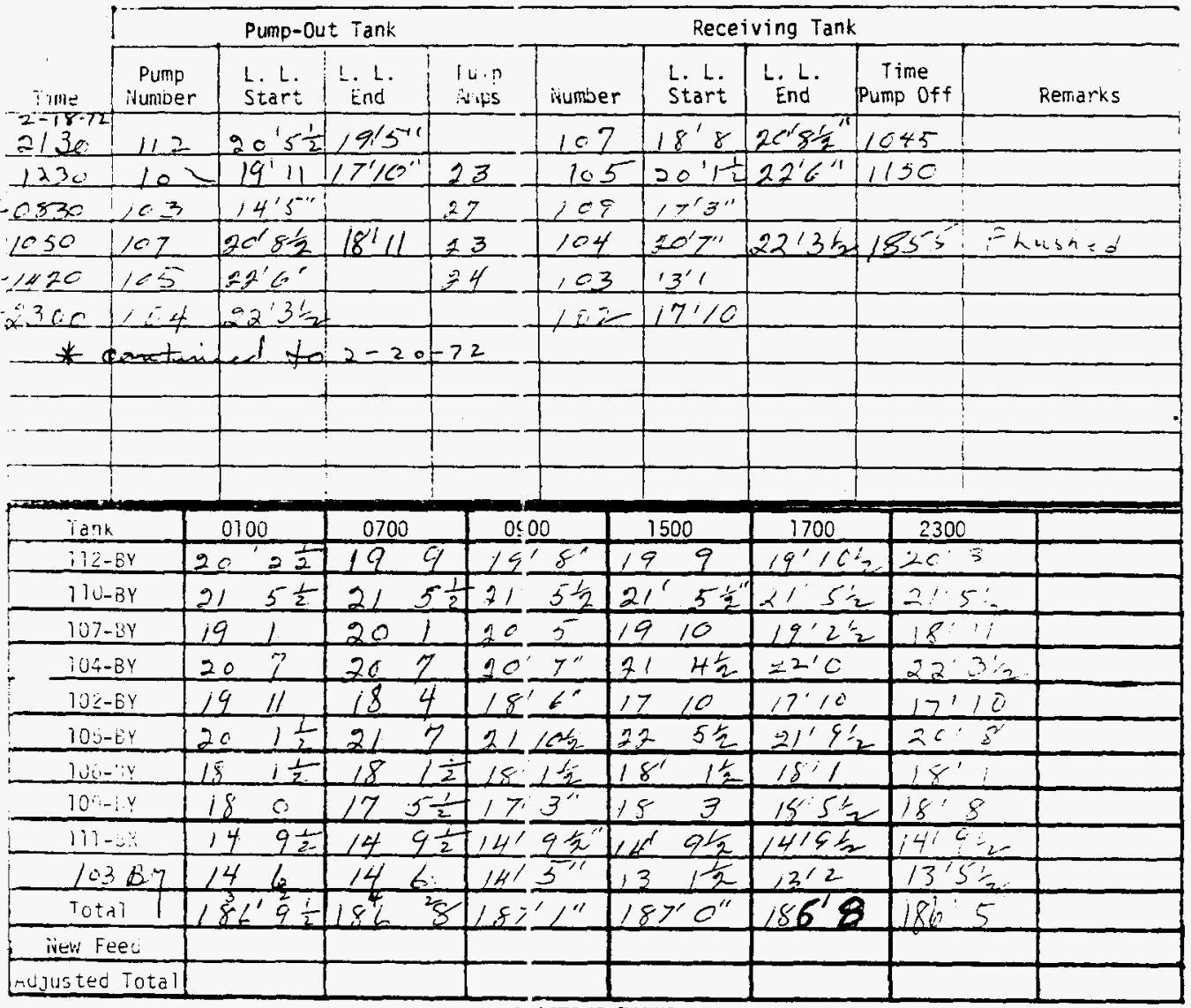

IIIACTIVE TANKS

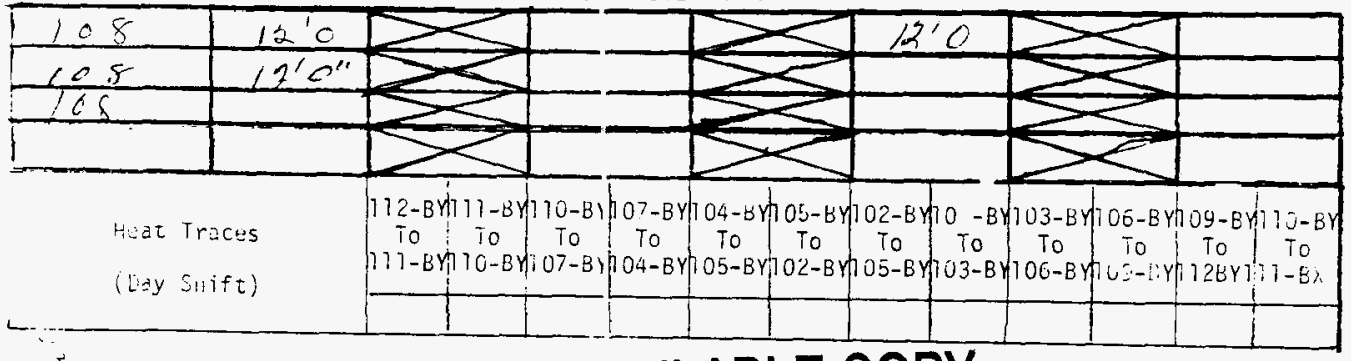




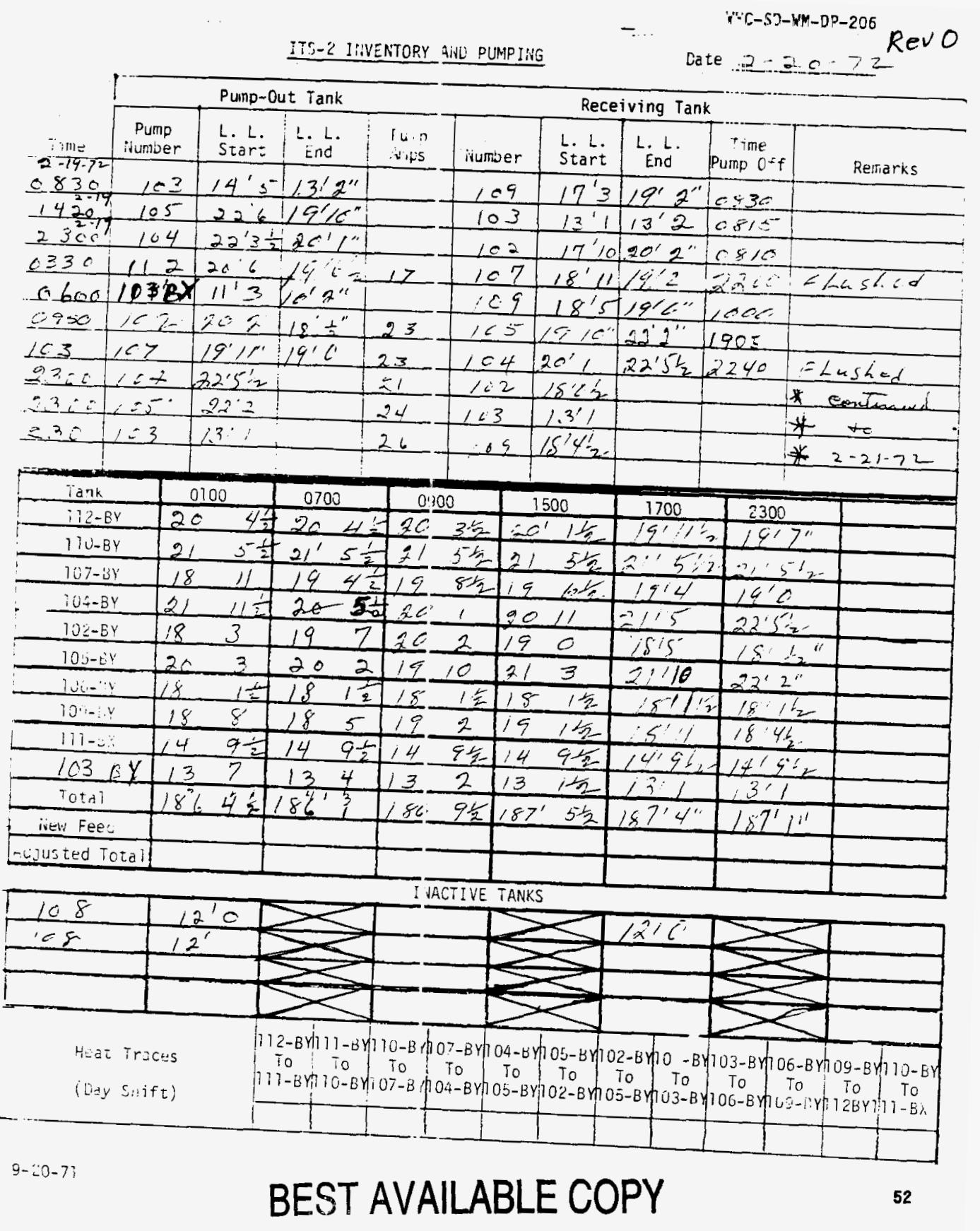


Cate $2-\therefore 1-7=$

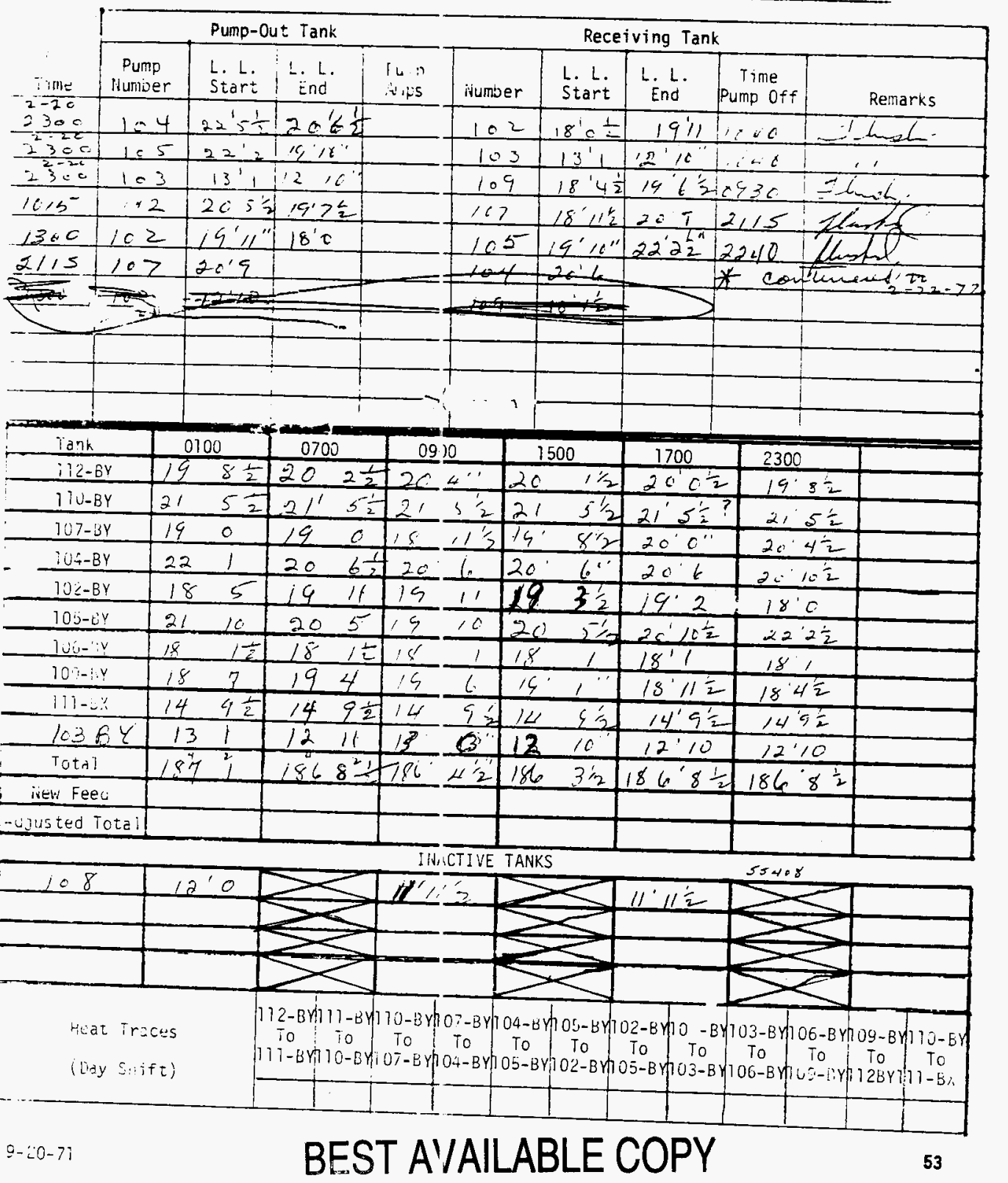




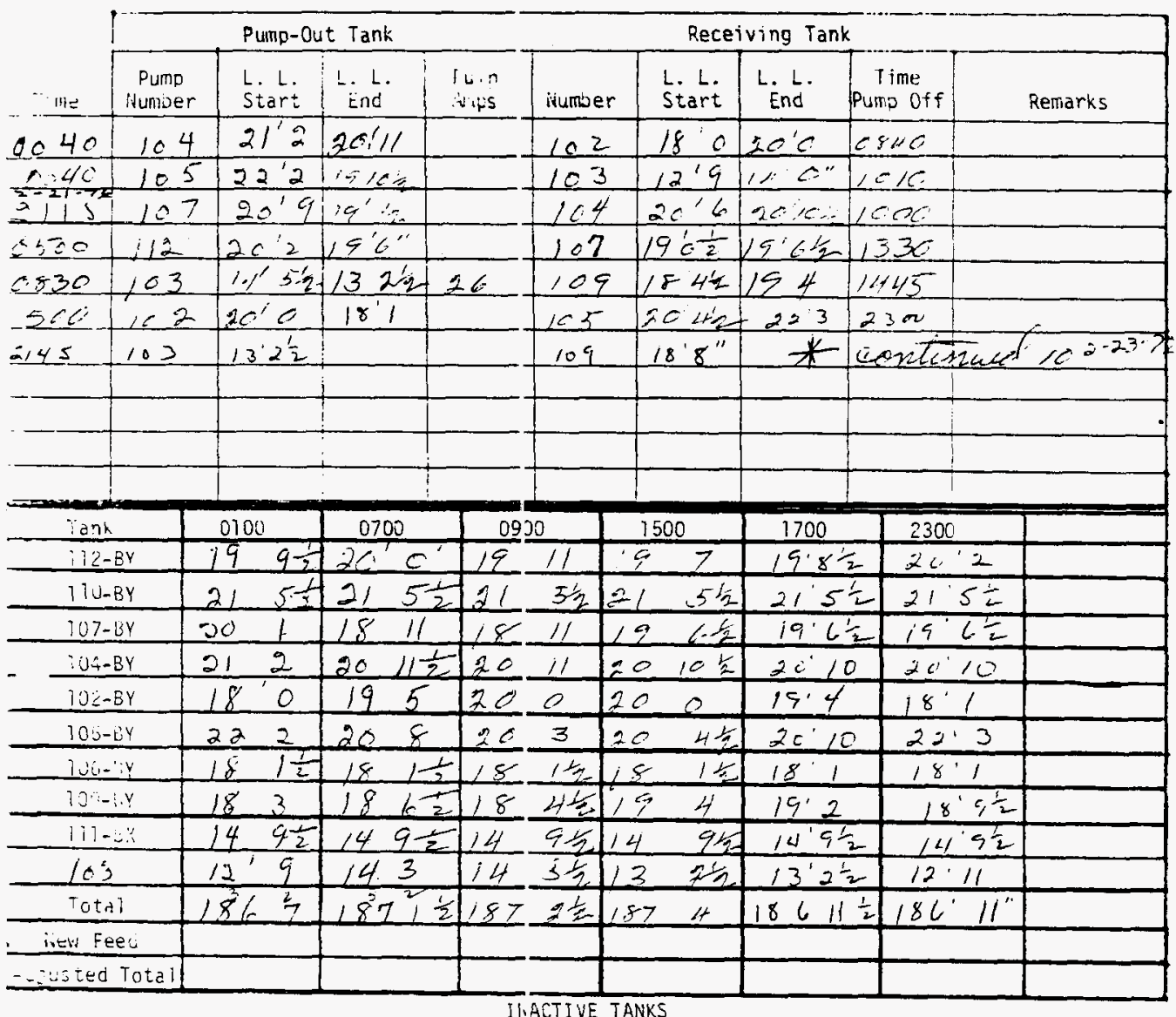

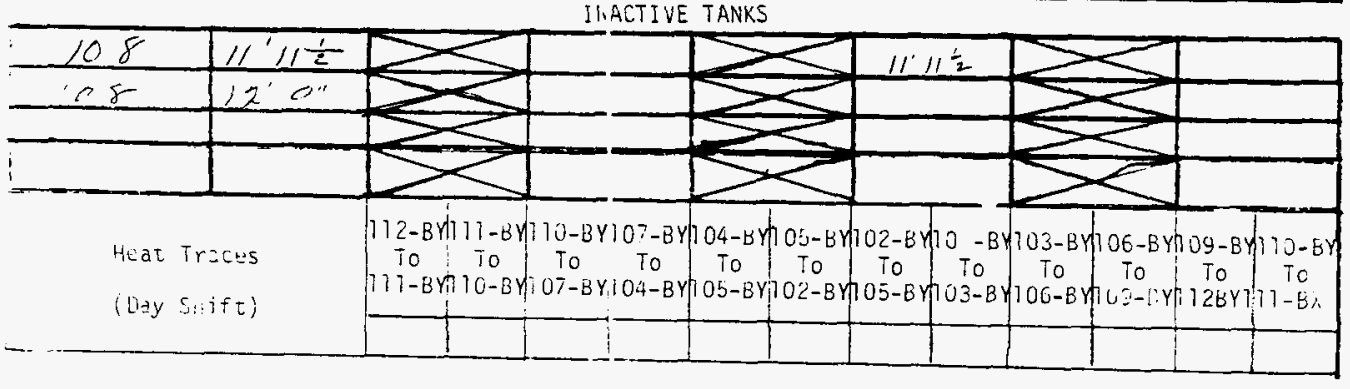




\section{- WHC-SD-WK-DP-206}

ITS- Z IRIVENTORY AINU PUMPING

Cate $2-23.72$

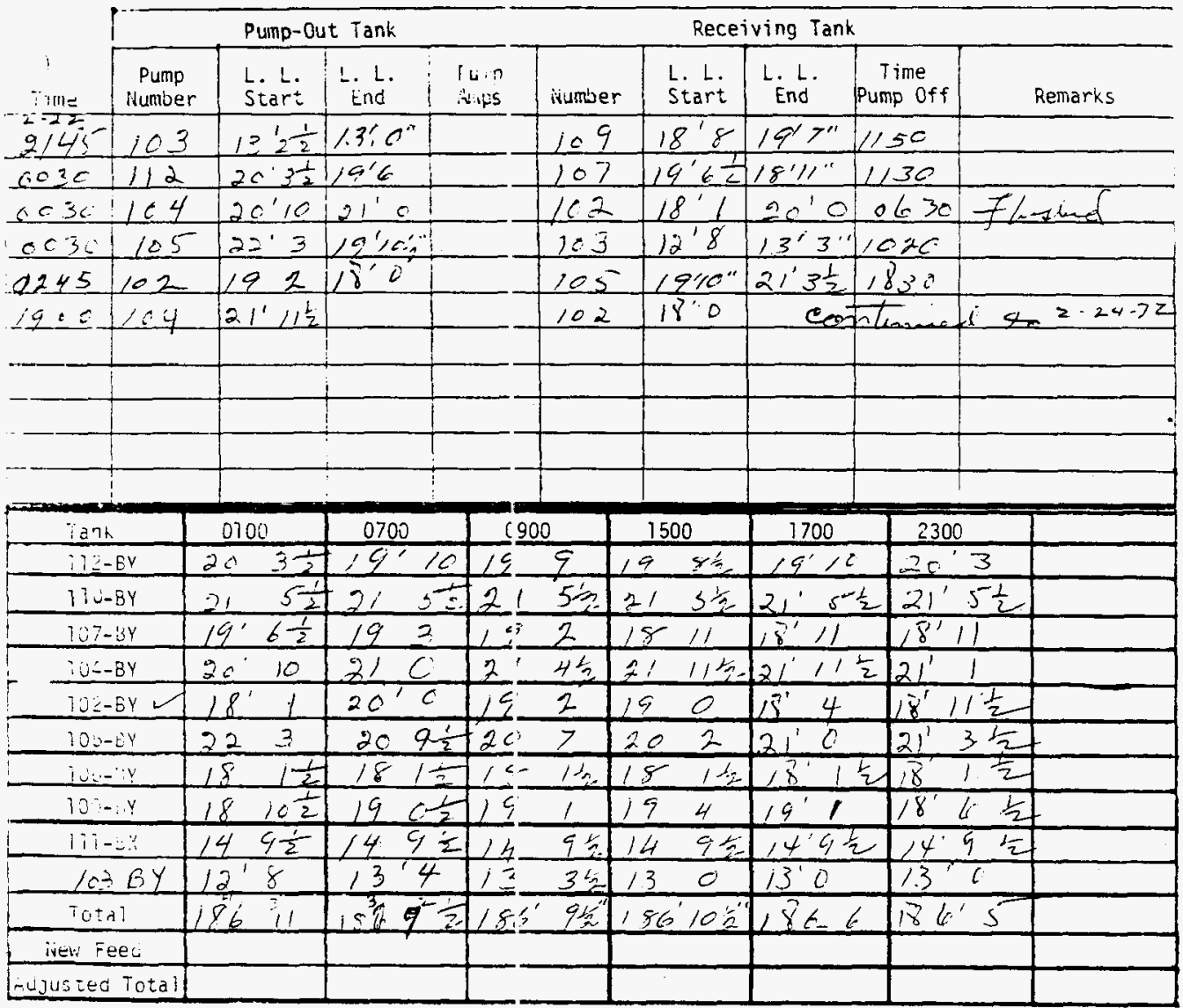

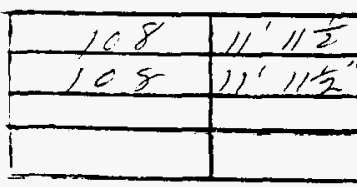

Heat iraces

$(L i a y s i f t)$

INACTIVE TANKS

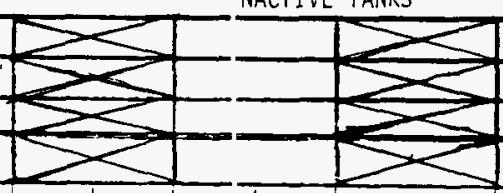

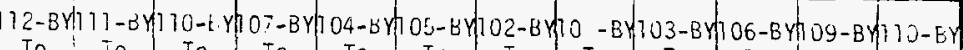

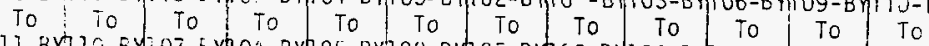

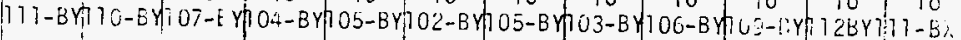

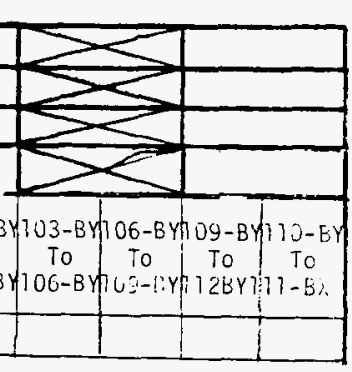

L.

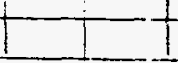

$9-20-7 i$ 
Pump-Out iank

\begin{tabular}{l|l|l} 
Pump & L. L. & L.L. L. \\
Number & Start & End
\end{tabular}

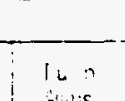

$2 \frac{a 11 \leq}{23-72}$

1900

$21^{\prime} 1 \frac{1}{2} x^{\prime} \mathrm{C}$

$00^{3} 1052^{\prime} 3$ 14\%

0245 112 20'6 2i

$0.6151107 .19^{\prime} 5 \frac{11}{2} 14^{\prime} 6$

xदse

1053

102

$\therefore 65$

112

$20^{\circ} 0 " 1 / 5^{\prime \prime} 1$

$\frac{1}{1}$

ivumer L L

Receiving Tank

\begin{tabular}{l|l|l|}
\hline $54 c$ & itc & 217 \\
\hline
\end{tabular}

$2006+22^{\prime 2}$

\begin{tabular}{l|l|l|} 
L. L. & L. L. & Timie
\end{tabular}

Start End Pump off

102 18'o $20^{\circ} \circ$ cjec Itimid?

103

$130: 140: 0160$

$20918111532 \cdot 15$

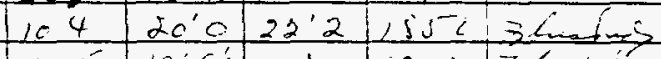

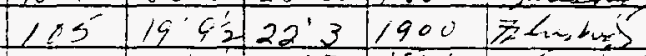

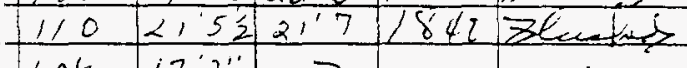

\begin{tabular}{|l|l|}
104 & $17^{\prime} 2^{\prime}$ \\
\hline 167 & $19^{\circ} 6$ \\
\hline 108 & 181 \\
\hline
\end{tabular}

3

eontrimed

78

$2=-215=72$

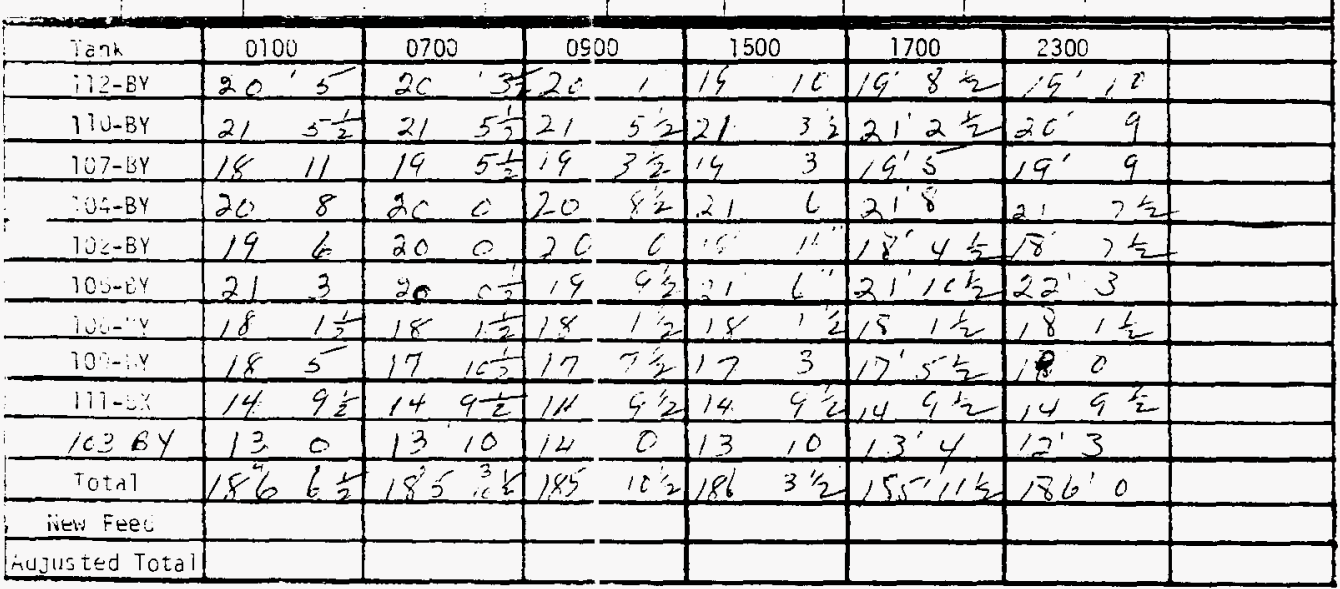

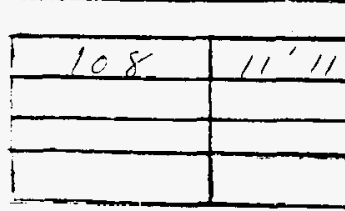

II.ACTIVE TANKS
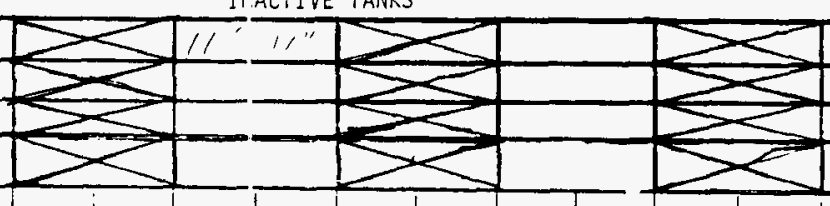

Heat Iraces

(Liay $S_{i}$ ift)

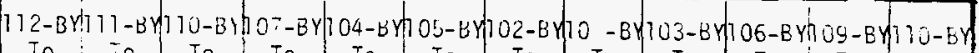

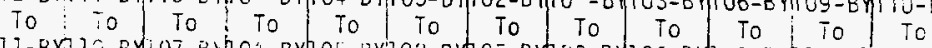

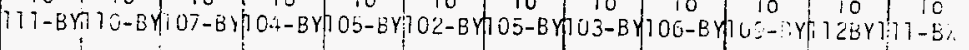

L.

$+1$

1

$9-20-7 i$ 


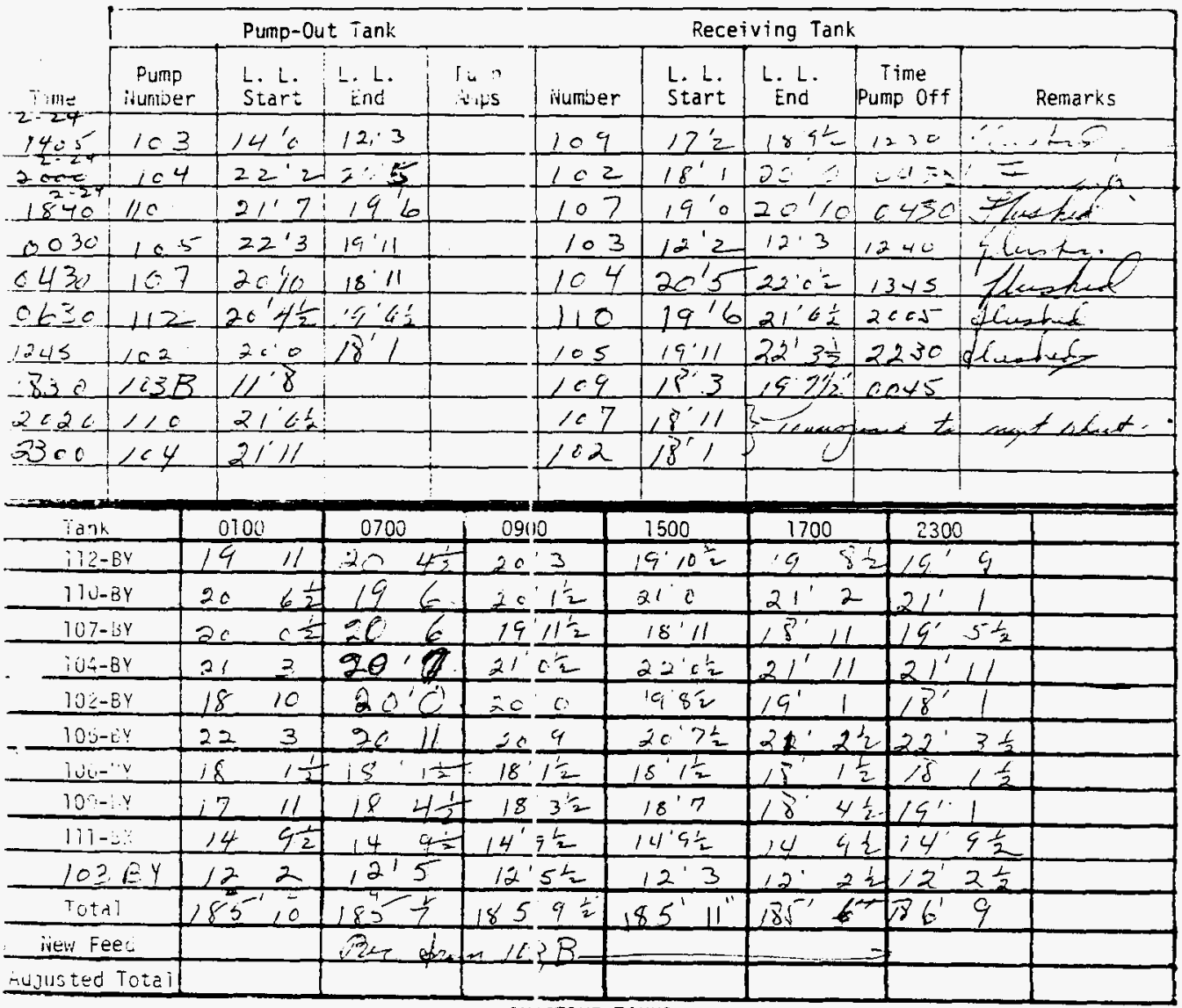

IN.ICTIVE TANKS

\begin{tabular}{l|l}
108 & $11^{\prime 11}$ \\
& \\
\hline
\end{tabular}
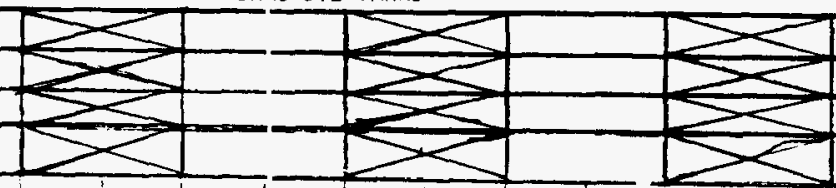

Hä Tracts

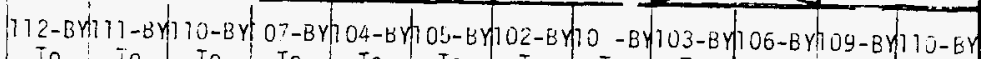

(Lay $S_{\text {: if }}$ if)

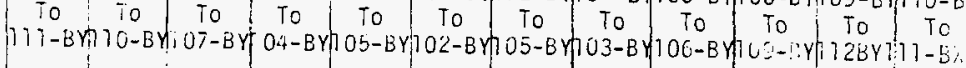


Pump-out Tank

\section{Pump}

26261 $\because 0$

$\frac{2300 i 64}{9425-163} 2^{\prime 2} 11^{\prime \prime} 14^{\prime \prime} 10^{\frac{1}{2}}$

0850.112 \#ct立 $/ 96$

$0910: 107 \quad 2: 9 \frac{1}{2} \mid 1810 \frac{1}{2}$

$15001102+201 \frac{1}{2}$

$-1505103 \quad 14^{\prime} 4 / 2^{\prime} 2$

$2250<1$
$2, \cdot 8$

\begin{tabular}{|c|c|c|c|c|c|c|c|}
\hline Tan. & 0100 & 0700 & 0900 & 1500 & 1700 & 2300 & \\
\hline$i 1:-E v$ & 74 & $2644^{\prime \prime}$ & $20^{\prime} \leq \frac{1}{2}$ & $20^{\circ} 1$ & $\therefore 4^{\prime} 1<\frac{1}{2}$ & $19^{\circ}<$ & \\
\hline $110-5 Y$ & $21^{\prime} ?^{\prime \prime}$ & $19^{\prime} 7^{\prime \prime}$ & $196 \frac{1}{2}$ & $20^{\circ} 7^{\prime \prime}$ & $2011 \frac{2}{2}$ & $21^{\circ} 8$ & \\
\hline $107-24$ & $19^{\prime} / 1^{\prime \prime}$ & $3 \varepsilon^{\prime} 16^{\circ}$ & $264 \frac{1}{2}$ & $19 \cdot 7$ & $19^{\prime} 1$ & $15 \times \frac{1}{2}$ & \\
\hline$\therefore \div-36$ & $21.622^{\prime \prime}$ & $20^{\prime} 2 \%$ & $19110 \frac{1}{2}$ & $21 \cdot 1 \frac{1}{2}$ & 2,1 & $31.8 \frac{1}{2}$ & \\
\hline $30-5:$ & $18 \cdot 6 \frac{2}{2}$ & $14^{\prime} 4 \frac{1}{2}$ & 2012 & $20 ; \frac{1}{2}$ & $\bar{y}^{\prime} 7$ & $18^{\circ} 6$ & \\
\hline $105-26$ & $2 \hat{x}^{\prime} \quad 4 t^{\prime \prime}$ & $37^{\prime} 4^{\prime \prime}$ & $213 \frac{1}{2}$ & $19^{\circ} 10$ & $26 \quad 6 \frac{1}{2}$ & $21^{\prime 11}$ & \\
\hline $16-2$ & $18^{\prime} / \frac{1}{2}$ & $18^{\prime} / \frac{1}{2}$ & $18^{\prime} 12$ & $181 \frac{1}{2}$ & $1 \vec{x}^{\prime} 1 \div$ & $18,1 \frac{1}{2}$ & \\
\hline$i 6-y$ & $14^{\prime} 7^{\prime \prime}$ & $14^{\prime \prime} 1^{\prime \prime}$ & 1811 & $18 \cdot 1 \frac{1}{2}$ & $18^{\circ}$ & $19^{\prime} 4$ & \\
\hline $11 i-$ & $8 \frac{1}{x}$ & $14^{\prime} 6^{\prime \prime}$ & 14.92 & $149 \frac{1}{2}$ & $\left\langle x^{\prime} 5 \frac{1}{2}\right.$ & $14 \quad 5 \div$ & \\
\hline 10361 & 12.3 & $12^{\prime} 10^{\circ}$ & $133 \frac{1}{2}$ & 1442 & $13 \quad 5$ & 12,5 & \\
\hline Tota & $188 \%$ & 1572 & $187,3 \frac{1}{2}$ & 1870 & $186^{\circ} 10$ & $166^{\prime} 16$ & \\
\hline ivew Feed & $Z^{\prime} / 1 \frac{1}{2}$ & & & & & & \\
\hline idjusted io:al & & & & & & & \\
\hline
\end{tabular}

II.ACTIVE TANKS

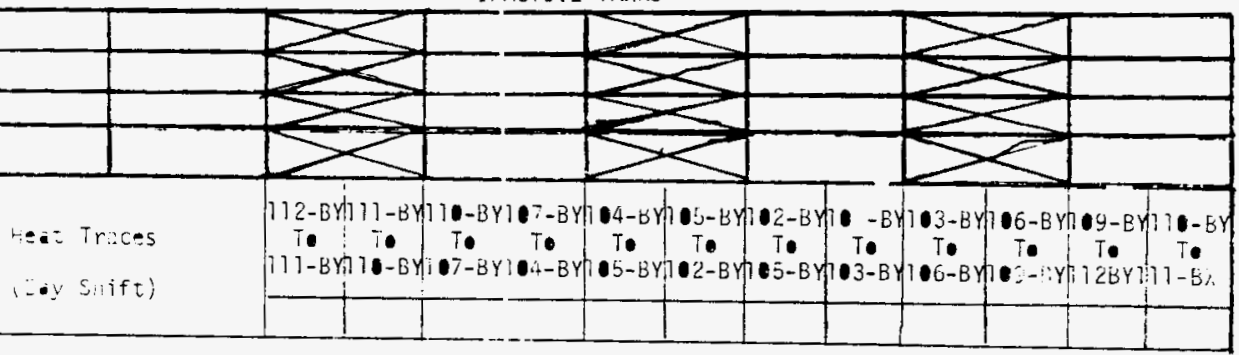

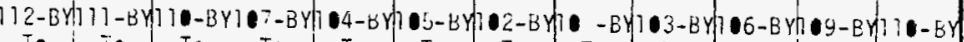

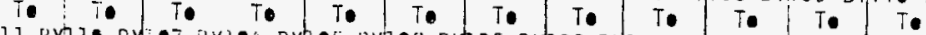
-

\section{$9-9-7 i$




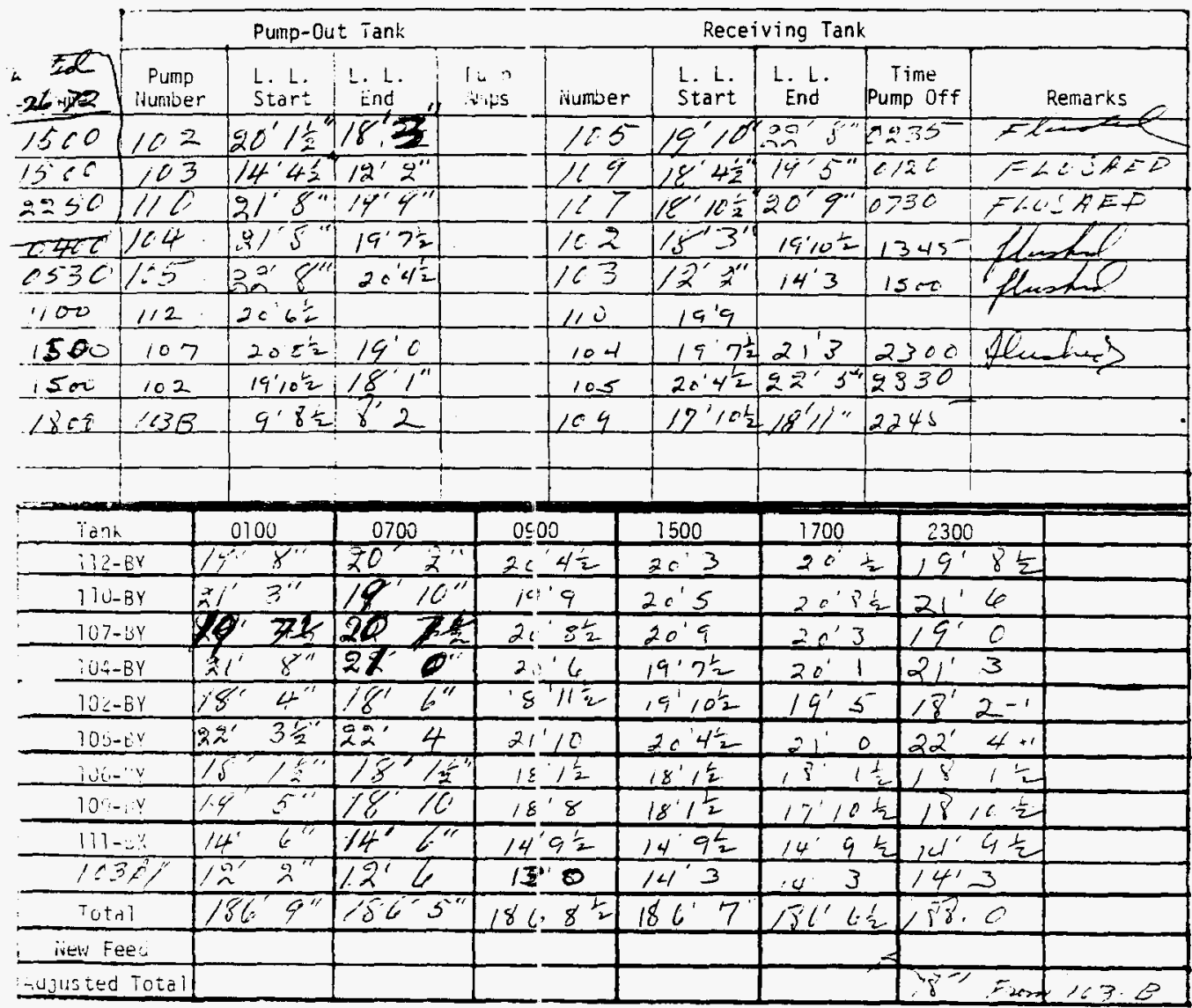

II ACTIVE TANKS

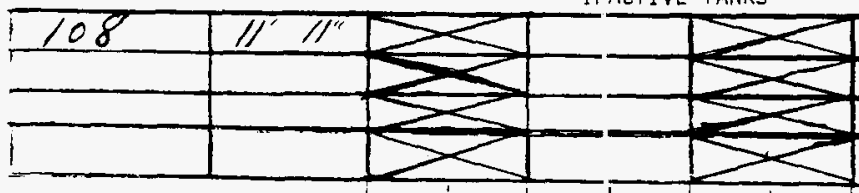

Heat Traces

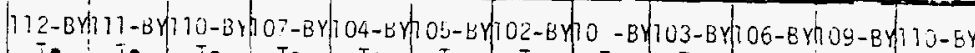

(Loy Sifift)

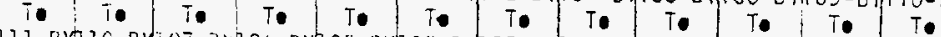

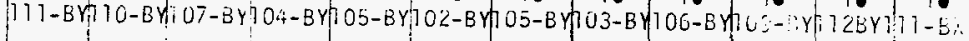




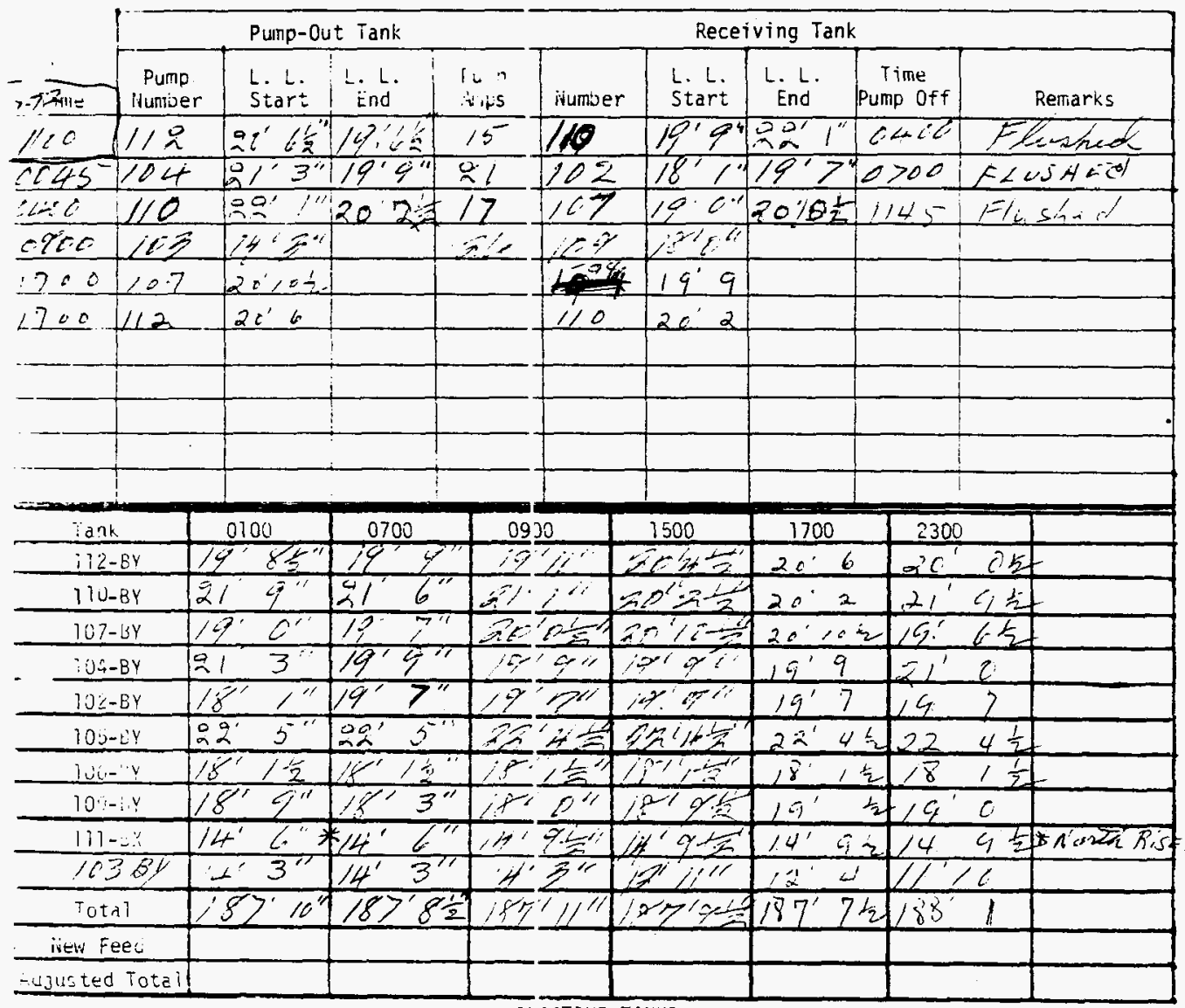

II.ACTIVE TANKS

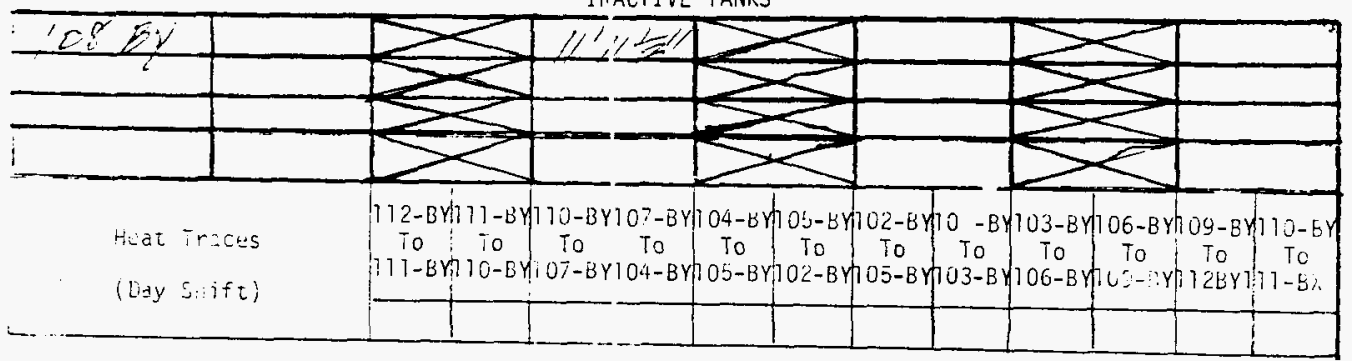




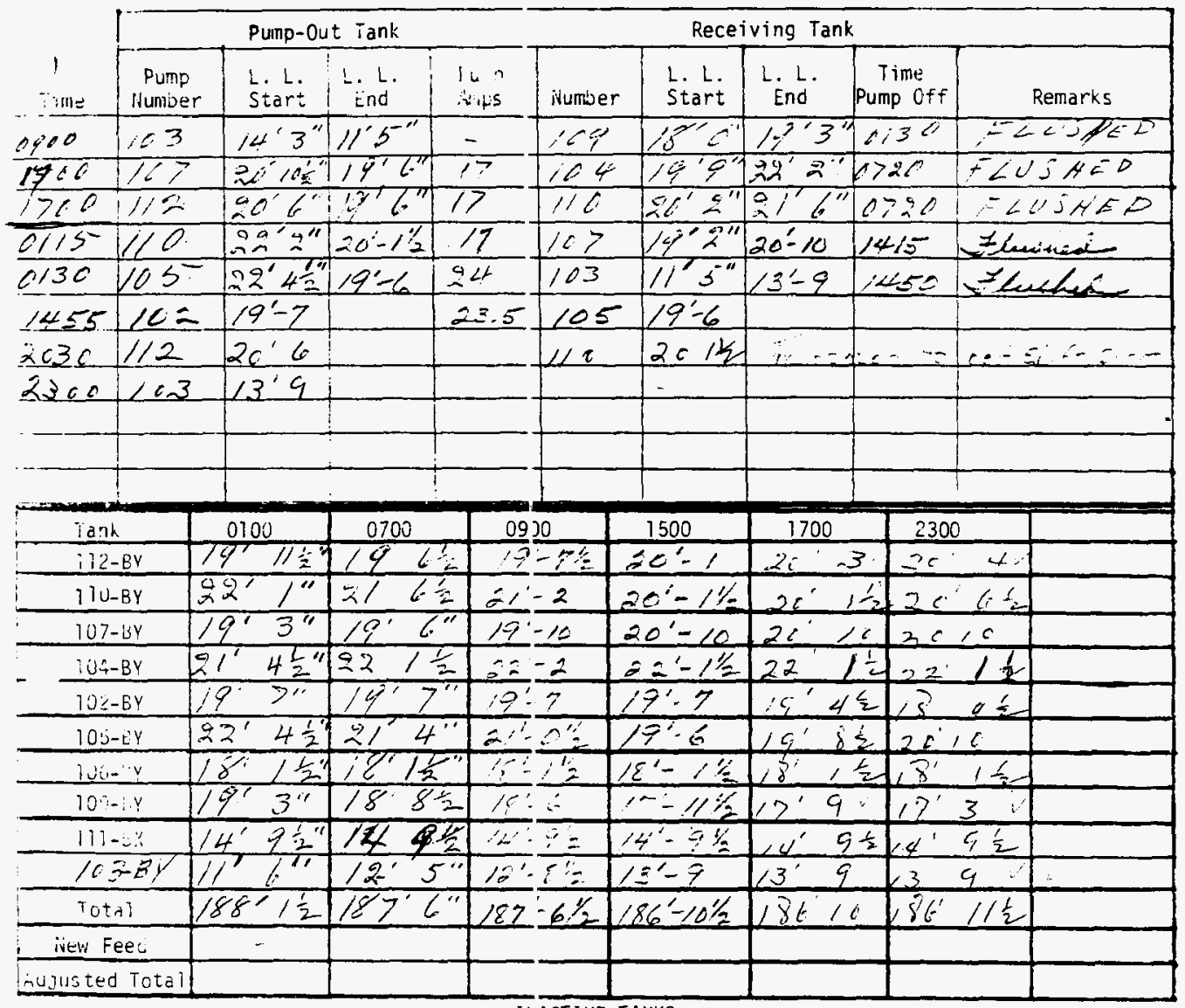

IWACTIVE TANKS

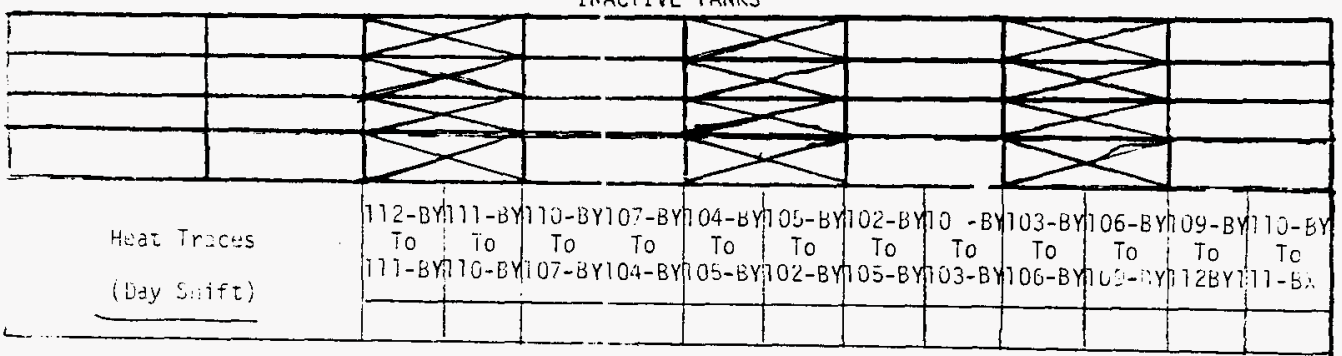


Puinp-Out Tank

Pump

ituniber L. L. I. L.

$2+27-7: 2=$ 102 1455

2030

115

0200

2.200103

$0 \times 1004$

0630

105

$12=0127$

人

1,120

1900102

(n)

2030,112

$22^{\prime} 3^{\prime \prime} / 9^{\prime}-7$

$20 \div 10$
Start ind

$19^{\prime} 7^{\prime \prime} 18^{\prime} I^{\prime \prime}$

$20^{\prime} 6^{\prime \prime} 19^{\prime}-8$

ints itumber

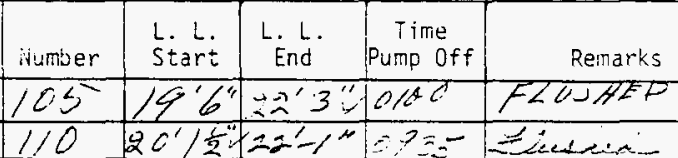

Receiving Tank

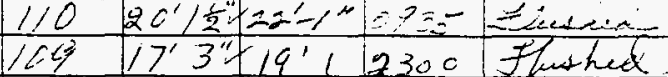

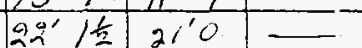

23

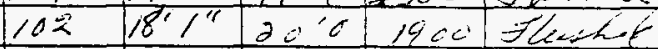
$103 / 2^{\prime} 1^{\prime \prime} 12^{2}-6 / 21500$ flenthe

\begin{tabular}{|c|c|}
\hline 104 & $20^{\prime}-9$ \\
107 & $19^{\prime 7}$ \\
\hline 105 & $19^{\prime 7}$ \\
\hline 110 & $21^{\prime 7}$ \\
\hline
\end{tabular}

\begin{tabular}{|c|c|c|c|c|c|c|c|}
\hline ark & 0100 & 0700 & 0900 & 1500 & 1700 & 2300 & \\
\hline i $12-B Y$ & $20^{\circ} 3^{\prime \prime}$ & $19^{\circ} 11^{\circ}$ & $19-8 \therefore$ & $60^{\prime}-1 \%$ & 20 & $20,4 \frac{1}{2}$ & \\
\hline $11 U-B Y$ & $20^{\circ} 10^{\prime \prime}$ & $2 /^{\prime} 8^{\prime \prime}$ & $2=-0=$ & $=2^{\prime}-0 / 2$ & $22^{\circ} 0 \frac{1}{2}$ & 21 & \\
\hline $107-34$ & $20^{\circ} 10^{\prime \prime}$ & $20^{\circ} 10^{\circ}$ & $32^{\prime}-12$ & $20^{\prime}-41 / 2$ & 20 & 1962 & \\
\hline $00_{4}^{n}-8 Y$ & na / / & $81^{\prime} 10^{\prime \prime}$ & $31=5$ & $20^{\prime}-10$ & $20 \quad 10 \frac{1}{2}$ & 212 & \\
\hline $102-B y,-3$ & $18^{\prime} / 1^{1 / 0^{2}}$ & 1845 & $183=$ & $19^{\prime}-31 /$ & $19^{\prime} 7^{\prime \prime}$ & 197 & \\
\hline 10u-EY. & $2 \leq-510$ & $22^{\prime} 3^{\prime \prime}$ & $21 \div:$ & $19^{\prime}-7$ & 7 & $90^{\prime} 6$ & \\
\hline 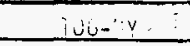 & 451 & $1.8 \quad 1$ & $18-i=$ & $16-1 \%$ & $1 \frac{1}{2}$ & $181 \frac{1}{2}$ & \\
\hline $10:-i . y$ & $17^{\prime} 6$ & $18^{\prime} 1$ & $18=3$ & $18^{\prime}-10$ & $1 \frac{1}{2}$ & 1911 & \\
\hline $111-2$. & $14^{\prime} \quad \% \frac{1}{2}$ & $14^{\prime} 7 \div$ & $14 \div ?=$ & $14^{\prime}-9 \leq$ & $4=$ & 1492 & \\
\hline $103 B 1$ & $13^{\prime} 3^{\prime \prime}$ & $12^{\prime} L^{\prime \prime}$ & $11=3$ & $12^{\prime}-61 \%$ & $\angle 2$ & 112 & \\
\hline Total & $1880^{7}$ & $187^{1} 11^{\prime \prime}$ & $18:-31=$ & $16 b^{1}-6 / 2$ & $186 \div 2$ & 1863 & \\
\hline iven Fee' & - & & & & & & \\
\hline Aujus ted Tota & & & & & & & \\
\hline
\end{tabular}

\begin{tabular}{|l|l|}
\hline $105^{\circ}$ & $11^{\prime} 11^{\prime \prime}$ \\
\hline & \\
\hline & \\
\hline & \\
\hline
\end{tabular}

Hẽat Trictss

(Dey S!ifif)

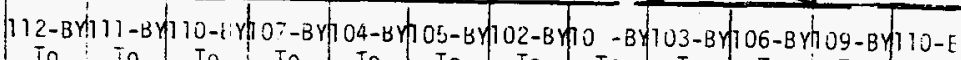
To To To To To To To To To To To To To

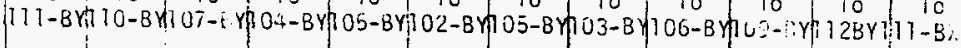

L.

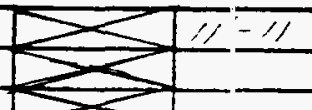

$\longrightarrow$
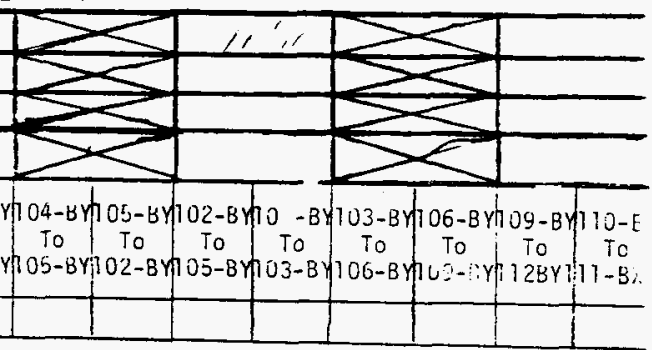

$9-3-7 i$ 


\section{ITS-2 IIVENTORY FINU PUMPIHG

Punp-Dut iank

\section{Pume L.L. L.L. P P e}

univer Start End

$1 / 2$

1900.110

1,13001107

m.1900 10:

0945104

$11100 \quad 105$

r. 10302

1,2030

or

12000

105

12230

110

112
Receiving Tank

\begin{tabular}{|c|c|c|c|c|}
\hline ivumer & $\begin{array}{l}\text { L. } L \text {. } \\
\text { start }\end{array}$ & L. L. & $\begin{array}{l}\text { Time } \\
\text { Pump ff }\end{array}$ & Remarks \\
\hline 110 & $27^{\prime \prime} 7^{\prime \prime}$ & $=\therefore=$ & 3452 & Flus: \\
\hline 107 & $14^{\prime} 7^{\prime \prime}$ & $20^{\prime}-10$ & $0 \leq 23$ & $=2-\ldots$ \\
\hline $10 \%$ & $20 \%$ & 256 & 0215 & FLCUAED \\
\hline $105^{\circ}$ & $19^{\prime} 7^{\prime \prime}$ & $m=\frac{1}{3}$ & $00^{3 *} 0^{-72}$ & Flushed \\
\hline 102 & $18 \div 0$ & $20^{\prime} 0$ & 2030 & find h \\
\hline 103 & $11^{\prime} 7^{\prime \prime}$ & $14^{\prime} 3^{-1}$ & 0045 & 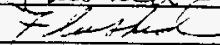 \\
\hline 104 & $2^{\prime \prime} 3$ & & & \\
\hline 105 & $19^{\prime}=$ & & & \\
\hline 107 & $200 \frac{1}{2}$ & & & \\
\hline 110 & & & & \\
\hline
\end{tabular}

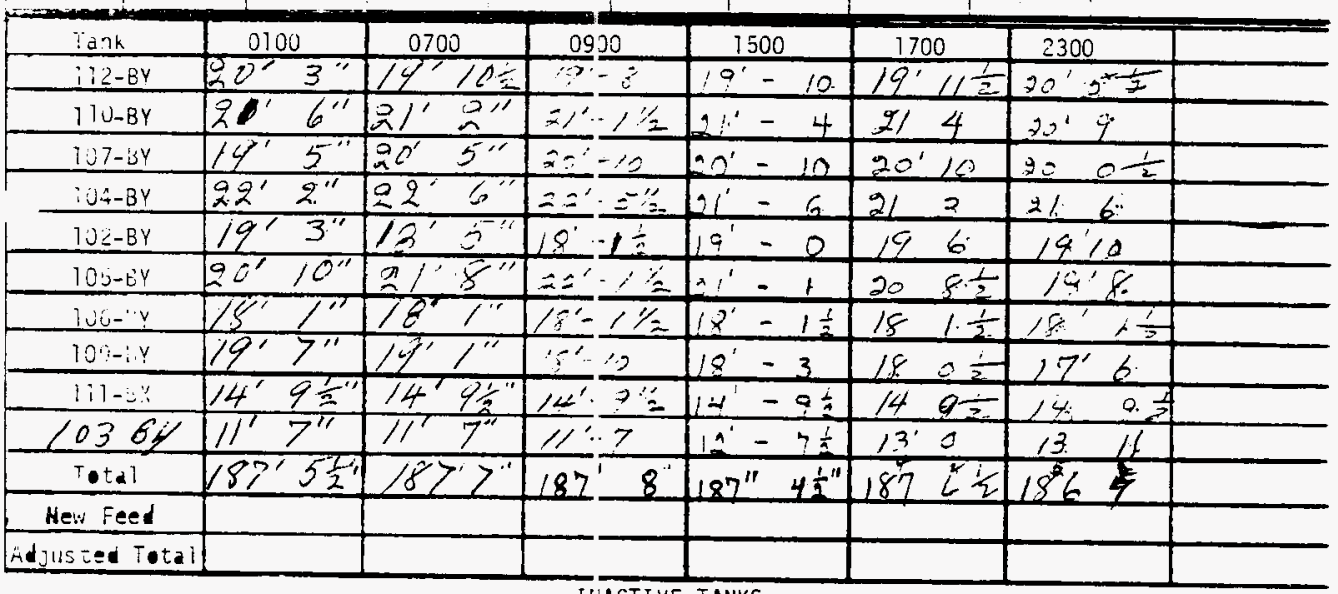

IIIACTIVE TANKS

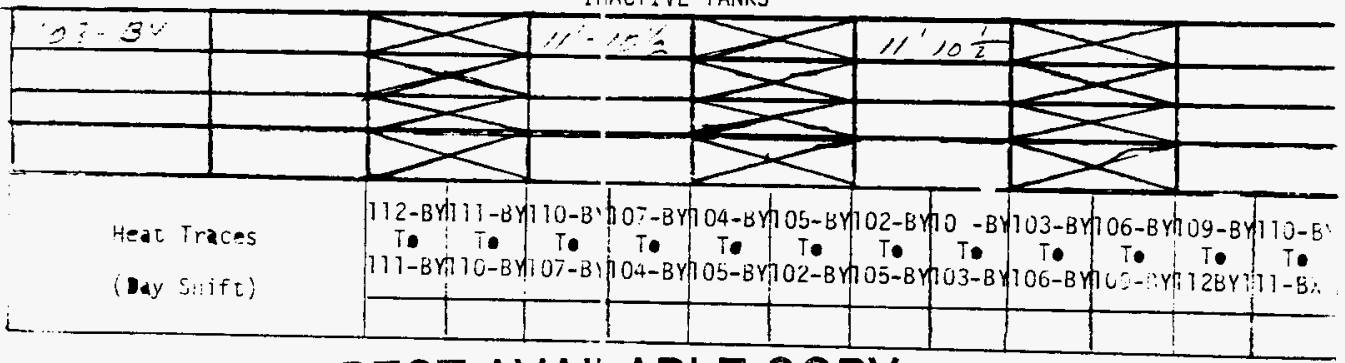




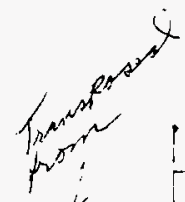

Cate

Pump-Out Tank

i Pump inte lumber $1630 \quad 107$ $2030 \quad 02$

$x \frac{2000110}{2000}$

122301112 0045 $\operatorname{arc} 430$ N.1030 103

M) $1630: 167$

(B) 1630 1630103

\section{L. L. L.} Start End $1911 / 2: 19^{\prime} 7$ $20^{\prime} 9 \frac{1}{2}+18^{\prime} 10^{\prime \prime}$
Receiving Tank

\begin{tabular}{|c|c|c|c|c|}
\hline ivumier & $\begin{array}{l}\text { L. L. } \\
\text { Start }\end{array}$ & $\begin{array}{l}\text { L. L. } \\
\text { End }\end{array}$ & $\begin{array}{c}\text { Timie } \\
\text { Pump off }\end{array}$ & Rentarss \\
\hline 104 & $213^{\prime \prime}$ & 926 & OSEC & $F \angle O S A E D$ \\
\hline 105 & $17^{\prime} 7^{n}$ & 246 & $?$ & Hicuside' \\
\hline 107 & $20^{\prime} 0 \frac{1^{\prime}}{2}$ & $20^{7} 16$ & 0.36 & $E \angle C \& H E O$ \\
\hline
\end{tabular}

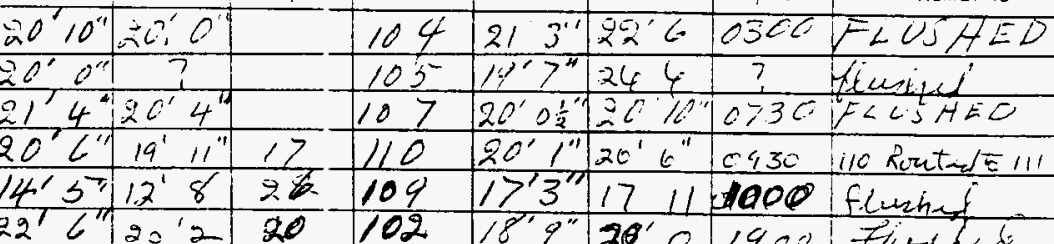
$22^{\prime} c^{\prime \prime} 22^{\prime} 280$ 102 $111 B \times 149^{\prime} / 25^{\prime \prime \prime} 2100$ Fluch

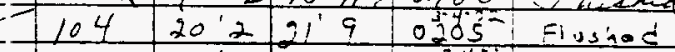

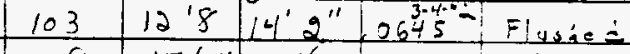
\begin{tabular}{l|l|l|l|l|}
109 & $17^{\prime} 4$ & $17^{\prime} 5$ & $2041^{\prime}$ the
\end{tabular}

\begin{tabular}{|c|c|c|c|c|c|c|c|c|}
\hline$d{ }^{2}$ & 0100 & 0700 & & & 1500 & 1700 & 2300 & \\
\hline$i 12-6 Y$ & $20^{7} 4$ & 306 & 19 & 11 & $19 \quad 10$ & $\angle 9,9 \frac{1}{2}$ & 1982 & \\
\hline $11 U-B Y$ & $20^{\prime} 9^{\prime \prime}$ & $50^{\circ} 4$ & $20^{\circ}$ & $5^{-11}$ & $20^{\prime} 10$ & $20 \quad 9 \frac{1}{2}$ & $20^{\prime} 91$ & \\
\hline $107-i 5$ & $20^{\circ} 0^{-1}$ & $20 \cdot 8$ & 20 & 10 & $20^{\prime} 9^{\prime \prime 2}$ & $20 \quad 9 \frac{1}{2}$ & $19^{\prime} 7$ & \\
\hline$: u^{n}+B^{v}$ & $21^{\prime} / 1^{\prime \prime}$ & $\begin{array}{rl}92 & 0 \% \\
x & \\
\end{array}$ & 21 & 6 & $20^{\circ} L^{\prime \prime}$ & 202 & $2 d^{\prime} 0$ & \\
\hline $10 \div-E^{Y}$ & 1465 & $1 S^{\prime} f_{2}^{2}$ & 18 & $8 i 2$ & $19^{\prime} 1^{\prime \prime}$ & 194 & $20^{\circ} 0$ & \\
\hline $10 y-E^{v}$ & $19^{\circ} 7^{\prime \prime}$ & $214^{\prime \prime}$ & 21 & $91 / 2$ & $22 \cdot 6$ & $22^{\prime} 6$ & $21 \quad+\frac{1}{2}$ & \\
\hline ivin.'v & 181 & $79^{\prime} 1$ & 18 & 1 & 18 & 181 & 181 & \\
\hline $10 \%-\ldots$ & $1)^{\prime} 3^{\prime}$ & $17.77^{\prime \prime}$ & 12 & 9 & $175 \frac{1}{2}$ & 174 & $177-\frac{1}{2}$ & \\
\hline $111-\ldots \%$ & $147 \frac{1}{2}$ & $14^{\prime} 96$ & 14 & $9 / 2$ & 151 & 154 & $1511 \frac{4}{2}$ & \\
\hline 1032 & $46 s^{-11}$ & $13^{\prime} 5$ & 13 & $c^{\prime \prime}$ & 12.8 & 12 & 1218 & \\
\hline Total & $15^{\prime}>$ & $186^{\prime} 11 \frac{1}{2}$ & 184 & $9 \%$ & $196^{\circ} 10^{\circ}$ & 1864 i & 14684 & \\
\hline iven Fee: & & & & & & & & \\
\hline mujusted Total & & & & & & & & \\
\hline
\end{tabular}

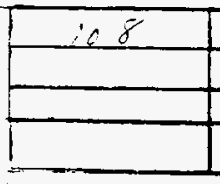

Hiat Tracus

$(1+y)$ stift $)$

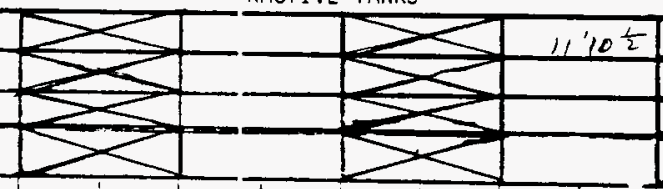

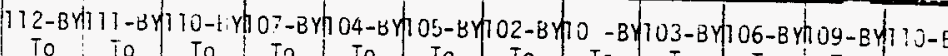
To To To To to To To To To To To To

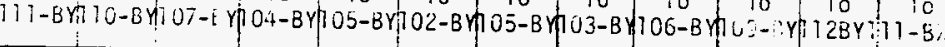




\begin{tabular}{|c|c|c|c|c|c|c|c|c|c|}
\hline \multirow[b]{2}{*}{ m } & \multicolumn{4}{|c|}{ Punp-Out iank } & \multicolumn{4}{|c|}{ Receiving Tank } & \multirow[b]{2}{*}{ Rerrarks } \\
\hline & $\begin{array}{c}\text { Pump } \\
\text { ilumber }\end{array}$ & $\begin{array}{l}\text { L. L. } \\
\text { Start }\end{array}$ & E. L. & $\begin{array}{l}14: 9 \\
4+5\end{array}$ & rumber & $\begin{array}{l}\text { L. L. } \\
\text { Start }\end{array}$ & $\begin{array}{c}\text { L. } L . \\
\text { End }\end{array}$ & $\begin{array}{c}\text { Time } \\
\text { Pump 0ff }\end{array}$ & \\
\hline 2200 & 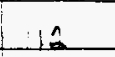 & $20^{\prime} 6^{\prime \prime}$ & $199^{\prime} 10$ & 18 & 107 & $18^{\prime} 10^{\prime \prime}$ & $20<1 \frac{1}{2}$ & 1630 & $-77 u+h e_{-1}$ \\
\hline 0202 & 102 & $10^{1} 9 \frac{1}{2}$ & $18^{\prime} 0$ & & 105 & $19^{\prime} 7 \div$ & & $\therefore 800$ & Ilustres \\
\hline 1315 & $111 B X$ & $15^{\prime} 5^{\prime \prime}$ & $144^{\prime} \quad 11$ & & $1103 x$ & $8 \cdot 2 \frac{1}{2}$ & $8^{\prime} 8 / 2$ & 1445 & 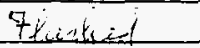 \\
\hline 1630 & 107 & $2014 \div$ & $19^{\prime} 0^{\prime \prime}$ & & 104 & $21^{\prime} 9$ & $2,10^{\prime \prime}$ & $2030^{-5}$ & Elusised \\
\hline 1630 & 104 & $22^{\prime} 0$ & $0 i^{\prime} 1^{\prime \prime}$ & & 102 & $18^{\prime} 0$ & $2 e^{1} c$ & $\begin{array}{l}3.5 .72 \\
0700\end{array}$ & Flushed \\
\hline & & & & & & & & & \\
\hline & & & & & & & & & \\
\hline & & & 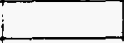 & & & & & & \\
\hline & & & & & & & & & \\
\hline & & & & & & & & & \\
\hline & & & & & & & & & \\
\hline
\end{tabular}

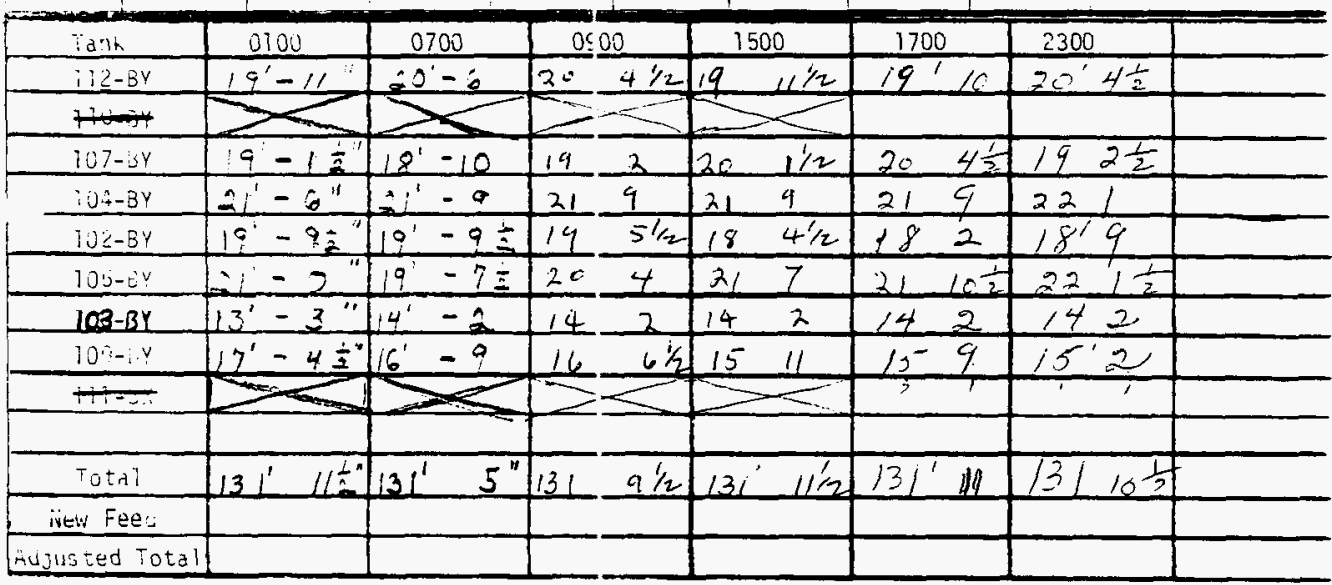
I.IACTIVE TANKS

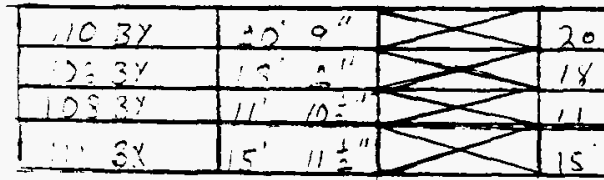

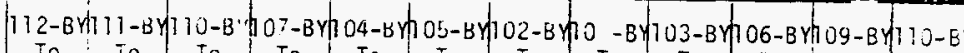

Heat Traces

(Dyy s:ift)

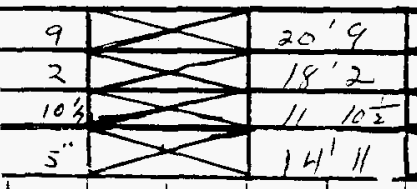

To to To To To to to to To To ito To To

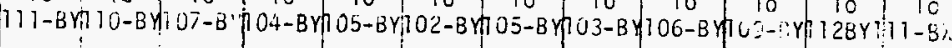




\section{- WHC-SD-WM-DP-206}

ITS-2 IIIVENTORY HINU PUMPING

Cate $z-5-7$, ReVO

Puinp-Out Tank

L. L. L. L.

Pump

itumber

$n i n=$

Start

ind

- ins

$20^{\prime} 8^{\prime \prime} 22^{i}=x^{\prime \prime}$ $20^{\prime}-\frac{1}{2}+19 \div 6$

112 $20^{\circ} 2,199^{\circ}$ 1230

107

$14^{\prime} 213^{\prime} 6$

$2230105 \quad 22^{\prime} / \frac{1}{2} / 9^{\prime} 6$

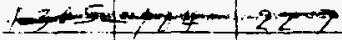

Receiving Tank

\begin{tabular}{|c|c|c|c|c|}
\hline irumber & $\begin{array}{l}\text { L. L. } \\
\text { Start }\end{array}$ & L. L. & $\begin{array}{l}\text { Time } \\
\text { Pump off }\end{array}$ & Remarks \\
\hline $1: 3 x$ & $1 \pm U^{\prime \prime}$ & 15 & $900-$ & -0101 \\
\hline 107 & $10^{1} 0^{\prime \prime}$ & $20-5$ & 0050 & $=2 \mathrm{~min}-2-7=$ \\
\hline 104 & $21^{\prime \prime} 1$ & $22 \div 7$ & 2000 & $F(U S H E D$ \\
\hline 109 & $18^{\prime} 2$ & $\angle 9^{\prime} 7$ & 1630 & F/usted \\
\hline 103 & 142 & $14^{\prime 3}$ & 1300 & \\
\hline+2 & $\ldots \ldots$ & & & \\
\hline
\end{tabular}

\begin{tabular}{|c|c|c|c|c|c|c|c|}
\hline ank & 0100 & 0700 & $0 \div 00$ & 1500 & 1700 & 2300 & \\
\hline$i 12-8 Y$ & 208 & $90^{\prime} 42$ & $31 / 2$ & $1911 / 2$ & $j=$ & $1917 \frac{1}{2}$ & \\
\hline For & - & & & & & & \\
\hline $107-3 y$ & $10^{\prime} 0^{\prime \prime}$ & $3-i$ & 19 & 20 & $19 \quad 11$ & 301 & \\
\hline$: 04-8 Y$ & $21^{\prime \prime} 10^{\prime \prime}$ & $21^{\prime}$ & 21 & 21 & $21 \quad 10 \div$ & $22^{\prime} 7$ & \\
\hline $10 \leq-B Y$ & $19^{\prime} 1$ & $22^{\circ} a^{\prime \prime}$ & 20 & 20 & $2 c$ & $20^{\prime} c$ & \\
\hline $10 j-64$ & $e^{\prime} ! \frac{1}{2}$ & $1 \div 1$ & 22 & 22 & $22,1 \frac{1}{2}$ & $22^{\prime}, 1 \div$ & \\
\hline $132-1$ & $14^{\prime} \Rightarrow 1$ & $\therefore-1$ & 14 & 14 & $14 \quad 2$ & $14^{\prime} 2$ & \\
\hline $10^{-}:-y$ & $14^{\prime} 11$ & 6 & $16 \quad 31 / 2$ & 17 & $15 \quad c \div$ & $18 \div$ & \\
\hline $111-2.1$ & $\angle 7$ & $\because$ & 4 & & & 1 & \\
\hline Total & $131^{\circ} 9 \frac{1}{2}$ & $133^{1} \frac{1}{2}$ & $x^{10} 3^{\prime}{ }^{2}$ & $135^{-1} 66^{\prime \prime}$ & $135^{\prime}, 1 \frac{1}{2}$ & 1369 & \\
\hline inew Fee: & & $109340=2$ & & & $103 \beta$ & & \\
\hline Aujus ted Total & & $=73 M: 02-3$ & & & $\therefore=109 b^{\circ} y$ & & \\
\hline
\end{tabular}

I VACTIVE TANKS

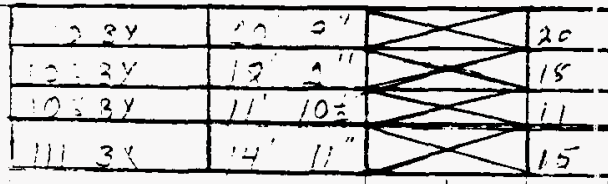

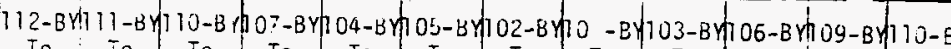

Heat Traces

(Doy Sifift)

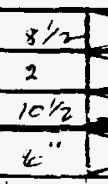

To : To

$11-8$ in $10-8$ 107-B To To To To To To To To To

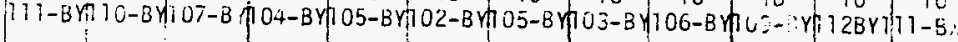

i. 
Pump-Out Tank

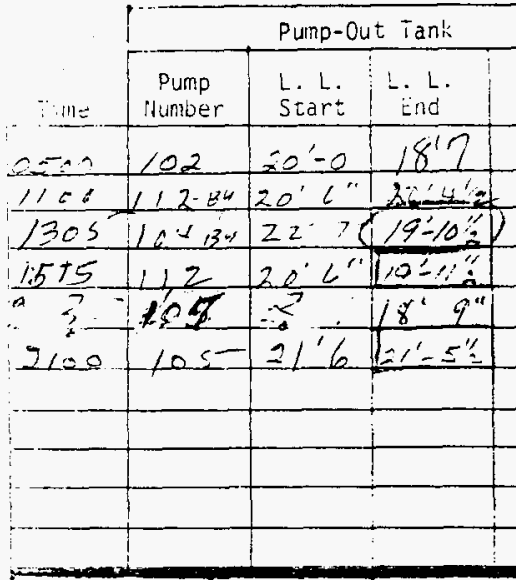

Receiving Tank

\begin{tabular}{|c|c|c|c|c|}
\hline iviniser & $\begin{array}{l}\text { L. L. } \\
\text { Start }\end{array}$ & L. L. & $\begin{array}{c}\text { Timie } \\
\text { Pump off }\end{array}$ & Remarks \\
\hline $10=$ & $50^{\prime}-6$ & 224 & 1205 & $2 x$ \\
\hline $111-13 x$ & $15^{\prime}=12$ & $-159 \%$ & 1326 & Adurben \\
\hline $10 \div-13 y$ & 10.2 & $60-0$ & $0 / 45$ & 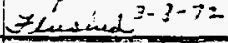 \\
\hline 107 & $205^{\prime} 2$ & $\because 7 \div 2 \frac{1}{2}$ & $053-$ & T2un $i^{3-7-72}$ \\
\hline 104 & 3 & & 1102 & $7 x^{2}+2<21$ \\
\hline $10^{7} 3$ & $136 \frac{1}{2}$ & $1+-61 / 2$ & $0: 25$ & $2.12: 2-7.32$ \\
\hline & & & & \\
\hline & & & & \\
\hline & & & & \\
\hline & & & & \\
\hline & & & & \\
\hline
\end{tabular}

\begin{tabular}{|c|c|c|c|c|c|c|c|}
\hline dak & 0100 & 0700 & 0300 & 1500 & 1700 & 2300 & \\
\hline$: i 2-B Y$ & $19-5$ & 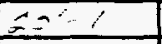 & $20^{\circ} ; \%$ & $26^{\prime} 6^{\prime \prime}$ & 20 & 2013 & \\
\hline$-\infty,-$ & 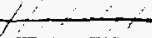 & מיח & & & & & \\
\hline $107-34$ & $20 \leq-51$ & $20^{\prime}-51 /$ & $205^{-1}=$ & $26 \quad 5^{\prime \prime 2}$ & 207 & $20^{\circ} 2 \frac{1}{2}$ & \\
\hline$: 0:-B Y$ & $\hat{x}=-?$ & $= \pm-1$ & 227 & 223 & 22,0 & .224 & \\
\hline $10 \div-B y$ & $20^{\prime}-0$ & $19-72$ & 197 & 1. 6 & $1 \frac{1}{2}$ & $18 \quad 10 \frac{1}{2}$ & \\
\hline $105-E^{Y} Y$ & $21 \div 6$ & $A^{\prime}=$ & $2 i \quad c$ & $\pm 2 \quad 1$ & $20^{i} 6$ & $01 \quad 6$ & \\
\hline $103-v$ & $2-1$ & $\therefore-0=$ & $1311^{1} 2$ & $13 \quad 16$ & $13^{\prime} 6-2$ & 139 & \\
\hline $10 \cdot 1-4 y$ & $y-2 y$ & $17-0$ & 19 & $14 \quad 6$ & 19 & $18 \quad 4$ & \\
\hline 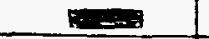 & & & & & 1 & " & \\
\hline iotal & $136^{\prime}-7^{\prime \prime}$ & $136^{\prime}-x^{\prime \prime}$ & $130^{\prime} 0^{\prime \prime} 2$ & 13.5111 & $135^{\prime} 10$ & $125^{\prime} 10$ & \\
\hline iven Feec & & & & & & & \\
\hline Hujusted Totall & & & & & & & \\
\hline
\end{tabular}

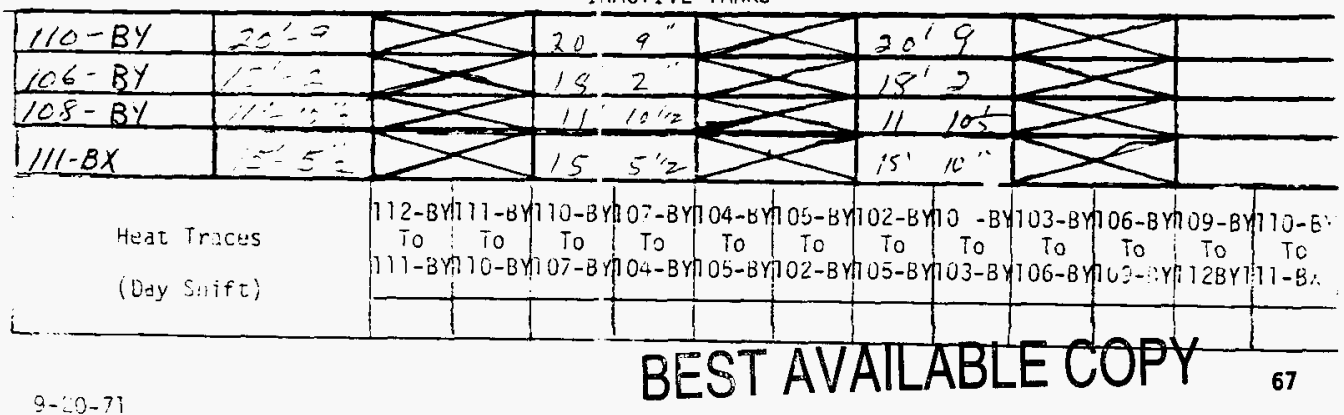




\section{$=\quad$ WHC-SD-WH-DP-206}

ITS-2 IIUENTORY IINE PUMP IIIG
Pump-Out Tank

L. L. I. L.

Start

Ënd ivits

$\therefore \therefore \quad 102$

$-2 \div 105$

$210=112$

\begin{tabular}{l|l|l}
$1^{\prime}-5: 34^{\prime}$ & $1^{\prime \prime}$ & $=\because$ \\
$22^{\prime}-5$ & $?$ & $=-1$
\end{tabular} $21^{\prime} 6 \quad 20^{\prime} 6$
Receiving Tank

\begin{tabular}{|c|c|c|c|c|}
\hline ivumber & $\begin{array}{l}\text { L. L. } \\
\text { Start }\end{array}$ & $\begin{array}{l}\text { L. L. } \\
\text { End }\end{array}$ & $\begin{array}{c}\text { Time } \\
\text { Pump Off }\end{array}$ & Rerriarks \\
\hline $10=$ & 103 & $19^{\circ} z$ & 1330 & $\hat{f}_{1} x_{2} 4$ \\
\hline 103 & $14-1$ & $=$ & $?$ & 3 \\
\hline 107 & $78^{\prime} 9$ & $19^{\prime} 2 \frac{1}{2}$ & 152 & 11 \\
\hline
\end{tabular}

\begin{tabular}{|c|c|c|c|c|c|c|c|c|}
\hline$\because a 7 k$ & 0100 & 0700 & $0 s$ & & 1500 & 1700 & 2300 & \\
\hline$\lcm{72-8 y}$ & $\Rightarrow=\therefore$ & $00^{\circ}-=$ & 20 & $3 / 2$ & $25^{\prime} \quad 11$ & 2111 & 5 & \\
\hline \multicolumn{9}{|l|}{ tevas } \\
\hline $107-3 Y$ & $30^{\circ}-10$ & $10-8$ & 19 & $31 / 2$ & is & 18 & $19 \quad 02$ & \\
\hline $\mathrm{O}) 4-B Y$ & $= \pm-5 \%$ & $22^{\prime}-4$ & 22 & 3 & $q^{\prime \prime}$ & $21 \quad 6$ & 25 & \\
\hline $10 \leq-B Y$ & $y^{\prime}-\therefore$ & $18 \div 7$ & 18 & $8^{\prime 1 / 2}$ & $7 !^{\prime \prime}$ & $15^{i} 2$ & 19 & \\
\hline $100-64$ & $21^{\prime} \cdots$ & $22^{\prime}-5$ & $\approx 2$ & $2 / 2$ & $22 \quad 5^{16}$ & 224 & 2216 & \\
\hline $3-4$ & $\angle \angle^{\prime}=-\angle$ & $14^{\prime}-1$ & 14 & 1 & 245 & $145 \frac{1}{2}$ & 14152 & \\
\hline $101:-1 . y$ & $18^{\prime}-7$ & $8^{\prime}-7$ & 18 & 9 & 194 & $191 \frac{1}{2}$ & $19^{\prime} 52$ & \\
\hline \multirow[t]{2}{*}{ 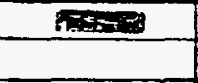 } & & & & & & 1 & 2 & \\
\hline & & & & & 135 & & & \\
\hline$T_{0 t a l}$ & $=0^{\prime}-0^{\prime \prime}=$ & $\angle 26-2$ & $135^{-1}$ & $7^{11}$ & $134^{\circ} / 1^{\prime \prime}$ & $135^{\prime \prime} / 0$ & $135^{-1} 102$ & \\
\hline ivew Fees & & & & & ? & & & \\
\hline Aujus zed Total & & & & & & & & \\
\hline
\end{tabular}

I IACTIVE TANKS

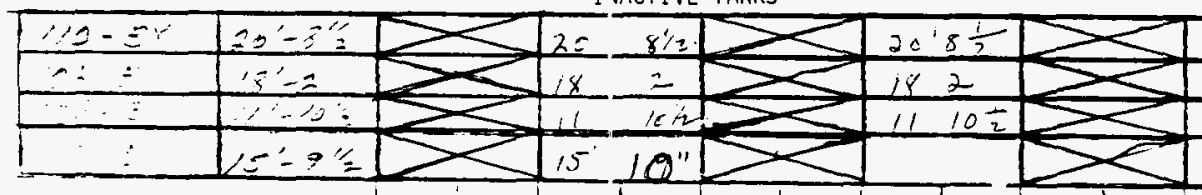

Heat Tracts

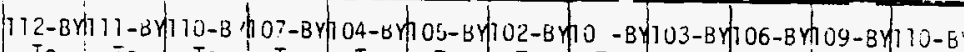

(bay s:ift)

i.

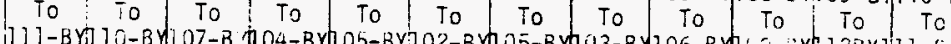

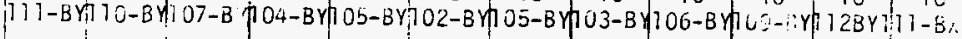




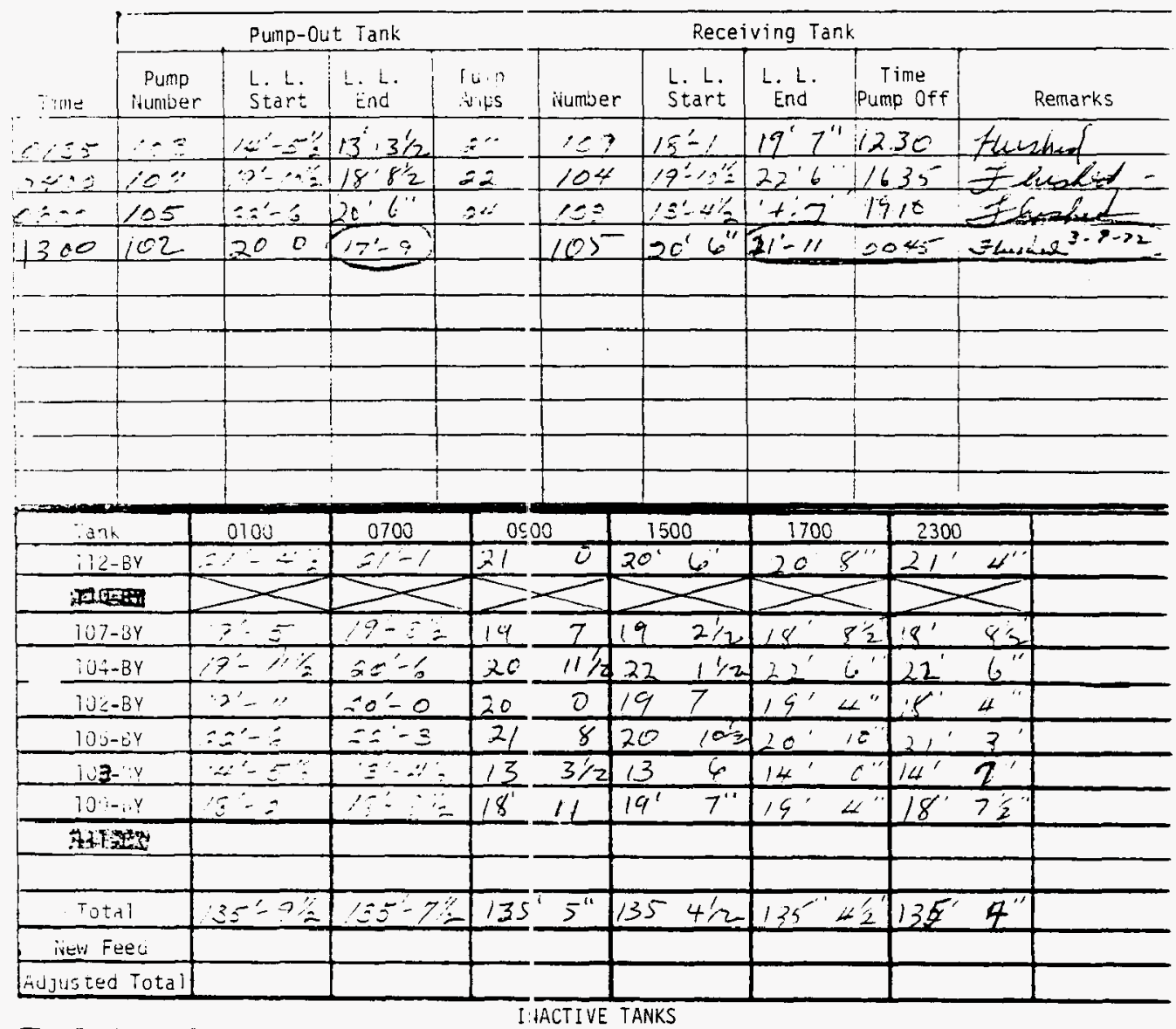

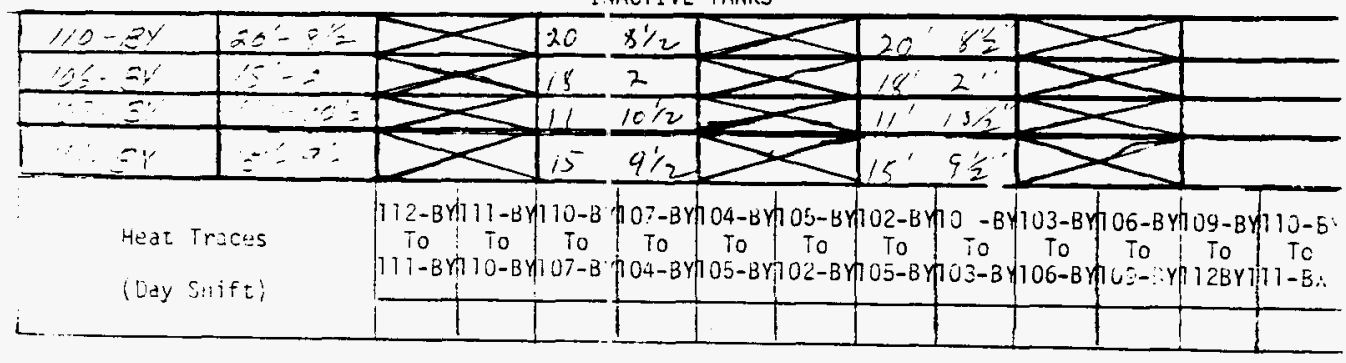




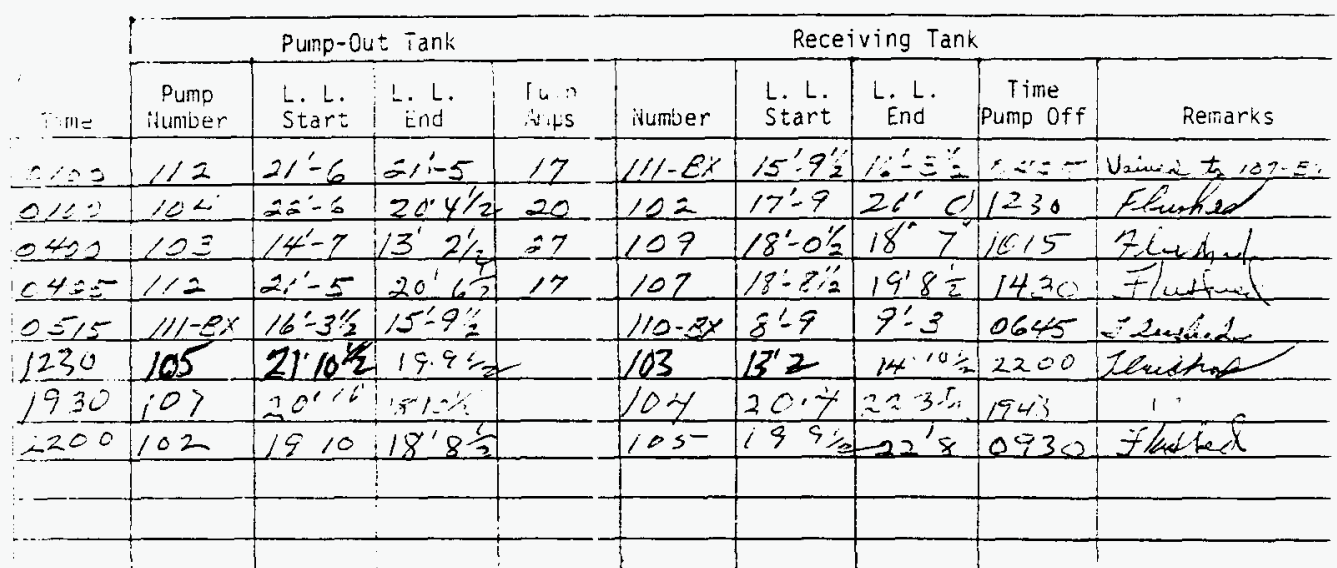

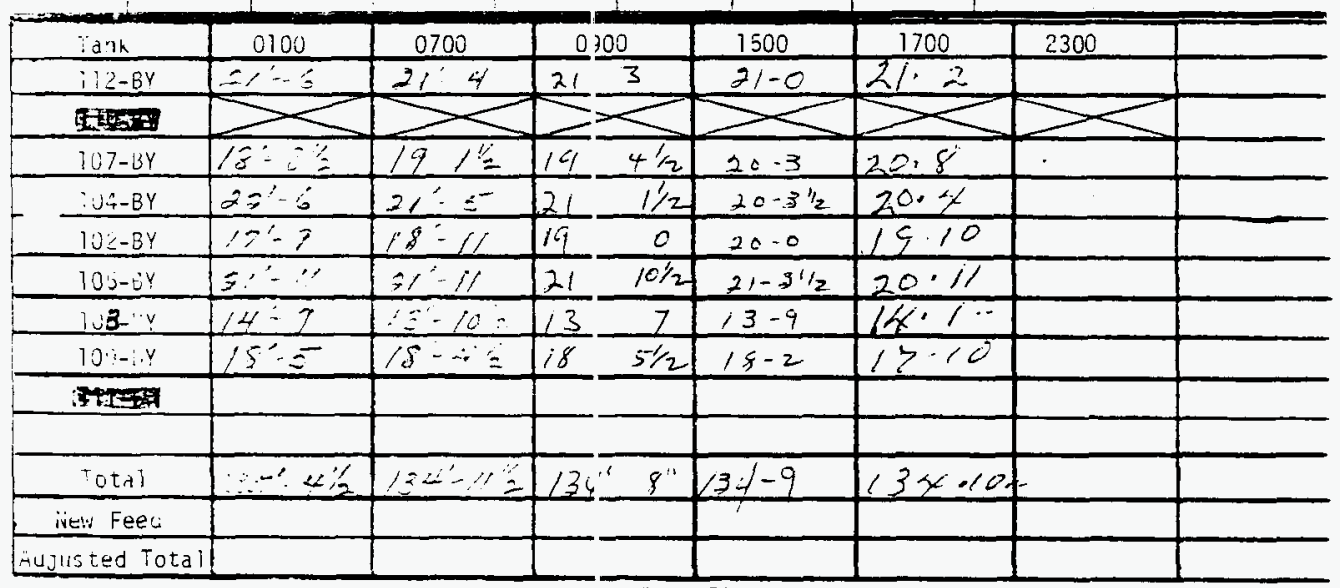
IVACTIVE TANKS

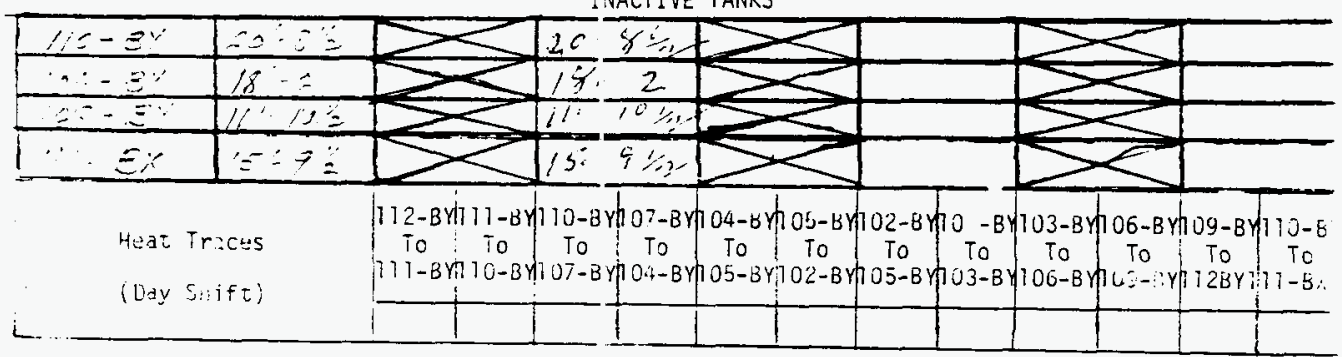




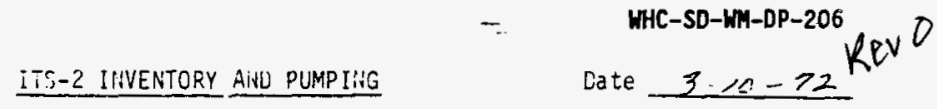

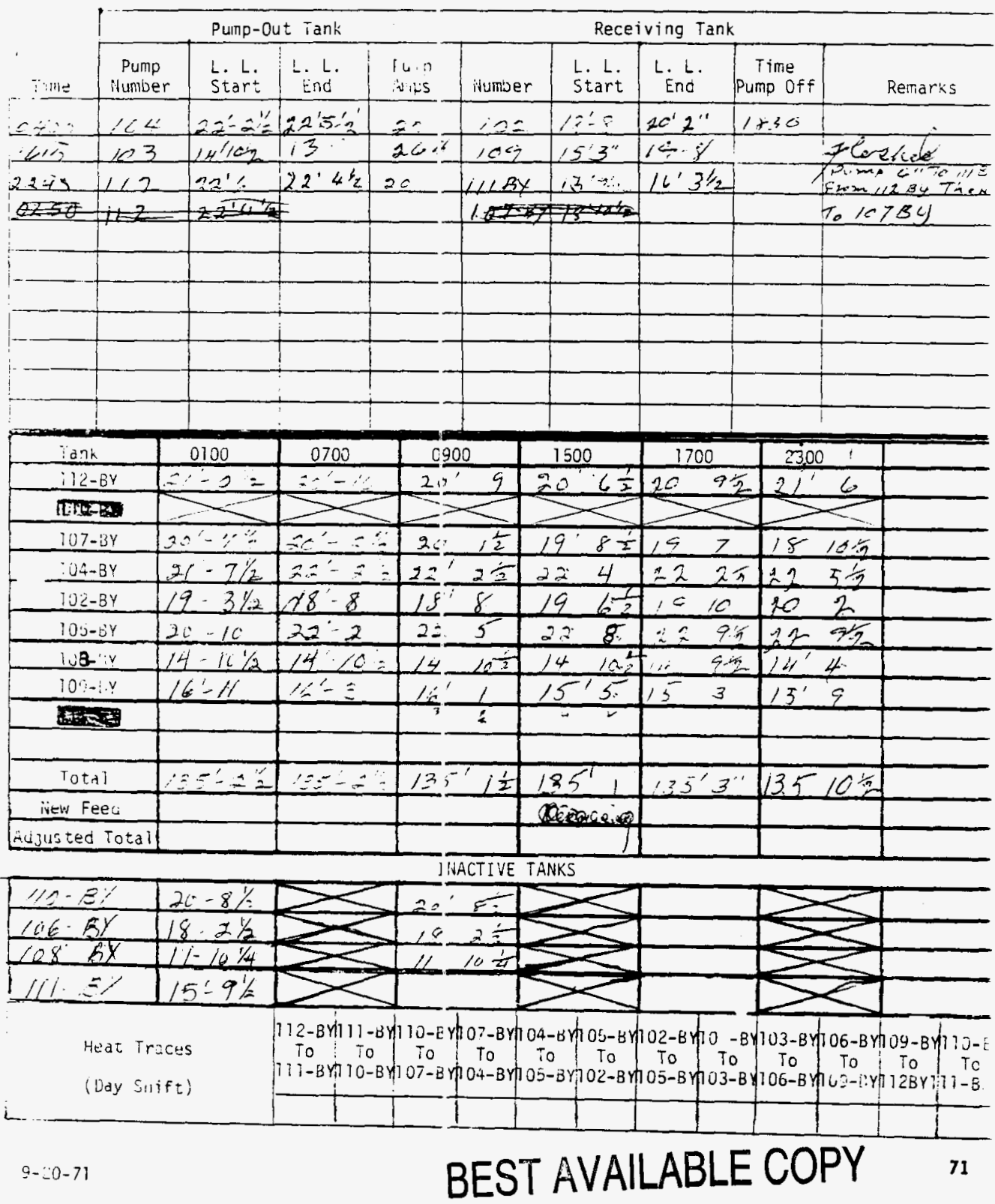




\section{-. $\quad$ WHC-SD-WH-DP-206}

ITS-2 IINENTORY ZINJ PUMPING

Date $3-11-72$

Pump-Out Tank

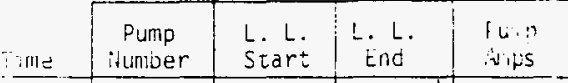

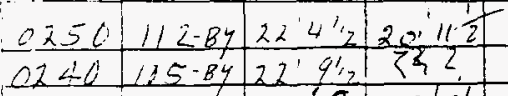

$\angle 10=$

1900

1900

102

$20^{\prime} \sigma: 18^{\prime} 5 z$

$1+\frac{1}{3-12}+112$

1000112

$1300 \quad 112$

$22 \cdot 5$

$20.7: \frac{20.0}{21.5 \%}$

$213+209$

$20^{\prime} 9: 20^{\prime} 8^{\prime \prime}$ $20^{\prime} 8^{\prime \prime} 20^{\prime} 6^{\prime \prime}$
Receiving Tank.

\begin{tabular}{|c|c|c|c|c|}
\hline isumber & $\begin{array}{l}\text { L. L. } \\
\text { Start }\end{array}$ & L. L. & $\begin{array}{c}\text { Time } \\
\text { Pump off }\end{array}$ & Remiarks \\
\hline 107.84 & $15: 10: 2$ & $20^{\prime} 7$ & 1430 & Thether \\
\hline $103 \mathrm{By}$ & $13^{\prime} 5^{\prime}$ & $13-7$ & 0040 & 7 LUSHED \\
\hline 105 & $20^{-3} \frac{1}{4}$ & 276 & 133 & I $\mid$ binit \\
\hline 102 & 19.0 & & & \\
\hline 104 & $22 \cdot 3 x$ & 22.8 & 0025 & 7cuSHED \\
\hline 107 & $3^{30} 6$ & $20^{\prime 4}$ & $\angle 0 O A$ & \\
\hline$\angle \angle B X$ & $15^{\prime} 11$ & $16^{\prime} 32^{\prime \prime}$ & 1300 & over $74^{\circ} \mathrm{C}$ \\
\hline 107 & $19^{\prime} 8^{\prime \prime}$ & $19^{i} 3$ & 0840 & Ele \\
\hline & & & & \\
\hline & & & & \\
\hline & & & & \\
\hline
\end{tabular}

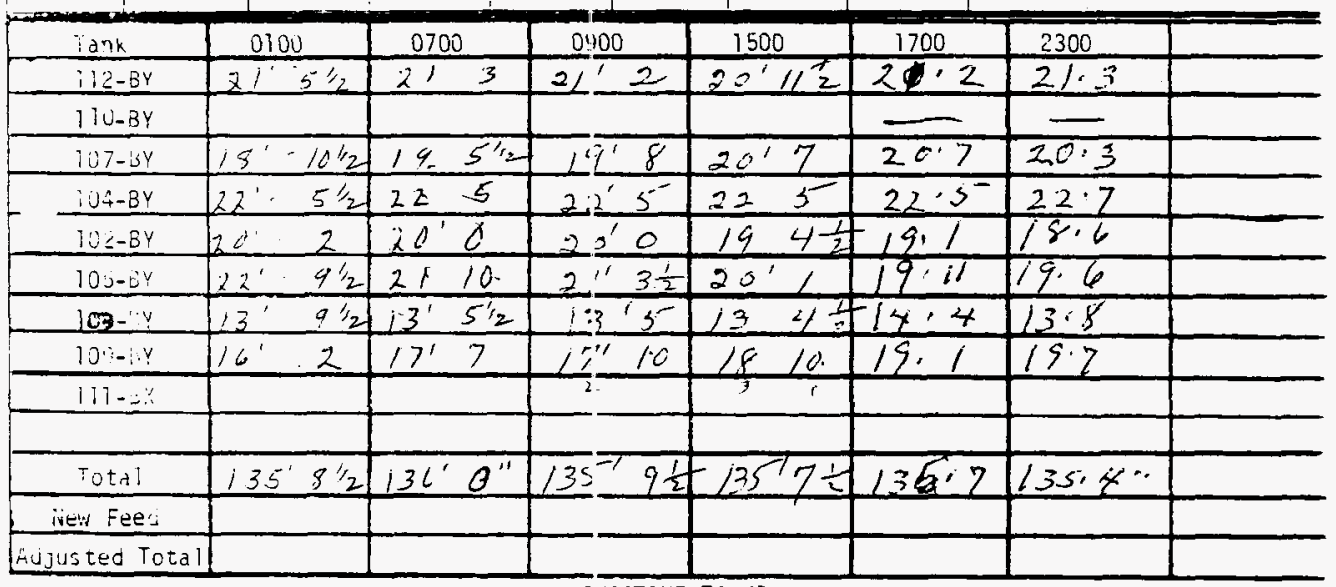

I VACTIVE TANKS

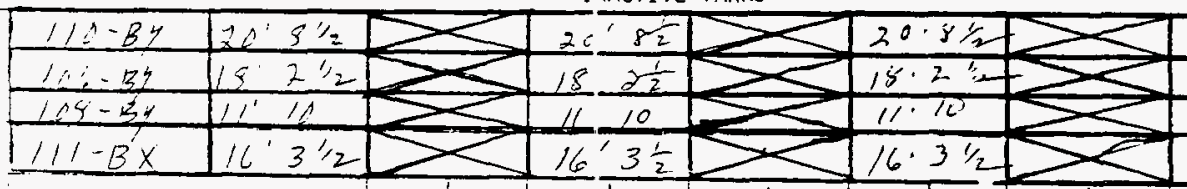

Heat Traces

(0)

L.

59516

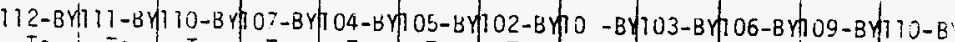

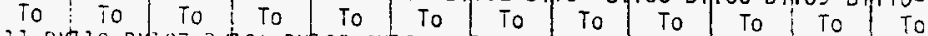

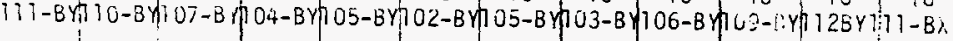




\section{- $\quad$ HHC-SD-MN-DP-206 ReND}

ITS-2 IINENTORY HWU PUMPING

cate 112121972

Pump-Out Tank

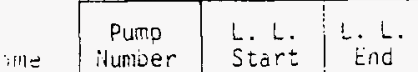

$044512720^{\prime} 9^{\prime \prime}+811$

\begin{tabular}{l|l|l|}
$6603-111$ & $10-3 / 2$ & $15-10 \%$ \\
\hline
\end{tabular}

18,51103

\begin{tabular}{l}
1830105 \\
\hline 510
\end{tabular}

2
$14.2 \% 14^{\prime} 00^{\prime \prime} 2^{\prime 25}$ ics

[u. n

Receiving Tank

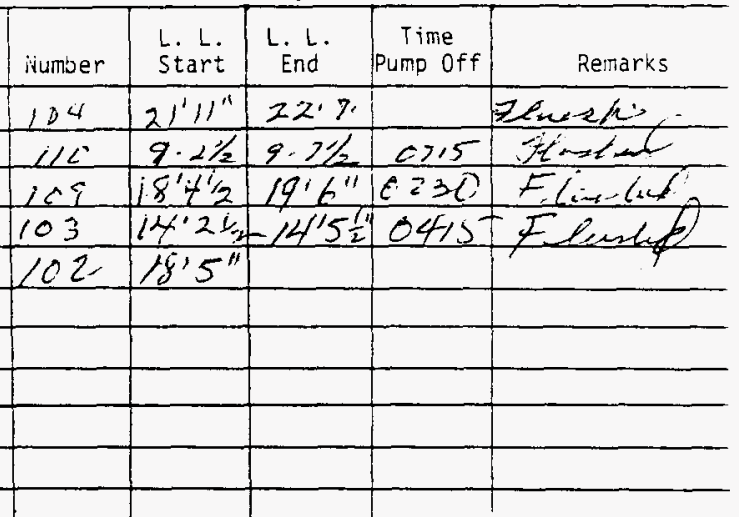

\begin{tabular}{|c|c|c|c|c|c|c|c|}
\hline \\
\hline & & & & & & & \\
\hline & & & & & & & \\
\hline & & & & & & & \\
\hline 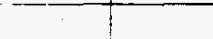 & & & & & & & \\
\hline & & & & & & & \\
\hline ink & 0100 & 0700 & $0: 00$ & 1500 & 1700 & 2300 & \\
\hline $112-8 Y$ & 212 & $20^{\prime} 11^{\prime \prime}$ & $20^{\prime} 10$ & 2472 & $-90^{\prime} 2^{\prime \prime}$ & $20 \quad 2$ & \\
\hline $1 \mathrm{U}-\mathrm{BY}$ & & & & & & & \\
\hline $107-3 Y$ & $20^{\prime} c$ & $20^{\prime} 7$ & $265-\frac{1}{2}$ & $19 \cdot 6 \frac{1}{2}$ & $19^{\prime} 4 \prime 2$ & $18 \div ; 1$ & \\
\hline$; j 4-B Y$ & $22-8$ & $21-11$ & $2=0 \frac{1}{2}$ & 2313 & $22^{\prime} x$ & 2217 & \\
\hline $1 J \angle-B Y$ & $18-61 / 2$ & $15-5$ & 18 & $18^{\prime} 87$ & $19: 8$ & 19,10 & \\
\hline $100-E^{\prime} Y$ & 19.512 & $21-1$ & $\approx 1<$ & $22^{\prime} 6$ & $22^{\prime} 6^{\prime \prime}$ & 2117 & \\
\hline$i v 3 y$ & $14-21 / 2$ & $141,7^{112}$ & $14,2 \frac{1}{2}$ & $14^{\prime} \quad 2 \frac{1}{2}$ & $14^{\prime} 2^{1 / 2}$ & $14+1$ & \\
\hline $100-i=2$ & 19412 & $18^{\circ} 9$ & $18 \quad 7 \frac{1}{2}$ & 1392 & $18^{\prime} 7^{\prime \prime}$ & $18 ! 21$ & \\
\hline $1 i 1-3$. & & & $3-2$ & 32 & & & \\
\hline & & & & & $136^{\prime} 7^{\prime \prime}$ & $1366^{\circ}$ & \\
\hline Total & $13.5^{1} .5^{\prime \prime}$ & 1351612 & $136 \div 1$ & 136 & $\Rightarrow 9^{\prime \prime}$ & & \\
\hline ive Fees & & & & $1 / 1_{2}^{\prime \prime}$ & & & \\
\hline Aujus ted Total & & & & & & & \\
\hline
\end{tabular}

\begin{tabular}{|c|c|}
\hline$\triangle C B 4$ & $0^{\prime} S^{1 / 2}$ \\
\hline $1=1,124$ & $\left.15^{\circ} z^{\prime \prime}\right)^{\circ}$ \\
\hline 11984 & $11^{\prime} 9$ \\
\hline $11: B$ & $16^{\circ} 3 \frac{2}{2}$ \\
\hline
\end{tabular}

Heat Tracts

(Dey Suife)

\section{0}

$9-60-7 i$
BEST AVAILABLE COPY 
\begin{tabular}{ll|l}
\hline Pump & L. L. L. L.
\end{tabular}

$\therefore$ itumber

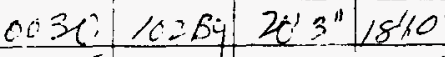

\section{inites}

100

\begin{tabular}{l|l}
1030 & 105 \\
105
\end{tabular}

$1032 \quad 103$

$1215-107$

$2000104200^{\circ} 2.20104$

\begin{tabular}{|c|c|c|c|c|}
\hline irumber & $\begin{array}{l}\text { 1. L. } \\
\text { Start }\end{array}$ & $\begin{array}{l}\text { L. L. } \\
\text { End }\end{array}$ & $\begin{array}{c}\text {-imét } \\
\text { Furnp Cff }\end{array}$ & Remarks \\
\hline 10389 & $27^{\prime} \frac{1}{2}$ & $22^{\prime} 3 \frac{1}{2}$ & 1645 & \\
\hline $102 \pi$ & 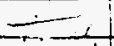 & 1911 & & \\
\hline 107 & 1933 & Cen & Eurices & $0 n 3-14.72$ \\
\hline 103 & $145 \%$ & 14,5 & $1<15$ & \\
\hline 109 & $18-7$ & $12^{\prime 2}=$ & 1713 & \\
\hline 104 & $1910^{\circ}$ & $21^{\prime} / 2$ & $\div 506$ & Zentesco \\
\hline $\cos 2$ & $18^{\prime} 10^{4}$ & 2010 & $c \leq 345$ & 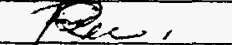 \\
\hline
\end{tabular}

\begin{tabular}{|c|c|c|c|c|c|c|c|}
\hline$a n k$ & 0100 & 0700 & $Q: 00$, & 1500 & 41700 & $2300 \div$ & \\
\hline i $12-B Y$ & $20^{\prime} 6^{\prime \prime}$ & $21^{\prime 2} 2^{\prime \prime}$ & 215 & $2 i \cdot 6$ & 215 & 21 & \\
\hline$-+10-B Y$ & & & & & & & \\
\hline $107-34$ & $14^{13} 3^{\prime \prime}$ & $14^{\prime} 3^{\prime \prime}$ & 193 & is 712 & 1311 & $5 \frac{1}{2}$ & \\
\hline UUद-BY & $22^{\prime 2} 2^{\prime \prime}$ & $21^{\prime} 211$ & $\therefore 09$ & $199 \mathrm{j}_{2}$ & 2910 & 20 9\% & \\
\hline $10 Z-B Y$ & $20^{\prime 3}$ & $20^{\circ} C^{\prime}$ & $19 / 12$ & $199^{\prime \prime}$ & $16 \quad 3 \div$ & 19112 & \\
\hline $100-0 y$ & 2110 & $21^{\prime}+t^{2}$ & $\because 110$ & $21 \quad 10$ & 21.10 & $\pm 2 \quad 32$ & \\
\hline ius iv & $144^{\prime} \frac{1}{2}$ & $1415 \frac{1}{21}$ & \pm 54 & $14 \quad 5$ & 145 & $14 \quad 4 \sqrt{2}$ & \\
\hline $10 \cdot-i . y$ & $19^{\prime} 2^{11}$ & $1 x^{\prime} 11 \frac{1}{2}$ & $-94 \%$ & $19 \quad 3$ & 196 & 192 & \\
\hline $411-\infty$ & & & & 131 & & & \\
\hline & & & & & & & \\
\hline Total & $136^{\prime} 4^{\prime \prime}$ & $136^{\prime}+f_{2}^{\prime \prime \prime}$ & $136.5 \frac{1}{2}$ & $136^{\circ} 20^{\circ}$ & $136 \cdot 4=$ & $13 c^{\prime} 3 \frac{1}{2}$ & \\
\hline ivew Fees & & & & & & & \\
\hline Mujus ted Total & & & & & & & \\
\hline
\end{tabular}

I VACTIVE TANKS

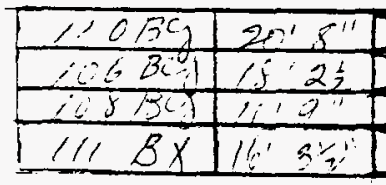

Heat Tracts

(Day Stifi)

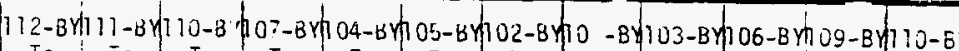
To To To To To To To To To To To To

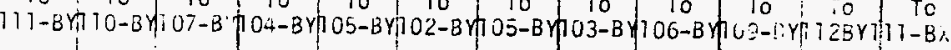

L.

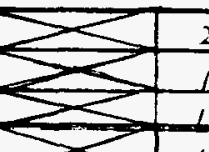

2. 5320

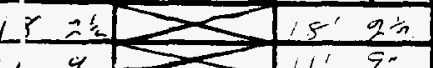

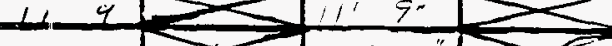

$16 \% \%$ 


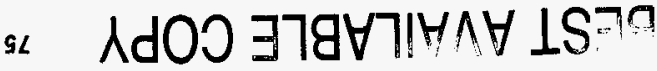

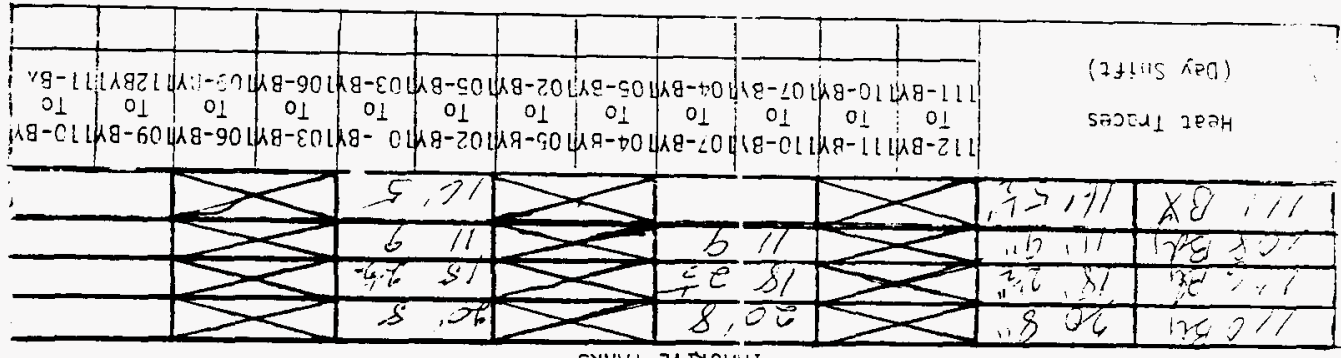

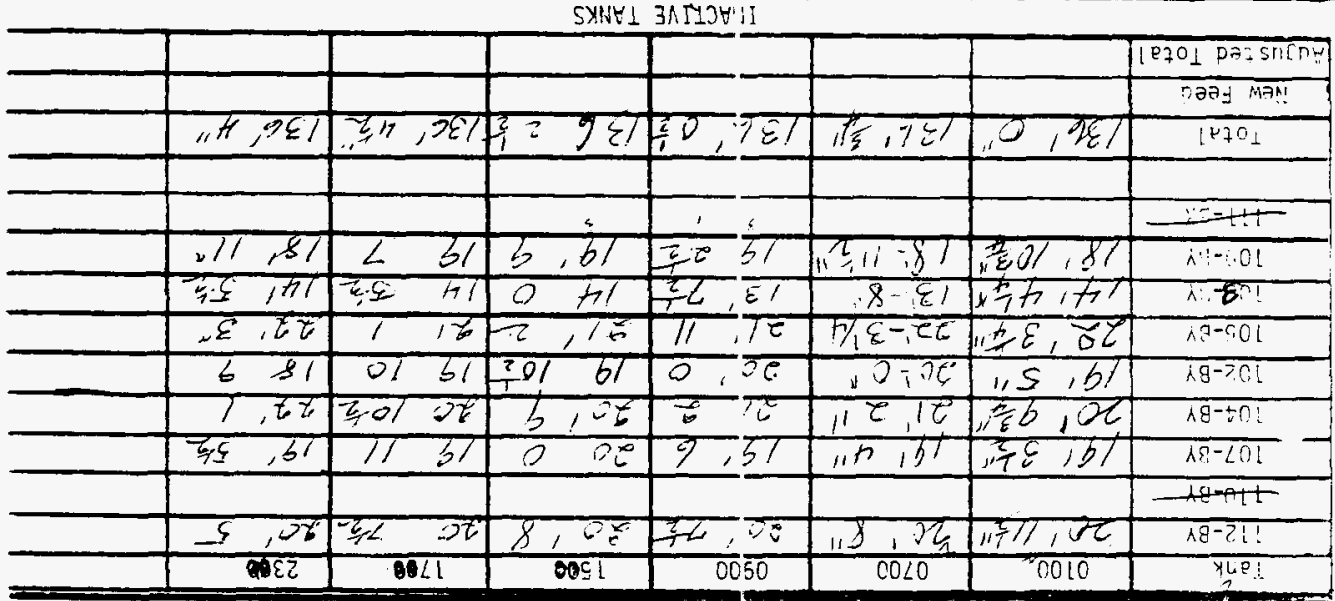

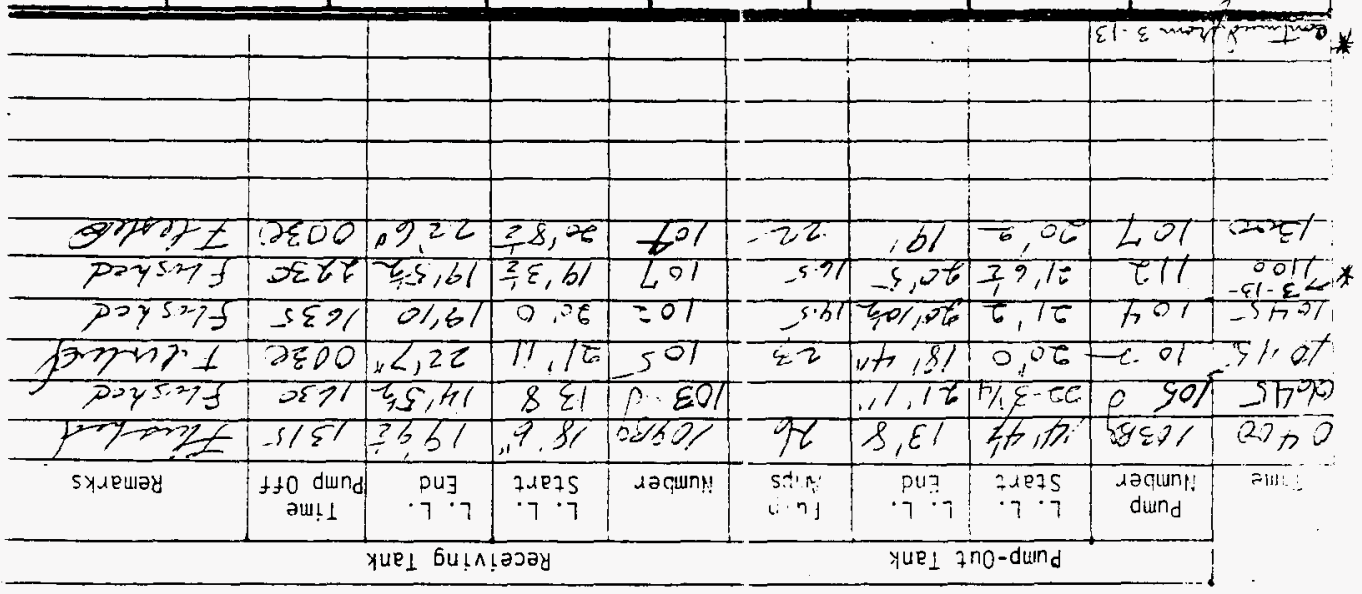

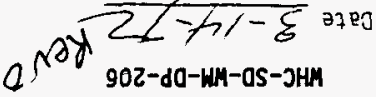

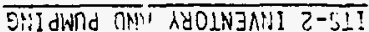


Punp-0ut Tank

\begin{tabular}{|c|c|c|c|c|}
\hline \multirow[b]{2}{*}{$\therefore m=$} & \multicolumn{4}{|c|}{ Puinp-0ut iank } \\
\hline & $\begin{array}{l}\text { Pump } \\
\text { Mumper }\end{array}$ & $\begin{array}{l}\text { L. L. } \\
\text { Start }\end{array}$ & E. L. & $\begin{array}{l}14,0 \\
40,5 \\
\end{array}$ \\
\hline 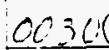 & 2 & $22^{\prime} 6^{\prime \prime}$ & $20^{\prime}$ & 20 \\
\hline 00.30 & 713 & $14 t^{\prime} 5^{-11}$ & $13^{\prime} 5^{\prime \prime}$ & 25 \\
\hline$C 416$ & $10 \leq 2$ & $2217^{\prime}$ & $2 c^{\prime} / i^{\prime \prime}$ & 26 \\
\hline 10936 & 102 & $19^{\prime} 5^{-}$ & $19^{\prime} 2^{\prime \prime}$ & \\
\hline 1215 & 12 & $21^{\prime} 6 \frac{1}{2}$ & & \\
\hline 1032 & 703 & $14^{\prime} 1 \frac{1}{2}$ & $i^{\prime \prime} / w^{\prime \prime}$ & \\
\hline 1510 & 107 & $119^{1} \mathrm{~s}^{-}$ & $1 p^{\prime \prime} / c^{\prime \prime}$ & \\
\hline SEEC & $11 \angle B X$ & & $10^{\prime} a^{\prime \prime}$ & \\
\hline
\end{tabular}

Receiving Tank

\begin{tabular}{|c|c|c|c|c|}
\hline ivumber & $\begin{array}{l}\text { L. L. } \\
\text { Start }\end{array}$ & L. Lnd & $\begin{array}{c}\text { Time } \\
\text { Pump off }\end{array}$ & Remarks \\
\hline 102 & $18^{1} / 4_{2}^{\prime}$ & $191 / \frac{11}{2}$ & 0545 & $F_{i<3}$ \\
\hline 109 & $15 \cdot e^{\prime}$ & $19^{16} 5$ & 0636 & Felco \\
\hline 103 & $13^{\prime} 5^{\prime \prime}$ & $1411 / \frac{1}{2}$ & 1262 & Ethws \\
\hline 105 & $21^{\prime} 4$ & $225 \div$ & $003 x$ & \\
\hline 107 & $19^{\prime} 0$ & ev & terive & 3 \\
\hline 109 & $18 / \frac{1}{2}$ & 19 & 11700 & Ekteskeil \\
\hline 104 & $20 \%$ & 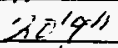 & 2100 & FHKGPELD \\
\hline$\angle i D-\theta$ & $812 \pi$ & $x^{\prime} / 1$ & $2 \angle 2 /$ & FLLPL \\
\hline
\end{tabular}

\begin{tabular}{|c|c|c|c|c|c|c|c|}
\hline iank & 0100 & 0700 & 0930 & 1500 & 1700 & 2300 & \\
\hline$\overline{i z-B^{Y}}$ & $x^{\prime} 7 \frac{111}{2}$ & 211211 & $2144 \frac{1}{2}$ & $2^{\prime}<\frac{1}{2}$ & $21 \mathrm{k}^{\prime \prime}$ & 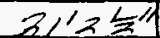 & \\
\hline 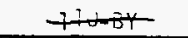 & & & & & & & \\
\hline $107-3 Y$ & $19^{7} C$ & $10^{\prime} 0^{\prime \prime}$ & $19^{\prime} 0$ & $19^{\prime} 3$ & $19^{\prime} 3-57$ & $14^{\prime}{ }^{\prime}{ }^{\prime}{ }^{\prime \prime}$ & \\
\hline$\because O \hat{4}-B Y$ & $22^{\prime} 5^{2-4}$ & $21^{\prime} 10^{\prime \prime}$ & $6 \cdot \frac{1}{2}$ & $20^{\circ} 6 \frac{1}{2}$ & $2 E^{\prime} L^{\prime \prime}$ & $3 n^{\prime}+71$ & \\
\hline $70<-B Y$ & $18^{1} 43$ & $1911^{11}$ & 195 & 15:5: 5 & $19^{\prime} \overline{2}^{\prime \prime}$ & $19^{\prime} z^{\prime \prime}$ & \\
\hline $100-6 Y$ & $22^{\prime} 72^{n}$ & $22^{\circ} 0^{\prime \prime}$ & 21.7 & $21^{\prime} c$ & $Z E^{\prime} \angle E^{\prime \prime}$ & $3 n^{\prime}<1$ & \\
\hline is-y & 1432 & $13^{\circ} 5^{\prime \prime}$ & $14^{7} 0$ & 1411 & $14,11-\frac{1}{2}$ & $\left.\angle H^{\prime}, 1\right\rangle$ & \\
\hline $10 \div-\div$ & $18^{\prime} 9^{\prime \prime}$ & $19^{\prime}\left(0^{2}\right)^{\prime \prime}$ & 19 & $19^{\prime} 3$ & $19^{\prime} \mathrm{p}^{\prime \prime}$ & 'ots' & \\
\hline$-+1+=\ldots$ & & & 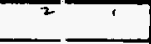 & 1 & & & \\
\hline Total & $13 h^{\prime}{ }^{3} z^{\prime \prime}$ & 136127 & $1366^{7}$ & $13511 \frac{1}{2}$ & |si' $\left.\right|^{\prime \prime}$ & $1314^{\prime \prime \prime}$ & \\
\hline iven Feec & & & & & & & \\
\hline djusced Total & & & & & & & \\
\hline
\end{tabular}

II ACTIVE TANKS

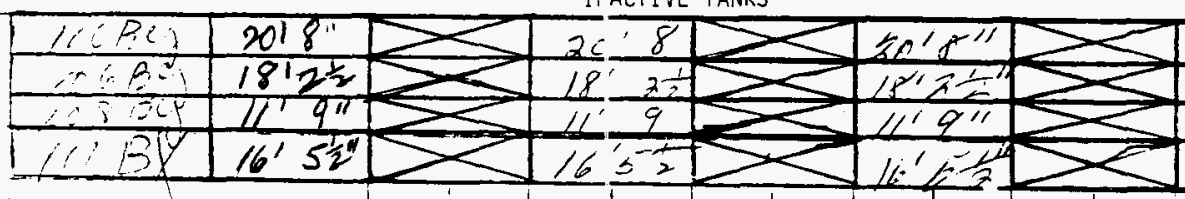

Heat Traces

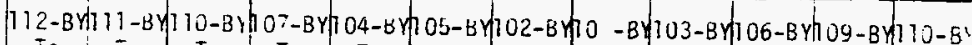

(Day Silft)

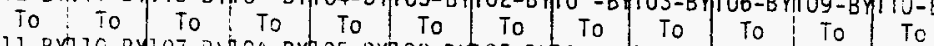

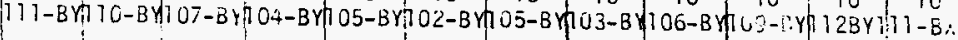

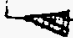




\section{-. $\quad$ WHC-SD-WM-DP-206}

ITS-2 IIIVENTORY AINU PUMP ING

Date $3-16-72 B \mathrm{C}^{\circ}$

Puinp-Out Tank

Receiving Tank

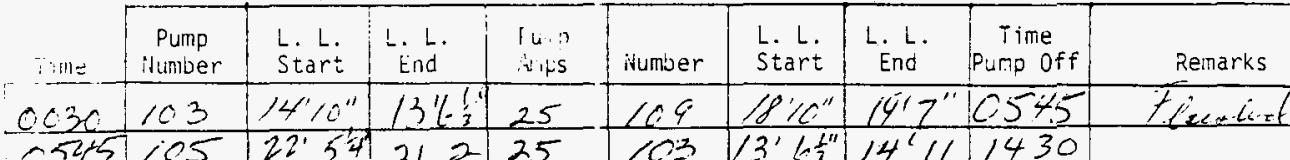

$652+5] 105$

$0730 / 107$

$103,13^{\prime} 4 \frac{1}{7} \mid 14^{\prime} / 1430$

$3+5,12112$

$20^{\prime} 4 \frac{1}{2} / 9^{1 / 10^{11}} 22$

1000102

$216 \frac{1}{2}=055$

1132.103

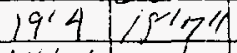

1400104

$14^{\prime} 11+141$

$215-\frac{i}{2 i}$

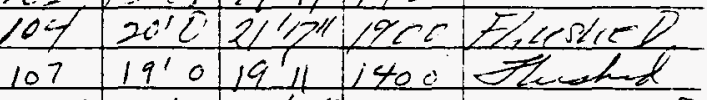

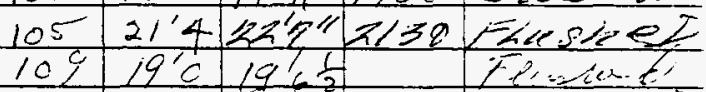

$10218^{\prime} 7($ Ininflend Fstuct 3-17-72)

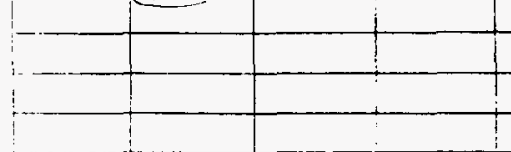

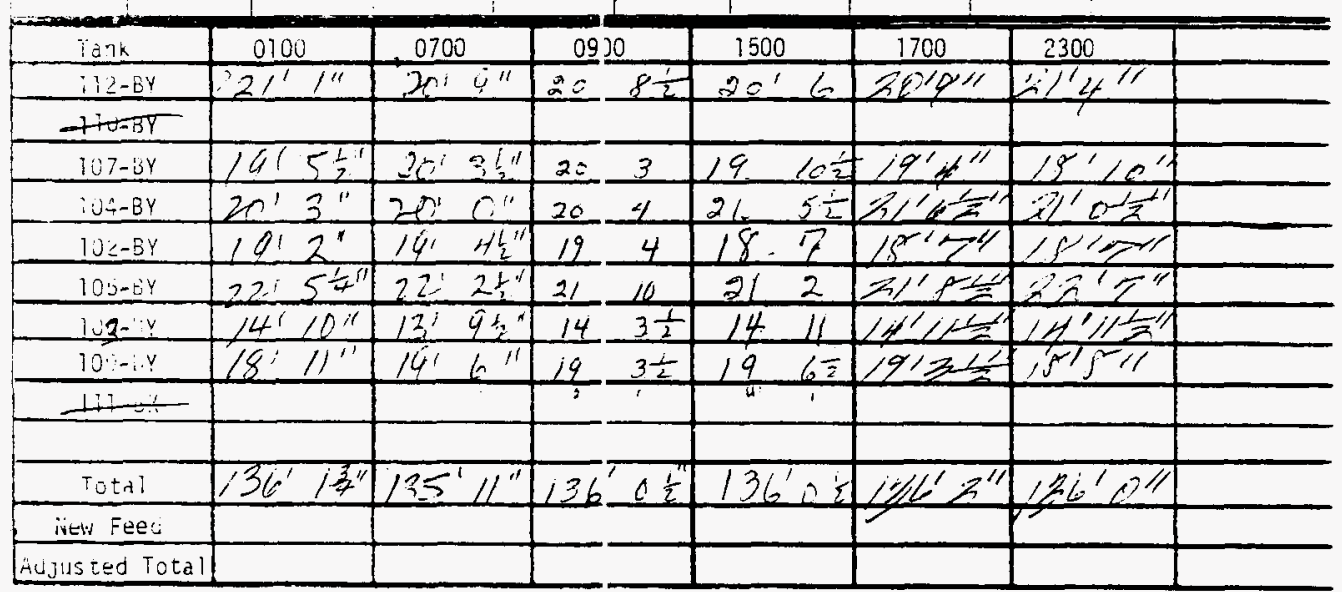

IIIACTIVE TANKS

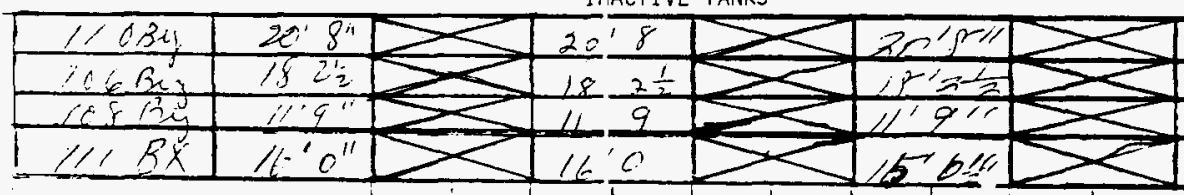

Heat Traces

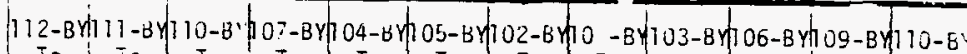

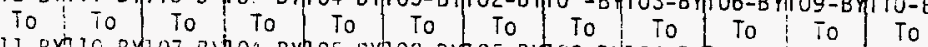

(Day Silift)

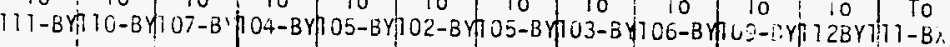

L. 


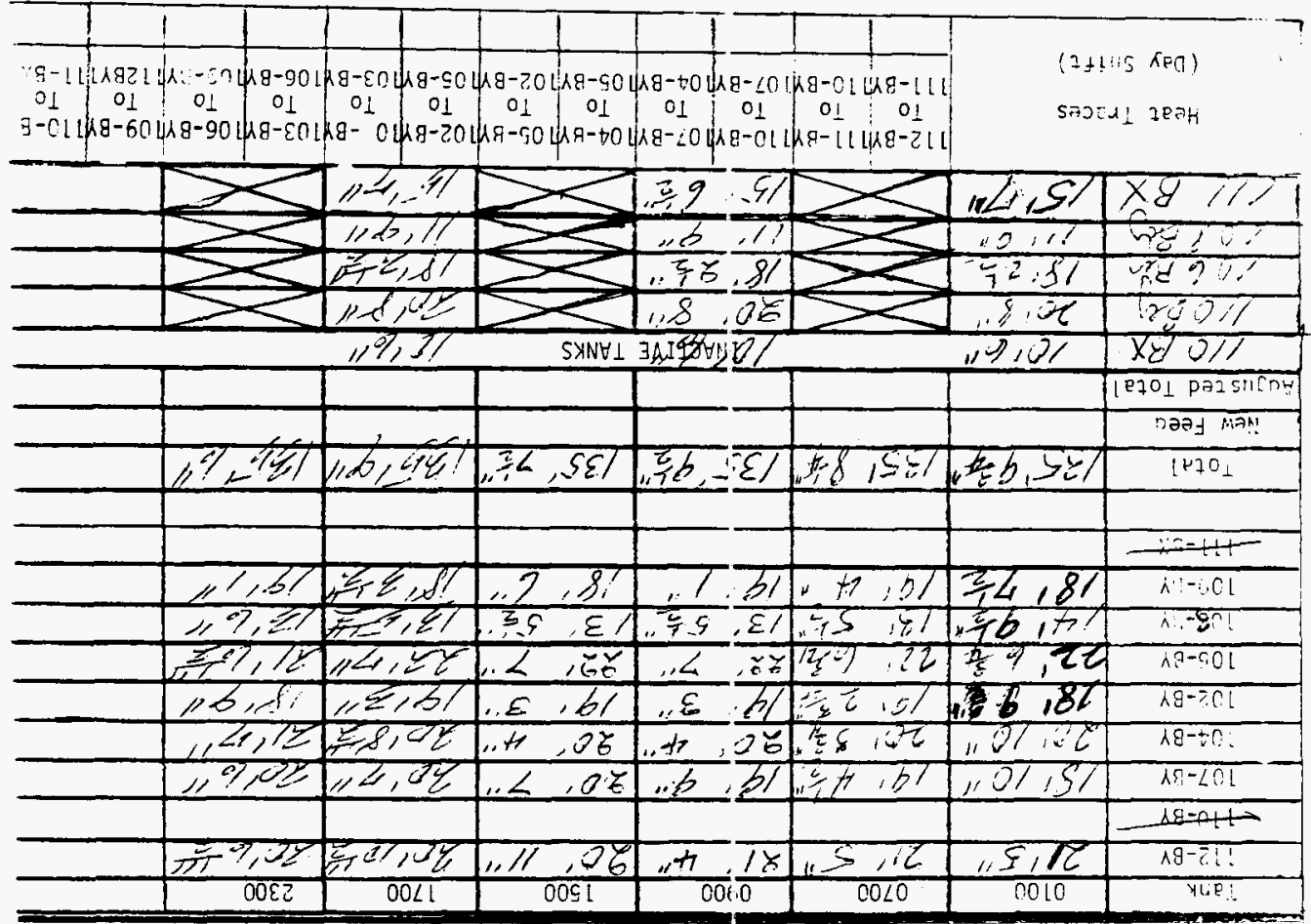

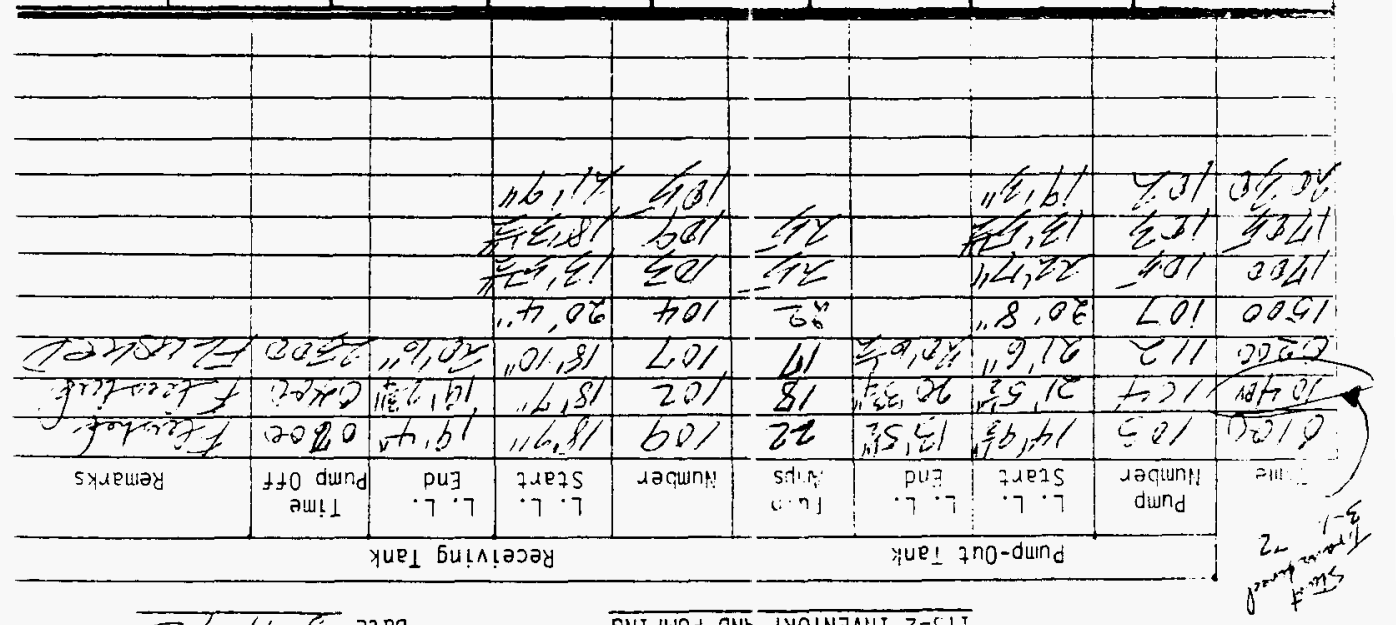


Punp-out iank

IIIL \begin{tabular}{|l|l|l|l} 
Pump & L.L. & L.L. & I L. \\
idumber & Start & Lnd & ivirs
\end{tabular}

$1500 \quad 167 \quad 20,18111 \quad 2=$ $2=\rightarrow 20 \cdot 8 \% 25$

\begin{tabular}{|c|c|c|c|}
\hline ivumber & $\begin{array}{l}\text { L. L. } \\
\text { Start }\end{array}$ & $\begin{array}{l}\text { L. L. } \\
\text { End }\end{array}$ & $\begin{array}{c}{ }^{n} \text { me } \\
\text { Pump } 0=f\end{array}$ \\
\hline
\end{tabular}

10420421036

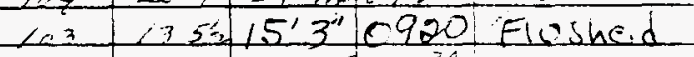
$1260 ; 105$ $1705 \div 1+3$ 2030 1020 13 5\%, $136125^{-1}$ 1009 i8 $7 \frac{1}{2}$ $1 \% 3$ $18^{\prime} 1^{\circ}, 1^{\circ}$ $+2 \%$

$1100 \quad 104$ $1000+112$ $2+10$ 19 $19^{\prime \prime}$

$\frac{j 4}{17}=$

$20 \% 8 \%$ c920 Flushol

सEC $2 \%$

ancl 12

$\frac{21^{\prime} 6 "}{14^{\prime} 6 " 1}$

$2{ }^{m i 4}$

2,

\begin{tabular}{|c|c|}
\hline & 196 \\
\hline 107 & $18^{\prime} 10^{\prime \prime}$ \\
\hline- & $=11 \mathrm{~L}^{\prime}$ \\
\hline 6 & \\
\hline
\end{tabular}

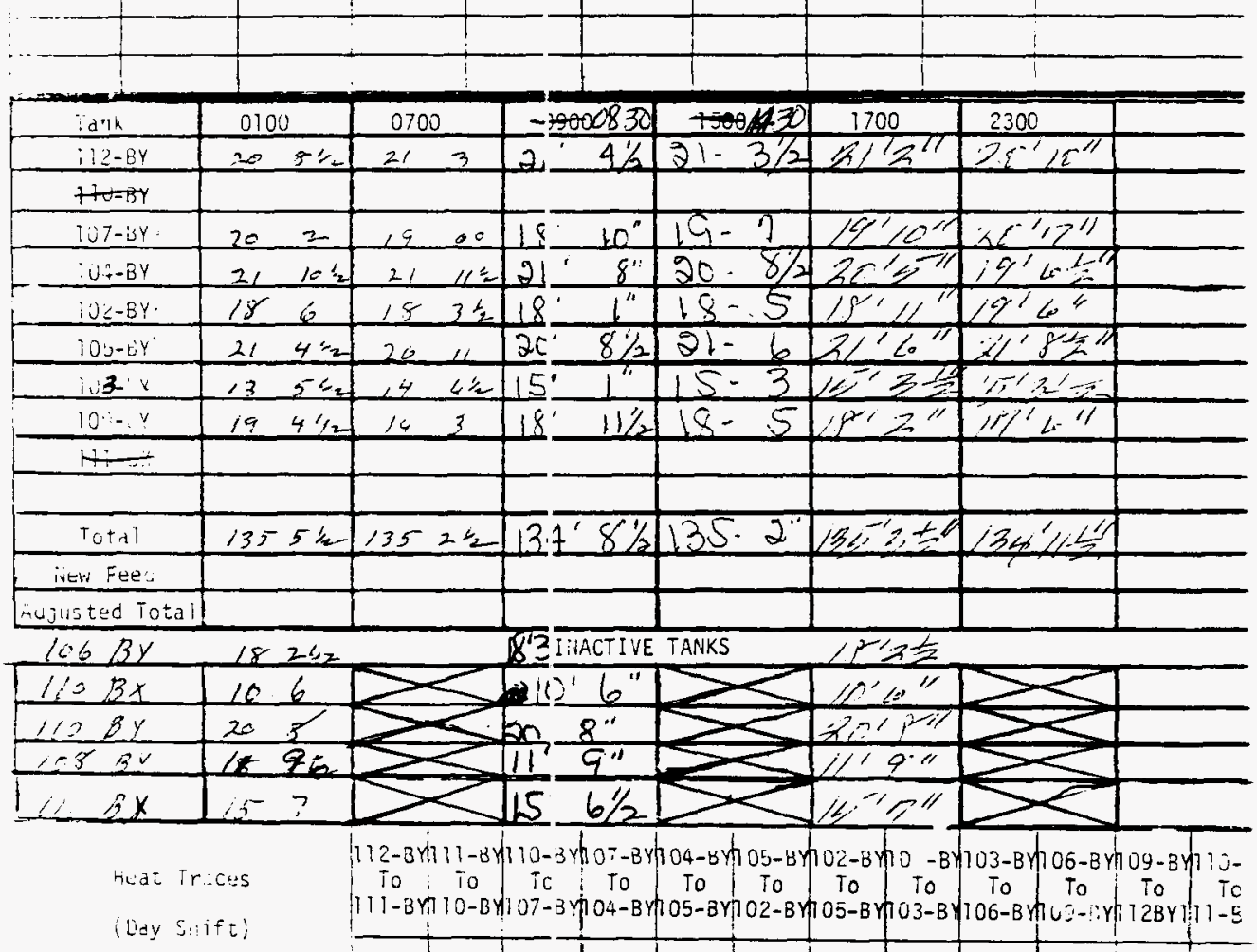

4

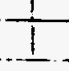

$3-2 .-7)$ 
Puinp-Out iank

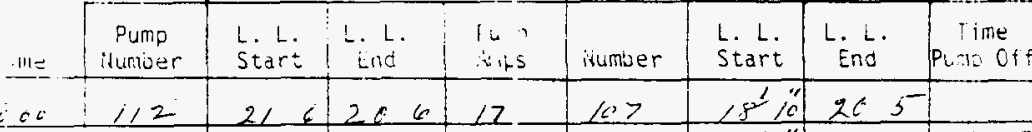

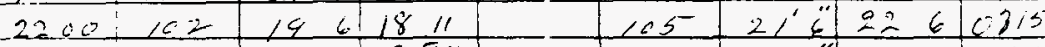

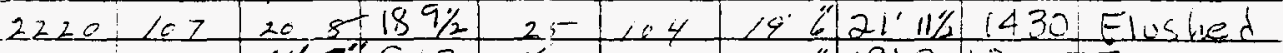

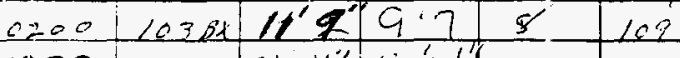

1430 104 $2111 \% 2$ 公作

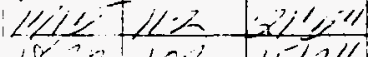

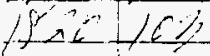

1,211

$14-\frac{102}{16}$

$17^{\prime 2}$

17218.3 is 25

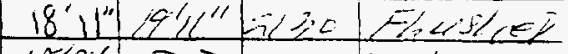

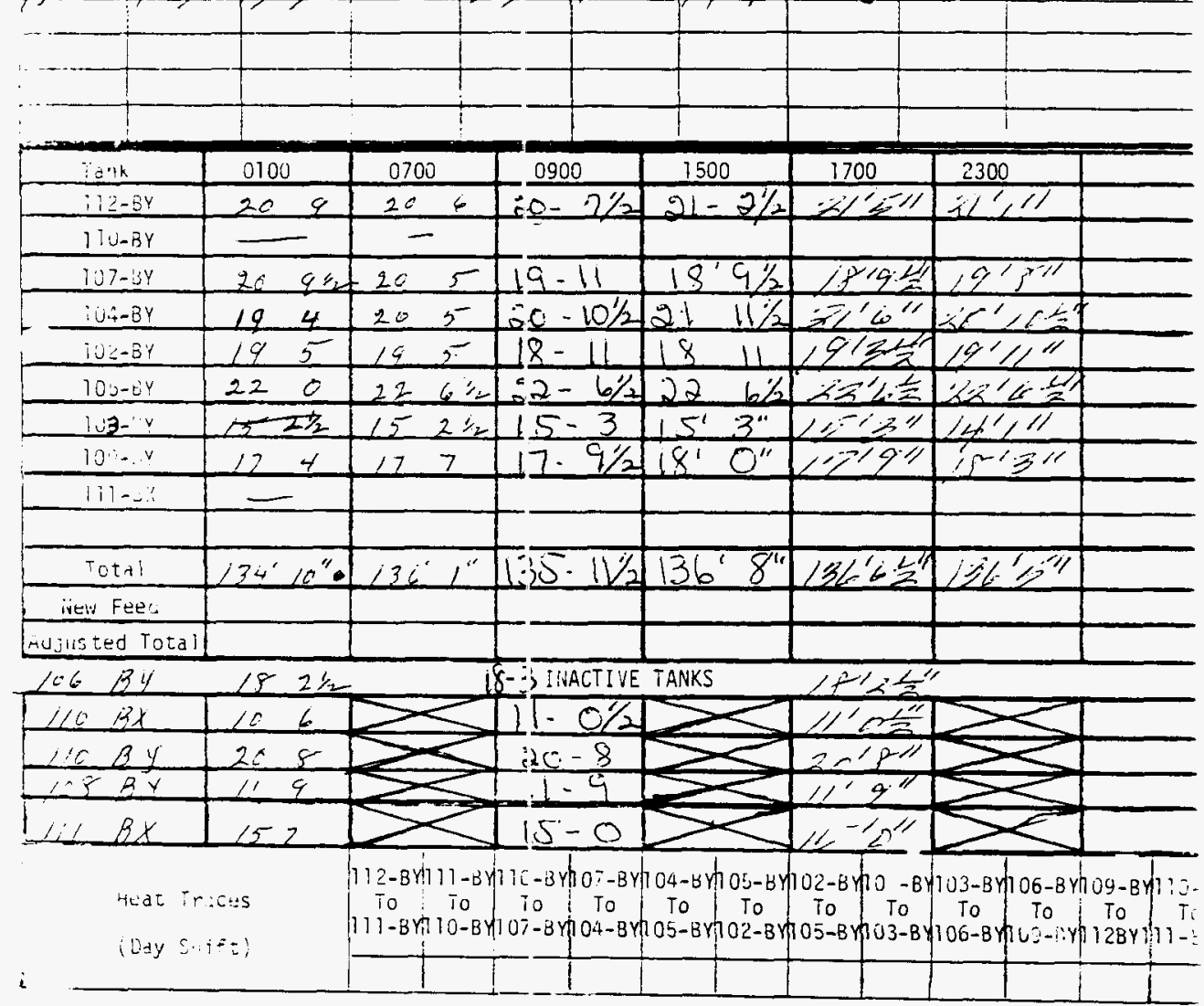




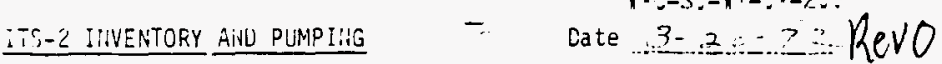

Pump-Out Tank

Receiving Tank

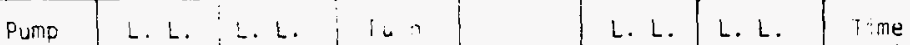

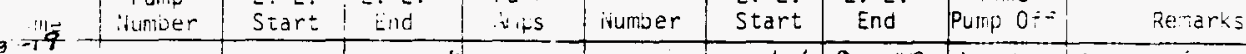

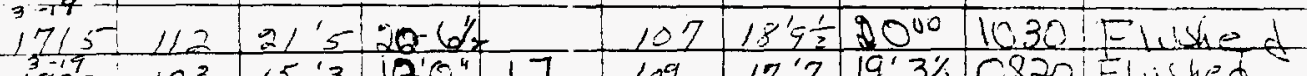

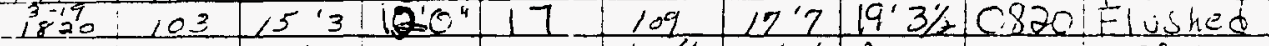

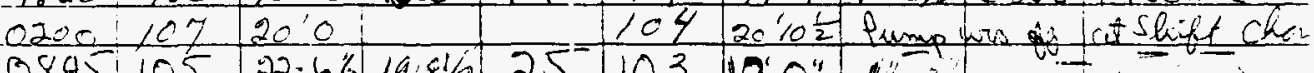

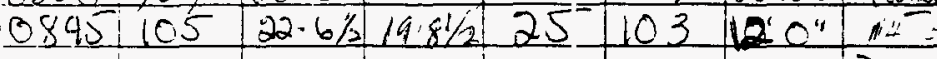

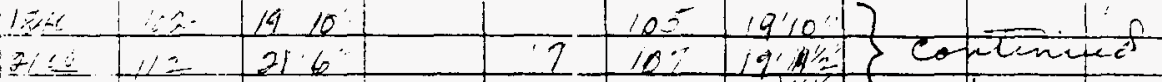

$\therefore=104220$

18.102

$19 \cdot 3\}$

22

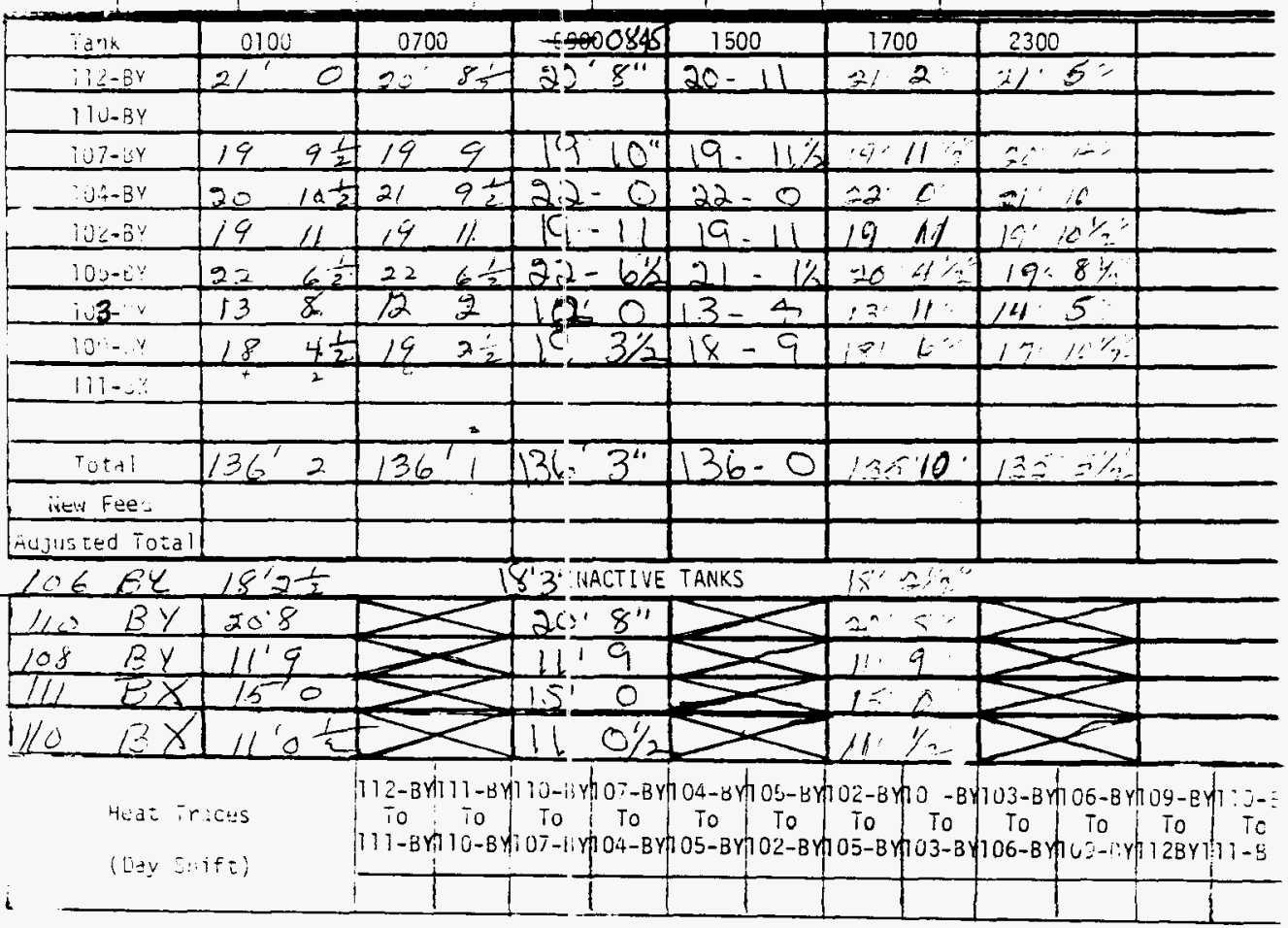

$9--0-7 i$ 


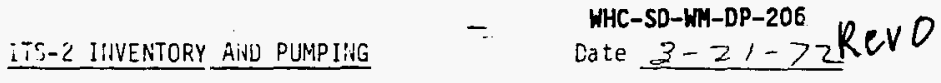

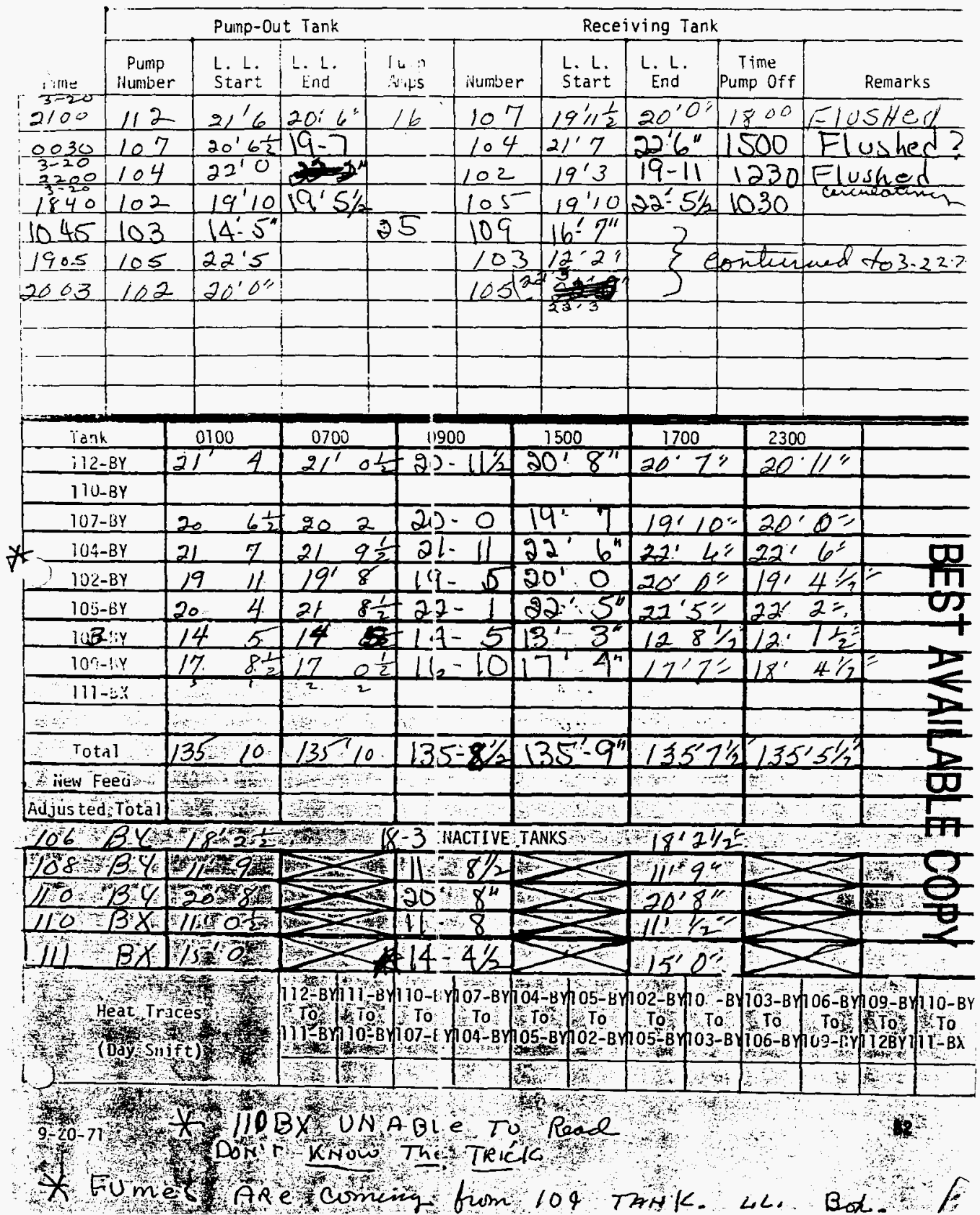




\section{ITS-2 IIVENTORY AIN PUMPING $\quad-\quad$ WHC-SD-WM-DP-206 $\mathrm{KeV} O$}

\begin{tabular}{|c|c|c|c|c|c|c|c|c|c|}
\hline & \multicolumn{4}{|c|}{ Pump-Out Tank } & \multicolumn{4}{|c|}{ Receiving Tank } & \multirow[b]{2}{*}{ Remarks } \\
\hline met & $\begin{array}{l}\text { Pump } \\
\text { inumber }\end{array}$ & $\begin{array}{l}\text { L. L. } \\
\text { Start }\end{array}$ & $\begin{array}{l}\text { E. L. } \\
\text { End }\end{array}$ & $\begin{array}{l}\text { I } 6: " \\
\text { Nits } \\
\end{array}$ & ivumber & $\begin{array}{l}\text { L. L. } \\
\text { Start }\end{array}$ & $\begin{array}{l}\text { L. L. } \\
\text { End }\end{array}$ & $\begin{array}{c}\text { Time } \\
\text { Pump 0ff }\end{array}$ & \\
\hline & 103 & $14^{\prime} 5$ & 12,0 & & 109 & $16^{\prime} 7$ & $18^{\prime} 8$ & 1330 & Flem hes \\
\hline 1905 & 105 & $22^{\prime} 5$ & $?$ & & 103 & $12 \cdot 2$ & $?$ & $?$ & ? \\
\hline 2003 & 102 & $20^{\circ} 0$ & $18^{\prime} 7$ & & 105 & $22^{\prime} 3$ & $2110 \%$ & tA & $1+\sqrt{15}$ \\
\hline 0500 & 112 & $221^{\prime} 6$ & $21^{\prime} 4$ & & 107 & $20^{\prime} 0$ & $20^{\prime}$ & 0200 & \\
\hline 0200 & 109 & $19^{1} 05$ & $19^{\prime} 1$ & & 112 & $21^{\prime} 4$ & $21^{\prime} 4$ & 0200 & Therate \\
\hline 1350 & 112 & $21^{\prime} 6^{\prime \prime}$ & $21-4$ & & 107 & & $21-0$ & 1825 & $\partial_{L S}<H \leq D$ \\
\hline 1490 & 104 & $22 ! 53$ & & 17 & 102 & $18^{\prime} 0^{\prime \prime}$ & & & Sont. TO 3.23 \\
\hline 1495 & 105 & $21-10 /$ & 19.10 & 25 & 103 & 132 & 15.0 & 2235 & $7<L 5 E[)^{\prime}$ \\
\hline 1955 & $107-8 y$ & $21-0$ & & 21.0 & $104-3 y$ & $22-1$ & & & cont To $3-23$ \\
\hline 2005 & $1 / 2 \cdot \beta^{2}$ & $21-5$ & & & $111 \cdot B x$ & $1 z-10$ & & & $\begin{array}{lll}\| & \| & 4\end{array}$ \\
\hline $21-30$ & $10 z-8 y$ & $18-2$ & & & $105-34$ & $19-1 \mid$ & & & .1 \\
\hline
\end{tabular}

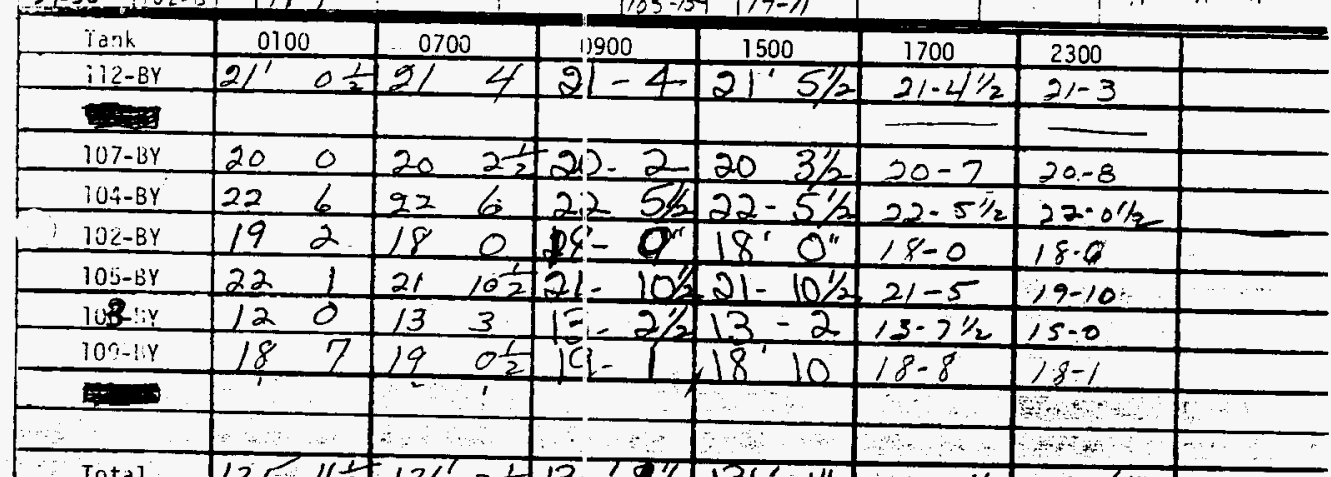

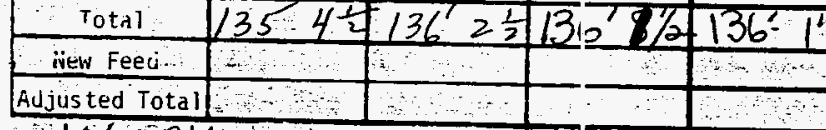

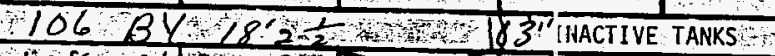

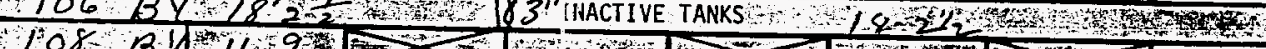

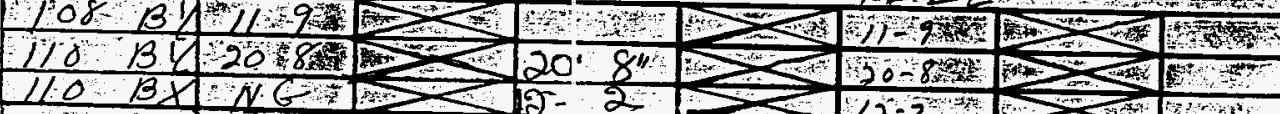

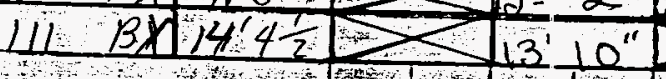

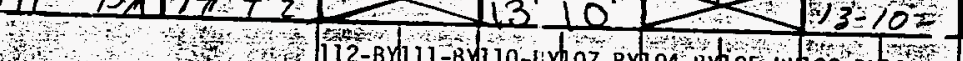

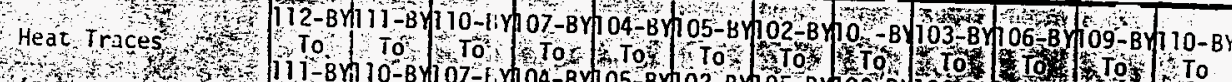

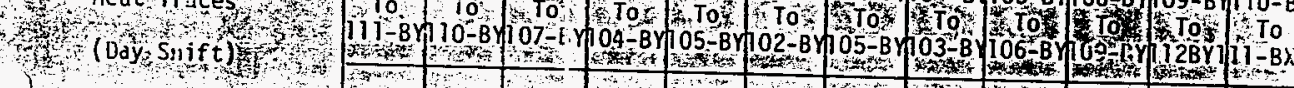

10 r

$9-\div 0-71$

BEST AVALABLE COPY

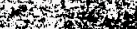

$0 \%$ tot

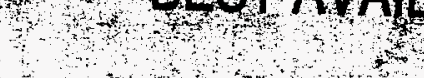


Pump-Out Tank

Pump

llumber

1440104

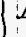

$1955+107$

12005112

$2 / 30$

$0145-112$

0.2301103

$1013-105$

1313 107
22 's $\frac{1}{2}$

$21^{\prime} 0199^{\prime \prime 1}$

\begin{tabular}{l|l}
$21^{\prime} 5^{-}$ & $21^{\prime} 2$ \\
\hline 1817 & 17.11
\end{tabular}

\begin{tabular}{|l|l|}
$18^{\prime} 7$ & $17-11$ \\
\hline $21^{\prime 2}$ & $20.5 / 2$ \\
\hline
\end{tabular}

\begin{tabular}{l|l|l}
$15^{-1} \mathrm{O}$ & 13.0 \\
\hline
\end{tabular}

$22^{\prime} 5 \frac{1}{2} ; 21-8,26$

$2010: 18 \%$
Receiving Tar

L. L. L. L. Time

Start End Pump off

Remarks

\begin{tabular}{|c|c|c|c|c|}
\hline 102 & $18^{\circ} 0$ & & & \\
\hline 104 & $22^{\prime} 1$ & $223 \frac{1}{2}$ & 0630 & \\
\hline $1 B X$ & $13^{\prime} 10$ & $14)^{\prime}-\frac{1}{2}$ & $0 / 45$ & 11 \\
\hline
\end{tabular}

\begin{tabular}{l|l|l|l|l}
105 & $19 \%$ & $21-8$ & 2300 & RECIRR
\end{tabular}

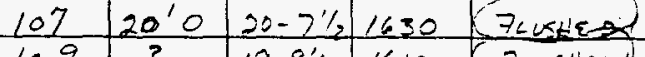

\begin{tabular}{l|l|l|l}
109 & $19-812$ & 1010 & $(74-54 \varepsilon 2)$
\end{tabular}

$103\left|3^{\prime} \frac{1}{2}\right| 1 /-1 \mid 2300$ TLushed

$10421^{\prime \prime 2} 21^{\prime 2} \frac{1}{2} 0030$ CONT. To 3-20122

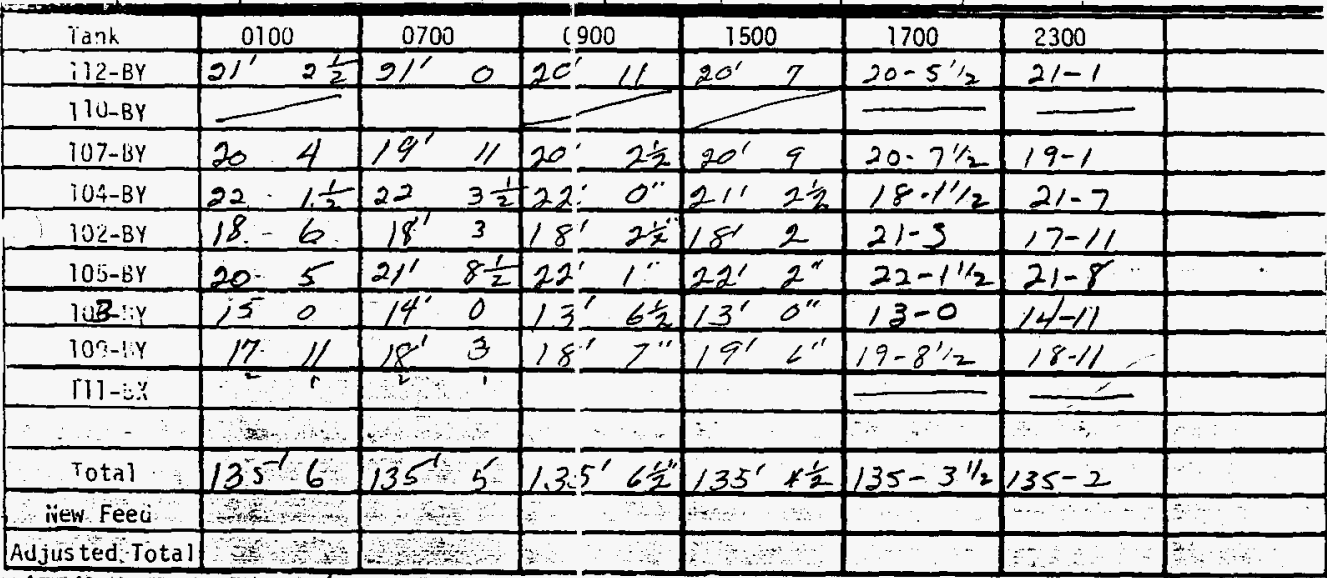

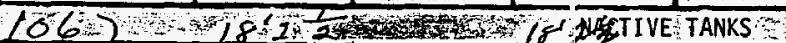

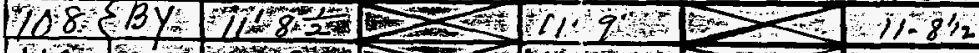

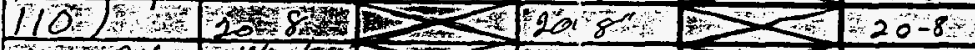

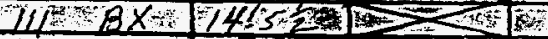

$1 / 0, B X / 2+2+2=1$

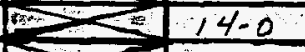

$3-24-72$

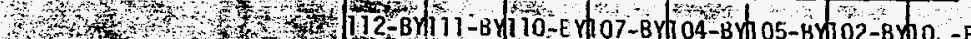

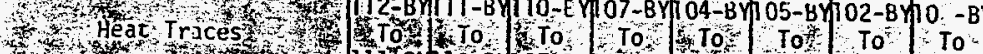

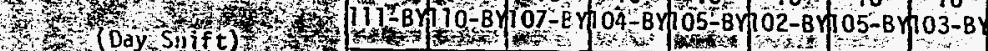

$\mathrm{H}_{\mathrm{r}}$ (Day Silf t)

(1)

+2.

and

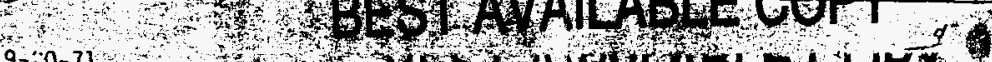

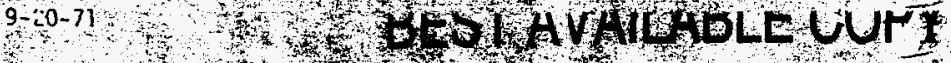

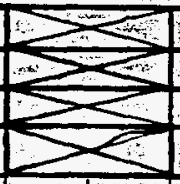

sto

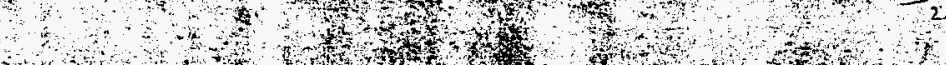

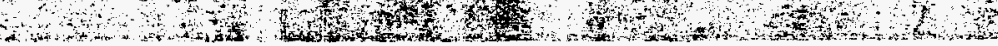

03-BYTO6-BYIOQ-BYI10-BY

To ToE To To $106-8 Y 00-[Y][2 B Y][1-B \lambda$ 


\section{iTs-2 IIIVENTORY AINU PUMPIHG}

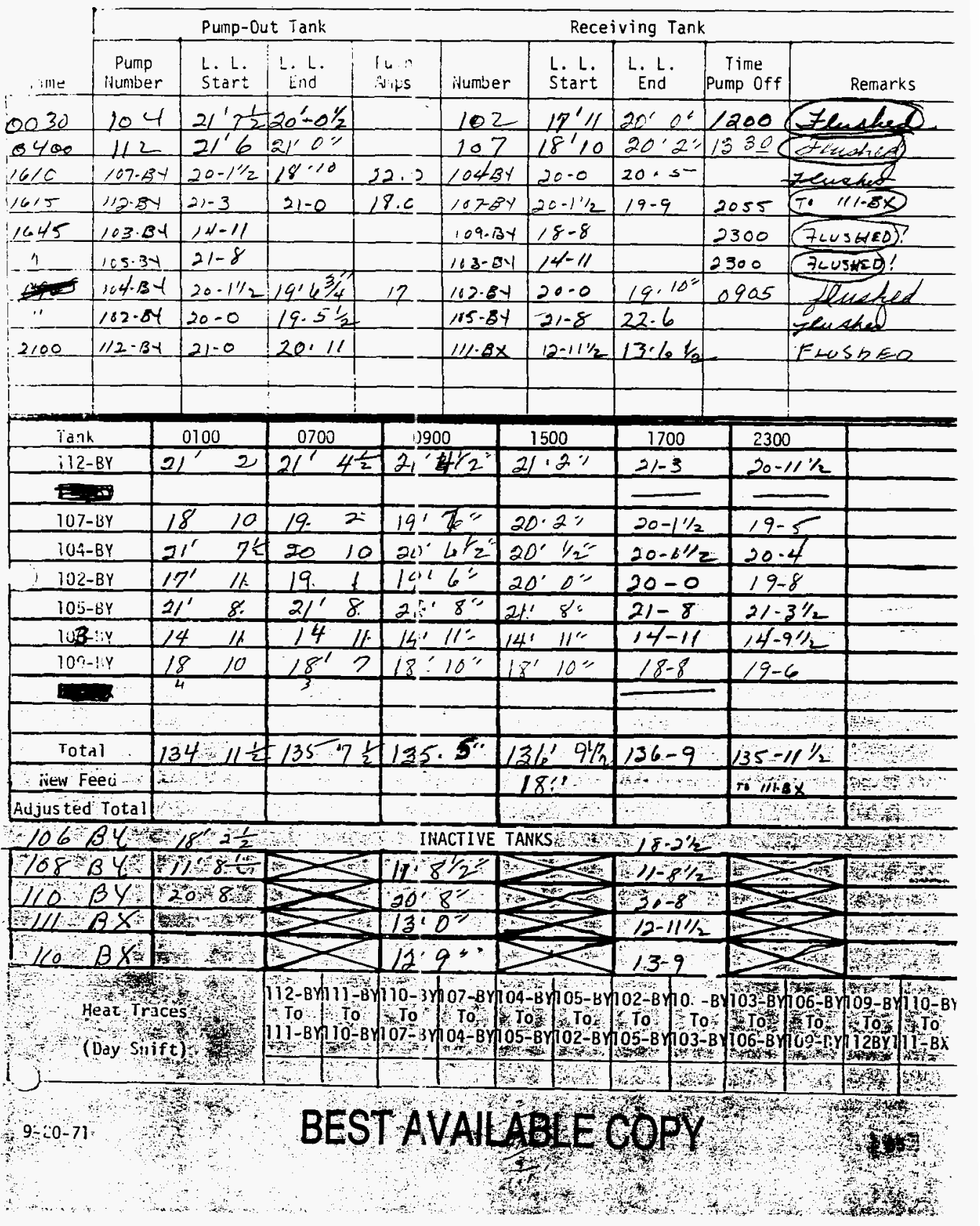




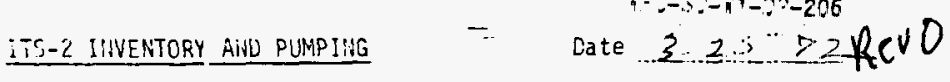

Pump-0ut Tank

\begin{tabular}{|c|c|c|c|c|c|c|c|c|c|}
\hline HE & $\begin{array}{c}\text { Pump } \\
\text { iunber }\end{array}$ & $\begin{array}{l}\text { L. L. } \\
\text { Start }\end{array}$ & E. L. & $\begin{array}{l}\text { lu : } \\
\text { nitits } \\
\end{array}$ & dumber & $\begin{array}{l}\text { L. L. } \\
\text { start }\end{array}$ & L. L. & $\begin{array}{c}\text { Time } \\
\text { Pump Off }\end{array}$ & Remarks \\
\hline 0315 & $112-$ & 210 & $20^{\prime} \cdot t^{r}$ & 17 & 107 & 1810 & & 13305 & zespod \\
\hline 20 & 103 & $14110 \%$ & $13-10$ & 24 & 109 & $18^{\prime 43 / 4}$ & $19-8$ & 1945 & $7<454=0 !$ \\
\hline 1350 & 105 & $226^{\prime 3} / 4$ & 21.7 & 25 & 163 & $13^{\prime} / 11$ & $14.41 / 2$ & 2210 & 7 Lus $\neq \varepsilon D !$ \\
\hline 1620 & $103-84$ & $20-1$ & $|8-1|$ & 22.0 & $104-0 Y$ & $19-63 / 4$ & $20-8$ & 2215 & ZLUSACQ! \\
\hline 2225 & $110-84$ & 20.9 & & $20 . c$ & $107-B Y$ & $|8-1|$ & & & Sont, ro 5.26 \\
\hline
\end{tabular}

Receiving Tank

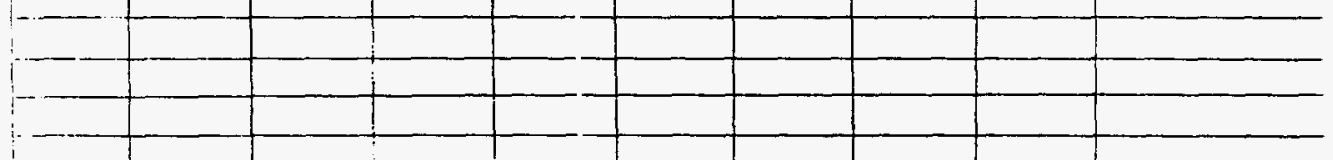

\begin{tabular}{|c|c|c|c|c|c|c|c|}
\hline Tank & 0100 & 0700 & 1) 900 & 1500 & 1700 & 2300 & \\
\hline i) $2-B Y$ & $20 \cdot 11$ & $20 \cdot 11$ & $21 \cdot 9=$ & $20^{\prime} 6 \%=$ & $20-91 / 2$ & $2 /-31 / 2$ & \\
\hline IlUs & & & & $20^{\prime} 9 \frac{1}{2} i^{\prime \prime}$ & $20-9$ & $20-9$ & \\
\hline $107-13 Y$ & $18111 \frac{1}{2}$ & 19 & $19,5^{\circ}$ & $20^{\prime} 11 / 2=$ & $20-1$ & $18-+11^{1 / 2}$ & \\
\hline $10 \div-B Y$ & $20.4 \%$ & 19,11 & $19^{\prime}<3 / 4$ & $19^{\circ}, 43 / 4^{\prime}$ & $19-6 \frac{1}{2}$ & $20-81$ & \\
\hline $102-8 Y$ & $19.7 \frac{1}{2}$ & $19.5 \frac{2}{2}$ & $14110^{\circ}$ & $19^{\circ} 10^{\circ}$ & $19-10$ & $18-9$ & \\
\hline $105-6 Y$ & 21.9 & $22-6$ & $22: \quad 63 / 4$ & $22^{\circ} 3 \div 7$ & $2 f-9$ & $21-7=$ & \\
\hline lues:iy & $14.19 \frac{1}{2}$ & $14.91 / 2$ & $14^{\prime} \quad 10^{\prime \prime}$ & $13^{\circ} \quad 10 \frac{1}{2}$ & $13-10$ & $44 \cdot 4 ! 1 / 2$ & \\
\hline $10 r-1 y$ & $19,4 \cdots$ & $18 ; 9$ & $18, \quad 5 \frac{1}{2}$ & $19^{\prime} 0^{\prime \prime}$ & $19-3$ & 19.5 & \\
\hline$+21 \div$ & at 20 & $\therefore 4$ & 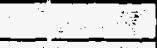 & & & & \\
\hline 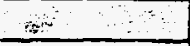 & 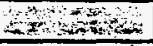 & $\tan 6$ & 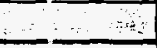 & 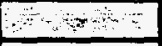 & 8 & $\therefore 1$ & $\because$ \\
\hline Total $\because$ & 13278 & 135.4 & $1=5^{\prime} 3^{7}$ & $156^{\circ} 14^{\prime \prime}$ & $135-10$ & $155-21 / 2$ & \\
\hline inew Feed & $6 x^{2}, y^{2}$ & $x, 4$ & 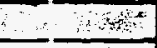 & $x+x+4 x$ & 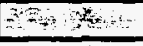 & सy & 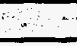 \\
\hline Adjusted Total & 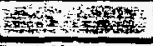 & 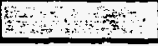 & 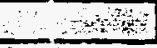 & $x+4 y$ & 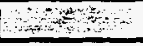 & मnन & 32 \\
\hline
\end{tabular}

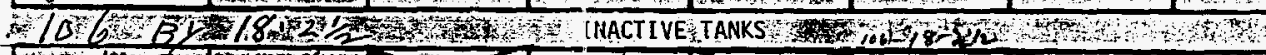

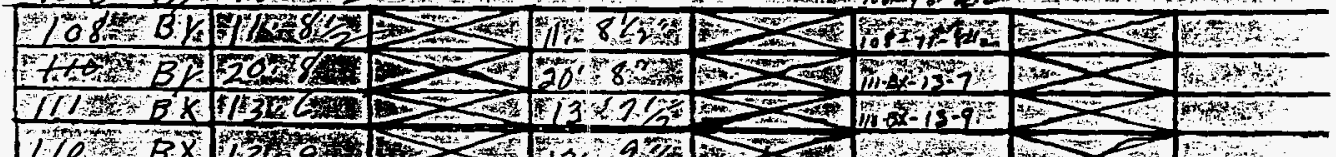

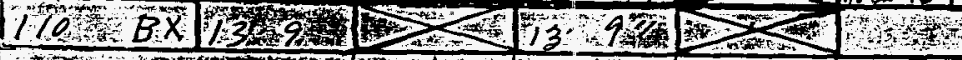

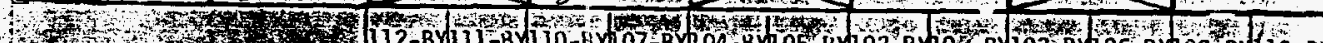

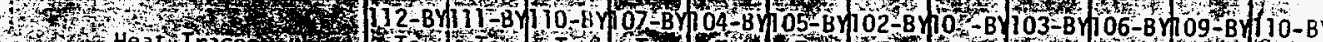
4 (1) Heat Trcesh

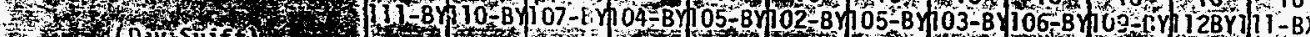
3 is (paysing (3)

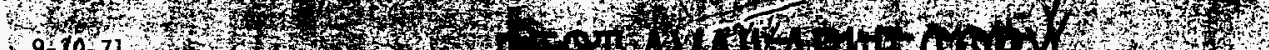

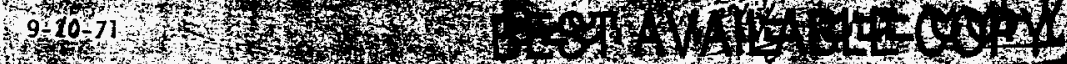
f th

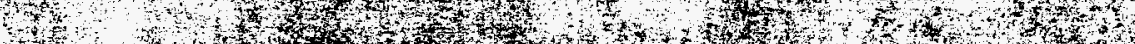

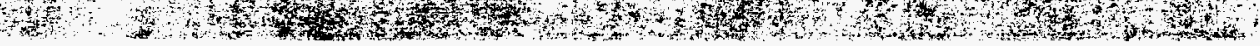




\section{WHC-SD-WM-DP-206 \\ ITS-2 IIVENTORI AWU PUMPING \\ Dale 326.27

Pump-Out Tank

Receiving Tank

\begin{tabular}{|c|c|c|c|c|c|c|c|c|c|}
\hline$\therefore$ Int & $\begin{array}{c}\text { Pump } \\
\text { Humber }\end{array}$ & $\begin{array}{l}\text { L. L. } \\
\text { Start }\end{array}$ & E. L. & $\begin{array}{l}\text { IL : : } \\
\text { : diles } \\
\end{array}$ & isumber & $\begin{array}{l}\text { L. L. } \\
\text { Start }\end{array}$ & L. $\mathrm{L}$. & $\begin{array}{c}\text { Time } \\
\text { Pump off }\end{array}$ & Remarks \\
\hline 0100 & 112 & $2 / 611$ & $20 \cdot 6$ & 17.6 & 110 & 20111 & $20 \cdot 9$ & $06 / 5$ & \\
\hline $10^{\circ}=$ & 103 & $14^{\prime} 5^{\prime \prime}$ & 13412 & & 109. & $18^{\prime 2}=$ & $19^{\prime} 7^{\prime \prime}$ & 1938 & OPluche \\
\hline 1460 & 105 & $2215 / 4$ & $19-9$ & & 103 & $13^{\prime} 5^{\prime \prime}$ & 14.2 & 2300 & $=0:$ \\
\hline $\begin{array}{l}\text { Frem } \\
3=25-22\end{array}$ & 110.84 & 20.9 & & 20.3 & $107 . \Delta y$ & $|8 \cdot 1|$ & & & $-s c$ \\
\hline 1620 & $107 \cdot-8 y$ & $20-3$ & & 22.11 & $104-8 y$ & 20.2112 & & & $3 / 27 / 72$ \\
\hline 1200 & $104-B 1$ & $20-8$ & & 17. & $112-B y$ & $(7-1) 1 / 2$ & & & sheet \\
\hline & & & & & & & & & \\
\hline & & & & & & & & & \\
\hline & & & & & & & & & \\
\hline & & & & & & & & & \\
\hline
\end{tabular}

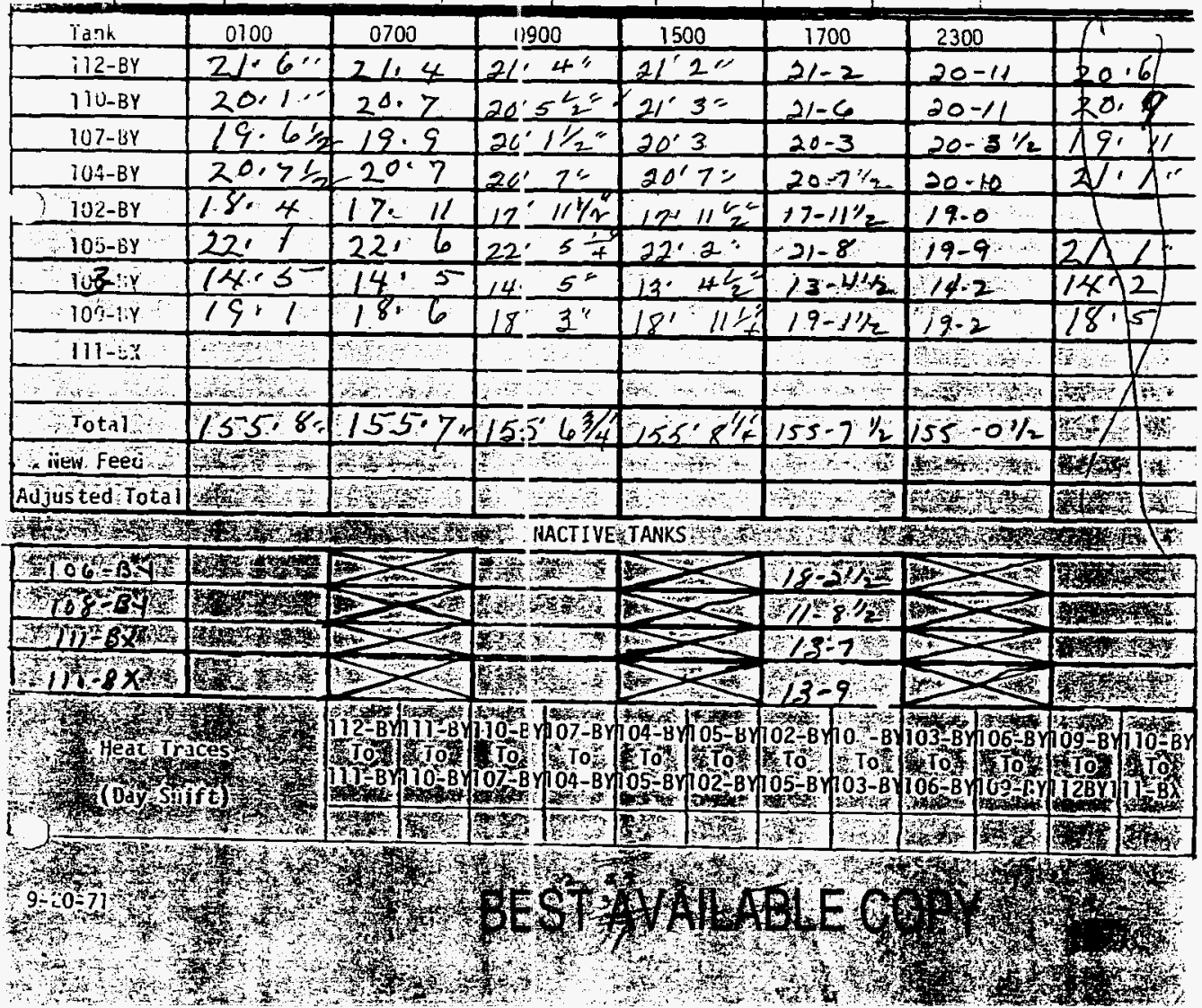




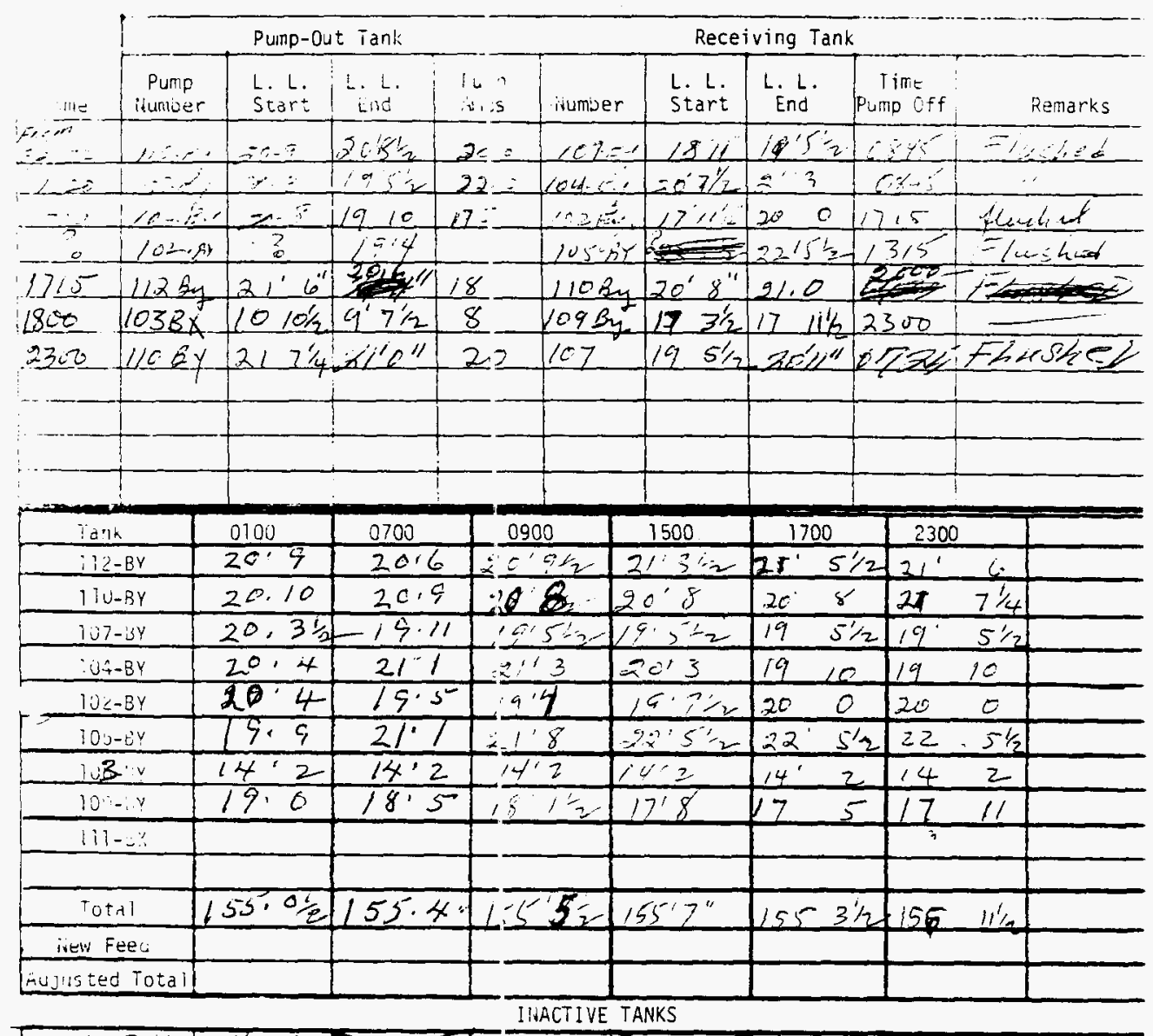

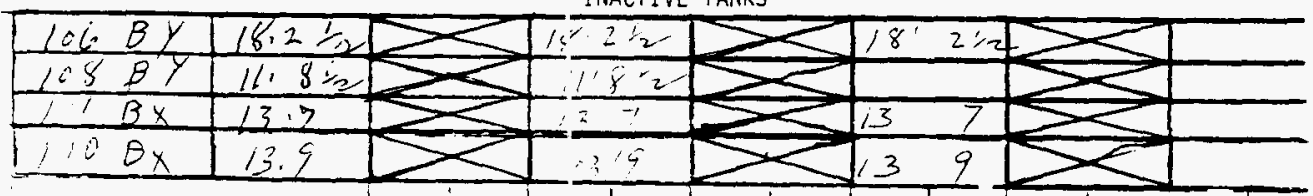

Heat Traces

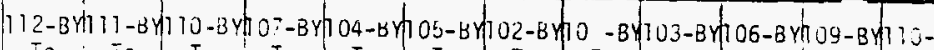

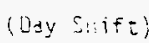

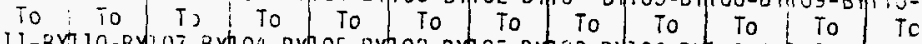

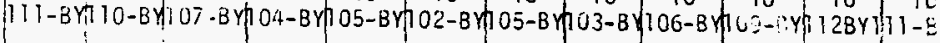

i 1

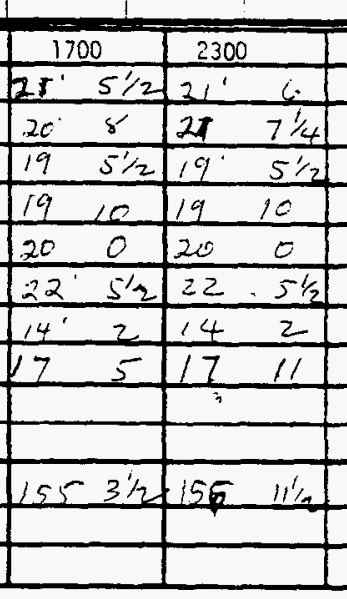




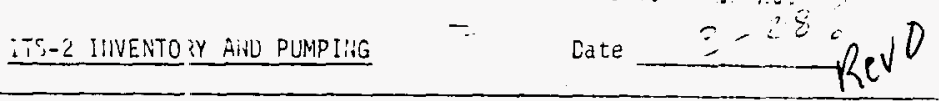

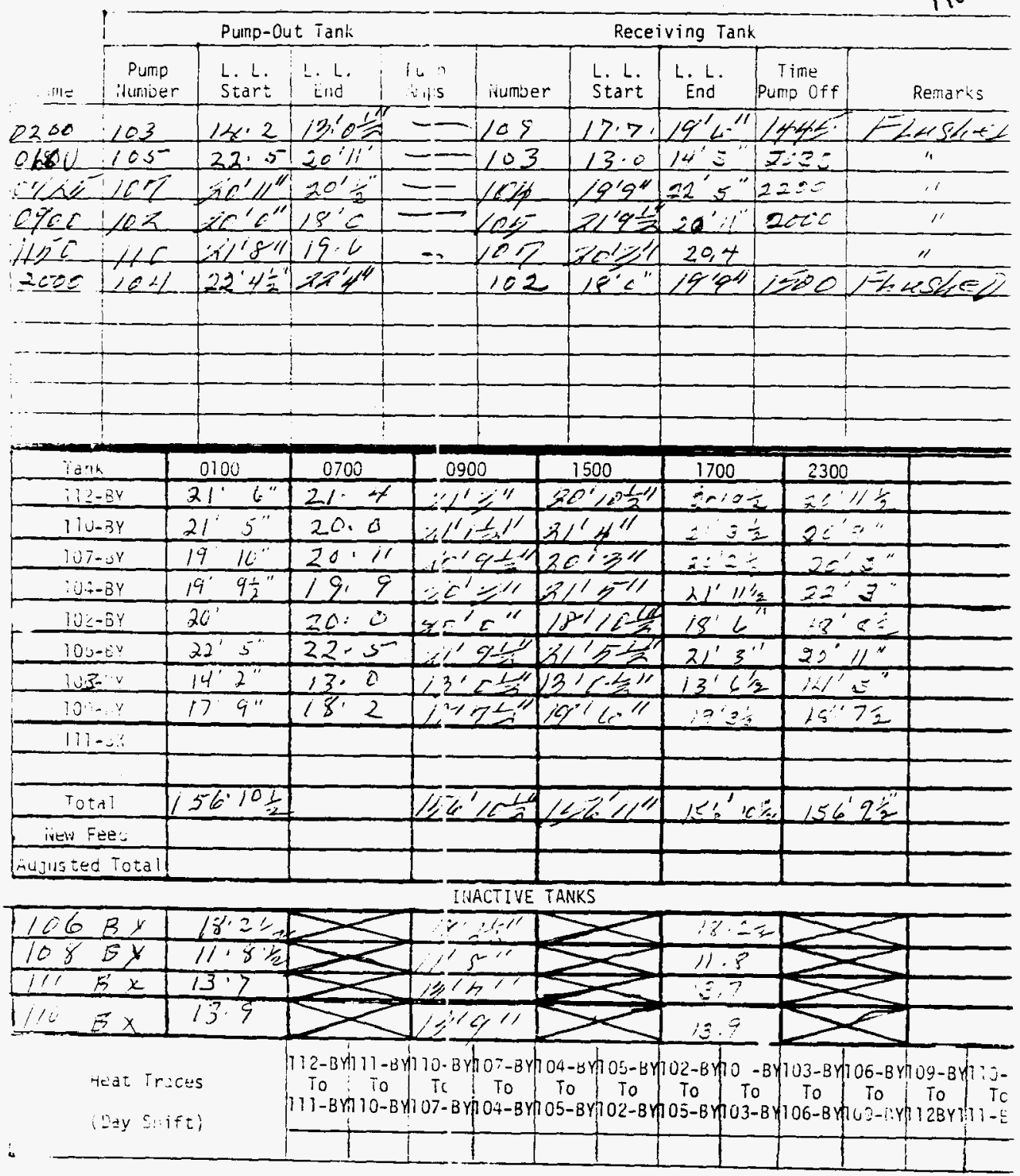




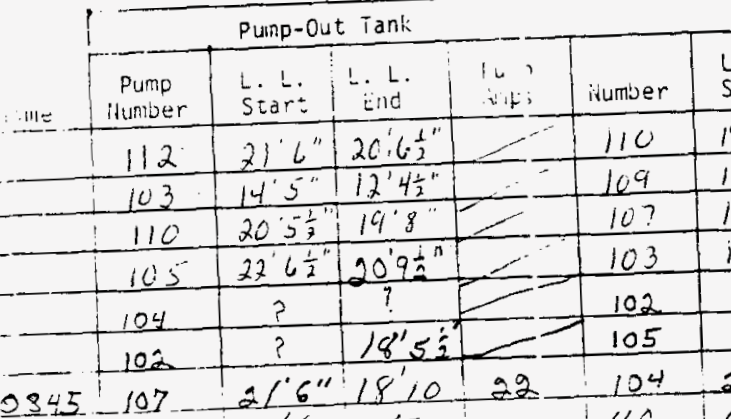

Receiving Tans.

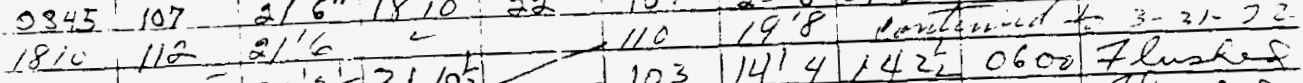

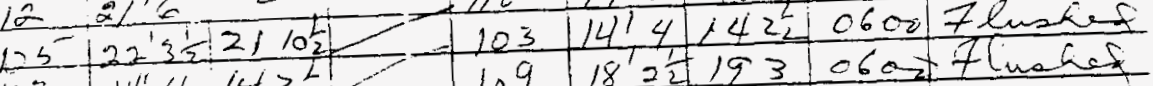

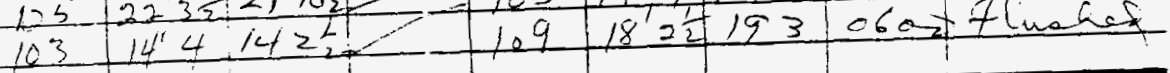

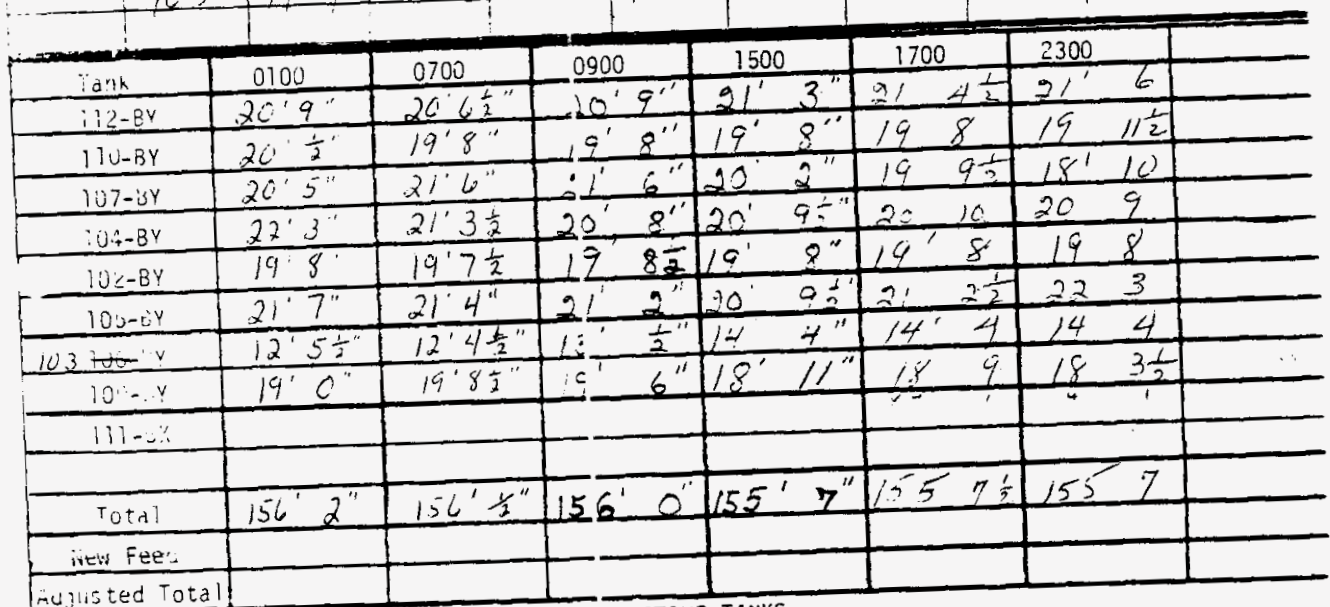

nujlisted iotal

\section{INACTIVE TANKS}

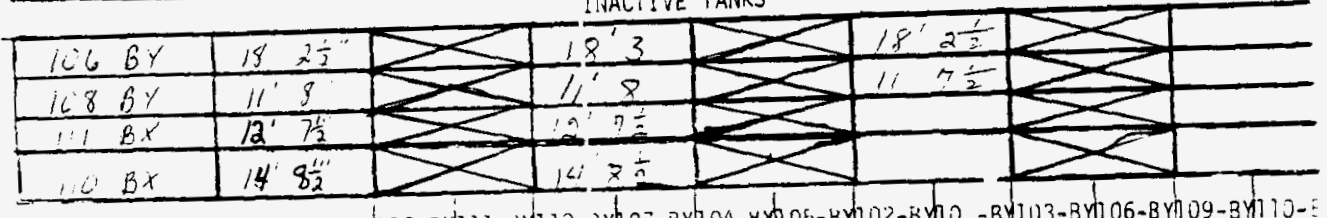

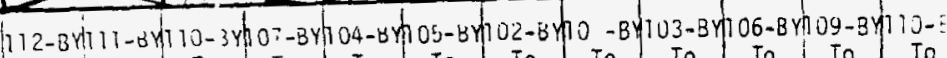

Heat Tr.aces

(bey Sift)

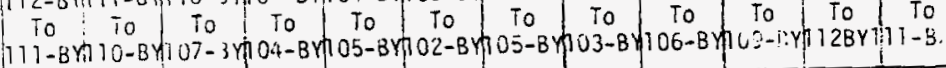

L

$9-20-7 i$ 


\begin{tabular}{|c|c|c|c|c|}
\hline & \multicolumn{4}{|c|}{ Punp-Out iank } \\
\hline$m=$ & $\begin{array}{c}\text { Pump } \\
\text { itumiber }\end{array}$ & $\begin{array}{l}\text { L. L. } \\
\text { Scart }\end{array}$ & 1. L L & $\begin{array}{l}\text { ln } \\
\text { anis }\end{array}$ \\
\hline $0<C$ & 110 & $2 /{ }^{\prime} c$ & $491 / 2=1$ & \\
\hline $0 / \sigma=3$ & $1 C 4$ & $2 i, 4$ & 1262 & \\
\hline $2=0$ & 167 & $19^{\prime} 6^{\prime \prime}$ & $18^{\prime} 9$ & . \\
\hline $16 C 6$ & $\angle 04$ & $19^{\prime} x^{\prime \prime}$ & $20^{\circ} c$ & \\
\hline
\end{tabular}

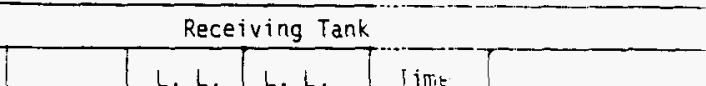

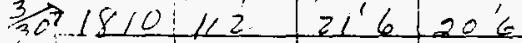

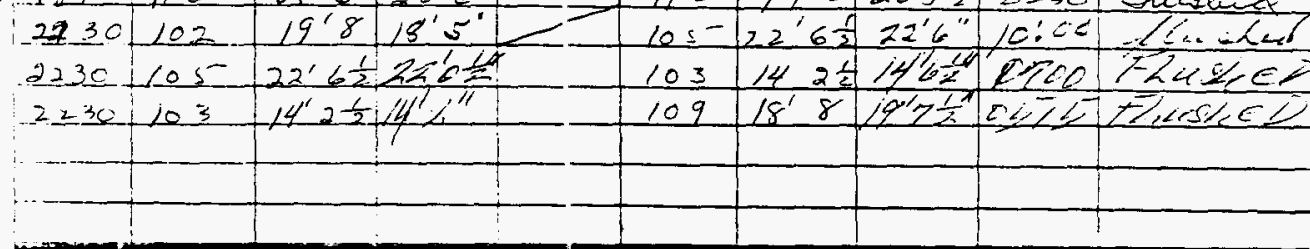

\begin{tabular}{|c|c|c|c|c|c|c|c|}
\hline alk & 0100 & 0700 & 0900 & 1500 & 1700 & 2300 & \\
\hline i12-8v & $21 \cdot 6$ & $21-2 \div$ & $21^{\prime} 1^{\prime \prime}$ & $20^{\circ} 10^{\circ}$ & $20^{\circ} 9 \div$ & $20^{\prime} 6$ & \\
\hline $11 U-B Y$ & $\therefore 0 \quad 4$ & $21-0$ & $2 \varepsilon^{\prime}>2$ & $=0^{\prime} 4^{\prime \prime}$ & $29^{\prime} 5$ & $5 \frac{1}{2}$ & \\
\hline $107-5 Y$ & $18+3$ & $18-9 \frac{1}{2}$ & $1 a^{\prime}=\frac{1}{2}$ & $19^{\prime} 39$ & $19 \quad 1 \frac{1}{2}$ & 18 & \\
\hline$i U:-B Y$ & 20.4 & $19-6 \frac{1}{2}$ & $10^{\circ} 6 \frac{1}{3}$ & $1=0^{\prime} \therefore$ & $19<1 \frac{1}{2}$ & 20 & \\
\hline $10 \leq-8 Y$ & 166 & $29-3$ & $18^{\prime} 35$ & $101=\frac{1}{3}$ & $19^{\prime} 6 \frac{1}{2}$ & $19^{\prime} 8$ & \\
\hline $100-e^{4}$ & 22,1 & $21 / 10 \frac{2}{2}$ & 2214 & $32^{\prime}=$ & $22^{\prime} \quad 6 \frac{1}{2}$ & $2926 \frac{7}{2}$ & \\
\hline$i_{t} v$ & $14 \cdot 4$ & $141-2 \div$ & $\angle 2^{\prime} \geq 2^{\circ}$ & $14^{\prime} 22$ & $14^{\prime} 2 \div$ & $1412 \frac{1}{3}$ & \\
\hline $10 \cdot:-y$ & $18 \cdot 8$ & $19-3$ & $19^{\prime} \div$ & $18^{\prime} 6$ & 184 & 18 & \\
\hline $111-\ldots$ & & & & & 3 & 7 & \\
\hline & & & & & & & \\
\hline$T_{0+41}$ & 135.6 & $155-1$ & $155^{\circ}=$ & $15410^{\frac{1}{2}}$ & $154^{\prime} 11$ & $1955^{\prime} 8 \frac{1}{2}$ & \\
\hline ivew Fee: & & & & & & & \\
\hline Eujusted Totall & & & & & & & \\
\hline
\end{tabular}

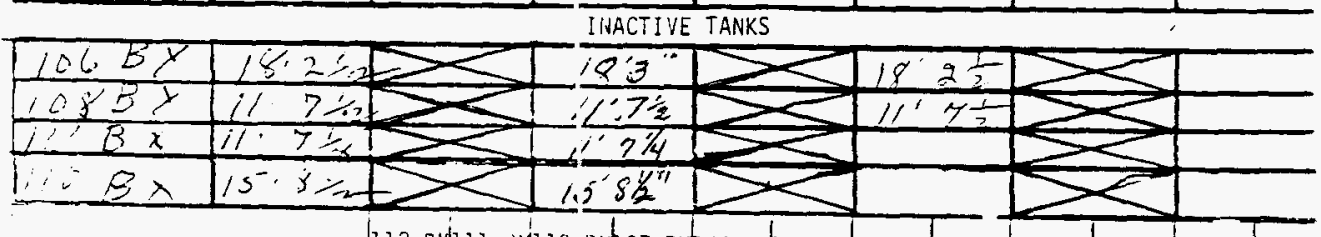

He'al iriaces

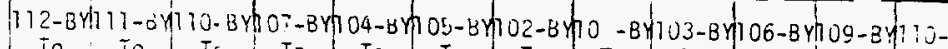

(Ligy s! ift)

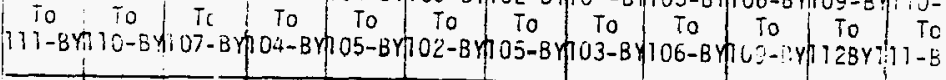

$\therefore$

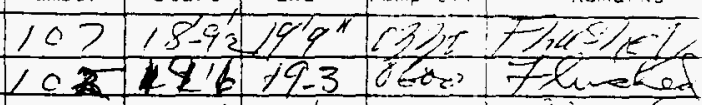

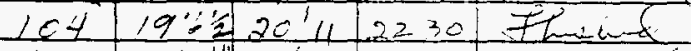

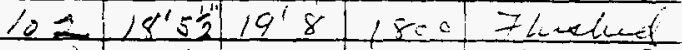

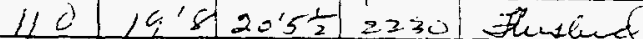




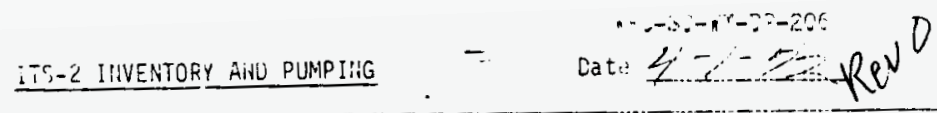

0

\begin{tabular}{|c|c|c|c|c|c|c|c|c|c|}
\hline & \multicolumn{4}{|c|}{ Puinp-Out Tank } & \multicolumn{5}{|c|}{ Receiving Tank } \\
\hline $11=$ & $\begin{array}{c}\text { Pump } \\
\text { ilumber }\end{array}$ & $\begin{array}{l}\text { L. L. } \\
\text { Start }\end{array}$ & $\begin{array}{c}\text { 1. } 1 . \\
\text { Lind }\end{array}$ & $\begin{array}{l}\text { In, } \\
\text { viti }\end{array}$ & itumber & $\begin{array}{l}\text { L. L. } \\
\text { Start }\end{array}$ & L.L. & Tinis & Remarks \\
\hline ¿if & 1017 & $14^{\prime} q^{\prime \prime}$ & $18^{\prime} Q^{\prime \prime}$ & $\pi=\bar{z}$ & 1017 & $2011^{11}$ & $213^{21}$ & -15 & $7 !$ \\
\hline 26 & 124 & $24^{\prime} z^{2}$ & & $\angle 8$ & $1 c^{2} / 2$ & $j p^{\prime} \mid-11$ & ? & & \\
\hline 30 & $1 / 3$ & $215 "$ & 3. & & 110 & $19^{\prime}:$ & 2 & & \\
\hline$\equiv \underline{E}$ & $1 E j$ & $E^{\prime} z^{\prime}$ & $19^{\prime} 9$ & & $1=2$ & $15^{\prime}=$ & $14^{\prime} / 0^{\prime}=$ & $f f_{0}::$ & $\leq 1 \leq 1$ \\
\hline 10 & 103 & $14^{\prime} 6 \frac{1}{2}$ & $14^{\prime} 6^{\prime \prime}$ & & 109 & 181 & $19^{\prime \prime} 7^{\prime \prime}$ & 0200 & Eluzhed \\
\hline$=1$ & 105 & $22^{\prime} 6 \frac{1}{2}$ & $2110 \%$ & & 103 & $14^{\prime} 6 \frac{1}{2}$ & $14^{\prime} 6^{\prime \prime}$ & $=300$ & Llecaket \\
\hline & 102 & $19^{\prime} 10 \frac{1}{2}$ & $.171^{\prime \prime}$ & & 101 & $226 \frac{1}{2}$ & $216 \%$ & 020.0 & Lzecte. \\
\hline
\end{tabular}

\begin{tabular}{l|l|l|l|l|l|l|} 
& & & & & \\
\hline
\end{tabular}

\begin{tabular}{|c|c|c|c|c|c|c|c|}
\hline ienk & 0100 & 0700 & .0900 & 1500 & 1700 & 2300 & \\
\hline$\therefore: 2-B V$ & $-10^{\prime} q^{\prime \prime}$ & $\because x^{\prime} y^{\prime \prime}$ & $=115$ & $\because \div 2$ & $\left.2\right|^{\prime} \quad 1 \frac{1}{2}$ & $20^{\prime} / c^{\prime}$ & \\
\hline 1$] \cup-B Y$ & $30^{\prime} 0^{\prime \prime}$ & $1 q^{\prime},-2$ & 195 & $2=13$ & 206 & $2 i^{\prime} 3$ & \\
\hline $137-3 y$ & $14^{\prime} 3^{4}$ & 19'C" & $\angle Q^{\prime}$ & $1 x^{\prime 2}=$ & $18 \quad 8 \div 7$ & $18 \quad 32$ & \\
\hline $0 \div-B Y$ & $200^{\circ} / 4$ & $x^{\prime}<^{\prime} z-y^{\prime \prime \prime}$ & $\because 13$ & $26^{\prime \prime}$ & $20 \quad 0$ & $19^{\prime} \%$ & \\
\hline$j 0 \leq-0 Y$ & $\angle 4{ }^{\prime \prime}$ & $\angle 2^{\prime}<\frac{1}{2}$ & $1616^{\prime \prime}$ & 193 & 1913 & $1616 \frac{1}{2}$ & \\
\hline $100-6 \%$ & $\operatorname{Rin}^{2} x \leq 4$ & $22^{\prime} \leq 1 \leq$ & $\therefore 1 \leq$ & $2+16$ & $226 \frac{1}{2}$ & 225 & \\
\hline $2 v$ & $z^{\prime} z^{\prime \prime}$ & $\angle H^{\prime} \leq 0$ & $17^{\prime}$ & $126 \div$ & $146 \frac{7}{2}$ & $146 \frac{1}{2}$ & \\
\hline $131-. y$ & $A^{\prime}<^{\prime \prime}$ & $\left.19^{\prime}\right)^{-1}=7$ & $1=2 \div$ & $18:$ & $15^{\circ} 16$ & 18 & \\
\hline$+i+=$ & & & & & 2 & 1 & \\
\hline & & & & & & & \\
\hline Total & $12078 \leq$ & $11^{2} 2^{-1} q^{11}$ & $\therefore=1$ & $15 x^{\prime}+7^{\prime \prime}$ & 1357 & $1553=$ & \\
\hline ivew Feec & & & & & & & \\
\hline Hujusted Total & & & & & & & \\
\hline
\end{tabular}

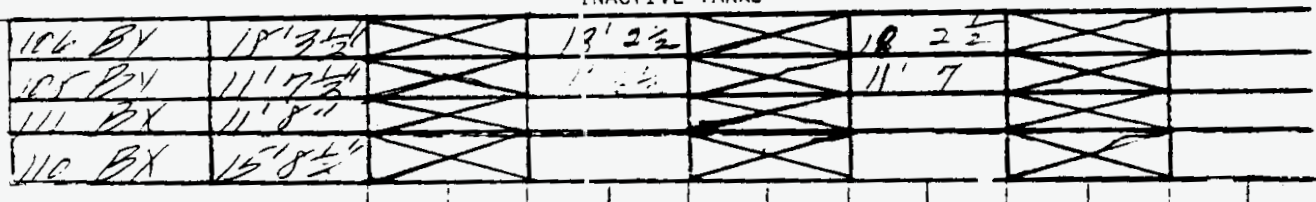

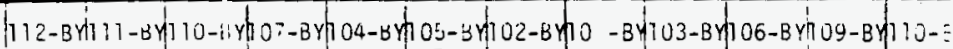

Heat ir.aces

To : io to to to to To to To to To To

(Day Stift)

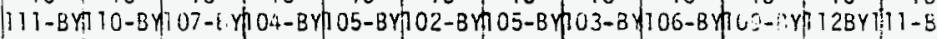

i

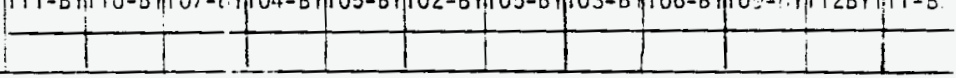

$9+-0-7 i$

BEST AVAILABLE COPY 


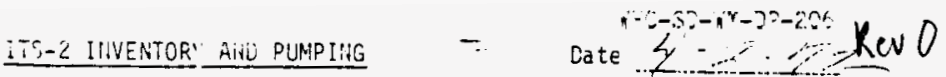

Punn-Out Tank

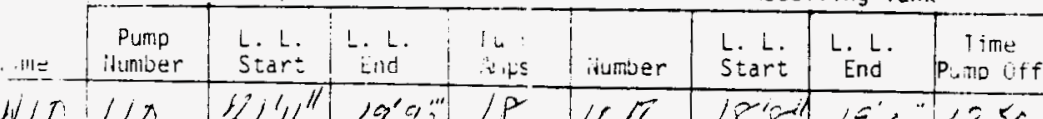

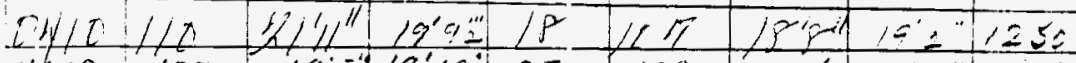
$1310 \quad 107+145^{\circ} 19^{\prime} 16^{\circ} 23$

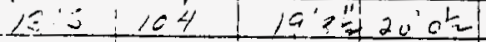
$180^{\circ} 01119 \quad 215027 \frac{16}{2}$ $0031102 \quad 19^{\prime} 2+19 \cdot 3$ 2230 120s $22^{\prime} 2 \frac{1}{2}, 22^{\prime 2}$ $2235103 / 4^{\prime} 5: 14^{\prime} 5$ 104 19 7\% $3210 " 1040$

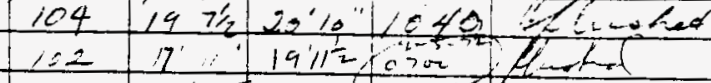

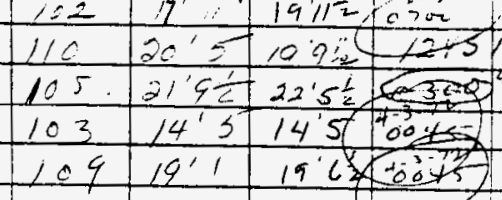

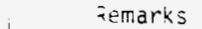

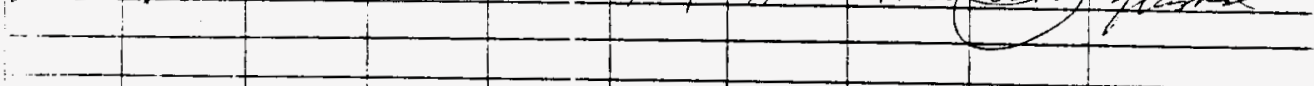

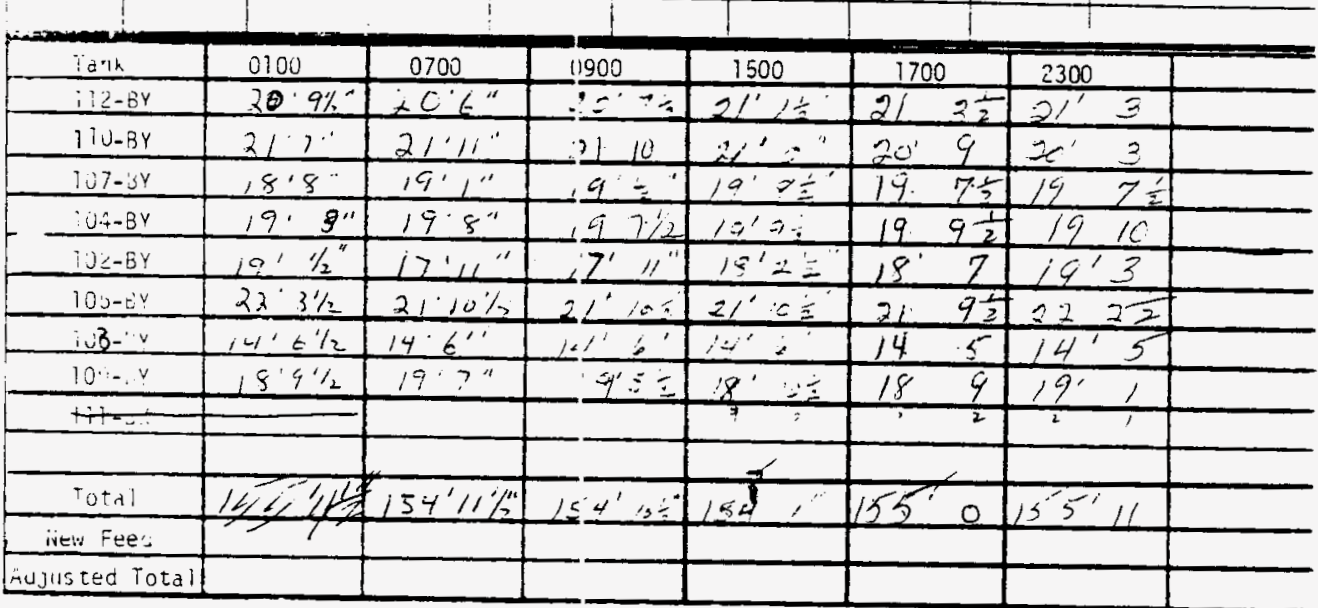

\begin{tabular}{|c|c|c|}
\hline 126 & $3 x$ & \\
\hline 105 & $B x$ & \\
\hline 1,0 & $.3 x$ & $15.8 \%$ \\
\hline 111 & $3 x$ & 1190 \\
\hline
\end{tabular}

Heia Tr:ces

(Day sitifi)
INACTIVE TANKS
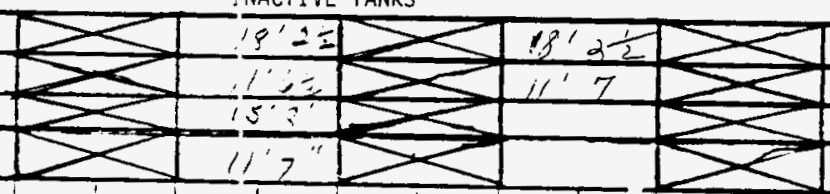

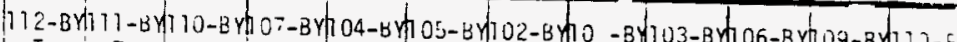
To: To To to To To To To To

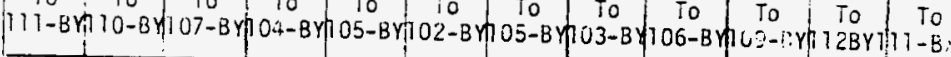

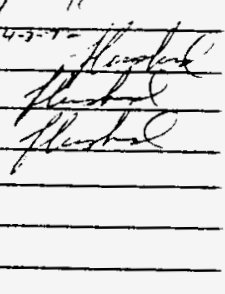


Puinp-out iank

Receiving Tan.

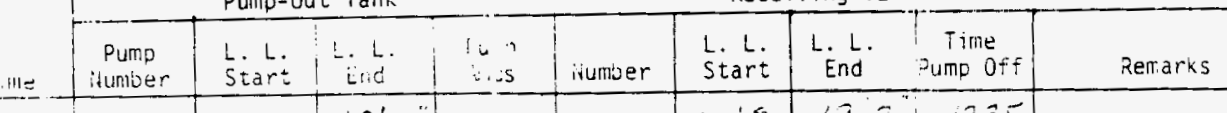

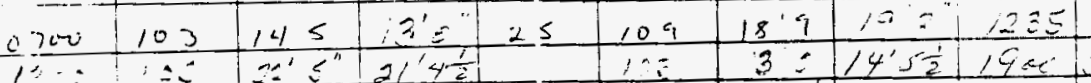

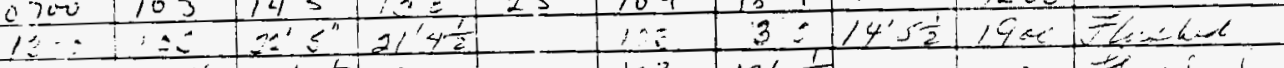

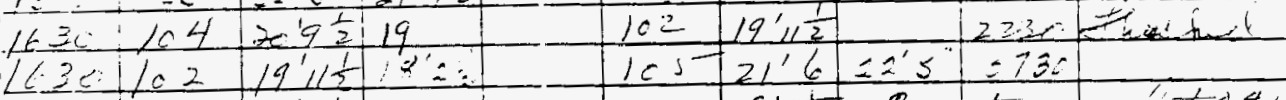

$2230112+215 \frac{1}{2}$

$2230+102-22^{\prime}<\frac{1}{2}+219$

$22301102,145 \frac{1}{2}+145$

$105721^{\prime} 6=2501 \leq 35$

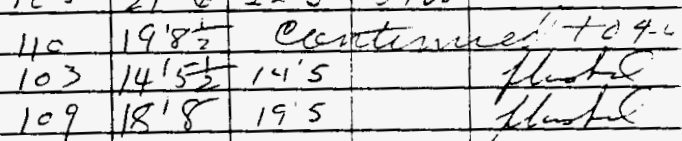

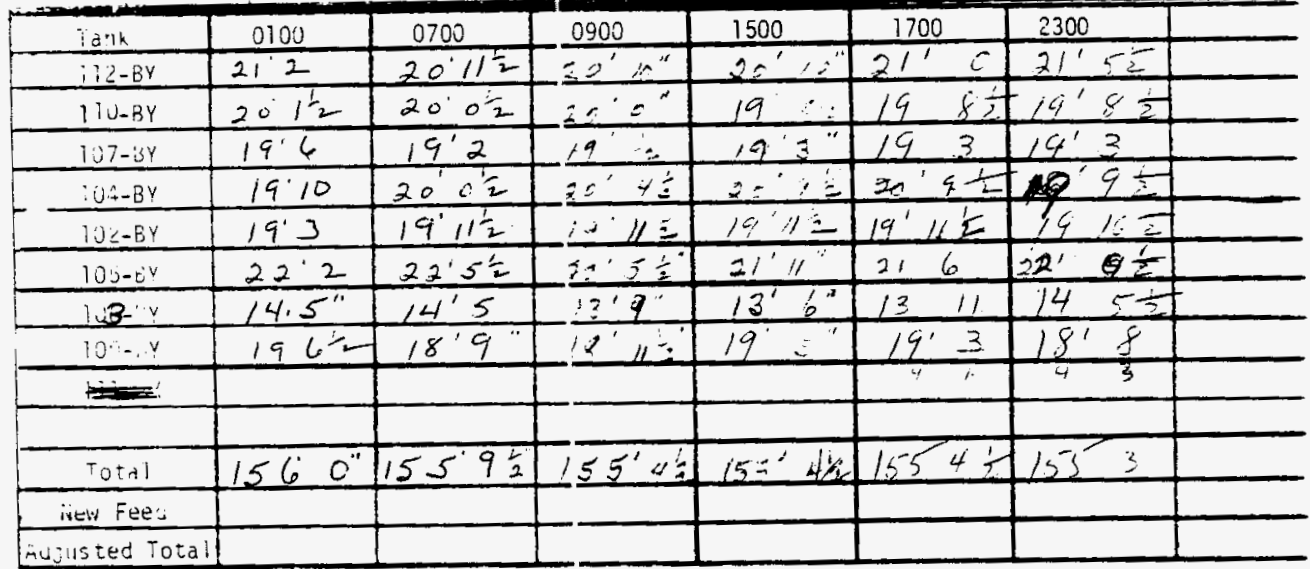

\begin{tabular}{|c|c|}
\hline $106 B y$ & $18^{\prime 2}$ \\
\hline $108 B y$ & $11^{\prime} 2$ \\
\hline $10 B X$ & 158 \\
\hline $111 B X$ & $11^{\prime} 7^{\frac{1}{2}}$ \\
\hline
\end{tabular}

Heat ir.acus

INACTIVE TANKS

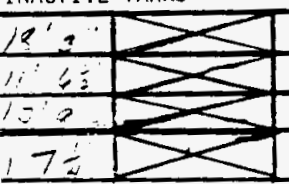

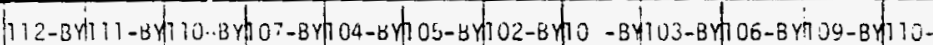

(Day sififi)

To To To To To To To To To To

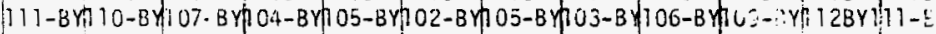

L
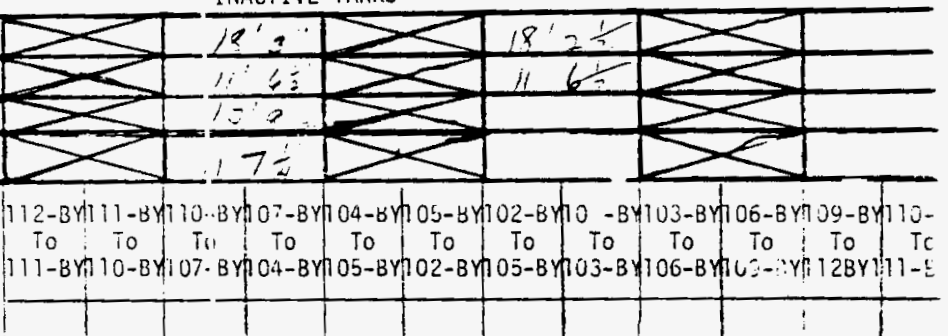

BEST AVAILABLE COPY

$9--0-73$ 


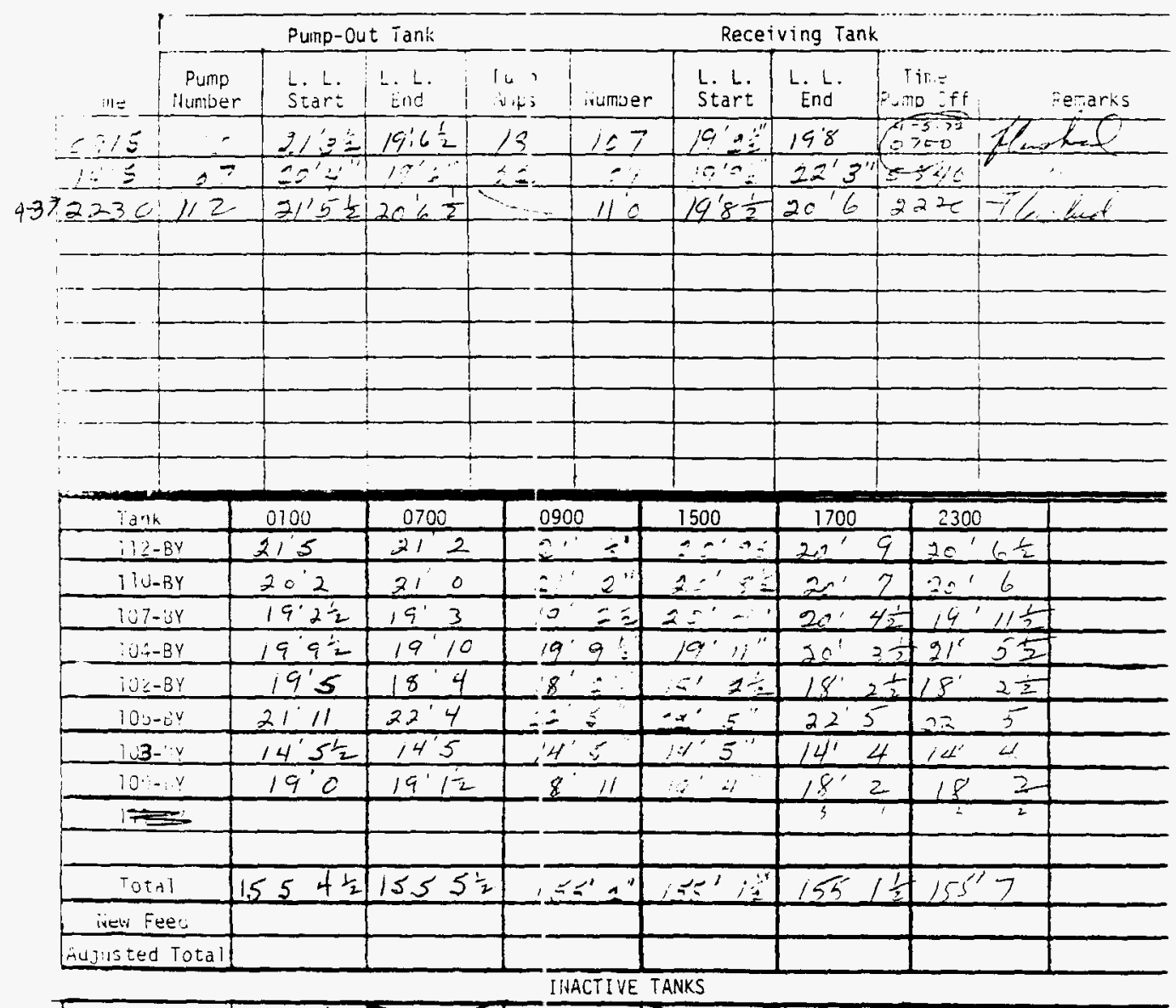

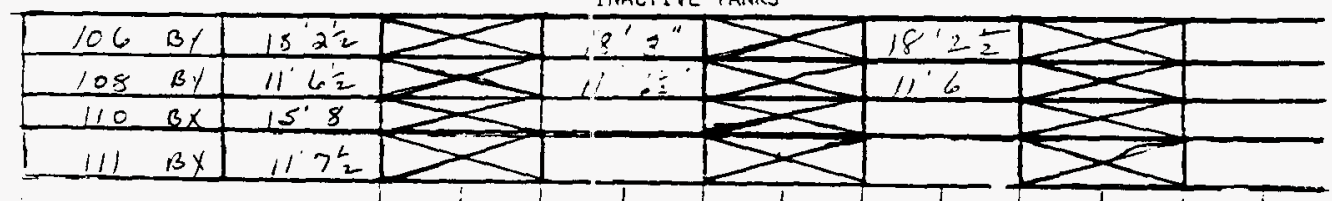

Hidi iricts

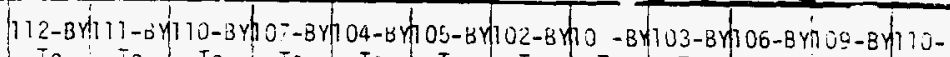

(Dey S:ift)

i

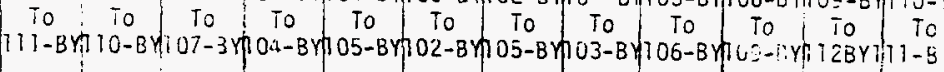

\begin{tabular}{lll}
1 \\
\hline
\end{tabular}

1 
$,-4,-\cdots--1$

RerO

Pump-Out Tank

me \begin{tabular}{c|c|c}
\hline Pump & L. L. & L. L. \\
Humber & Start & End
\end{tabular}

0030103

ocjo 105

c)3ec ! 104

$0710 \quad 112$

$13 \hat{3}$

$1-\because=$

$1566 \quad 122$

$1,83<1 / 2<1$

22451103
144

$2,2.5$

$22^{\prime 2}$

$2,4 \times 20^{\circ} \div$

$10^{\prime} \cdot 18^{\prime} 7^{\prime \prime}=$

$0 \div 6192 \%$

$19^{\prime} 112^{\circ} 18^{\prime} / 1^{\prime \prime}$

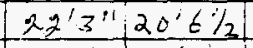

$14^{\prime} 3 \frac{1}{13} 3^{\prime} 2$
$=$

Receiving Tank:

\begin{tabular}{l|l|l|l} 
L. L. & L. L. & Time
\end{tabular}

Start End Pump off Remarks

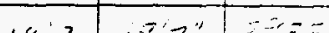

$18^{\prime 3} \therefore 7^{\prime \prime} \quad \because=5$

147 14-21/2/2/5 7LLSAED io 3

102

$182 \div 1911+4-6=2$

110

$196 \frac{1}{2} 199 \frac{1}{4}-10700$

$\because \operatorname{lin}$

$\therefore<4$

$x^{\prime} \leq \frac{1}{3}$

$22^{\prime 2}$

4315

$\operatorname{lnch} \sec$

$\because \cdots \quad 0^{\prime} \because 19^{\prime} 3^{\prime} 2$ (1030) shedes

$1=5$ 19'10:226"

$1=5$
162

$19^{\prime} 3^{\prime \prime}$

108

120

$\left(\begin{array}{l}0.02 \\ 07-6.72 \\ 0700\end{array}\right.$

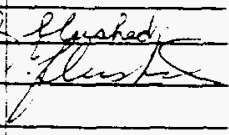

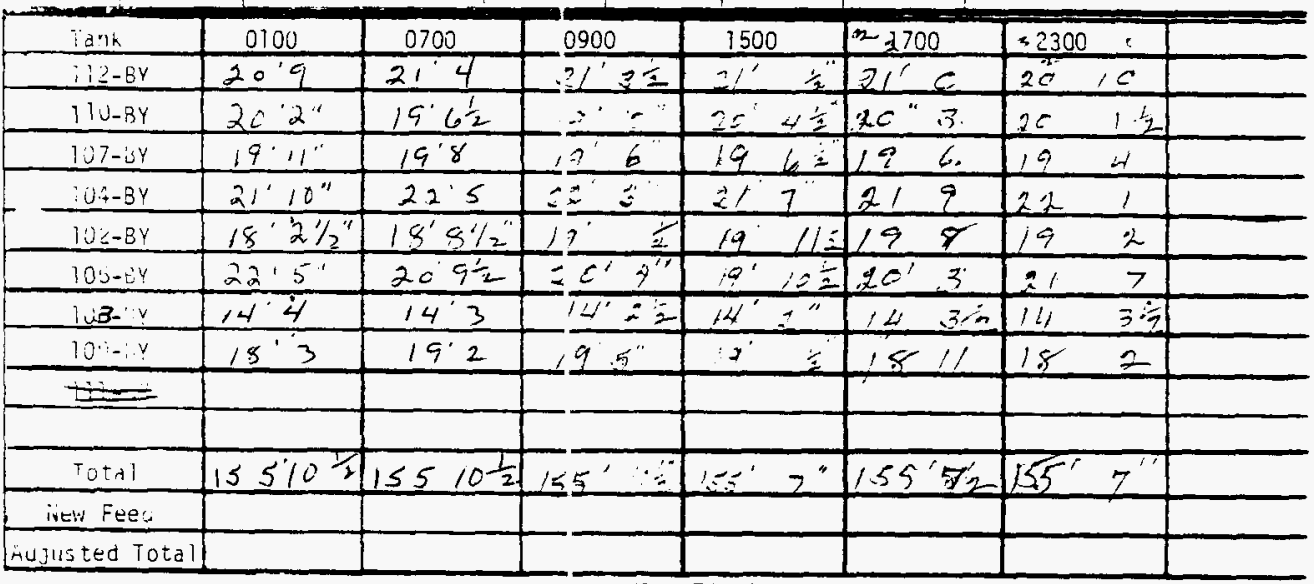

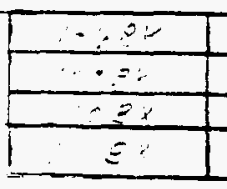

Hea: Tr:ass

(Day S:ift)

INACTIVE TANKS

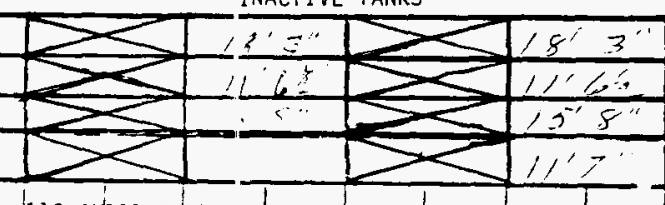

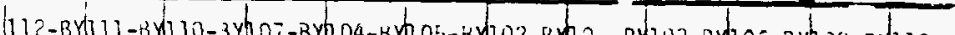
2-BYh - 0 H 111 io To To To To To To to To To

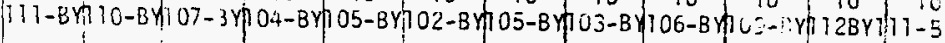




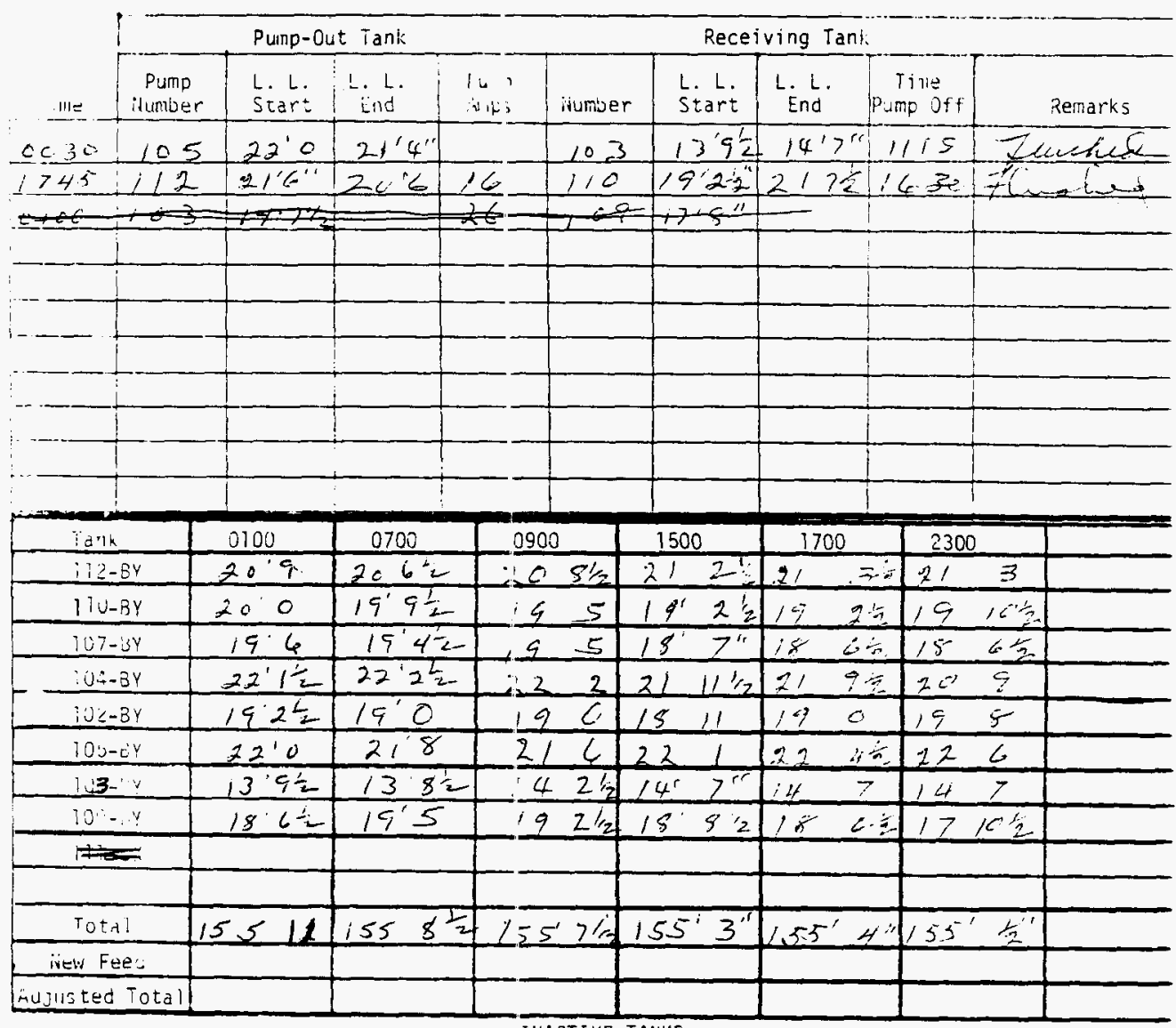

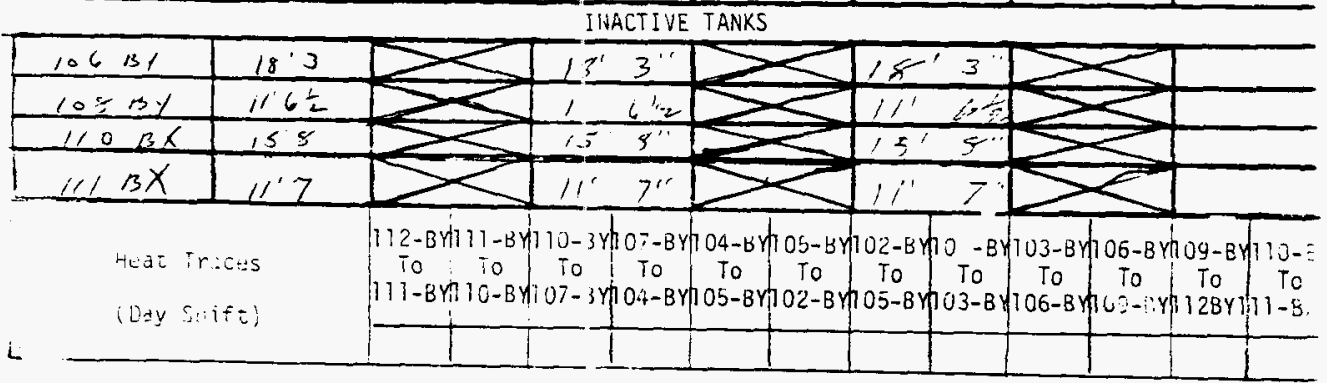
Oa....n
BEST AVALABLE COPY
$104 \rightarrow 102$
$102 \rightarrow 105$ in meter

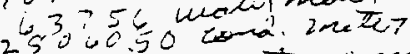

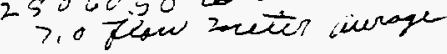




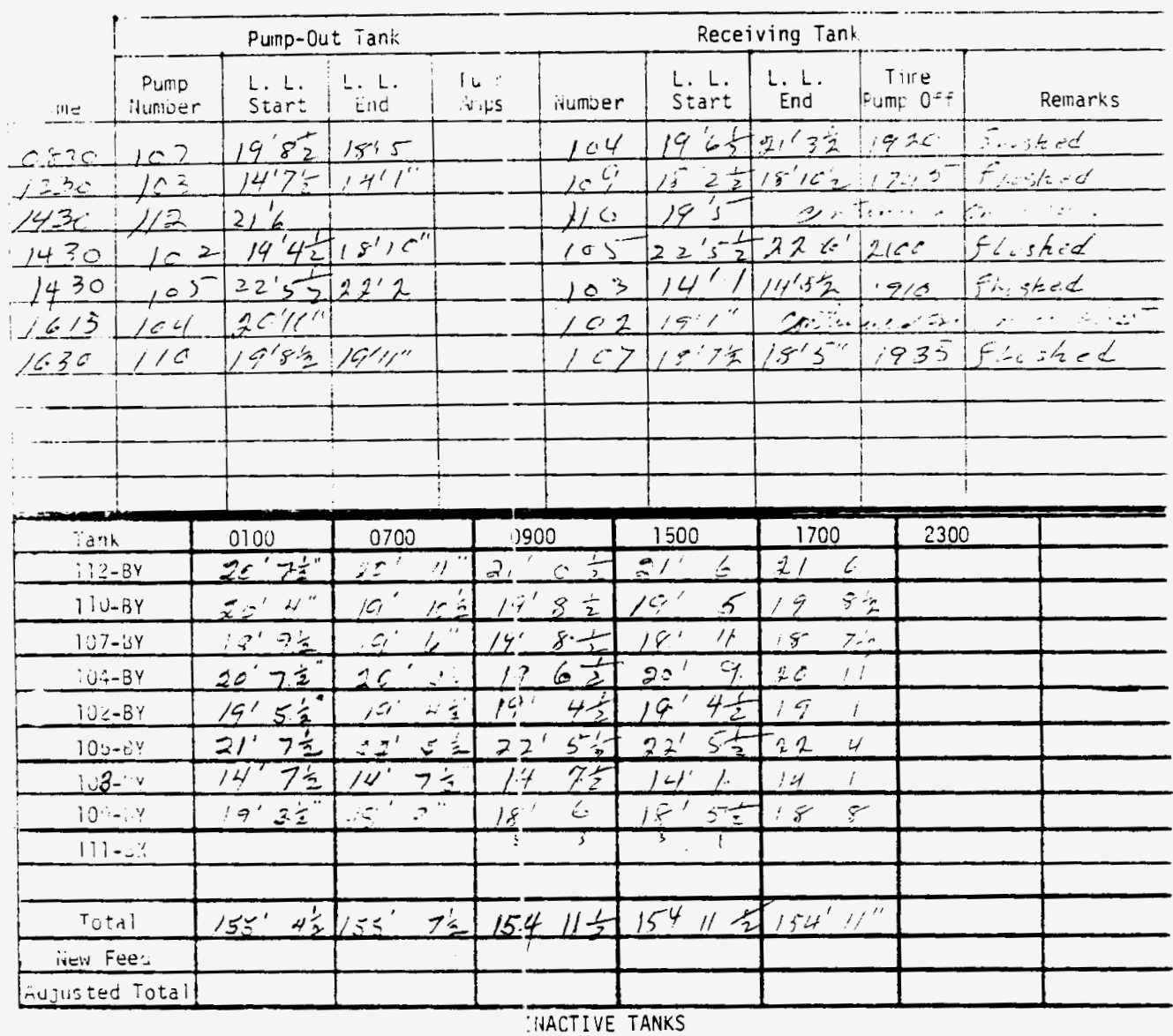

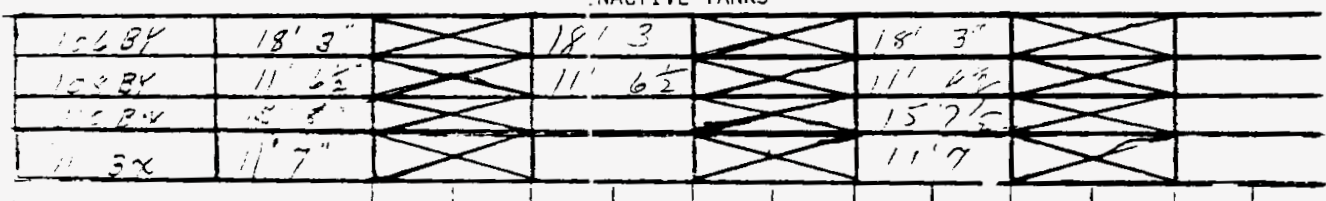

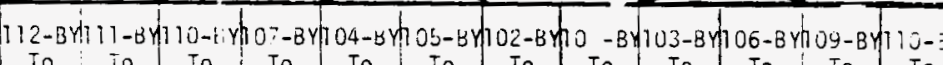
Hede Iraces To to To To To To To (Day S:ift)

i

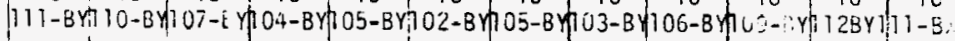


$90-52-h^{2}-79-206$

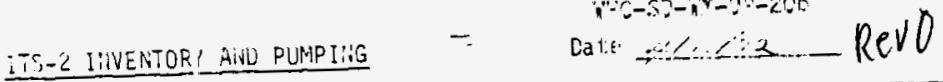

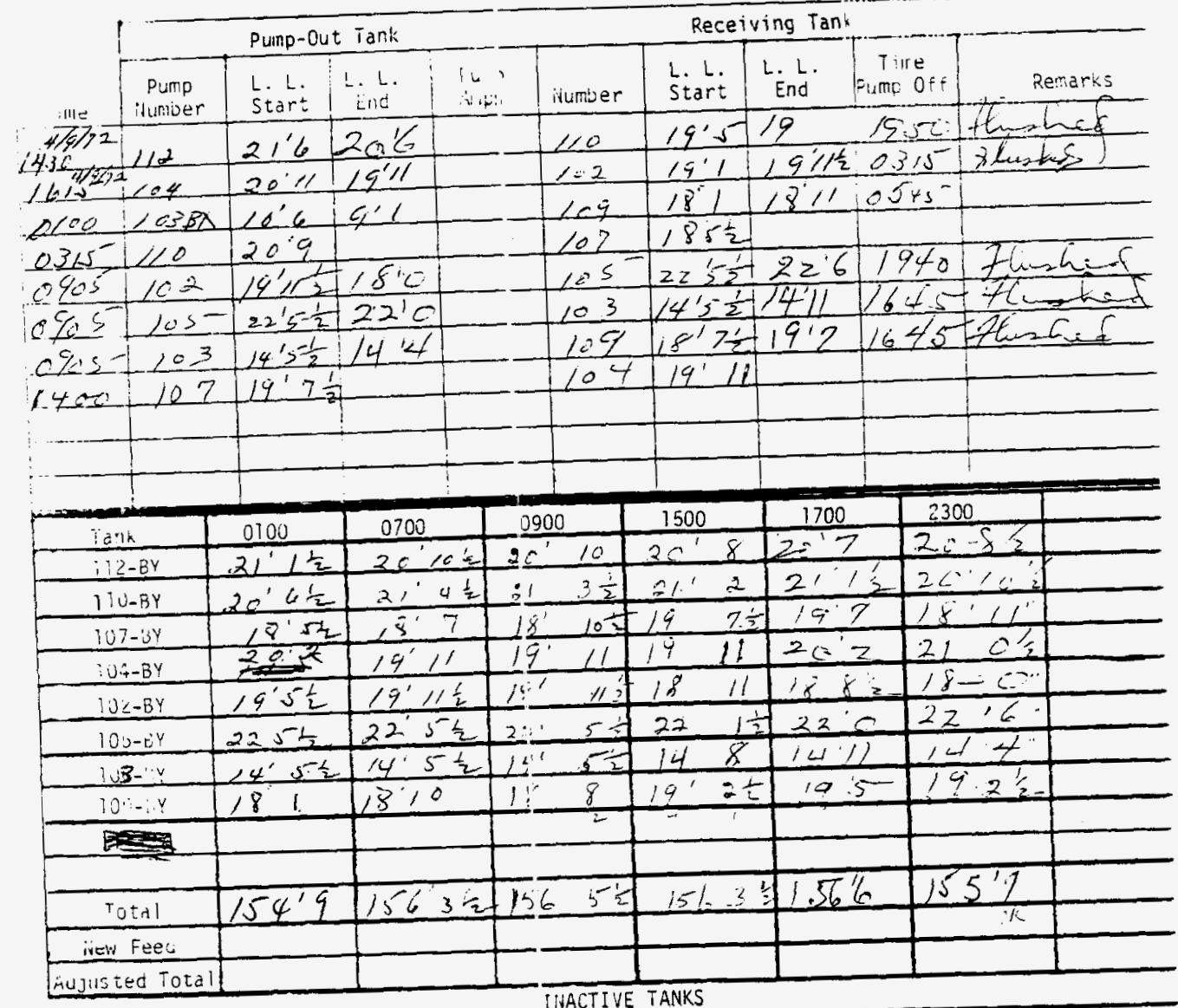

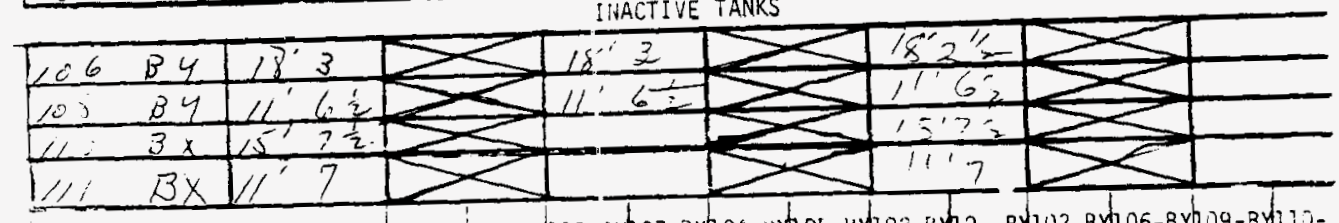

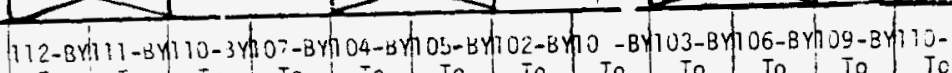

Heat ir.acss

(Day 5 !: ifi)

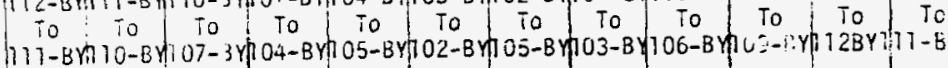

(DAY Si, if

$1+1$

$9-20-71$

BESTT AVAILABLE COPY 


\section{ITS-2 IIVENTOFY AINU PUMPIIG $\quad$ CETE}

Puitp-Out Tank

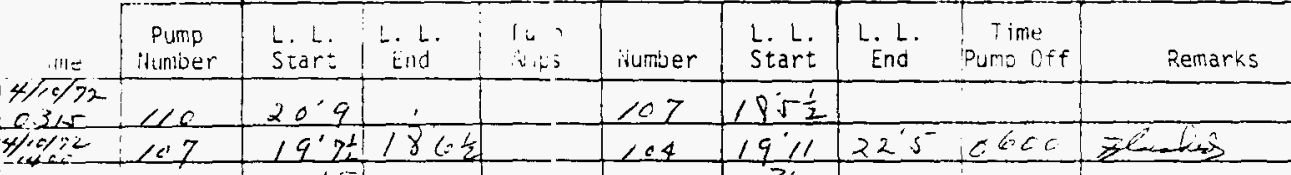

Receiving Ta-k

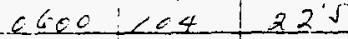

$0 \operatorname{lis} 21 / 1221.5$

\begin{tabular}{l|l|l|l|l}
0900 & 103 & $14^{\prime} 3 \Sigma^{\prime} 14^{\prime} 2 \dot{2}$
\end{tabular}

$1035105-222^{\prime \prime} 2^{\prime} 5^{\prime 2}$

$120=1107 \quad 1915$

$12.53 \quad 102 \quad 15^{1} 11.5$

$+$

102180

$110 \quad 19^{\prime} 11$

$109.19^{i} 2 \frac{1}{2} 196 \frac{1}{2} 1230$ firiked

$10313 \% " 14^{\prime 2} 2$ i2 45 Fruned

1042,4

$105222^{\circ} 0$

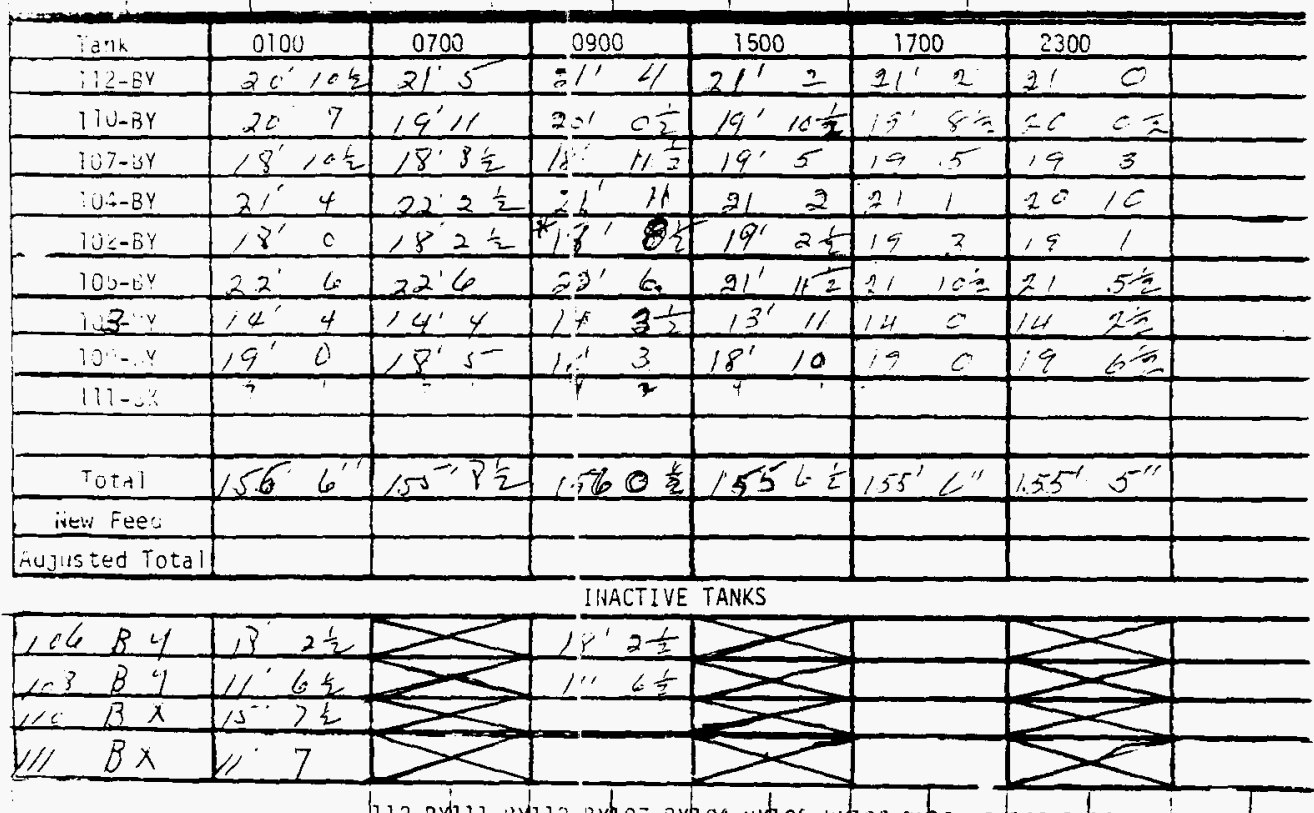

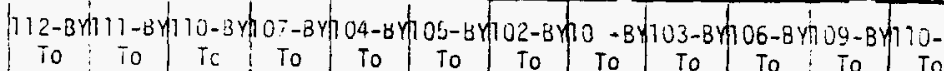

Heat Tricus

(Day S!ift)

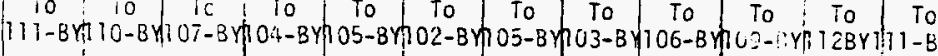

i

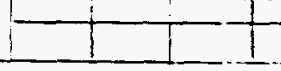

$+$ 
Ci.

\begin{tabular}{|c|c|c|c|c|c|c|c|c|c|}
\hline \multirow{2}{*}{$\frac{111=}{2}=$} & \multicolumn{4}{|c|}{ Puinp-Out Tank } & \multicolumn{3}{|c|}{ Receiving Tar: } & \multirow{2}{*}{\multicolumn{2}{|c|}{ Remarks }} \\
\hline & $\begin{array}{c}\text { Pump } \\
\text { itumber } \\
\end{array}$ & $\begin{array}{l}\text { L. L. } \\
\text { Start }\end{array}$ & L. L. & $\begin{array}{l}\text { In } \\
\therefore 40\end{array}$ & ivumber & $\begin{array}{l}\text { L. L. } \\
\text { Start }\end{array}$ & $\begin{array}{c}\text { L. L. } \\
\text { End }\end{array}$ & & \\
\hline $\begin{array}{l}4 / 1072 \\
03,5 \\
\end{array}$ & 110 & $2 c^{\prime} 9$ & $19: 2 \div 4$ & & 167 & $13^{\prime} 5 \frac{1}{2}$ & $i 0^{\prime} \div$ & $\therefore 000$ & $E 1: 5:=0$ \\
\hline 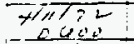 & 104 & 225 & & & $\angle<2$ & $18^{\prime} 0$ & & & \\
\hline$+1162^{2}$ & 112 & 715 & $20^{\circ} 6 \div^{\prime \prime}$ & & $1<0$ & $19^{\prime} 11$ & $19^{\prime}>1$ & 1000 & Elusisd. \\
\hline $\begin{array}{l}71172 \\
12 \times 0\end{array}$ & 10.2 & $19 v^{-}$ & $10^{\prime} 10 \div 2$ & & 104 & 214 & $20^{\prime} 2^{\prime \prime}$ & $x<12$ & ELingi \\
\hline $\begin{array}{l}41 / 122 \\
1250\end{array}$ & 102 & $181, \frac{1}{2}$ & $18^{\prime} 10$ & & $105^{-}$ & $22^{\prime} 0$ & 227 & 0.30 & Zlewbin \\
\hline $2545^{2}$ & 10.3 & $14^{\prime} 3$ & $14^{\prime} 3^{\prime \prime}$ & & 109 & $18 \cdot 8$ & $19^{\prime} 6^{\prime \prime}$ & 1205 & Elushed \\
\hline 0900 & 105 & $22^{\circ} 7$ & $22^{\prime} 10 \frac{1}{n}$ & & 103 & $13^{\prime} 2$ & $14^{\prime} \leq \frac{1}{2}^{\prime \prime}$ & 1245 & $=i, 34=i$ \\
\hline $10<0$ & 162 & $19^{\circ} 5 \frac{-1}{2}$ & $19^{\prime} 42^{11}$ & & 105 & $22^{\circ} 4$ & $22^{\circ} \div \div$ & 2020 & Elosie i \\
\hline & & & & & & & & & \\
\hline & & & & & & & & & \\
\hline & & & & & & & & & \\
\hline
\end{tabular}

\begin{tabular}{|c|c|c|c|c|c|c|c|}
\hline$a \eta k$ & 0100 & 0700 & .0900 & 1500 & 1700 & 2300 & \\
\hline$i 13-34$ & 2011 & $20.9 \frac{1}{2}$ & $\therefore 0^{\prime} 5 \frac{1}{2}$ & $28^{\circ} 7 E$ & $20^{\circ} 7 \div$ & $\$ 0^{\circ} 10$ & \\
\hline $1 Y U-B Y$ & $1 c^{\prime} \quad \xi \varepsilon$ & 14,9 & $19^{\prime} 82$ & $19^{\prime} 7 \frac{1}{2}$ & $10^{\prime} 2^{\prime \prime}$ & $10^{\circ} 8$ & \\
\hline $107-3 Y$ & $\angle 9^{\prime} \quad 3 \frac{1}{2}$ & $19^{\circ} 4$ & $193 \frac{1}{2}$ & $19^{\prime} 2$ & $10^{\prime} 2$ & $19 \div$ & \\
\hline $0.7-3 y$ & $20^{\circ} 9$ & $20^{\prime} 11$ & $2 c^{\prime} 10 \frac{2}{2}$ & $20^{\prime} 9$ & $\pm 2^{\prime} 8^{\prime \prime}$ & $20^{\prime} \quad 4$ & \\
\hline $10=-0 y$ & $19^{\prime} 3$ & $18^{\circ}, 0$ & \pm 212 & .9 .4 & $19^{\prime} \quad 4 \frac{1}{2}$ & $10^{\circ}$ & \\
\hline $100-y^{2}$ & $21^{\prime} 9$ & $22^{\prime} 7$ & $22^{\prime} 7$ & 2211 & $21^{\circ} 142$ & $4 \div$ & \\
\hline $33^{2} \cdot$ & $\angle 4^{\prime} 3$ & $14^{\prime} 3$ & $13^{\prime} 7$ & $13^{\prime} 11$ & $14^{\prime} 3^{\prime \prime}$ & $141^{\circ} 5$ & \\
\hline $10 \div-y$ & $19^{\prime} 3$ & 1810 & $14^{\prime} 1$ & $19^{\prime} 6$ & $15^{\prime} 6^{\prime \prime}$ & $18^{\circ} \quad 9$ & \\
\hline $1 i 1-?$ & $=-1$ & & $\pm D$ & & & & \\
\hline Total & $15 j^{\prime \prime 3}$ & $155^{\prime} 3 \frac{1}{2}$ & $1.55^{\prime} 1$ & $155^{\prime} 0$ & $155^{\prime}$ & $155^{\circ} \quad \frac{1}{2}$ & \\
\hline ivew Fees & & & & & & & \\
\hline تujusted Total & & & & & & & \\
\hline
\end{tabular}

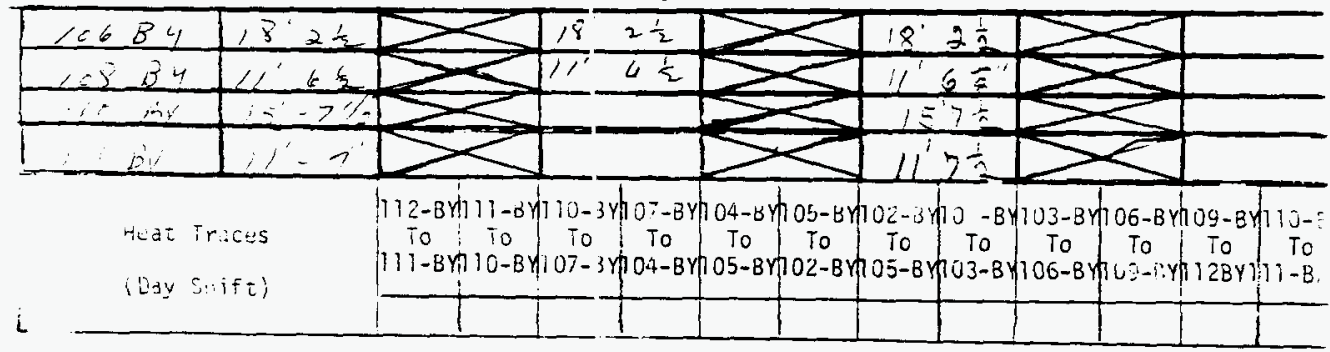




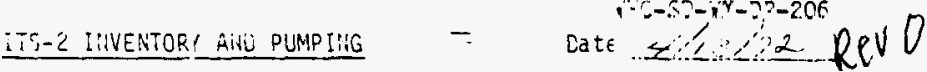

\begin{tabular}{|c|c|c|c|c|c|c|c|c|c|}
\hline \multirow{3}{*}{ 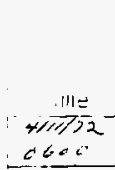 } & \multicolumn{4}{|c|}{ Pump-Out Tank } & \multicolumn{4}{|c|}{ Receiving Tank } & \multirow[b]{2}{*}{ Pemarks } \\
\hline & $\begin{array}{l}\text { Pump } \\
\text { Plunber } \\
\end{array}$ & $\begin{array}{l}\text { L. L. } \\
\text { Start }\end{array}$ & 1. L. L. & $\begin{array}{l}\text { in }, \\
\text { inti }\end{array}$ & iumber & $\begin{array}{l}\text { L. L. } \\
\text { Start }\end{array}$ & $\begin{array}{l}\text { L. L. } \\
\text { End }\end{array}$ & Purip off & \\
\hline & 104 & $22 v-1$ & $20+c^{1 / 2}$ & $=$ & $102^{\circ}$ & 180 & 20.0 & {$[2: 4,-$} & $z_{i}^{i} i x$ \\
\hline$\therefore \therefore$ & $=3.24$ & .1 .5 & 1319 & $\Longrightarrow$ & $\Leftrightarrow=$. & $14-6$ & $19^{\prime} 8$ & $14<S^{-}$ & i \\
\hline COE & $1 / 2$ & 216 & & & $\angle 2 \theta$ & $15 \cdot 3$ & & & \\
\hline$=5:=$ & $\cos 3$ & $\because 21$ & $91^{\prime} 0^{\prime \prime}$ & $\approx$ & $\therefore 2$ & $13-3:$ & $14^{\prime} 5^{\prime \prime}$ & 720 & $=\therefore$ \\
\hline c 900 & 102 & $20^{\circ} 0$ & $18^{\prime} 0^{\prime \prime}$ & & 105 & 218 & $218 \frac{1}{2}$ & 2100 & Fivsines \\
\hline$\angle 230$ & 110 & $21^{\prime 2} 2^{\prime \prime}$ & & 20 & 107 & $18^{\prime} 10$ & & & \\
\hline
\end{tabular}

\begin{tabular}{|c|c|c|c|c|c|c|c|}
\hline Yank & 0100 & 0700 & 0900 & 1500 & 1700 & 2300 & \\
\hline i12-BY & $2^{\prime} \quad 0$ & $21: 6$ & $\therefore 1: 5$ & $211 \quad 3$ & $2 i^{\prime}$ & $=$ & \\
\hline $11 U-B Y$ & $19^{\prime} 3$ & 198 & $19^{\prime} 11.5$ & $20^{\prime} \quad 8.4$ & $=110$ & $\hat{\Delta \hat{O}^{\prime}}$ & \\
\hline $307-3 y$ & $\angle 3^{\prime}, c$ & $\angle \overrightarrow{8} 10$ & $11^{\prime} 10$ & $\angle 8: \quad 10$ & $18^{\prime} \quad 12$ & $10^{\circ}$ & \\
\hline $0 .+-B Y$ & $20^{\circ}=\frac{1}{2}$ & $20 \div 2$ & $\Rightarrow 002$ & $30^{\prime}$ & $10: 1=$ & $10: 11 \div$ & \\
\hline $10 \leq-B Y$ & $20^{\circ} \quad 6$ & $20^{\circ} \mathrm{c}$ & $2 C$ & $\angle 2$ & $18^{\circ} 8$ & i2: & \\
\hline $300-e^{Y}$ & $22^{\circ}$ & $22^{\circ} 31$ & 21 & $31^{\prime} 8$ & $21^{1}$ & $21 \quad 2 \div$ & \\
\hline 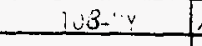 & $14^{\prime}$ & $10^{\circ} 3 \frac{1}{2}$ & 132 & $13^{i} 5$ & $14^{\prime} \quad 3$ & $4^{\circ}=$ & \\
\hline $10-y$ & 186 & $15^{\prime} 1$ & $1^{\prime \prime} 1 \div$ & 148 & $10^{\circ} 5$ & $18^{\prime} \quad 8$ & \\
\hline $111-\therefore$ & & & & $y$ & & & \\
\hline Total & $154^{\prime} 9 \frac{1}{2}$ & $154^{\prime} 82$ & 15492 & 15456 & $154^{\prime} 4 i$ & $154^{\prime}$ & \\
\hline iven Fees & & & & & & & \\
\hline Aujus ted Totall & & & & & & & \\
\hline
\end{tabular}

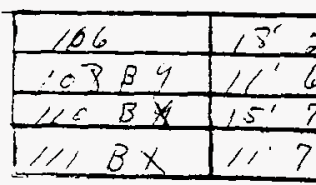

Huat Tr.aces

(0dy S:ift)

\section{INACT IVE TANKS}

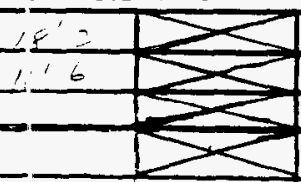

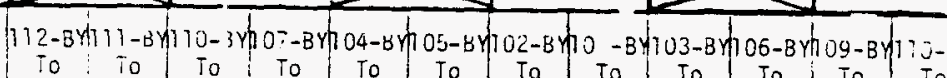

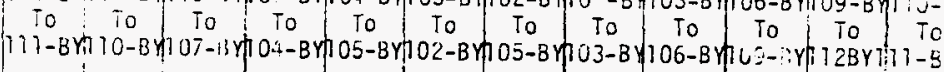

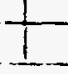

$$
1
$$

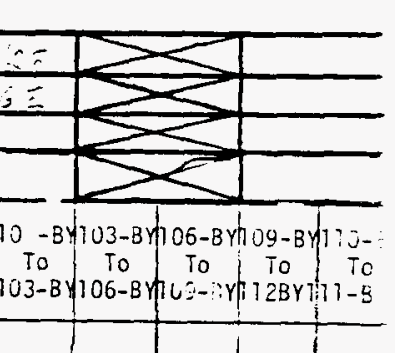

$9-20-7 i$ 


\section{IIT-2 IIVENTOR": ALV PUMPIIG \\ $Y=C-57-W M-D P-206$}

Puinp-Out iank

Receiving Tank

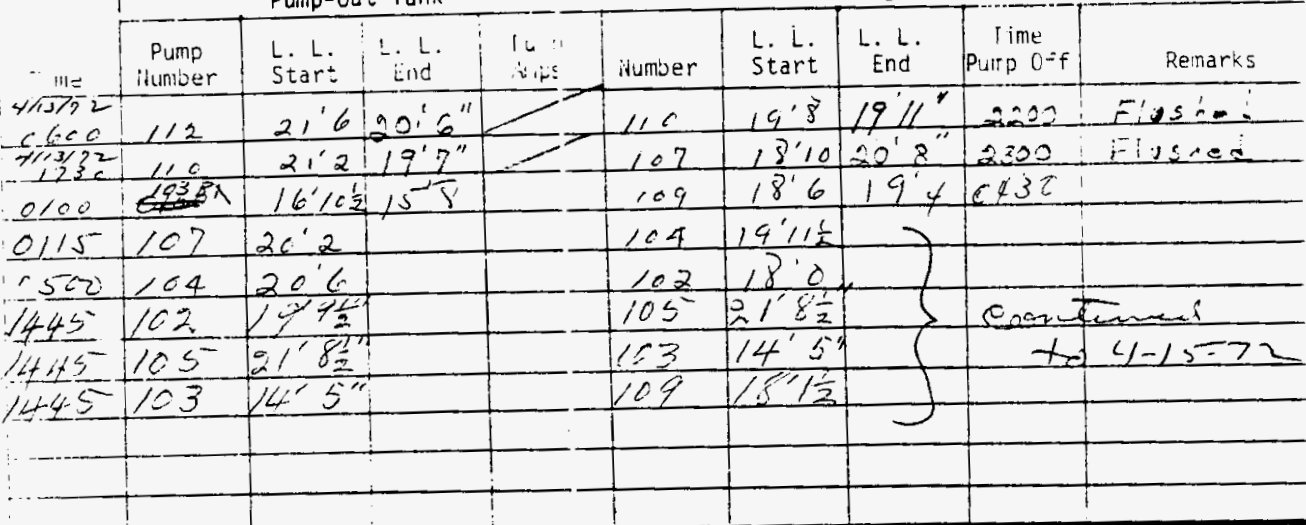

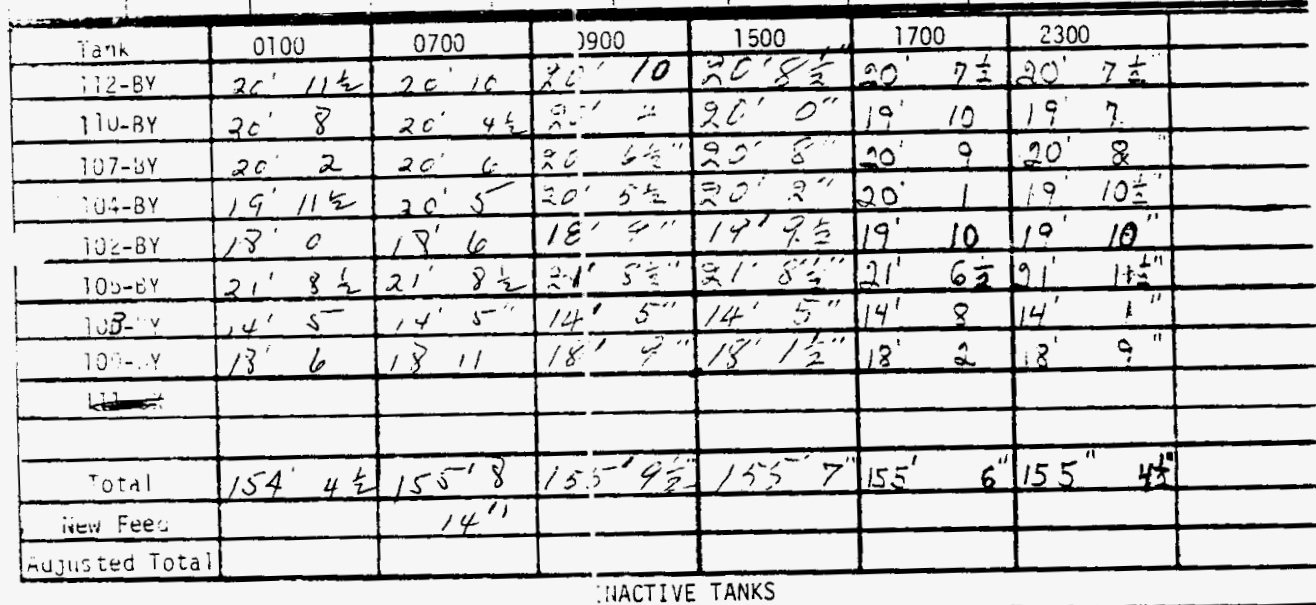

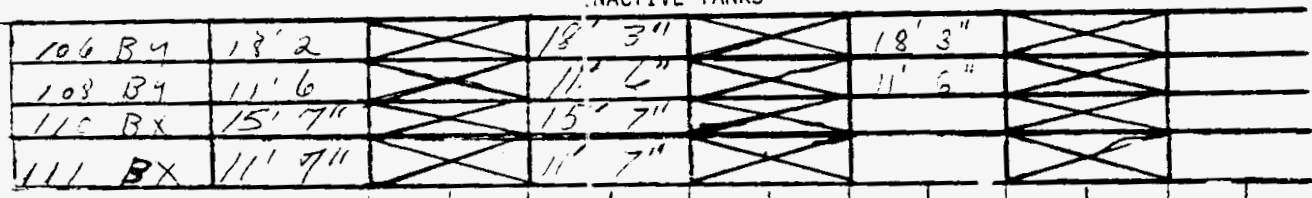

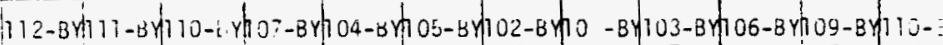

Heat ir:ct:s

To: To To To to to

(Doy Saifi)

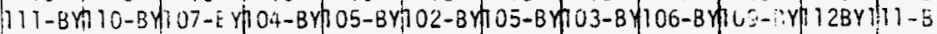

i 


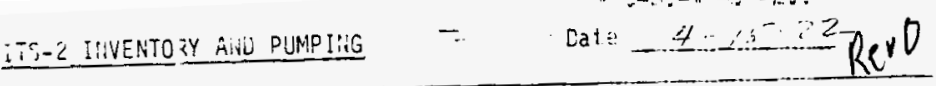
Punp-Qut Tank

Receiving Tarik

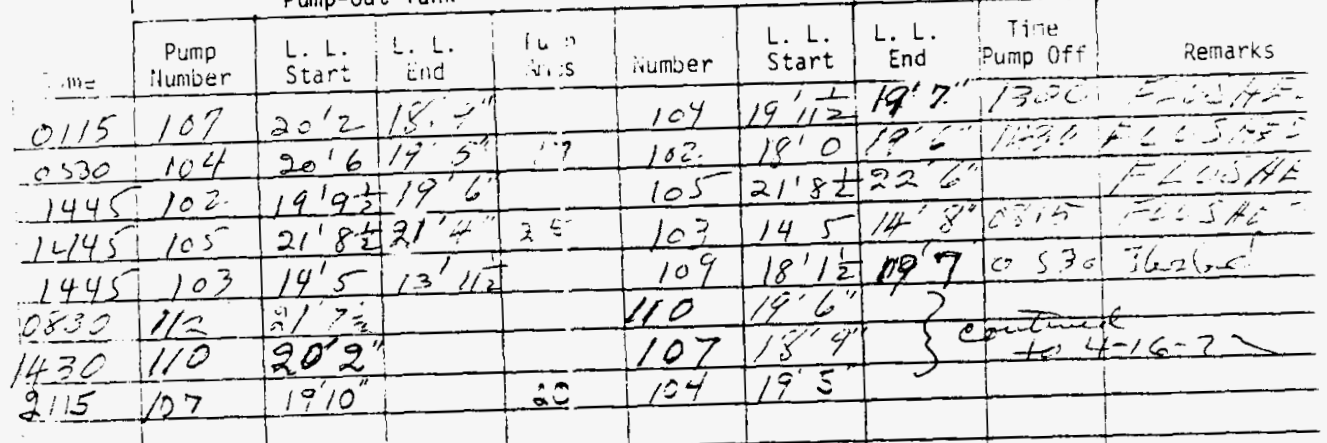

\begin{tabular}{|c|c|c|c|c|c|c|c|}
\hline 列x & 0100 & 0700 & 0900 & 1500 & 1700 & 2300 & \\
\hline $32-8 v$ & $2019+1$ & 214 & 27 & $2 i$ & $91^{\prime} \quad 4 \div$ & $9 i^{\prime} \hat{i}$ & \\
\hline $11 \cup-8 Y$ & $19^{1} 6$ & $19 ; 6$ & 7 & $50^{\prime}$ & $10^{\prime}$ & $10^{\prime} 11$ & \\
\hline $137-3 \gamma$ & $20^{1} 55$ & $14^{\prime} \quad 7.1$ & 19 & 17 & $19^{\prime}$ & 1011 & \\
\hline$: 0 \div-B Y$ & $19^{\prime} \quad 4 \div 2$ & 198 & 19 & $16 \%$ & $19^{\circ}$ & $a^{\prime} 7 \equiv$ & \\
\hline$i 0 \leq-8 y$ & $191 \quad 10$ & 14182 & 14 & 19 & $19^{\prime}$ & $19^{\prime} 6 \div$ & \\
\hline $102-e^{2}$ & $21 \quad 10$ & $5=$ & $\overline{2}$ & 岁至 & $22^{\circ}$ & $\ln 6$ & \\
\hline 3.4 & $14^{\prime} \quad 01$ & 142 & iin & $1 \angle$ & $14^{\prime}$ & 14 & \\
\hline $10-i v$ & $15^{\circ} \quad 112$ & 19,6 & 11 & $18^{\prime}>$ & $18^{\prime}$ & $17^{\prime}$ & \\
\hline $11 i-2$ & $?$ & 3 & & & & & \\
\hline & & & & & & & \\
\hline Total & $155 \quad 2 \frac{1}{2}$ & $1,-4115$ & 154 it & $154^{\prime} 11^{\prime \prime}$ & 155 & 154 & \\
\hline ivew Fees & & & & & & & \\
\hline Aujusted Tota? & & & & & & & \\
\hline
\end{tabular}

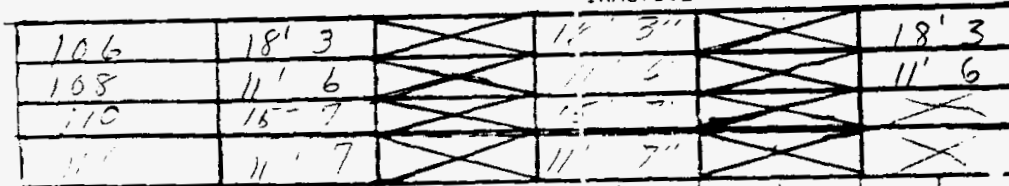

Heat ir:cts

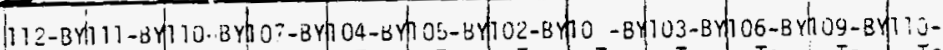

(D)y S:ift;

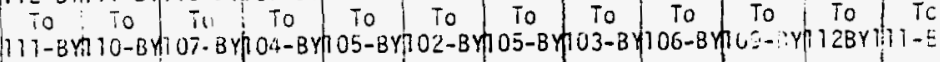

i.

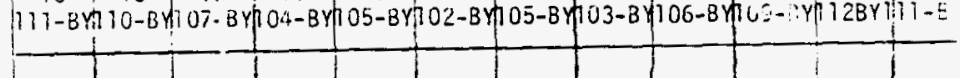

$9--0-7 i$

BEST AVAILABLE COPY 


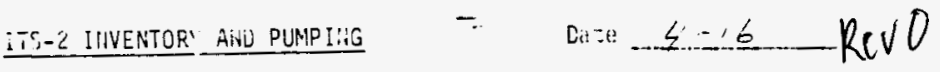

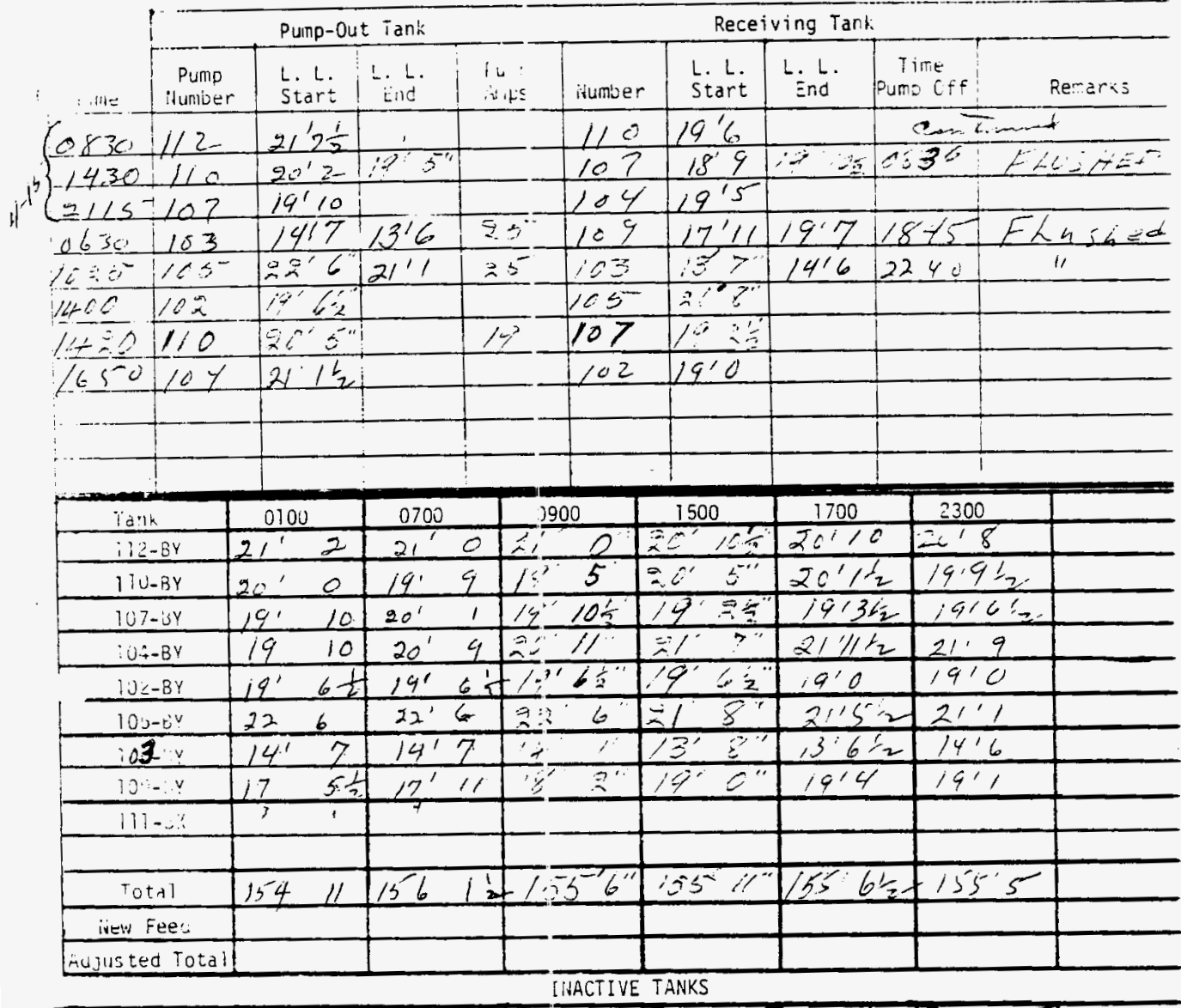

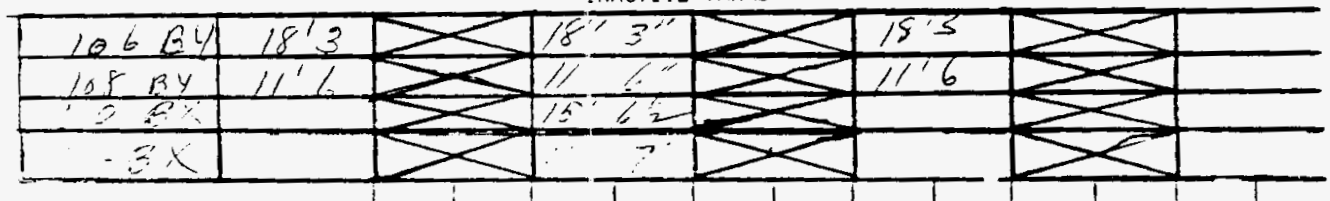

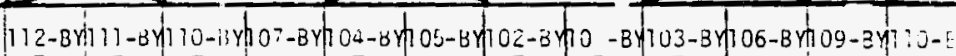

Heiat Ir:cus

To To To To To To To

(Day stift)

L

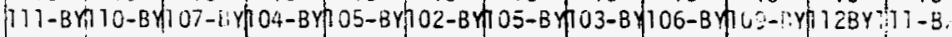

$9-\div 0-7 i$

BEST AVAILABLE COPY 


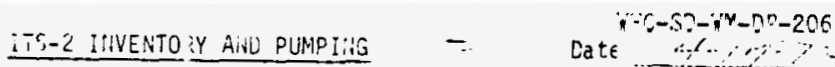

Red

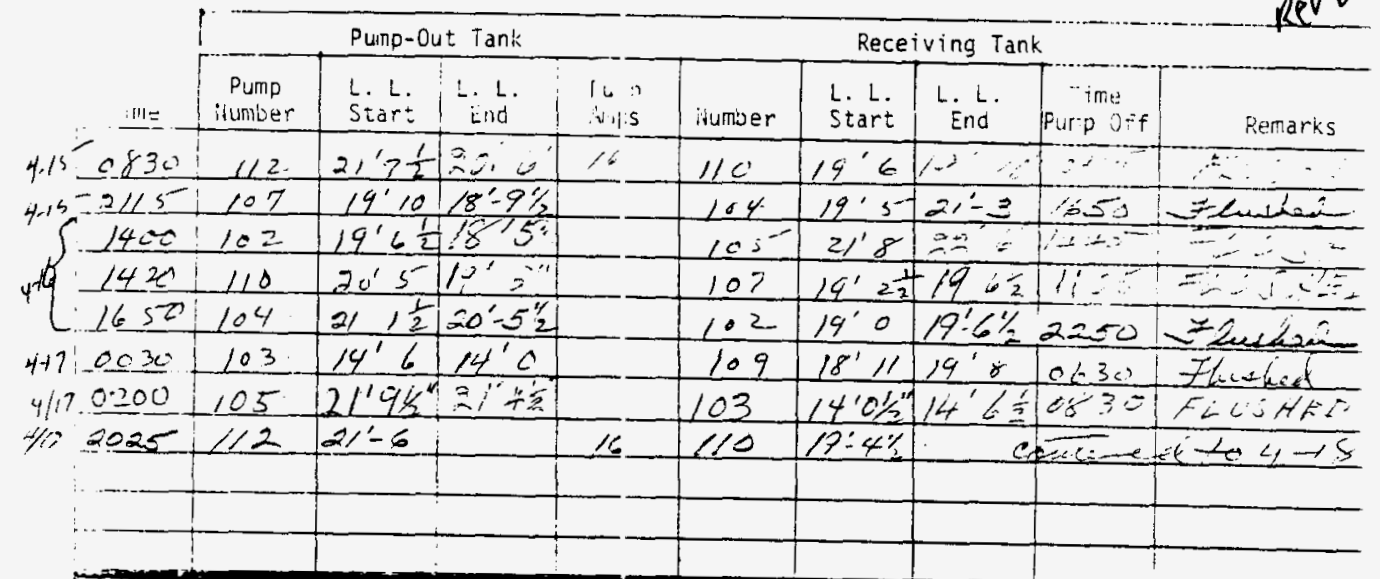

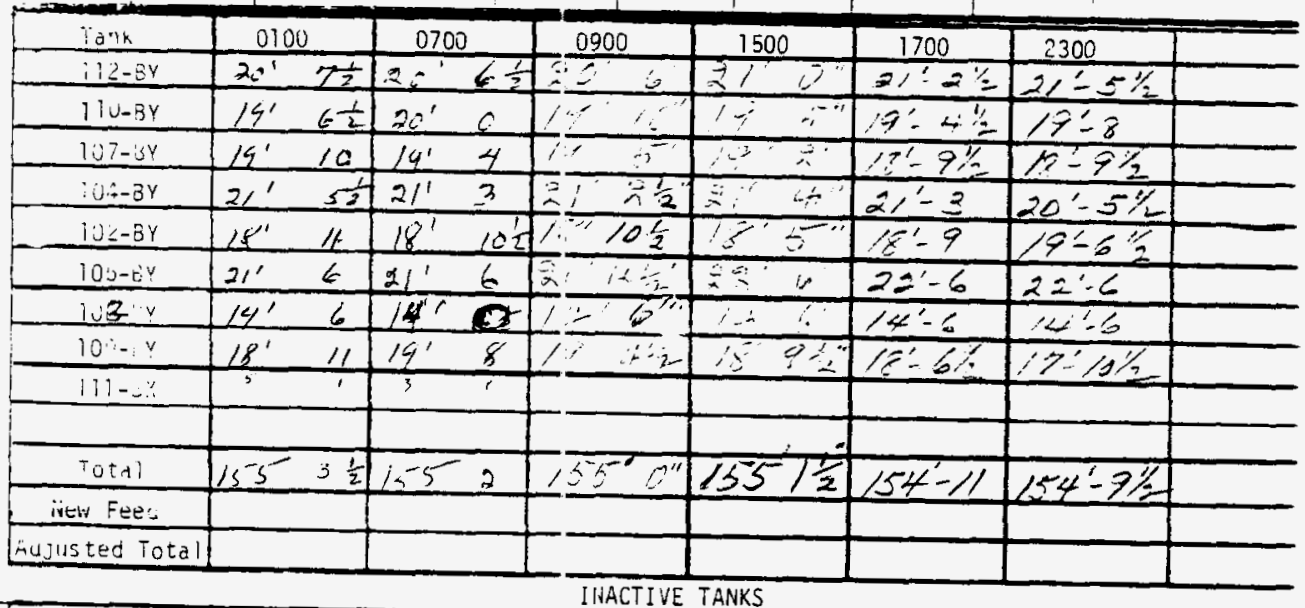

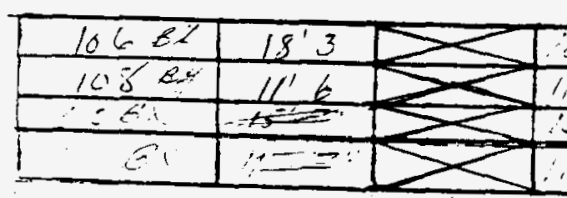

NACTIVE TANKS

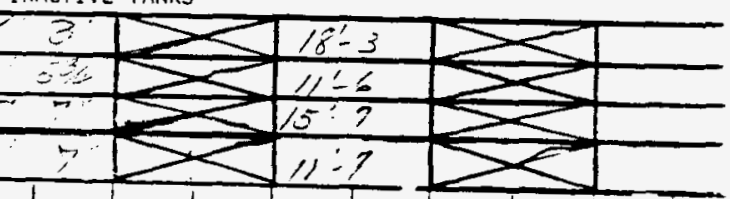

Heat ir.aces

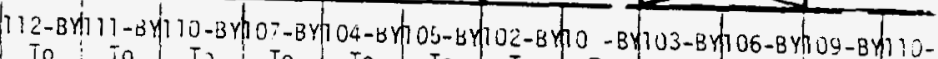

(Day S!ift)

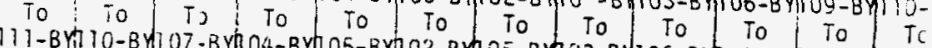

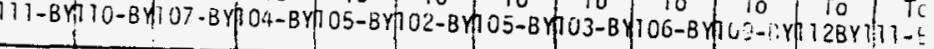

\section{-

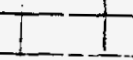 \\ $9-50-7 i$


Date: $\geq \cdots, f \cdots>2$

Punp-out iank

Receiving Tank

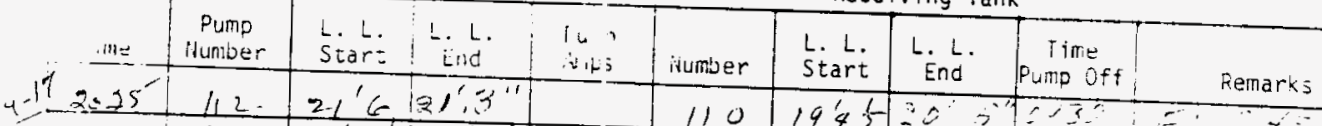
$023010219^{\prime} 618^{\prime} 92$

c $22011052 z^{\prime} 622^{\prime} 3$

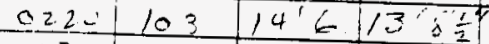

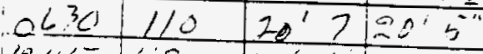

$\left.1=45^{-} \angle 2 \quad 5\right)^{\prime} 62^{\prime}$

$1330 \sqrt{16} 1206$

$182=10720^{\circ}-1$

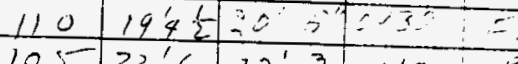
$105222^{\prime} 62^{\prime} 3$ 1630! Inatent

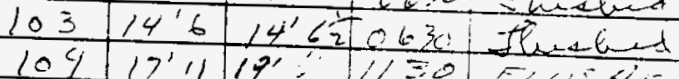

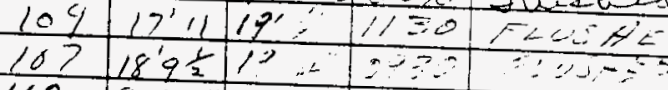
$189 \frac{1}{2} 17+2>?$ 110 250" \begin{tabular}{|l|l|l|l|}
1 & $20-1$ \\
\hline & &
\end{tabular} $10420 \div 5 \int_{0}-4$ की

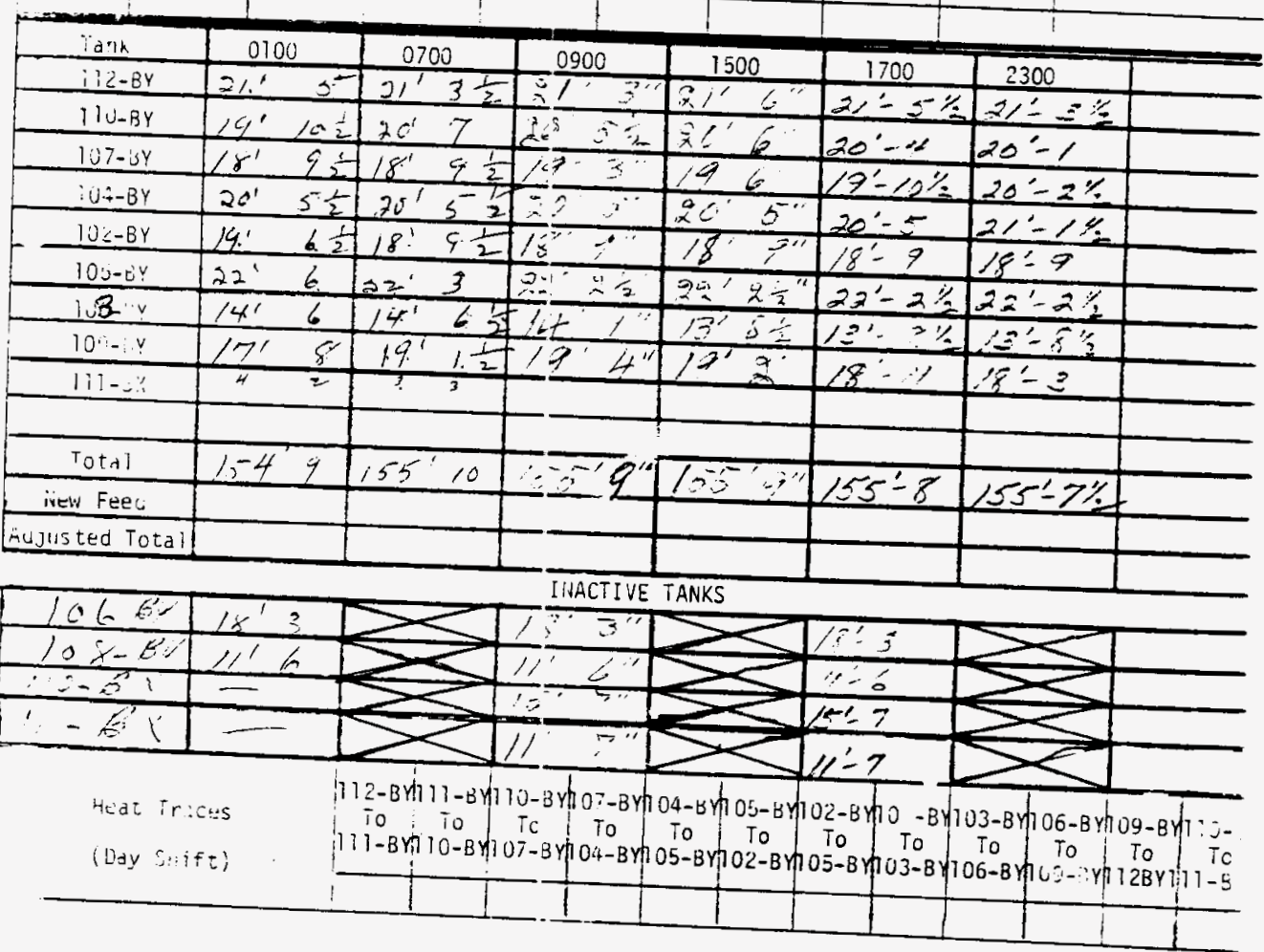

$9--0-7 i$ 


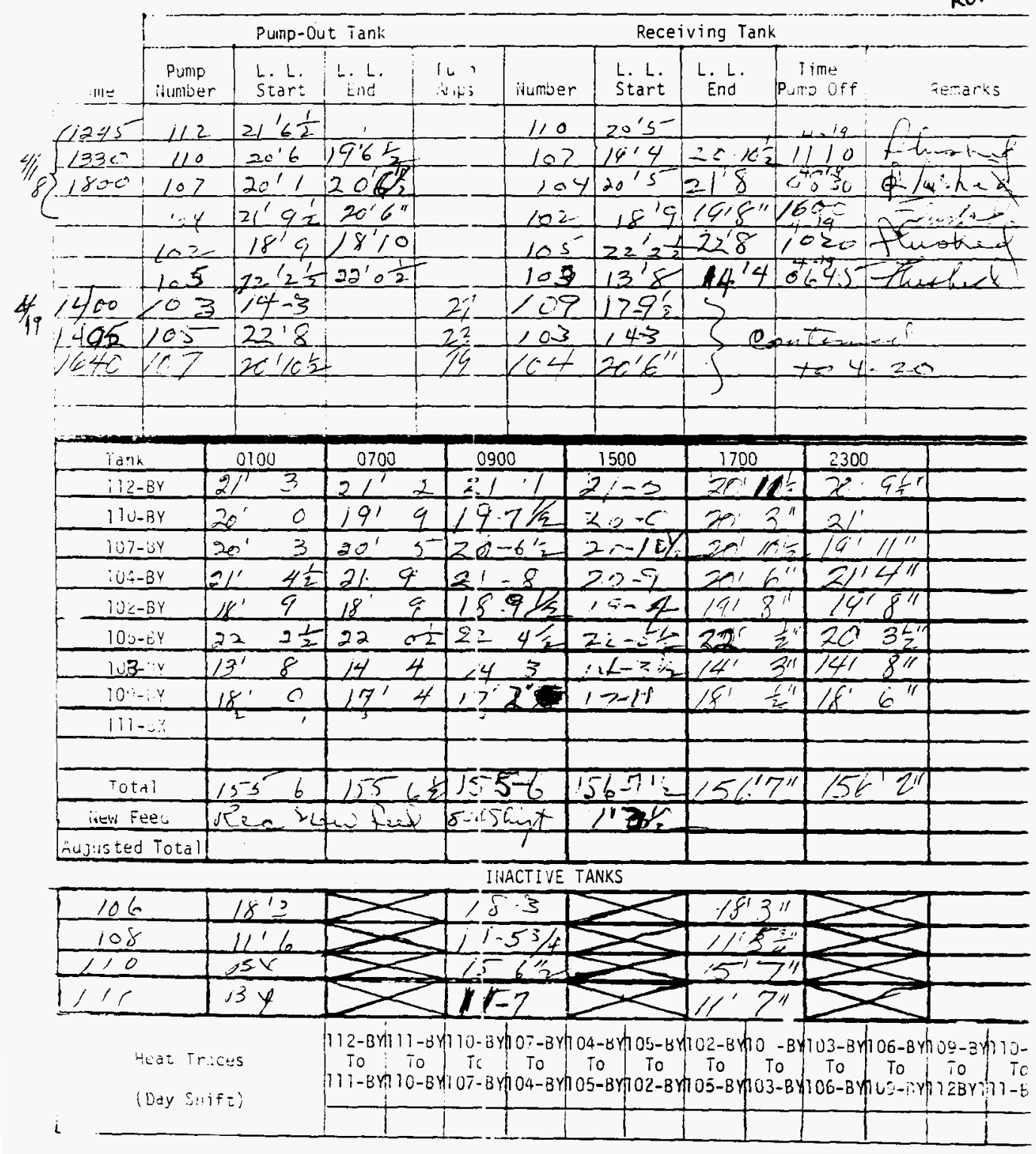

$9-20-71$ 


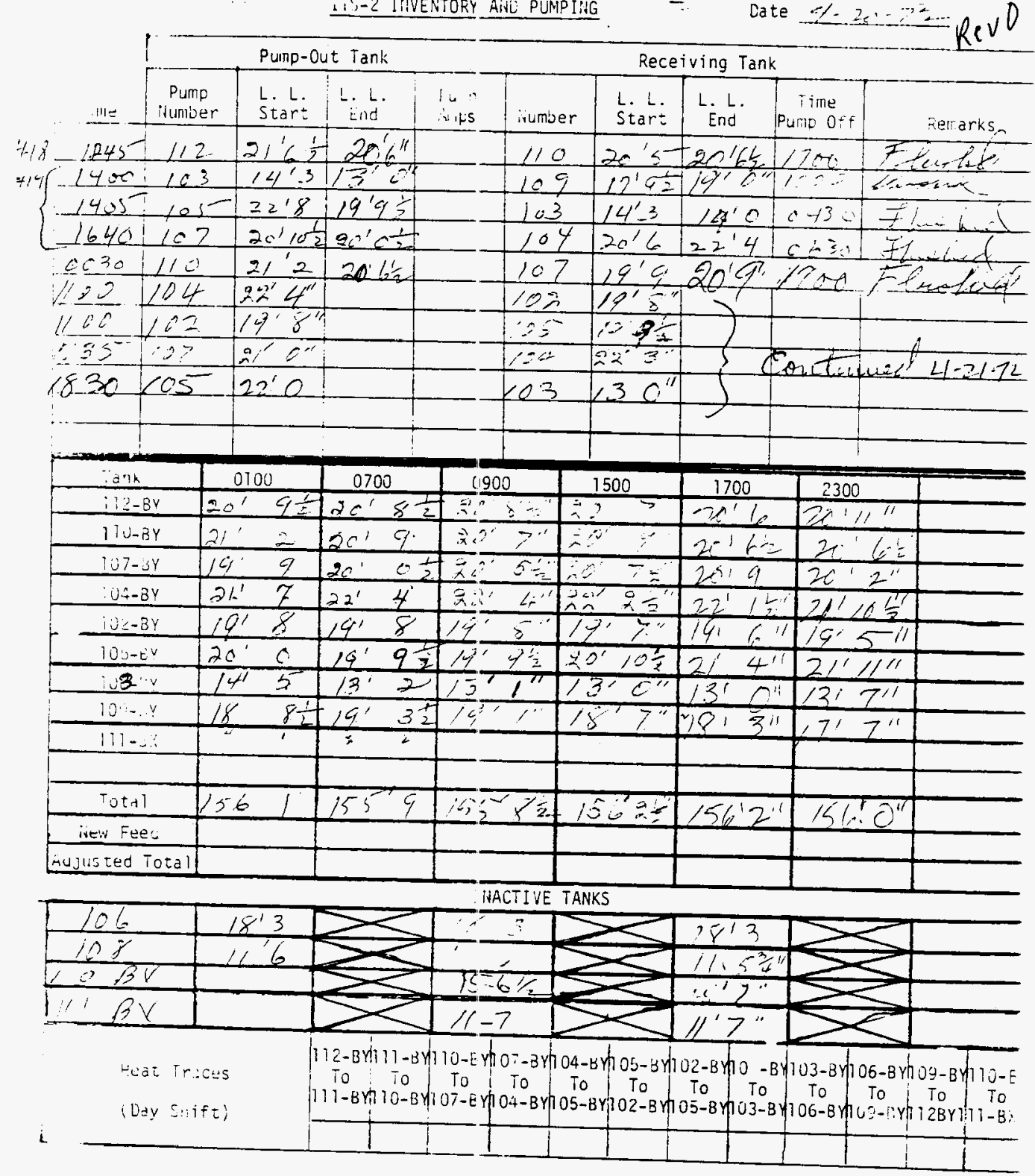

$9--0-71$ 


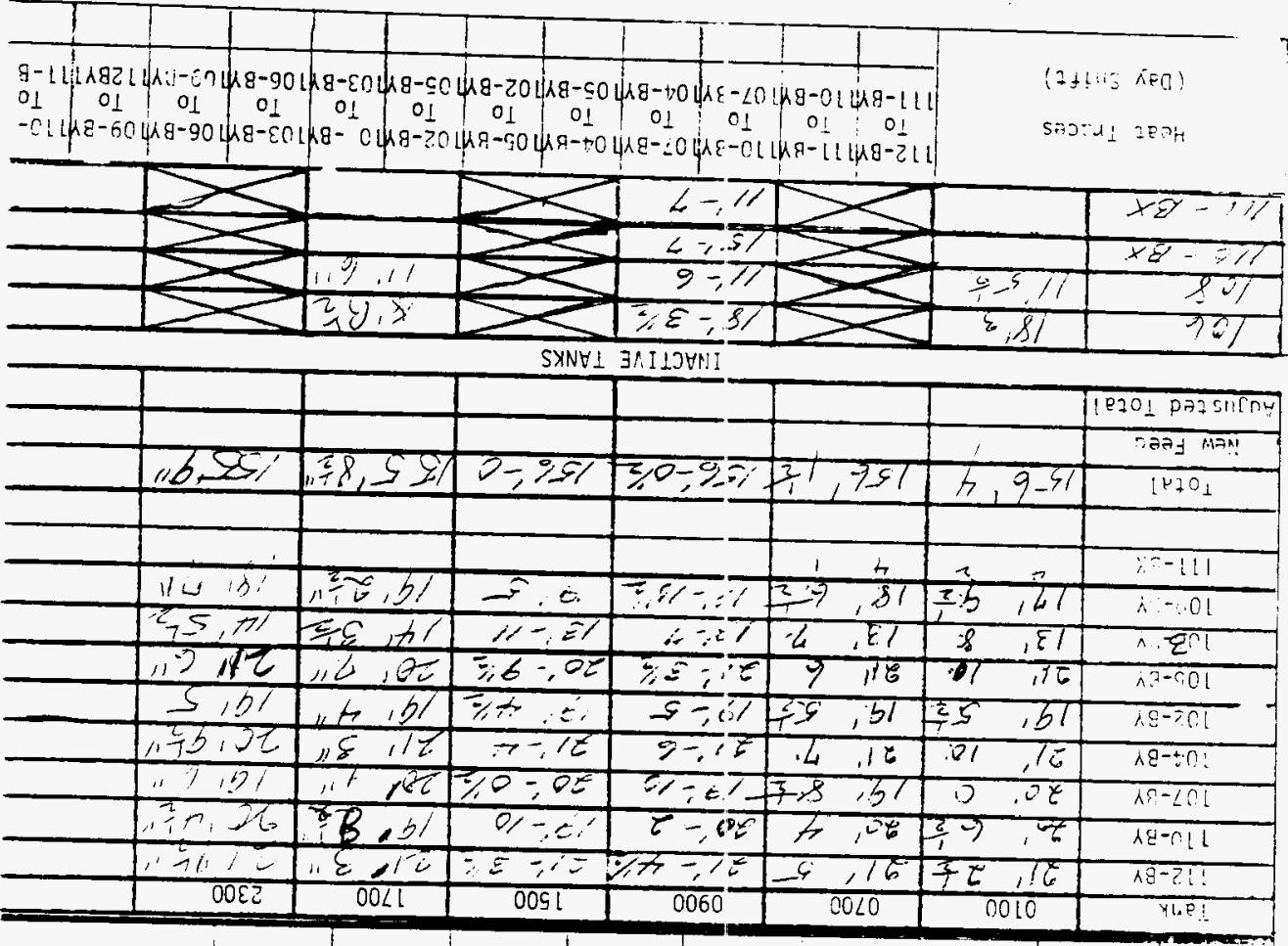

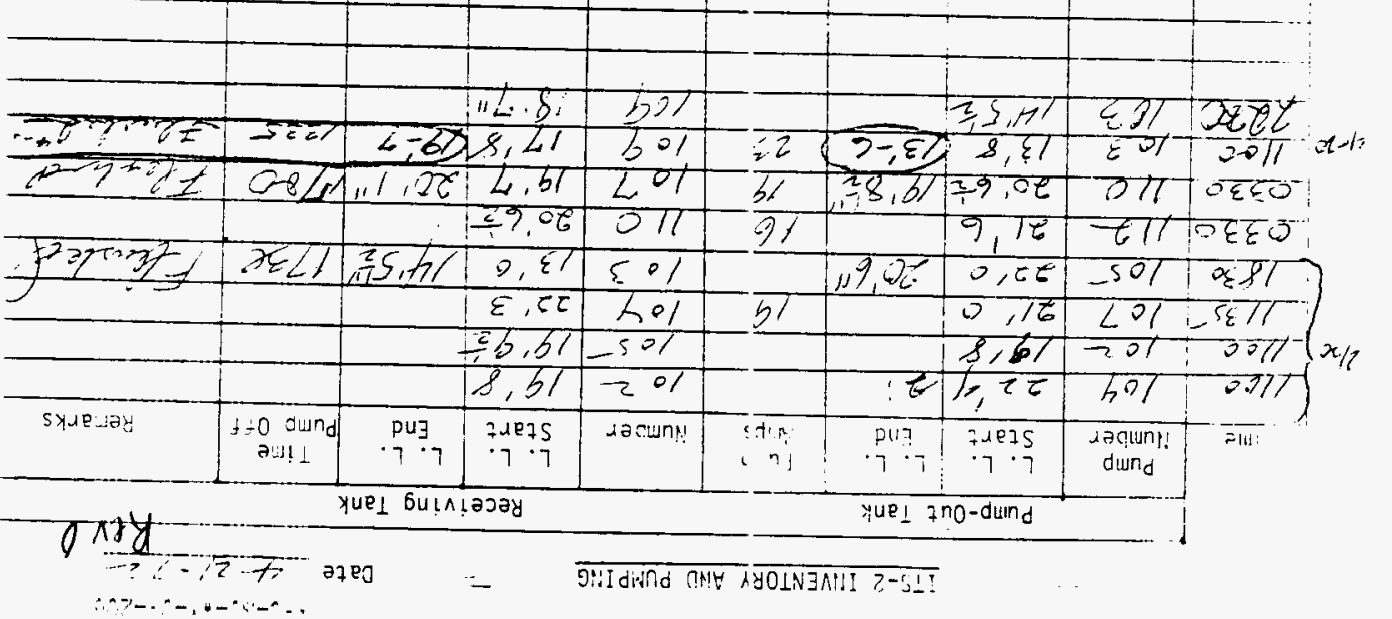




\section{RPN?}

Punp-Out Tank

Pump

$t s$

iof $\frac{100}{1,00} 1+2$

k

1.2-i, 2

$\{0330 \mid 1 / 2$

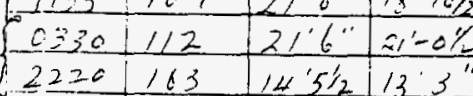

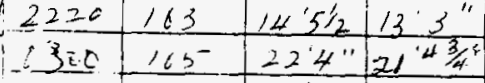

$133511=12116$

$\angle 36,101 / \frac{2}{2}$

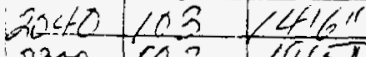

$230 \quad 2021415$

220 8550.51

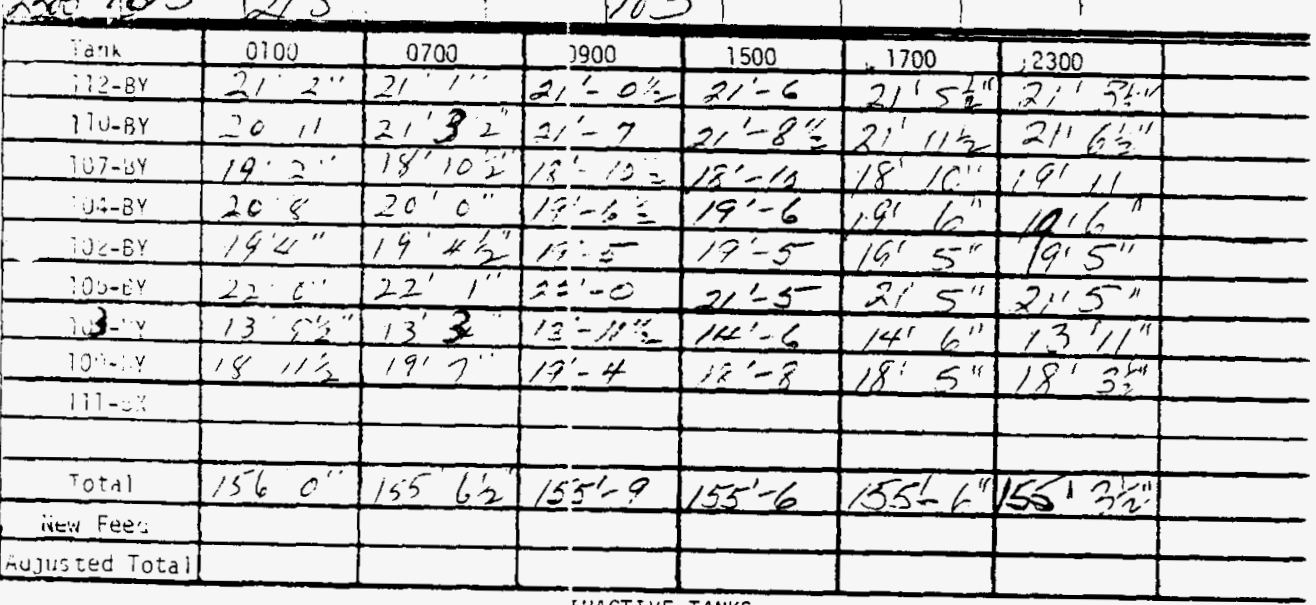

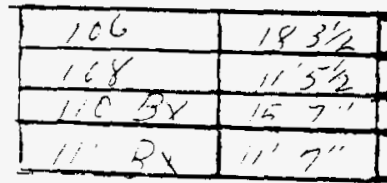

Heat Tr:a:s

(Day Sitifi)

Receiving Tank

Rerriarks

$\therefore+$ inumer Start End Pump off

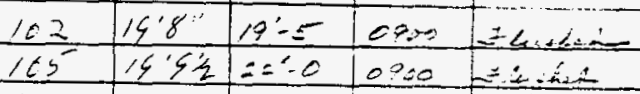

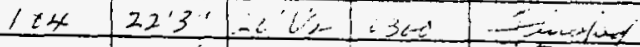
$110 \quad 206 \frac{1}{2}=1-7$ opes mankmen wiv

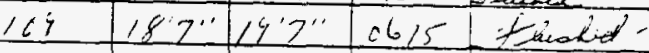
\begin{tabular}{l|l|l|l|l|l|}
163 & $134^{\prime \prime}$ & $14166^{\circ}$ & $1122^{\circ}$ & Fin
\end{tabular}

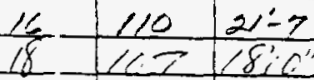
36 $109 \mid \frac{1 g^{\prime} a^{\prime \prime}}{4}$

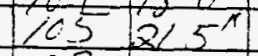
103 INACTIVE TANKS

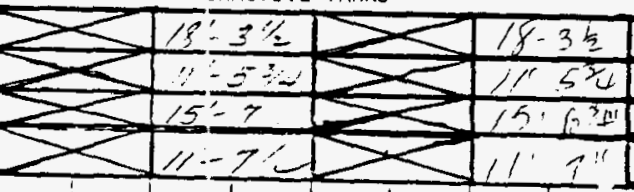

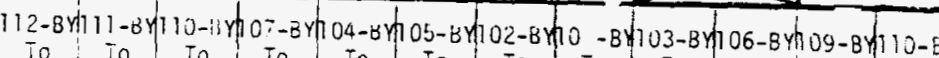
To To To To To To To To To To To To To

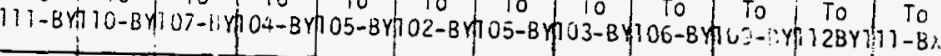

i $1+1$

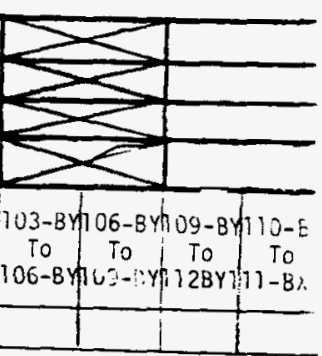

$9-20-71$ 


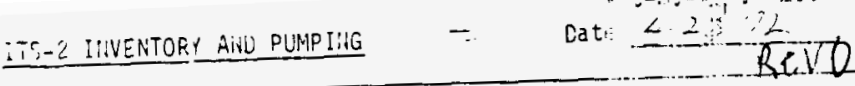

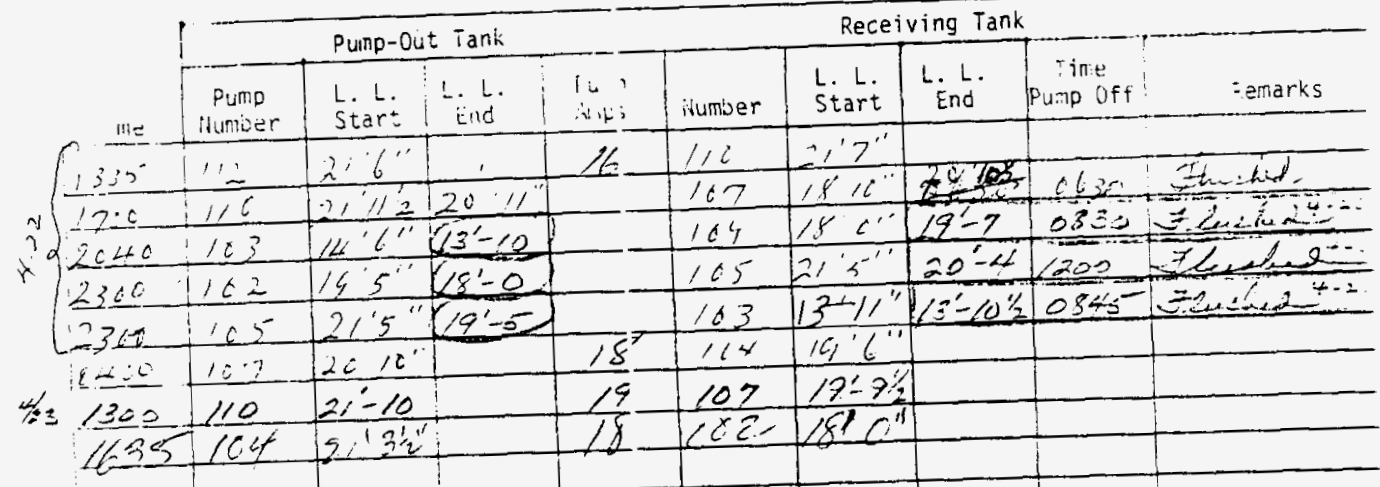

\begin{tabular}{|c|c|c|c|c|c|c|c|}
\hline lapk & 0100 & 0700 & 0900 & 1500 & 1700 & .2300 & \\
\hline 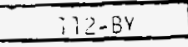 & $21^{\prime \prime}$ & 2142 & $=1-2 \%$ & $21-2$ & $21^{\prime} 1^{\prime}$ & $2111 \frac{1}{2}$ & \\
\hline $11 \cup-B Y$ & $2 ! 51 / 2$ & $26 \quad 16$ & $2:-4$ & $2=-2 \because$ & 2311 & $21 \% 8$ & \\
\hline $107-3 y$ & $20 \cdot 2 \frac{1}{2}$ & $2010 \%$ & 230 & $19: 10^{\prime}$ & $10^{\prime} 11^{\prime \prime}$ & $21: 3^{\prime \prime}$ & \\
\hline $04+8 y$ & 16 & $19 \quad 1 \frac{1}{2}$ & $20: 2$ & $21 \div 112$ & 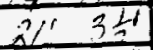 & 211721 & \\
\hline $10 x-8 y$ & 19.4 & 19 & $P^{\prime}-6$ & $16^{\prime}-0$ & 0.1 & $18^{\prime} 6^{\prime \prime}$ & \\
\hline $10-C^{2} Y$ & $10 \frac{1}{2}$ & 158 & 5 & $20^{\prime}-4$ & $2033^{1}$ & $20^{\prime}$ & \\
\hline$v_{3}=v$ & +613 & 1310 & $16-10=$ & $13 \div-10 \%$ & $13^{\prime} 165$ & $13: 102$ & \\
\hline $10-y$ & 18613 & 153 & -6 & $=-10^{\prime \prime}$ & $\left(8^{\circ} 60\right.$ & 1413 & \\
\hline$H=$ & & & & & $6 \frac{12}{2}$ & & \\
\hline & & & $-4 t-8 \%$ & & $155^{5}-1^{11}$ & $156^{\prime} 7^{\prime \prime \prime}$ & \\
\hline Total & $1<5$ & 1549.2 & $154-81=$ & $155-5$ & & 1562 & \\
\hline Aujus ted Tota & & & & & & & \\
\hline & & & & & & & \\
\hline
\end{tabular}

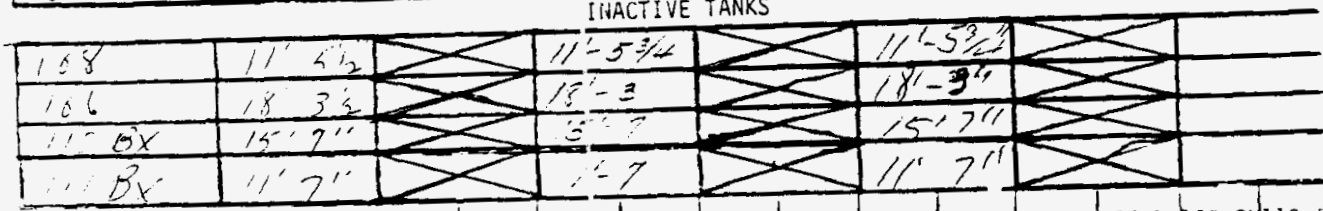
D

Hede ir:a:s

(Dy S:if:)

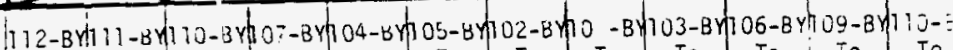
To To 10 To 10 To To

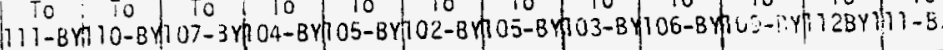
$1+1$ 
ITS-2 IIIVENTORY ANV PUMPIIG $\quad-$ Cate $46.24-72$

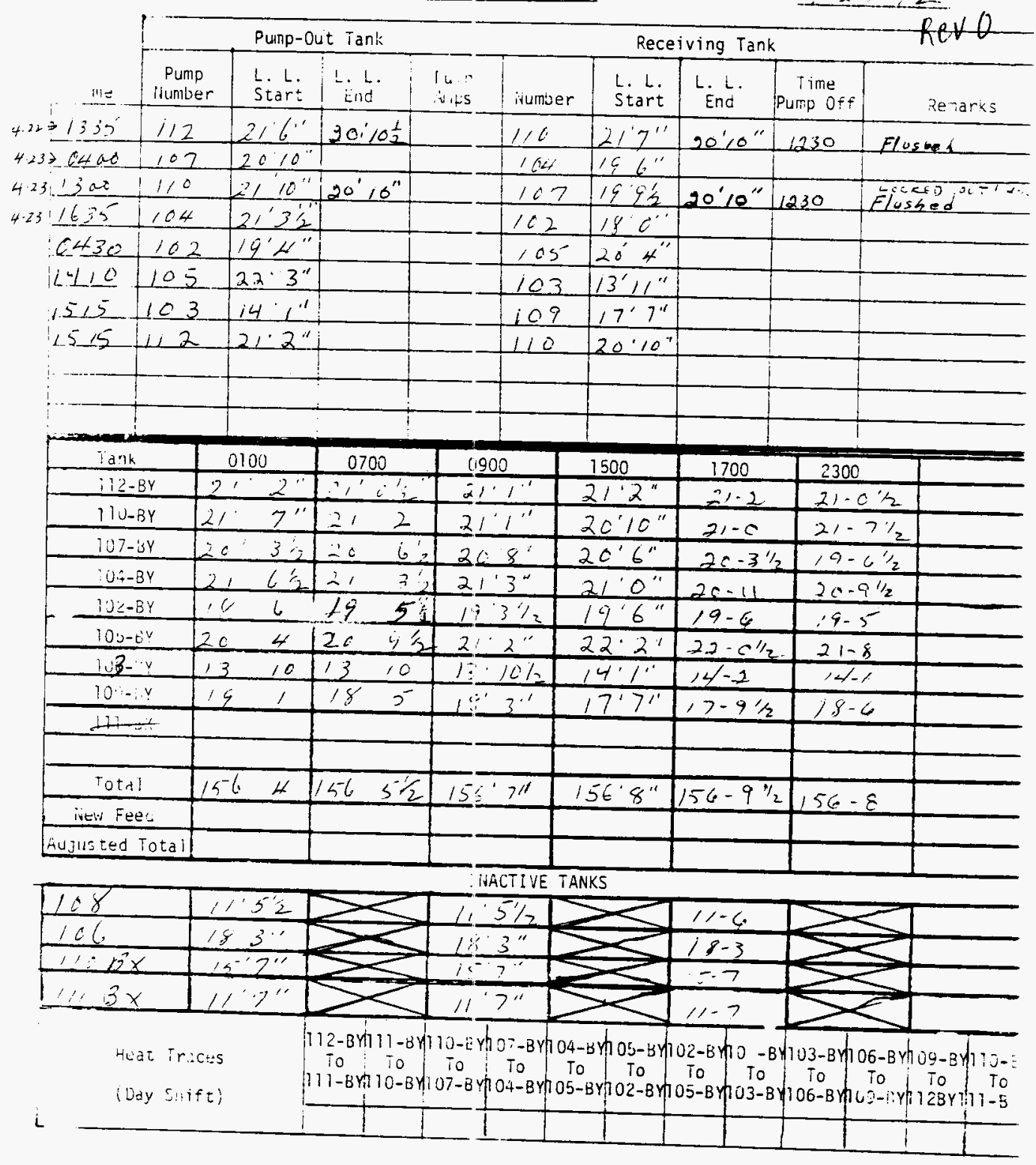

$9-20-73$ 


\begin{tabular}{|c|c|c|c|c|c|c|c|c|c|}
\hline \multirow[b]{2}{*}{ HIE } & \multicolumn{4}{|c|}{ Puinp-Out Tank } & \multicolumn{4}{|c|}{ Receiving Tank } & \\
\hline & $\begin{array}{c}\text { Pump } \\
\text { idumber }\end{array}$ & $\begin{array}{l}\text { L. L. } \\
\text { Start }\end{array}$ & L. L. L. & 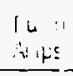 & itumber & $\begin{array}{l}\text { L. L. } \\
\text { Start }\end{array}$ & $\begin{array}{l}\text { L. L. } \\
\text { End }\end{array}$ & $\operatorname{limit}_{\text {Pinp }}$ & Remarks \\
\hline 423 ingi & $\therefore 7$ & $\therefore c \quad 1 c^{\circ}$ & $19: 2{ }^{\prime}$ & & 16 & $8^{\prime} 6^{\prime \prime}$ & $266^{\prime \prime}$ & 1896 & 7 inoher \\
\hline $43 \times 1635^{\circ}$ & 164 & 2132 & $19^{\prime} 8 \%$ & & 162 & $15^{\prime} 6$ & $18 \cdot 5^{\prime \prime}$ & 1400 & Elosked \\
\hline$\therefore 430$ & 102 & $194 "$ & $17-111 / 2$ & & 105 & $20^{\circ} 4^{\circ}$ & $22-2$ & $\therefore 6,5$ & 7 Lustr2! \\
\hline $7 \sqrt{1410}$ & 165 & $22^{\circ} 3^{\prime \prime}$ & $20^{\circ} 10 \%$ & & 103 & $13^{\circ} 11^{\prime \prime}$ & $148^{\prime \prime}$ & 0935 & Ilecatied \\
\hline$x\{15 \div 5$ & 103 & $14^{\prime}, 1$ & $14^{\prime} 0^{\prime \prime}$ & & 107 & $.7^{\prime} 7^{\prime \prime}$ & $19^{\prime} 6^{\prime \prime}$ & $6 \cdot 20 x$ & $I(i-x)$ ivel $=$ \\
\hline $415 \div 5$ & 112 & $21^{\prime} 2^{\circ}$ & $20^{\prime} \|^{\prime}$ & & 110 & $20^{\circ} 10^{\circ}$ & $\because 1, ;$ & 6345 & $1 \cosh \alpha$ \\
\hline 0630 & 107 & $19^{\prime} 2^{\prime \prime}$ & $18^{\prime} 9^{\prime}$ & & 104 & $159^{\prime}$ & $1916 \% 2$ & 0930 & H-dushed \\
\hline 1030 & 112 & $212:$ & $21.6 "$ & & 107 & $18^{\circ} 9^{\prime \prime}$ & 1911 & 1330 & 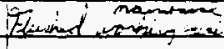 \\
\hline 1500 & 103 & $14.8^{\prime}$ & $121-4$ & & 109 & $18^{\circ} 9^{\circ}$ & $48-11$ & 160 & $7 \leq<\leq H \subset D$ \\
\hline 1500 & 112 & $21 \cdot 8 "$ & & 24 & 107 & $191^{\circ}$ & & & \\
\hline $210-$ & 107 & $10.51 / 2$ & & 30.6 & 104 & 19. $8^{1} 12$ & & & i \\
\hline
\end{tabular}

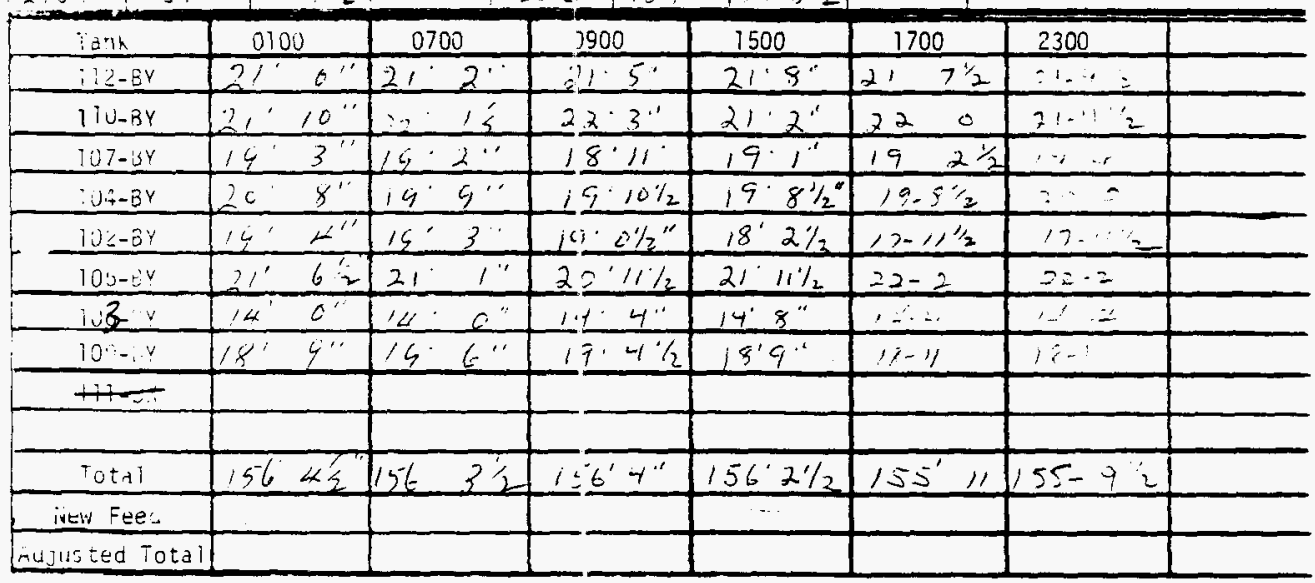

[NACTIVE TANKS

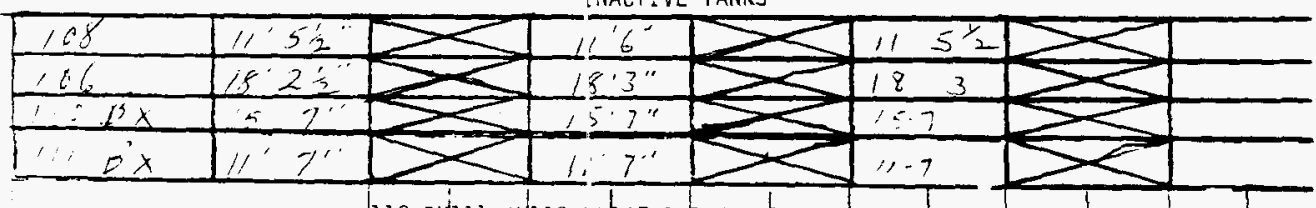

$H_{\mathrm{t}^{2}} a$ T Tr.aces

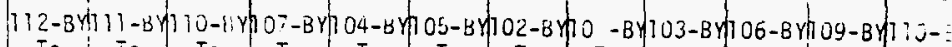

(boy S:ift)

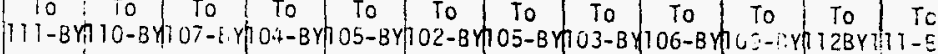

\begin{tabular}{l}
1 \\
\hline
\end{tabular}

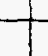

$9-20-7 i$ 


\section{$1-6-52-i^{4}-35-206$}

iT,-2 LIVENTO IY AINU PUMPIIIG

Late $\measuredangle 1-\cdots$

\begin{tabular}{|c|c|c|c|c|c|c|c|c|c|}
\hline \multirow[b]{2}{*}{ N IIE } & \multicolumn{4}{|c|}{ Punp-Out Tank } & \multicolumn{5}{|c|}{ Receiving Tank } \\
\hline & $\begin{array}{c}\text { Pump } \\
\text { ivumiver }\end{array}$ & $\begin{array}{l}\text { L. L. } \\
\text { Start }\end{array}$ & L. L. & $\begin{array}{l}\text { In: } \\
\text { itis }\end{array}$ & ivumber & $\begin{array}{l}\text { L. L. } \\
\text { Start }\end{array}$ & $\begin{array}{l}\text { L. L. } \\
\text { End }\end{array}$ & $\begin{array}{l}\text { Time } \\
\text { Puts off }\end{array}$ & Rerarks \\
\hline$n+150$ & 1112 & $2.8^{\prime \prime}$ & $21: 8^{\prime \prime}$ & & 167 & 4,1 & $19^{\prime} 3^{\prime \prime}$ & & 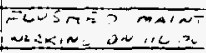 \\
\hline $216=$ & 189 & 19821 & $18^{\prime} 7^{\prime}$ & & 162 & 1982 & $21 \cdot 2^{\prime \prime}$ & 1400 & Elingiat \\
\hline .520 & 1104 & $20^{\circ} 10^{\circ}$ & & & 102 & 1711 年 & & & \\
\hline 1320 & 112 & $21.8 \%$ & & & 167 & $18^{\prime} 7 \%$ & & & \\
\hline 2043 & 103 & $14^{1} 4$ & & & 109 & $18^{1} 0^{\prime \prime}$ & & & \\
\hline 2115 & 105 & $22^{\prime} 2$ & & & 103 & $14^{\prime} z$ & & & \\
\hline 250 & 102 & $1911 / 2$ & & & 105 & $22 \frac{1}{2}$ & & & \\
\hline 2243 & 107 & $19^{\circ} 7^{\circ}$ & & & 104 & $19^{\prime} \%$ & & & \\
\hline & & & & & & & & & \\
\hline & & & & & & & & & \\
\hline$\vdots$ & & & & & & & & & \\
\hline
\end{tabular}

\begin{tabular}{|c|c|c|c|c|c|c|c|}
\hline$\because 21 k$ & 0100 & 0700 & 0900 & 1500 & 1700 & 2300 & \\
\hline i $12-8 Y$ & $2 \cdot 4 \frac{1}{2}$ & $\because 1 \quad 80$ & 218 & $21 " 6 \%$ & $21161 / 5$ & $21 \%$ & \\
\hline $1: U-5 Y$ & 22,12 & 12 & $271 / 2$ & $22^{\prime} \mathrm{Hi}^{\prime}$ & 2,10 & $21^{\prime} 10^{\circ}$ & \\
\hline $107-3 y$ & $15^{\circ} 5$ & $19^{\circ} 3^{\circ}$ & 19.3. & $18^{\prime} 8 \% "$ & $18^{\prime} 10$ & $19^{\prime} 7^{\prime \prime}$ & \\
\hline $0 \div-3 Y$ & $20^{\circ} 26^{\prime \prime}$ & 112 & $211^{\prime \prime}$ & $21^{\prime} 0 \% "$ & $20^{\prime} 10^{\prime}$ & $19^{\prime} 1^{\prime}$ & \\
\hline $10 \leq-3 v$ & $19^{\prime}+12$ & $18 \quad \angle$ & $18.2 \%{ }^{4}$ & $18^{\prime} 11 \%$ & $1921 / 2$ & $19^{\circ} 1 c^{1 / 2}$ & \\
\hline $100-5$ & 2211 & 2212 & $22^{\prime \prime}$ & $2.21 \%$ & $22 \cdot 2$ & $2111 \% "$ & \\
\hline i3- & $1+$ & 14 & $14 \cdot 4^{\prime \prime}$ & $14: 4$ & 144 & $14^{1} 2^{1 / 2}$ & \\
\hline $10 \div-v$ & $17 \quad 10 \%$ & $8 \quad 11$ & $18^{\prime} 7^{\prime \prime}$ & $15^{\circ} 8^{\circ}$ & $1 \varepsilon^{\prime} i^{\prime}$ & $18^{\prime 3}$ & \\
\hline $111-\ldots$ & & & & & & & \\
\hline Total & $155^{\circ} 10^{\circ}$ & $15 \% 5 / 2$ & $157^{\circ} 7^{\circ}$ & $15 \% 5 \%$ & $157^{\prime} 5^{\prime \prime}$ & $157^{\circ} 14_{2}^{\prime \prime}$ & \\
\hline iiven Fee' & & 192 & & & & & \\
\hline mujusted iotal & & & & & & & \\
\hline
\end{tabular}

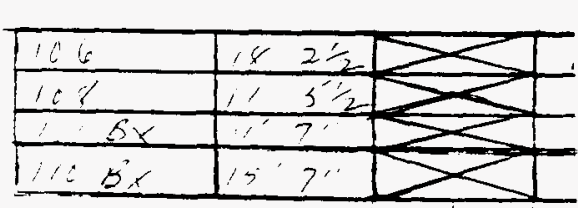

IINACTIVE TANKS

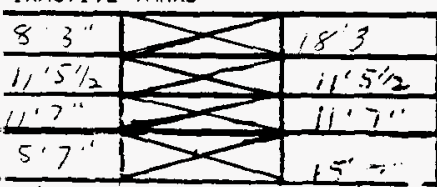

Heat ir.aces

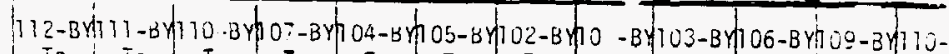

(ity S:ift)

i

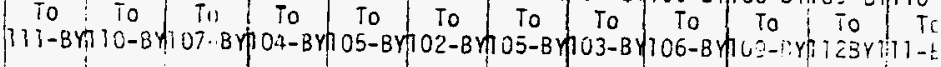

\begin{tabular}{l|l|l|l|l}
\hline & \\
\hline
\end{tabular} 


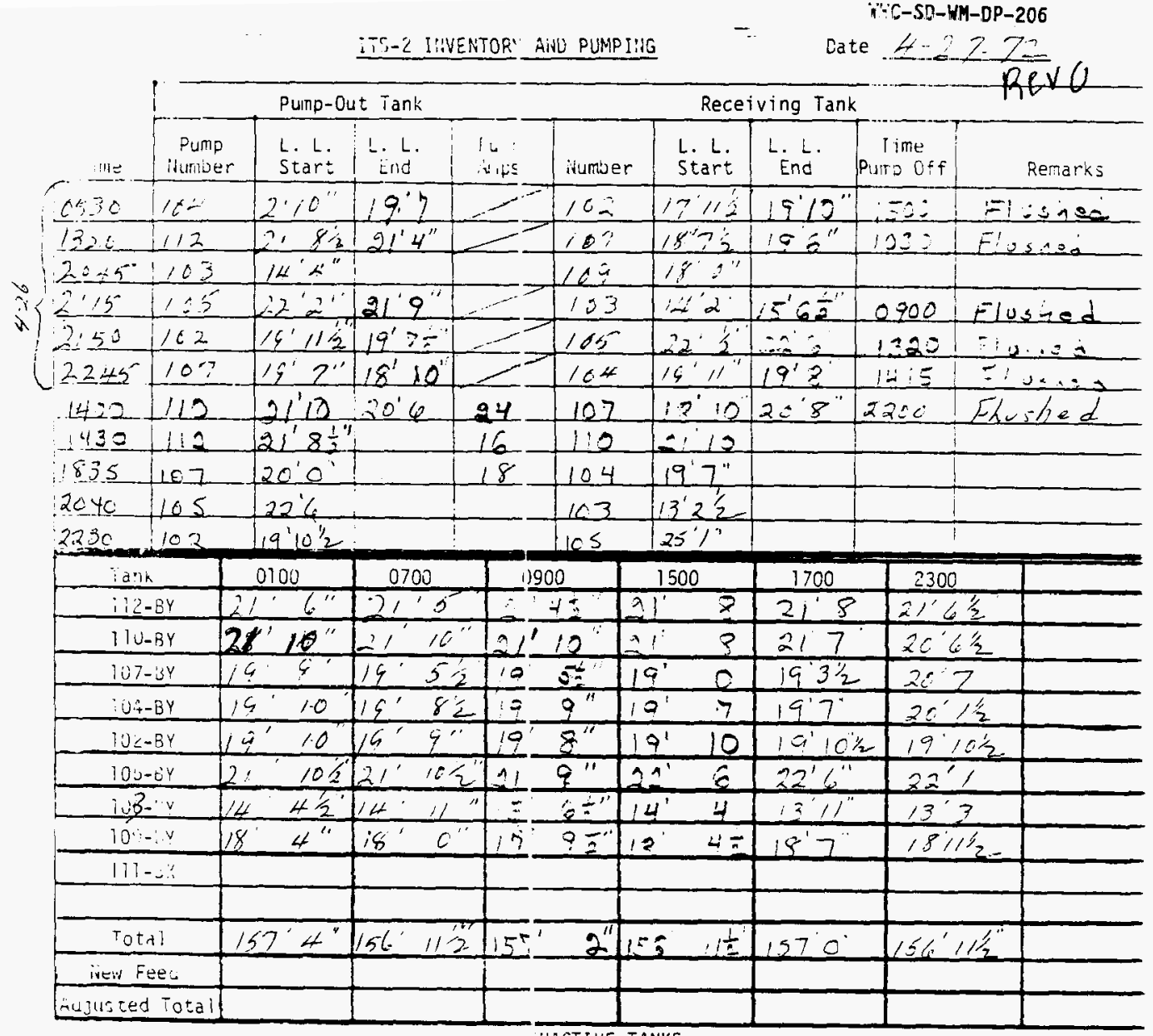

\begin{tabular}{|l|l|}
\hline 106 & $18: 3$ \\
\hline 163 & $11^{\prime} 52$ \\
\hline $116 \times$ & 113 \\
\hline $100 x$ & 15 \\
\hline
\end{tabular}

Heat Tr.:ces

(Day S:ift)

\section{WACTIVE TANKS}

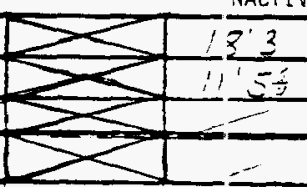

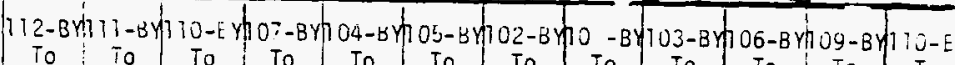

To To To To To To To to To To To To

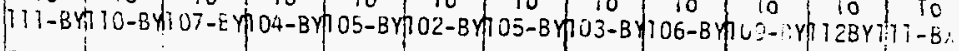

i

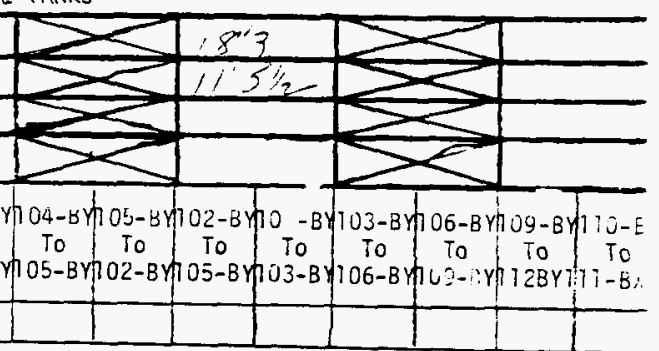

$9-20-71$ 
$\because-5-62-n^{2}-32-206$

ITS-2 IIIVENTOR' AIU PUMPIIIG

Cate

Revo

\begin{tabular}{|c|c|c|c|c|c|c|c|c|c|}
\hline & \multicolumn{4}{|c|}{ Puinp-Out Tank } & \multicolumn{5}{|c|}{ Receiving Tank } \\
\hline 에는 & $\begin{array}{l}\text { Pump } \\
\text { iluniber } \\
\end{array}$ & $\begin{array}{l}\text { L. L. } \\
\text { Start }\end{array}$ & $\begin{array}{c}\text { I. L. } \\
\text { End }\end{array}$ & $\begin{array}{l}i_{u}:= \\
\text { vifs }\end{array}$ & irumber & $\begin{array}{l}\text { L. L. } \\
\text { Start }\end{array}$ & $\begin{array}{l}\text { L. L. } \\
\text { End }\end{array}$ & I ime & Remiarks \\
\hline $4-2 \dot{0} \sim 6165$ & 16 & 124 & $13: 7 \cdots$ & & $\therefore<$ & $180^{\prime \prime}$ & $15^{\prime} 8^{\prime \prime}$ & {$[2 z$} & 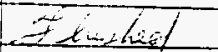 \\
\hline 1630 & 112 & $21^{\prime} \bar{y}^{\prime \prime}$ & & & 116 & $\therefore 10^{\circ}$ & & & \\
\hline$x^{3} 1835$ & 102 & 206 & & & 164 & $14^{\prime} 7^{\prime \prime}$ & & & \\
\hline $20 \div 0$ & 105 & $22^{\prime} 6^{\prime \prime}$ & 213 & & 103 & 13212 & $14<$ & $\therefore i 2 c$ & $k^{\prime} / w s h e d$ \\
\hline 2236 & 102 & 191012 & $178^{\prime \prime}$ & & 105 & $2 z^{\prime} / 1$ & $22^{\circ} 6^{\prime \prime}$ & 0110 & fluminid \\
\hline $0 Z 30$ & 104 & $212 \% 2$ & & & 102 & $1851 / 2$ & & & \\
\hline 1045 & 110 & $\frac{21,11}{14}$ & $20^{\prime} 6^{\prime \prime}$ & & 107 & $18 \quad 11$ & $20^{\prime} / 1 / 2^{\prime \prime}$ & 0430 & Fesind \\
\hline 1500 & 10.3 & 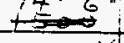 & $13^{491 / 2}$ & & 109 & 1810 & $19.7^{\prime \prime}$ & 0310 & Rousnat \\
\hline 1530 & 105 & 22.2 & $21^{\prime} 92$ & & 103 & 145 & $14^{\prime} 7$ & 2115 & Fhushe \\
\hline
\end{tabular}

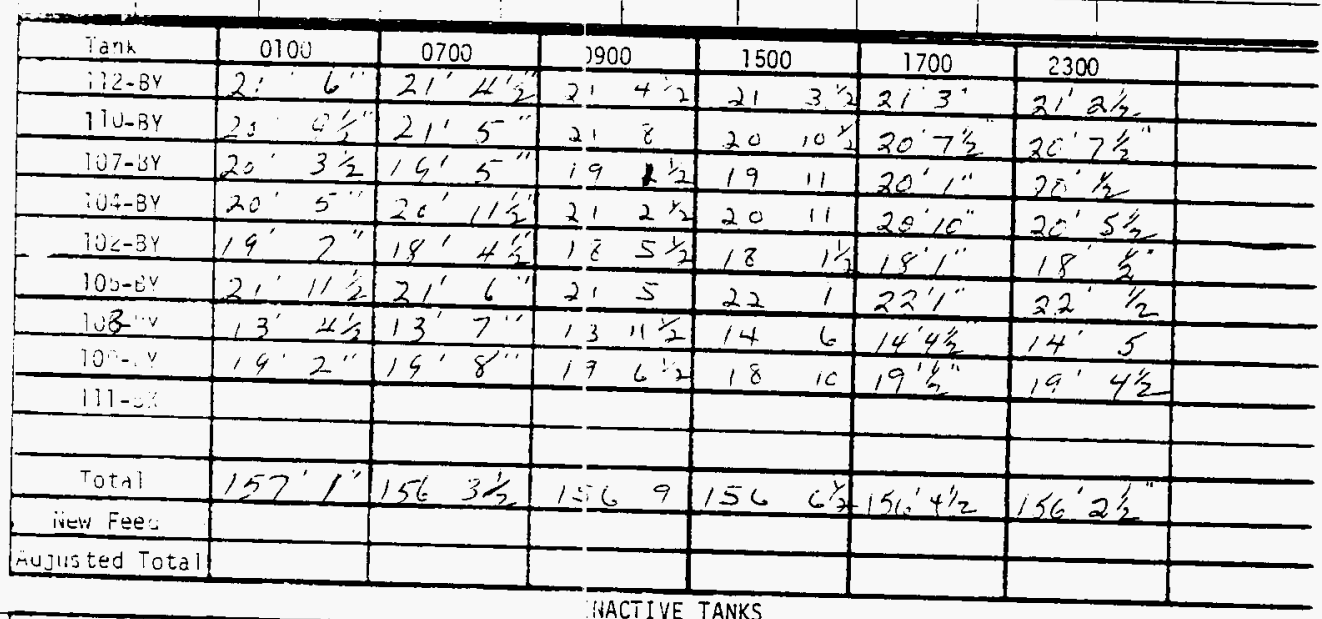

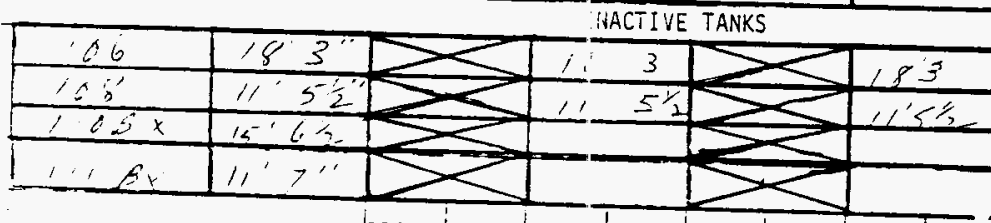

Heat ir.aces

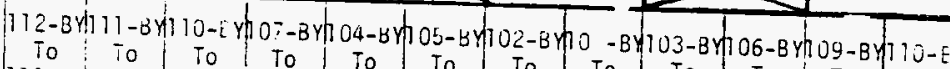

(2)y S:ift)

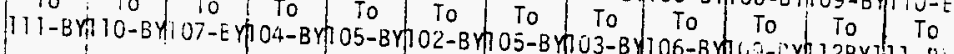

i

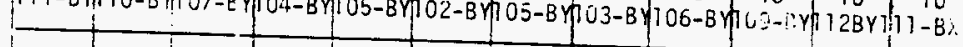

$9-20-71$ 


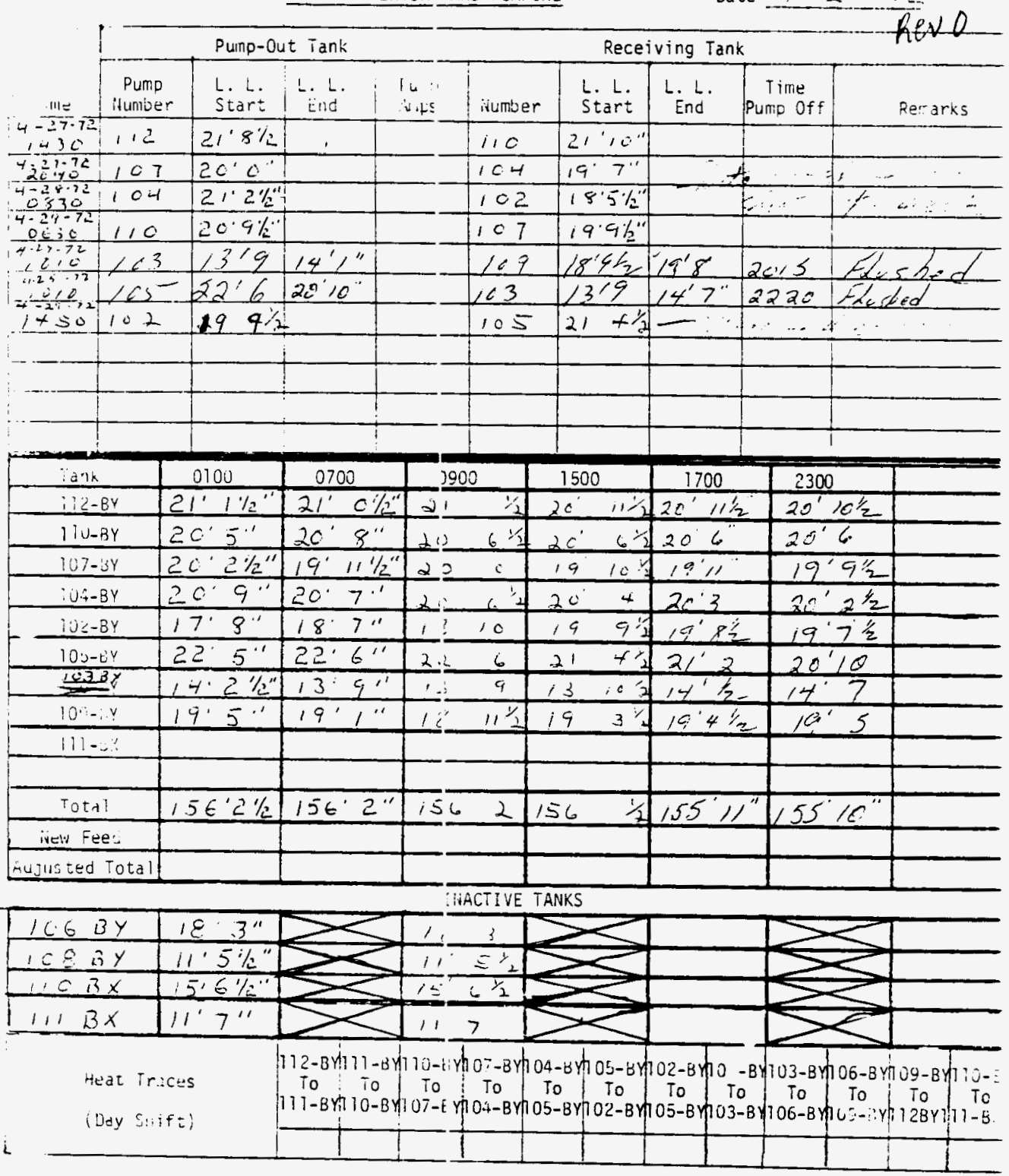

$9-20-71$ 


\section{$1=5-3 .-74-2 P-206$}

iTs-2 IIIVENTORY AINU PUMPIIIG

Cate $1.30-72$

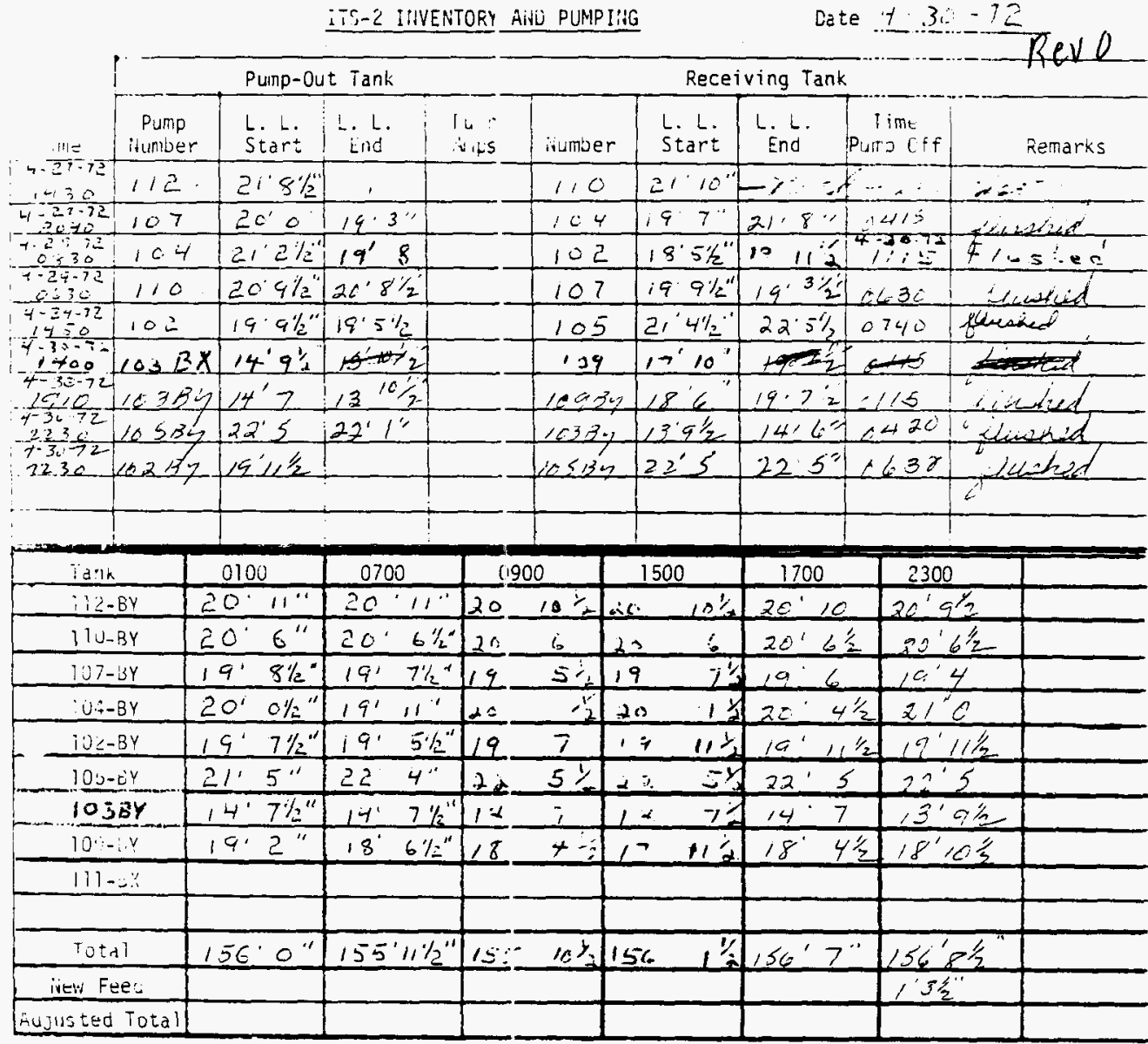

\section{WACTIVE TANKS}

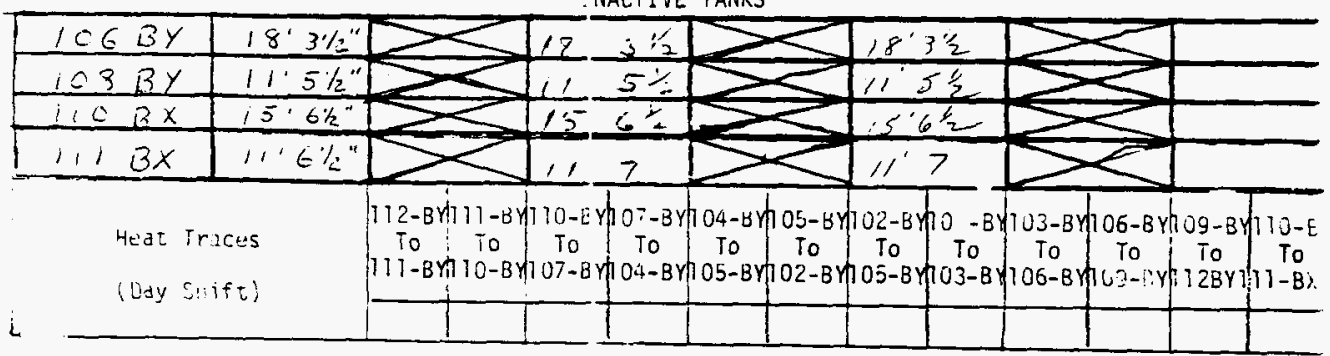

$9-20-7 i$ 
IT,-2 IINENTOFY AiNU PUMPIHO $\quad-$ Date $5-\ldots, 2$

Puinp-Out Tank

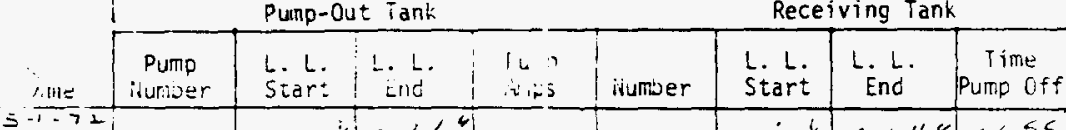

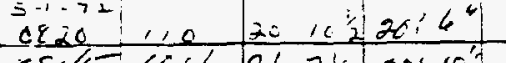

$6.851 / 6412176.120101$

$126: i c \Rightarrow$

\begin{tabular}{l|l|l|l|}
22 & 255 & 225 & 2612 \\
$5-1-72$ & 26
\end{tabular}

\begin{tabular}{l|l|l|}
$5-152$ & 45 & $203 \frac{1}{2}$
\end{tabular}

$-\frac{736}{372} 162-1911 \frac{1}{2}$

$430^{2}+12+218 / 2 \cdot 26 c^{\prime \prime}$

$5.272165 \quad 22^{\prime}-40^{2}+1124$

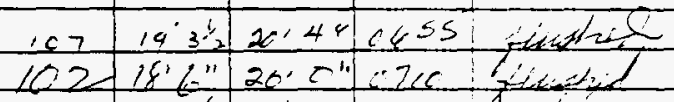

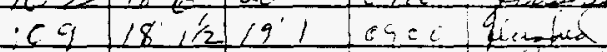

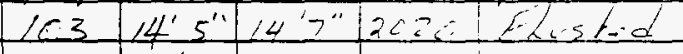

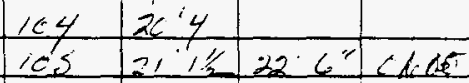
$110 \quad 21^{\prime} 10^{\circ} 20^{\circ} 6^{\prime}=0<10$

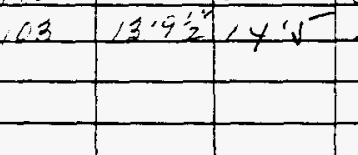

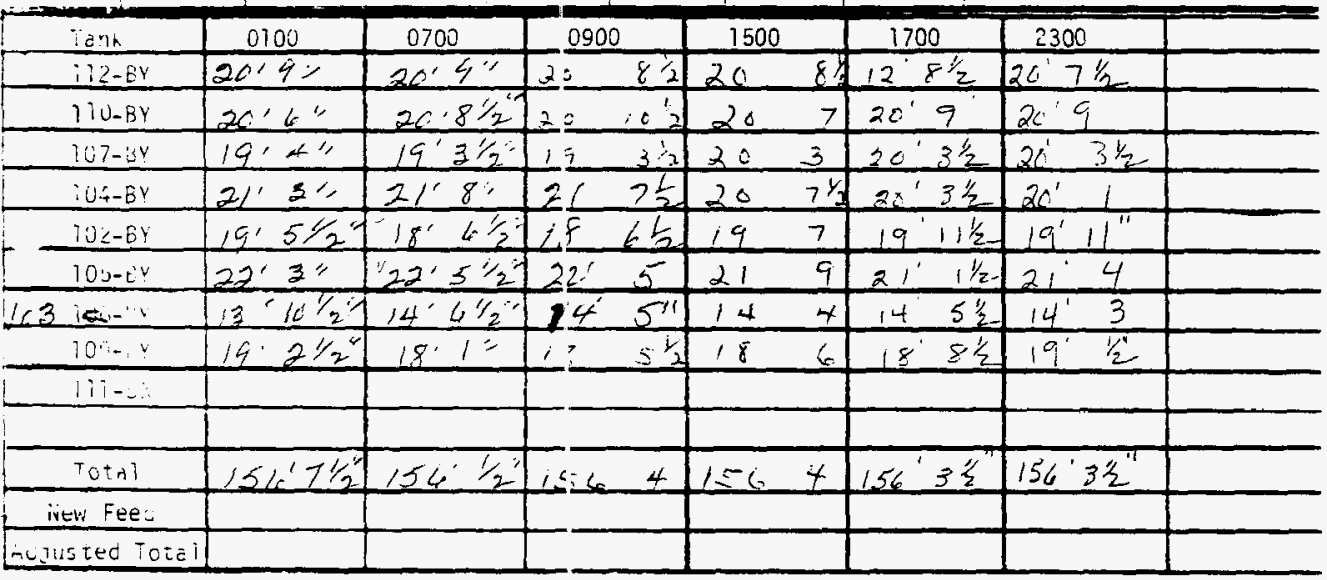

\section{INACT I VE TANKS}

\begin{tabular}{|c|c|}
\hline$\angle C 1-B y$ & $28^{\prime 3}$ \\
\hline $105-\beta C$ & 111 \\
\hline $110.3 x-$ & $15<1 / 2$ \\
\hline $111-\beta x$ & $11^{\prime}$ \\
\hline
\end{tabular}

Hedi Tracus

(bay $\{$ ift)

\section{INACTIVE TANKS}

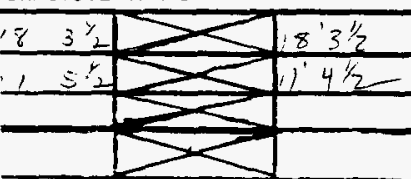

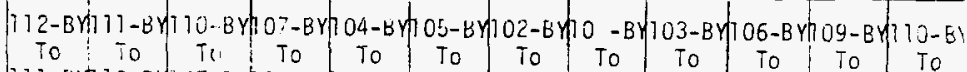

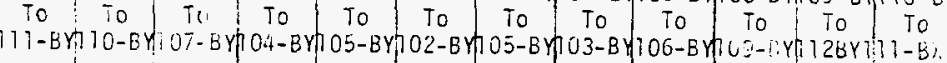

i $1+1$

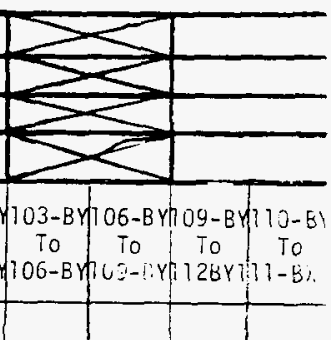

$\Xi-\div 0-7 i$

BEST AVALABLE COPY 


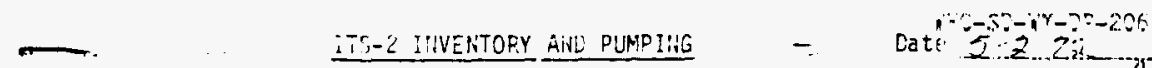

reve

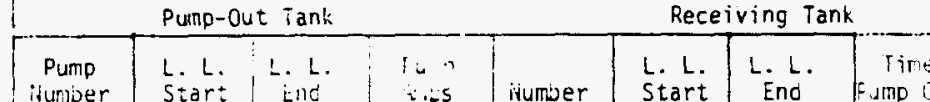

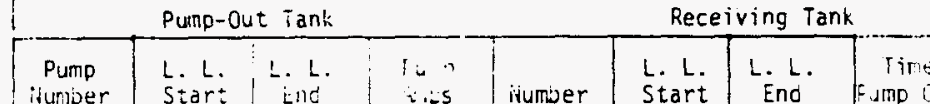

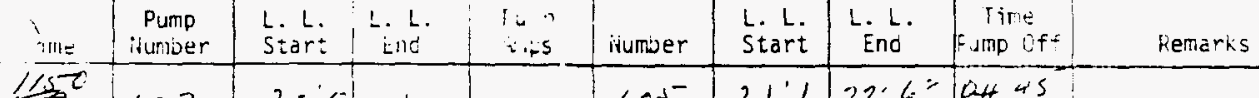

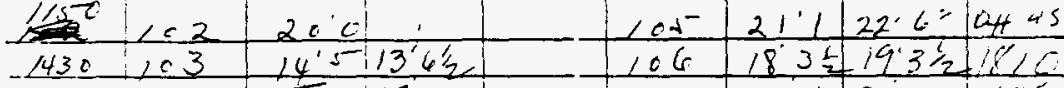

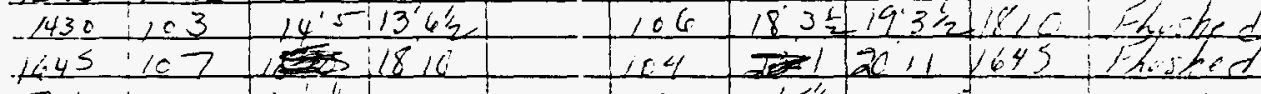

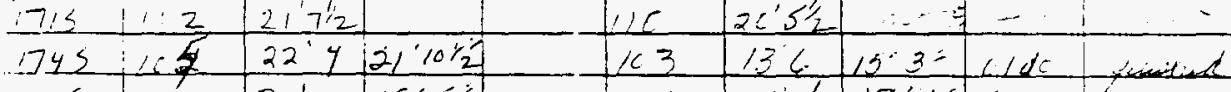

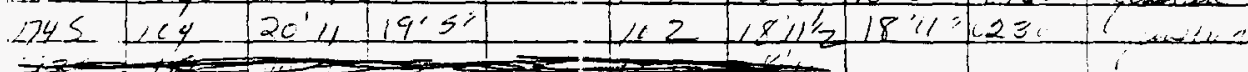

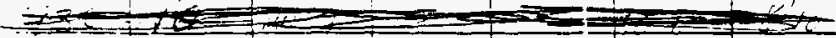

\begin{tabular}{l|l}
$\ldots$ & \\
$\ldots \ldots$ & \\
\hline &
\end{tabular}

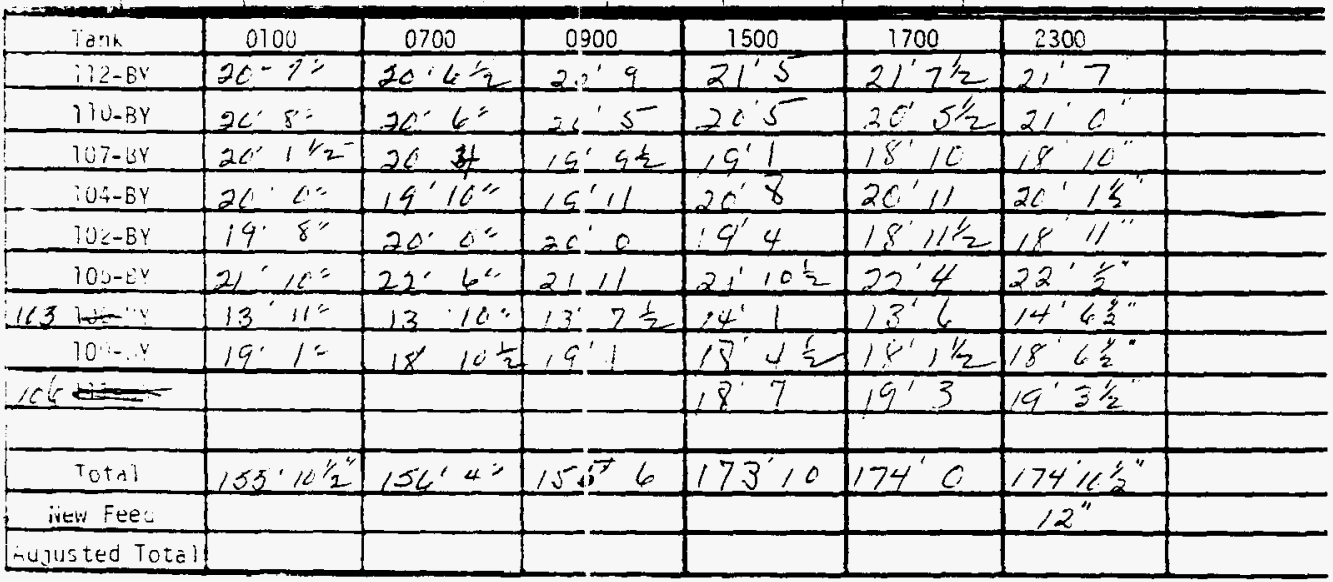

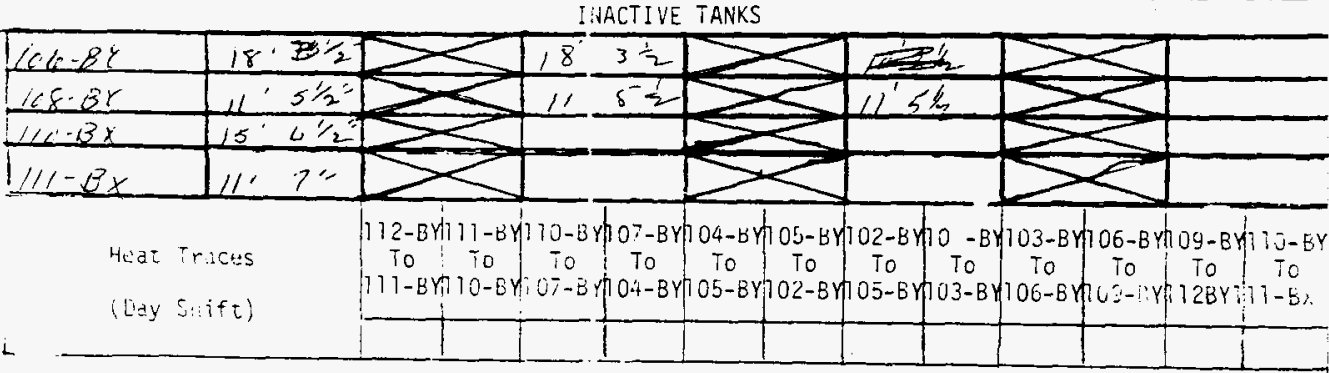

$9.0-71$

BES I AVAILABLE COPY 


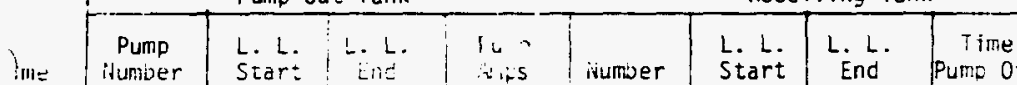

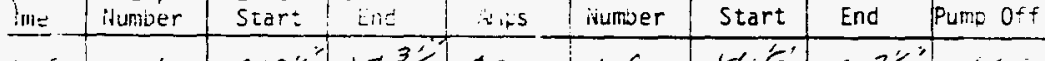

Reriarks

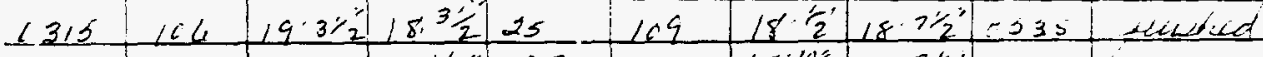

0261160

$2111 \% 13 c^{\prime} 4 \% 2 " 22$

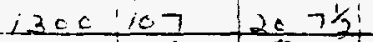

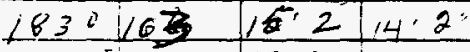

230

\begin{tabular}{|c|c|}
\hline 9,3 & $1 / 7^{\prime} 1 c^{2}$ \\
\hline $21^{\prime} 7 / 2$ & \\
\hline
\end{tabular}

107

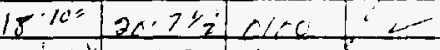

.715

\begin{tabular}{l|l|l}
112 & $2,7 / 2$ \\
\hline
\end{tabular}

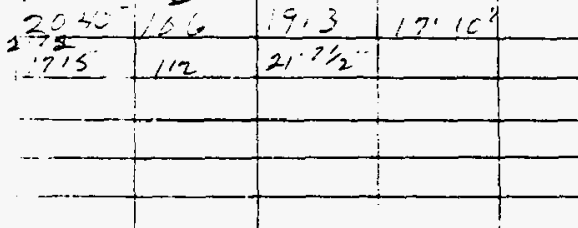
104

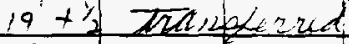

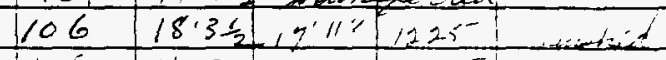

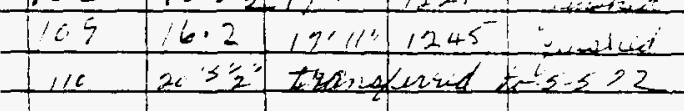

\begin{tabular}{|c|c|c|c|c|c|c|c|c|c|}
\hline Tank & 0100 & 0700 & 09 & & 15 & & 1700 & 2300 & \\
\hline ii2-EY & $21 \cdot 2 \%$ & $216 "$ & 21 & 6 & 21 & 6 & $21: 2$ & 21.37 & \\
\hline $1 T U-B Y$ & $21^{\prime} 3$ & $21 \cdot 11=$ & 21 & $8 \frac{1}{2}$ & 20 & 71 & $20: 7$ & $20.6 \frac{1}{2}$ & \\
\hline $107-64$ & $18^{\prime} \quad 10^{\prime \prime}$ & $18^{\prime} 12=$ & 19 & $2 \frac{1}{3}$ & 20 & 8 & $20 \cdot 10$ & $20: 7$ & \\
\hline$\therefore U \div-B Y$ & $19.8=$ & $19 \cdot 5=$ & 19 & $+\%$ & 19 & 7 & $19-10$ & 20.7 & \\
\hline $10 \leq-B Y$ & $18^{\prime} 11$ & $18^{\prime} 7 \%$ & 18 & 7 & 18 & 7 & $18^{\prime} 7$ & $18 \cdot 7$ & \\
\hline $100-E \mathrm{Y}$ & $21.10 \div 3$ & $22^{\prime} 6^{\prime \prime}$ & 22 & 1 & 22 & 6 & $22 \cdot 51 / 2$ & $22 \cdot 5 \div$ & \\
\hline $163+0$ & 15,31 & $15^{\prime} 3 \prime$ & $i s$ & 3 & 15 & 2 & $15-2 \cdots$ & $14 x-x$ & \\
\hline $10 \cdot-i y$ & $18^{\prime} 2=$ & $18^{\prime} 4^{\circ}$ & 18 & $31 / 2$ & 17 & $7 \%$ & $17: 4 \frac{1}{2}$ & 18 & \\
\hline$\angle C \dot{G} H-\ldots$ & 19.319 & $18^{\prime} 31 / 2$ & 18 & $3 \%$ & 18 & $3 \%$ & $18 \cdot 3$ & 18.8 & \\
\hline & & & & & & & & & \\
\hline Totai & $124^{\prime} / k^{\prime \prime}$ & $174^{\prime} 3 \prime$ & $i 74$ & 812 & $i 74$ & $6 \frac{1}{2}$ & 1241 & 174.7 & \\
\hline iven Fees & & & & & & & & & \\
\hline aujusted Total & & & & & & & & & \\
\hline
\end{tabular}

INACTIVE TANKS

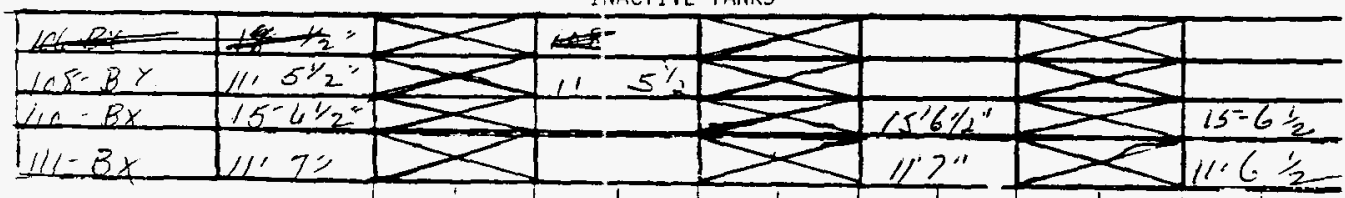

Heat Tr.icts

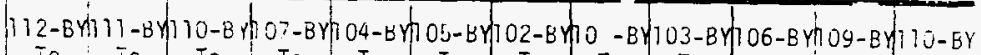

(Doy $s: i f \tau$ )

i.

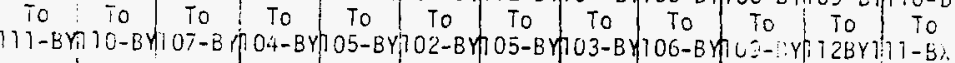

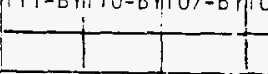

$9-\because 0-7$ ir $223>6$

BEST AVAll_ABLE COPY 


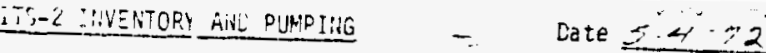

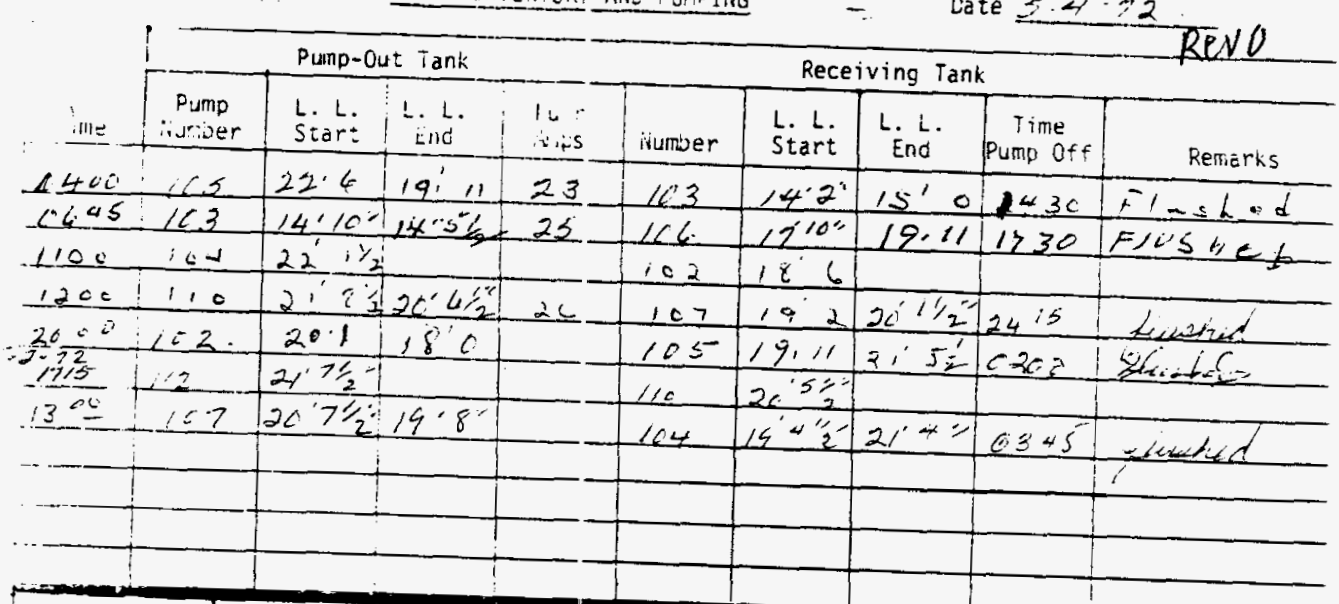

\begin{tabular}{|c|c|c|c|c|c|c|c|c|}
\hline ank & 0100 & 0700 & & & 150 & & 1700 & 2300 \\
\hline IYZ-EY & $21.4=$ & $21.5=$ & 21 & 5 & +1 & $5 \%$ & $21.5^{-3}$ & $21 \cdot 5$ \\
\hline $170-B Y$ & $21.4 \%$ & $21 \cdot 2=$ & +1 & 4 & 11 & $2 \frac{7}{3}$ & 20,10 & $20: 7$ \\
\hline $107-6 \times$ & 20,712 & $19^{\prime} 10^{\prime 1 / 2}$ & 19 & 73 & 19 & $7 \times$ & 19.10 & 20,2 \\
\hline $30 \div-E Y$ & $21 \cdot \quad 1 / 2$ & $21.8^{\circ}$ & 21 & 11 & 22 & $c$ & 21611 & 2117 \\
\hline $306-E^{4}$ & $18^{\circ} 61 / 2$ & $18, \quad 6 \%$ & 18 & 6 & 19 & 1 & $19 \cdot 5-2$ & -20.0 \\
\hline $105-$ & $22^{\prime} 6^{\circ}$ & $21^{\prime} 10^{\circ}$ & 21 & 2 & 19 & 11 & $19 \cdot 11$ & 20.5 \\
\hline 106 & $17 \cdot 10$ & $17^{\prime} 16^{\prime \prime}$ & 12 & $2 \frac{1}{1}$ & iq & & $19+11$ & $15-11$ \\
\hline
\end{tabular}

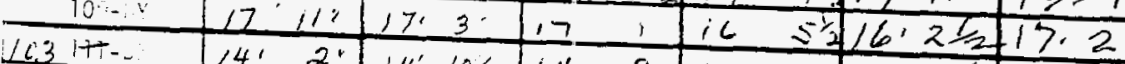

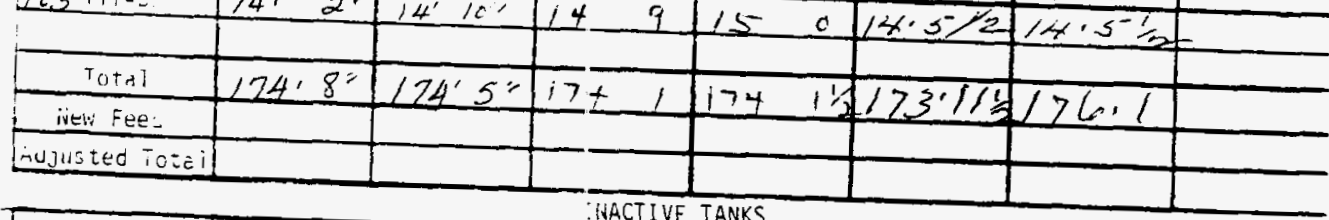

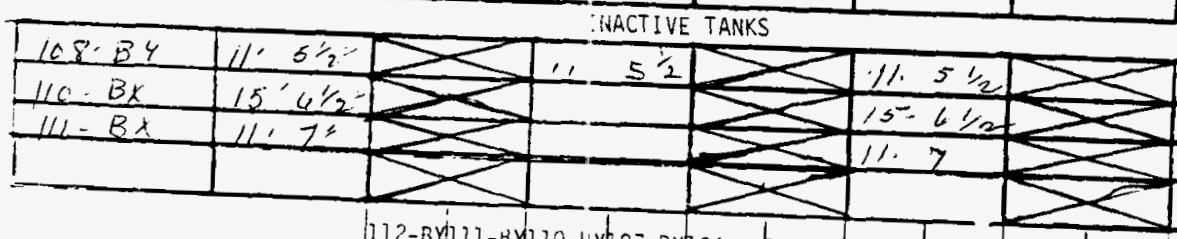

Heaz ir.aces

(Liey $5: ; i f t$ )

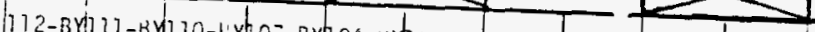

jo

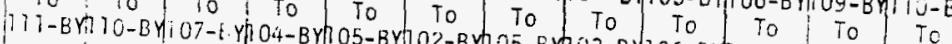

L.

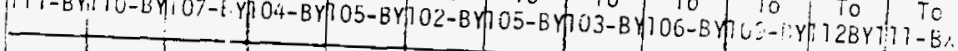

$9-20-77$ 


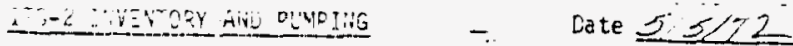

Revo

Puinp-Out Tank

Receiving Tank

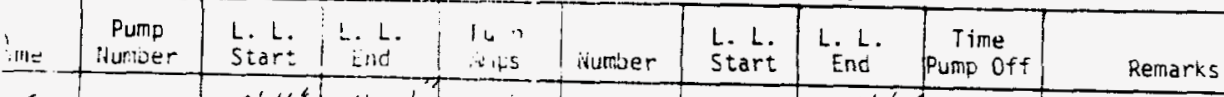

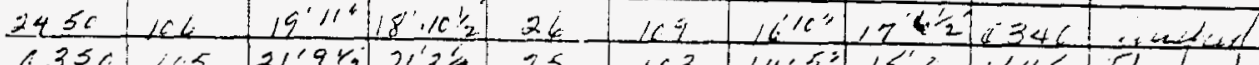

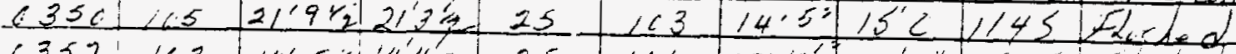

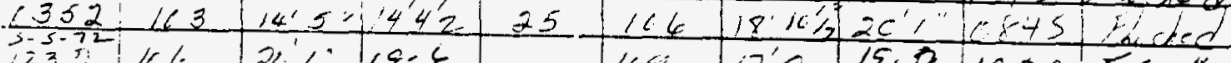

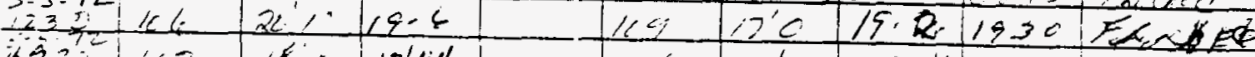

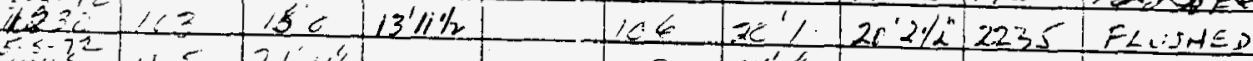
$\frac{5-s^{2}}{5-7^{2}}+12=21+1 / 2$ $4+3^{2}+164+20196 \%$

$2030,10 \quad 21,10$ 4930107

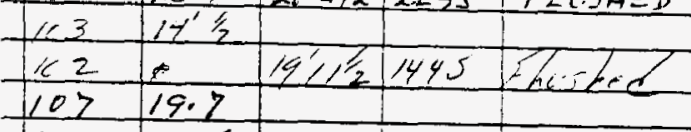
$10 x+19.6$

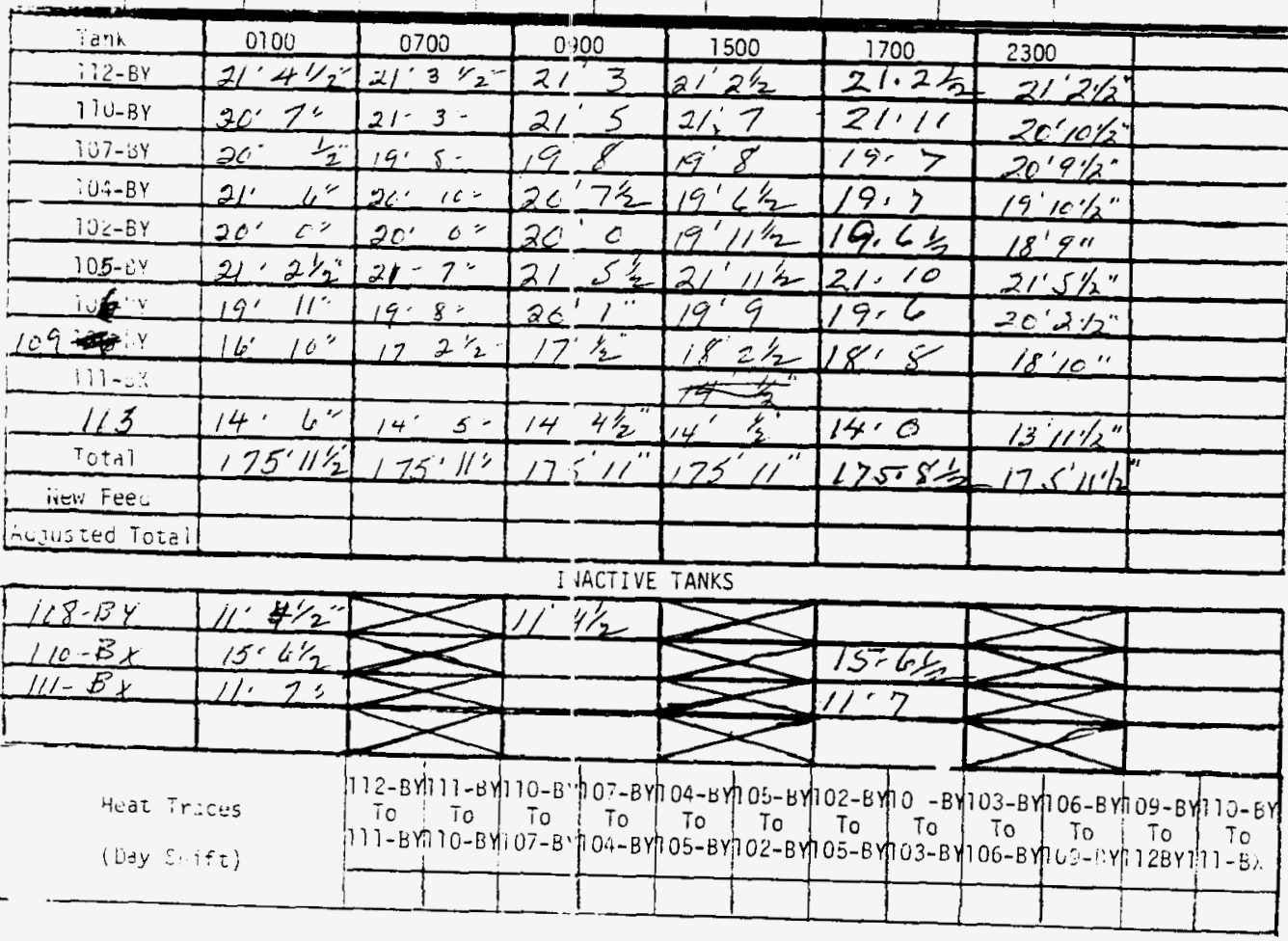

$9-0-7 i$ 


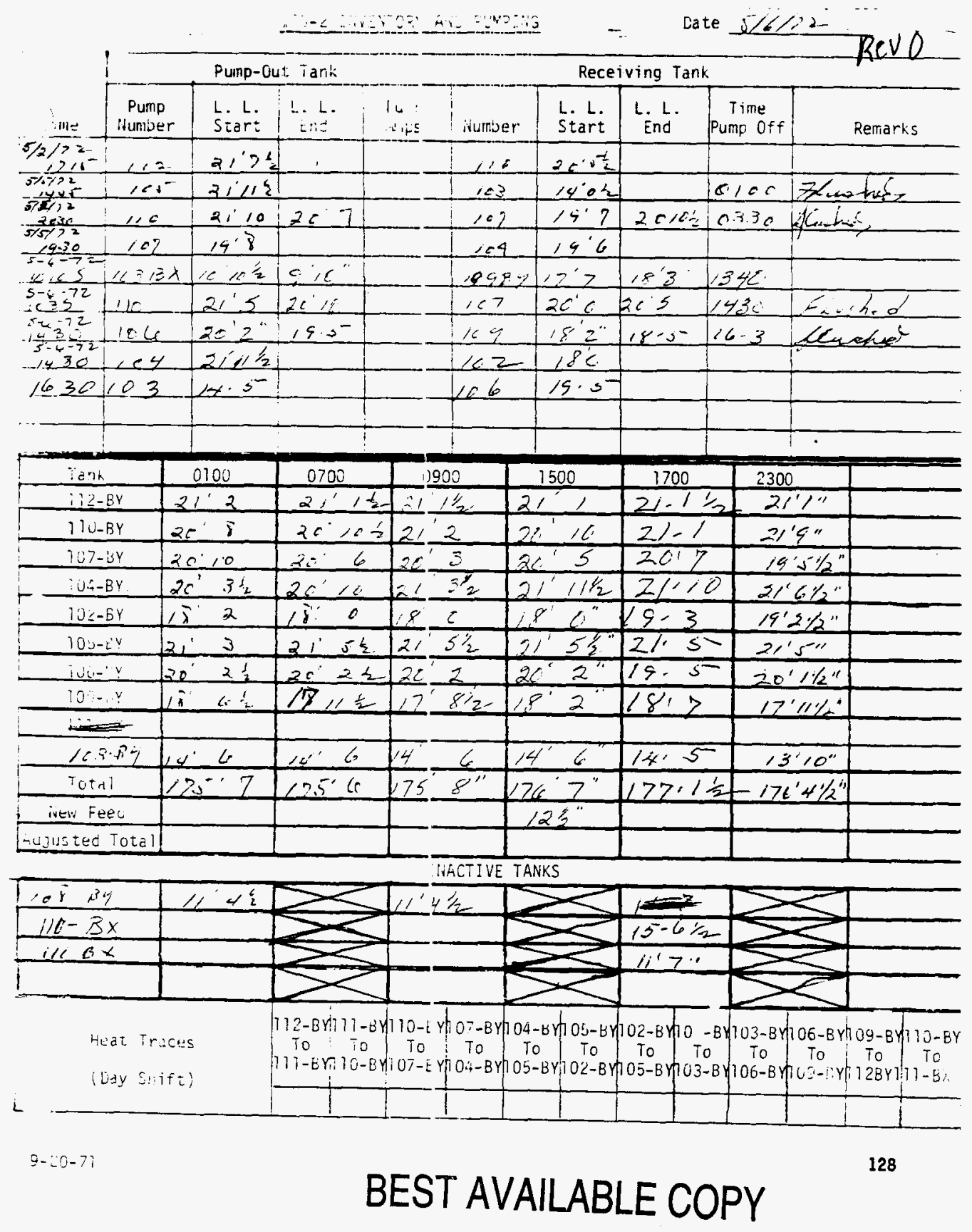


Cate 5

Revo

Pump-Out Tank

Receiving Tank

\begin{tabular}{|c|c|c|c|c|c|c|c|c|c|}
\hline , $11=$ & $\begin{array}{l}\text { Pump } \\
\text { ilumber }\end{array}$ & $\begin{array}{l}\text { L.t. } \\
\text { Start }\end{array}$ & 1. L & $\begin{array}{l}\ln , \\
u+5\end{array}$ & ivumber & $\begin{array}{l}\text { L. L. } \\
\text { Start }\end{array}$ & L. 1. & $\begin{array}{c}\text { Tine } \\
\text { Pump of: }\end{array}$ & Rer:arks \\
\hline $\begin{array}{l}5 / 5722 \\
15.12\end{array}$ & 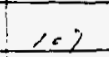 & $14^{\circ} 3$ & 1 & & $<=4$ & $19^{\prime} 6$ & & & \\
\hline$+(2))_{2}$ & 1,2 & $2,3 \frac{1}{2}$ & $2+\frac{1}{2}$ & convis: & 110 & $2-2,-\frac{1}{1}$ & $z$ & & \\
\hline $\begin{array}{l}5 / 6 / 32 \\
-\angle 412\end{array}$ & $1,1<4$ & $2,11 \frac{1}{2}$ & $915 \frac{1}{2}$ & & $<\leqslant$ & $17^{-0}$ & $20^{\circ} \mathrm{C}$ & $\operatorname{coc} i$ & $x^{x}+x<-2$ \\
\hline $5 / 4020$ & 1183 & $i<.=$ & $1301 / 2$ & & 106 & 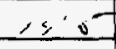 & $217 \%$ & $S 0 \mathrm{C}$ & $1 / \lambda_{2}-a^{\prime}$ \\
\hline$\leq 106$ & 116 & 31113 & $\because 6$ & & 2 & $15^{\prime} C$ & $9 ; 1$ & $\therefore<5$ & 1. \\
\hline $6.7 e^{-}$ & $\angle \cos ^{\circ}$ & 215 & $\rho 5^{\prime} / 1 \frac{5}{2}$ & & $\angle \leq 3$ & $13^{\circ} 91$ & $13^{\circ} 11$ & 5200 & \\
\hline$\varepsilon \varepsilon t$ & 104 & $=221$ & & & $1 \leq 2$ & 20 & & & \\
\hline 0841 & ic? & 211 & & & 115 & $\angle S^{\prime} 11$ & & & \\
\hline Lngsc & 105 & 산ㄷㄴ & $19^{\prime} 6 / 2$ & & 163 & $13^{i} \angle L^{\prime \prime}$ & $122^{\prime} / 6$ & 1445 & \\
\hline 1440 & 106 & $2,21 / 2$ & $14-6$ & & 109 & $16^{17} / 2$ & 17,9 & 1860 & gense !' \\
\hline 1900 & 103 & $14.9 \%$ & & & 1106 & 19 & & & \\
\hline
\end{tabular}

$1900103 \quad 14.91 / 2$

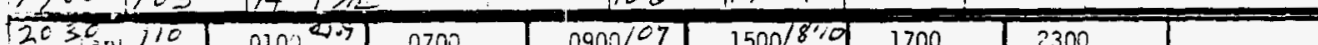

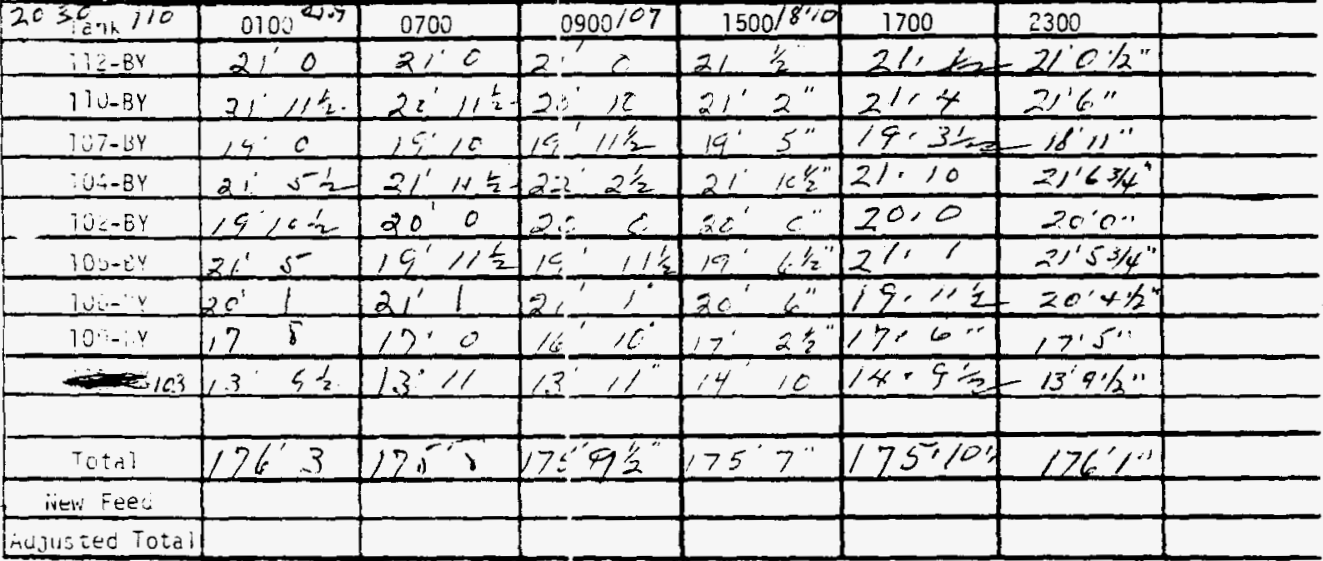

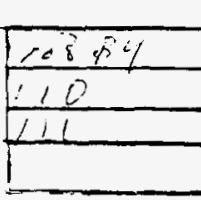

$4: a t$ Tr.:ces

(liay S!ifi) INACTI VE TANKS

$11 \times 5$

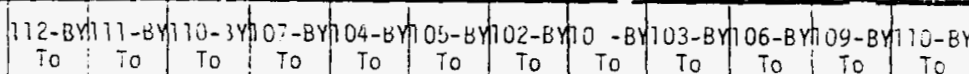

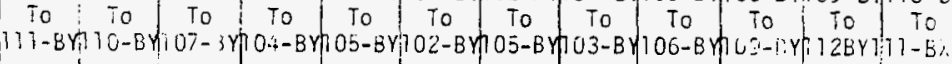

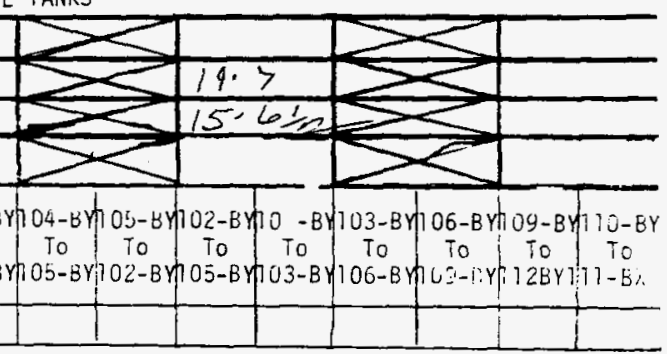

\section{BEST AVAILABLE COPY}




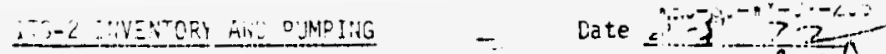

Pump-Out Tank

Receiving Tank

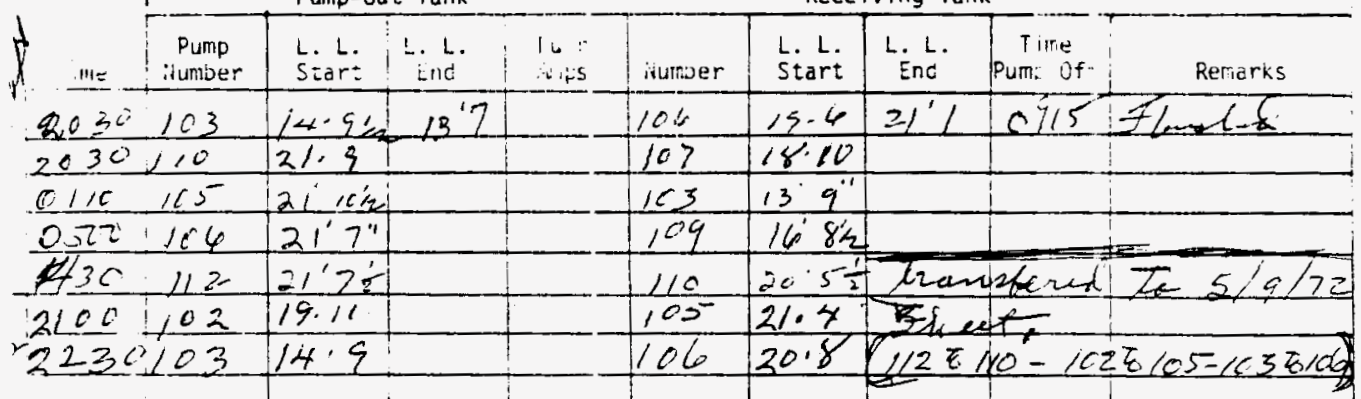

\begin{tabular}{|c|c|c|c|c|c|c|c|}
\hline ic\% & 0100 & 0700 & $(1900$ & 1500 & 1700 & 2300 & \\
\hline i)-EY & $2 i$ & $11 \%$ & $2=11$ & $20^{\prime} 9 \frac{1}{2}$ & $20.8 \frac{1}{3}$ & 20.8 & \\
\hline $11 \cup-B Y$ & 21 & $2 c^{\prime} \quad$ is & $20^{\prime} 6$ & $20^{\prime} 6$ & $20.5 \%$ & -20.3 & \\
\hline $10 i-3 y$ & 19 & 19 & $167 \frac{1}{3}$ & 197 & 15.2 & 19,7 & \\
\hline$: \cup \div-3 Y$ & $21 \quad 5 / 2$ & 211 & 211 & 2011 & $20,10 \frac{1}{2}$ & 20110 & \\
\hline$B \in \leq-E x$ & $19 \quad 10^{\prime} / 2$ & $11 / 2$ & $17^{\prime} 1 x$ & 1916 & $19^{\prime} 11^{\circ}$ & 15.10 & \\
\hline $10-i v$ & $21 \quad 1 c^{\prime} / 2$ & 21 & $2 \div 6$ & 214 & 21.4 & $21 \cdot 6$ & \\
\hline 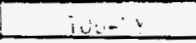 & $20 \quad 6^{\prime} / 2$ & 21 & $2 t^{\prime} t$ & $20^{\prime} 8$ & $20.8^{\prime \prime}$ & 20.8 & \\
\hline $10 \ldots$ & $17^{\prime} \quad 7^{*}$ & 17 & $\angle Z^{\prime} . T$ & $18^{\prime} 2 \frac{1}{2}$ & 18,3 & $18 \cdot 0$ & \\
\hline $103-6 y$ & $13^{\prime} \quad 9^{4}$ & $13^{\circ} 6$ & $1 \therefore 7$ & 1217 & 13.9 .1 & $13 \cdot 9$ & \\
\hline & & & & & & & \\
\hline Tetai & $176 \quad 1 / 2$ & $175 \quad 8 \%$ & $17: 99$ & 1756 & 1175.7 & $175 \cdot 611$ & \\
\hline ivew Fees & & & & & & & \\
\hline iujus isec Total & & & & & & & \\
\hline
\end{tabular}

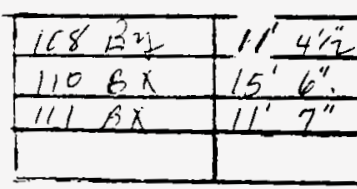

$$
\text { IIIACTIVE TANKS }
$$

Há Traces

$i=v \operatorname{Sift)}$

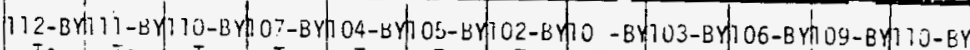
To to to to to to To $_{0}$ To

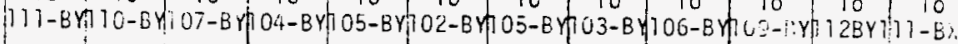

L
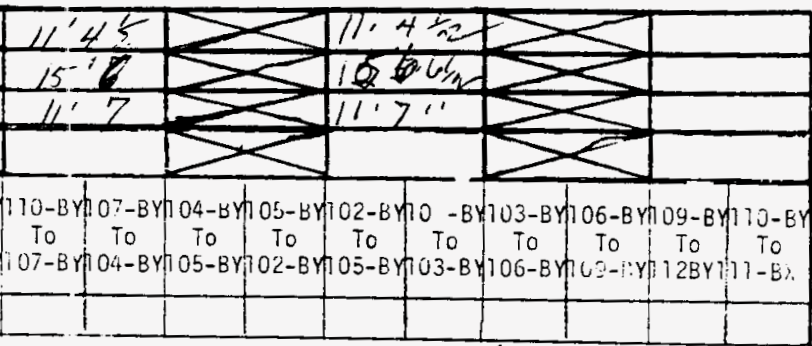

$9-20-7 i$ 
from $5 / 8 / 72$

inect *

III= \begin{tabular}{c|c|c|c} 
Pump & L. L. & L. L. L. \\
Uumer & Start & End \\
\hline
\end{tabular}

Ill $=$\begin{tabular}{l|c|c} 
Pump & L. L. & L. L. L. \\
Uuniber & Start & End \\
\hline
\end{tabular}

$\div 1430.112121720 .6$

$-2100102+1911.178$

$-2230 / 13144^{\prime \prime} 139$

C.115 105 22, $0^{\prime} \mathrm{g} 1^{\prime} 6$

$65+1038 x$. $9^{\prime} 10^{\prime \prime} 9^{\circ}$

$1225+106$

$.3=5116 \quad 1216 \frac{1}{2} 20^{\prime} 6 "$

$+343^{2} 107$

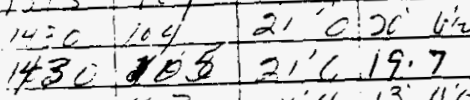

$1421 / 631,44134 \%$

\begin{tabular}{|c|c|c|c|c|c|c|c|}
\hline c7k & 0100 & 0700 & 0900 & 1500 & 1700 & 2300 & \\
\hline$\because Z-B Y$ & $20^{\prime} 8^{\prime \prime}$ & $20^{\prime} \quad 2^{\prime \prime}$ & $20: 2$ & 2,0 & $2 / 3$ & $21 \cdot 5$ & \\
\hline $10-8 Y$ & $20 \quad 10 / 2$ & $21^{\circ}$ & $\therefore 18 \div$ & 217 & $21-10$ & $20: 4$ & \\
\hline $7-3 y$ & $19^{\prime} 7^{\circ}$ & 1967 & 1917 & 198 & $19.11 \frac{1}{3}$ & $20 \cdot 0$ & \\
\hline j4-EY & $2 c^{\prime} \quad 10 \%$ & $2 e^{\prime \prime} \quad k \cdot / 2$ & 0.10 .2 & 210 & $20011 \frac{1}{2}$ & 2019 & \\
\hline $2-E Y$ & $19^{\prime} 2^{\prime}$ & $18 \quad 4 "$ & 49 & $17^{\prime} 10$ & $18^{\prime} 21 / 2$ & 1810 & \\
\hline $125-t^{2}$ & $22 \mathrm{c} / 2$ & $21 \quad 81 / 2$ & 21 & 21 & $24.10 \%$ & 2011 & \\
\hline$i v a-v$ & $21 \quad 0$ & $22^{\circ} \quad 1 / 2$ & 22 & 21 & 21,16 & $21 \cdot 11$ & \\
\hline $39-$. & 17.912 & 17 & 1718 & 7 & $12,63=$ & $18 \cdot 3$ & \\
\hline $1 r^{3} \equiv 0 y$ & 135 & $139^{\prime \prime}$ & $17^{\prime}, \frac{1}{2}$ & 14 & $14 \cdot 3 \cdots$ & $13-9$ & \\
\hline & & 3 & $=$ & & & & \\
\hline 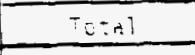 & $175^{-1} 5^{\prime}$ & $175 \quad 5 / 2$ & $17 \mathrm{~B}^{\prime} \mathrm{O}$ & 176,1 & $1760^{\circ} 5$ & 176.41 & \\
\hline iner: Fees & & 17638 & & & & & \\
\hline iujusted Total & & & & & & & \\
\hline
\end{tabular}

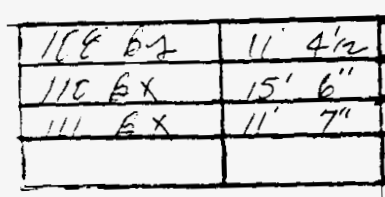

Heat Traces

Day sifift

$i$

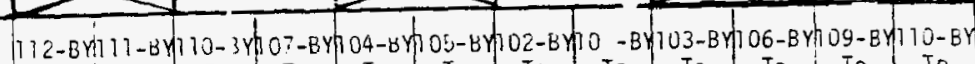
To 10 To

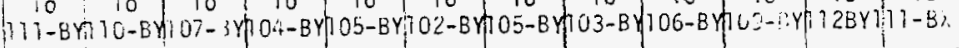

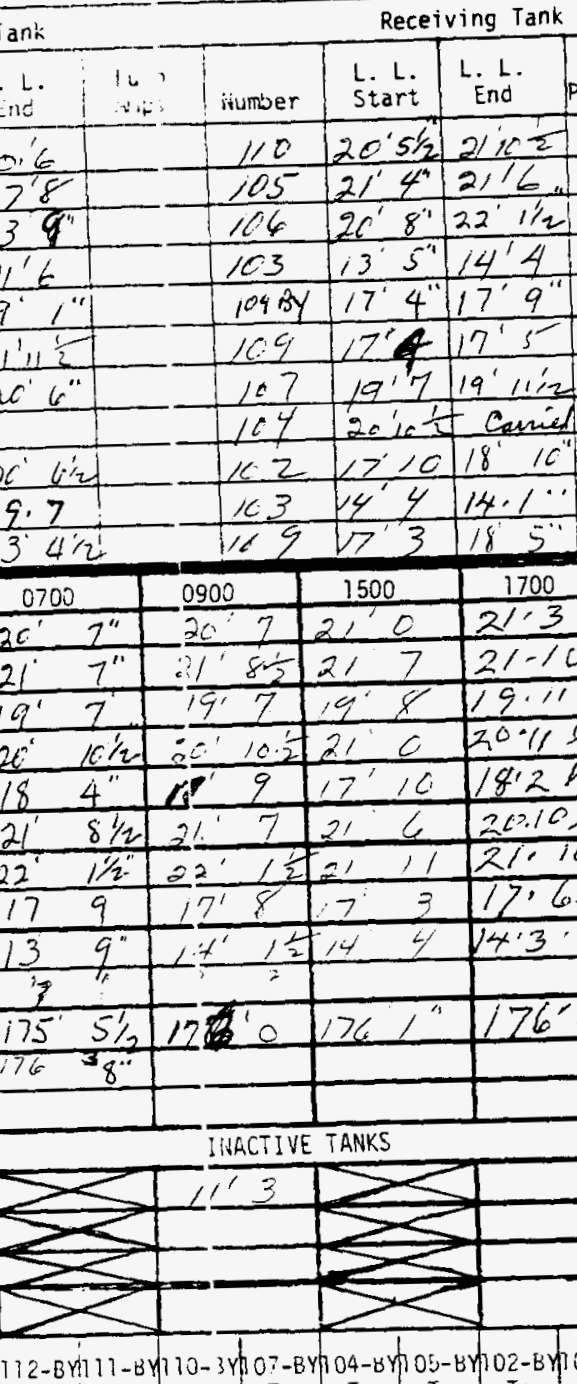




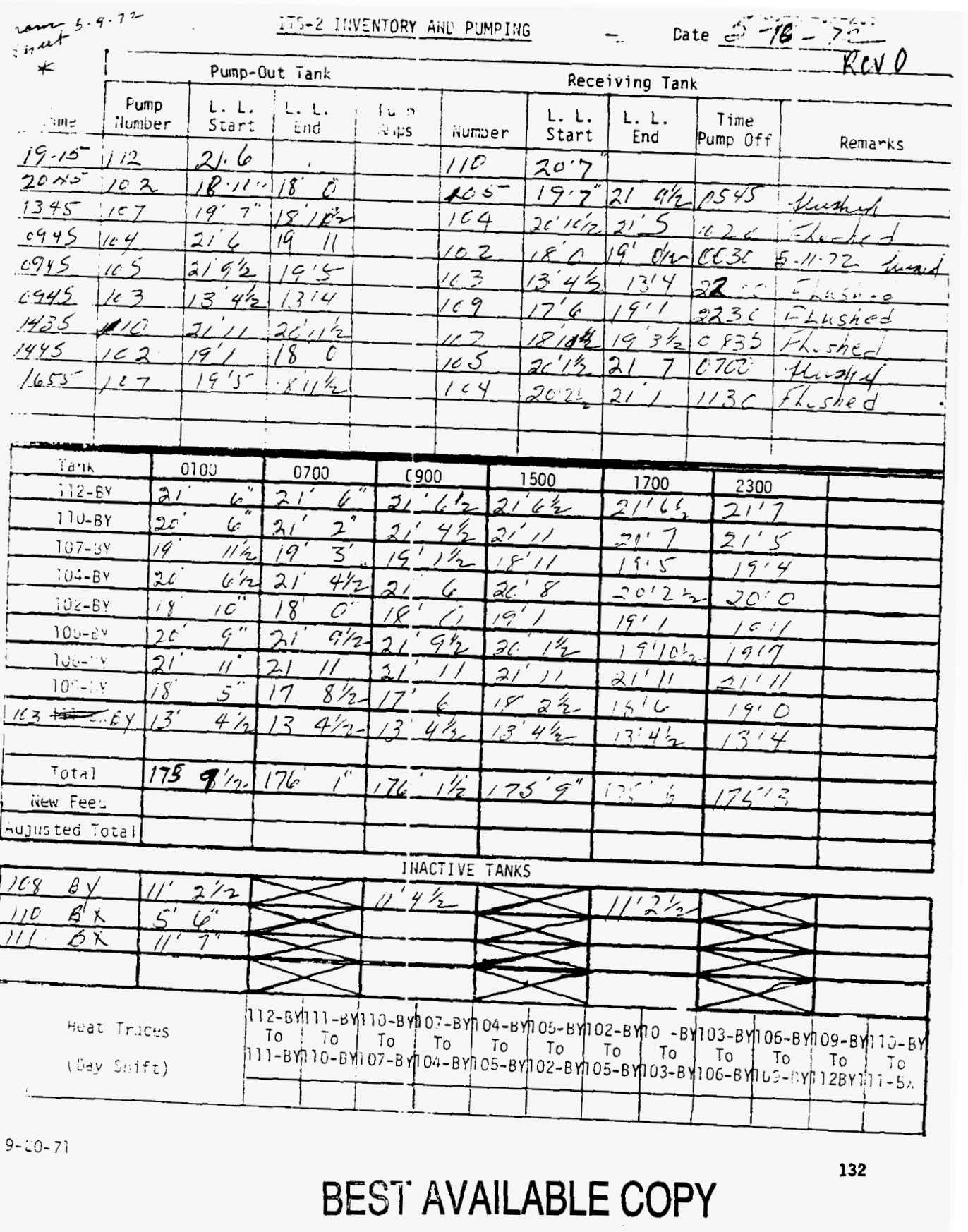




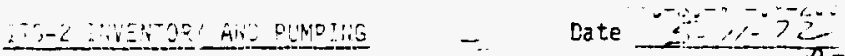

Puinp-Out Tank

Receiving Tank

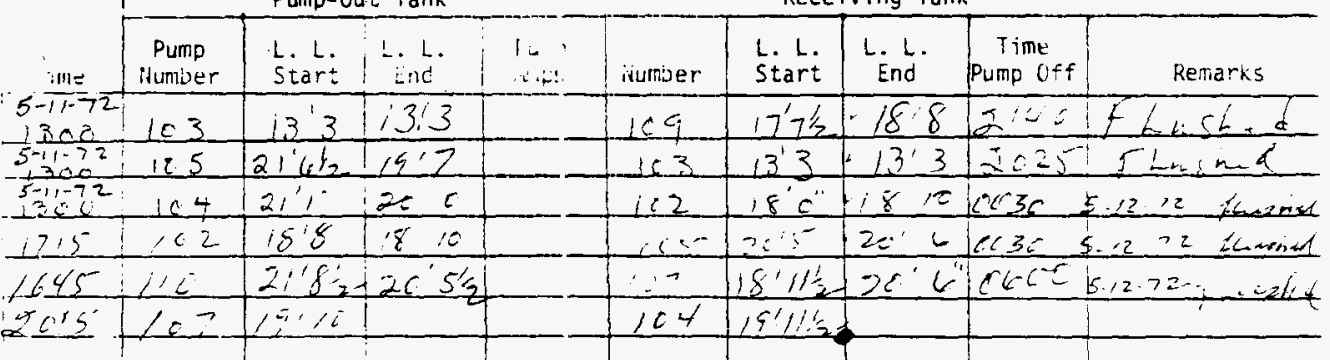

\begin{tabular}{|c|c|c|c|c|c|c|c|}
\hline$\overline{a j h}$ & 0100 & 0700 & 3900 & 1500 & 1700 & 2300 & \\
\hline$i 12-8 Y$ & 21912 & $91 / 2$ & 2110 & $21^{\prime} 9$ & $2118 \div=$ & $2161=$ & \\
\hline PUU BY & 212 & 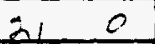 & $x=1 / \frac{1}{2}$ & $2 \cdot 6 \frac{1}{2}$ & $318 \frac{1}{2}$ & $a^{\prime \prime} 2 c^{\prime}=$ & \\
\hline $137-3 Y$ & 194 & 194 & $c_{3} 31 / 2$ & $18^{\prime 11 / 2}$ & $18111 \frac{1}{2}$ & $1483=$ & \\
\hline $.04-B Y$ & 1911 & $20^{\prime} \quad 0^{\prime} / 2$ & $2041 / 2$ & $2 c^{\prime} 9$ & 20.7 & $19^{1} / 012$ & \\
\hline$B J \leq-B Y$ & 19012 & 180 & 8 & $18^{\prime} 4 / 2$ & $28^{\prime} 8^{\prime}$ & $19^{\prime} 0$ & \\
\hline $100-E y$ & 20 & 21 & $21,61 / 2$ & $24^{1} 1$ & $=0.5$ & $x^{4} 0^{\circ} 2.42$ & \\
\hline$i v i-y$ & 2111 & 11 & $=1711^{\circ}$ & 2,11 & 21111 & 2111 & \\
\hline $10 \cdot-y$ & $18 \quad 11$ & $18 \quad 2$ & 2711 & $7^{\prime} 9 / 2$ & 1812 & $18: 6$ & \\
\hline $103-2 y^{\prime}$ & 3 & 134 & 23 & $13.3 \%$ & 32 & $\angle 3 i 3$ & \\
\hline 21 & & & & & & & \\
\hline Total & $175^{\prime} 8^{\prime \prime}$ & $175^{\prime} \quad 8^{\prime \prime}$ & $: 76^{\prime \prime}$ & $175^{\circ} 5 / 2$ & $175^{\prime 4}$ & 11 & \\
\hline ivew Fees & & & & & & & \\
\hline injusted Totel & & & & & & & \\
\hline
\end{tabular}

\section{TWACTIVE TANKS}

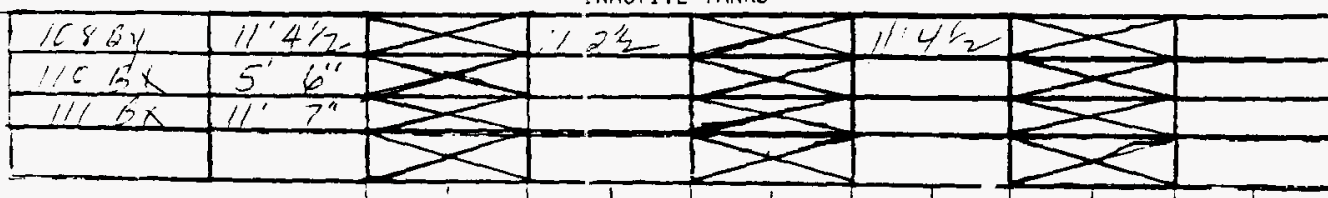

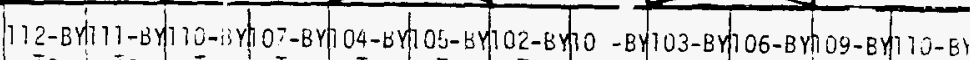
Heat Tr:ous To to to to to To (Lat $s: i f t$ )

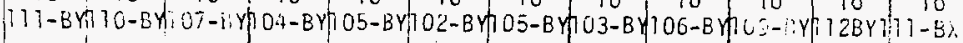
i

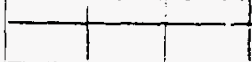

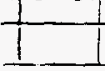

$9-: 0-7 i$ 


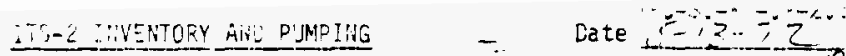

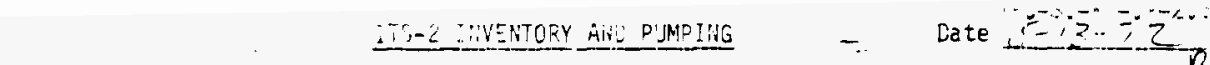

\begin{tabular}{|c|c|c|c|c|c|c|c|c|c|}
\hline & \multicolumn{4}{|c|}{ Pump-Out Tank } & \multicolumn{4}{|c|}{ Receiving Tank } & \multirow[b]{2}{*}{ Remarks } \\
\hline ill & $\begin{array}{l}\text { Pump } \\
\text { ilumber }\end{array}$ & $\begin{array}{l}\text { L. L. } \\
\text { Start }\end{array}$ & $\begin{array}{c}\text { I. L. } \\
\text { End }\end{array}$ & $\begin{array}{l}160 \\
21,5\end{array}$ & ifunder & $\begin{array}{l}\text { L. L. } \\
\text { Start }\end{array}$ & E. L. & $\begin{array}{c}\text { Time } \\
\text { Pumf off }\end{array}$ & \\
\hline $\begin{array}{l}-13.12 \\
156 \\
\end{array}$ & 1110 & 217 & $26: 5$ & & 167 & $193 \%$ & $19^{\circ} 16$ & 6.52 & \\
\hline$=30$ & 103 & $14^{\prime} 1$ & & & $\angle C 5$ & $1511^{\circ}$ & $440^{\prime \prime}$ & 1415 & \\
\hline$?$ & 104 & $21^{\prime} 7$ & $19^{7} 11$ & & $1 c^{2}$ & 1811 & $19^{7}$ & 1500 & $c S \dot{A} \leqslant$ \\
\hline 3 & ic 2 & $19^{\circ} 52$ & & & 145 & $2 c^{\prime} 6$ & $\cdot-$ & & \\
\hline$?$ & 105 & $21^{\prime} 6$ & 196 & & 103 & 133 & $14^{\prime} 5$ & $2 x=5$ & $\mu \leq 6$ \\
\hline$\because 1<0$ & 110 & $211^{\prime} \not 4^{\prime \prime}$ & & & 107 & $\angle 9^{3} 1^{\prime}$ & & & \\
\hline$\angle 6 ! 3$ & $\therefore 3$ & $14^{\prime} 8:$ & & & 169 & $171 / 2$ & & & \\
\hline
\end{tabular}

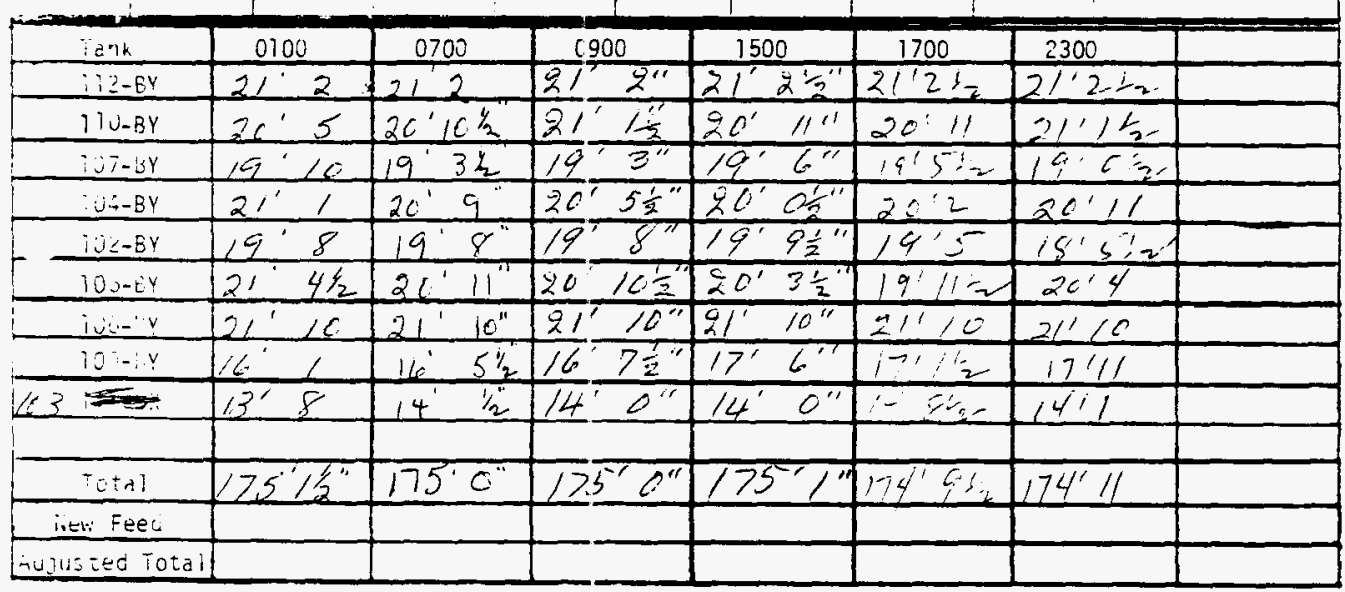

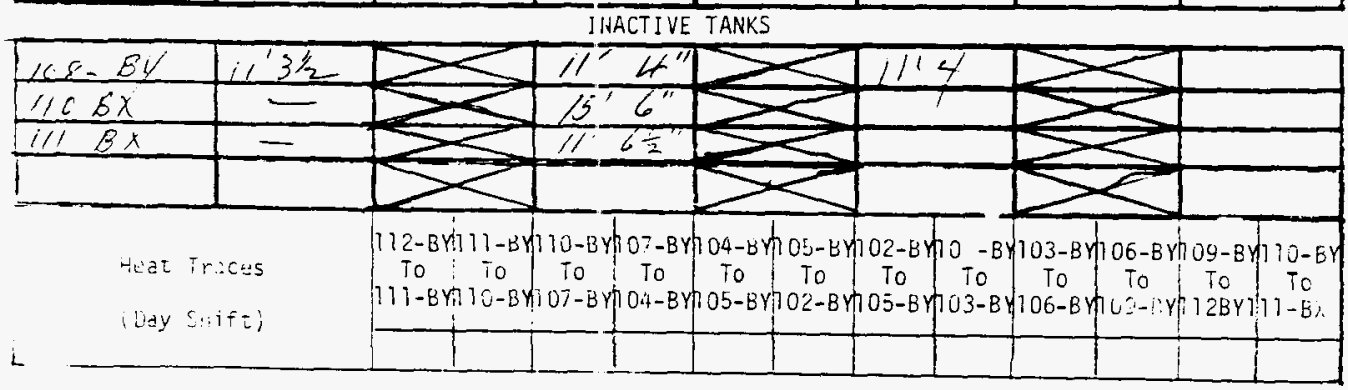

$9-20-7 i$ 


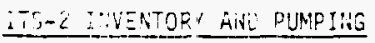 \\ Date $5-14-72$}

Puinp-Out Tank

Receiving Tank

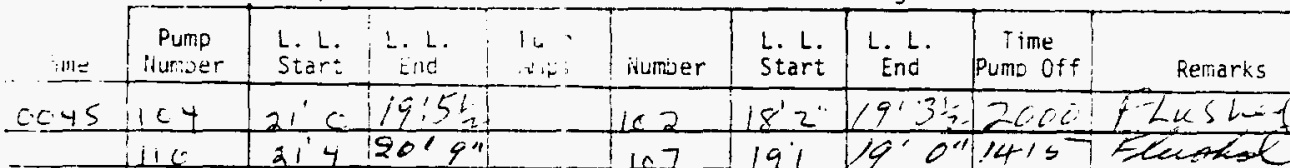

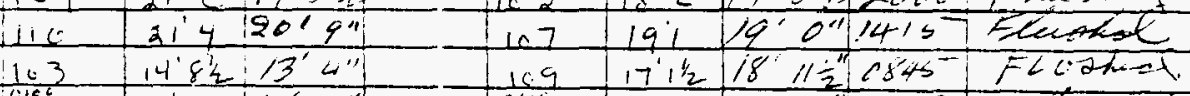

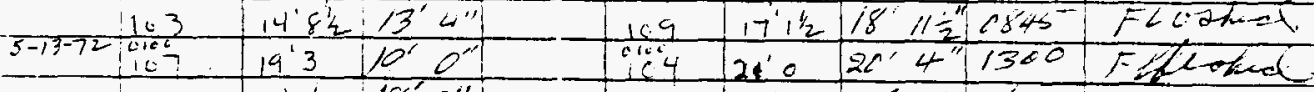

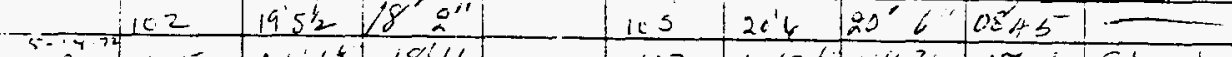

$-22^{2}+105$

$1400100.10^{\prime} 3^{\prime \prime}$

1460 103 $/ 4^{\prime} 6^{\prime \prime} / 3^{\prime} 3 / 2$ $1520-1 C 6$

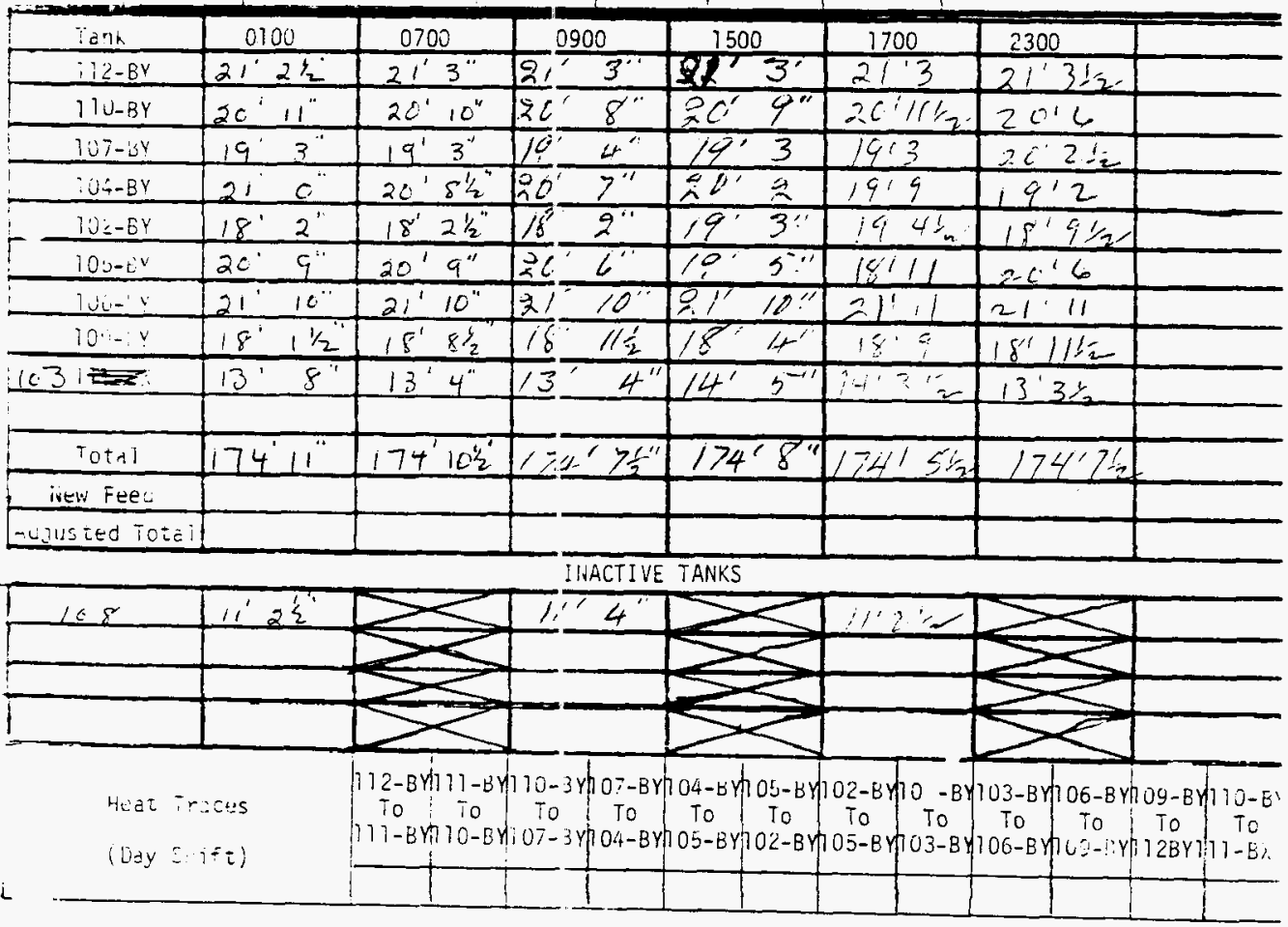


Cate $5-15-7$

Pump-Out Tank

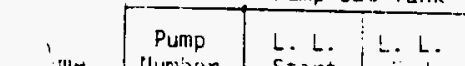

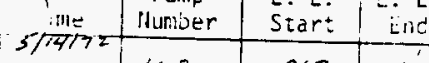

\begin{tabular}{l|l|l|l|}
1420 & 162 & 193 & 19.0
\end{tabular}

\begin{tabular}{|l|c|c|c|}
\hline 0830 & 165 & $20^{\prime} 11$ & $14^{\prime} 6$ \\
\hline
\end{tabular}

6f4s 110 21 $30^{\prime} 0^{\prime} 10^{\prime \prime}$

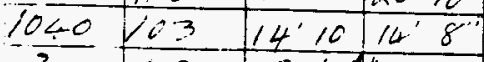

- $35-\mu-7 / 1 / 2$

$1410+10$

1.845106 \begin{tabular}{ll|l|l}
1520 & Lic & $211 / 2$ & 2062 \\
\hline 51.5172 &
\end{tabular} $7,21^{\prime 2} 2^{2}$

只1"3" 3 " $20^{\prime} 5 \div$

$220^{\circ} 20^{\circ} \div 25$

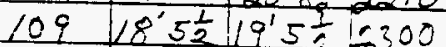

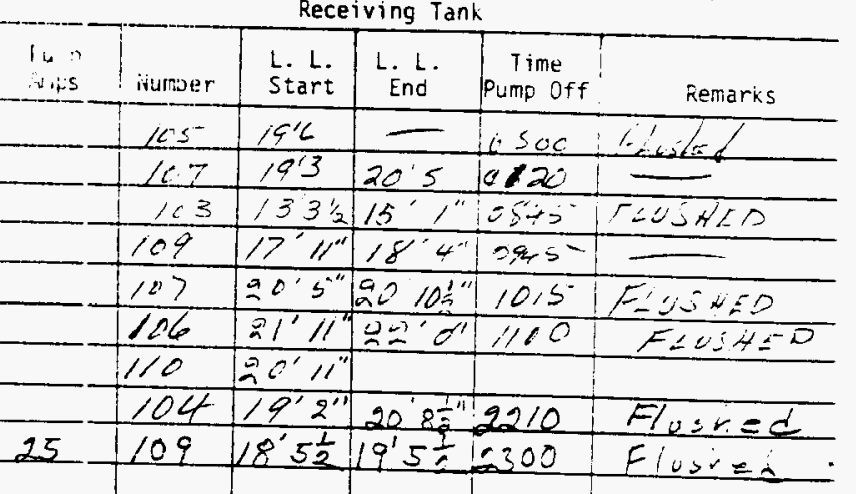

Receiving Tank

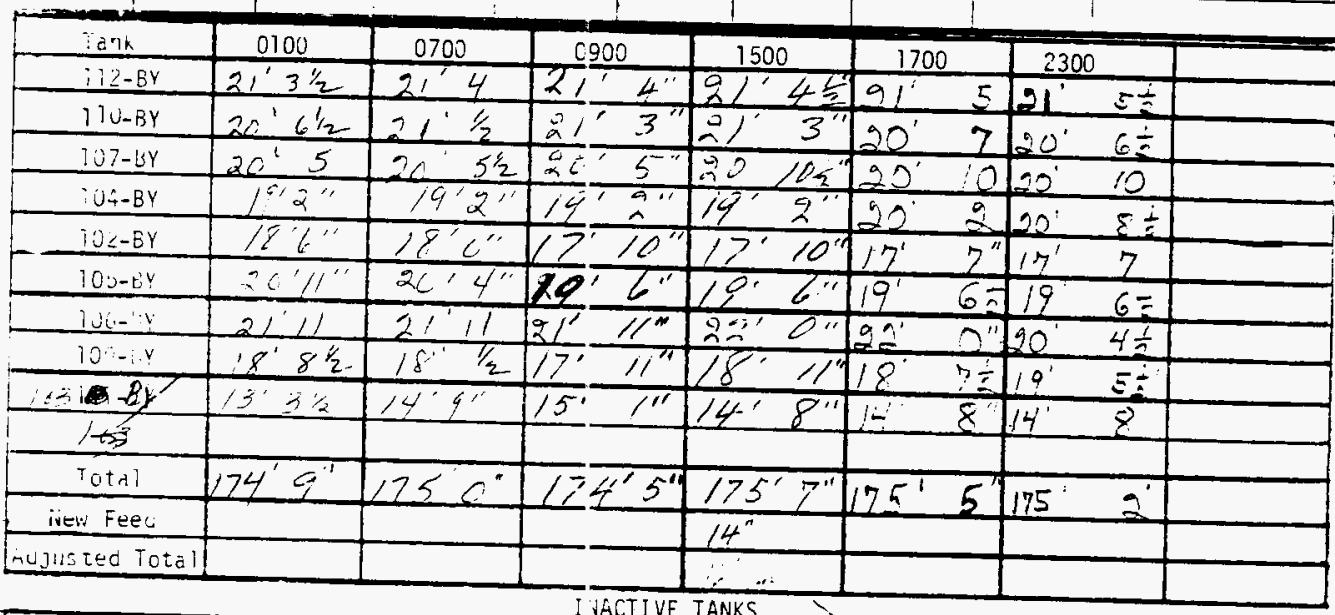

I VACT IVE TANKS

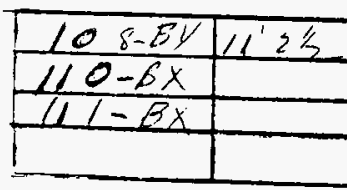

Heat Tr:act:s

(Lity s:ift)
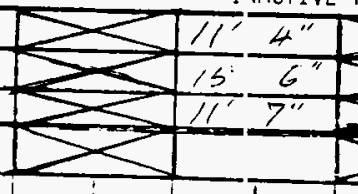

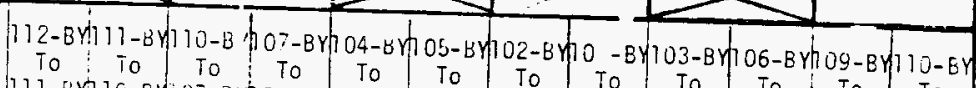

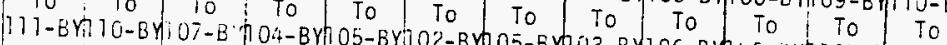

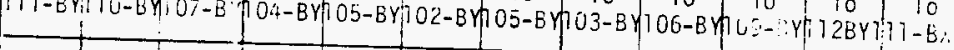




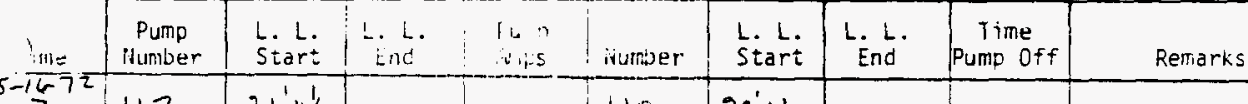

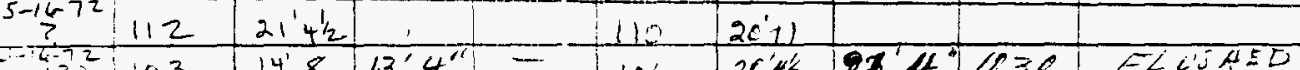

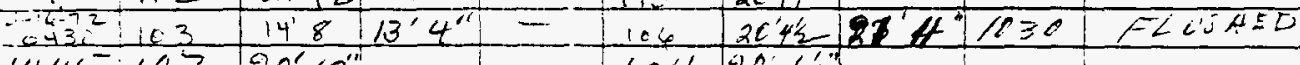

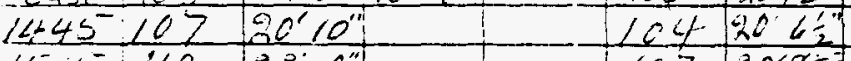

$15^{-}$is 110 in ${ }^{\circ} 0^{\prime \prime}$

$21 \leq=\quad 104 \quad 22^{\prime 2 \frac{1}{2}}$

$21001106-2111$

\begin{tabular}{l|l|l|l}
2145 & 103 & $13^{\prime} 4$ & $13^{\prime} 2$ \\
\hline
\end{tabular}

107 20082

$102,17^{1} 7$

$105183 \div$

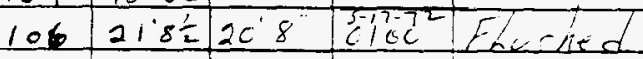

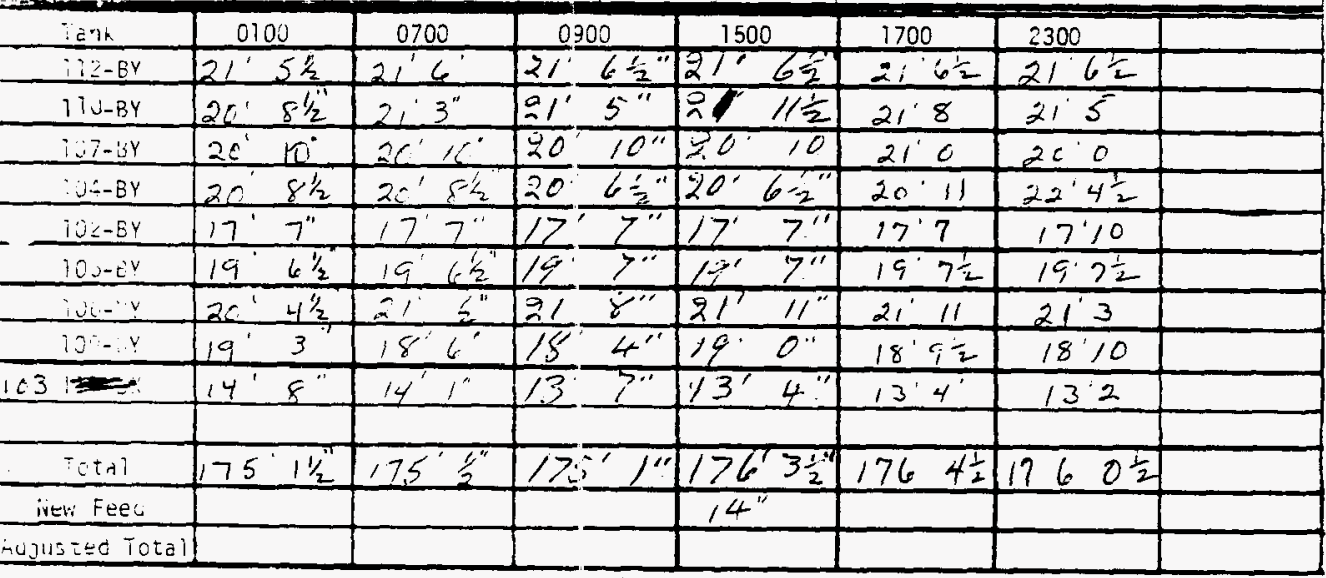

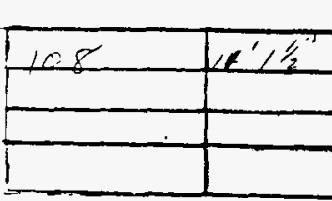

Heat Tracts

Ley Sifift)

\section{VACT IVE TANKS}

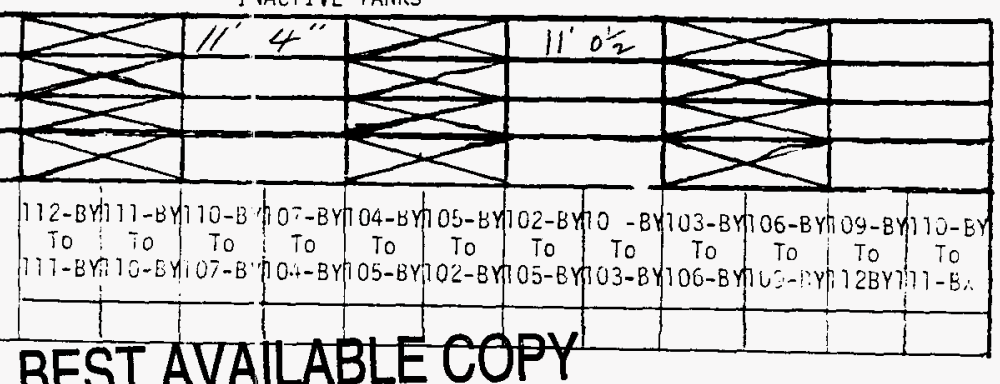




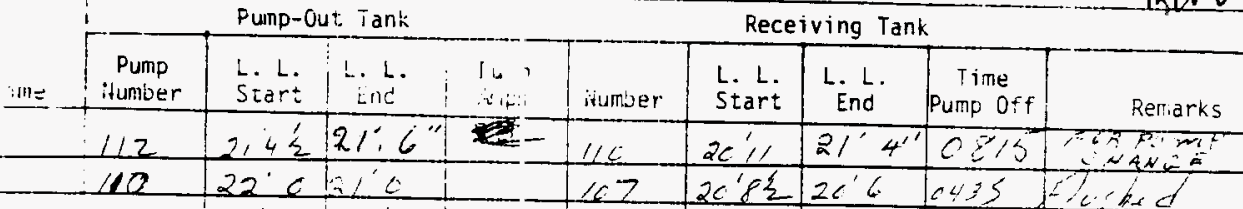

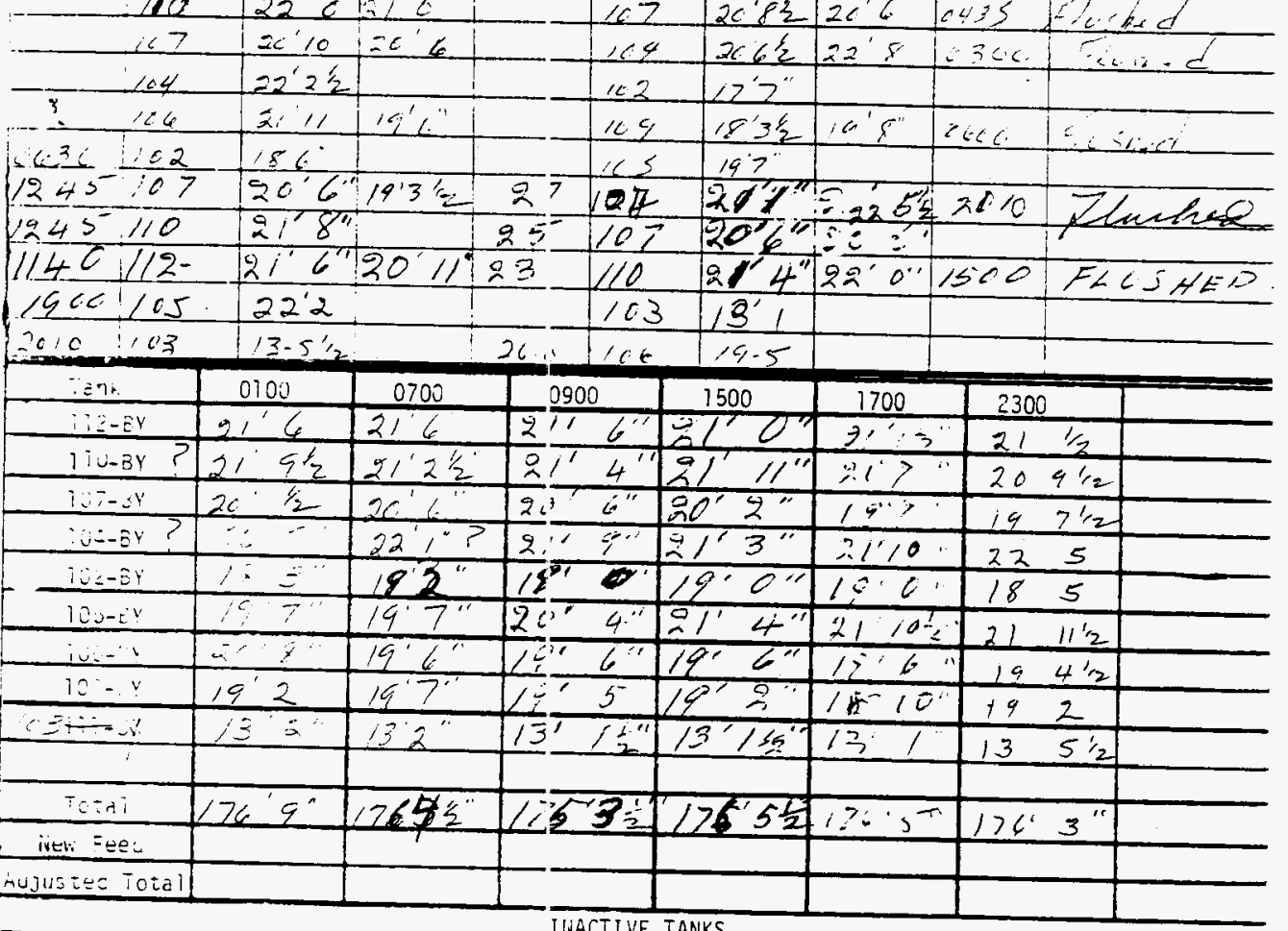

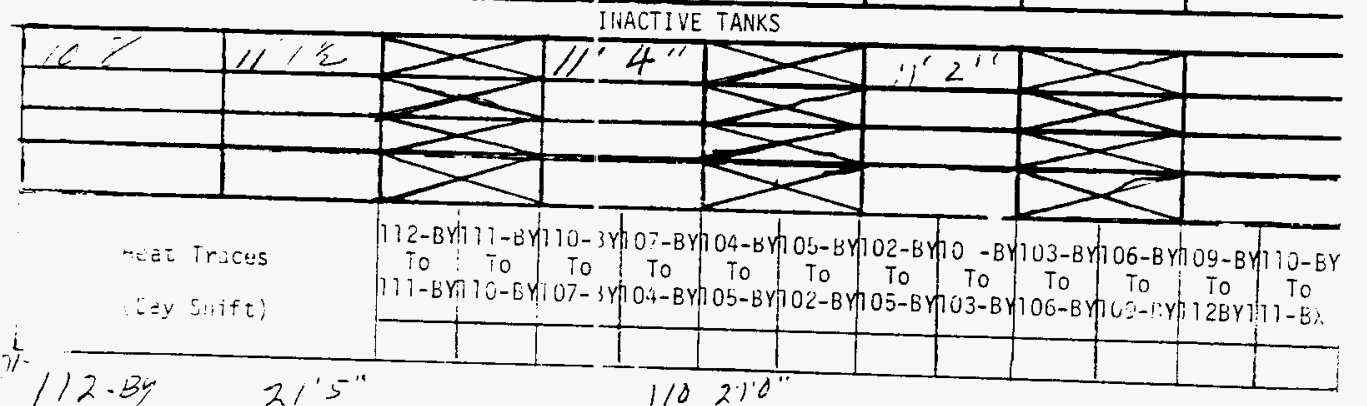




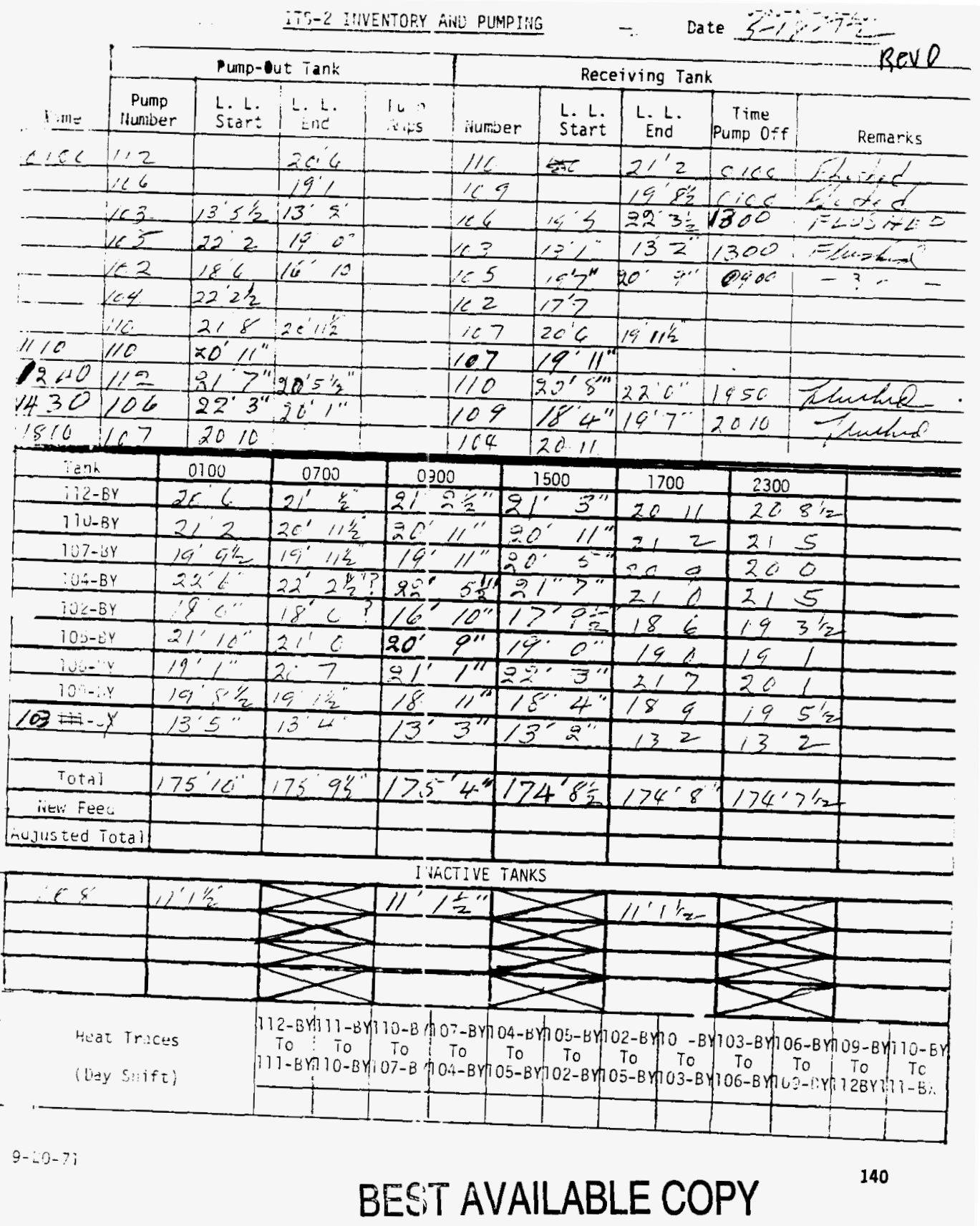




\section{iIr-2 ::iVENTORY AiNL PUMPING $\quad-$ Date}

Rev 0

Puinp-Out Tank

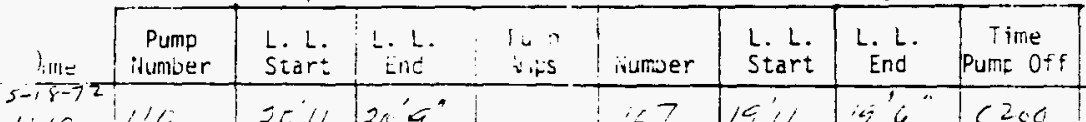

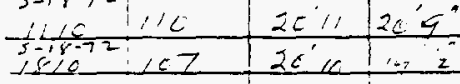

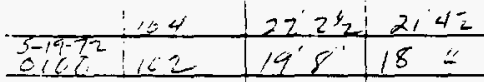

$\therefore 73 c: 112$

$1115+110$

$1830, i<7$

1835103

16351106

2300,164
$2{ }^{\circ} \mathrm{C} 20^{\circ} \mathrm{C}$

$21^{\prime} 920^{\circ} 4^{\circ}$

2091972

$13^{\prime} 2^{\prime}+12^{\prime} 1 !$

$20^{\prime 1} 195^{\prime}=$ $22^{\prime} 7^{11} 23^{1 /}$
Receiving Tank

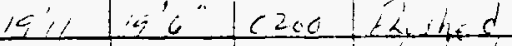

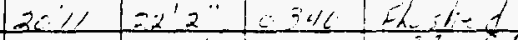
$17>201 \%, 0530$ filete $10,2-225^{-1} 2213010$ tenes \begin{tabular}{l|l|l|l}
110 & $20^{\prime}$ & $2110^{\circ}$ & 310 \\
107 & $10^{\prime} 0$ & 19114 & 2120 \\
\end{tabular} $1<0+2$ 1 inche

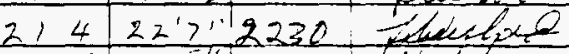

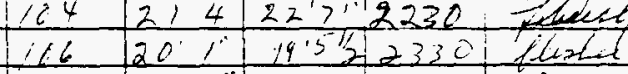

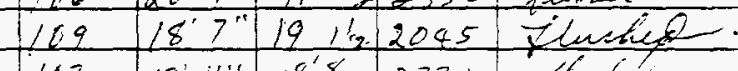

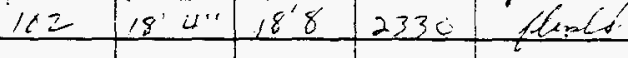

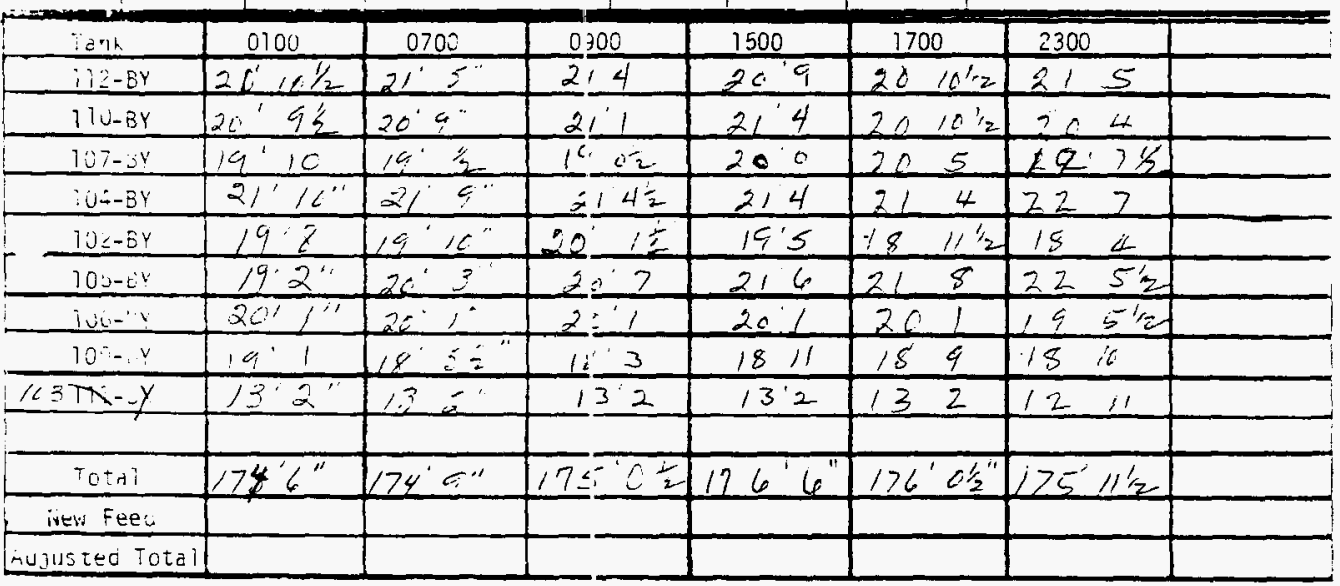

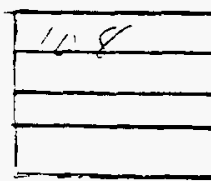

Hat Tracus

(Lizy Stifi)
I IACTIVE TANKS

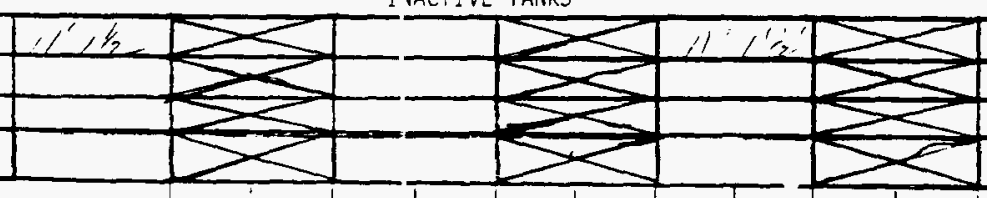

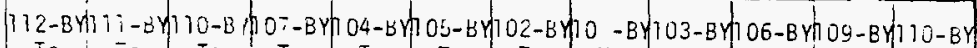
To To To to to To to To To To To To

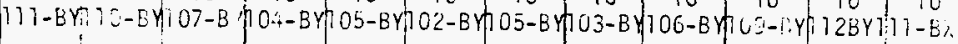

$9-20.71$ 


\begin{tabular}{|c|c|c|c|c|c|c|c|c|c|}
\hline & & Pump-Ou & 1. Tank & & & Rece & ving Tank & & \\
\hline$b=\frac{1 n !}{25}$ & $\begin{array}{c}\text { Pump } \\
\text { ilumber }\end{array}$ & $\begin{array}{l}\text { L. L. } \\
\text { Start }\end{array}$ & $\begin{array}{c}1 . L \text { L. } \\
\text { End }\end{array}$ & $\begin{array}{l}16: 9 \\
\therefore+5 \\
\end{array}$ & Number & $\begin{array}{l}\text { L. L. } \\
\text { Start }\end{array}$ & $\begin{array}{l}\text { L. L. } \\
\text { End }\end{array}$ & 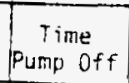 & Remarks \\
\hline $\begin{array}{r}0.26 \\
-c c 30\end{array}$ & , s: & 225 & $193 / 2$ & 23. & 103 & 1211 & 14412 & 2310 & \\
\hline 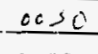 & 104 & $203 / 2$ & 2042 & 18 & 102 & 1818 & 156 & 1215 & \\
\hline
\end{tabular}

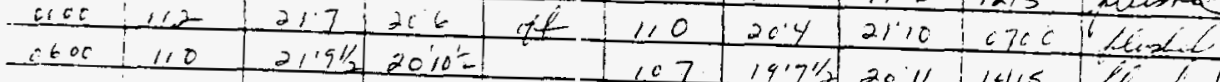

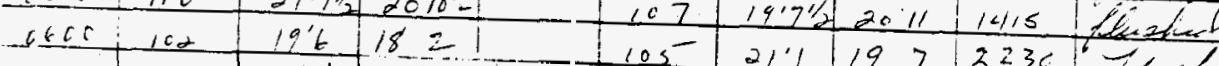

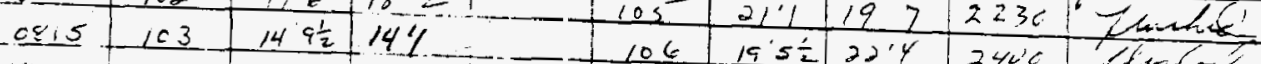
$1215+106 \quad 269 \quad 202 \frac{1}{2}$ $1415: 127+2011+19213$

$12001112 \quad 216206$

$1700: 103 B Y 1141210^{\prime} 0$

$1740110 \quad 12010^{1} 2214$

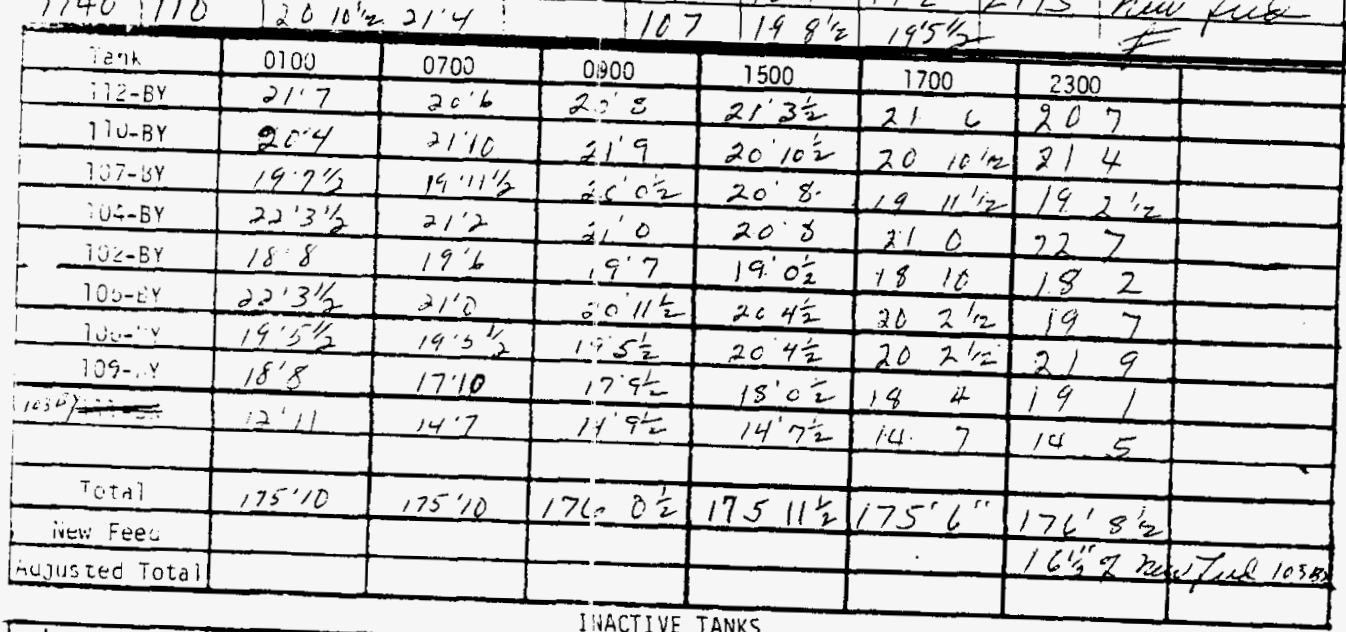

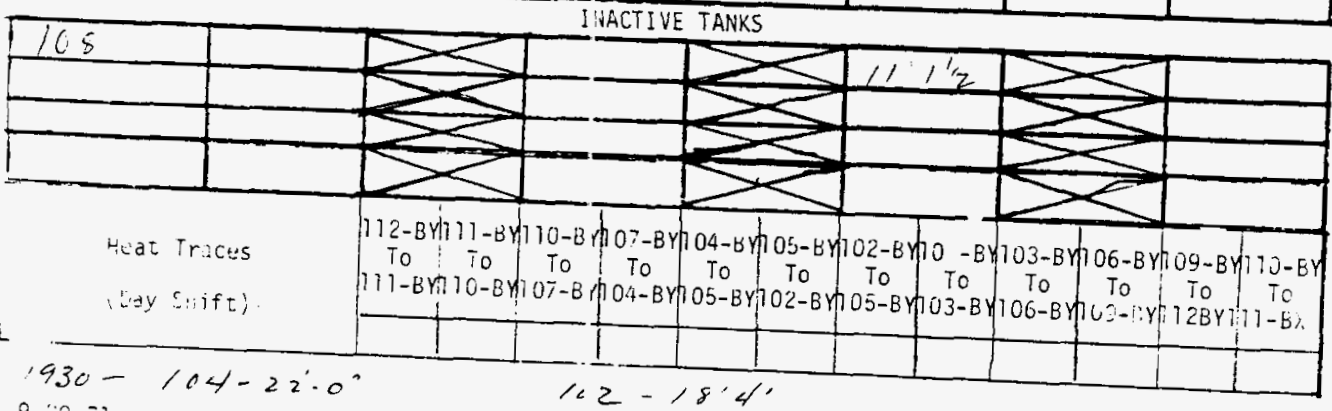

$9-20-71$

\section{BEST AVAILABLE COPY ${ }^{192}$}


Punp-Out Tank

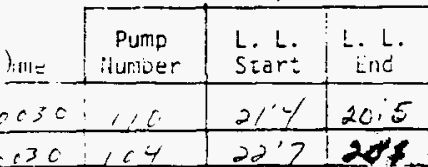

$0902: 112 \quad 21^{\circ} 6: 20^{\circ} 6$

$09001.107 \quad 26510^{\prime} 11$

eqsi

1003

C $23 i+16 i$

150

110

$141+12 \%$

$22^{\circ} 3 \frac{1}{2} 203 / 2$

$19^{\prime} 8^{\prime} 19^{\prime}$

$22^{\circ} 0+20^{\prime} 6 \frac{11}{2}$

Receiving Tank

\begin{tabular}{l|l|l}
\hline L. L. & L. L. & Time \\
\hline
\end{tabular}

Start End Pump off

Remiarks

\begin{tabular}{l|l|l|l}
102 & $193 \%$ & 70.5 & 040 \\
102 & 182 & 19.8 & 2400
\end{tabular}

110

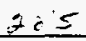

2111

104

212

106

$223 \div$

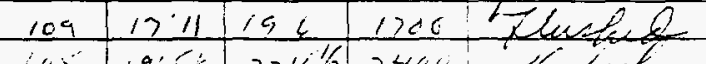

1530

0 !

Alued?

Cluctel.

tewet.

$11+x+6$

107

1811006

\begin{tabular}{|c|c|c|c|c|c|c|c|}
\hline$i a \cdot k$ & 0100 & 0700 & $(1900$ & 1500 & 1700 & 2300 & \\
\hline$\overline{i T 2-E V}$ & 20.8 & 2113 & $=16$ & $20^{\prime} 7$ & 208 & 203 & \\
\hline $11 U-B Y$ & 3114 & $20 \cdot 7$ & $\therefore c s$ & $22^{\circ} 0$ & 218 & 207 & \\
\hline $307-36$ & $14,5 \%$ & $20^{\prime} 2 / 2$ & $2 c 5$ & 1811 & 104 & $20 \quad 312$ & \\
\hline$U \div-B V$ & 2217 & $21 \cdot 7$ & $\therefore 12$ & $21^{\prime} 8$ & $\therefore 14$ & 203 & \\
\hline $10 \leq-6 Y$ & $15^{\prime 2}$ & $19^{\prime 2}$ & $\angle$ & $19^{\prime} 7 \frac{1}{2}$ & $1 \mathrm{C} ? \mathrm{~m}$ & 19 & \\
\hline $1010-e^{\circ}$ & $19^{\prime} 5$ & $19 \cdot 5$ & $19.5 \frac{1}{2}$ & $20110 \frac{1}{2}$ & $21,1 / 2$ & 22212 & \\
\hline lici- : & $32+4$ & $22 \cdot 4$ & 2232 & $21^{\circ} 2$ & $203 \%$ & $20 \quad 312$ & \\
\hline $10 \div-2$ & $18 \% \frac{1}{2}$ & $18 \%$ & 1711 & $19 \cdot 4$ & 1911 & 19212 & \\
\hline $123-\beta Y$ & $14 \%$ & $14 \%$ & 141 & $12 \% 11$ & 1211 & 1211 & \\
\hline & $176 \cdot 10$ & & & & & & \\
\hline Total & $\Rightarrow$ & $17681 / 2$ & $176^{\circ} 11$ & 177 & $176^{\prime} 9^{\prime} 2$ & $176 \quad 6$ & \\
\hline ivew Fee: & & & & & & & \\
\hline Hujusted Totall & & & & & & & \\
\hline
\end{tabular}

INACTIVE TANKS

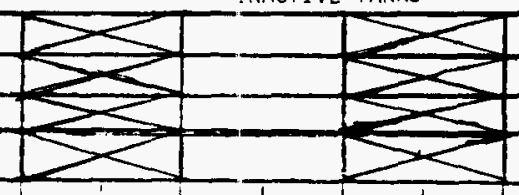

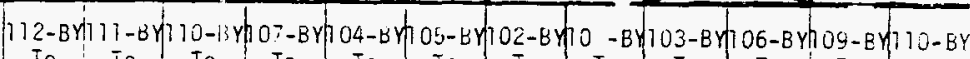

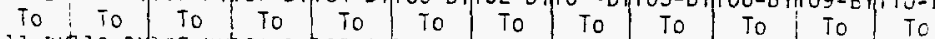

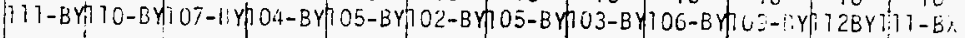

i

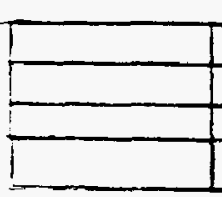

Hicat ir:cess

(idy $s:$ if $t$ )

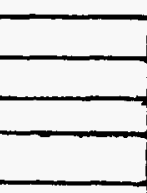

)

(1)

$9-i 0-7 i$ 
iTS-2 IIVENTOR AIND PUNPIIIG $\quad-$ Cate $5-22-7=$

Punp-0ut iank

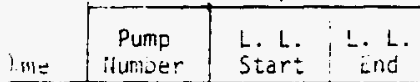

Receiving Tank

\begin{tabular}{c|c|c|c|} 
L. L. & L. L. & Time & \\
Start & End & Pump off & Remarks \\
\hline
\end{tabular}

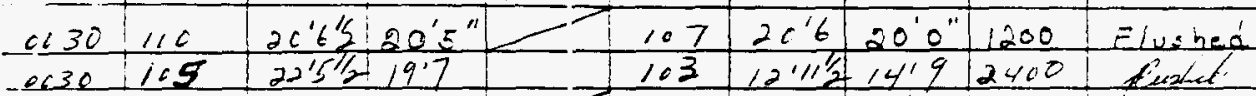

$6366^{1127}: 21^{\prime 6}: 20^{\prime} 6^{\prime \prime}$

\begin{tabular}{l|l|l|l|}
0.001 & $10 ?$ & $20^{\prime} 6$ & $19^{\prime} 2 \frac{1}{2}$ \\
\hline
\end{tabular}

$=600+10 \quad 209+20^{\prime} 2$

$-\operatorname{cic} i=2$

$\therefore 001103$

$1100: 106$

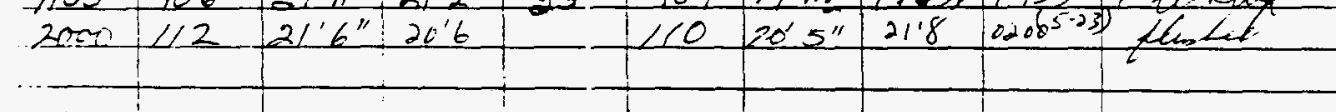

\begin{tabular}{l|l|l|}
\hline $19^{\prime 8}$ & $19^{\prime 2}$ \\
\hline & 3 \\
\hline
\end{tabular}

110 26L $c^{\circ} 010 \% 0030$ Elushea

$12420112{ }^{\prime} 6^{\prime \prime} 1630$ Ipentel!

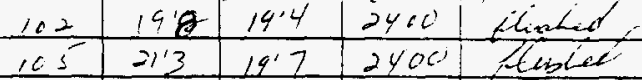

$14^{\prime 3} 13^{\prime} 11^{\prime \prime}$

\begin{tabular}{l|l}
105 & 213 \\
\hline 106 & $70 \% 3 \frac{1}{2}$
\end{tabular}

\begin{tabular}{l|l|l}
\hline $1^{\prime} / l^{\prime \prime}$ & $21^{\prime} 2^{\prime \prime}$ & 25 \\
\end{tabular}

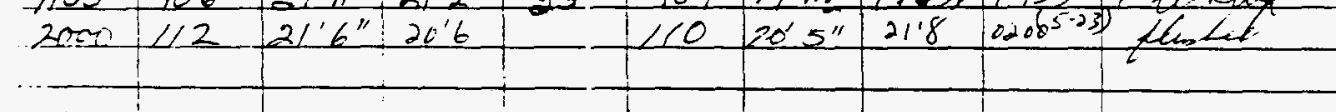

109

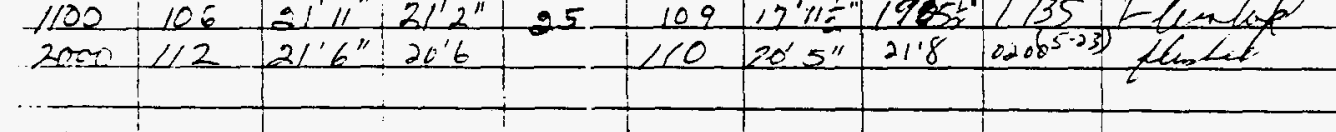

$222^{\prime \prime 2} 2200$

Fientibs

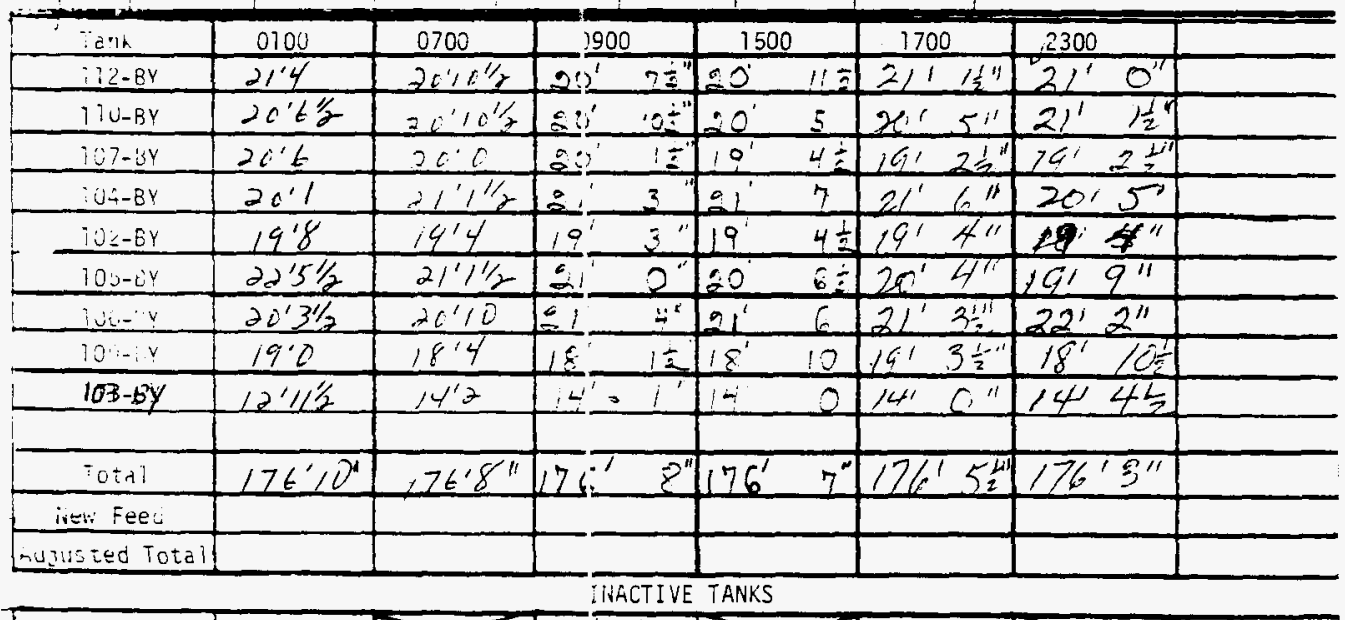

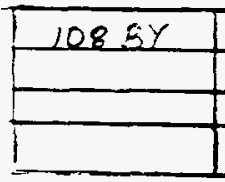

Heat Tracus

(bay $S: ; \neq z)$

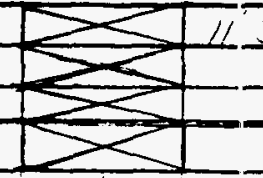

$112-B$ Y $1111-B Y 110$
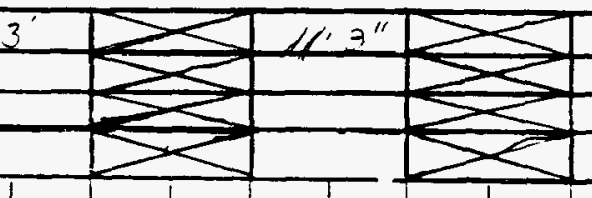

1

Yh 07-BY 04-6Y 05-BY102-BY10 -BY703-BY 06-BYh 09-BY110-EY

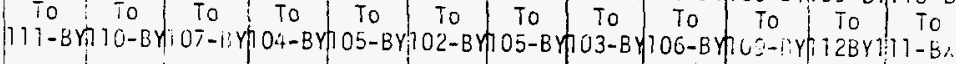

L 1

1

$+$

$1+1$ 
ITS-2 IIUEETORY ANU PUMPING $\quad-\quad$ Cate

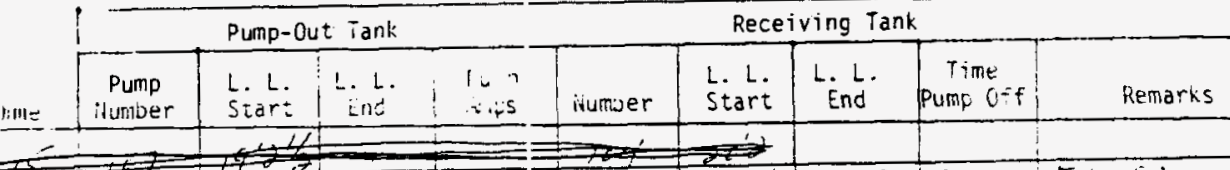

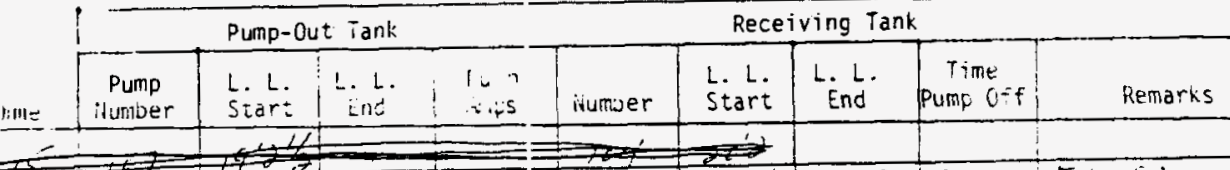

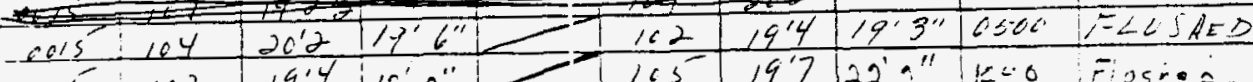

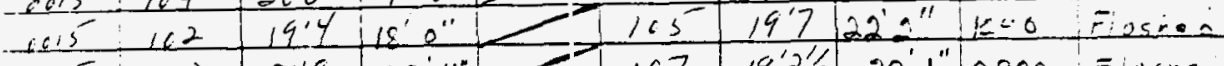

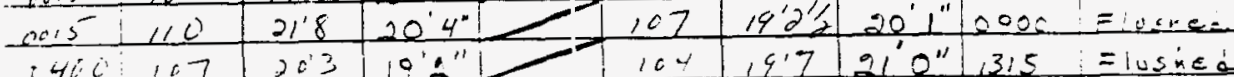

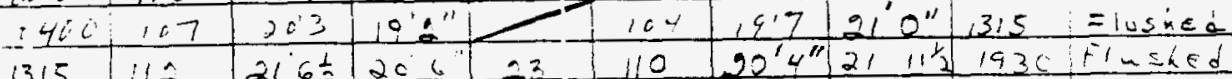

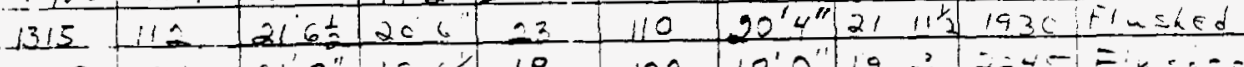

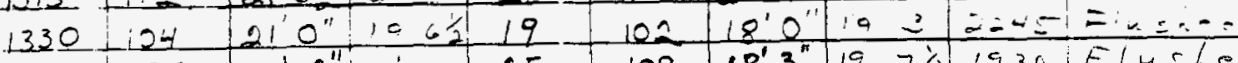

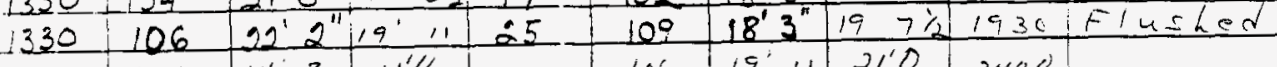

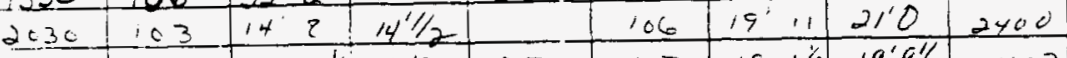

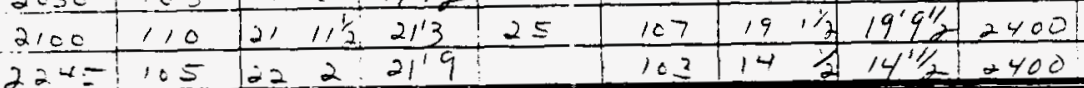

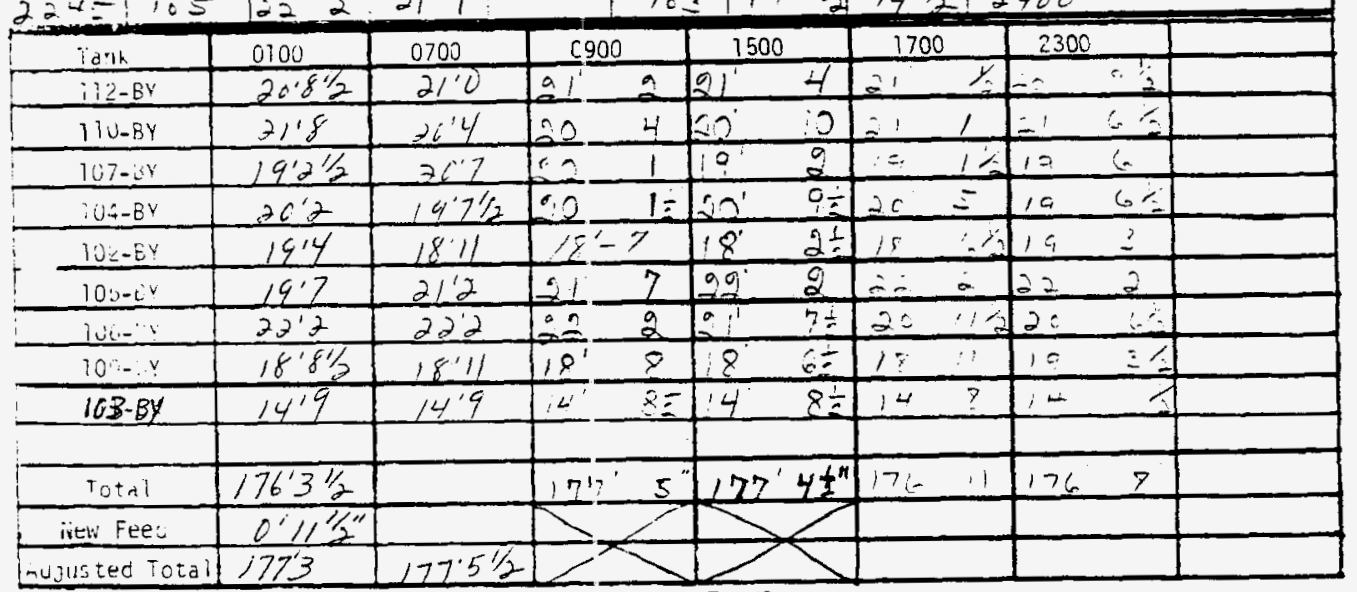

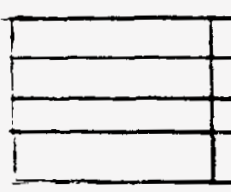

Heat ir:ces

(Lizy 5 : ift)

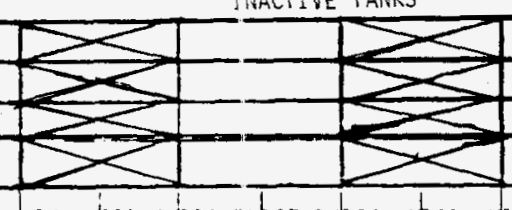

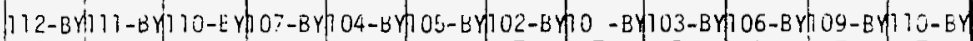
To to to to to to

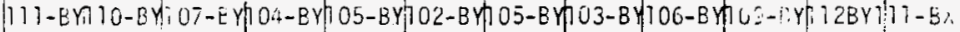

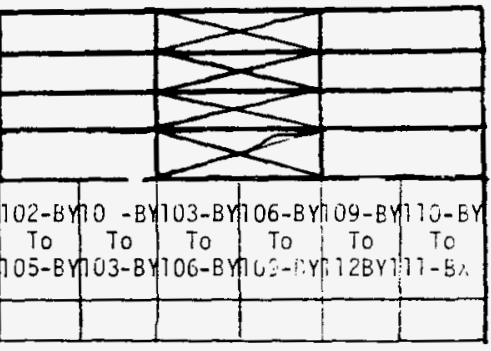

\llcorner

\begin{tabular}{c|c|c|}
\hline \\
\hline
\end{tabular}

\section{$9-20-71$ \\ BEST AVAILABLE C,OPY}


ITS-2 IINENTOR" AIUU PUMPING $\quad-\cdots$ Cate

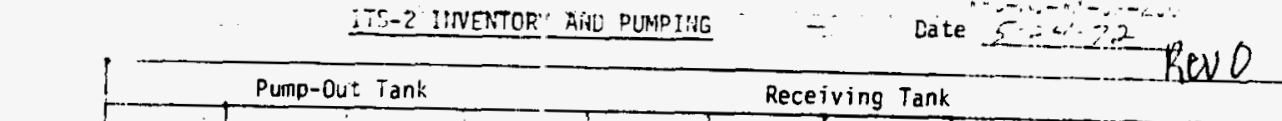

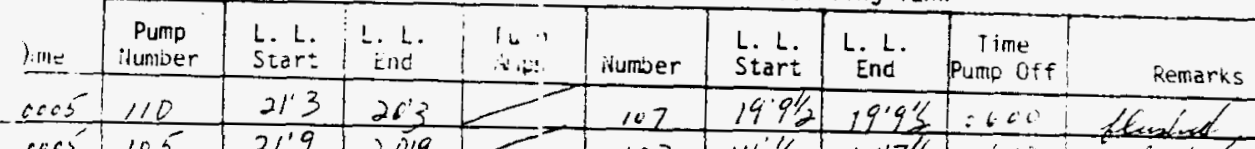

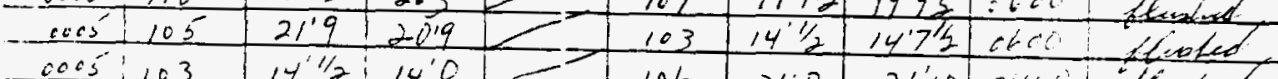

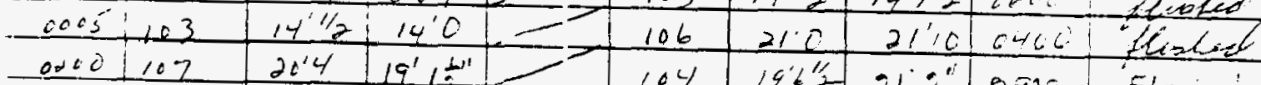

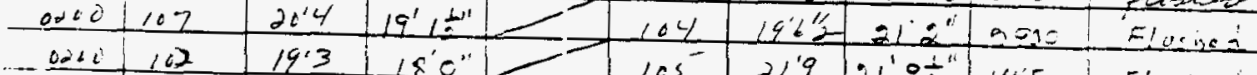

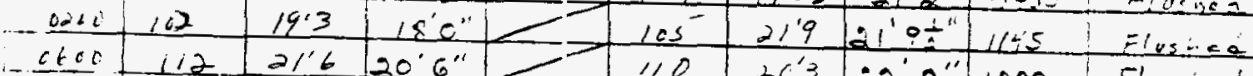

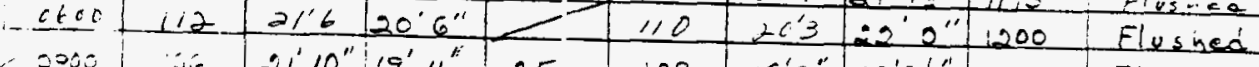

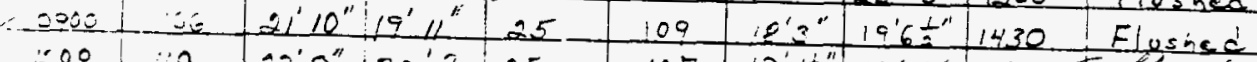

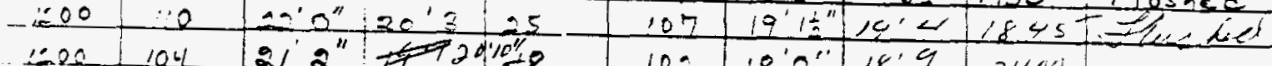

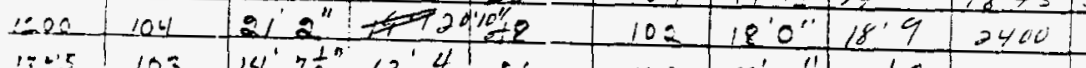

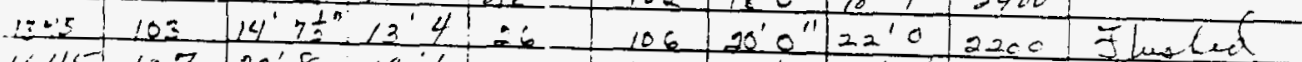

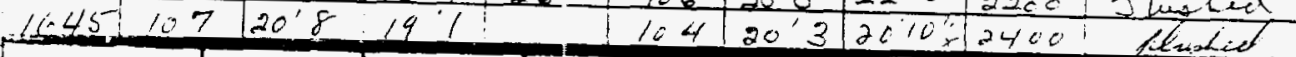

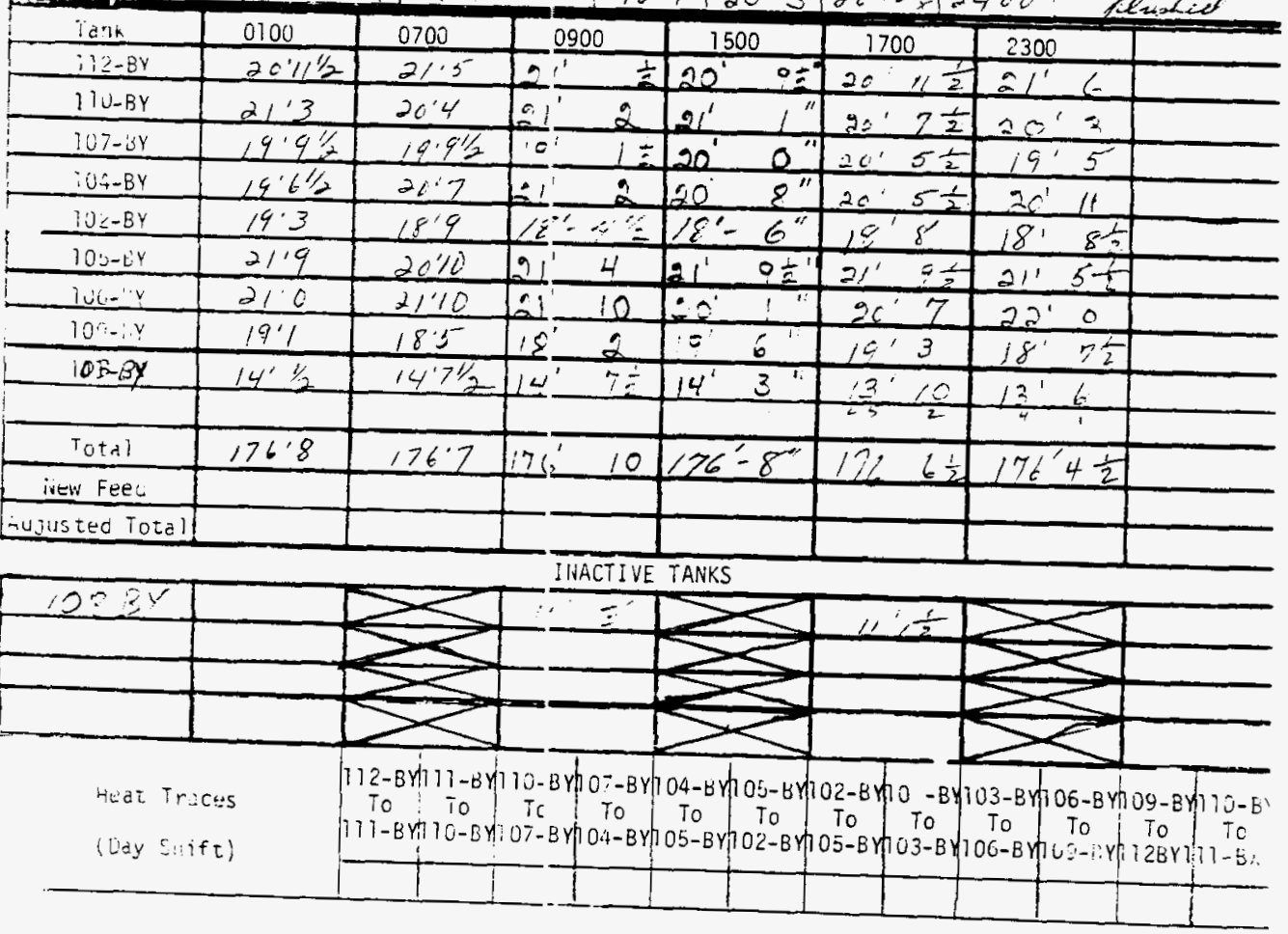

$9-20-7 i$

BEST AVAILABLE COPY' 


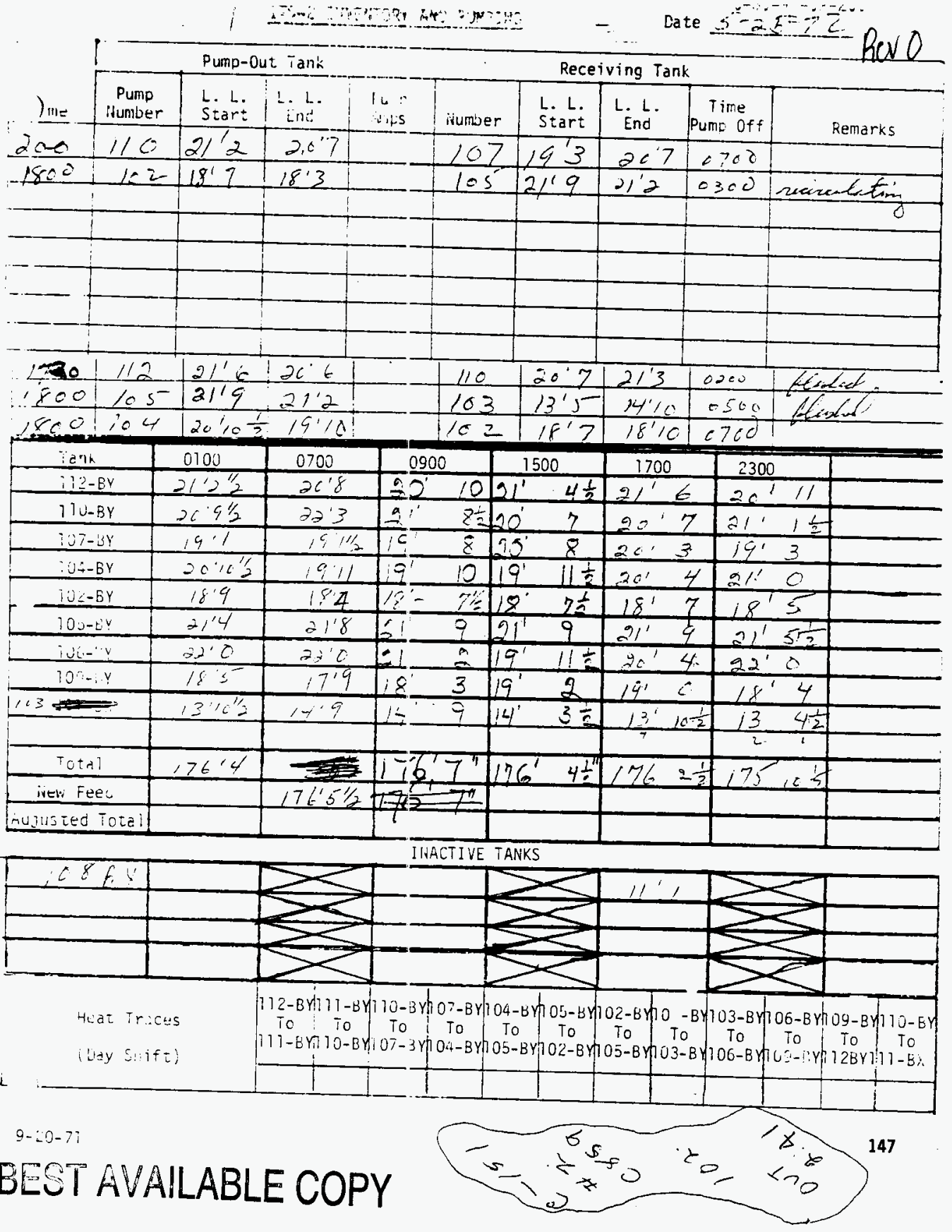


$-\quad$ Cate ${ }^{n-5}=x^{4}-2-206$ Revl Punp-Out Tank Receiving Tank

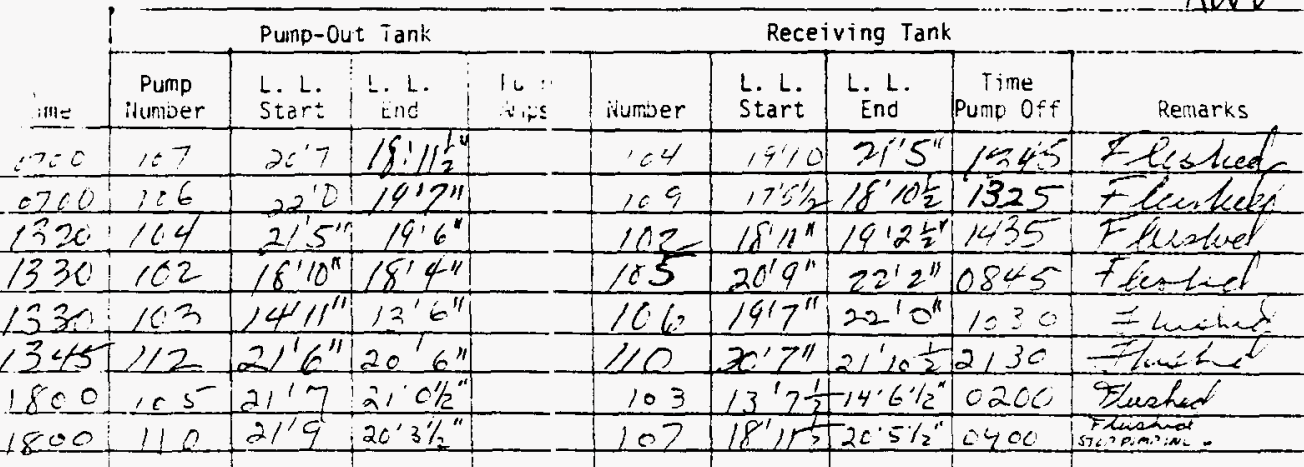

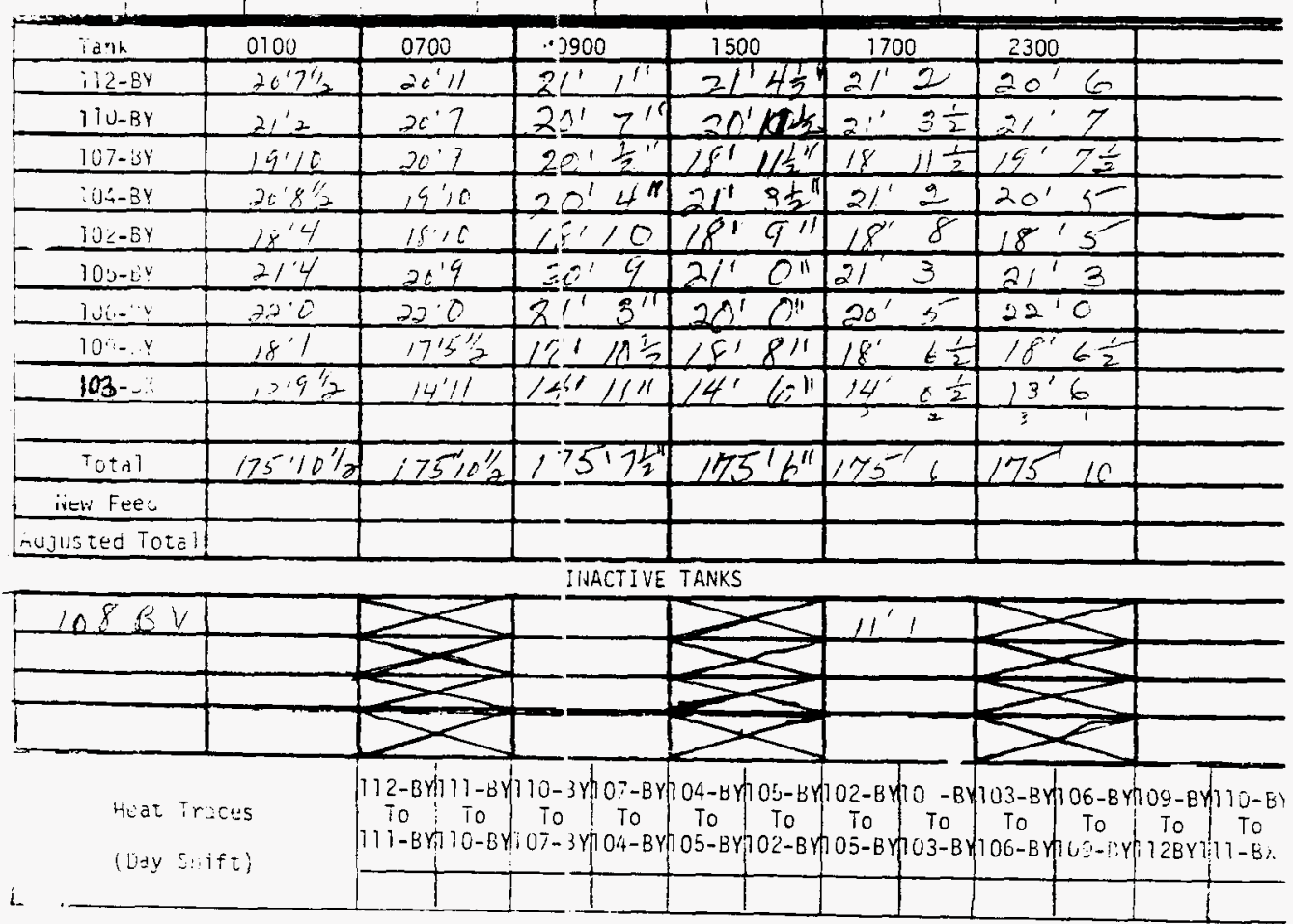




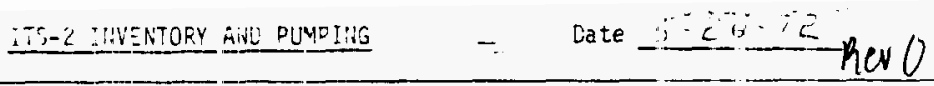

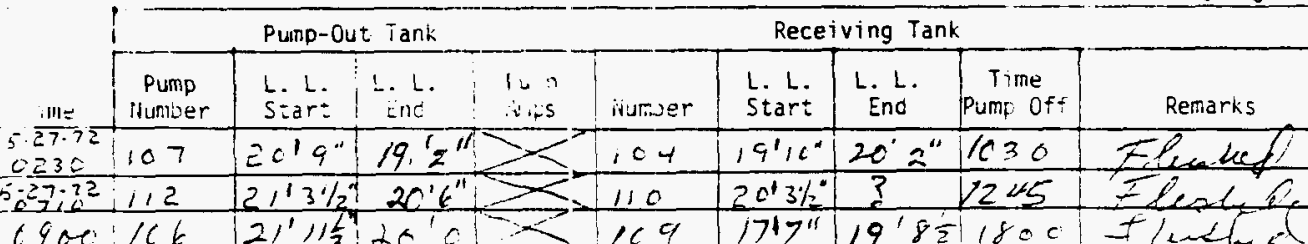

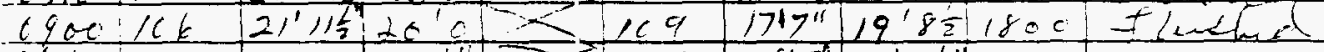

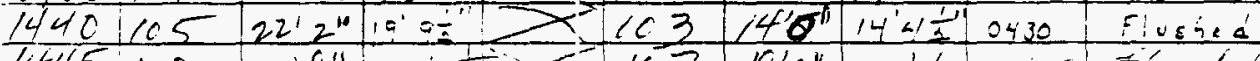

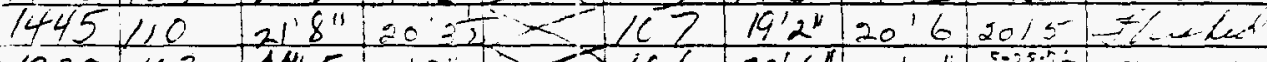

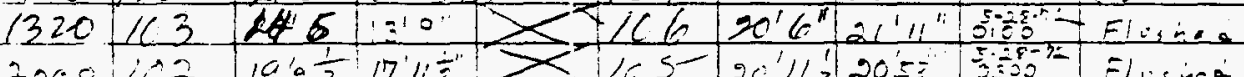

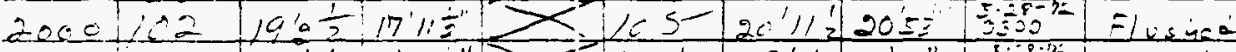

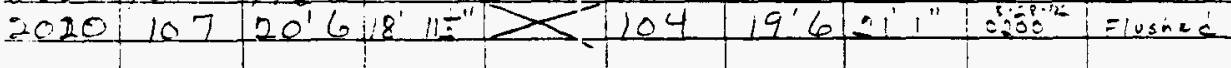

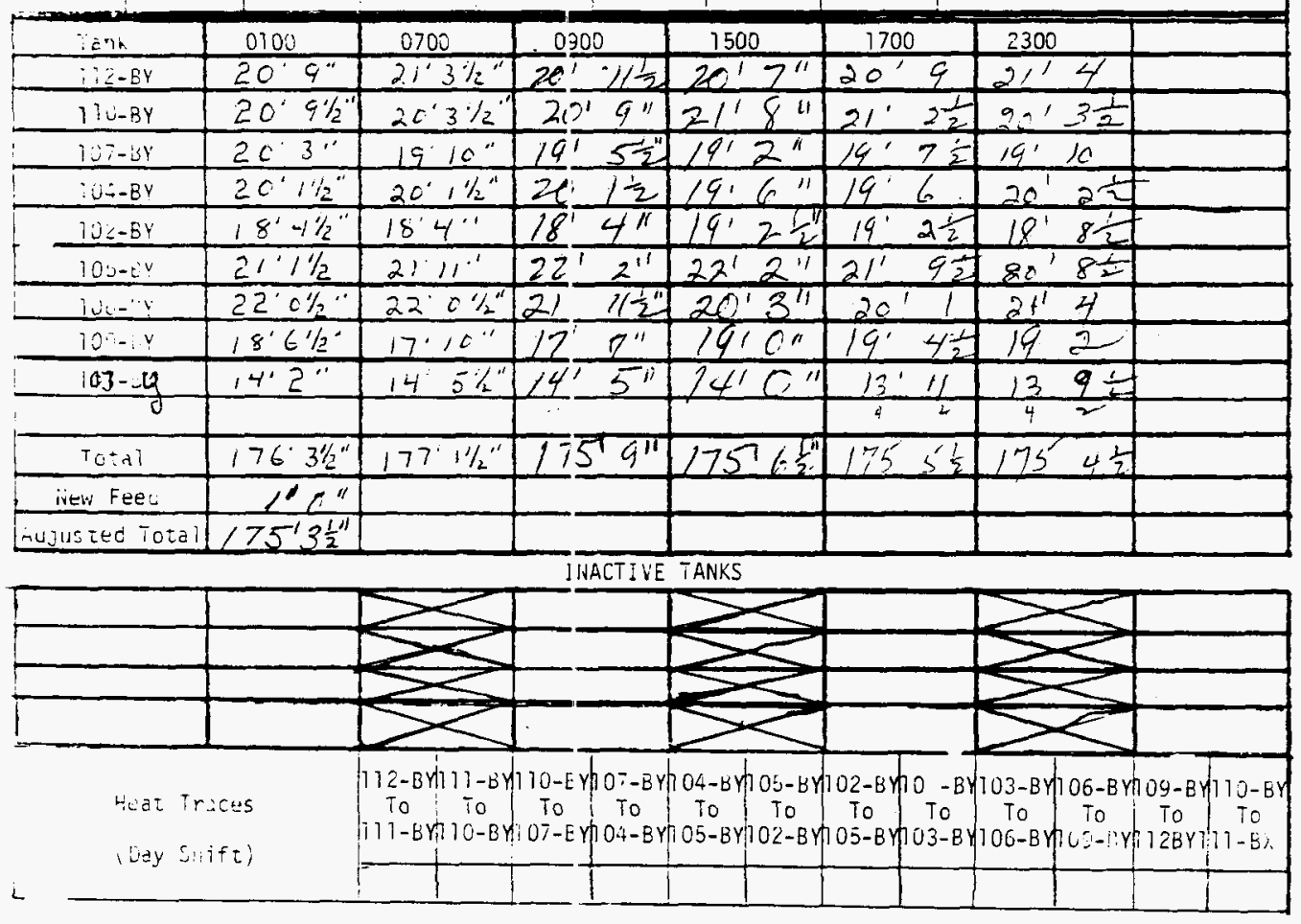

$9-20-7 i$ 
Pump-Out Tank

Receiving Tank

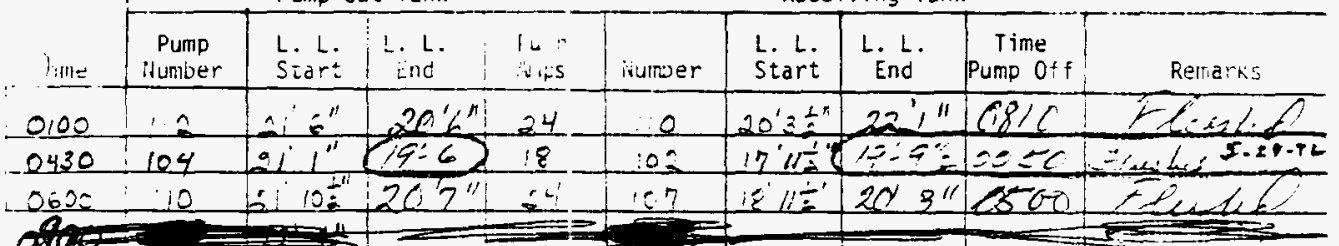
$\operatorname{coc} 21038 \times 12^{2}-11^{\prime} 7^{\prime \prime}$

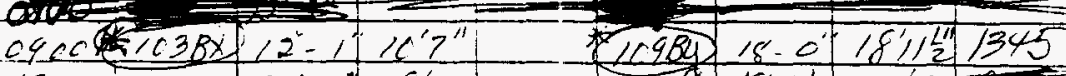

1300 - $107-\frac{x^{\prime}-7 \mid 190}{19}$

1040 $10=19$ ' 10 :-2:

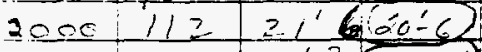

$2232: 110 \quad 21^{\prime 3}=0: 9:$

$\Rightarrow$

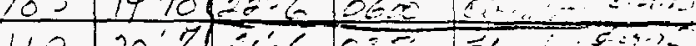

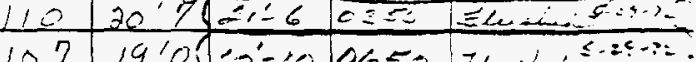

\footnotetext{
'...
}

\begin{tabular}{|c|c|c|c|c|c|c|c|c|}
\hline anth & 01 & & 0700 & $1) 900$ & 1500 & 1700 & 2300 & \\
\hline$i Z 2-E Y$ & 2.1 & G & $50^{\circ}$ & $=0^{\prime} 6^{\prime \prime}$ & $31^{\prime \prime} 2^{\prime \prime}$ & 2114 & 2,13 & \\
\hline$I T U-B Y$ & 23 & $\vdots \bar{\vdots}$ & $\therefore: 1$ & $62^{\prime} 0^{\prime \prime}$ & $20^{\prime} 7^{\prime \prime}$ & $20^{\prime} 7$ & 213 & \\
\hline $107-30$ & 19 & $3^{\prime \prime}$ & $\bar{y}$ & $13^{\prime} 4^{\prime \prime}$ & $20^{\prime} 3^{\prime \prime}$ & 19111 & 1910 & \\
\hline$: 04-B Y$ & 22 & $c^{\prime \prime}$ & $E$ & 201471 & $19^{\prime} 10 \frac{1}{2}$ & 20 & $19^{\circ} \quad 107$ & \\
\hline $10<-B Y$ & $18^{\circ}$ & 43 & $5 \div$ & $1819^{\prime \prime}$ & 19 & $19^{\prime}$ & $19,10 \%$ & \\
\hline $100-e^{2}$ & $60^{\circ}$ & $7^{\prime \prime}$ & $\bar{t}$ & $14 \cdot 10^{\prime \prime}$ & $19^{\prime} 10^{\prime \prime}$ & $19 \quad 10$ & 21,2 & \\
\hline $1 v i-\cdots$ & $21^{1}$ & $11^{\prime \prime}$ & $6:$ & $2: 11 \frac{1}{2}$ & 21115 & $21^{\prime} \quad 11$ & 21116 & \\
\hline $10 \div-y$ & $8^{\prime}$ & $\pi$ & $y$ & i $C^{\prime \prime}$ & $18^{\prime} 92^{\prime \prime}$ & $18^{\prime \prime} \quad 8^{\prime}$ & 1915 & \\
\hline $103-34$ & 13 & 9 & 4 & $14+14^{\prime \prime}$ & $14^{\prime}<t^{\prime \prime}$ & 1414 & $14^{\prime} 4$ & \\
\hline & & & & & & 2 & 71 & \\
\hline Tothl & $4: 5$ & $4^{\prime \prime}$ & $1 \div 5^{1}$ & $1751 \frac{1}{2}$ & $176^{\prime} 6^{\prime \prime}$ & $17167 \frac{1}{2}$ & $128^{1}$ & \\
\hline ivew Fee: & & & & & $86^{\prime} 6^{\prime \prime}$ & & & \\
\hline mujusted Totall & & & & & 175 & & & \\
\hline
\end{tabular}

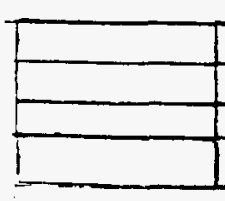

Heat Traces

(Lity Sifift)

INACTIVE TANKS

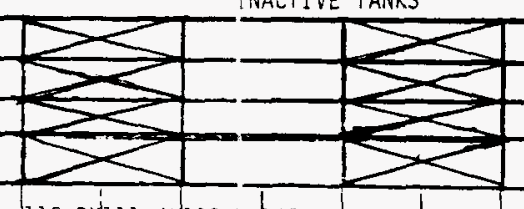

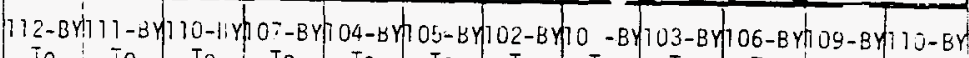
To To To To To To To To To To To To

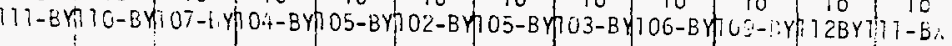

L

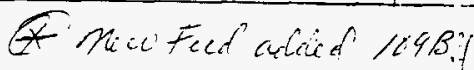

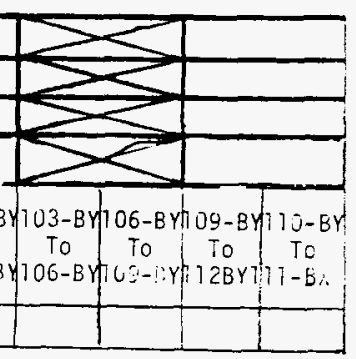

$9-20-7 i$

BEST AVAILABLE COPY 


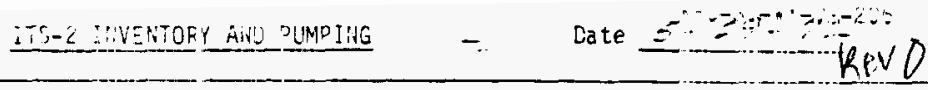
Pump-Out Tank Receiving Tank

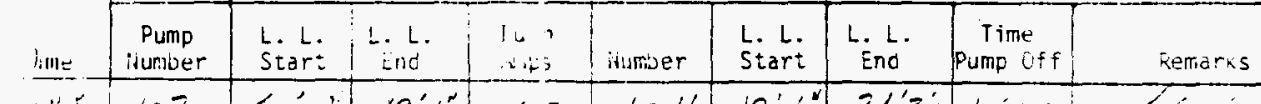

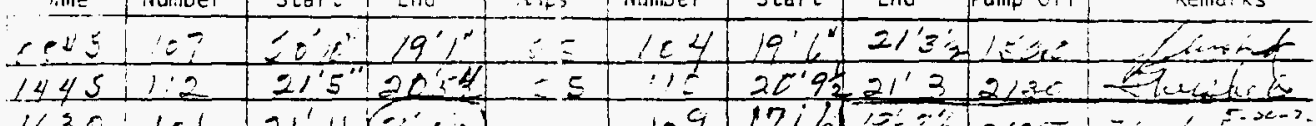

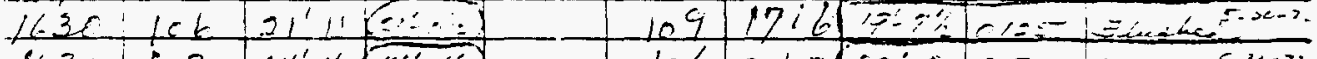
$163010314^{\prime} 40$ 163,105 22. 10.10 $1225111021.52=215$ $\left.2230-10720^{\prime} 77^{\prime}: 0\right)$ -2230162 18'1128120 $2230104213 \div(19-6)$

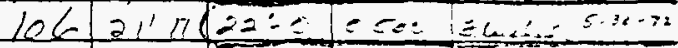

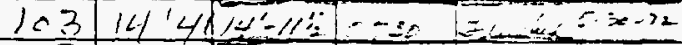
$10 ? 19^{\prime} 01=0=0.50=0.36$

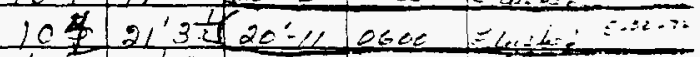

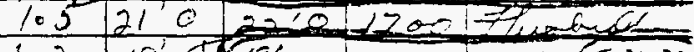

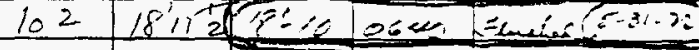

\begin{tabular}{|c|c|c|c|c|c|c|c|}
\hline Tark & 0100 & 0700 & 0900 & 1500 & 1700 & 2300 & \\
\hline $\mathrm{i} i 2-\mathrm{BV}$ & $=6-1, \%$ & $200-7$ & $=0.10^{\prime \prime}$ & $2 r^{\prime \prime}{ }^{\prime \prime}$ & $21^{\prime} \geq \frac{1}{2}$ & $20^{\prime} 6 \frac{1}{2}$ & \\
\hline $11 U-B Y$ & $\because 2=$ & $E=-2$ & $2=-9 k$ & $2019:$ & $21^{\prime} 3$ & 2110 & \\
\hline $107-34$ & 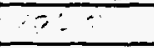 & $=-\therefore \quad \cdots$ & $20-10^{\prime \prime}$ & $19^{\prime}{ }^{\prime}$ & $19^{\prime} \mathrm{c}$ & 2617 & \\
\hline$: 04-8 Y$ & $\because \div$ & $19^{\circ}-1=$ & $1916^{\prime \prime}$ & $21^{\prime} 3 " 1$ & $21^{\prime}=1 \div$ & $21 \cdot 3 \frac{1}{2}$ & \\
\hline $10 \div-B Y$ & $\because:$ & $3 \div-11$ & $1 E^{\prime}-112^{\prime \prime}$ & $8 \quad \because 5$ & $28^{\prime} 11+\frac{1}{2}$ & $18^{\prime \prime} 11^{3}$ & \\
\hline $100-e^{2}$ & $\because$ & 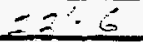 & $22-6$ & $=2^{\prime} \quad 6$ & 221 & $21^{\prime} 0$ & \\
\hline $10 x-2$ & $=$ & $=1-1$ & $\leq y \div 11$ & 2 & 2111 & $21^{\prime} \quad 3 \cdot \frac{1}{2}$ & \\
\hline $10-i, y$ & $\because 11$ & $-\because$ & $15^{\prime}-5^{\prime}$ & $12^{\prime} 8^{\prime \prime}$ & $17^{\prime} 6$ & $18^{\prime} 11$ & \\
\hline 103-BY & $\approx-2$ & $\because \because 4$ & $4^{\prime}-4^{\prime \prime}$ & $14^{\prime} 4^{\prime \prime}$ & $14^{\prime} 4$ & $141 \%$ & \\
\hline & & & & & 3 & 2 & \\
\hline$T_{0}[A]$ & $178^{\prime}-1$ & $7 \varepsilon^{\prime}-1$ & $1^{\prime \prime} 8^{\prime}-1^{\prime \prime}$ & $177^{\prime} 111^{\prime \prime}$ & $17711 \frac{1}{2}$ & $177^{\prime} 4$ & \\
\hline inew Fees & & & & & & & \\
\hline Ujusted Totall & & & & & & & \\
\hline
\end{tabular}

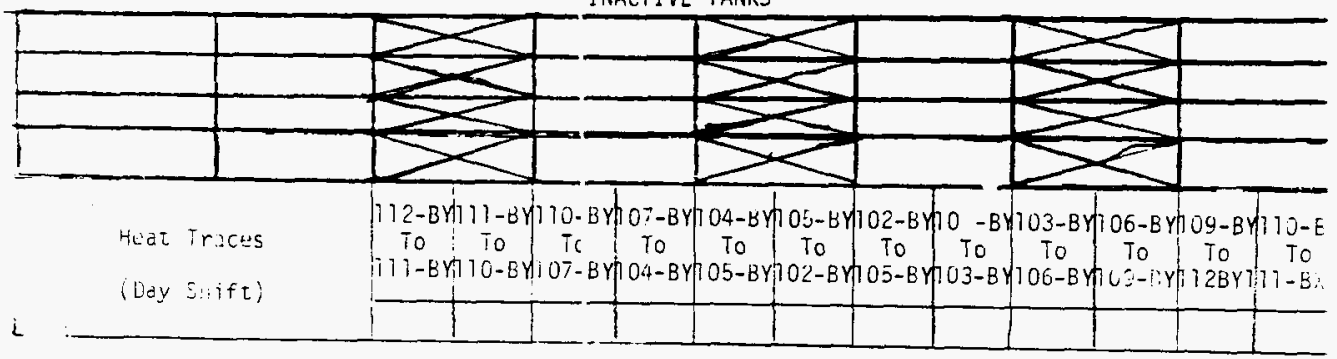




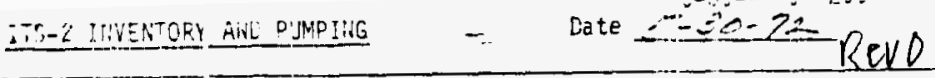
Puinp-Out Tank

Receiving Tank

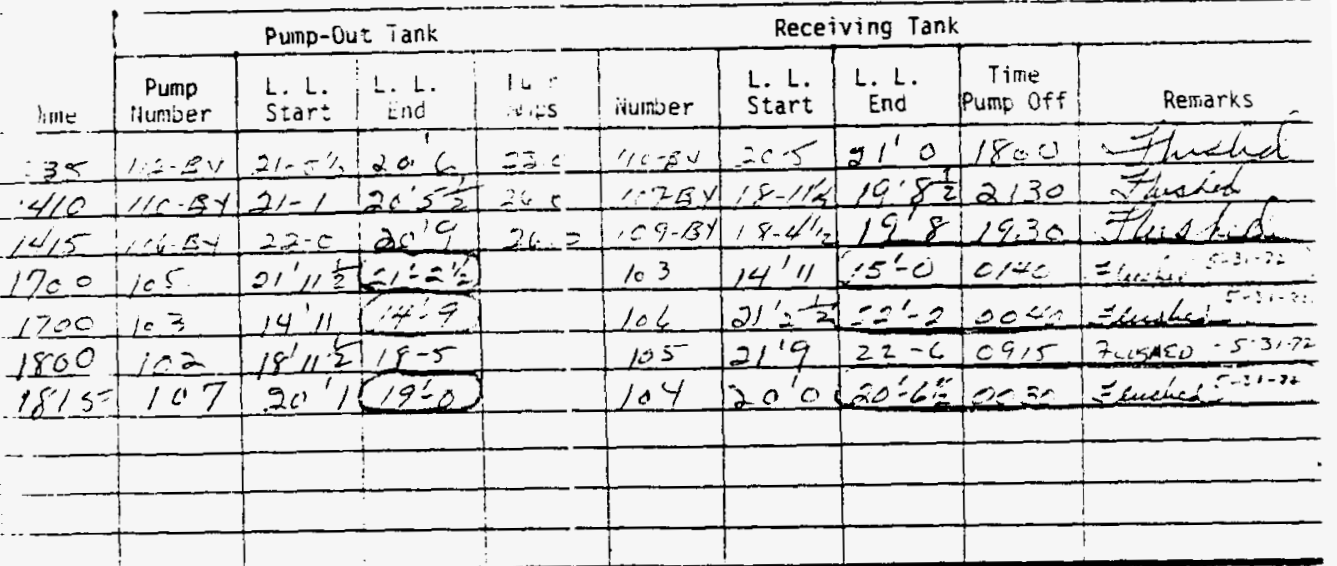

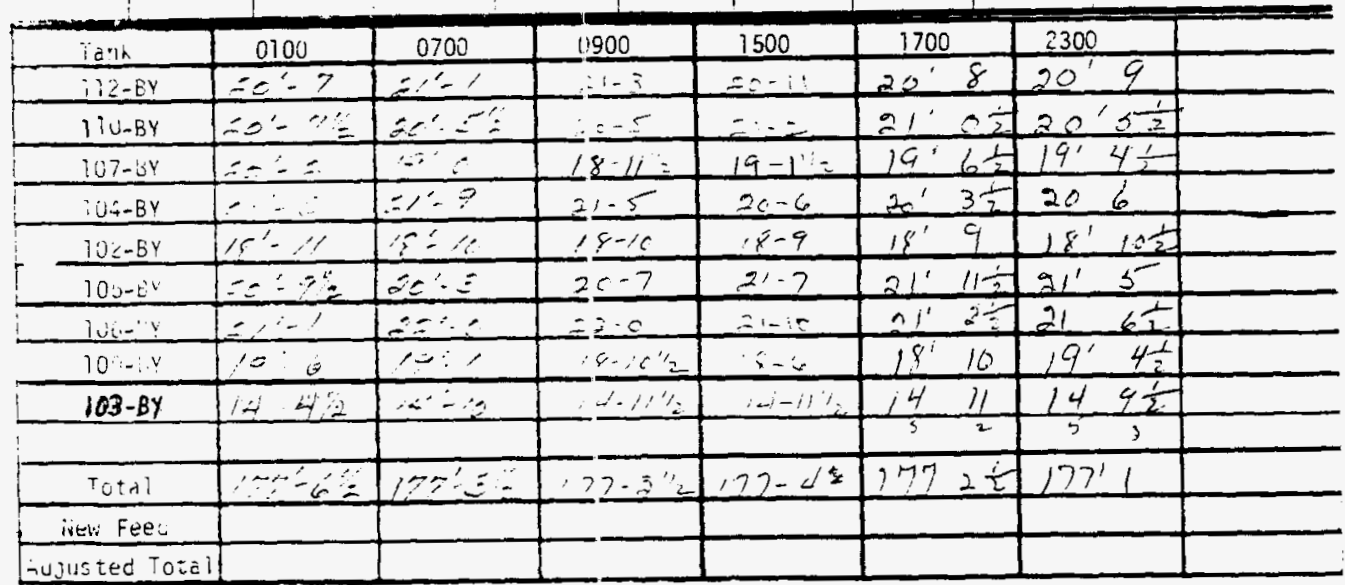
IIACT IVE TANKS

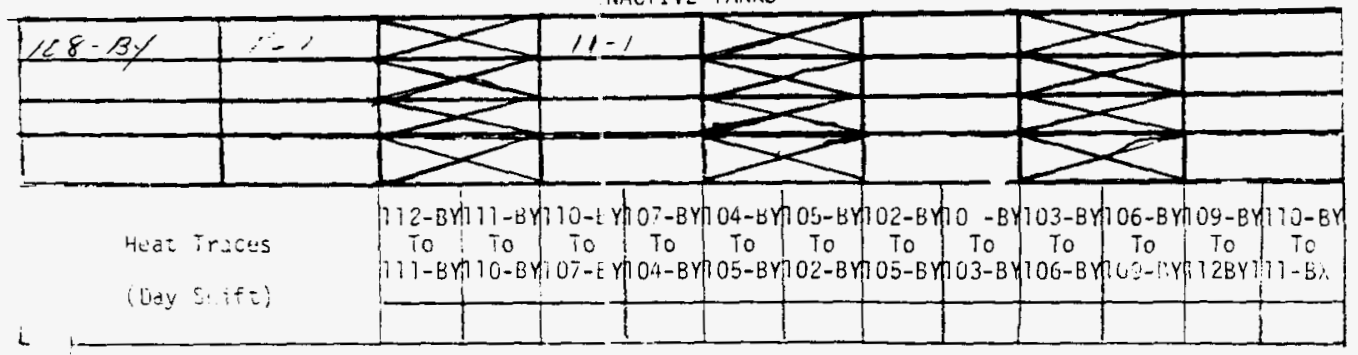

$0-20-7 i$ 
IT-2 IINVENORY AINi PUMPING $\quad-$ Date Punp-Out Tank

Receiving Tank

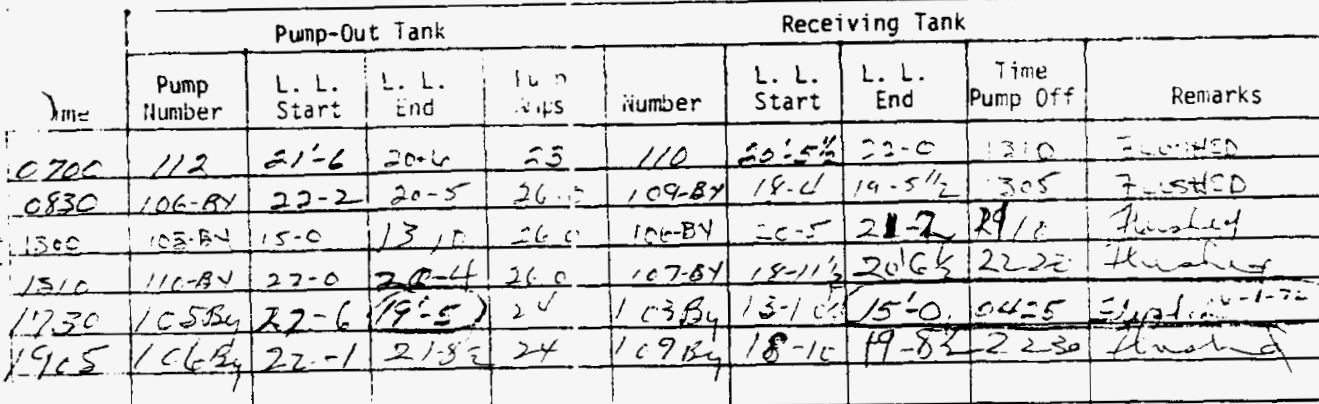

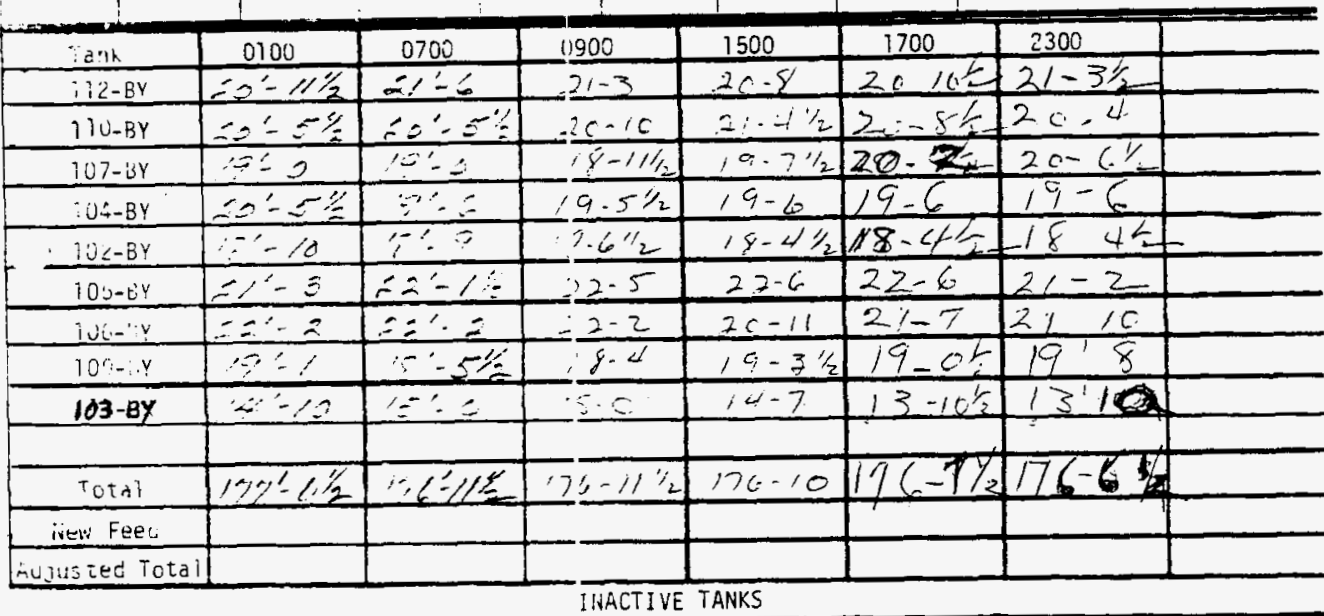

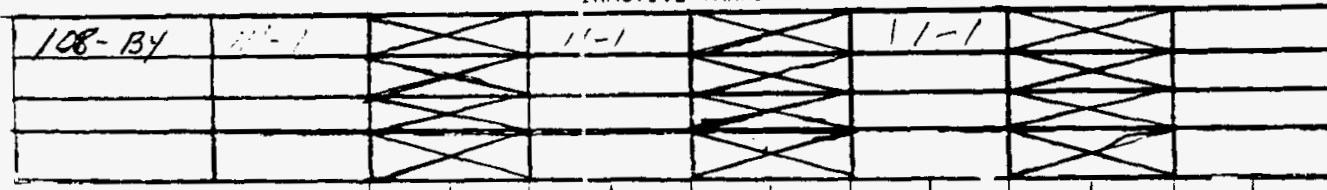

Hetat Tricts

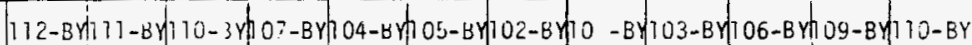

(Dey Slifi)

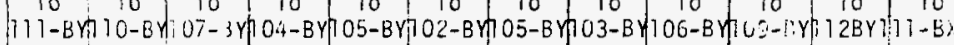

i

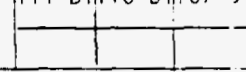

\begin{tabular}{l|l|l|l|}
\hline & & & \\
\hline
\end{tabular}

(2) 


\section{IT,-2 IHVENTORY Aive PUMPING $\quad-$ Cate}

Pump-0ut Tank

\begin{tabular}{l|l:l} 
Pump & L. L. & I. L L.
\end{tabular}

ins ilumber Start ind
Receiving Tank

\begin{tabular}{l|c|c} 
L. L. & L. L. & Tilre \\
Start & End & Pume oft
\end{tabular}

Reriarks

\begin{tabular}{|c|c|c|c|c|c|c|c|c|c|}
\hline$=110$ & 17 & $2^{2} 0^{2}-6$ & $19 \leq 0$ & $=6$ & $\angle C \div$ & 1916 & $\because \therefore 2$ & $0: \therefore$ & $\angle r<C$ \\
\hline$\therefore \angle E O$ & $\because=$ & $21 \div 6$ & $20 \cdot 6 \%$ & 24 & lis & $20: 4$ & $22^{\prime}, 1 ' s$ & 0,5 & $22 x+20$ \\
\hline 0,000 & $16^{4}$ & 210 & $2 m-$ & & 102 & $15^{\prime} 4 \prime^{\prime} 2$ & $i q=i c$ & 1718 & $\therefore$ \\
\hline 0,000 & 110 & $222-$ & $21-0$ & & 167 & $14^{\circ} 6^{\circ}$ & $19 \%$ & 223 & $72=1+4 U_{2}$ \\
\hline 13 & 106 & $2111^{\circ}$ & 19512 & 24 & 109 & 182 & 19.7 & $192 \varepsilon$ & $=16+2=1, c+f_{i}^{\prime}$ \\
\hline 15 & 107 & 203 & g-1 & & 104 & $199 / 2$ & $21-3$ & $22 Z_{0}$ & $11+2+6 c$ \\
\hline & $\angle \leq 312$ & $14-11^{2}$ & 7 & $\geqslant 6$ & $16 G i$ & $19-5 / 2$ & $=1-\angle$ & $0, \ldots$ & $=6 \cos ^{--2-8=}$ \\
\hline & $\mid 12+t_{3}$ & $2+5 / 2+$ & $20=$ & & $11 \cos _{3}$ & $20 \% 6:$ & $2,10^{\prime \prime}$ & $E=0$ & 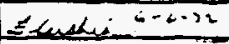 \\
\hline & $\therefore 0=1$ & $? ?$ & $\angle 8=0)$ & & 105 & $? ?$ & $21: 9$ & 0500 & seis $=\cos _{0} x-2=$ \\
\hline
\end{tabular}

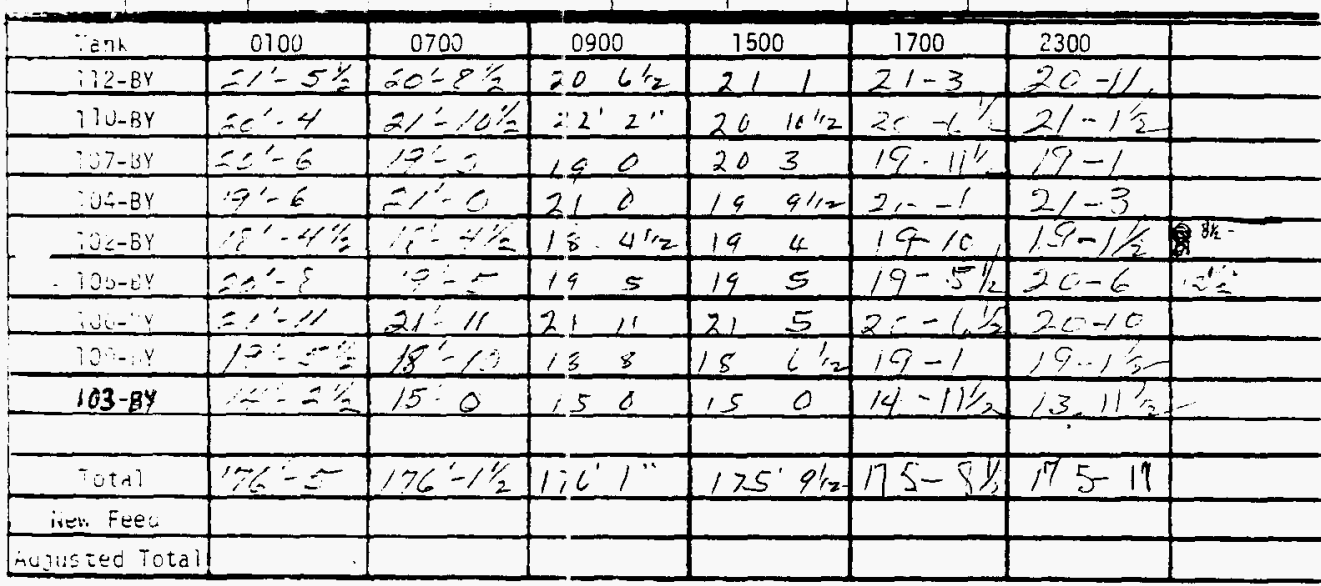

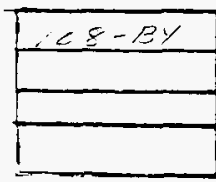

Heat Tricus

(Wdy S: ift)

\section{INACT I VE TANKS}

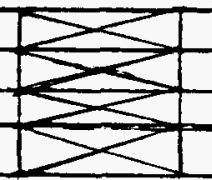

$112-8$ หri $111-8 Y 110-8$

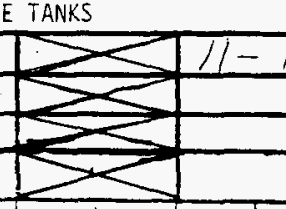

the

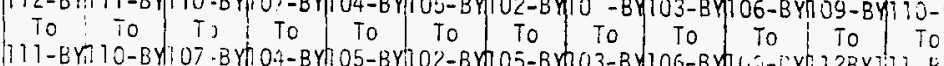

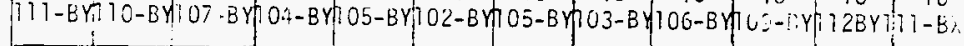

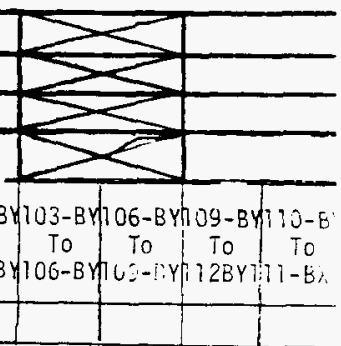

i

$$
9 \begin{array}{r}
354850 \\
72261 \\
10.5
\end{array}
$$


Date ...E

\section{Pump-Out iank Receiving Tank}

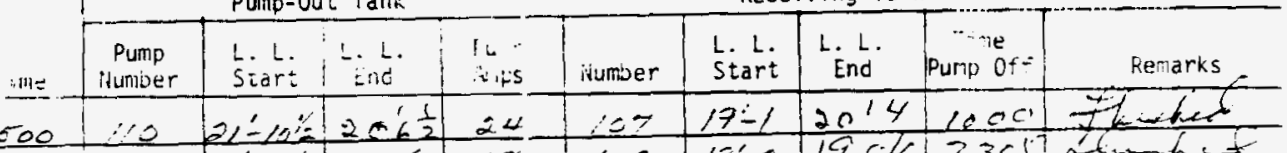

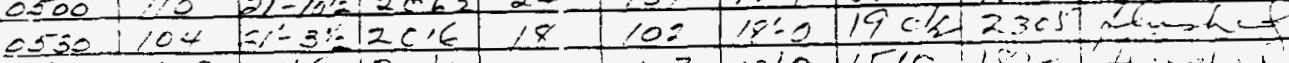

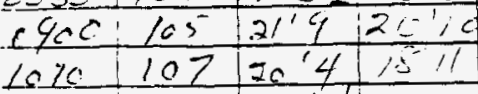

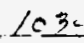

$1=30102 \frac{1812}{103} 11 / 1310 \frac{1}{2}$

$\frac{610}{2235 / 16}-216 / 2$

$2731 / 66122 i$

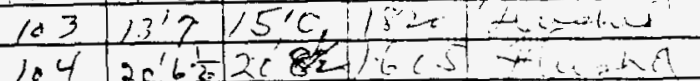
$105 \sqrt{21} 4 \frac{1}{2}$

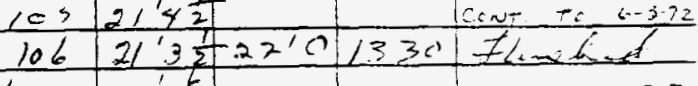

\begin{tabular}{l|l|l|} 
& & \\
& &
\end{tabular}

\begin{tabular}{|c|c|c|c|c|c|c|c|}
\hline lank & 0100 & 0700 & 3900 & 1500 & 1700 & 2300 & \\
\hline$i 12-B Y$ & $-0,-7 \%$ & $30-11$ & 216 & $21^{\prime} 5$ & 216 & $20^{\prime 83}$ & \\
\hline $110-B Y$ & $21-2 \%$ & -1232 & $20^{\circ} 10 \frac{7}{2}$ & $20^{1}<\frac{2}{2}$ & 209 & 21,111 & \\
\hline $107-3 Y$ & $\therefore=1$ & $9 \leq 7 \div$ & $7 \div=0 \frac{1}{2}$ & $19 \quad 3-\frac{1}{2}$ & 18 & $14^{\prime} 11 / 2$ & \\
\hline$: 0 \div-8 Y$ & $21^{\circ}-2 \div$ & $21-0$ & $20^{\prime}$ & $30^{\prime} 8 \frac{1}{2}$ & 212 & $17^{\prime}\left(1 V_{2}\right.$ & \\
\hline $10 \angle-B Y$ & $1 \varepsilon^{\prime}-\because$ & 1515 & $147 \frac{1}{2}$ & $19^{\prime}$ & $196 \mathrm{k}$. & $19,01 / 2$ & $t$ \\
\hline $10 j-1 Y$ & $21-5$ & $=1-9$ & 2,4 & $27^{7}-1$ & $20^{\circ} 11$ & $2118 \frac{1}{2}$ & $E$ \\
\hline $106-\cdot v$ & $E \therefore-\therefore$ & $21: 2$ & $3 \frac{1}{2}$ & {$\left[22^{\prime} C\right.$} & 226 & 2119 & \\
\hline $10 \%-y$ & $\because ! \because$ & 525 & $16^{11} 1 ?$ & $118^{\prime} \alpha$ & $13^{\circ} 15^{15}$ & $18: 4$ & \\
\hline $103-B y$ & $\therefore \div \div$ & $\because \div \div$ & 1.72 & $14^{\prime} 1 \frac{1}{2}$ & 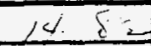 & $15-0^{3}$ & \\
\hline & & & 6 & 22 & & 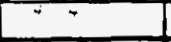 & \\
\hline Totat & $176 \div=$ & 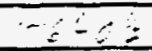 & 1711 & 1764 & $197-3$ & 117 & \\
\hline ivew Feec & & & & 51 & $12^{\prime \prime}$ & $\rightarrow$ & \\
\hline Eujusted Total & & & & & & & \\
\hline
\end{tabular}

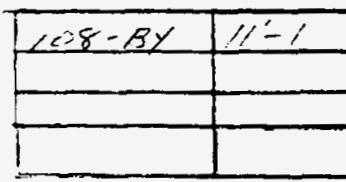

Heat Trices

(Dey Stift)

\section{IINACT I VE TANKS}

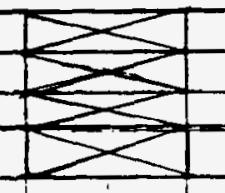

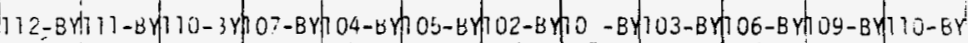

ic to To To To To

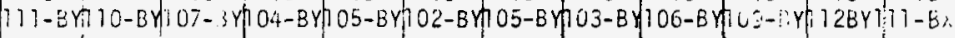
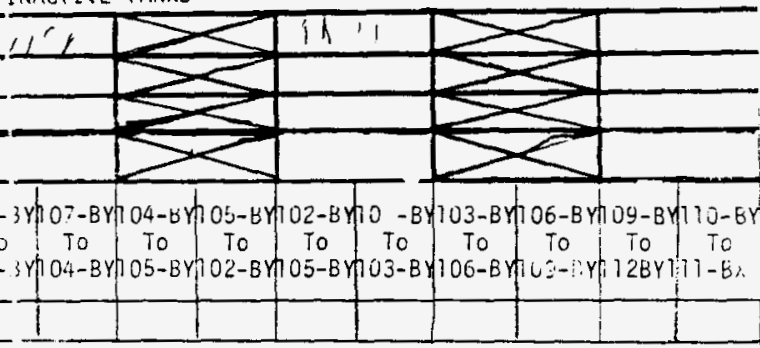

i

$9-20-71$ 


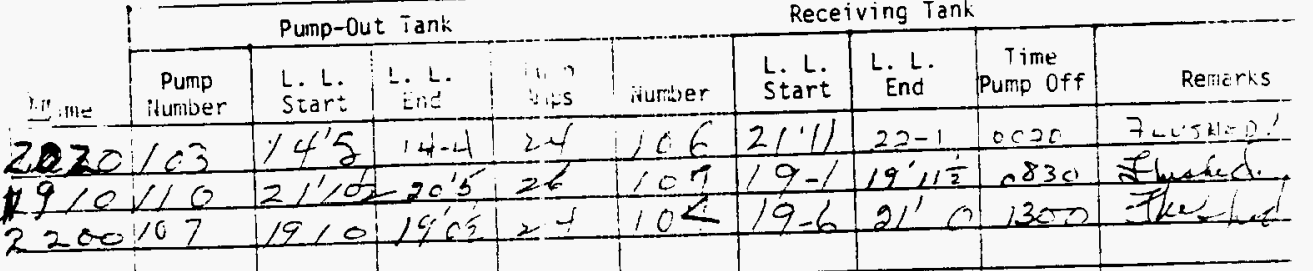




\section{ITT-2 IHVENTOR" AiNU PUMPIHG - Cate "-57"}

Puinp-out Tank

Receiving Tank

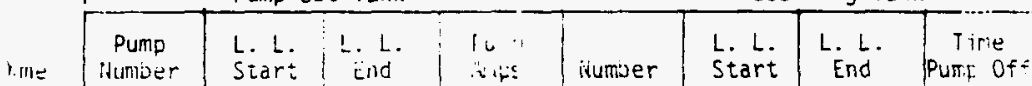

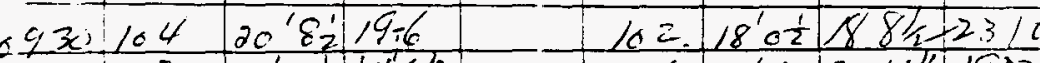

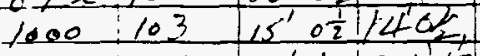

$1600.106277^{\prime} 1214$

$104.22^{\prime} 12211 / 1900$

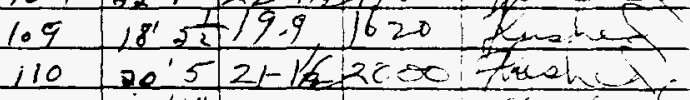

\begin{tabular}{l|l|l|l|}
\hline 0 & 30112 & 216 & $20^{\prime} 6$ \\
\hline
\end{tabular}

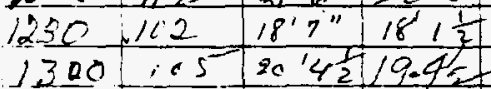

$430-110+21^{\prime 4} / 20^{\circ} 6^{\circ}$ $105.21^{\prime \prime} 22^{\prime} 0$ 1030 fkuthed

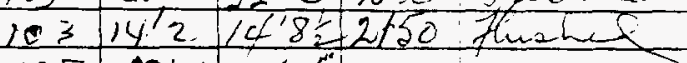
$107.492 / 20^{\circ} 5 \mathrm{c} / \mathrm{co}$

$14301107 / 19-\overline{8} 18^{\prime} 11$

104

203

20

$0830:$

then he

\begin{tabular}{|c|c|c|c|c|c|c|c|}
\hline ank & 0100 & 0700 & 3900 & 1500 & 1700 & 2300 & \\
\hline$\square, 2-8 Y$ & $2 c-9$ & $21-41 / 2$ & $24^{\prime} .5 \frac{1}{2}$ & 21.1 & $20-8 / 2$ & $20-8 / 2$ & E \\
\hline$Y T U-B Y$ & $21-3$ & $20-5^{1 / 2}$ & $20^{\prime} 5$ & $21^{\prime \prime} 4 k$ & $2 /-3$ & $20-7$ & \\
\hline $107-5 Y$ & ss-c. & $46-11 / 2$ & $1 i^{\prime} \quad 11 \frac{1}{2}$ & $0101 / 2$ & $18-7 / s$ & $20-\pi$ & \\
\hline$\therefore 4-B Y$ & $2 c-c$ & $5=-8$ & $22^{1} 8$ & 2018 & $20-4$ & $-(6 / 2)$ & \\
\hline $73 \leq-E Y$ & $i \varepsilon-c$ & $18 \cdot c$ & $18 \subset 2$ & $18^{\prime} 7 \%$ & $18-8$ & $18-81 / 2$ & \\
\hline $10 j-1 Y$ & $21-0$ & $20-0$ & $20^{\circ} 1$ & $20^{\prime}=1 / 2$ & $19-11$ & & 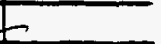 \\
\hline$i v e-v$ & $22-1$ & $32 \cdot 1$ & $\div 2^{1} 1$ & $2 \times 17$ & $2 !-71$ & $C / 2$ & \\
\hline $10 \%-y$ & $19-11 / 2$ & ${ }^{15-2 \mid}$ & $1 g^{\prime} 3 \frac{1}{2}$ & 154 & $19-8$ & -1 & \\
\hline $10 E-3 y$ & $121-4$ & $15-1$ & $15 \cdot 6 \frac{1}{2}$ & $14 t^{\prime} / 1 / 2$ & $14-1$ & & \\
\hline & & & 32 & 32 & & & \\
\hline Total & $76-c^{\prime \prime}{ }^{\prime}$ & $70-5 \%$ & $16^{1} 0 \frac{1}{2}$ & $17 b^{\prime} 0 \frac{1}{2}$ & $175-10$ & $1 / 2$ & \\
\hline ivew Feec & & & & & & & \\
\hline Guinsted Totail & & & & & & & \\
\hline
\end{tabular}

IUACTIVE TANKS

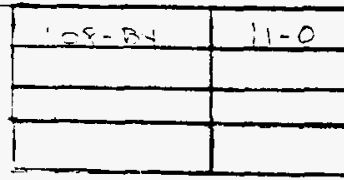

Heat Tr:ces

(Liay si: i

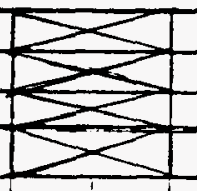

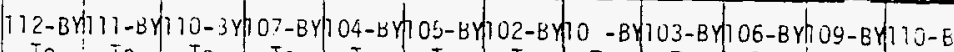
To to to To To to to to to to To to To To

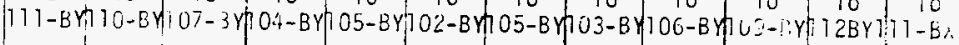

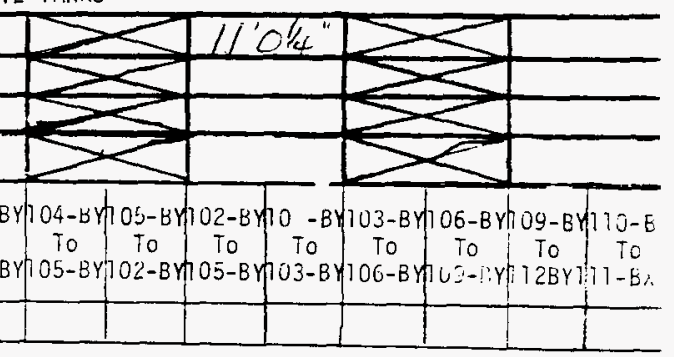


Punp-Out Tank

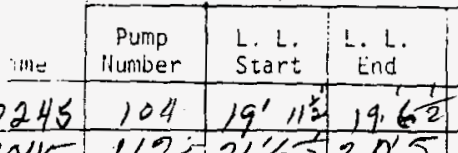
$10451 / 2,216 \frac{21205}{2}$ $1430110.21^{\prime} 3,20.5 \frac{1}{3}$ 183510622062094 $2010107 \quad 19 \% 10 \frac{1}{7} 18^{\prime} 11$ 2. $5^{3} / 13 \times 4-914^{\prime} 4$ $224016523^{\prime} 019^{\prime} 10$
Catet

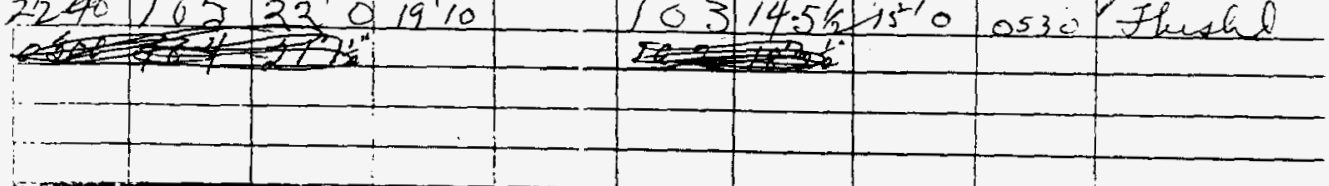

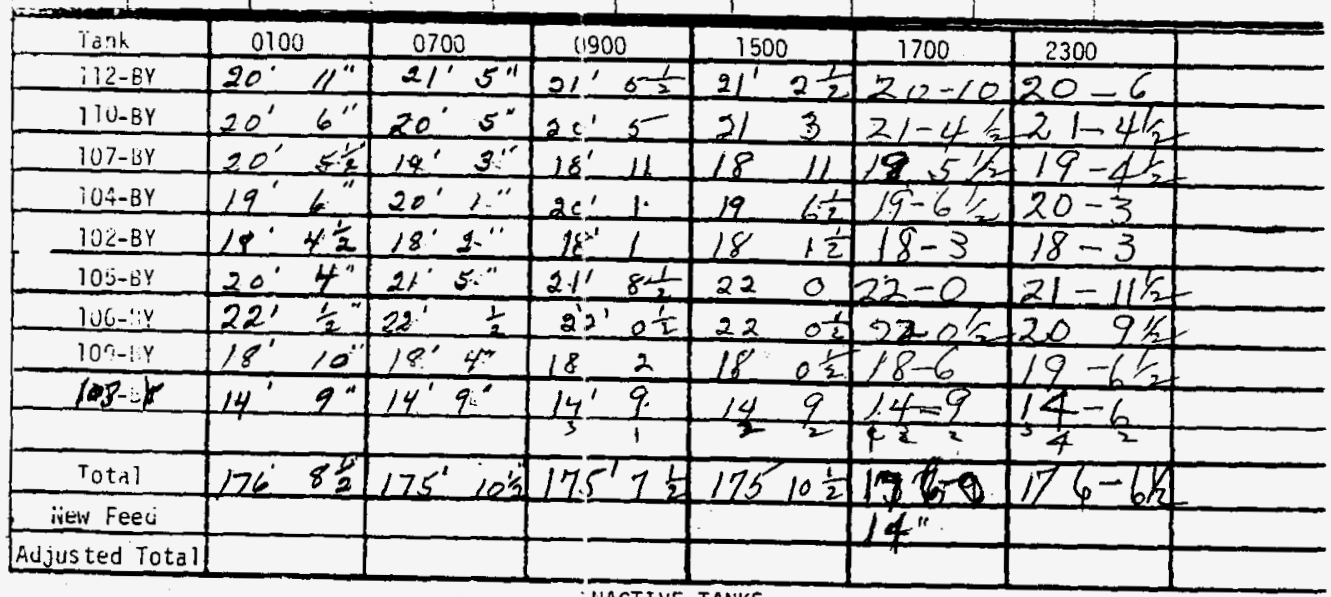

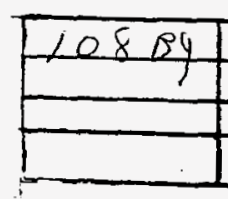

Heat Traces

(Day Stiff)

NACTIVE TANKS

Receiving Tank

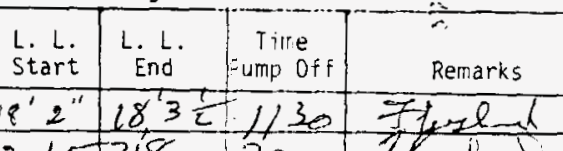
$1102015+218$ 2000 Fhushed $10718^{\prime} 11$ ogos $19^{\circ}$ thand 109 18-4 9723172

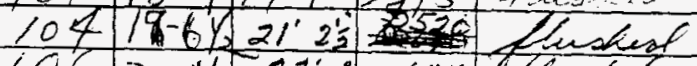
$10620-14207^{\circ} 0355$ thentar

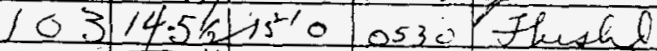
$=2=2.10$ 


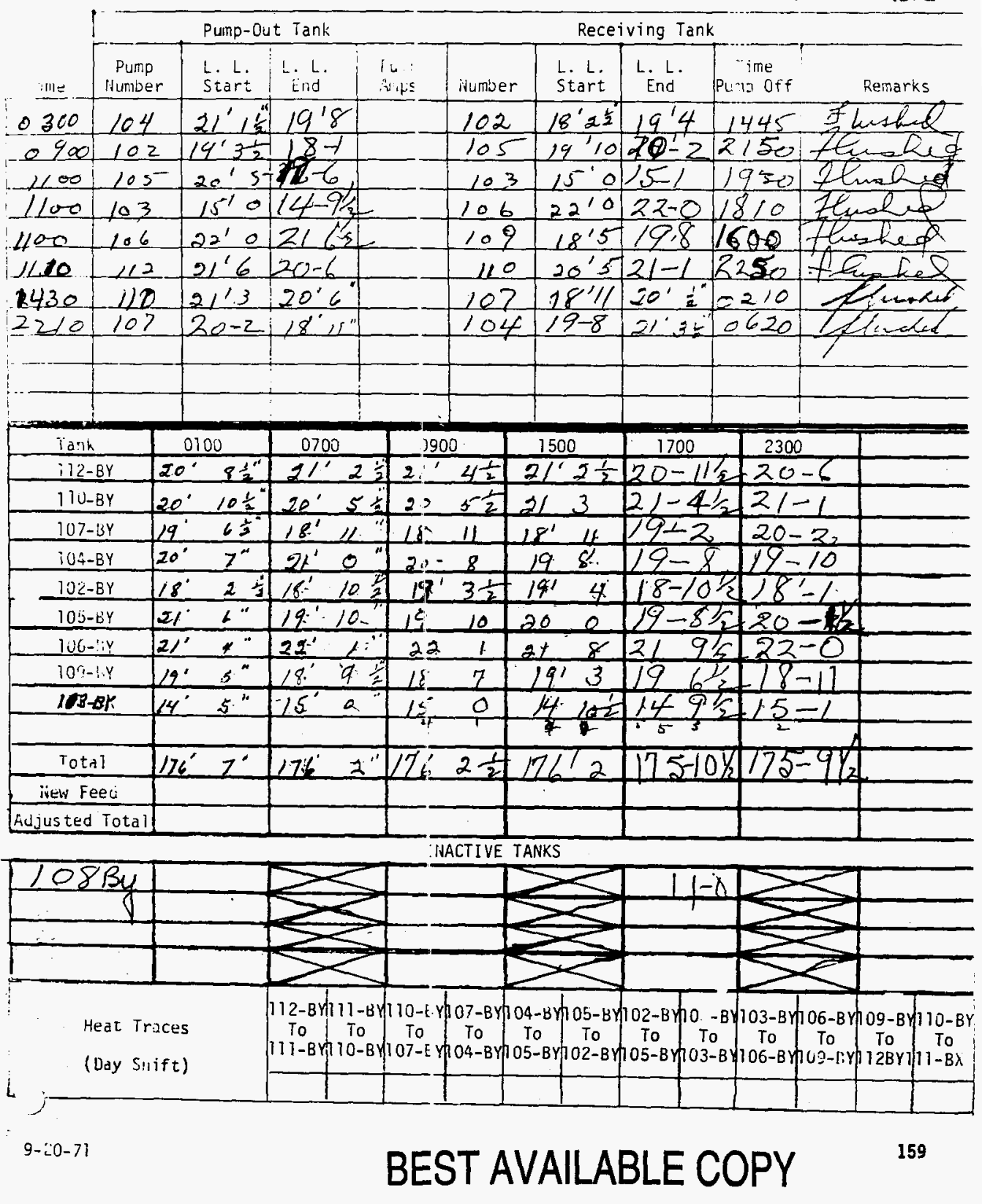




$$
00-52-n^{2}-2 p-206
$$

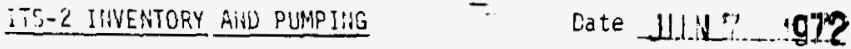

Revo

\begin{tabular}{|c|c|c|c|c|c|c|c|c|c|}
\hline \multirow[b]{2}{*}{ iml } & \multicolumn{4}{|c|}{ Pump-Out Tank } & \multicolumn{5}{|c|}{ Receiving Tank } \\
\hline & $\begin{array}{l}\text { Pump } \\
\text { llumber }\end{array}$ & $\begin{array}{l}\text { L. L. } \\
\text { Start } \\
\end{array}$ & $\begin{array}{l}1 . \mathrm{L} . \\
\text { End }\end{array}$ & $\begin{array}{r}\text { In: } \\
\text { ints } \\
\end{array}$ & Humber & $\begin{array}{l}\text { L. L. } \\
\text { Start }\end{array}$ & L. L. & $\begin{array}{c}\text { Tine } \\
\text { Pump } C-f\end{array}$ & Remarks \\
\hline 0145 & $1038 x$ & $14^{\prime} 5 \frac{1}{3}$ & $13^{\prime}, 3 \frac{1}{6}$ & & $1098 \mathrm{~B}$ & $18^{\circ} 7^{\prime}$ & $19^{\prime}$ & $0<20$ & a.tal \\
\hline 0430 & 10484 & $21^{\circ} 3$ & $19^{\prime} 7^{\prime \prime}$ & & $1028 Y$ & $18^{\circ} 1^{\prime \prime}$ & & 1645 & GOAED \\
\hline 1041 & 102 & $19^{\prime} 3$ & $18^{\circ} 0^{\circ}$ & & 105 & $20 ' 2$ & $21^{\prime}$ & $\operatorname{coc} 0$ & 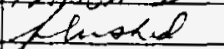 \\
\hline 1130 & 112 & $21^{\prime} 6$ & $20^{\prime} 6 "$ & & $\angle \angle O$ & $20^{\prime} 6$ & & $20 \geq 0$ & $\angle 4 S<E$ \\
\hline$\angle 300$ & 103 & $15^{1} 1-\frac{1}{2}$ & $\left\langle y^{\prime} q^{\prime \prime}\right.$ & & 106 & 220 & 23 & 1980 & $2 / 4 s<<0$ \\
\hline 1300 & 166 & $22^{\prime} 0$ & 只些究 & & 109 & $18^{\prime} 8$ & 12 & $\angle P Q D$ & FAHSHED \\
\hline$\angle 400$ & 105 & $20^{\prime} 111 / 2 i$ & $20^{\prime}=\frac{1}{2}$ & & 103 & $14^{\prime} 10^{\prime}$ & 15 & seze? & Facsh \\
\hline 1430 & 110 & $21^{\prime 2}$ & $20^{\circ} 6^{\prime \prime}$ & & 107 & $18^{\prime} 11$ & $19^{\circ}$ & 0045 & \\
\hline 324 & $\angle P\rangle$ & $30^{\prime} e^{\prime}$ & & & 104 & 19171 & & & 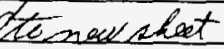 \\
\hline & & & & & & & & & \\
\hline
\end{tabular}

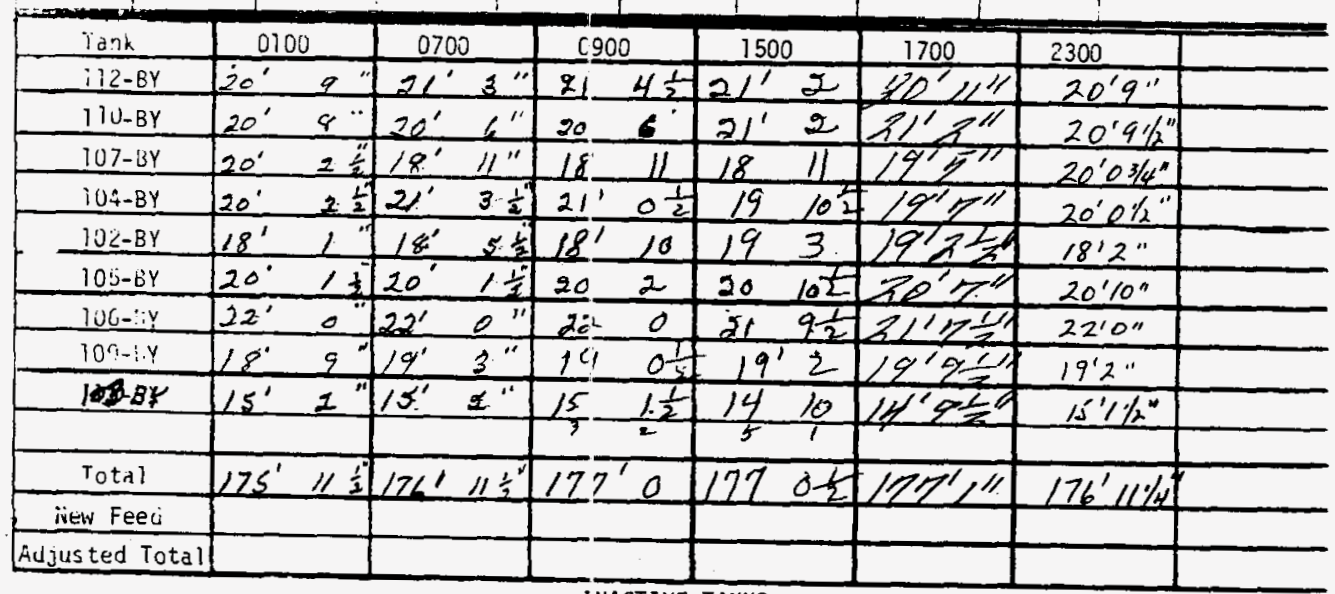

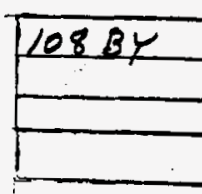

Heat Traces

(Day Silift) i

$9-: 0-71$

\section{INACTIVE TANKS}

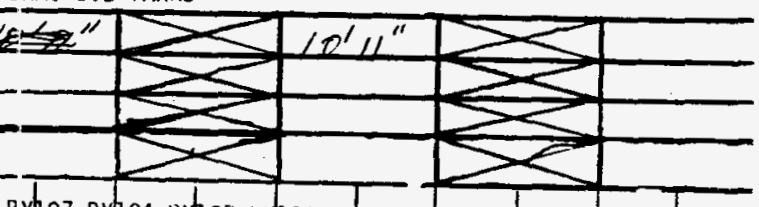

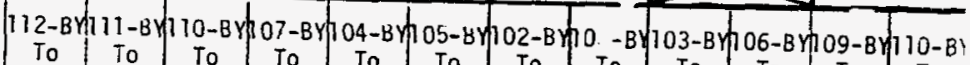

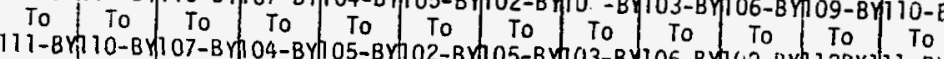

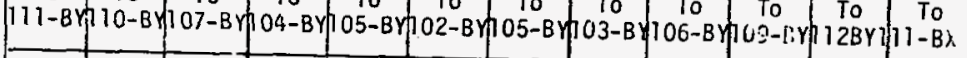
$\rightarrow$ 
Receiving Tank

\begin{tabular}{|c|c|c|c|c|c|c|c|c|}
\hline \multicolumn{4}{|c|}{ Punp-Out Tank } & \multicolumn{5}{|c|}{ Receiving Tank } \\
\hline $\begin{array}{l}\text { Pump } \\
\text { number }\end{array}$ & $\begin{array}{l}\text { L. L. } \\
\text { Start }\end{array}$ & $\begin{array}{l}\text { L. L. L. } \\
\text { End }\end{array}$ & $\begin{array}{l}1,9 \\
\text { sitis }\end{array}$ & iuminer & $\begin{array}{l}\text { L. L. } \\
\text { start } \\
\end{array}$ & $\begin{array}{l}\text { L. L. } \\
\text { End }\end{array}$ & $\begin{array}{c}\text { Time } \\
\text { Purre } 01 f\end{array}$ & Vermarks \\
\hline 107 & $20^{\circ} 0^{\circ}$ & 'q.o" & & 104 & $1 e^{\prime} 7$ & 20 & 5 & Tineleyn \\
\hline 127 & $21^{\prime} 0^{\prime \prime}$ & 1962 & & 102 & $18^{\prime} 0$ & $195 \%$ & 1245 & Sncted \\
\hline$\frac{1}{1<3}$ & $21^{\prime} 7^{1 / 2}$ & $206 k^{\prime}$ & & 116 & $20^{\prime} 6$ & 218 & 1010 & Ex+ad \\
\hline c3 & $15^{\prime} / 1 / 2$ & $\psi^{\prime} z^{\prime \prime}$ & & 106 & $22 \frac{1 / 2}{2}$ & $22^{\prime} 0 \frac{14}{3}$ & 11802 & Ehesicel \\
\hline 103 & $22 \%$ & 3ite & & 109 & 1812 & $1 q^{\prime \prime q 1}$ & LPeE & FUUSHED \\
\hline 103 & 2,0 & $20^{\circ} 42^{\prime \prime}$ & & $\frac{103}{105}$ & 149 & $\frac{15^{\prime 1 / 2 x}}{\left.20^{\prime} 10^{\prime \prime}\right|^{*}}$ & $\frac{2045}{2300}$ & $\begin{array}{l}E \angle U S A E D \\
F L U S I E D\end{array}$ \\
\hline 110 & $\frac{1992}{218}$ & $\frac{13^{\prime}+1 y^{2}}{3 c^{\prime}}$ & & 107 & 96 & $20^{\prime \prime \prime}$ & 1214 & Flisted \\
\hline 107 & $20^{\prime} 1^{\prime \prime}$ & $19^{\prime} 0^{\prime \prime}$ & & 104 & $1 q^{\prime} 7^{\prime \prime}$ & $20^{\prime} 7^{\prime \prime}$ & 2315 & $E \leqslant U j t \leq D$ \\
\hline 112 & $21^{\prime} \sqrt{1} / 2$ & & 2 & 110 & $20^{\prime} 6^{\prime \prime}$ & & & \\
\hline & & & & & & & & \\
\hline
\end{tabular}

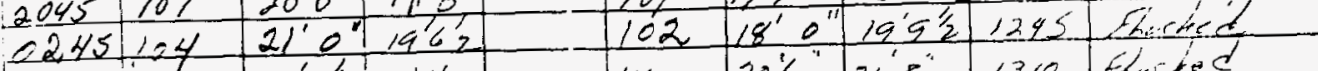

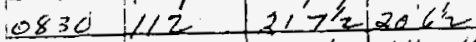

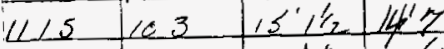

IIIS $106 \quad 22^{\prime 2}$ 23'6"

1245103 2.0 $20^{\circ} 4 \%$

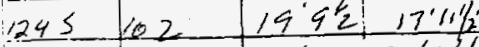

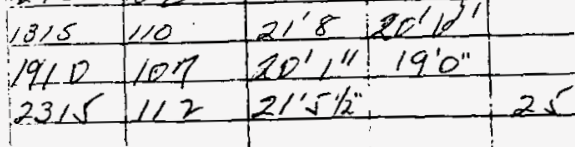

\begin{tabular}{|c|c|c|c|c|c|c|c|}
\hline Tan & 0100 & 0700 & 9900 & 1500 & 1700 & 2300 & \\
\hline$i 12-B Y$ & $20^{\circ} 11$ & $2 L^{\circ} 5$ & $21^{\prime} 7 / 2$ & $30^{\prime} 8$ & $2 x^{\prime} e^{2}$ & $21^{\prime} 5^{1 / 2}$ & \\
\hline 1 IU-BY & $20^{\circ}$ & $20^{\prime} 6$ & $20^{\prime} 6^{\prime \prime}$ & $21^{\prime} 4^{\prime \prime}$ & $80^{\prime} 115=$ & $20^{\prime} 6^{\prime \prime}$ & \\
\hline $107-3 Y$ & $19^{\circ}$ & $19^{\circ}$ & $\Sigma^{\prime \prime} 0^{\prime \prime}$ & $19^{\prime} 4^{\prime \prime}$ & $14 e^{\prime} e^{\prime \prime}$ & $19^{\circ} 0^{\prime \prime}$ & \\
\hline $104-B Y$ & $20^{\prime}$ & $20^{\prime} \quad 6 x^{\prime}$ & $242 \frac{2}{2}$ & $19^{\prime} 4 \frac{1}{2}$ & $14^{\prime} 8^{\prime \prime}$ & $20^{\prime} 7^{\prime \prime}$ & \\
\hline $10 \angle-B Y$ & $18^{\circ} e$ & $18^{\prime}$ & $19^{\prime}, 1$ & $19^{\prime} 5$ & $\angle q^{\prime} \Delta$ & $17^{\prime} 11 / 2^{n}$ & \\
\hline $105-B Y$ & 21 & $22^{\prime}$ & $2^{\prime \prime} 0^{\prime \prime}$ & $20^{\prime} 10^{\prime / 2}$ & $50^{\prime} 8-\frac{1}{2}$ & $20^{\prime} 10 \% 2^{\prime \prime}$ & \\
\hline $100-1: 2$ & $21^{\prime}$ & $23^{\prime}$ & 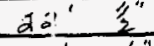 & $2^{\prime} 8^{\prime \prime}$ & 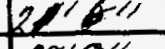 & $22^{\prime} 0^{\prime \prime} z^{\prime \prime}$ & \\
\hline $10 r_{-1.1 . y}$ & $18^{\prime}$ & $18^{\circ}$ & 18 & $19^{\prime} 2$ & $16 q^{\prime \prime}$ & $19^{\prime}, 1$. & \\
\hline $108-8 Y$ & $15^{\circ} 1 \frac{1}{2}$ & $1 \frac{1}{2}$ & 15 & $14^{\prime} 8^{1 / 2}$ & $46 \times 11$ & $15^{1} 0 \%$ & \\
\hline$T_{0 t a 1}$ & $17 i^{\prime} a^{\prime}$ & $1 / 6^{\prime} \quad 8^{-\frac{4}{2}}$ & $\sqrt{176^{\prime} 8^{\prime \prime}}$ & $1767^{\prime \prime}$ & $17^{\prime} q^{\prime \prime}$ & $176^{\prime} 6 \% 2^{\prime \prime}$ & \\
\hline iivew Feec & & & & & $\hat{a}$ & & \\
\hline & & & & & & & \\
\hline
\end{tabular}

aujus ted Total L WACTIVE TANKS

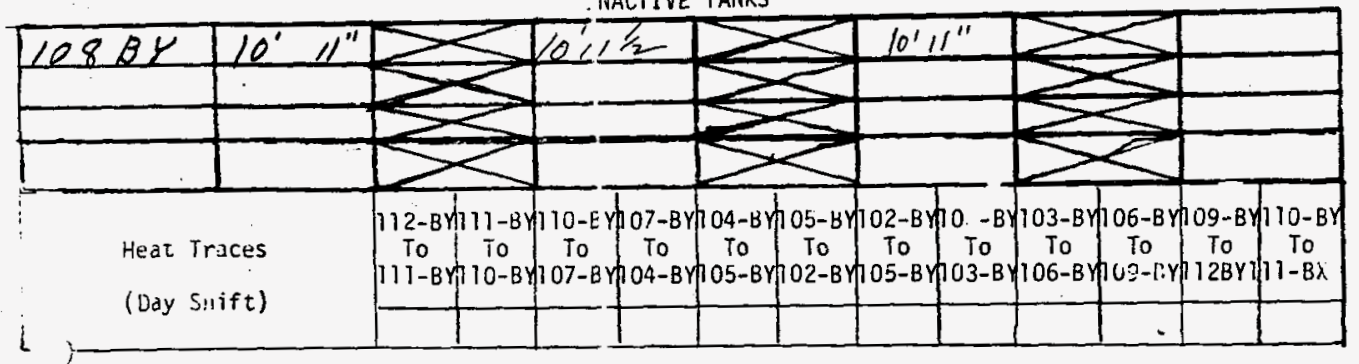

$9-20-7)$ 
Puinp-Out Tank

Receiving Tank

\begin{tabular}{|c|c|c|c|c|c|c|c|c|c|}
\hline \multirow[b]{2}{*}{. ․ㅣㄷㅡ } & \multicolumn{4}{|c|}{ Puinp-Out Tank } & \multicolumn{5}{|c|}{ Receiving Tank } \\
\hline & $\begin{array}{l}\text { Pump } \\
\text { Number }\end{array}$ & $\begin{array}{l}\text { L. L. } \\
\text { Start }\end{array}$ & E. L. & 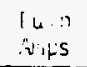 & ivumber & $\begin{array}{l}\text { L. L. } \\
\text { Start }\end{array}$ & L. L. & $\begin{array}{c}\text { Tine } \\
\text { Pump of }\end{array}$ & Renarks \\
\hline $\begin{array}{l}6-8 \cdot 72 \\
2515\end{array}$ & 112 & $2 L^{\prime}+\frac{1}{2}$ & $20^{\circ} 6$ & 25 & 110 & $20^{\prime} 6^{\prime \prime}$ & $21<\frac{1}{2}$ & Q 7 70 & \\
\hline 5245 & 110 & $21^{\prime} y^{4}$ & 20,8 & 25 & 107 & $19^{\circ} 0^{\circ}$ & 15.9 & 1400 & Lenshe \\
\hline 81030 & 1207 & $20 \cdot 0$ & $19^{\prime} 0 / 2$ & 25 & 104 & 2017 & $2 z^{\prime \prime \prime \prime}$ & 1830 & $F \angle 054 \leq 2$ \\
\hline 1400 & 106 & $22-1$ & $19.99^{\prime \prime}$ & & 109 & $17 \cdot 6$ & $19^{\prime} 4 \%$ & 2330 & $F_{L U S A O S}$ \\
\hline 1830 & 103 & $150 \%$ & $13 \cdot 8$ & 24.5 & 106 & $20^{\prime} 3^{\prime \prime}$ & 22.0 & 0845 & $F 205 * E D$ \\
\hline 1830 & 104 & $22^{\prime} 1^{\prime \prime}$ & & 18 & 102 & $1711 / 21$ & $20 \cdot 0$ & 1300 & $=4054 E 0$ \\
\hline 1845 & 110 & $20^{\prime} 8^{\prime} / 2^{\prime \prime}$ & 214 & 25 & 107 & $19^{\prime} 012^{\prime \prime}$ & 19.0 & 01030 & stredes \\
\hline 1045 & $1 / 2$ & $21^{\prime} \mathrm{C}^{\prime \prime}$ & $20 \%$ & 25 & 110. & $20^{\prime} 81 / 2$ & $218 \frac{1}{2}$ & 0330 & Eluabed \\
\hline & & & & & & & & & \\
\hline & & & & & & & & & \\
\hline & & & & & & & & & \\
\hline
\end{tabular}

\begin{tabular}{|c|c|c|c|c|c|c|c|}
\hline jank & 0100 & 0700 & 1900 & 1500 & 1700 & 2300 & \\
\hline i $12-B Y$ & $2 \prime^{\prime} 3^{\prime \prime}$ & $20^{\circ} 66^{\prime \prime}$ & 20.7 & 2111 & $21^{\prime} 4^{\prime \prime}$ & $20^{\prime} / 1^{\prime \prime}$ & \\
\hline $110-B Y$ & $20^{\prime}$ & $21^{\prime}<1$ & $21 \cdot 4$ & 20.8 & $20^{\prime} 8 \% 2^{\prime \prime}$ & 2111 & \\
\hline 107-BY & $19^{\prime}$ & $19^{\circ} \quad 9 y^{\prime \prime}$ & 19.11 & 15.9 & $19^{\prime} 0 \% 2^{\prime \prime}$ & $19^{\prime} 8 \% / 2 \%$ & \\
\hline $104-B Y$ & $20^{\prime} 7^{\prime \prime}$ & $20^{\prime} \mathrm{T}$ & 20,7 & $21+4$ & $2 z^{\prime} /{ }^{\prime \prime}$ & $21^{\prime} 3 / 2 "$ & \\
\hline $10 \angle-B Y$. & $17^{\circ} 112^{2}$ & $17^{\circ} 11 \ldots$ & 17.11 & $17-11$ & $17^{\prime} / 1 / 2 "$ & $18^{\prime} 10^{\prime \prime}$ & \\
\hline $105-6 Y$ & $20^{\prime} 10 \frac{1}{2}$ & $20^{\circ} 10 \frac{1}{2}$ & $2.6) 9$ & 20.9 & $20^{\prime} 10^{\prime \prime}$ & $20^{\prime} 91 / 2 \prime$ & \\
\hline $100-1 y$ & $22^{\prime}$ & $22^{\prime} \quad 0^{\frac{1}{2}}$ & $2: 1$ & $22 \cdot 1$ & $20 \% 10 \%$ & $19^{\prime} 9 . \prime$ & \\
\hline $105-1 . y$ & $19^{\prime}$ & $18^{\circ} 2 \frac{1}{2}$ & $18^{\prime} 9$ & $17-6$ & $18^{\prime} 31_{2}^{\prime \prime}$ & $19^{\prime} 8 / 2 "$ & \\
\hline $103-2 x$ & $15^{\prime} \quad 0 \frac{1}{2}$ & $15^{\circ}$ & $1 \leq 0$ & $15 \div 0$ & $15^{\prime} / 11$ & $140^{\prime \prime}$ & \\
\hline Total & $176^{\prime} 9^{\prime \prime}$ & $176^{\prime} \quad 63$ & 176.11. & $176 \cdot 4$ & $176^{\prime} 2 \% 2^{\prime}$ & $176^{\circ} 1^{\prime \prime}$ & \\
\hline iivew Feeci & & & & & & & \\
\hline Aujusted Total & & & & & & & \\
\hline
\end{tabular}
NACTIVE TANKS

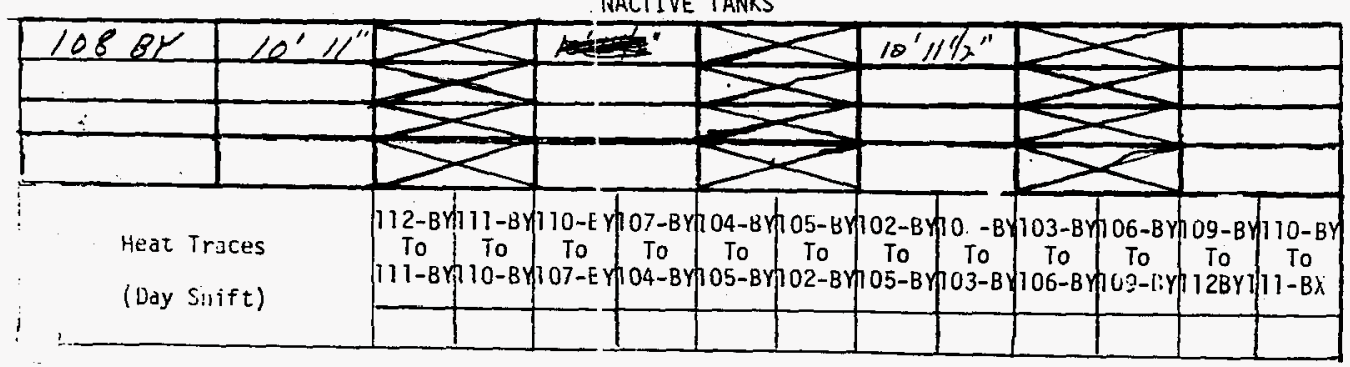

$9-20-71$ 
$W=C-S D-W M-D P-206$

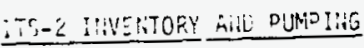

Lat: $E-C_{-2}-\mathrm{ReV}^{2}$

\begin{tabular}{|c|c|c|c|c|c|c|c|c|}
\hline \multirow[b]{2}{*}{$i: n=$} & \multicolumn{3}{|c|}{ Punp-Out Tank } & \multicolumn{3}{|c|}{ Receiving Tank } & \multirow{2}{*}{\multicolumn{2}{|c|}{ Remarks }} \\
\hline & $\begin{array}{c}\text { Pump } \\
\text { ifumber }\end{array}$ & \begin{tabular}{|l|l} 
L. L. & $1 . \mathrm{L}$. \\
Start & End \\
\end{tabular} & $\begin{array}{l}160 \\
i n+5 \\
\end{array}$ & ivumber & $\begin{array}{l}\text { L. L. } \\
\text { Start }\end{array}$ & L. L. & & \\
\hline$c<00$ & $1 e z$ & $14^{\prime}, \frac{1}{2}, 20,0$ & & 105 & 2010 & 20.6 & 1300 & \\
\hline clos & $105^{-}$ & $20^{\prime}(0) / 198$ & & 103 & $13^{\prime} 9 \frac{1}{2}$ & $13 \cdot 8$ & 130 & \\
\hline 0330 & 107 & $20^{\prime} 3: 19.0$ & & 104 & $20^{\prime} 9$ & 20.9 & 101632 & "xache \\
\hline 300 & 102 & 200 & & $105^{-}$ & 18.8 & $\cos$ & Uvining & $t+6-12$ \\
\hline $17 \pm 15$ & 410 & सi $4 k^{\prime \prime} 20^{\prime} 8^{\prime \prime}$ & 25 & $i<7$ & $190^{\circ}$ & $19^{\prime} 1 / 2$ & $1645 k$ & $(x-1)=1-x+1+0 v$ \\
\hline $21 \theta 0$ & 164 & $20^{\prime} \epsilon^{\prime \prime 2} 20.9$ & 18 & 102 & $196 \%$ & 20.0 & 600 & 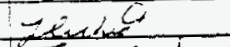 \\
\hline $173 t$ & 112 & $217^{\prime \prime}=054$ & 24 & 110 & $21^{\prime} 4 \%$ & $20^{\prime} 8$ & 6300 & 5 \\
\hline & & & & & & & & \\
\hline
\end{tabular}

\begin{tabular}{l|l|l|l|l|l|l|}
\hline & & & & & & \\
\hline
\end{tabular}

\begin{tabular}{|c|c|c|c|c|c|c|c|}
\hline icik & 0100 & 0700 & 6900 & 1500 & 1700 & 2300 & \\
\hline$i 12-84$ & $20^{\prime} 9$ & $20^{\prime} 7$ & 30.5 & $2 / 3$ & $21^{\prime} 7^{\prime \prime}$ & $20^{\prime} 10 \% 1 / 2$ & \\
\hline $11 \cup-B Y$ & $21 \quad 3 \frac{1}{2}$ & 2155 & $21 \cdot 4$ & $21-4 \frac{1}{2}$ & $24^{\prime} 4 / 2 "$ & $21^{\prime} 5^{\prime \prime}$ & \\
\hline $107-1 Y$ & $19 \quad 9.5$ & 199 & $17 \cdot 3$ & 19.0 & $19^{\prime} 0^{\prime \prime}$ & $20^{\prime} 3 / 2^{\prime \prime}$ & \\
\hline$B U=-B Y$ & $20 \quad 11 \frac{1}{2}$ & 208 & $2 \% 10$ & $20 \cdot 6$ & $20^{\prime} 6^{\prime \prime}$ & $20^{\prime} 0^{\prime \prime}$ & \\
\hline $10 \leq-5 Y$ & $1 \frac{8}{2}$ & $19.2 \frac{1}{2}$ & 17 & $20 \cdot 0$ & 19262 & 19.211 & \\
\hline $700-i 4$ & $20 \quad 10$ & 204 & $2: 0$ & 19.8 & $20^{\prime} 61 / 2 \prime$ & $21,3 \prime \prime$ & \\
\hline luiver & 1911 & $215 \frac{1}{2}$ & $22 \cdot 0$ & 22.0 & $22^{\prime} 6^{\prime \prime}$ & $22^{\circ} 6^{\prime \prime}$ & \\
\hline $10:-y$ & 198 & $19: 6$ & 18.8 & $18 \cdot 6$ & $18^{1} 7 \%$ & $18^{\prime} 1$ & \\
\hline $103-\therefore$ & 1392 & $131 ?$ & 13.8 & $13 \cdot 8$ & $13^{\prime} 8^{\prime \prime}$ & $13^{\prime} 8^{\prime \prime}$ & \\
\hline - & 2 & 4 & & & & & \\
\hline Totat & 17612 & 121.07 & 176.6 & $175 \cdot 114$ & $=176^{\prime} 10^{\prime \prime}$ & $176^{\prime} 4^{\prime \prime}$ & \\
\hline ivew Fees & & & & & & & \\
\hline rujusted Total & & & & & & & \\
\hline
\end{tabular}

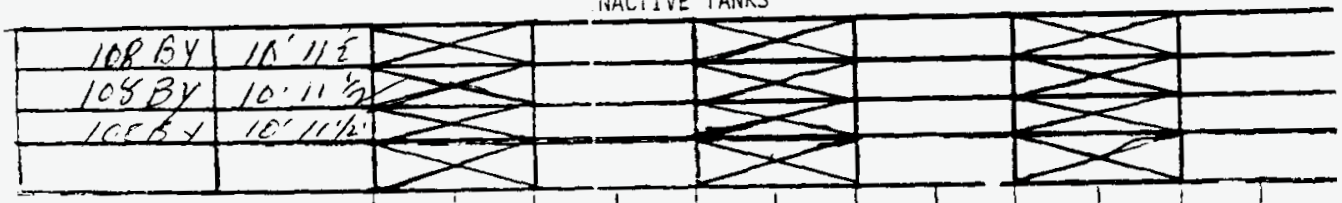

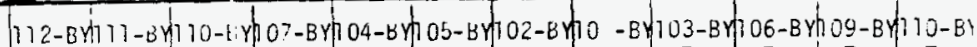

Heat Trices

(') $3 y$ sifis)

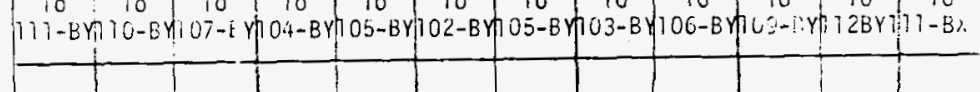

L. 


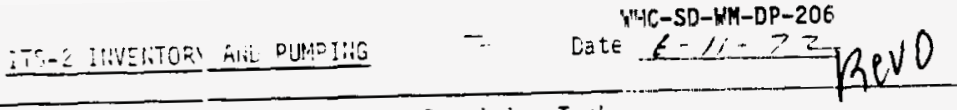

\begin{tabular}{|c|c|c|c|c|c|c|c|c|}
\hline \multirow[b]{2}{*}{ T:In:= } & \multicolumn{3}{|c|}{ Punp-Out Tank } & \multicolumn{5}{|c|}{ Receiving Tank } \\
\hline & $\begin{array}{l}\text { Pump } \\
\text { Numiver }\end{array}$ & $\begin{array}{l}\text { L. L. } \\
\text { Start } \\
\end{array}$ & $\begin{array}{l}16: \\
\therefore+5 \\
\end{array}$ & intumer & $\begin{array}{l}\text { L. } 1 . \\
\text { Start }\end{array}$ & $\begin{array}{l}\text { L. L. } \\
\text { End }\end{array}$ & P. Time off & Remarks \\
\hline 0030 & 107 & $2013 \frac{1}{2} 19.0$ & & 104 & $19^{\prime} 9$ & 20.9 & 1560 & $I E_{2}$ \\
\hline 4036 & 105 & $21^{\prime}, 1 / 5.2$ & & 103 & 1382 & 14.1 & $\angle 3<0$ & 200 \\
\hline 0330 & 103 & $14^{\prime} \geq \frac{1}{2}: 14: 1$ & & 1.6 & $21^{\prime} 11 \frac{1}{2}$ & 215 & 13020 & thenes \\
\hline 0330 & $\operatorname{lec} 6$ & $2^{\prime}, 11 \frac{1}{2} 211$ & & 107 & 12125 & \pm 19.7 & 1200 & 0 Cacters \\
\hline 1645 & $1 / 2$ & $21^{\prime} 72^{\prime \prime 28^{\prime}}$ & 26 & 110 & $26^{\prime} 8 \prime$ & 2 & 2316 & FLU $-51+5$ \\
\hline 1645 & 164 & $20^{\prime} 9^{\prime \prime} 19^{\prime} 6 \%$ & 19 & 102 & $14^{\prime \prime}$ & & 23,5 & $f+U S t E$ E \\
\hline 2130 & 110 & $21^{\prime} 8 \%$ & 24 & 107 & $19^{\prime} 2^{\prime \prime}$ & & tainis & $6-12$ \\
\hline & & 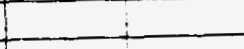 & & & & & & \\
\hline & & 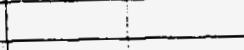 & & & & & & \\
\hline & & & & & & & & \\
\hline & & & & & & & & \\
\hline
\end{tabular}

\begin{tabular}{|c|c|c|c|c|c|c|c|}
\hline Jank & 0100 & 0700 & 9900 & 1500 & 1700 & 2300 & \\
\hline$i 12-8 V$ & $24^{\prime} 72$ & $20^{\prime} 7$ & 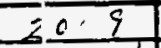 & 213 & $21^{\prime} 7^{\prime \prime}$ & $20^{\prime} s^{\prime \prime}$ & \\
\hline $1 I U-B Y$ & $21^{\prime} 8 \frac{1}{2}$ & $20^{\prime} 11$ & $20.10 \frac{1}{2}$ & $20: 5$ & $20^{\circ} 8^{\prime \prime}$ & $21^{\prime} 8^{\prime \prime}$ & \\
\hline $107-34$ & $2=3 \div 2$ & 2013 & 17.8 & $19 \cdot 6$ & $19^{\prime} 11 / 2$ & $19^{\prime} 61 / 21$ & \\
\hline$B U \div-B Y$ & $19 \quad 9$ & $20 \quad 93$ & $22+4$ & 20.9 & $26^{\prime} 9^{\prime \prime}$ & $19^{\prime} 61 / 2$ & \\
\hline $10 \angle-E Y$ & $19^{\prime} 3 \frac{1}{5}$ & $194 \frac{1}{2}$ & $1 \div 5$ & $20^{\circ} 0$ & $19^{\prime} 7^{\prime \prime}$ & $19^{\prime} 817$ & \\
\hline $100-E^{\prime} y$ & 215 & $24^{\prime} a$ & $\theta=2 \quad 30$ & $19 \cdot 6$ & $20^{\prime} 2^{\prime \prime}$ & $21^{\prime} 3 \prime$ & \\
\hline vir- $v$ & $21^{\prime} 152$ & $7 \frac{2}{2}$ & $3: 3^{\prime}$ & $21 \cdot 5$ & $21^{\prime} 41 / 2$ & $211412^{\prime \prime}$ & \\
\hline $10 \cdot-i v$ & 1711 & $18^{13}$ & $1: 7112$ & 19.6 & 19.11 & $18^{\prime} 61 / 2$ & \\
\hline $103 B y$ & $13 \quad 8 \div$ & 142 & 1h: & 13.1113 & $1+1,2$ & 1410 & \\
\hline & 3 & 3 & & & & & \\
\hline Totai & 1268 & $176^{\prime} 4$ & 176.21 & $176: 1 / 2$ & $\left.176^{\prime}+1 / 2\right]$ & $176^{\prime} 6^{\prime \prime}$ & \\
\hline ivew Feec & & & & & & & \\
\hline -cizus ted Total & & & & & & & \\
\hline
\end{tabular}

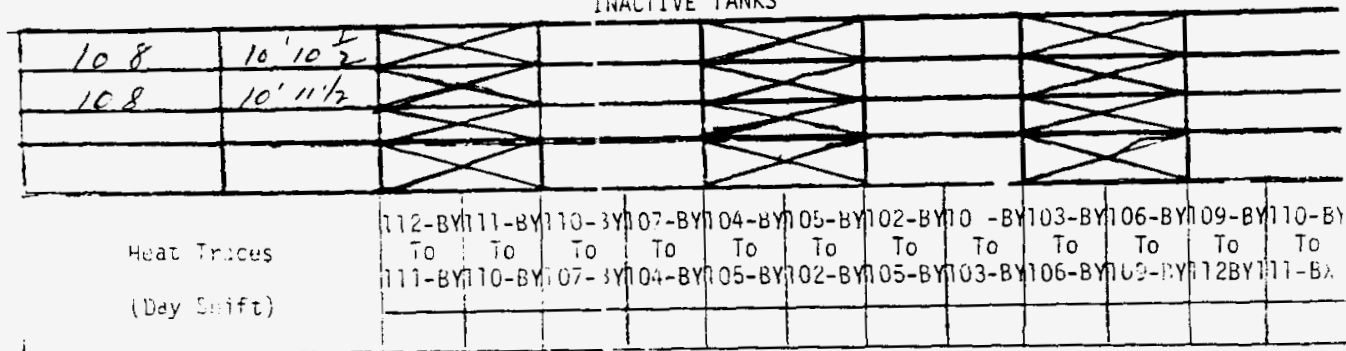

i 


\section{-}

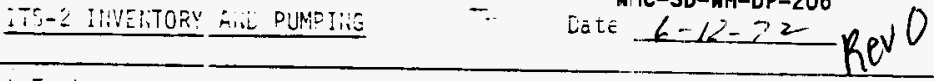
Punp-Out Tank

Receiving Tank

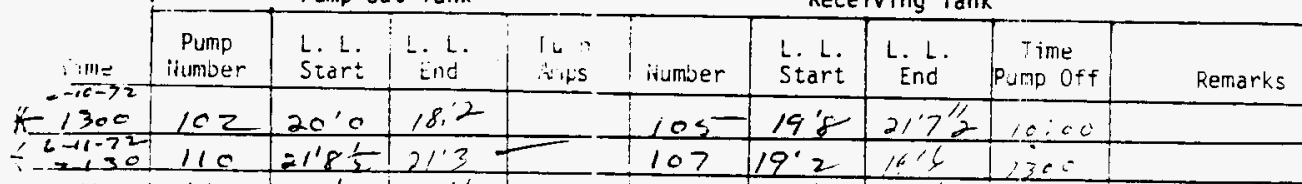

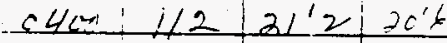

c4cil $105^{-22} \circ 211$

c.5ic $102145 \frac{1}{2} / 42^{2}$

$06-30+102.20^{\circ} 4 \mid 19^{\prime}$

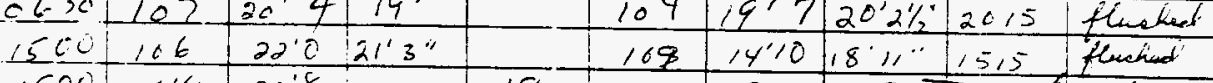

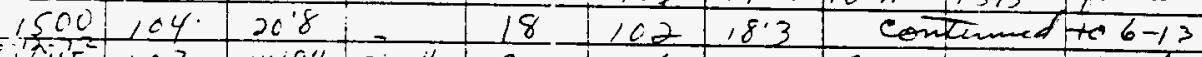

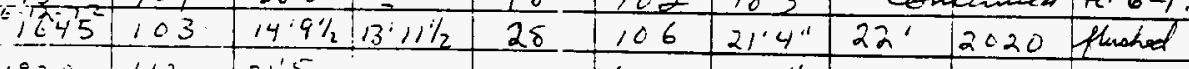

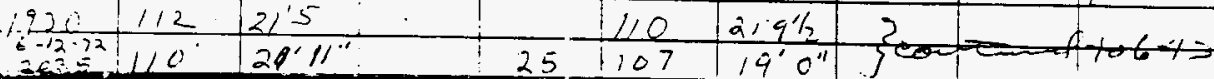

\begin{tabular}{|c|c|c|c|c|c|c|}
\hline$a n k$ & 0100 & 0700 & $(900$ & 1500 & 1700 & 2300 \\
\hline$i 12-6 v$ & $2 c^{\prime} 9 \frac{1}{2}$ & $20^{\prime} 10^{\prime}$ & $\therefore 0^{\prime} 6^{\prime \prime} 2$ & $20+1$ & $21^{\prime 2}$ & $21^{\prime 2} 2^{\prime \prime}$ \\
\hline $11 U-B Y$ & $21^{1} 6$ & $2 x^{\prime} x$ & $\because 1112$ & 2010 & $219 \%$ & $215 \%$ \\
\hline $707-3 y$ & 1918 & $2 c 4$ & $=6 \cdot 2$ & 19.8 & $19 \cdot 2 \%$ & $19^{\prime} 7^{\prime}$ \\
\hline$\because U \div-B Y$ & $14^{\prime} \quad 7 \frac{1}{2}$ & 1918 & $\therefore i$ & $2: 18$ & $20^{\prime} 8^{\prime \prime}$ & 19.11 \\
\hline$B U \leq-E Y$ & 196 & $18 \quad 6$ & 22 & $18^{\prime 3}$ & $18^{\circ} 7 \%$ & $19^{\prime} 8^{\prime \prime}$ \\
\hline $100-t^{\prime} Y$ & $21^{\prime} \quad 5$ & $219 \frac{1}{2}$ & $=1: 71 / 2$ & $20 \%$ & $20^{\circ} 11^{\prime \prime}$ & $20^{\prime} 11^{\prime \prime}$ \\
\hline ivi-ev & 21.4 & $21 \quad 7 \frac{1}{2}$ & $22^{\circ} i$ & 220 & $2,4^{\prime}$ & 22.01 \\
\hline $10-y$ & 185 & $18^{\prime} 9$ & 156 & $18 \cdot 3 / 2$ & $18^{\prime} 10 \%{ }^{\prime \prime}$ & $19.2 "$ \\
\hline $186 y$ & $\frac{14}{4}-1$ & $14^{\prime \prime} 5$ & 19 & 1410 & $14.9 \% 2^{\prime \prime}$ & $13^{\prime} 11 \%$ \\
\hline Totri & 1764 & 177 & $17: 31 / 2$ & $176^{\prime} 41 / 2$ & $177^{\prime} 4 / 2 \cdots$ & $178^{\prime} 2 \%$ \\
\hline ivew Fee: & & & & & $11 \%$ & $i_{1}$ \\
\hline jusied Total & & & & & $178^{\prime} 4^{\prime \prime}$ & \\
\hline
\end{tabular}

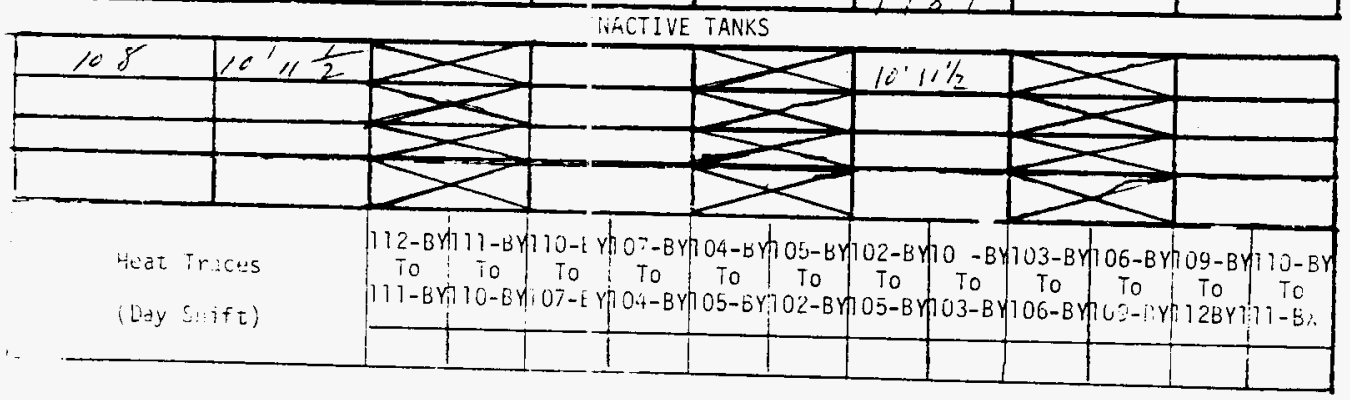

$9-20-7 i$ 


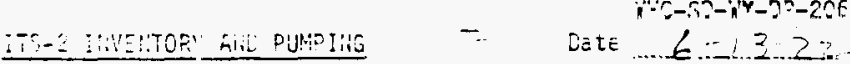

Date

Receiving Tank

Punp-Out Tank

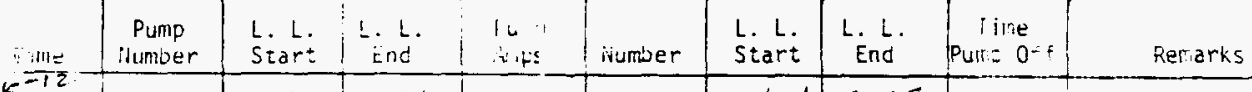

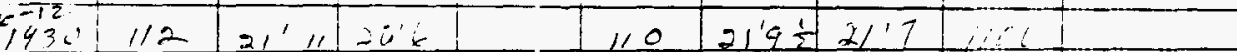

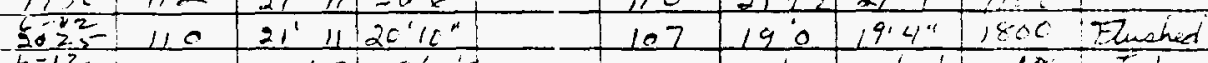

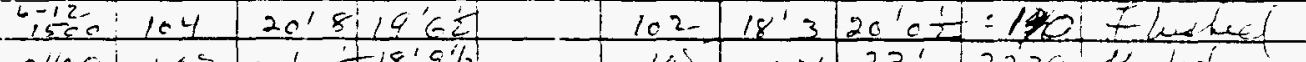

0 une $10 \sum 22^{\prime}=218^{\prime} 9^{\prime} \%$

E4ce: 10s $x^{\prime} 11$

- 6 .

Ofos: $104.20^{\prime} 5 \% 21^{\prime 9}$

$063010720^{\prime} 10+19^{\circ} 0^{\prime \prime}$

\begin{tabular}{l|l|l|l|}
300 & 164 & 209 & \\
\hline 400 & $1630 \times$ & 66 & 91
\end{tabular}

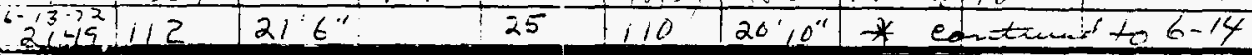

\begin{tabular}{|c|c|c|c|c|c|c|c|}
\hline lank & 0100 & 0700 & 0900 & 1500 & 1700 & 2300 & \\
\hline $112-8 Y$ & $21^{\prime} 12$ & 2116 & 2019 & c.11 & $21^{\prime 2}$ & $21^{\prime} 3 \% 2^{\prime \prime}$ & \\
\hline 1$) \cup-B Y$ & $21<\frac{1}{2}$ & $21^{\prime}=1$ & 217 & $20^{\prime} ! 1$ & $20^{\prime} 10^{\prime \prime}$ & $21.312^{\prime \prime}$ & \\
\hline $307-3 Y$ & $19 \div 2$ & $2 c$ & 2017 & $2-1 / 2$ & $19.7 \%$ & $19.0^{\prime \prime}$ & \\
\hline$B U_{4}^{n}-B Y$ & $19 \quad 9$ & 19.62 & $20^{\prime} 3$ & 2611 & $2 i^{\prime} 3$ & $20^{\prime} 8^{\prime \prime}$ & \\
\hline $70 \div-6 Y$ & $19 \quad 9 \frac{1}{2}$ & 197 & 42 & $18^{19}$ & $18^{\prime} 9^{\circ}$ & $18^{\prime} 10^{\circ}$ & \\
\hline $100-1 y$ & $2 c \quad 11$ & 208 & 2016 & $207 / 7$ & 211 & $22^{\prime} 0^{\prime \prime}$ & \\
\hline Fut- - & 220 & $21 \quad 103$ & $21: 9$ & 200 & $22^{\prime} 0^{\prime \prime}$ & $22^{\circ} c^{\prime \prime}$ & \\
\hline $10 \%-y$ & 196 & $5 \frac{1}{2}$ & 41 & $\therefore 71 / 2$ & $19 \%$ & $18^{\circ} 11^{\circ}$ & \\
\hline $10 B$ & $13 \quad 11 \frac{1}{2}$ & 144 & $14: 4$ & 150 & $15^{\prime} 0^{\prime \prime}$ & $15^{\circ} 0^{\prime \prime}$ & \\
\hline & 45 & $\frac{4}{5} 2$ & & & & & \\
\hline Total & $1786 \frac{1}{2}$ & 1778 & 180 & $17781 / 7$ & $178.9 \%$ & $179^{\prime} 1 / 2^{\prime \prime}$ & \\
\hline ivew Fees & & & & $+x$ & $1 \cdot 9 \cdot 1$ & & \\
\hline -ujusted Total & & & & $\Longrightarrow$ & $179^{\prime} 12^{\prime \prime}$ & & \\
\hline
\end{tabular}

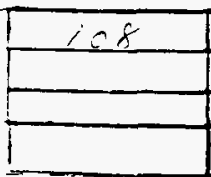

Heat Tr.actss

( Day Stifi)
IIACTIVE TANKS

$103,13 \%, 2+2,0$

$10918^{\prime}>14^{\prime}$ os 30

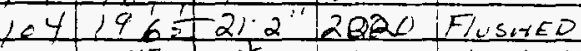

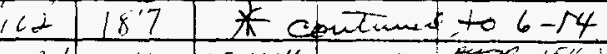

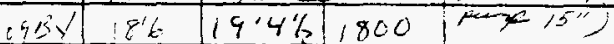

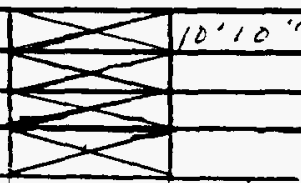

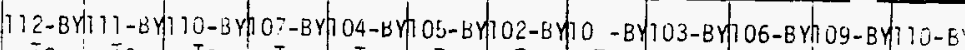
To To To To To To To To To To To To

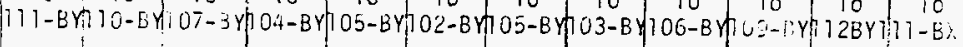

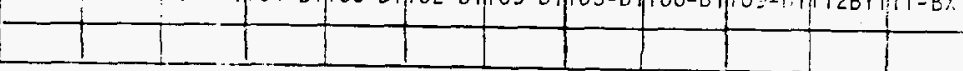




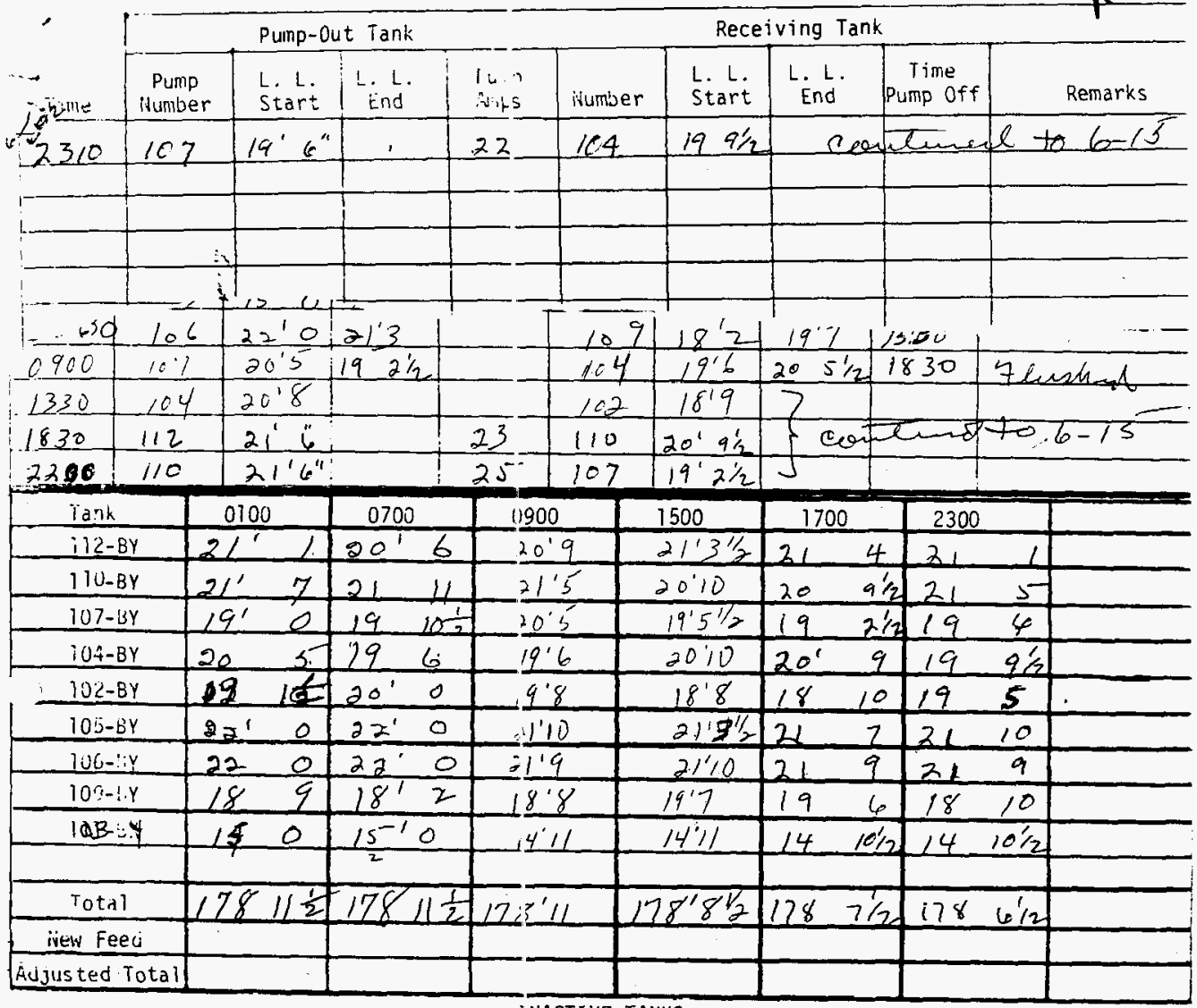

\begin{tabular}{|c|c|}
\hline 108 & $10 \%$ \\
\hline 108 & $10 \%$ \\
\hline & \\
\hline & \\
\hline & Heat Traces \\
\hline
\end{tabular}

INACTIVE TANKS

$9-20-71$

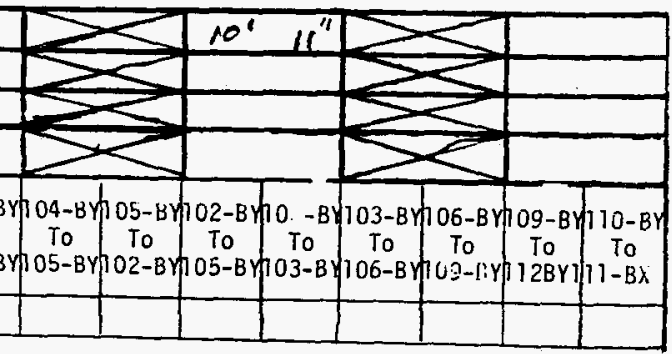

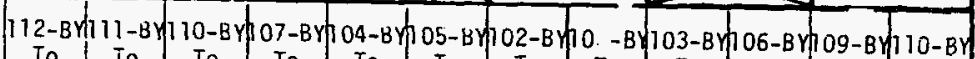

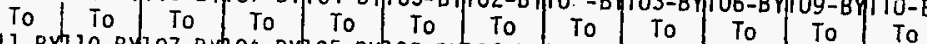

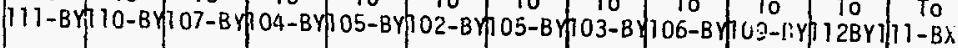




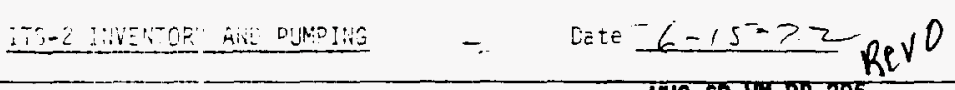

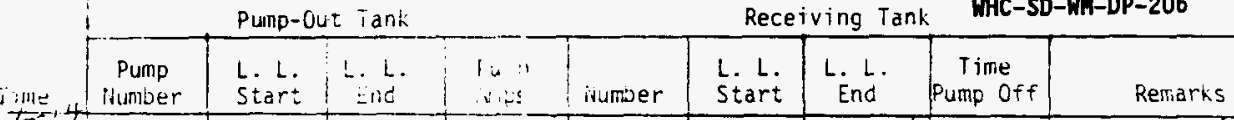

Receiving Tank WHC-SD-WT-DP-206

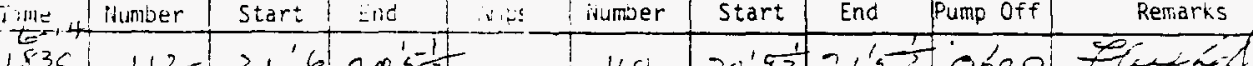

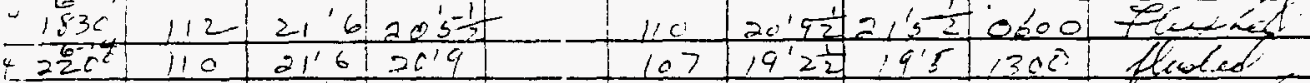

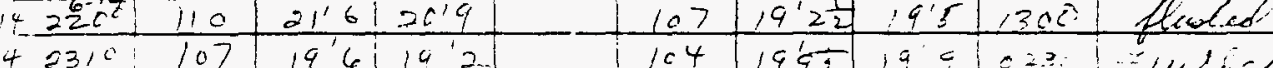

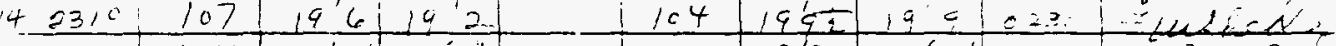

\begin{tabular}{l}
42301104 \\
\hline
\end{tabular}

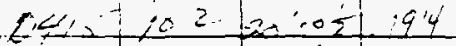

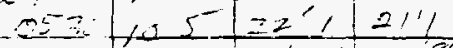

$\therefore \quad 123141,140 \%$

$=53023:=1 \%+21^{\prime} 1^{\prime \prime} 2$

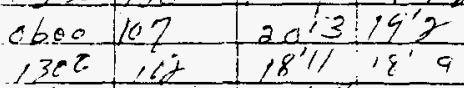

\begin{tabular}{l|l|l|l|l|l|}
150 & $1 \%$
\end{tabular}

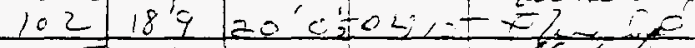

.

(1)

$125321 \%$ 2112 0.6

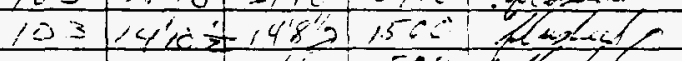

$10 \Leftrightarrow 2,90216$

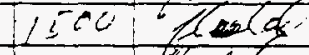

$1051280-1011$ 1300 Zecket

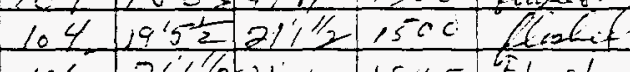

10s $211 / 2+21,1019+5$ Finshed

\begin{tabular}{|c|c|c|c|c|c|c|c|c|}
\hline lajk & 0100 & 0700 & 0900 & 7500 & .700 & & 2300 & \\
\hline$\overline{i 12-8 Y}$ & $20^{\prime} 11 \frac{1}{2}$ & $20^{\prime} 6$ & $20^{\prime} 9$ & 214 & 21 & $4: 2$ & 21 & \\
\hline $110-B Y$ & $3 \frac{1}{2}$ & $21^{\prime} 4$ & $20^{\circ} 10^{\prime \prime} 2$ & $2 c^{\circ} \%$ & 20 & 9 & 211 & \\
\hline $107-6 y$ & $4 \div$ & $=7$ & $2 c^{\prime} 2 \frac{1}{2}$ & $1912 \geq$ & 19 & 2 & 19 & \\
\hline$\therefore 0 . \div-B^{Y}$ & 19 & 19 & $26^{\prime} 4$ & $21^{1} 1 / 2$ & $2 c$ & 912 & $20^{\prime} 3$ & \\
\hline $10 \angle-B Y$ & $19 \quad 5=$ & $191 \quad 2 \frac{1}{3}$ & $19^{i 4}$ & $18^{\prime} 8$ & 18 & $T$ & 18 & \\
\hline $10 \nu-\mathrm{LYY}$ & 21 & $22 \quad 0 \frac{1}{2}$ & 2110 & $21 \%$ & 21 & 4 & 2110 & \\
\hline luci-.." & 21 & $2 \% 8$ & 216 & 216 & 21 & 6 & 21 & \\
\hline $10:-y$ & $18 \quad 8 \frac{1}{2}$ & $18^{\prime} \geq \frac{1}{2}$ & 1818 & 199 & 19 & 7 & iद & \\
\hline $103-\therefore$ & $14 \quad 10 \frac{2}{2}$ & $j 4 \quad 10^{2} \Leftrightarrow$ & $14^{\prime} i i^{\circ}$ & $1461 / 2$ & 17 & $\overline{8}$ & $1+$ & \\
\hline & 63 & 2 & & $17 \times 2{ }^{3}$ & & & & \\
\hline To:al & $17 x^{\prime} 5$ & $198^{\prime} 12$ & $178^{\prime} 3$ & $128 \%$ & 178 & & $1,77^{\prime} / 1$ & \\
\hline ivew Fees & & & & & & & & \\
\hline Iujus ted Total & & & & & & & & \\
\hline
\end{tabular}

168

IIACTIVE TANKS

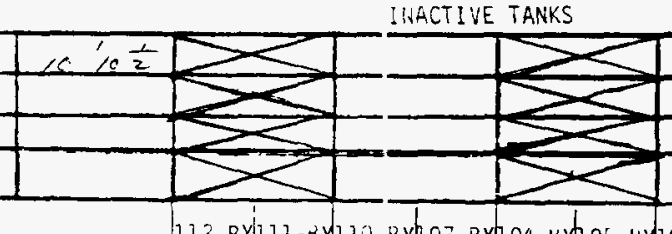

Heat Traces

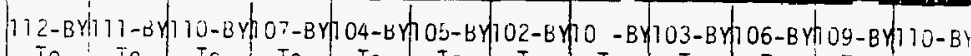

(bay S!ifi)

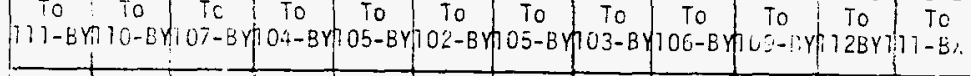
1

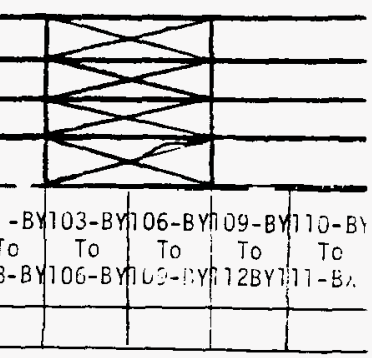




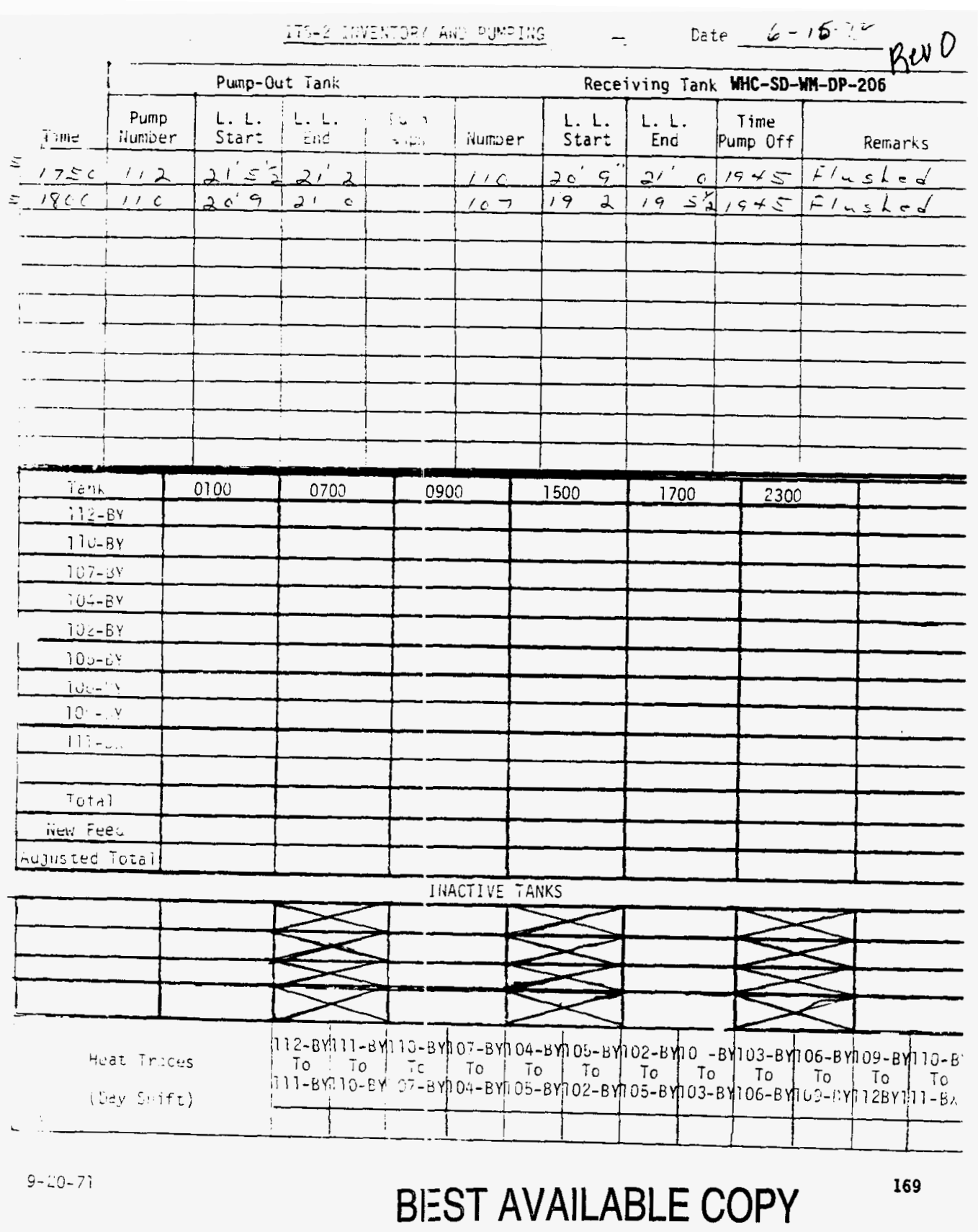




\section{I:- \\ Receiving Tank}

Puinp-Out Tanis

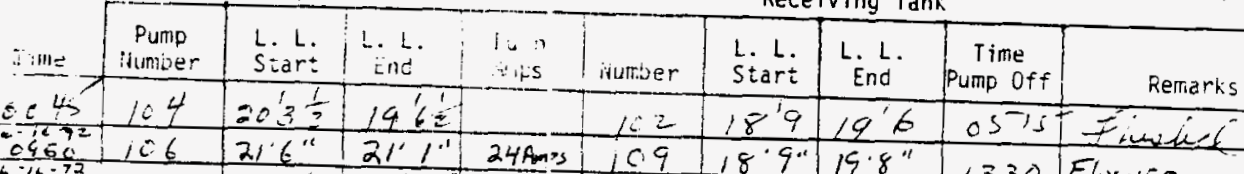

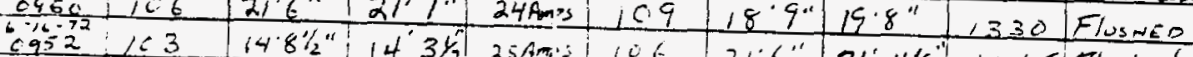

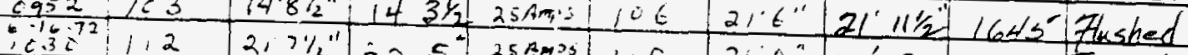

$4-16+721$

16: 105

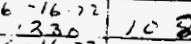

$2, \| 1 \% 1 \% 1 \%$

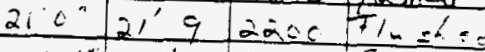

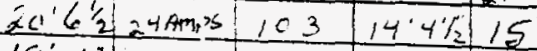

$\frac{330}{-16-72+12}$

$18^{\prime} 11^{\circ} \mid 23 A A^{\prime}=1,65$

$2,7 \% " 22^{\prime \prime}$

1920

$7 /$ usines

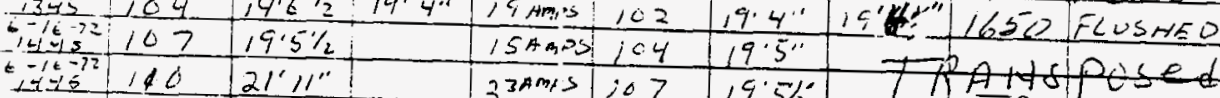

z 3 amis 107

$15^{\circ} 52^{\prime}$

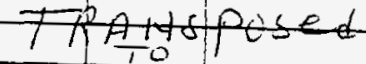

$6-17+72$

d) $T \mapsto F=e r$

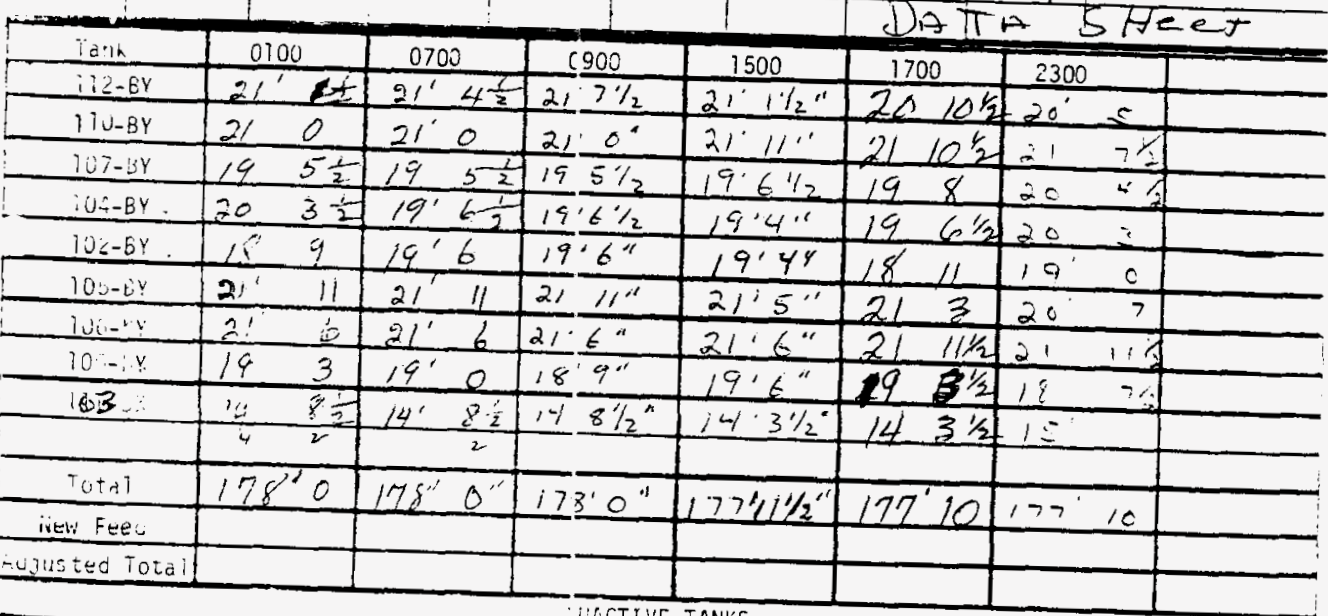

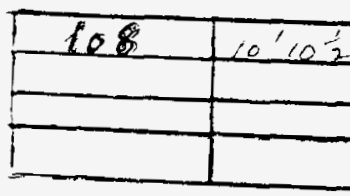

Heal Traces

liey s:ift)

IAACTIVE TANKS

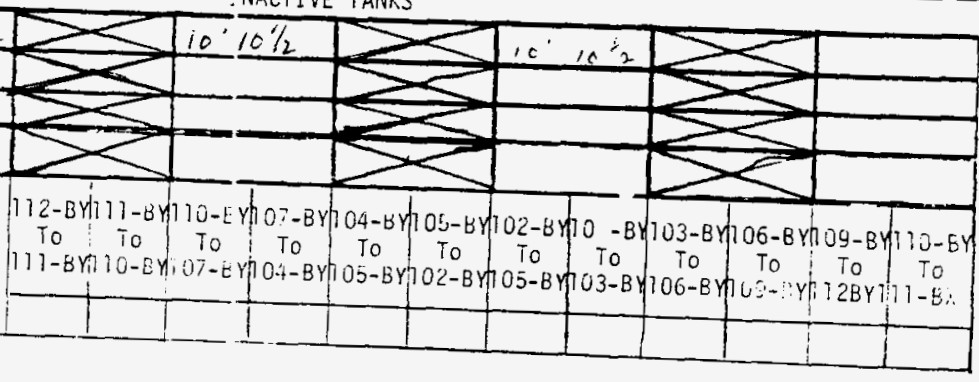

$9-20-7 i$ 


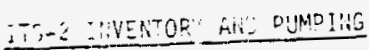 \\ Date $6-17-72$}

Receiving Tank

Punp-out Tarie

L. L. L. L. Time

Pump L.L. L. L. L L W Wumer Start End Pump Off

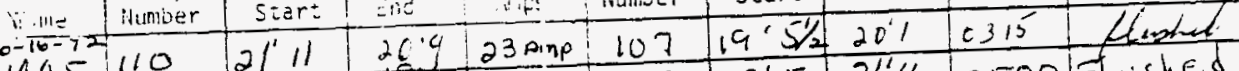

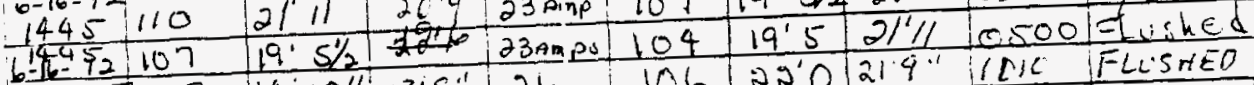

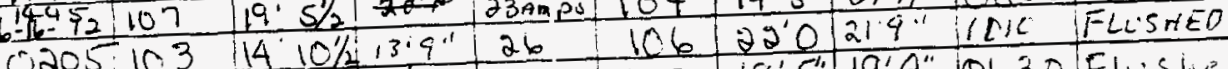

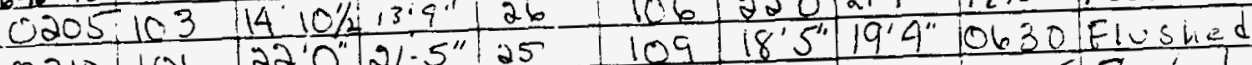

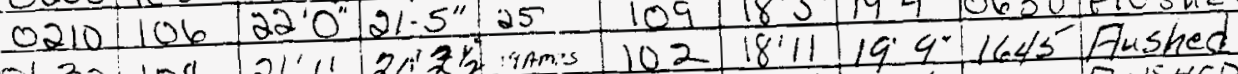

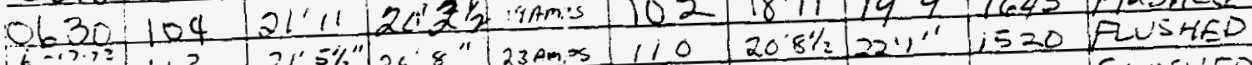

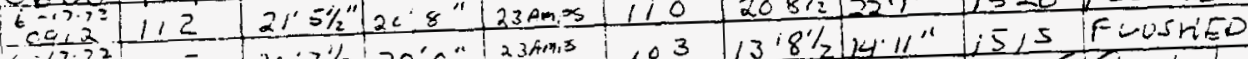

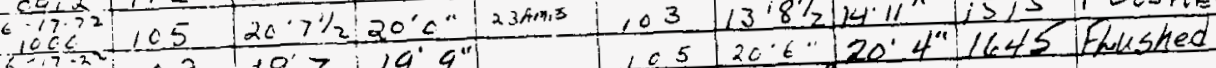
$6^{-172}+1 c^{2}+19^{\prime} 719^{\circ} 4^{\prime \prime}$

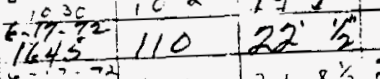

\begin{tabular}{l|l|l}
$2 \operatorname{coc}^{2}, 106$ & $21 / 20-0 \%$
\end{tabular}

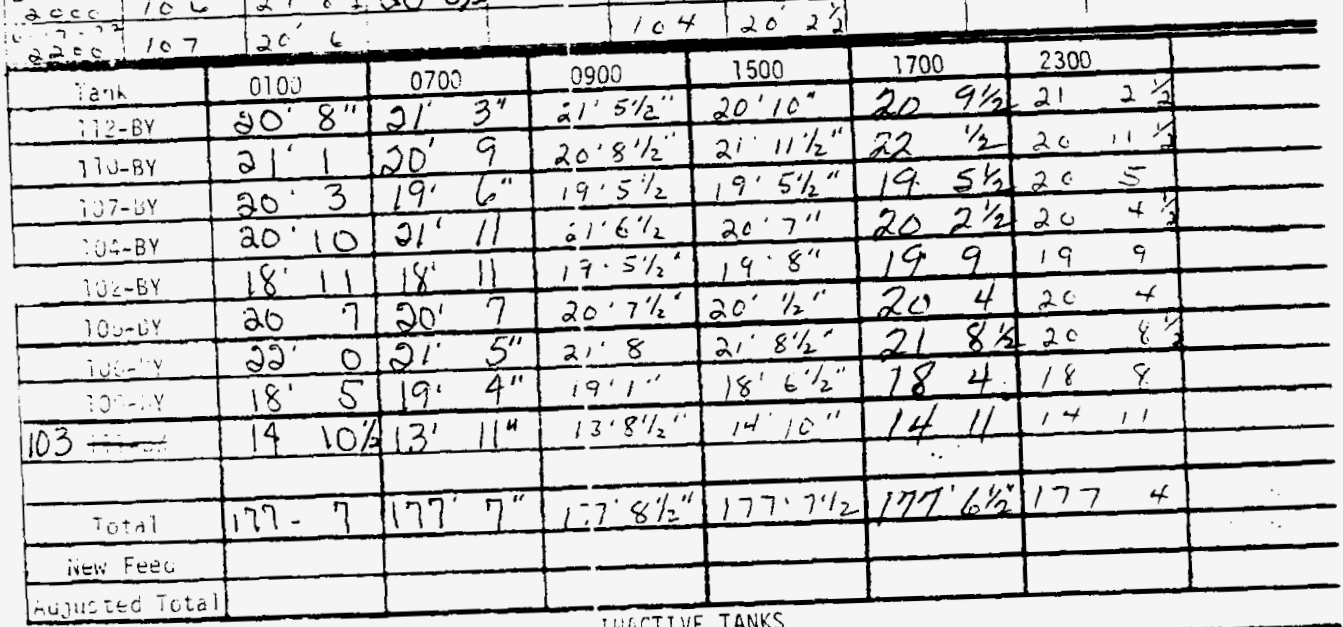

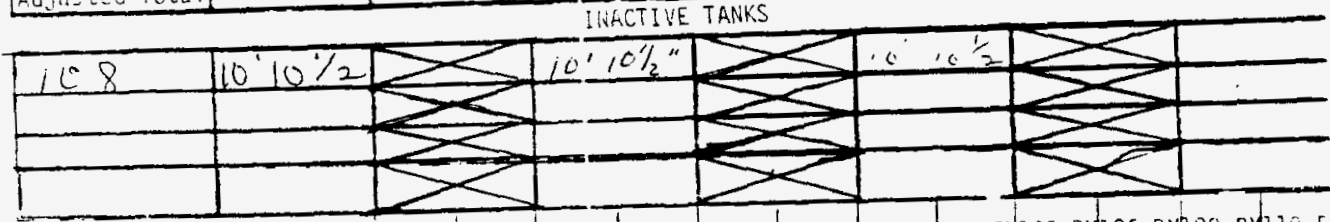

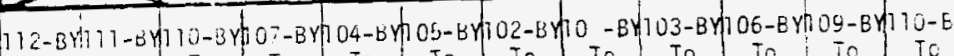

Het Tr:ous

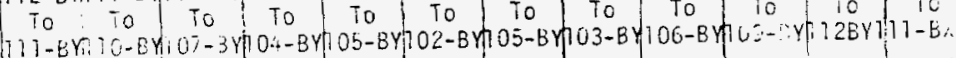

(bey Stift 


\section{IIT-2 liNenTORY mill pUMDIHG $\quad-. \quad$ Date $6-17-72$}

\section{Pump-Ou= Tank}

Receiving Tank

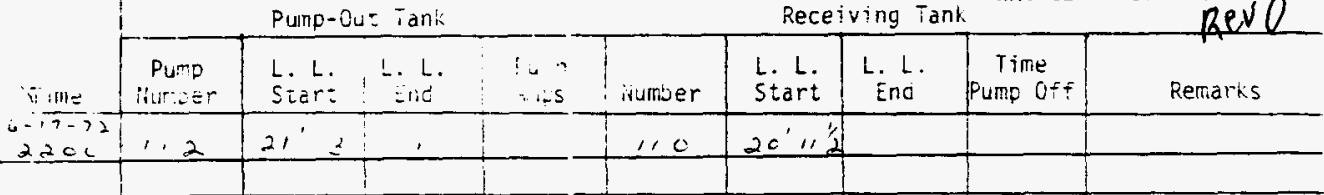

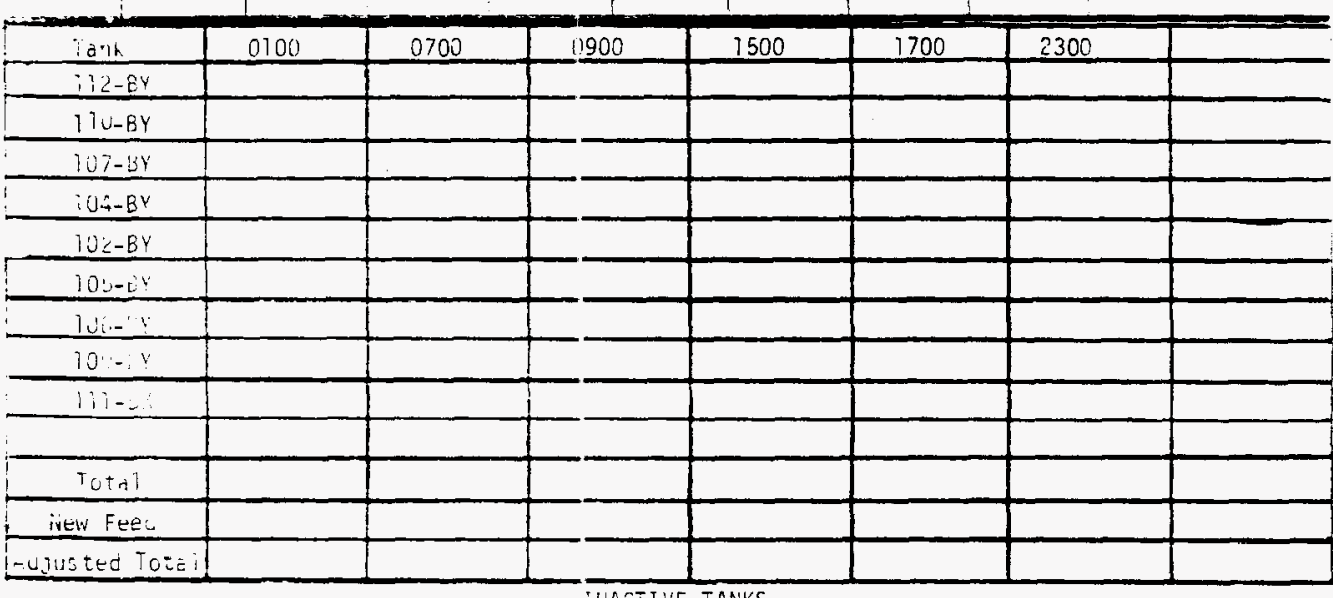

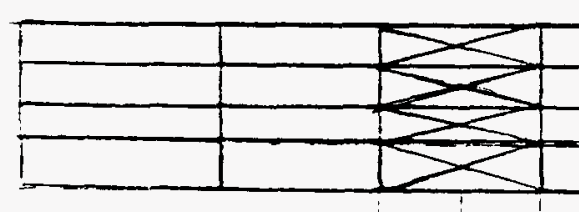

IIACTIVE TANKS

Hoci ir:ass
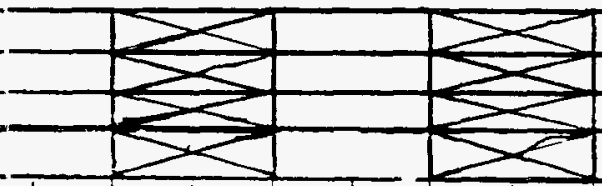

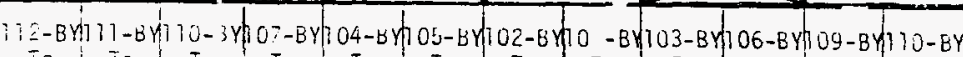
( Dey $\vdots i f \tau)$

\section{To to to to To to To To To To to to To To}

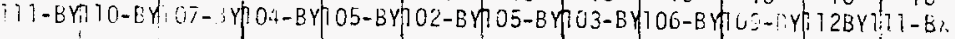

2 $+$

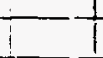




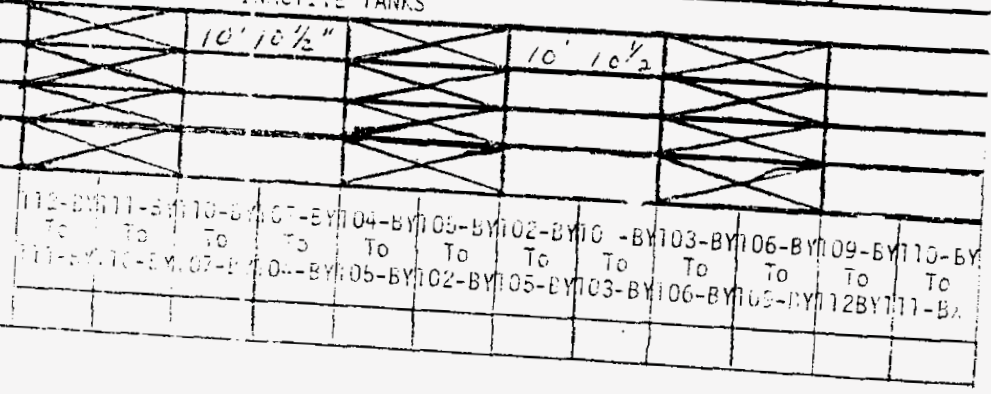




\begin{tabular}{|c|c|c|c|c|c|c|c|c|c|}
\hline & \multicolumn{4}{|c|}{ Pump-Out Tank } & \multicolumn{5}{|c|}{ Receiving Tank } \\
\hline mie & $\begin{array}{l}\text { Pump } \\
\text { Number }\end{array}$ & $\begin{array}{l}\text { L. L. } \\
\text { Start }\end{array}$ & 1. L. & $\begin{array}{l}\text { Lip } \\
\text { vips }\end{array}$ & rumber & $\begin{array}{l}\text { L. } L \text {. } \\
\text { Start }\end{array}$ & End & $\begin{array}{c}\text { Time } \\
\text { Pump Off }\end{array}$ & Remarks \\
\hline 00 & 102 & 19.4 & $19-6$ & & 105 & $19-7$ & $27-5 / 2$ & 2300 & 7 LuSdEO! \\
\hline 30 & 10.9 & $22-6$ & $19-5$ & 18 & 102 & $9-3$ & 19.10 & 0030 & Fushed \\
\hline 00 & 106 & $21 \cdot 11$ & 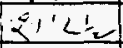 & 25 & 109 & $18-6$ & iq: & ILIC & $7 L 4 \leq 1<e^{-6}$ \\
\hline & 115 & $81-4$ & $20^{\prime} i 2$ & $00-23$ & 107 & 19.4 & 201 & $12 \mathrm{AT}^{2}$ & $11-x+2$ \\
\hline 40 & 103 & $15-1$ & 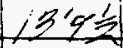 & 24 & 106 & $01-10 \%$ & $320^{\circ}$ & 侄必 & 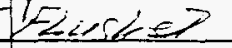 \\
\hline & 112 & $2: \leq$ & $\therefore 0-6$ & & 110 & $2 \div 7 \frac{1}{2}$ & $\div 3-2$ & $210 \mathrm{c}$ & 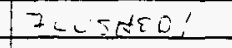 \\
\hline & $10-7$ & 2011 & $18-10$ & & 104 & $2.7=$ & $2 c-9$ & 1715 & $7_{1}=4501$ \\
\hline & 105 & 2019 & $21^{\circ} 9 \%$ & & 112 & $13 \div 0$ & 150 & 1845 & $F l u s h=d$ \\
\hline & $106-B y$ & 22.0 & $=0-8$ & & $109.8 Y$ & $18-10^{1 / 2}$ & $19.71 / 2$ & 2000 & IL:BSES: \\
\hline & 110.34 & $21-8$ & & & 107.54 & $18 \cdot 10$ & TRAMS & posed & $6 \cdot 20 \cdot 72$ \\
\hline & $10 \Xi-8 Y$ & $14-111 / 2$ & & & & $20-8$ & DATE & SHect & \\
\hline
\end{tabular}

\begin{tabular}{|c|c|c|c|c|c|c|c|}
\hline iank & 0100 & 0700 & $0 \leq 00$ & 1500 & 1700 & 2300 & \\
\hline$i 12-B Y$ & $20 \cdot 5 / 2$ & $20-10$ & $2,1 \%$ & 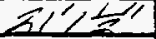 & $20-11$ & $20-91 / 2$ & \\
\hline $11 U-B Y$ & $31-4$ & $21=$ & 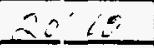 & $3, z \frac{1}{2}$ & $21-7$ & $2:-9$ & \\
\hline $307-B Y$ & $19-4$ & $19=$ & 101,16 & $1 q^{\prime} z=z^{\prime \prime}$ & $18-111 / 2$ & $19-\varepsilon$ & \\
\hline$: 04-B Y$ & $22-6$ & $1+16$ & $21: 2$ & $B E^{\prime} / L^{\prime \prime}$ & $20-4_{1} 1 / 2$ & $19-8$ & \\
\hline $7 U Z-B Y$ & $19-7$ & 3 & $194 \frac{1}{2}$ & $19^{\prime \prime} / 7^{\prime \prime}$ & $19-712$ & $19-6$ & \\
\hline $100-8 y$ & $20-0$ & 21. & $2=1.5 \% 2$ & $3 \pi<-\frac{1}{5}$ & $21-111 / 2$ & $2+51 / 2$ & \\
\hline ivu-iy & $21-11$ & $21-$ & 21,4 & $2 z \prime 01$ & $32-c$ & $20^{\circ} 8$ & \\
\hline $10 \div-i: y$ & 17.10 & $15-$ & $16: 2$ & $19 /=-\frac{1}{3}$ & $18-10 \%$ & $19-41 / 2$ & \\
\hline 103 & $15-1$ & $15=$ & 147 & $16^{\prime} z^{\prime \prime}$ & $14-61 / 3$ & $i 4-111 / 2$ & \\
\hline Total & 178.013 & 3 & 7 & $117817 \frac{11}{2}$ & $179-3$ & $178-10$ & \\
\hline iven: Feed & & & & & & & \\
\hline ajusted Total & & & & & & & \\
\hline
\end{tabular}

INACTIVE TANKS

Heat Tracts (Day suift)




\begin{tabular}{|c|c|c|c|c|c|c|c|c|c|}
\hline & \multicolumn{4}{|c|}{ Punp-Out Tank } & \multicolumn{5}{|c|}{ Receiving Tank } \\
\hline inle & $\begin{array}{c}\text { Pump } \\
\text { Numiver }\end{array}$ & $\begin{array}{l}\text { L. L. } \\
\text { Start }\end{array}$ & E. L. & $\begin{array}{l}\text { I w.n } \\
\text { nips } \\
\text { and }\end{array}$ & Number & $\begin{array}{l}\text { L. L. } \\
\text { Start }\end{array}$ & L. L. & $\begin{array}{c}\text { Time } \\
\text { Pump off }\end{array}$ & Remarks \\
\hline 120 & $110 \mathrm{BH}$ & $21 \cdot 8$ & $20+11$ & & $1078 y$ & 1810 & $20-6$ & 0545 & Floshed \\
\hline 300 & 10330 & $14-11 \%$ & 14.4 & 95 & $106 \mathrm{Bu}$ & $20-8$ & 220 & 0220 & \\
\hline 0115 & 10584 & $22-6$ & $21-6$ & 34 & 103 के & $14-4 / 2$ & $14-9 \%$ & 0505 & Elusked \\
\hline 2430 & 10.134 & 20.8 & $1 G^{\prime} / 11$ & 20 & $104 \mathrm{BY}$ & 19.5 & $2015 \frac{11}{2}$ & $20 \mathrm{CC}$ & FElutices \\
\hline 0440 & 11264 & $21-4$ & $20 \%$ & 24 & $110 \mathrm{By}$ & $20-9$ & $22 \%$ & 1110 & $F L U S A=?$ \\
\hline$c 530$ & $10 \mathrm{~b}$ BY & $21-11$ & $20^{\prime} z^{\prime \prime}$ & 25 & $109 \mathrm{BH}$ & $18 \cdot 8$ & $10^{\prime} 0^{\prime \prime}$ & 1020 & $F \leqslant u \leqslant t \leqslant 2$ \\
\hline 1020 & $103 B 1$ & $14^{\prime} 51 / 2$ & $13^{\prime} 9^{\prime \prime}$ & 21 & le $6 \mathrm{et}$ & $20 \%$ & $21 " 41 / 2 "$ & 1500 & $F \in u s \alpha E$ \\
\hline - & 10 & $2 a^{\prime}=1$ & 20161 & 24 & 122 & $19^{\prime \prime}$ & $14^{\prime} 4 t$ & 1830 & \\
\hline
\end{tabular}

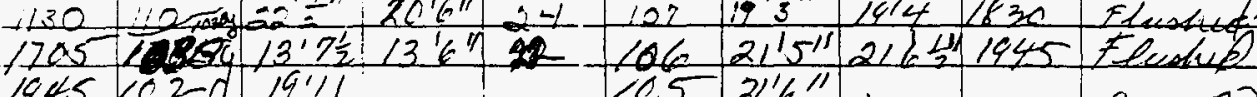

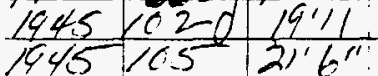

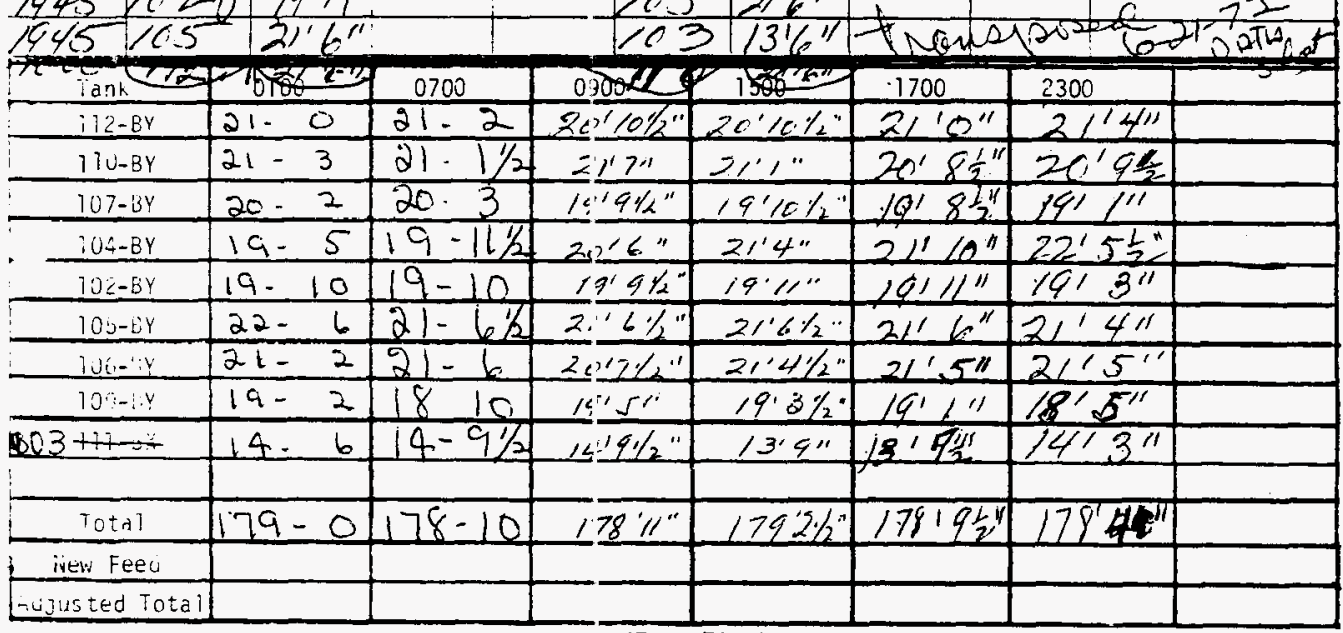

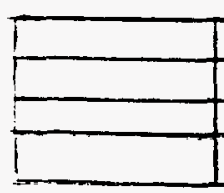

Heat Traces

(Lay Stift)

\section{NACTIVE TANKS}
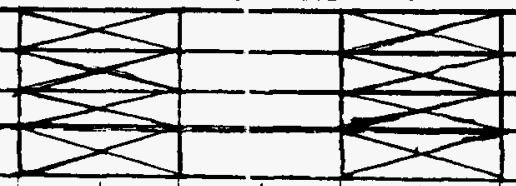

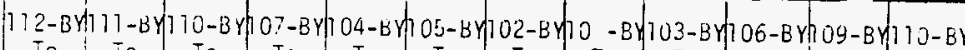

To To To To To To To To To to To To

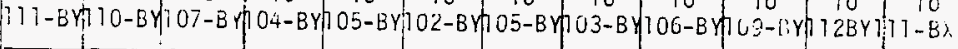

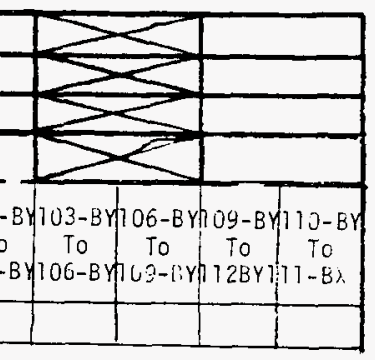




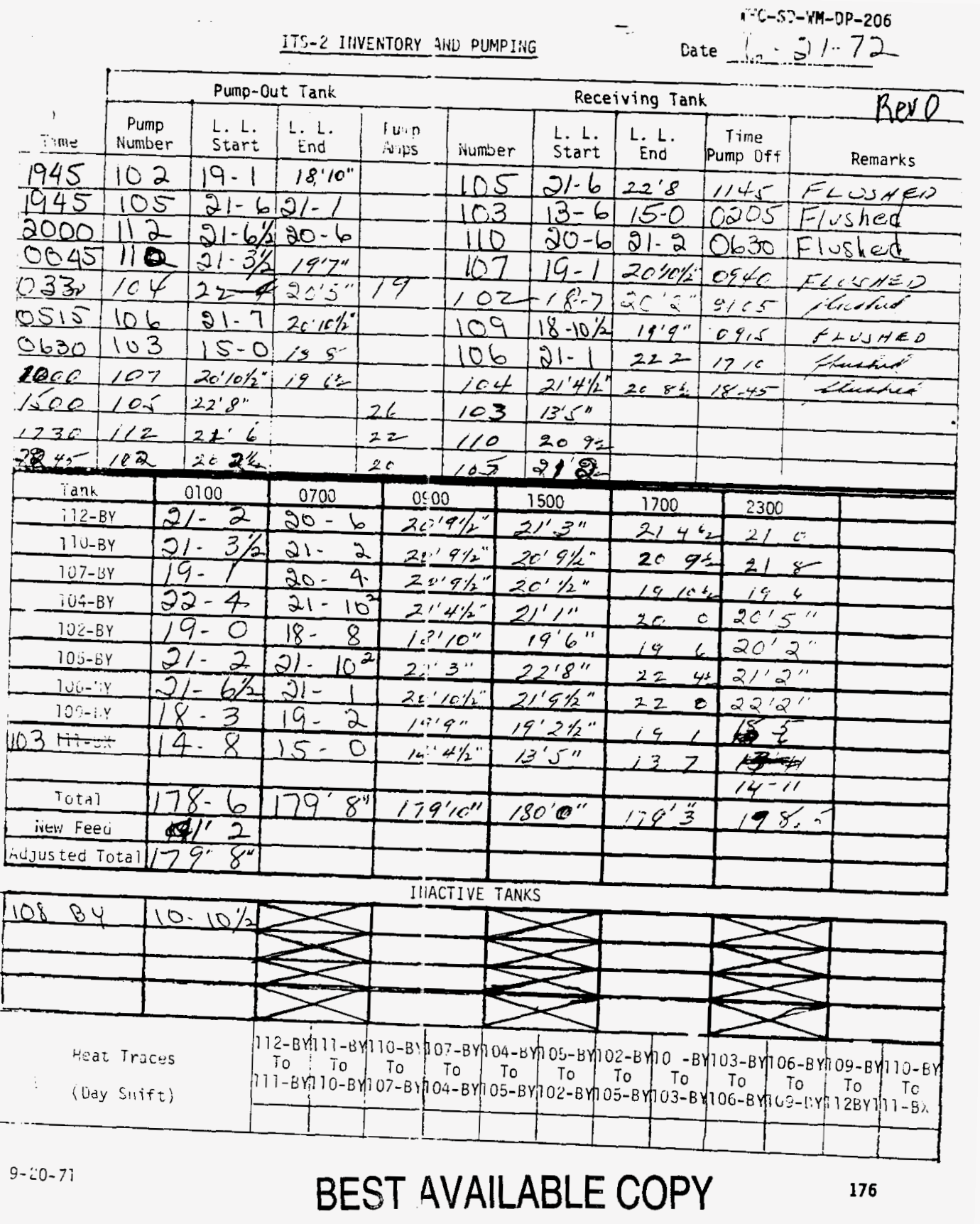




\begin{tabular}{|c|c|c|c|c|c|c|c|c|c|}
\hline \multirow[b]{2}{*}{$\begin{array}{c}1 \\
\vdots m=\end{array}$} & \multicolumn{4}{|c|}{ Pump-Out Tank } & \multicolumn{5}{|c|}{ Receiving Tank } \\
\hline & $\begin{array}{c}\text { Pump } \\
\text { Nuniver }\end{array}$ & $\begin{array}{l}\text { L. L. } \\
\text { Start }\end{array}$ & 1. L. & $\begin{array}{l}\text { lu, n } \\
\text { vips }\end{array}$ & Number & $\begin{array}{l}\text { L. L. } \\
\text { Start }\end{array}$ & L. L. L. & $\begin{array}{c}\text { Time } \\
\text { Pump off }\end{array}$ & Remarks \\
\hline 500 & $105 \mathrm{~B}$ & $25^{\circ} \cdot 8^{\prime \prime}$ & $21-0$ & 26 & 103 & $13^{\circ} 5^{\prime \prime}$ & $15^{\prime} 5^{\prime \prime}$ & 0030 & Flushed \\
\hline 1790 & 1120 & $21 \cdot 6$ & $20-6$ & 22 & 110 & $20.9 \%$ & 22.0 & 0300 & Flushed \\
\hline 2245 & $102 B$ & $202 / 2$ & 188 & 20 & 105 & $21^{\prime} 2^{\prime \prime}$ & $225 \frac{1}{2}$ & 0.960 & 11 \\
\hline 0030 & $1068 Y$ & $22 \cdot 2$ & $19-10 \%$ & 24 & 109 & $18^{\prime} 3^{\prime \prime}$ & $19-7$ & 10615 & $F \mid \cup s h \in d$ \\
\hline 0050 & 110 34 & $22-1$ & $?$ & 23 & 107 & $19^{\prime} 6^{\prime \prime}$ & & & \\
\hline 5.695 & $103 \mathrm{gy}$ & $5.4 \%$ & 2 & 24 & 106 & $19-10 / 2$ & & & \\
\hline$c 345$ & 107 & $20^{\circ} 4 v_{2}$ & $194 \frac{1}{5}$ & 15 & 16.4 & 20.4 & $42-3^{\prime \prime}$ & $2 x=0$ & Fin-shed \\
\hline $1 \sqrt{10}$ & 112 & $21^{\prime \prime \prime}$ & 20 lot & 24 & 110 & $20 \% 11 \%$ & $=24$ & 2315 & texex \\
\hline
\end{tabular}

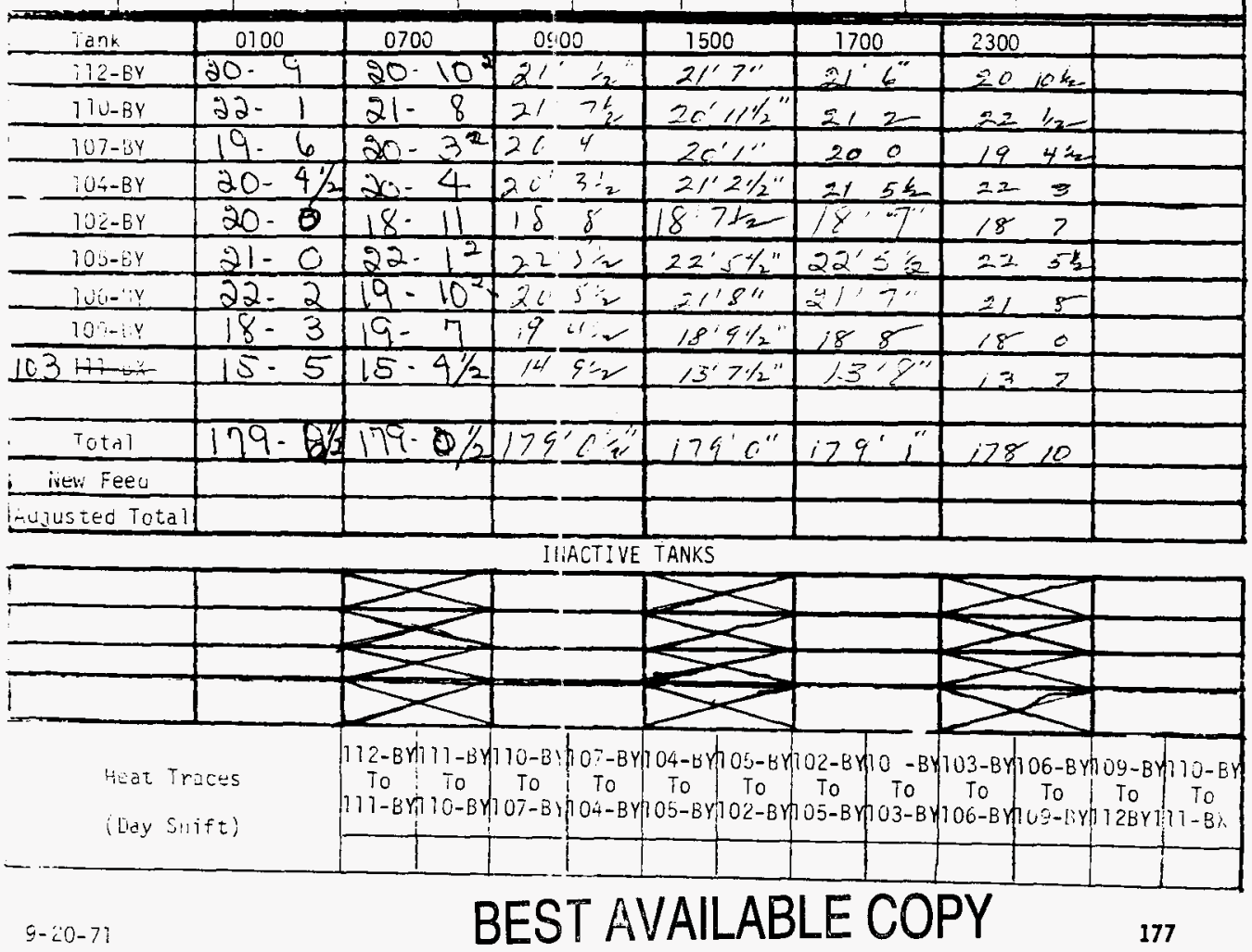




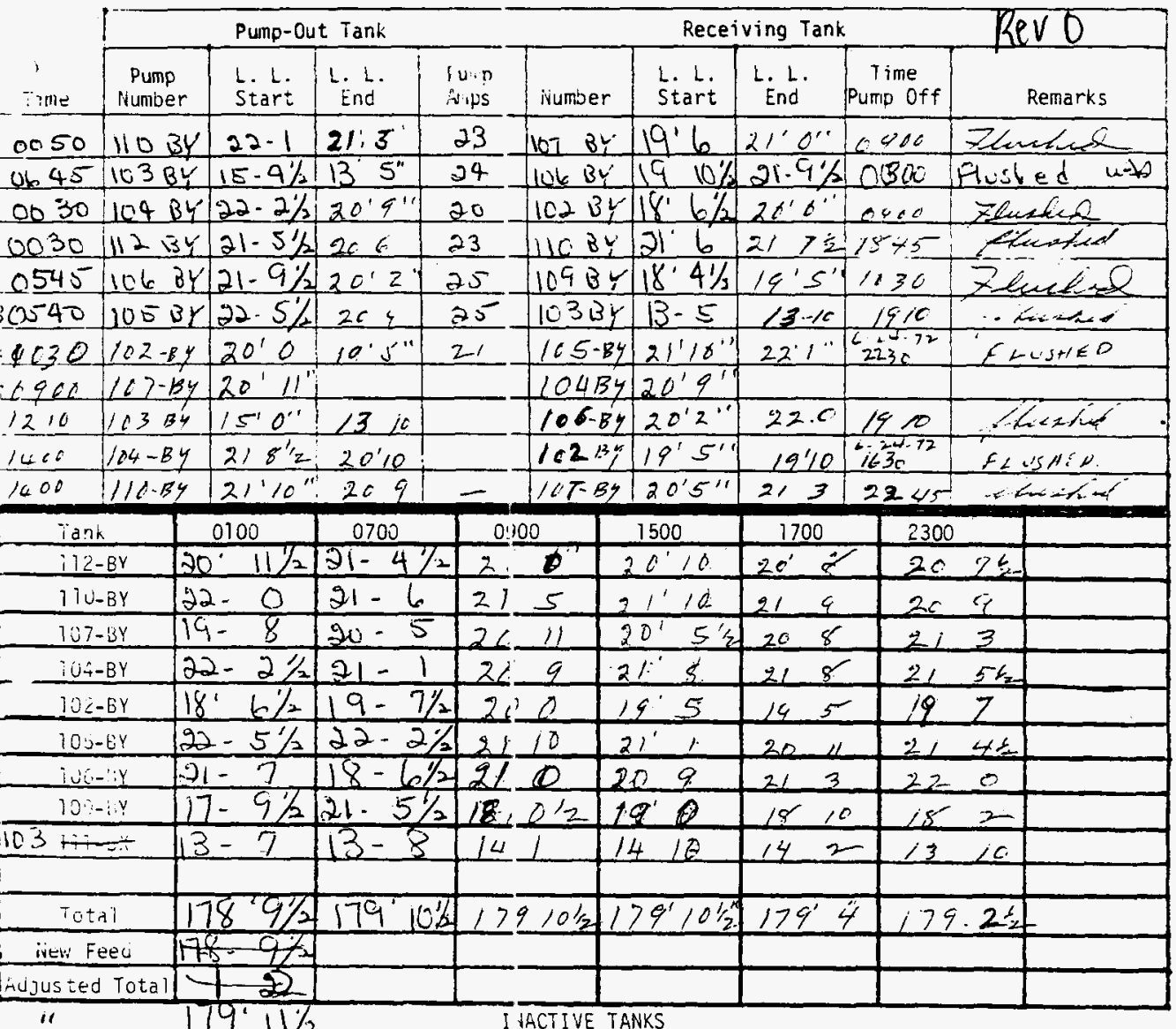

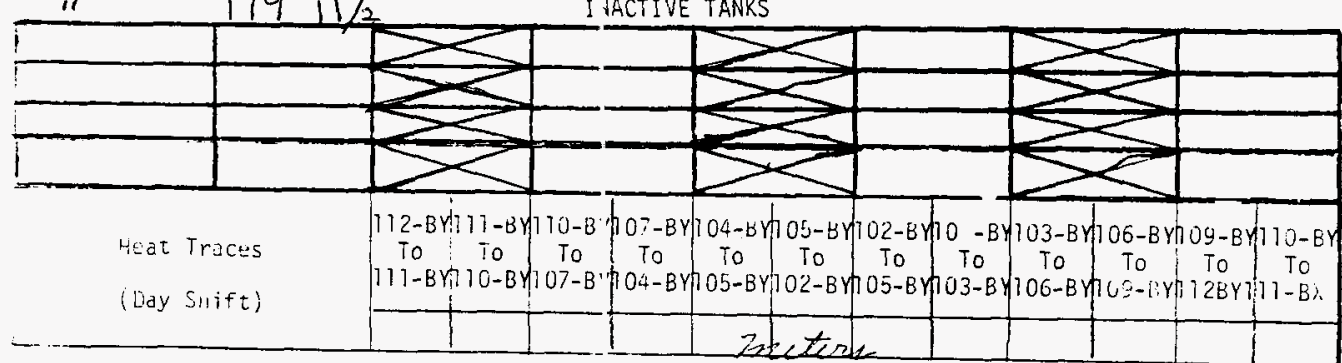

3725460

9.20-71 BEST AVAILABLE CIDPY $75 \times 998 \times 140$ 


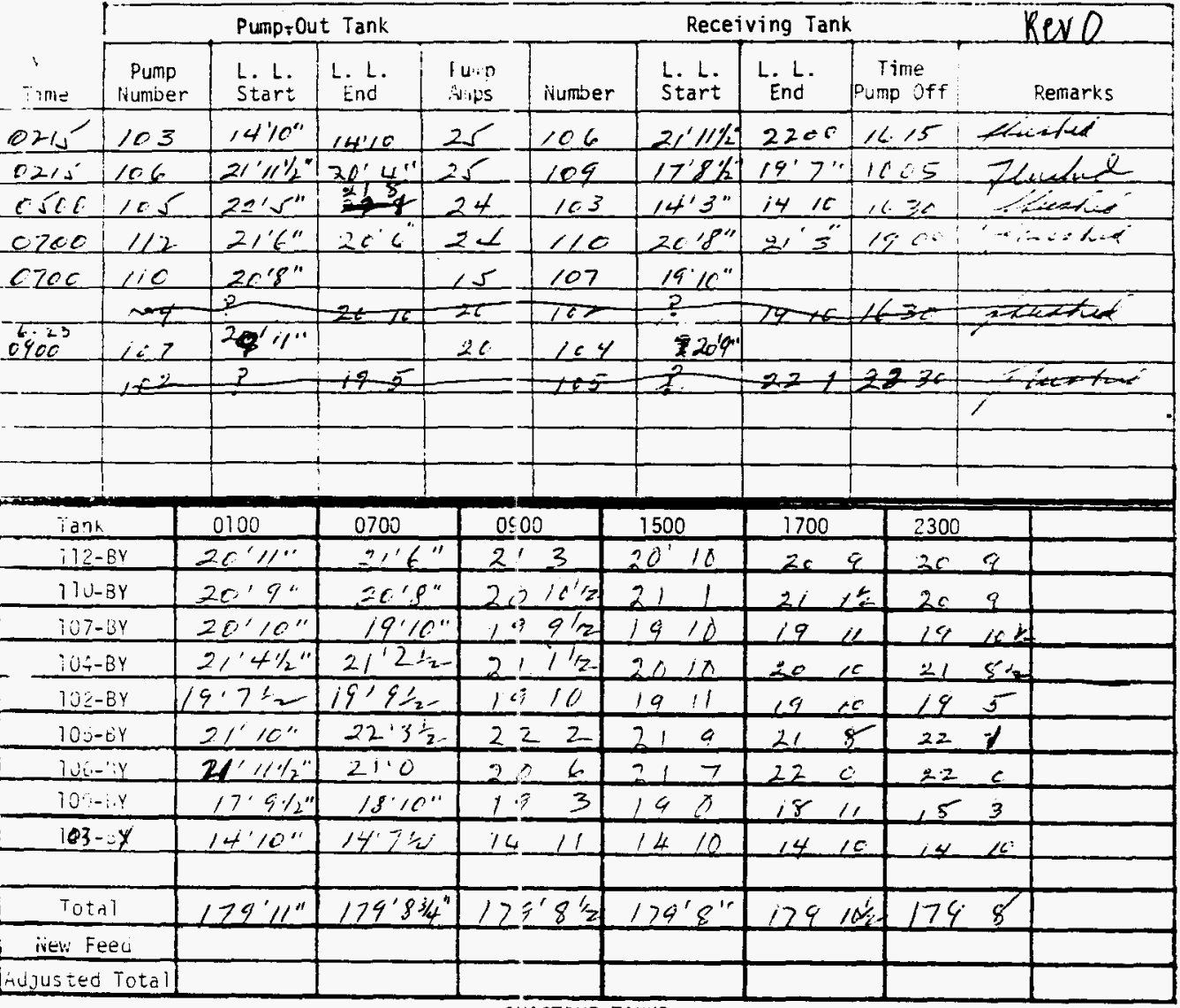

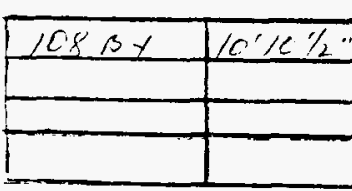

IIAACTIVE TANKS
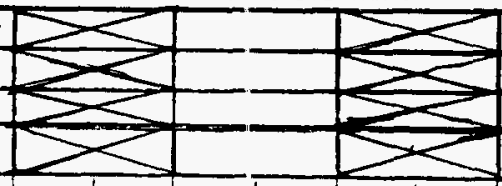

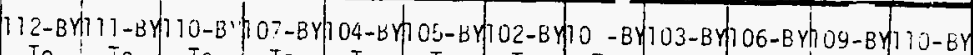

Heat Traces

To To To To To To to To To to To To

(bay Stift)

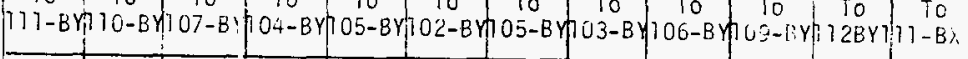

9:-20-71 BEST AVAILABLE COOPY 


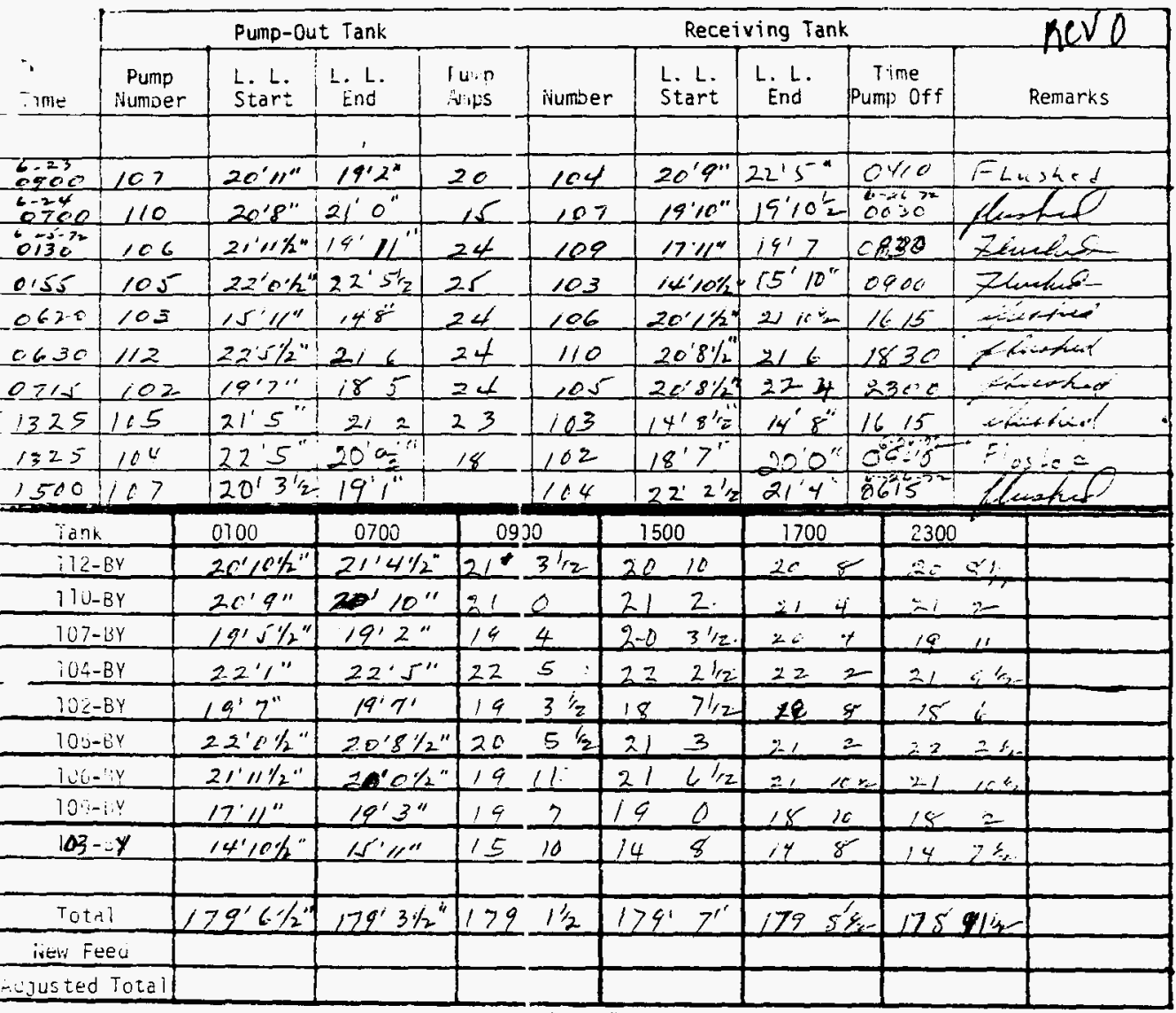

INACTIVE TANKS
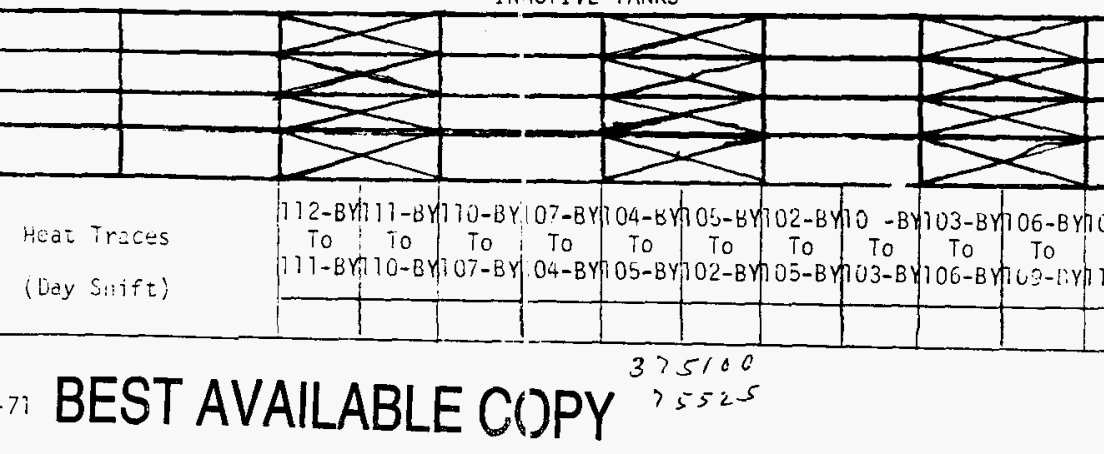

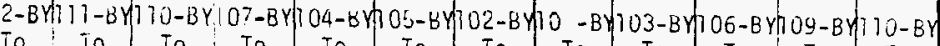

To To To To To To To

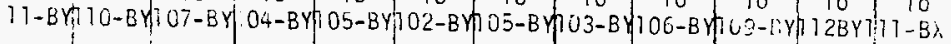




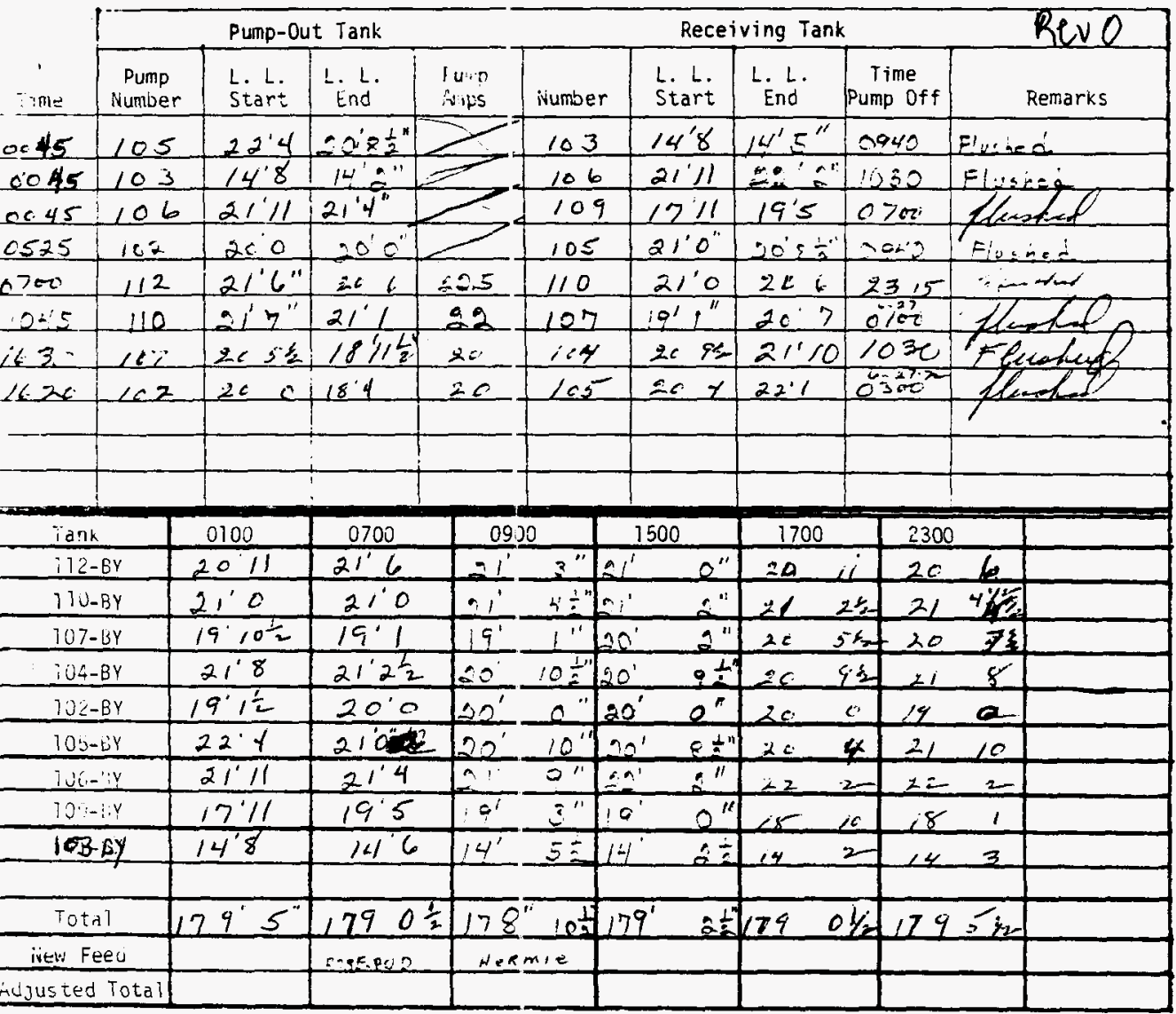

\section{INACTIVE TANKS}

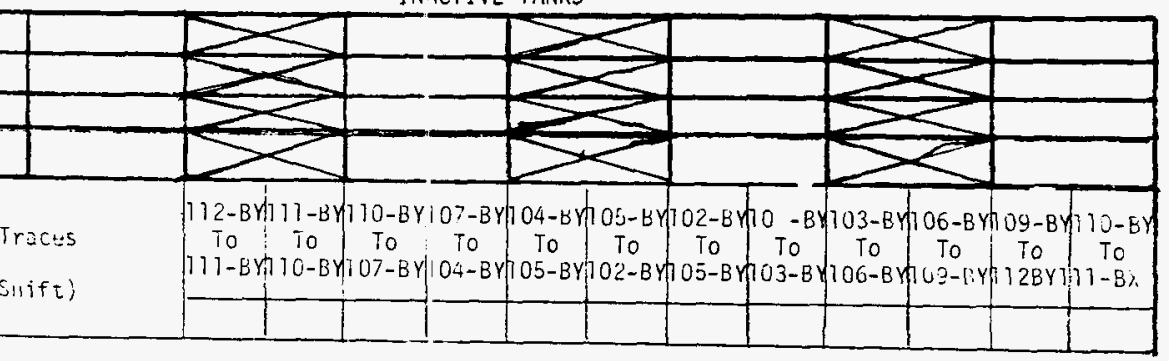

Heat Tracts

(Day suift) 


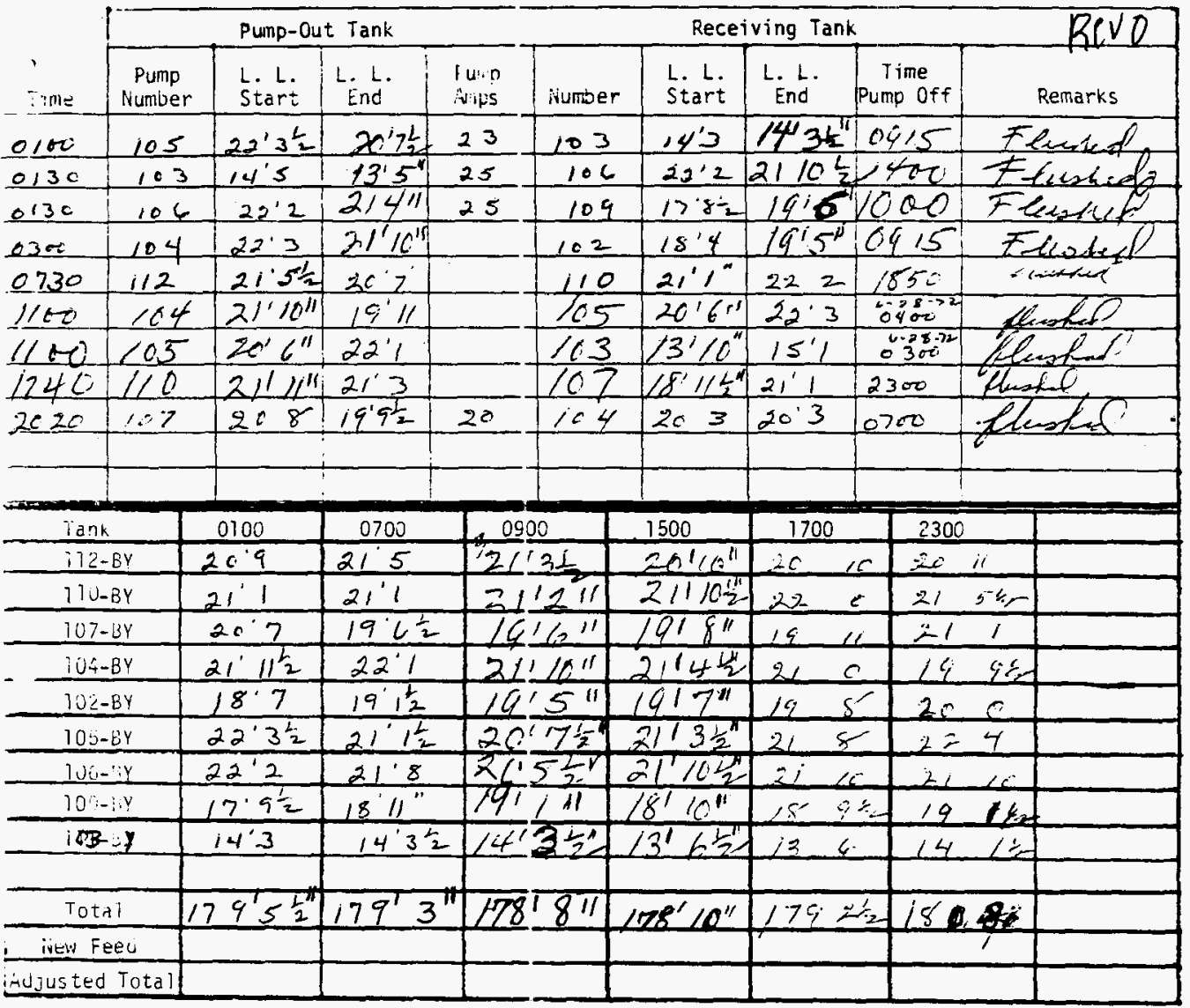

IT ACT IVE TANKS

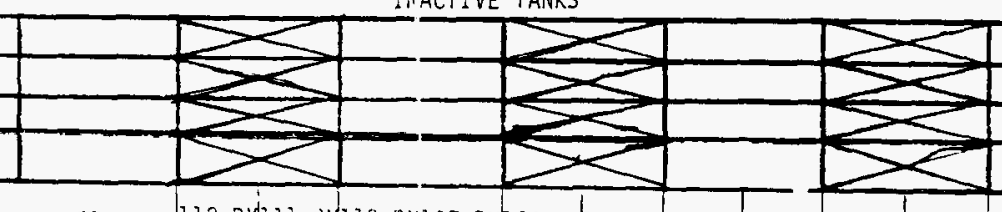

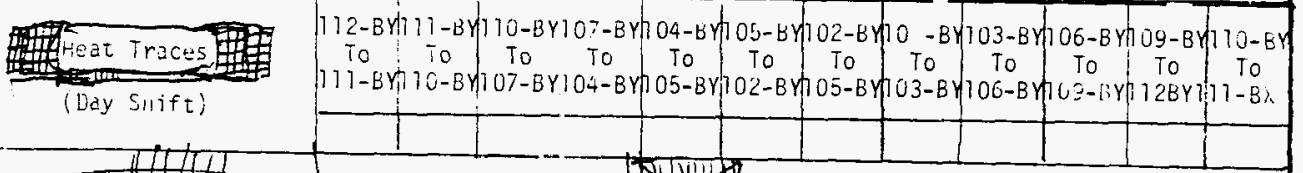

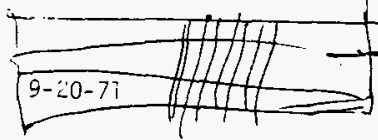




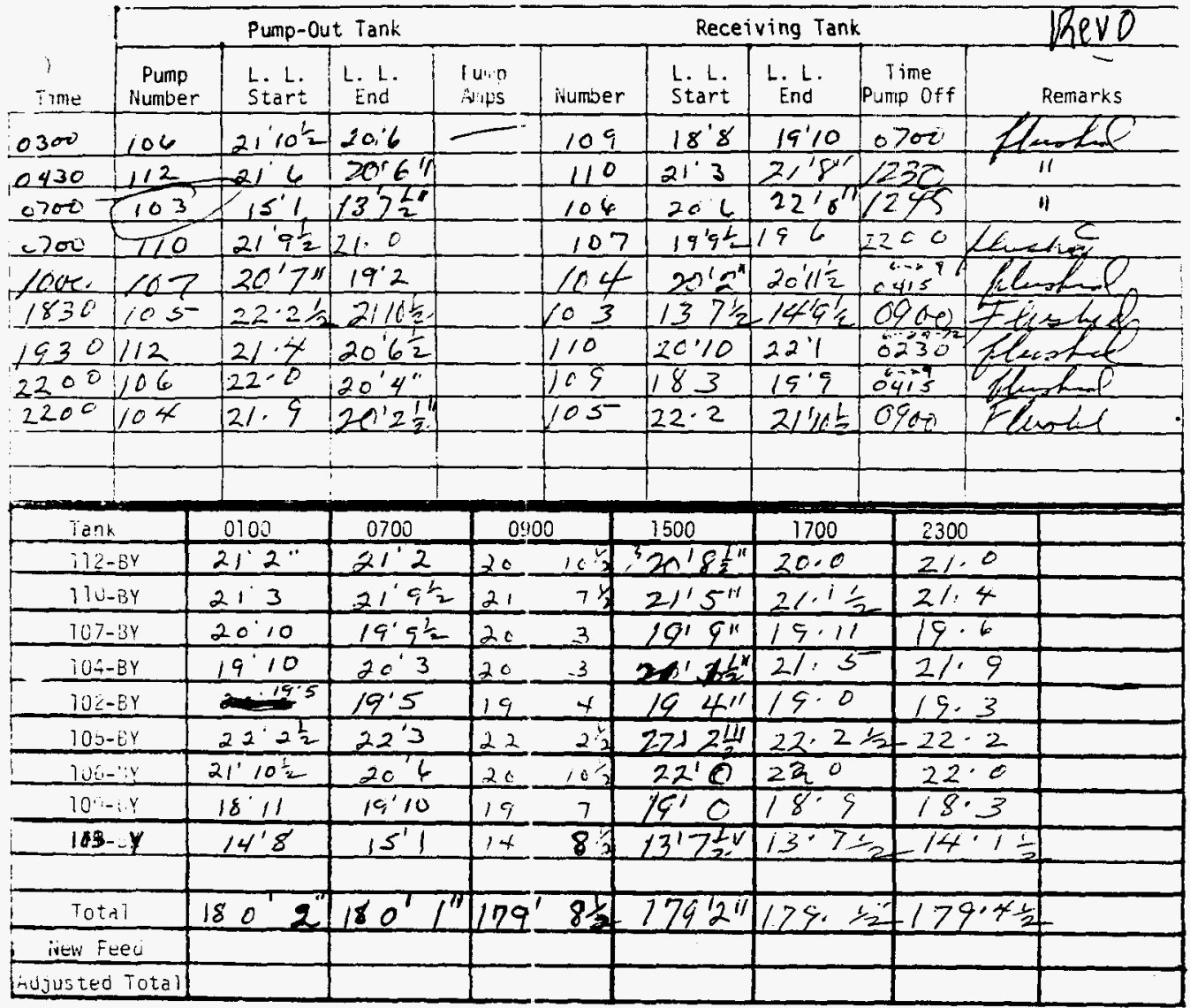

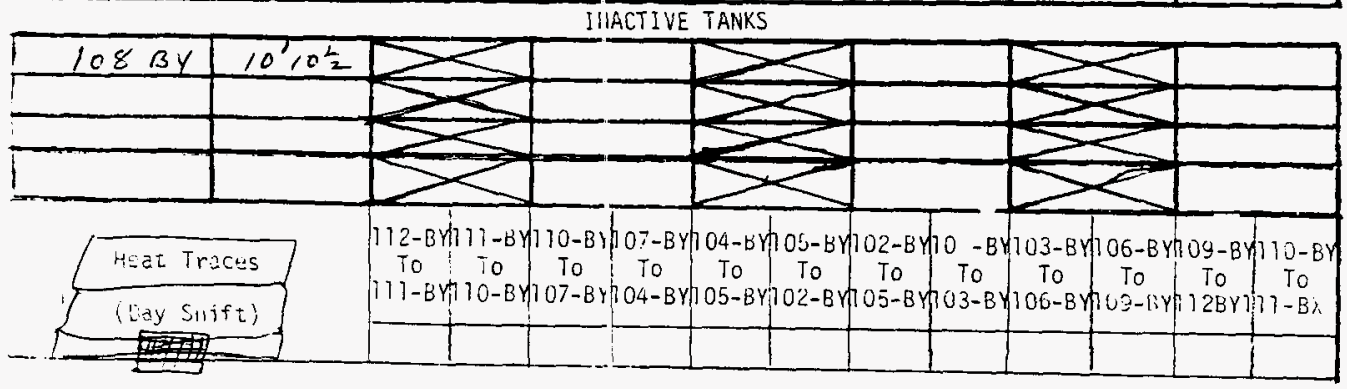

$-20-7 i$ 


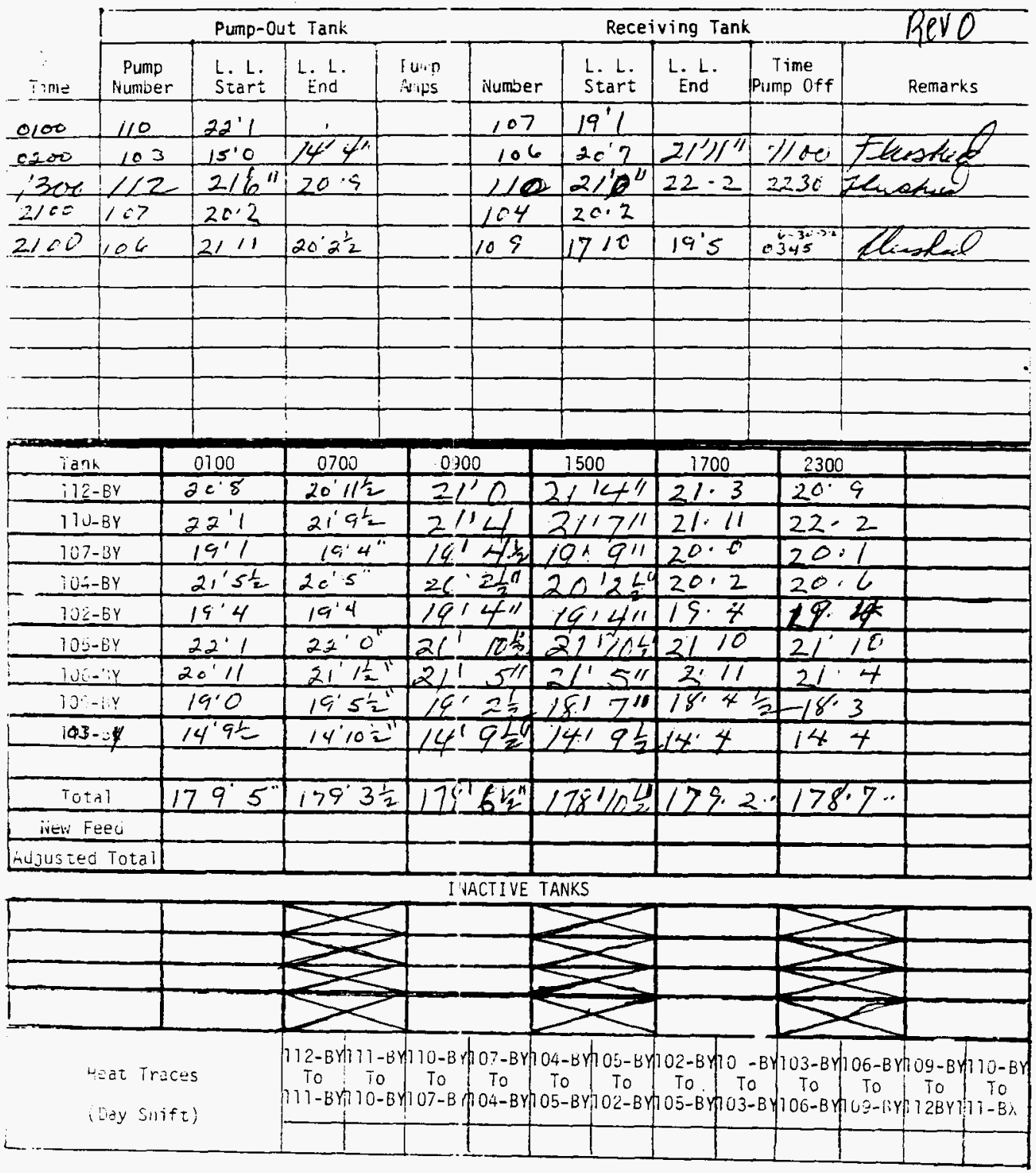


Date $:-30 \geq 2$

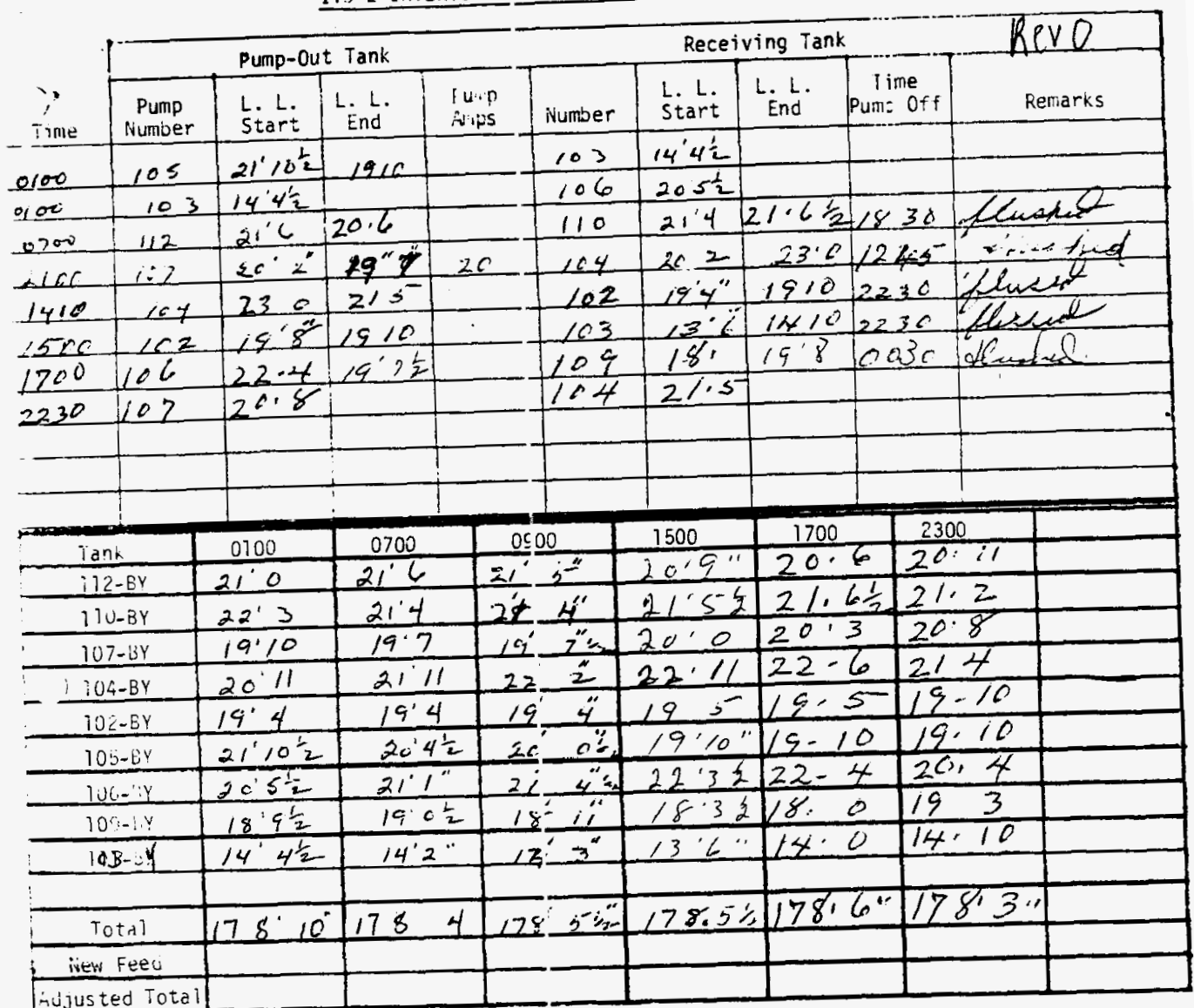

\section{IIIACTIVE TANKS}

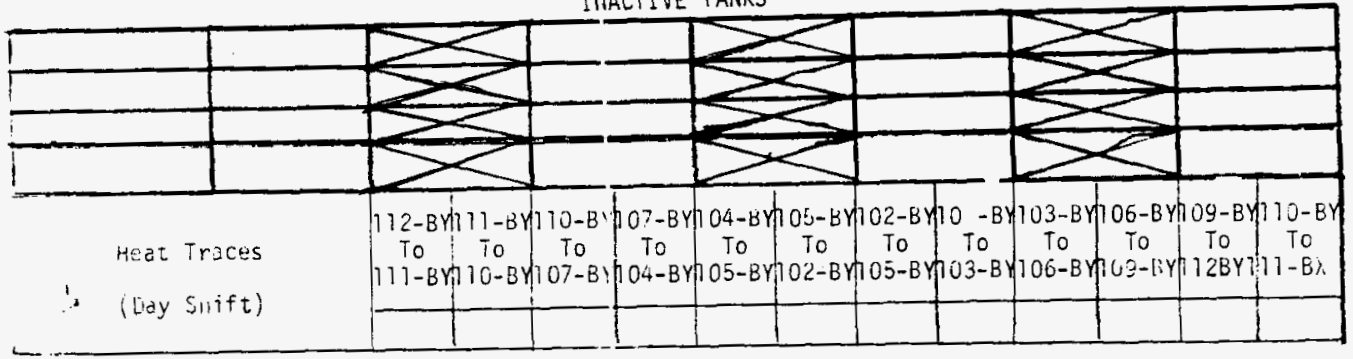




\section{DISTFIBUTION SHEET}

\begin{tabular}{|c|c|c|c|c|c|c|}
\hline To & \multirow{2}{*}{\multicolumn{4}{|c|}{$\begin{array}{l}\text { From } \\
\text { Remot } 2 \text { System and Sensor } \\
\text { Applizations }\end{array}$}} & \multicolumn{2}{|l|}{ Page 1 of 1} \\
\hline $\begin{array}{l}\text { Remote System and Sensor } \\
\text { Applications }\end{array}$ & & & & & \multicolumn{2}{|c|}{ Date $7 / 29 / 96$} \\
\hline \multirow{2}{*}{\multicolumn{5}{|c|}{$\begin{array}{l}\text { Project Title/Work Order } \\
\text { Sensor/Analytical Technology Projects }\end{array}$}} & \multirow{2}{*}{\multicolumn{2}{|c|}{$\begin{array}{l}\text { EDT No. } 617410 \\
\text { ECN No. }\end{array}$}} \\
\hline & & & & & & \\
\hline Name & & MSIN & $\begin{array}{l}\text { Text } \\
\text { With All } \\
\text { Attach. }\end{array}$ & Text Only & $\begin{array}{l}\text { Attach./ } \\
\text { Appendix } \\
\text { Only }\end{array}$ & $\begin{array}{c}\text { EDT/ECN } \\
\text { Only }\end{array}$ \\
\hline $\begin{array}{l}\text { H. Babad } \\
\text { W. B. Barton } \\
\text { C. H. Brevick } \\
\text { R. J. Cash } \\
\text { M. D. Crippen } \\
\text { D. R. Dickinson } \\
\text { D. W. Jeppson } \\
\text { N. W. Kirch } \\
\text { D. J. McCain } \\
\text { J. E. Meacham } \\
\text { F. R. Reich } \\
\text { D. A. Reynolds } \\
\text { Central Files (original +1) }\end{array}$ & & $\begin{array}{l}\text { S7-14 } \\
\text { R2-11 } \\
\text { S3-10 } \\
\text { S7-14 } \\
\text { L5-31 } \\
\text { L5-31 } \\
\text { L5-31 } \\
\text { R2-11 } \\
\text { R2-12 } \\
\text { S7-14 } \\
\text { L5-55 } \\
\text { R2-11 } \\
\text { A3- } 89\end{array}$ & $\begin{array}{l}x \\
x \\
x \\
x \\
x \\
x \\
x \\
x \\
x \\
x \\
x \\
x \\
x\end{array}$ & & & \\
\hline
\end{tabular}

